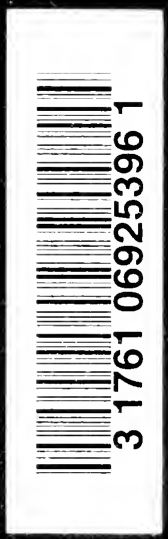





\title{
THE ART OF
}

\section{COUNTERPOINT}

\section{AND ITS APPLICATION AS A DECORATIVE PRINCIPLE}

\author{
BY \\ C. H. KITSON
}

M.A. (CANTAB.), D.MUS. (OXON.)

OXFORD

AT THE CLARENDON PRESS 
HENRY FROWDE, M.A.

PUBLISHER TO THE UNIVERSITY OF OXFORD

LONDON, EDINBURGH

NEW YORK AND TORONTO

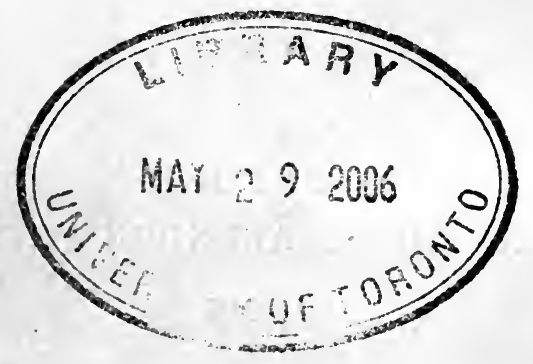




\title{
DEDICATED TO
}

\section{HUGH PERCY ALLEN}

\author{
M.A., D.MUS. OXON.
}




\section{Digitized by the Internet Archive in 2007 with funding from Microsoft Corporation}




\section{PREFACE}

THE appearance of a new book on Counterpoint, when there are already in existence numerous treatises on the subject, may seem to demand some justification. All books on Counterpoint must of necessity bear a certain resemblance to each other. Any difference must be sought in the point of view, in the relative prominence given to various phases of the subject, and in the treatment of detail, rather than in the statement of general truths or in novelty of arrangement. The history and the broad outlines of Counterpoint offer little scope for originality, and in these matters the present work will be found not unlike its predecessors.

The chief points of difference are :-

(a) The derivation of the rules of Scholastic Counterpoint from the practice of Palestrina, whose works exhibit the Art of Counterpoint in its perfection, rather than from a study of textbooks, the many discrepancies between which are due to the fact that theory has been built upon theory, often mingled with personal prejudice, so that the original basis of the earlier treatises has been forgotten or perhaps ignored. Little by little, harmonic resource has been restricted and melodic idiom enlarged. The result is a system of Counterpoint which has little connexion with the past and none with the present, containing a code of arbitrary rules which in many important features do not represent the technique of any period of musical art. To pretend that such a system has a certain disciplinary value 
implies a low view of the purpose of contrapuntal study. Moreover, a discipline which forbids the student to write what is correct both from a relative and an absolute point of view is illogical and useless. The true province of contrapuntal study is the attainment of the art of pure vocal part-writing. This art reached its zenith under the horizontal system of the Polyphonic Period, and it seems obvious that the best means of attaining it is to study the practice of the time.

(b) The prominence given to details commonly ignored or inadequately treated, such as the proper use of unessential notes, the importance of accent and rhythm, and the principles of criticism regarding consecutives. The difficulties which an intelligent student encounters are mostly matters of detail, and it is claimed that these have been more fully and consistently treated than in any book already in use.

(c) The treatment of Combined Counterpoint. Textbooks have usually little to say about this most interesting part of the subject, and the student who has mastered the rules of uncombined Counterpoint is expected to write combinations of the different Species without much more assistance. Experience shows that the special difficulties of Combined Counterpoint require ample explanation and illustration, and therefore these matters have been discussed at some length. An exhaustive analysis of the problems raised by the various possible combina. tions is well-nigh impossible, but sufficient has been said to enable the student to grasp the principles involved and to deal intelligently with any difficulties that are likely to arise.

(d) The application of Counterpoint to modern part-writing. It is hoped that the chapter on Counterpoint as a means of decoration will be useful to advanced students of Harmony. 
The following authorities have been consulted in the preparation of this book :-

Fux . . . . . Gradus ad Parnassum.

\begin{tabular}{|c|c|c|c|}
\hline Albrechtsberger & Counterpoint. & Pearce & Counterpoint \\
\hline Cherubini . & , & Prout & $"$ \\
\hline Ouseley . & " & Gladstone. & $"$ \\
\hline ekstro & , & Jadassohn & " \\
\hline lacfarren & , & Richter & ", \\
\hline
\end{tabular}

Bridge . . . "

Saunders . . Examples in Strict Counterpoint.

Grove . . . . Dictionary. Articles on Counterpoint (new edition),

Strict Counterpoint, Harmony, Musica Ficta,

Cadence, Hidden Consecutives, \&c.

Wooldridge . . The Oxford History of Music, vols. i and ii, The Polyphonic Period.

Parry . . . The Oxford'History of Music, vol. iii, The Music of the Seventeenth Century.

The Art of Music.

Prout . . . Musical Form.

The examples from Palestrina are usually given according to Messrs. Breitkopf and Härtel's edition ; occasionally the excerpts are transposed for convenience.

I have to thank the Syndics of the University Press, Cambridge, for their permission to print Canti Firm set at the Cambridge examinations for degrees in music, also Dr. Keeton for the use of a Cantus Firmus set by him at Durham University.

My especial thanks are due to Mr. G. G. Stocks, B.Mus. Oxon., of St. Edward's School, Oxford, for his invaluable help in revising the proof sheets, for many suggestions, for compiling an index, and for ungrudgingly devoting a great deal of his time to all sorts of matters connected with the publication of the volume.

C. H. KITSON. 


\section{CONTENTS}

CHAP.

PAGE

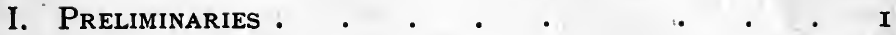

II. First Species in two Parts . . . . . . I4

III. Second Species in two Parts . • . . . 31

IV. Third Species in two Parts . . . . . 50

V. Fourth Species in two Parts . • • • . 76

VI. Fifth Species in two Parts. • . . . . 87

VII. Counterpoint in Triple Time . . . . . . IOZ

VIII. Counterpoint in three Parts . . . . . I Io

IX. The First and Second Species in one of three Parts ilg

X. The Third Species in one of three Parts • . 126

XI. The Fourth and Fifth Species in one of three PARTS . . . . . . . . . . . 130

XII. Combined Counterpoint in three Parts • • $\quad$ - 139

XIII. Counterpoint in four Parts - • • • • $~ I 92$

XIV. Counterpoint in five Parts. . . . . . $2 I_{4}$

XV. Counterpoint in six, seven, and eight Parts . 227

XVI. The Elementary Harmonic sub-structure of modern Contrapuntal Decoration . . . 250

XVII. The Application of Counterpoint as a Decorative

Principle . . . . . . . . . 290

XVIII. Counterpoint on a Chorale. • • • • 335

INDEX I $\quad \cdot \quad \cdot \quad \cdot \quad \cdot \quad \cdot \quad \cdot \quad \cdot 349$

INDEX II $\quad . \quad+\quad . \quad \cdot \quad \cdot \quad \cdot \quad \cdot \quad \cdot 35^{I}$ 


\section{CHAPTER I}

\section{PRELIMINARIES}

THE history of the art of Music falls into three main divisions :-

A. In the first period, represented chiefly by the music of the ancient Greeks, the expression of the emotions in terms of formulated sound, consisted of a succession of single utterances called Melody.

B. The next stage is that in which men began to experiment in the possibilities of combined sound. There were two lines of procedure :-

r. That of adding a part to a given part, called in Scholastic Counterpoint the Cantus Firmus, or Fixed Song.

2. That of forcing totally different melodies to be set one against the other.

The latter method was probably due to the fact that men found the task of adding three or more parts to a Cantus Firmus (C.F.), so as to produce euphonious combinations, so overwhelming, that out of sheer despair, or lack of the power of concentration, they selected a course which left such considerations to chance. Such a system, however, served the purpose of bringing before men's minds the effect of various harmonic intervals, and so helped them to form, in the process of time, a judicious selection of consonances.

It is true that among the Greeks there were signs of advance in the direction of combined sound. They perceived that the voices of men and children might be used to sing the same melody together at the distance of an octave; this they called 
Antiphony. But no progress could be made towards Polyphony, or the independent conception of parts, until men had found out other consonances. The Musica Enchiriadis of Odo, and the commentary upon it, called Scholia Enchiriadis, inaugurated this principle, as distinguished from the old Greek Antiphony, which was technically called Magadizing. It no doubt had its origin in the fact that when several men were singing the same melody together, some found it too high, others too low; and the distance of an octave usually caused difficulties at the other extreme. For reasons which need not be here detailed, the intervals of the fourth and fifth were the first to be selected as consonances-

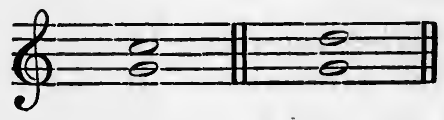

In the earliest attempts, men made their voices move in fourths or fifths in strict parallels. Sometimes, for technical reasons, the added part held the same note for a time, until it could proceed in parallel movement with the original part.

The fixed part was called the Principalis, and the added part the Organalis. This system was known as Diaphony or Organum. Odo's work belongs to the first half of the tenth century. The next work of which we know, dealing with the art of Diaphony, is the Micrologus of Guido d'Arezzo, written in the first half of the eleventh century. Here we see a freer sort of Diaphony, which admitted of sounds that had formerly been held as discordant; and also the use of oblique motion as a means of variety, as opposed to its adoption from technical reasons, in the older Diaphony.

Then, after the death of Guido (circa 1050) came a new Organum, in which the use of contrary motion and the interchange of concord with discord became characteristic features.

Finally, the constant use of combinations in which the Organalis was contrasted with the Principalis, not only in melodic curve, but also in time value, led to the formation of a system of musical standard of measure, called Discant or 
Cantus Mensurabilis. Discant in its turn merged into Counterpoint. During all these periods a process of elimination in reference to the selection of consonances resulted in the survival of the fittest, that is, those harmonic intervals which pleased the ear best. These were the third, fifth, sixth, and octave-

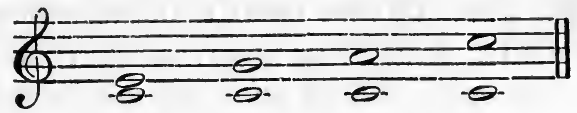

The fourth was discarded owing to experiments in three parts; this will be explained later.

The Polyphonic Period may be said to close with the death of Palestrina (I594). In him is seen the perfection of the method; and the technique of Counterpoint, as exhibited in his works, is the subject of this treatise. Certain modifications are of course necessary, in order to accommodate the system to modern conditions.

C. Now the work of Palestrina has, as one of its chief characteristics, simplicity of means. One of these was the frequent use of simultaneous movement in all the parts for extended periods. The following is the opening of his Stabat Mater :-

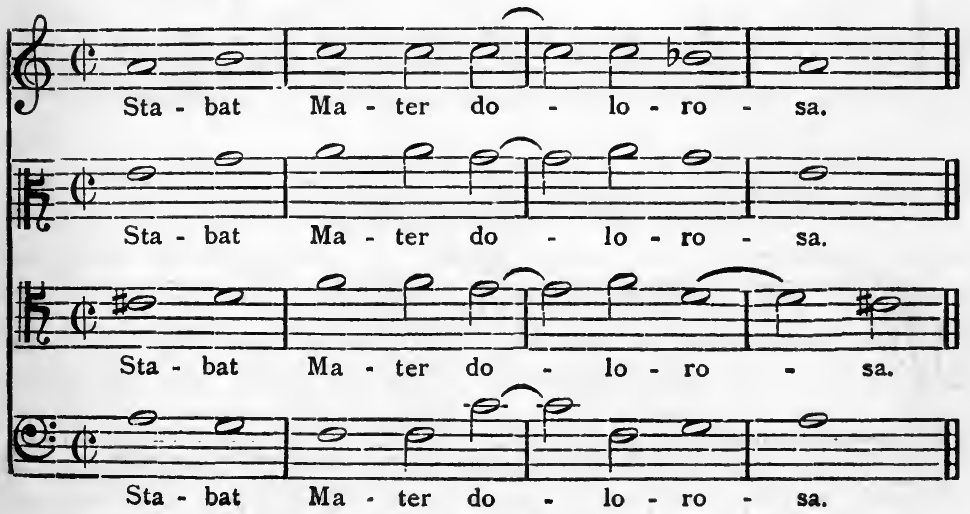

This brought before men's minds a greater possibility of beauty in combined melodies, by regarding the resultant sound 
vertically, that is, as blocks of harmony. And so the third or Harmonic Period was the natural evolution of the second. It may be said to begin with the dawn of the seventeenth century, and found its first culmination in J. S. Bach.

In the Polyphonic Period, the resultant harmony was the accident of the part writing. But in much of the work of Palestrina, the effect is very often that the whole is based on a simple harmonic structure; this was of course due to the awakening harmonic instinct, and natural judgement as to the fitness of things. Under modern conditions, the horizontal movement is entirely built up from a preconceived harmonic basis.

The following is an illustration :-

Fuga VIl.

Das Wohltemperirte Clavier.

J. S. BACH.

à 3 .

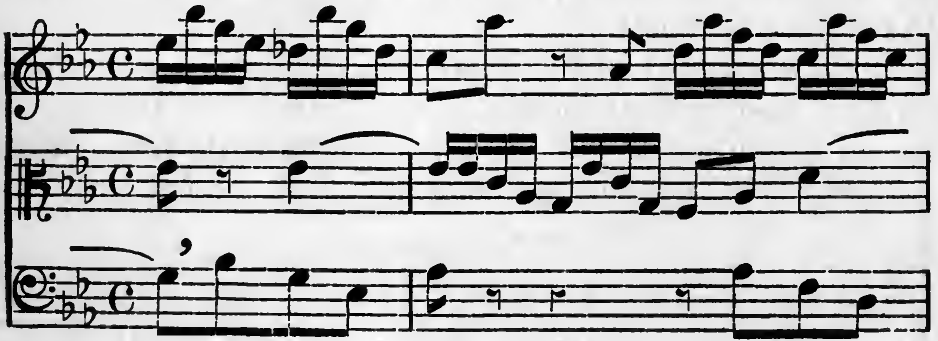

Harmonic Sub-structure.

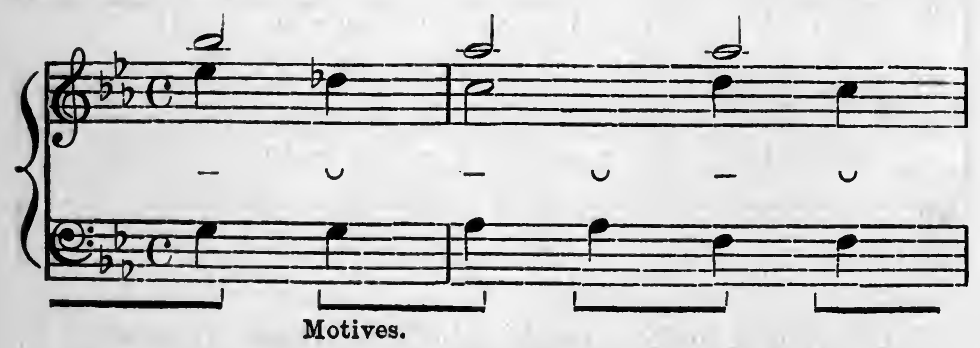



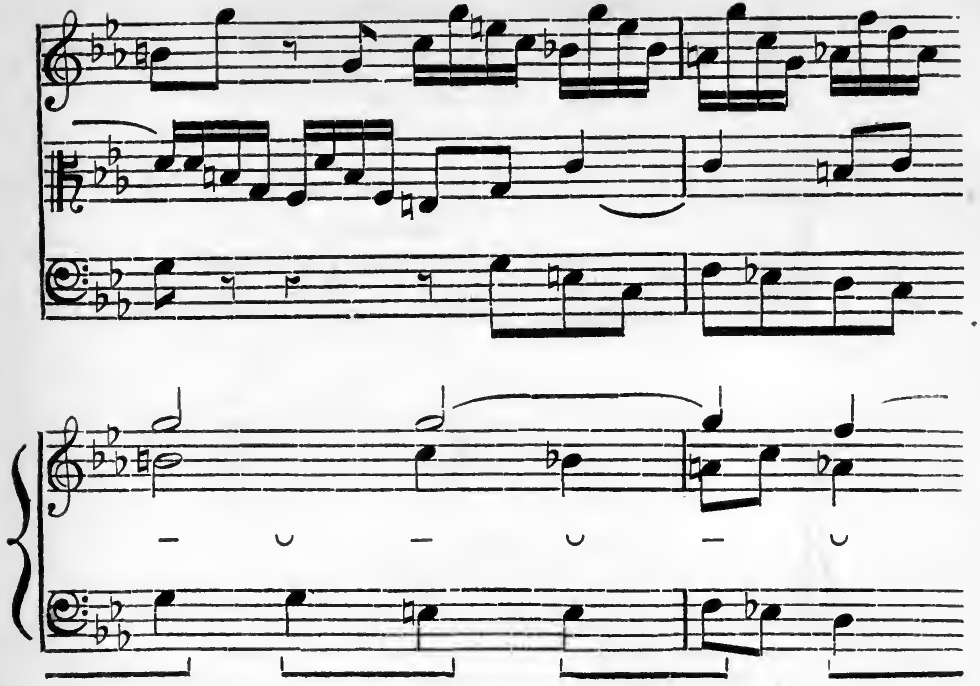

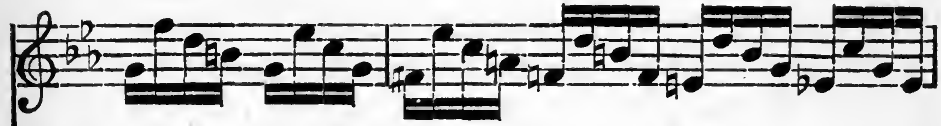

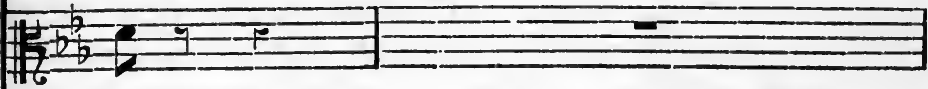
(e) $\frac{b}{b-b}=-2=0$

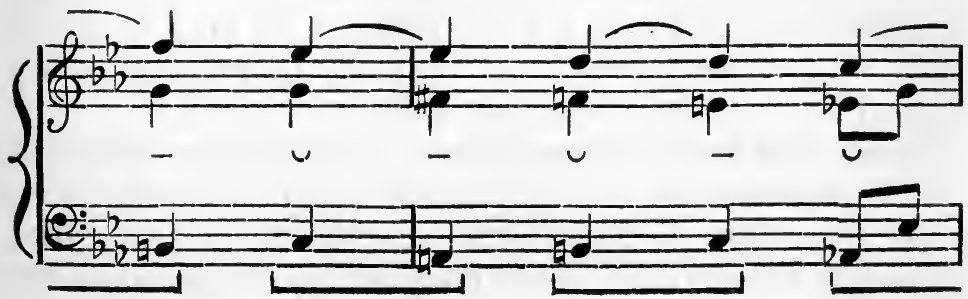



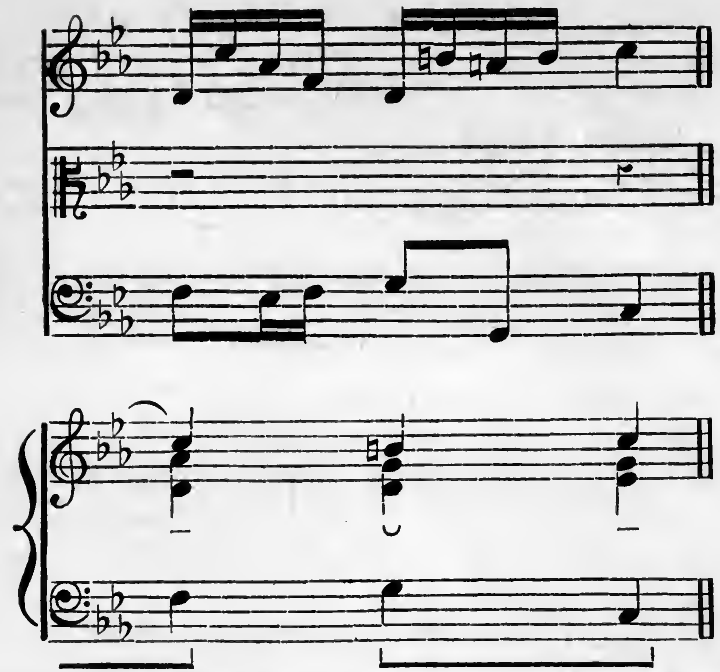

Each of these accents $u$ and - may be called a Chordal centre.

At first men were so much engaged with this vertical aspect of things, that they entirely ignored any questions dealing with the intrinsic merit of the individual parts.

The secularization of music, and the necessity of writing for instruments, tended at the outset to turn men's minds away from contrapuntal methods. They had to give their attention to the formation of melodies, in which rhythm was a characteristic feature ; and they had also to supply simple accompaniments to such melodies. Moreover polyphonic methods, which were entirely choral, were unsuited to a true instrumental style, and so the whole trend of things led to the enlargement of harmonic resource, and the formation of melody built upon such a substructure. All this progress was summed up in J. S. Bach, who adapted the principles of Harmony to Polyphony, and thus struck a just balance between the two.

It was only in the ordinary course of things, that when the art of Polyphony had come to maturity, theorists should devise a concise means of cataloguing its technique. The salient feature of Counterpoint is the independent conception of the 


\section{PRELIMINARIES}

parts, not only in melodic curve, but also in time value; so that it was only natural that Zacconi in his Prattica da Musica, published at Venice in 1596, should devise a means of considering complex movement under well defined heads. He used a C.F. consisting of notes of uniform length; to this a part had to be added in notes of the same time value, or in certain classified contrasted values, such as two notes to one, four to one, and so forth; these various ways of adding a part to a given C.F. are called Species or Orders, and Fux analysed the technique under five headings, called the Five Species of Counterpoint. This was not by any means an exhaustive analysis of the technique of the art; Zacconi wrote examples of Species which have not survived. But the classification as settled by Fux has been maintained to the present day, and it is adequate for its purpose.

There are then three chief questions in reference to this technique :-

I. What are the various sorts of complex movement arising from the independent conception of the parts?

2. What are the rules of vertical combination under these separate conditions?

3. What considerations regulate the melodic idiom of the parts?

It will thus be seen that Scholastic Counterpoint is a study of the technique of Polyphony under classified heads, just as Harmony is the study of the grammar of modern composition. And it will be shown how the latter was the natural evolution of the former, and how our rules for harmonic progression are framed on what the writers of the Polyphonic Period found out by experiment, or judged by natural instinct, to be the best ; and as we learn the principles of Counterpoint from the practice of the time, we shall see unfolded by degrees practically the whole of the diatonic fundamentals of modern harmony.

It is presupposed that the student has a knowledge of the elements of Harmony, including the use of fundamental discords, and the principles of modulation to nearly related keys. It is therefore unnecessary to define such terms as Perfect Inter- 
val, Tonic, Key, and so forth. Opinion is divided as to the order in which Harmony and Counterpoint should be taught. As the former was the result of the latter, the proper course would seem obvious: But there are grave reasons against a chronological study of Counterpoint and Harmony. The system of the ecclesiastical modes, under which the music of this Polyphonic Period was written, has of course given place to the use of the modern major and minor modes. The modern system demands a treatment of unessential notes quite different from that which obtained under the old régime. A great deal of the crudity of students' work is due to the fact that they use these unessential notes mechanically; this becomes such a habit of mind, that its pernicious influence on practical composition is often very difficult to eradicate.

The following diagram will give an idea of what is meant. The scale of $\mathrm{C}$ major is written horizontally and vertically; the latter forms the dominant thirteenth of the key of $\mathrm{C}$ major, that is, the complete series of diatonic dominant discords-

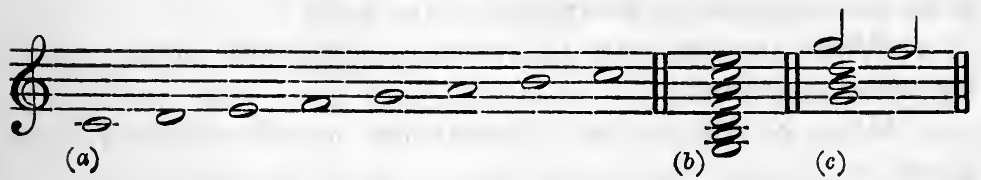

In $(c)$ the second minim is not merely an unessential note; it is felt to be the seventh of the dominant of $C$, and it must be treated as such.

The student will also be much better able to understand the treatment of prepared discords, and also their historic position, if he first grasp their modern classification.

The following is recommended as the course of study :-

(a) Harmony, up to the chord of the dominant seventh and simple modulation.

(b) Counterpoint--First to Third Species. Two parts.

(c) Harmony-Dominant ninth, eleventh, and thirteenth, Suspensions.

(d) Counterpoint. Fourth and Fifth Species. Two parts.

(e) The two subjects may then be studied concurrently. 
The student must work from the point of view of the sixteenth century; at the same time it is advisable that he allow his knowledge of harmonic conditions to influence his choice of progressions. Clear, smooth harmony, paradoxical though it may seem, is the foundation of good contrapuntal effect; and those progressions which the writers of the Polyphonic Period felt by instinct to be the best, we may also perceive and utilize by our experience of their adoption and classification in modern harmony. It is important to grasp the fact that Palestrina knew nothing of chords as we view them; his technique was not that of writing two, three, or four notes to one as representing one harmony, but two, three or four notes to one note, and their movements were limited by questions of consonance and dissonance, which have to be discussed.

A great diversity of opinion exists as to the use of modulation in strict Counterpoint. There is no inherent harm in it, of course ; but some modern theorists do not recommend it, as they consider Counterpoint a means of learning the diatonic resources of one key. This however is hardly its province, and the use of modulation has the support of the majority of theorists. The matter is one which requires the exercise of cautious discretion, especially when writing in the minor mode, and it will be discussed fully as occasion demands. No one has a right either to forbid or to impose modulation as essential ; of course, under the conditions of Musica Ficta (see Art. in Grove on Musica Ficta) by the use of cadences in related modes, men found out a principle of contrast, which gives to modern ears the impression of a modulation. Such an idea, however, was quite alien to the modal system. Artistic sense should help the student to decide for himself whether a modulation is desirable or not. The introduction of an occasional accidental will often enable him to make a progression smooth in effect, whereas a prohibition of such a use would cause the harmony to be crude.

Some theorists say that the harmonic resources of Counterpoint are restricted to the use of common chords and their first inversions, chiefly that the student may devote as much attention as possible to the melodic interest of his parts. But the use of 
the terminology of Harmony in reference to these essential combinations, without an adequate explanation of its real significance, puts the student at once in the wrong attitude of mind, and makes it possible for him to confuse contrapuntal with harmonic analysis.

A modern would analyse the following passage according to the figuring :-

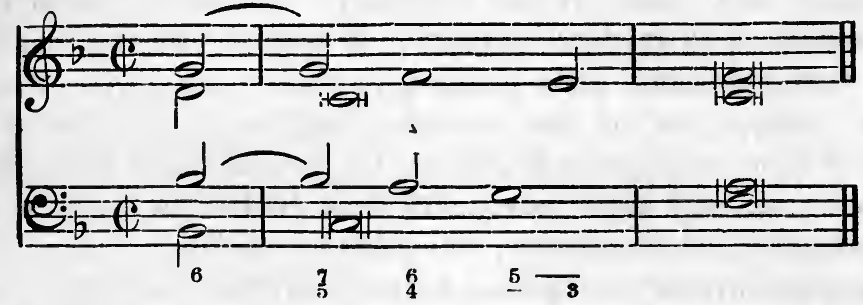

This is an extract from Palestrina's Mass, Aeterna Christ Munera. The bass (C) is a point d'orgue (pedal point). In the tenor the seventh (B flat) after preparation resolves on to a concord (A). The fifth in the treble moves with it, forming a sixth, the tenor being regarded as the real bass.

A student trained on historic principles will never in practical work use six-fours and essential discords crudely, because he has in this technique the origin of all our rules for the treatment of six-fours and fundamental discords.

But the student may ask by what process was the use of the common chord and its first inversion, as we moderns term the combinations, settled?

We have shown that experiments in combined sound must have led to the formation of laws, and classification of intervals. We have also shown that when only two parts were tried, the third, fourth, fifth, sixth and octave all ranked as concords. But when three parts were sung, it was found that the fourth would not combine with the fifth, the most satisfactory interval except the octave :-

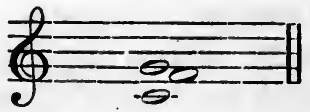




\section{PRELIMINARIES}

The fourth is first classified as a discord in a work by Marchetto of Padua (thirteenth century). He explains that the part which offends the ear by the use of this discord must make amends by passing to a concord, while the other parts stand still.

Theoretically, mediaeval musicians were guided by the 'ratios of the vibrational numbers of the limiting sounds', and the fourth came next to the fifth in excellence. The following table quoted from Dr. Davies' article on Counterpoint in Grove, shows how it comes to be said that in strict Counterpoint only common chords and their first inversions are available-

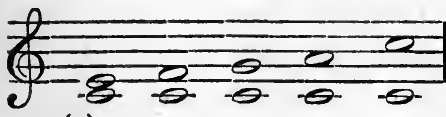

(a)

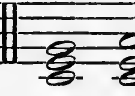

(b)

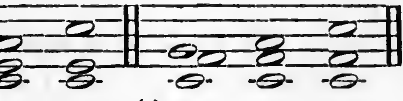

(c)

(a) All the available concords. (b) Combinations which ultimately formed the foundation for the art of Counterpoint. (c) The fourth displays the reason for its rejection in failing to do what the third does so well.

In a piece of four-part Counterpoint by Dufay, the earliest example of its kind extant ( 1360$)$, there is a frequent use of what we term the common chord and its first inversion. As time went on, though music was written as a combination of individual parts, the harmonic result of which was a matter of secondary importance, the greatest composers were feeling towards pure harmonic effect. We have pointed out that in Palestrina this is a marked feature. The effect of some of his music is that it is as clearly founded on a simple succession of chords as any modern music. His success lay in his recognition of such principles; and he stands on the highest point to which art considered horizontally could rise.

All modern music, like modern poetry, is formed on principles ' requiring regularity of accent and system in cadence' (Prout, Musical Form, p. 7).

The most complicated modern musical texture can be reduced to two forms of progression :-

I. The Iambus. Duple Time $\checkmark-$.

2. The Anapaest. Triple time $v \cup-$. 
(I)

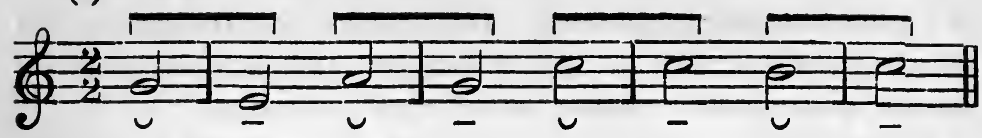

(2)

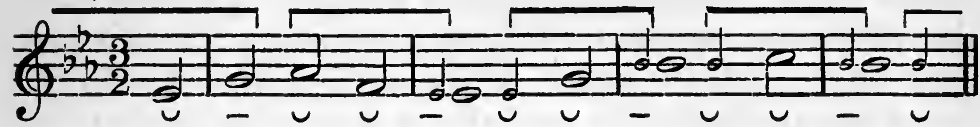

In duple time, should the first note be accented it forms the second half of an incomplete foot or motive. And in triple time, should the first note occur on a strong accent, or should the first two notes occur on a weak accent followed by a strong one, the motive is incomplete. As in literature, so in music, it is necessary to have some means of punctuation, so as to make one's thoughts intelligible. This end is gained by the idiomatic use, at the end of a phrase or sentence, of two harmonies, in the relation of weak to strong, called the cadence. During the period that men paid the greatest attention to the melodic side of the art, they regarded the progression of the last note but one of the Principalis one step downward to the Final as the salient feature of the Perfect Cadence or Clausula Vera.

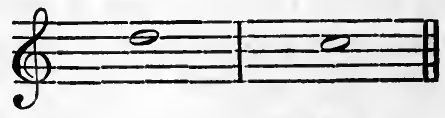

This was accompanied as follows:-

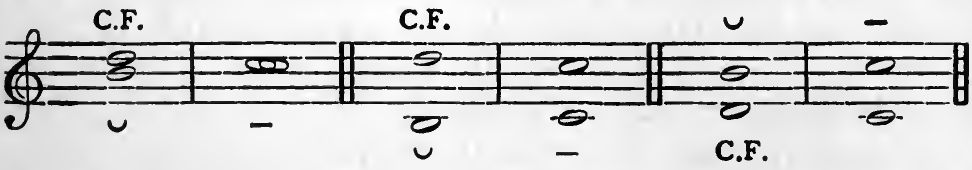

Now the rhythmic principle of all Scholastic Counterpoint is that of duple or triple time; and it depends partly on the nature of the Counterpoint, partly on the way in which we can group the harmony, as to whether we regard the grouping of feet as divisible into bars or parts of bars. The distinction will be drawn as occasion demands. It is of the highest importance that 
the sense of rhythm in chordal progression should never be lost, and a due appreciation of this will greatly help, both in choice of harmony and melodic curve. It is of course true that all the music of the Polyphonic Period was unrhythmic, but the use in Scholastic Counterpoint of a C.F. in notes of uniform length serves as the link between the old art and the new; that is, it enables men not only to study the methods of the sixteenth century, so far as technique is concerned, but also to use them as a training in the decoration of a preconceived harmonic structure. 


\section{CHAPTER II \\ COUNTERPOINT IN TWO PARTS}

First Species.

THE first way of adding a part to a C.F. is to write it in notes of the same time value, so that the two parts move simultaneously. The C.F. may be either the higher or the lower part. Formerly it was always in the bass, later it was put in the tenor, and eventually it found its way to the top ; but there is now no restriction as to its position.

As Scholastic Counterpoint is concerned with the art of vocal part writing, certain limitations in reference to melodic progression are obviously essential.

I. The governing principle of melody, in all Species, is that of conjunct movement, judiciously varied by the use of disjunct intervals. An excessive use of either is inartistic.

2. A part should not proceed by any augmented or diminished interval. Modern theorists allow the leap of a diminished fifth or fourth, if the melody return immediately to some note within such interval

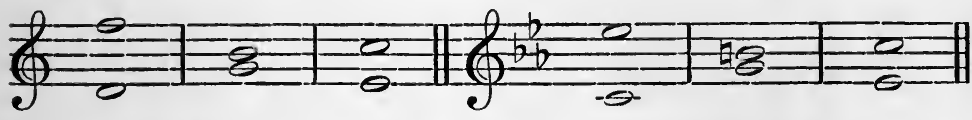

Though the use of such intervals is contrary to the practice of the sixteenth century, most modern theorists allow their occasional employment.

3. A part should not proceed by the interval of a seventh, nor by any compound interval, either directly or with one note between- 


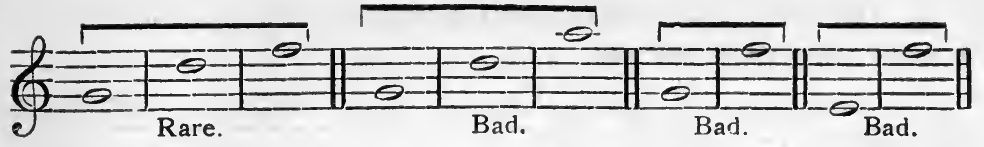

The following passage from the Kyrie of Palestrina's Mass, Iste Confessor, is worthy of consideration :-

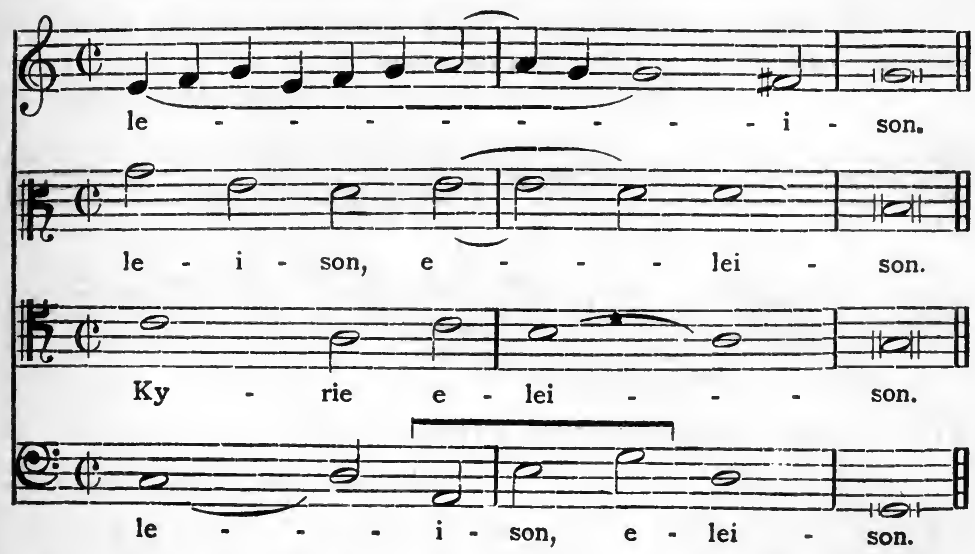

Here in the bass we have the leap of a seventh, with one note between : but the phrasing makes it entirely unobjectionable-

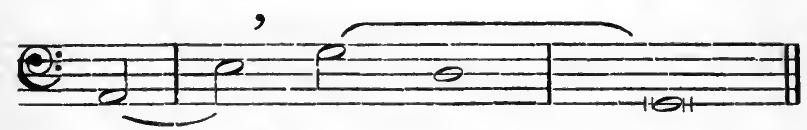

Again, in his Stabat Mater, Palestrina writes:-

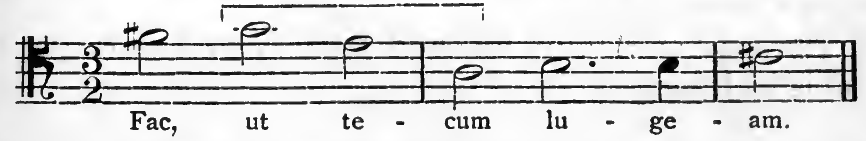

If such a melodic progression be used, care should be taken that the leap of the seventh is followed by some note within the interval. The following is unjustifiable :-

Fux.

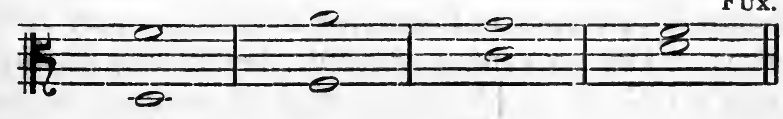


4. The leap of an octave should be preceded and followed by notes within that interval.

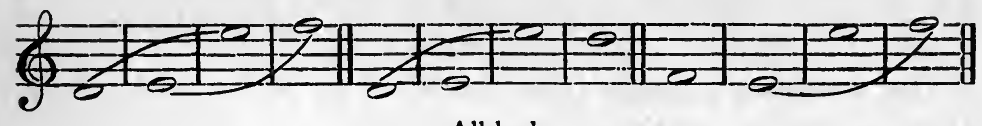

All bad.

The following is interesting as being analogous to the previous example quoted from the Iste Confessor:-

Kyrie, Iste Confessor.

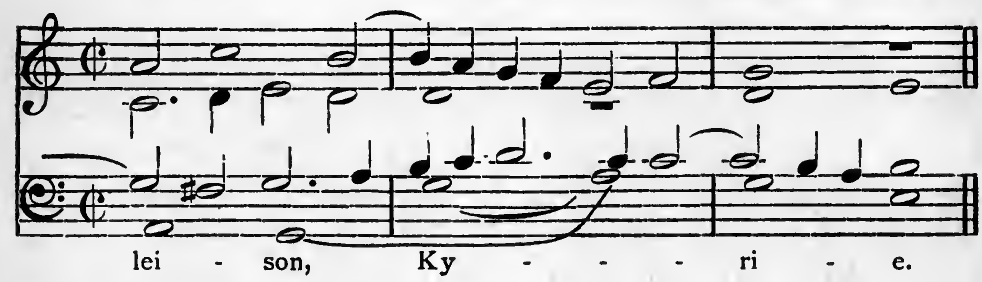

The example quoted below is a rare exception-

Palestrina. Magnificat, Tone vii.

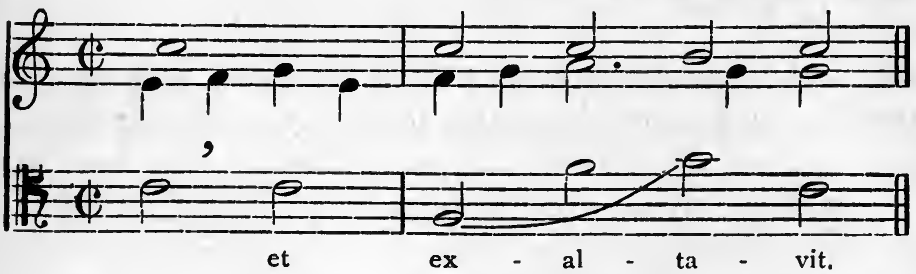

5. The leap of a major sixth should be avoided in First Species Counterpoint.

6. The leap of a third followed by that of a sixth (or vice versa) is inelegant-

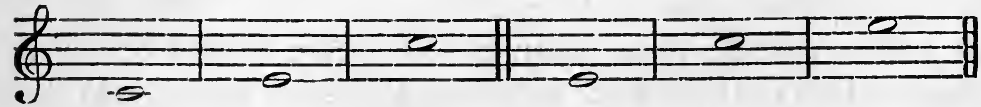

Professor Wooldridge's remarks on the principles of melodic progression in reference to Palestrina's technique may be quoted in extenso: 'The governing principle, technically speaking, of Palestrina's melody is of course that of conjunct movement; 
this, however, is beautifully varied by the constantly changing value of the notes, and also by occasional disjunct intervals, which are permitted upon the condition of not continuing in the direction of the leap, but immediately returning by gradual motion towards the point of departure.

' This rule may also, of course, be deduced from the methods of Palestrina's predecessors since I450, but there is in his application of it a certain final elegance, representing the ideal in such matters, which had been aimed at generally hitherto, but was now for the first time attained.

'Yet though Palestrina's method finally settles the questions respecting conjunct movement, and the general beauty and expressive qualities of the contrapuntal melody, exceptions to his rules may be found even in his own work, and especially as regards the continuance of the melody in the direction of a leap, a movement which sometimes, and most frequently in the bass or lowest part, is so to speak thrust upon him, from its occurrence in the given subject or from some other necessity. Palestrina's conduct in such circumstances is often interesting, and remarkably so in the Kyrie of the Mass Aeterna Christi Munera, where the leap first occurs in the tenor or subject: here he seems, by frequent allusion, forcibly to draw our attention to the unusual figure, also showing (at A) how it might have been brought within the rule, and afterwards accepting it and (at B) repeating it again and again, in the bass, until we are quite reconciled to it.' (Oxford History of Music, vol. ii, p. 376.)

(I)

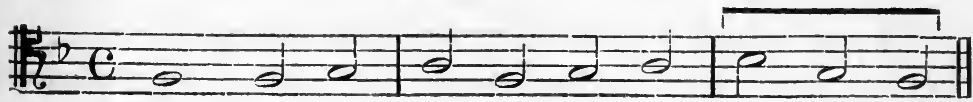

(2)
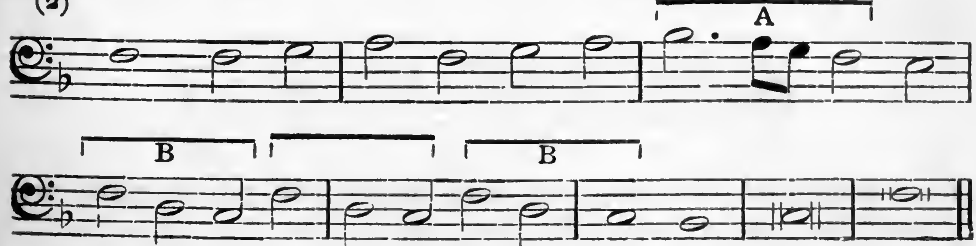
7. The leading note may never leap an octave.

8. More than two leaps in the same direction are inelegant, unless forming the arpeggio of a chord limited by the extreme interval of the octave.

9. Melodic monotony, whether arising from repetition of formula, or want of variety of range, should be carcfully avoided-

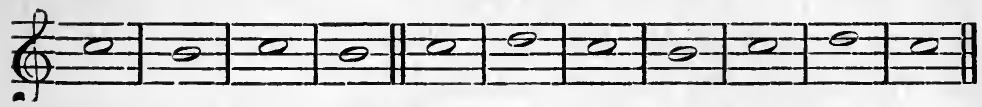

ro. The repetition of a note in the bass (at the same pitch) is liable to cause a halting effect; the leap of an octave is of course entirely free from this objection-

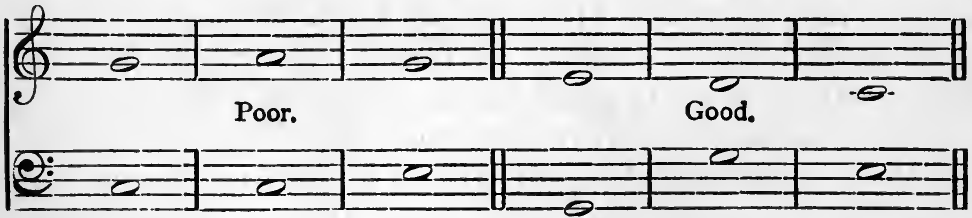

If, however, the repetition occurs between bars forming the end of one phrase and the beginning of another, there is no valid objection-

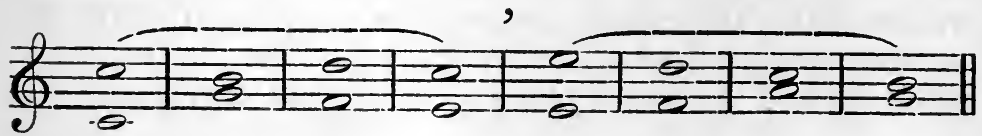

The following are the principles of Harmonic Progression :-

I. It has been indicated that experiments led men to catalogue harmonic intervals as concordant and discordant in the following way: the octave (or unison) and fifth are termed Perfect Concords-

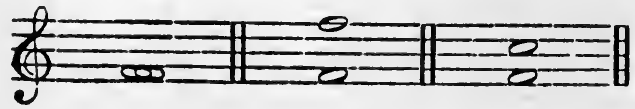

The major and minor third, and major and minor sixth are termed Imperfect Concords - 


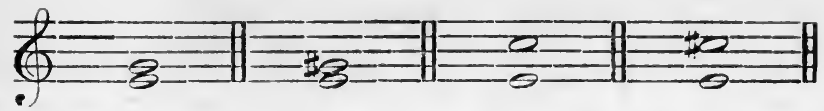

The second, fourth, seventh and their compounds are discords-

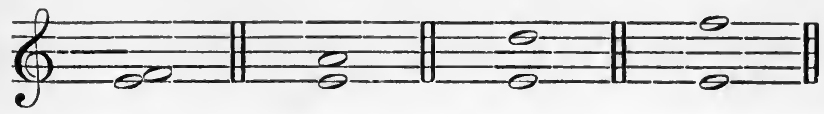

In two-part Counterpoint in the First Species only concords are allowed. Now any idea of a chord, as such, is entirely alien to the principles of the Polyphonic Period. At the same time, it is impossible for a combination of two sounds not to imply to modern minds some harmonic substructure, and it would be unwise to ignore the light which the modern system has thrown on such matters. It has been shown that in three parts the interval of a perfect fourth might occur between two upper parts. The same is true of the diminished fifth; but the diminished fourth is not permissible as an essential harmony under any circumstances-

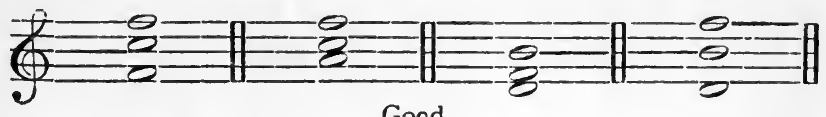

Gocd.

In all cases the lowest part must be concordant with each of the others.

Now let us experiment with the concords available, by adding a third part and using a separate sound, that is, not duplicating either of the other two.

First, the major scale will be taken-

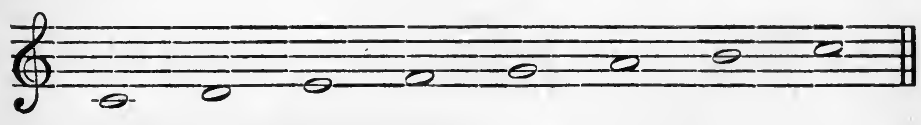

I.

Third. Fifth. Sixth. Octave.

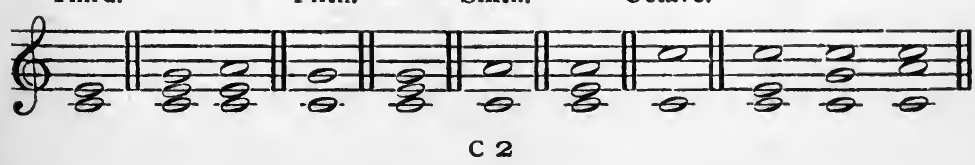


II. Third. Fifth. Sixth. Octave.

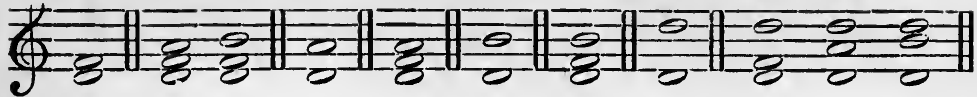

III.

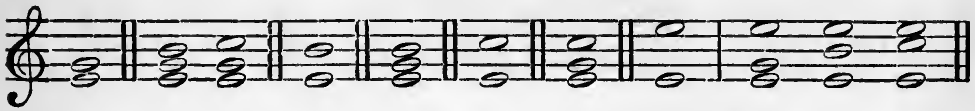

IV.

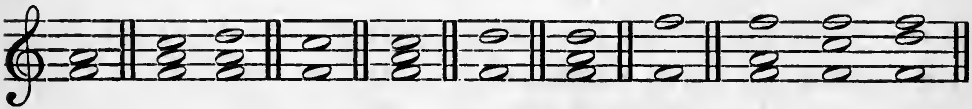

v.

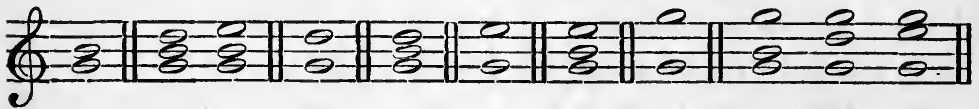

VI.

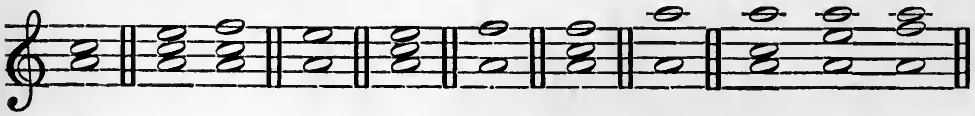

VII.

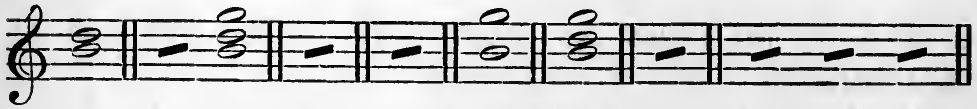

The result of this may be stated as follows :-

The Roman numerals will be used to indicate the seven triads :

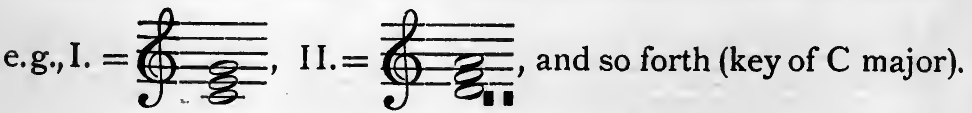
' $b$ ' written after a numeral indicates what we term the first inversion of the triad, e.g. I b. = So that it is true to say that the only harmonies that can be derived from the 
major scale are the diatonic triads with their first inversions, with the exception of VII. for the highest and lowest sounds of this combination form a diminished fifth, i. e. a discord.

Next take the minor scale.

Of these there are three forms :-

A. The Aeolian Mode.

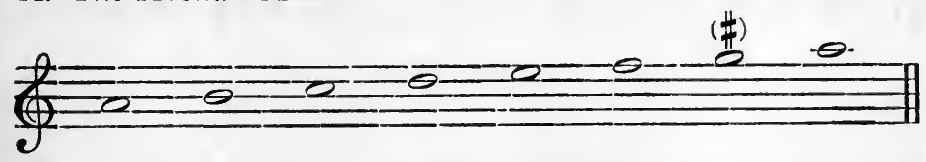

The early contrapuntists derive their harmony from this, raising the seventh in the cadence, by the principles of Musica Ficta.

Applying the same principles of combination as seen in deriving harmonies from the major scale, that is, the Ionian mode, it will at once be seen that among others we get :-

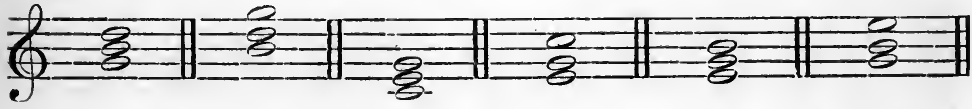

that is, from the modern point of view, they are harmonies proper to the relative major, and not to the minor mode. Any fusion of the two systems, with one or two exceptions, is impossible, and we should write either from the one standpoint or the other.

B. The Harmonic Minor Scale.

If we compare any major scale with its relative harmonic minor-

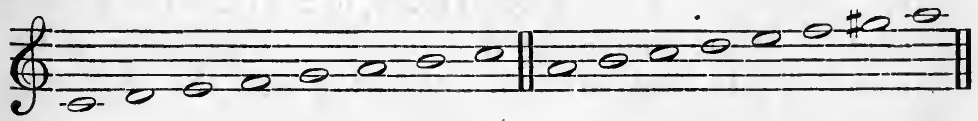

we shall see that the one point which differentiates the minor from the major mode, so far as the formation of harmonies is concerned, is the chromatic alteration of the seventh of the former, raising it a semitone. 
Now let us add a part to three C.F.'s according to the old system-

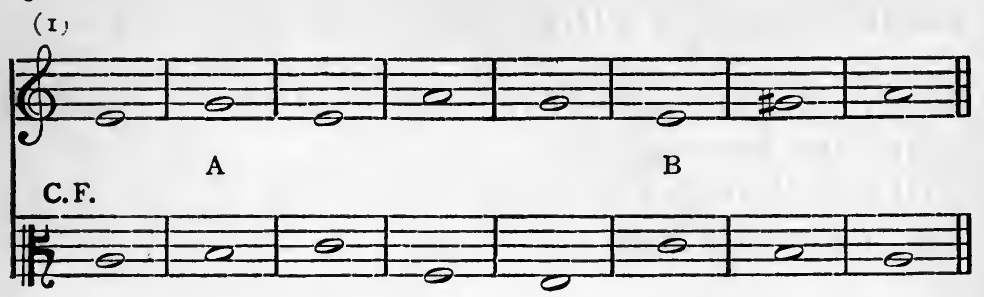

(2)

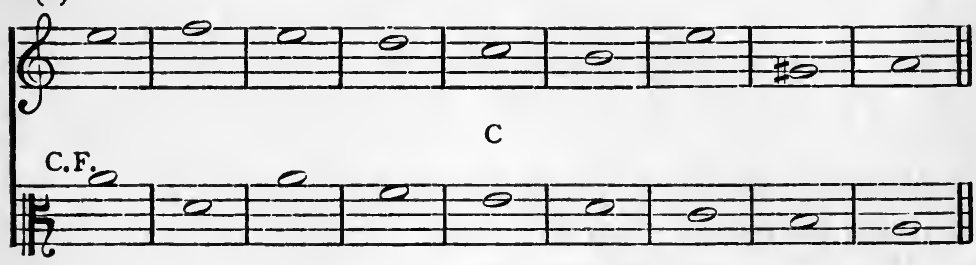

(3)

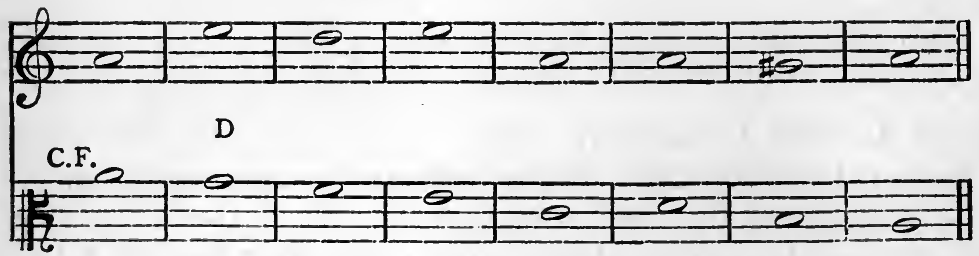

In (I) the mental effect from a modern standpoint is that from $A$ to $B$ we are in the key of the relative major.

Any such treatment therefore, from the standpoint of our tonal system is out of place, for the minor key is a very different thing from its parent, the Aeolian mode, in the matter of derived harmony. At the same time, it is possible to use some of the harmony of this mode without interfering with the characteristics of modern tonality.

In (2) is seen a case in point (C). Here there is no effect of confused or changed tonality. 
and under modern conditions it has been catalogued as a chromatic triad, that is, it can be used without necessarily producing modulation.

In (3) $D$ is another case of an implied chord which has been catalogued in modern times as chromatic.

When the bass moves down by step from the tonic to the submediant, the minor seventh may bear a first inversion. See (3).

Modern theorists say that in any other case the minor seventh should be regarded as the fifth of the relative major, and a modulation be made to that key-

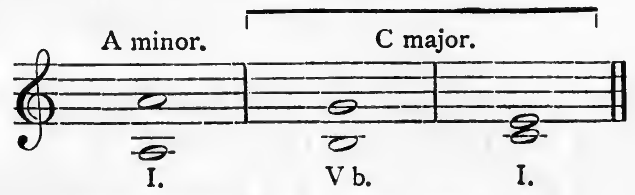

But if (3) be allowed, it is hard to condemn :-

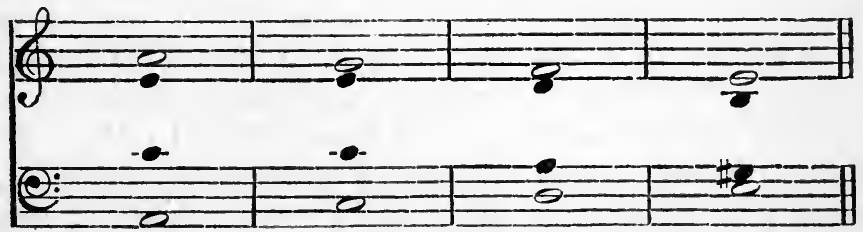

for again there is no impression of modulation. This is merely a harmonization of the descending form of the melodic minor scale.

Were it said, that if the minor seventh be approached or quitted by leap it should be regarded as the fifth of the relative major, the limitation ought to satisfy any fair-minded critic.

In deducing harmonies from the harmonic minor scale it will be found that II. III. III[b. and VII. are unavailable-

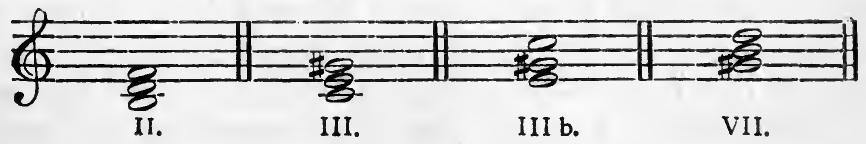


The harmonic intervals of the diminished fourth and augmented fifth are never permissible as essential harmonies.

C. The Melodic Minor Scale.

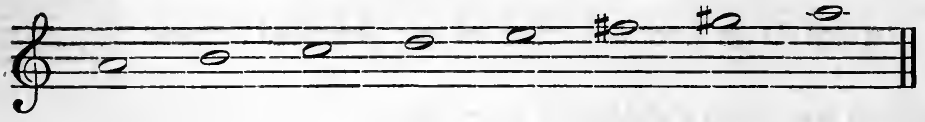

This will produce among others the following combinations :-

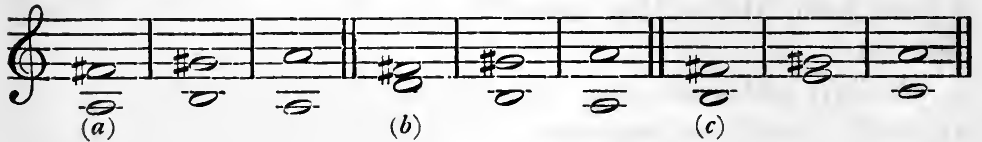

(b) and (c) would be regarded by a modern as the implied harmonization of the Dorian sixth, by the use of triads on the supertonic and subdominant.

As regards $(a)$ it is unnecessary to insist on modern analysis ; it is a concord, and the effect is quite good.

It should be pointed out that it is unwise to maintain a slavish adherence to modern harmonic analysis of chordal resource. At the time of Palestrina the process which led to our modern system was not in a state of classification; the use therefore of harmonies, which, besides being perfectly legitimate from the old standpoint, do not obliterate the characteristics of the present tonal system, will not be condemned by broad-minded critics.

It may be thought by some that a consideration of implied harmony is out of place in two-part Counterpoint. Nevertheless it is quite possible to write technically correct Counterpoint, and yet obtain very crude effects : this is largely due to a mechanical use of concords, without reference to the implied harmonic substructure, which should always be in the minds of present day students. The cases mentioned above, however, are illustrations of the legitimate application of original principles.

2. Except in the two opening bars it is unwise to have the same harmony for a weak accent followed by a strong one. Although such a rhythmic principle does not come within the scope of the Polyphonic Period, it cannot be said that such a recommendation is out of place. It should be our aim to 
make contrapuntal study as useful as possible as a preparation for Free Counterpoint.

3. Consecutive octaves and fifths are forbidden, whether by similar or contrary motion-

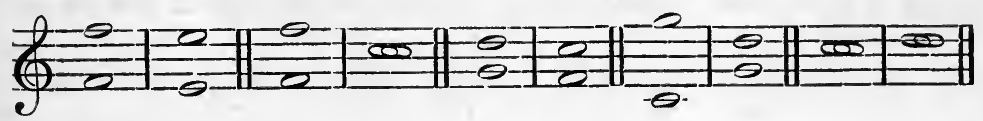

4. Except in the Cadence no perfect concord may be approached by similar motion; the objectionable effect is due to the exceptional prominence given to the perfect concord-

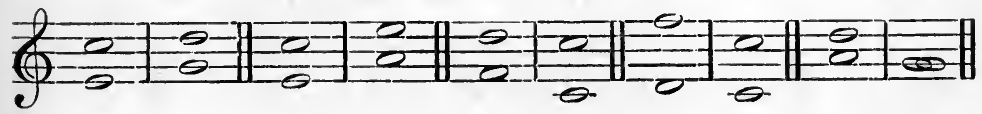

These are termed exposed fifths and octaves.

Exceptions to this rule will be dealt with as occasion demands.

5. The unison is only to be used in the first and last bars.

6. The leading note should never be doubled in two parts.

7. Of the three kinds of motion, similar, oblique, and contrary, the last is preferable, as tending to more independent movement.

Similar and contrary motion, however, should be judiciously alternated.

8. Not more than three consecutive thirds or sixths should be used, except when the parts cross, in which case the undue prevalence of parallel movement is avoided-
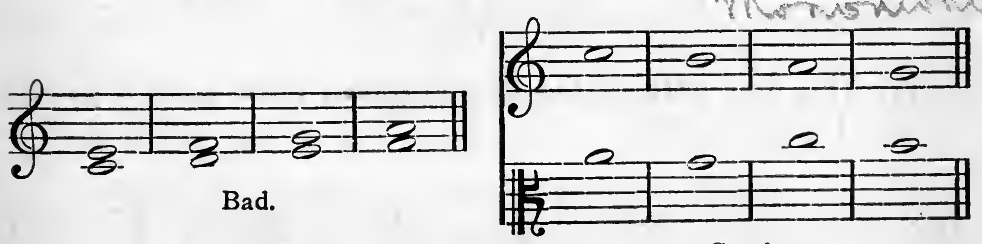

Good.

9. If both parts move by step, the interval of the augmented fourth should not exist between the C.F. and its Counterpoint in successive bars- 


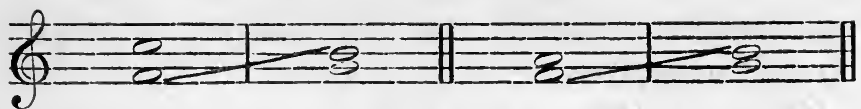

This is called the False Relation of the Tritone. If, however, one of the parts, or both, move by leap no bad effect results -

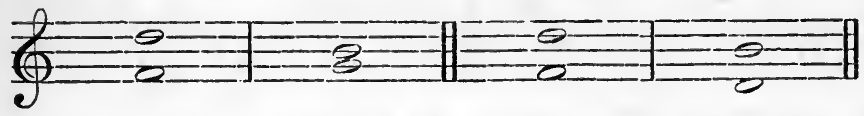

The mediaeval system of avoiding a False Relation was to alter the second note forming the interval by the addition of a flat, according to the principles of Musica Ficta-

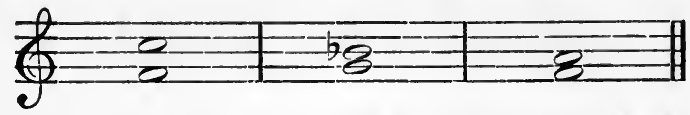

To modern ears this implies a modulation to $F$ major; though any such idea was quite alien to the Modal System, this procedure may be adopted in a case of emergency. Here is a case in which transient modulation may be introduced for the sake of euphony. Modulations may always be made to nearly related keys, provided that their use is not exaggerated so as to produce the effect of restless tonality.

Io. The parts should not overlap, but they may cross freely, in order to improve the melodic flow or to avoid objectionable progression-

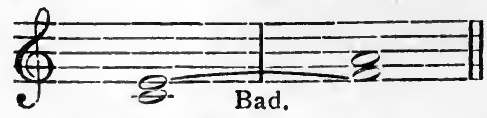

II. The first note of the Counterpoint must form a perfect concord with the C.F.
C.F.
C.F.
C.F.

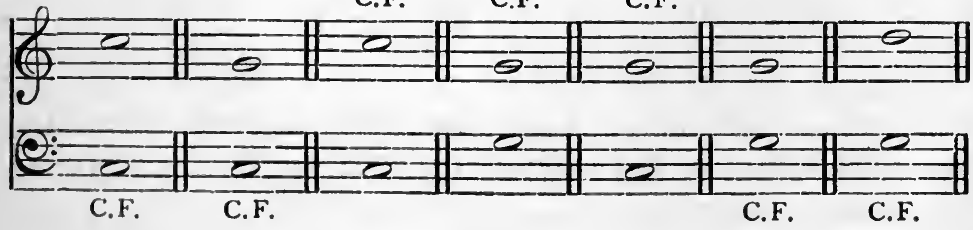


In the last four cases, the C.F. begins on the dominant; this is the only possible variation from the tonic as a first note.

12. Because of the implied harmony a third should not be followed by a fifth in contrary motion when both parts move conjunctly, except in the following cases:-

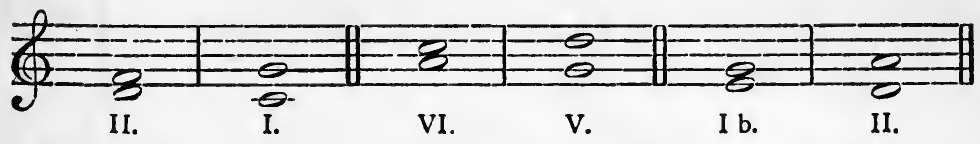

Palestrina, Kyrie, Iste Confessor.

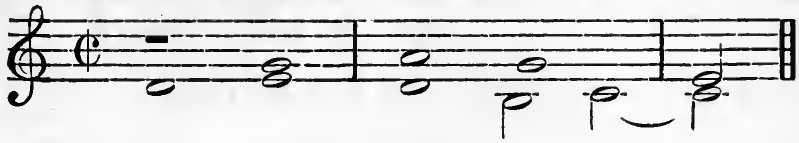

13. Use imperfect in preference to perfect concords.

I4. In approaching the Cadence avoid if possible anticipating the final of the lower part in the ante-penultimate bar-

C.F.

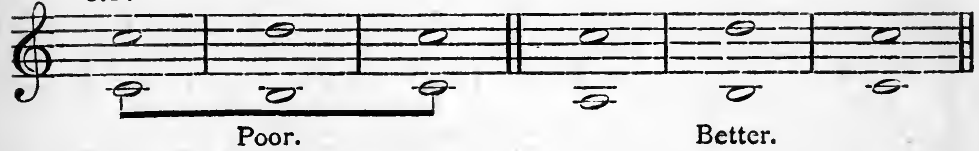

15. No note may be chromatically altered in the same" part or in different parts in consecutive bars.

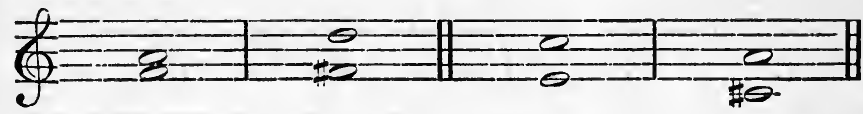

EXAMPLES.

It is quite true that Exercises in Counterpoint are for the most part written in Duple or Triple Time, that is with strong and weak accents in each bar. But at any rate in uncombined Counterpoint, groups of bars form the motives. Every bar marked thus' is the end of a motive, that is it represents a strong accent. The others represent weak accents- 
C.F.

\% I. VII b. Ib. IV. Vb. I. IV b. Vb. I.

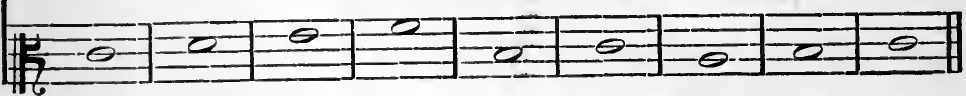
In practical terms

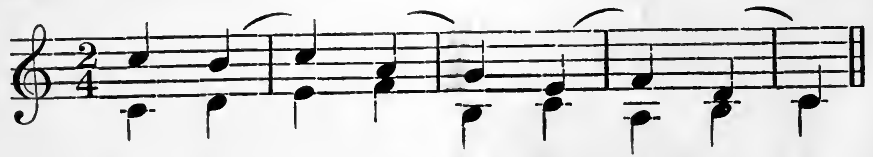

or it might have been grouped in triple time-
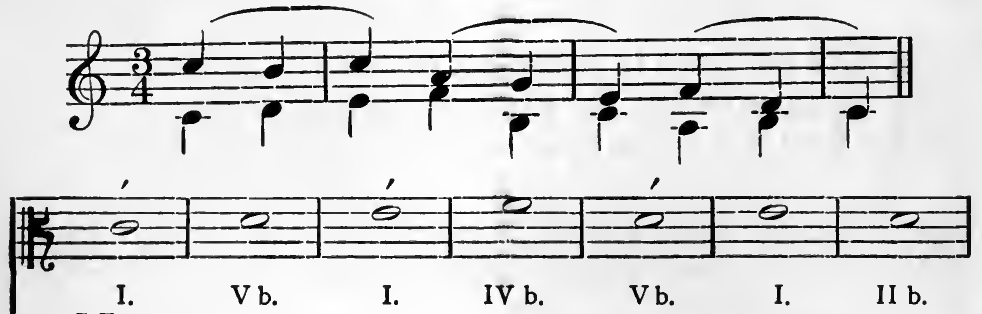
C. F.

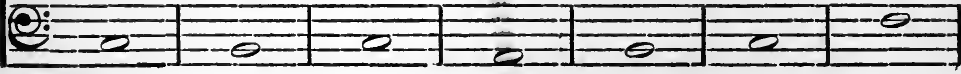

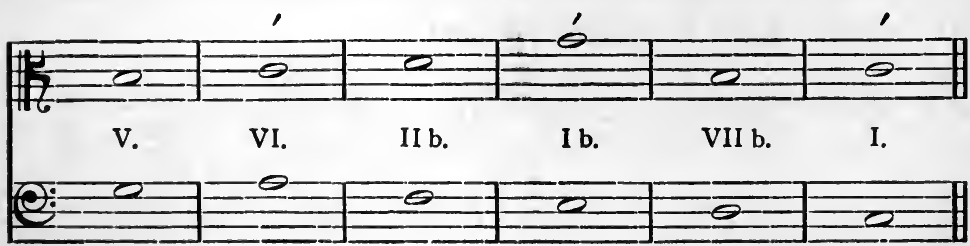

C.F.

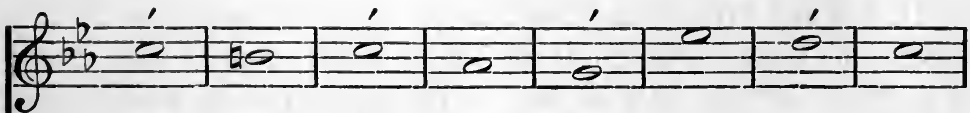

I. VII b. I b. IV. I b. I. V. VI.

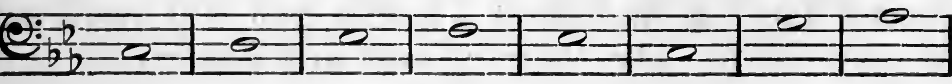




\section{COUNTERPOINT IN TWO PARTS}

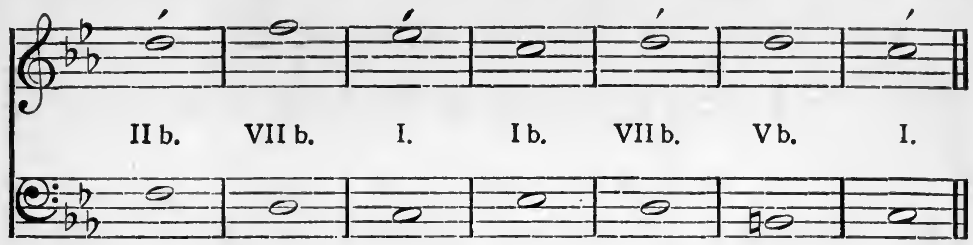
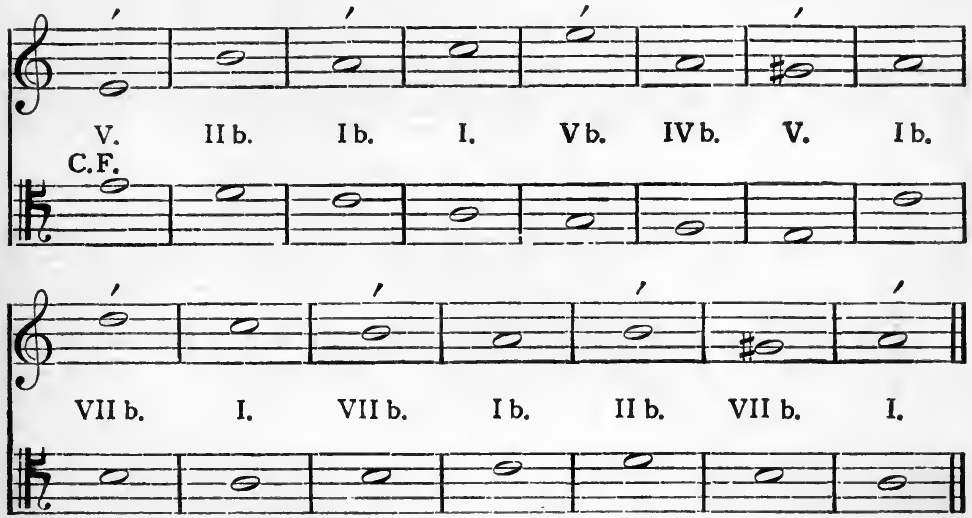

Example introducing modulation, and the use of the melodic minor scale -

G maj.
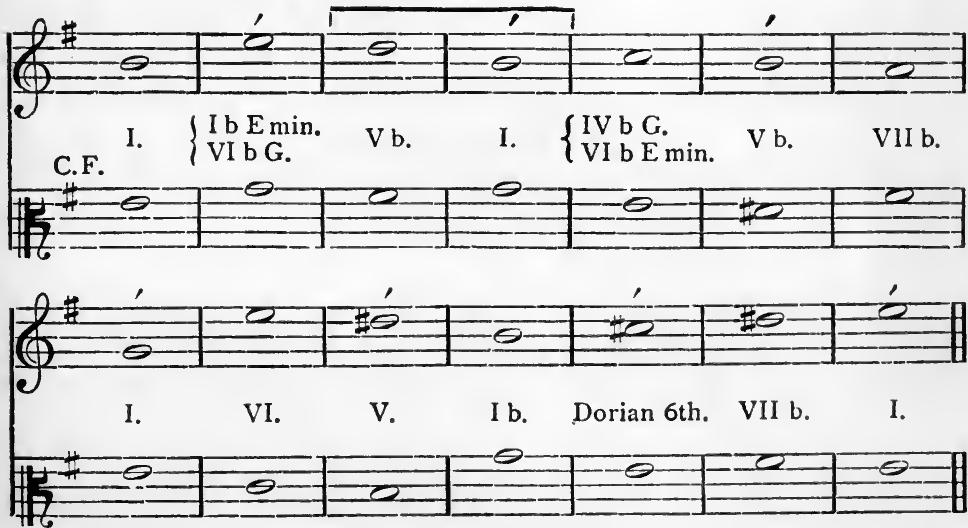


\section{THE ART OF COUNTERPOINT}

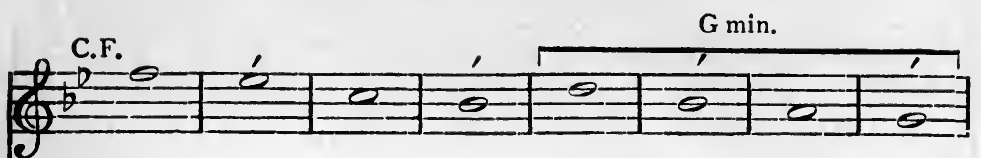

I. II. V b. I b. V b. I. VII b. I.
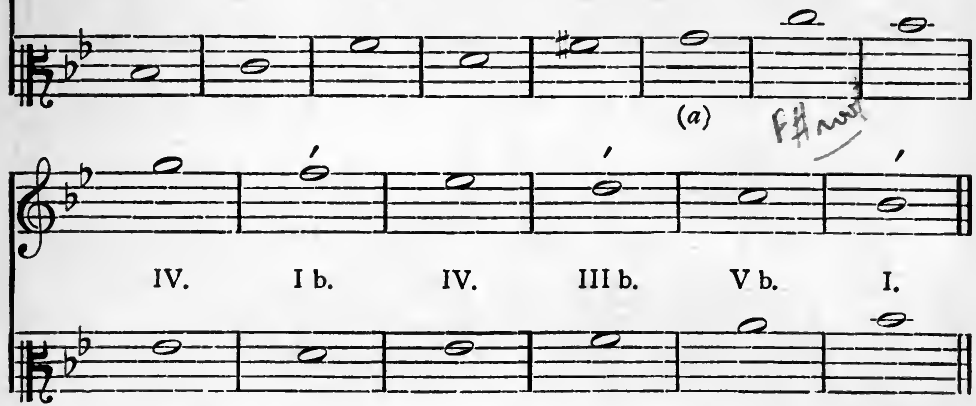

(a) if the parts cross, more than three thirds are allowable, as the prevalence of parallel movement, which is the cause of the rule, is avoided-

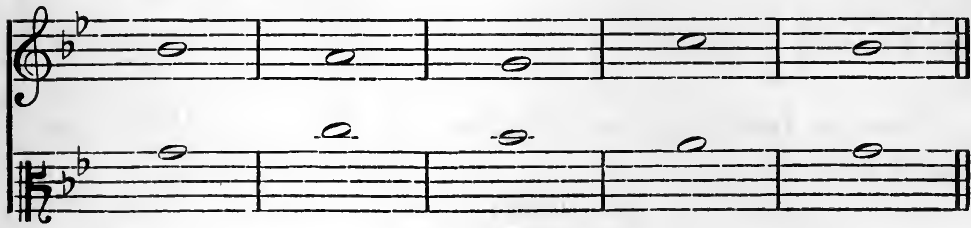




\section{CHAPTER III}

\section{SECOND SPECIES IN TWO PAR'TS}

\section{Two Notes against one.}

I. IT has been shown that the earliest attempts at Polyphony consisted of what we now know as First Species Counterpoint in two parts. It was also seen that one of the first means of variety was to make the parts move in notes of unequal value. Clearly, the simplest form of this variety was the writing of two notes to one. As it is the universal procedure in Polyphonic writing, that, except in the First Species, no two parts should commence at the same instant, the Counterpoint will begin on the second minim of the bar-

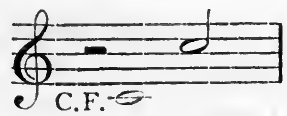

In the last bar, the Counterpoint has a note of the same duration as the C.F., obviously because the final of a Cadence must be on the strong accent. The Cadence then, is identical, as regards the sounds employed, with that in the First Species-

C.F.

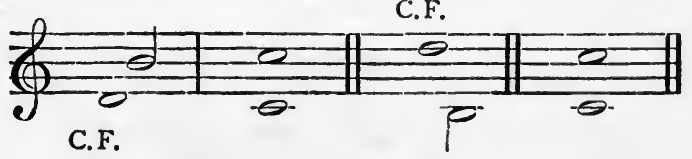

2. The first note of the Counterpoint must form a perfect concord with the C.F.- 
C.F.

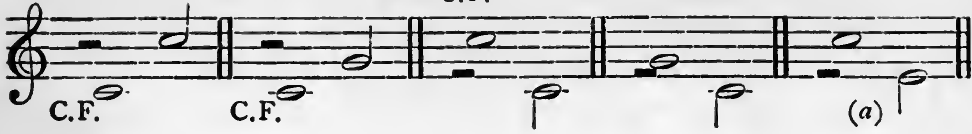
C.F.
C.F.

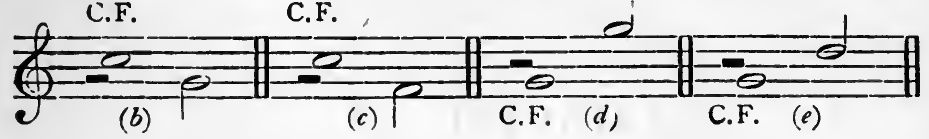

(a) is an imperfect concord.

(b) is a discord.

(c) is undesirable, as likely to give a false impression of tonality.

$(d)$ and $(e)$ are open to no objection.

3. The first note of the Counterpoint in all the intermediate bars, that is between the first and last, must form with the C.F. a perfect or imperfect concord, preferably the latter.

4. The second minim of all intermediate bars may be :-

(a) Another concord :-

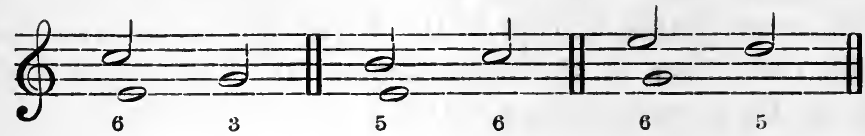

if the second minim of the bar be another concord, and if it is approached conjunctly from the first concord, e. g., 5 to 6,6 to 5 , it may leap to another concord, provided the leap is taken in the opposite direction to the previous conjunct movement. For a leap in the same direction to an accented note is inelegant-
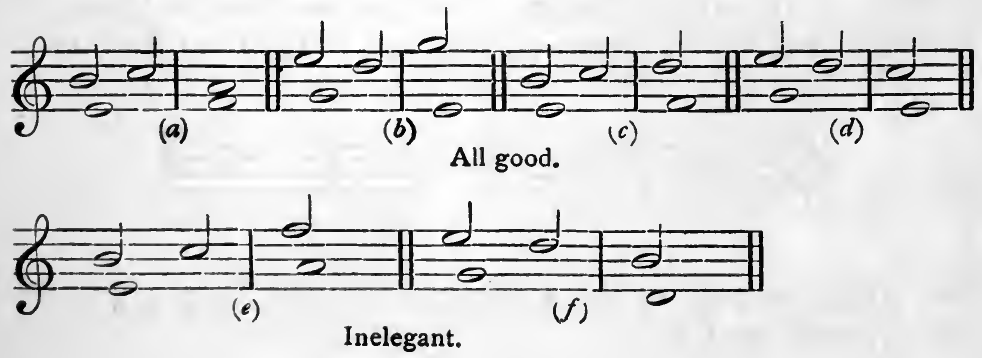
It may, of course, proceed conjunctly as at $(c)$ and $(d)$; in this case the second minim, though concordant with the C.F., is generally felt to be unessential.

Another concord taken by leap may proceed to a third concord by conjunct or disjunct movement, the selection depending upon the previous and succeeding movement. The best Second Species Counterpoint is that in which conjunct movement is judiciously varied by the use of occasional leaps. Generally speaking, the leap of the major sixth should be sparingly used, and the notes forming the interval should be portions of the same harmony. The leap of a minor sixth may be used in moderation.

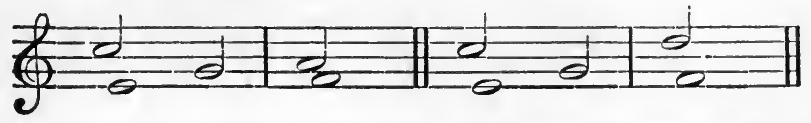

(b) A discord, which must be approached and quitted conjunctly-

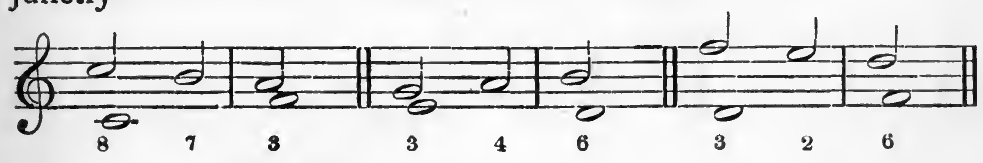

In the following examples:-

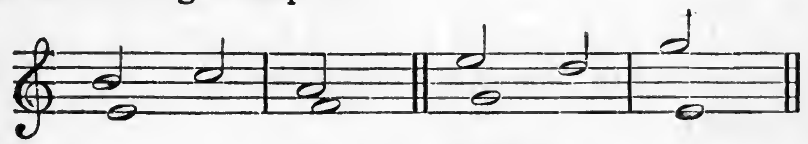

a change of harmony has been made on the second minim. Whatever arguments theorists may adduce to prove that the above are wrong are based on a false principle, and are quite inconsistent with practice.

Three illustrations from Palestrina will prove this :-

(a)

Sicul cerius.

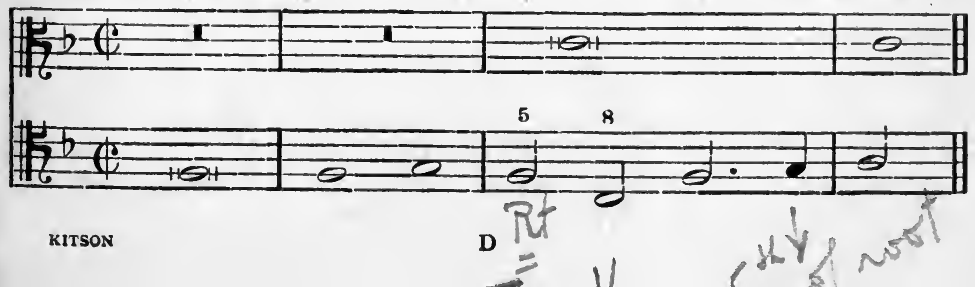




\section{THE ART OF COUNTERPOINT}

One theorist says the fifth of the root must never be written in the lowest part as the second note of the bar, as it implies the use of a second inversion.

But we have no right to suppose that Palestrina was using one chord. His view is $\mathrm{F}$ to $\mathrm{C}$ is a concord, so is $\mathrm{C}$ to $\mathrm{C}$.

Contrapuntal practice does not forbid what to us is the mental effect of a six-four, nor even its actual use from a modern analytical point of view, provided, of course, the fourth is prepared.

Another example is clearer still :-

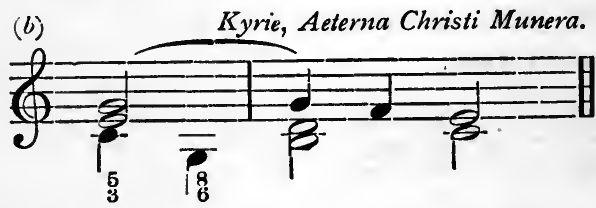

No fourth is present.

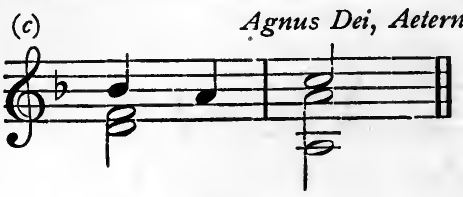

It is absurd to argue that because $A$ is no portion of the harmony of the first crotchet it cannot be quitted by leap. This is a clear case of the use of two harmonies.

Of course :-

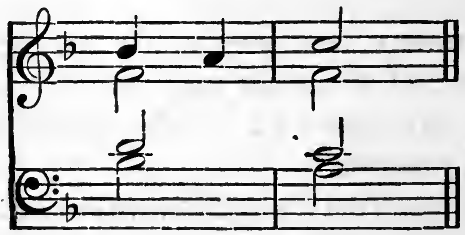

would be obviously incorrect. The following will make things quite clear :-

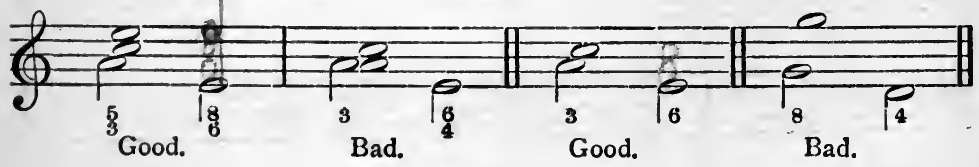


A few more examples (all taken from Palestrina's Stabat Mater), are added :-

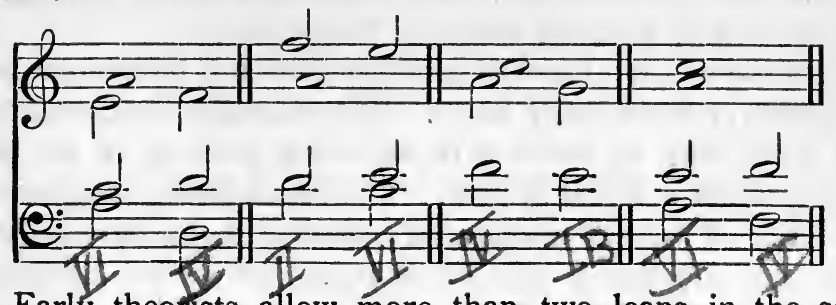

5. Early theorists allow more than two leaps in the same direction-

Albrechtsberger.

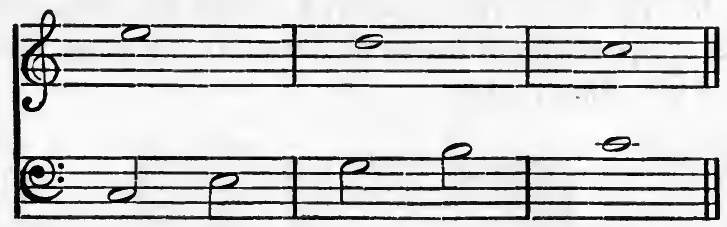

Such a progression is at best inelegant, and is contrary to general principle. If the C.F. were in the middle of a problem, the following would be better:-

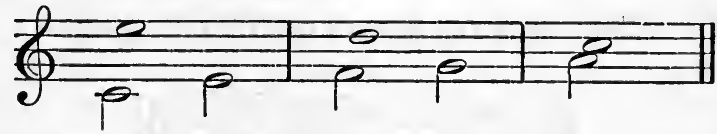

and if at the end :-

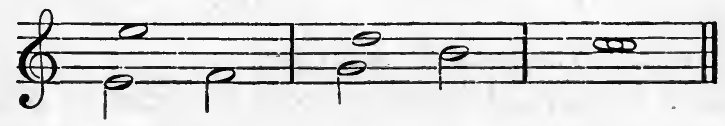

6. Unessential Notes.

It has been said that a discord may be used as a second minim in the bar, if approached and quitted conjunctly.

The theory that the second minim in the following example is to be considered unessential on account of the implied harmony of the first minim, cannot possibly find a place in Strict Counterpoint-

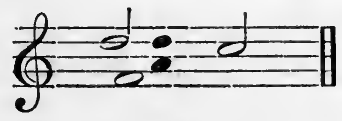

D 2 
Therefore, consideration will only be given to true unessential notes, that is, those that are discordant with the C.F., the second, fourth, seventh, and their compounds.

Now the modern tonal system brings with it limitations in the treatment of unessential notes. The mistake commonly made has been that of treating these notes just as if we were writing under a modal system. The result is, the student forms the habit of writing unessential notes in a purely mechanical way, and this has a pernicious influence on his attempts at modern composition. The use of unessential notes may be thus classified :-

Case I. When the two concords which the unessential note connects are portions of the same harmony, or as we should say, factors of the same root-

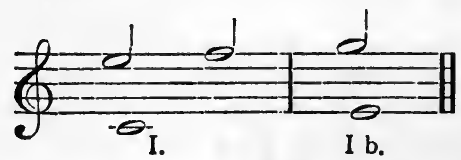

Musical sense prevents one from analysing the second bar as III.

Case II. When they are factors of different roots-

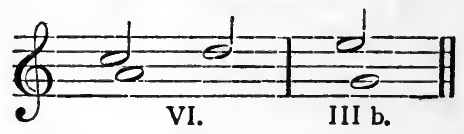

There are three aspects of each case.

(a) When the second harmony note is higher in pitch than the first (see above).

(b) When it is lower-

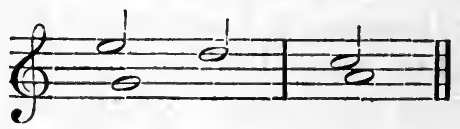

(c) When it is the same-

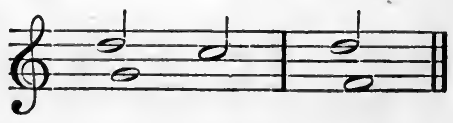


Case I. When the two concords which the unessential note connects are portions of the same harmony-

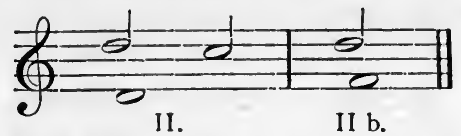

The above is bad, because the second minim $(C)$ has the mental effect of being the minor seventh of $\mathrm{D}$, with a tendency to fall ; the same applies to parallel cases in III, V, and VI -

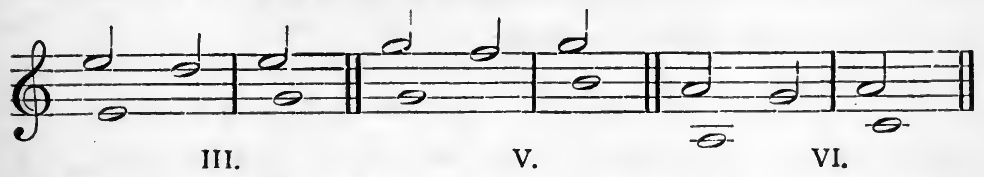

An observance of the following rule will prevent such crudities.

In writing true unessential notes between different positions of the same chord, such notes should be common to the scale of the Tonic, and that of the root of the chord used.

It will, of course, be seen that the above does not condemn such a case as :-

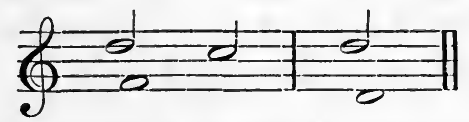

In this, the second minim should be regarded in its contrapuntal light, that is, as being essential. But even in such cases crude effects may be produced.

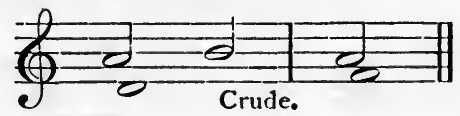

In order to make the matter perfectly clear, it will be well to explain a flaw in detail.

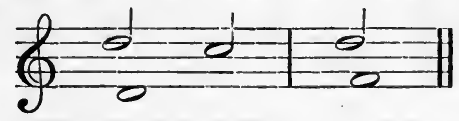

The key is $\mathrm{C}$ major.

Now here the triad of $\mathrm{D}$ minor is used, and the ascending 
scale of $\mathrm{D}$ minor requires $\mathrm{C}$ sharp; but $\mathrm{C}$ sharp is foreign to the scale of $\mathrm{C}$ major, the Tonic, therefore $\mathrm{C}$ must be omitted, and another note chosen-

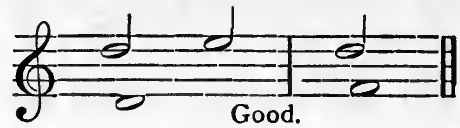

Case II. When the two concords/which the unessential note connects/are portions of different harmonies.

Consider the following cases :-

G maj.

C maj.

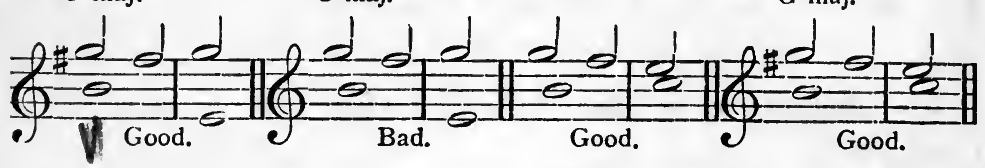

^ Hence we get a further rule:-In writing an unessential note between different harmonies, if such note is not common to the scale of the first chord and that of the Tonic, it should be capable of analysis as forming with the C.F. a good implied harmonic link with the next chord, whether such implied harmony be another concord, or a fundamental discord. The $X$ following examples will make this clear:-

F maj. C maj.

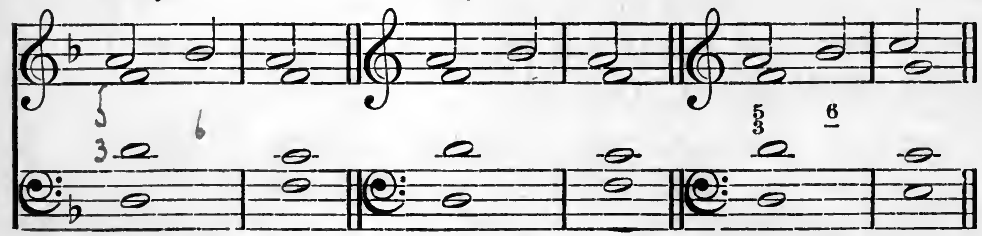

Good.

Poor harmony.

Good.

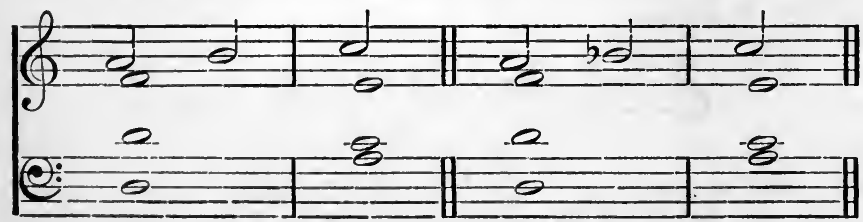

Goud.

Poor harmony. 
G maj.

C maj.

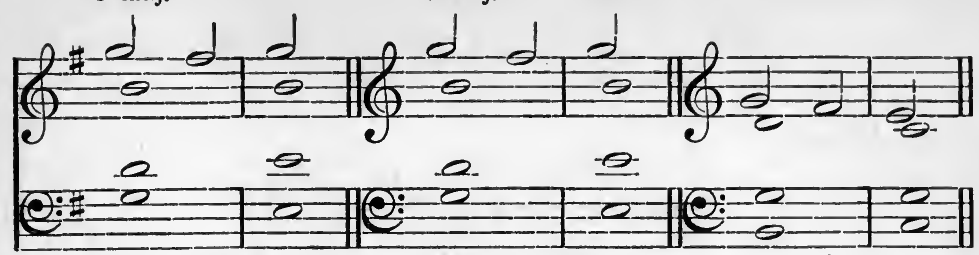

Good.

Poor harmony.

Good.

A min.

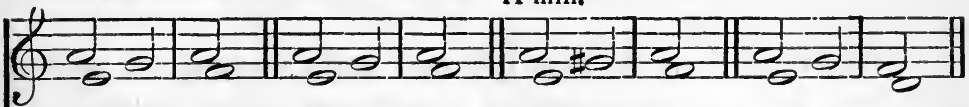

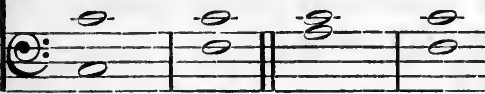

Good.

Bad.

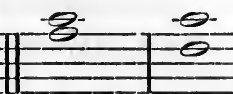

Good.

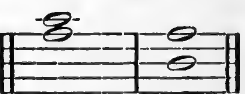

Good.

The following cases deserve careful observation :-

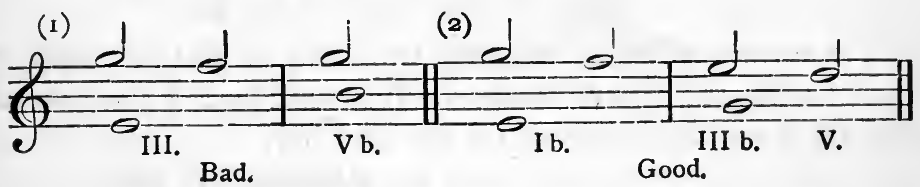

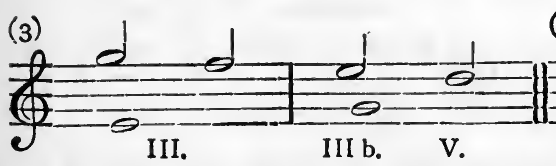
Bad.

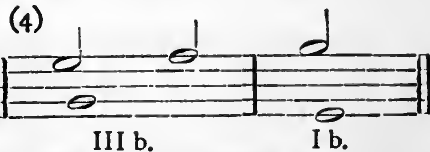

Bad.

None of these cases are objectionable as they stand : but only when the implied harmony is clearly defined. Nevertheless it is necessary to draw attention to the matter at the very outset.

(I) is unsatisfactory because the harmonic progressions belong to the key of $\mathrm{G}$ major rather than $\mathrm{C}$ major-

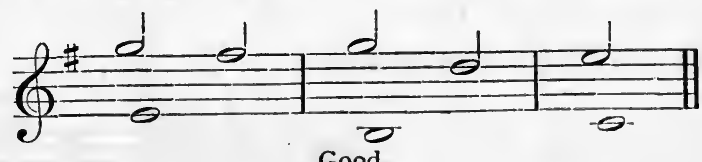

Good.

(3) is subject to the same criticism- 


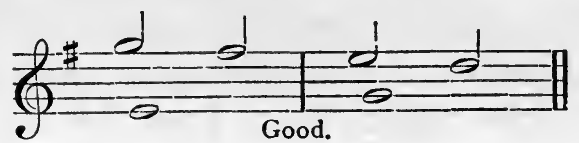

(4) likewise-

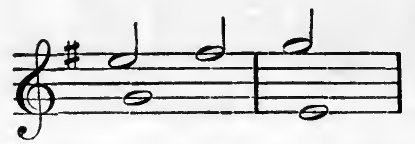

But in (2) a clear definition of another harmony would remove the bad effect.

It will thus be seen that the real point at issue is mental harmonic effect, under modern conditions. For instance, no objection can be urged against the following :-

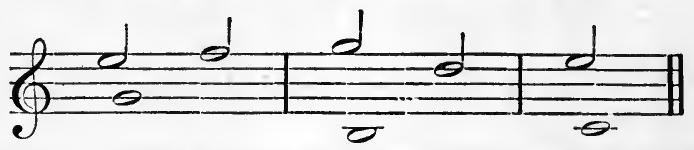

for the mental effect of the first two bars is that the whole is based on the root $\mathrm{G}, \mathrm{E}$ being the thirteenth, and $\mathrm{F}$ the seventh with the resolution deferred till the next bar.

It is also to be noted that both the following are good:-

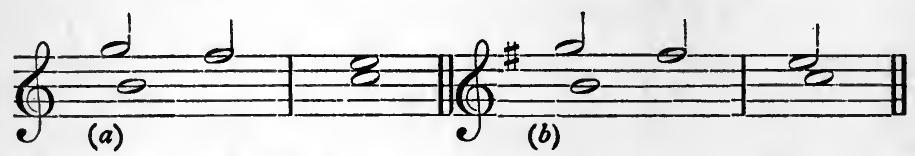

In $(a)$ the modern ear accepts the $F$ as the seventh of the dominant. In (b) the $\mathrm{F}$ sharp is accepted as an ordinary unessential note.

Common use has accustomed the ear to the following :-

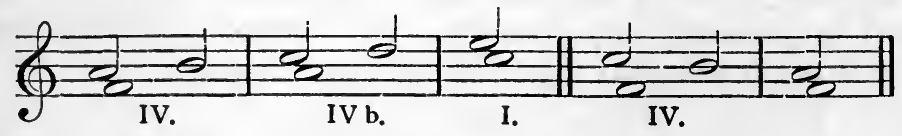

But one's ear revolts against :-

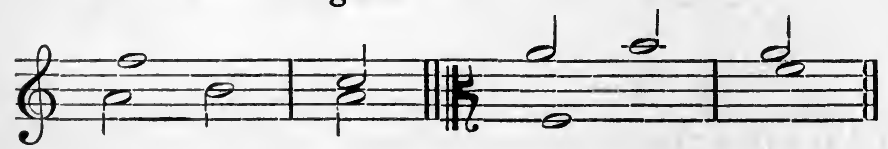


The following, however, is quite good in effect, because the mental impression is that of an implied $\frac{6}{2}$ on the second minim, and the roots of the two chords are different-

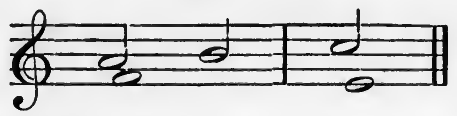

And so the following will be clearly understood :-

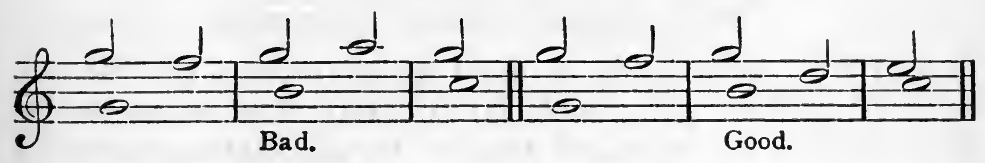

In the latter case the mental effect is that the resolution of the seventh is merely deferred.

The remaining cases will require no further comment-

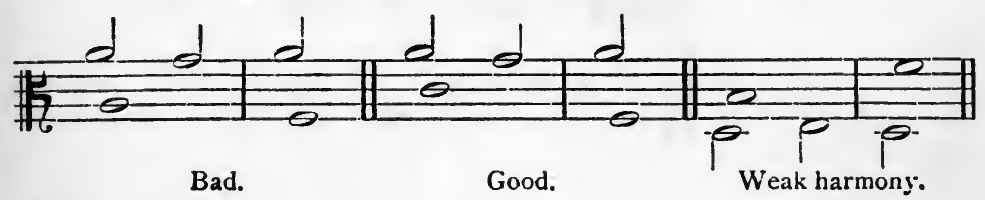

The minor key presents the same sort of cases, requiring intelligent as opposed to mechanical treatment. A discussion of it may be conveniently deferred till we treat of the Third Species. The salient point that comes out from all this is that in modern times notes that were formerly unessential have merged into the essential, are felt as such, and must be likewise treated.

Palestrina writes :-

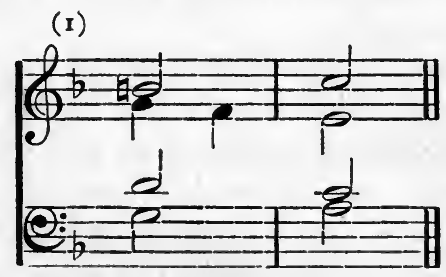

Now such a case is not the ultimate origin of the essential 
seventh ; this will be seen when we come to treat of prepared discords. But the use of the prepared seventh:-

(2)

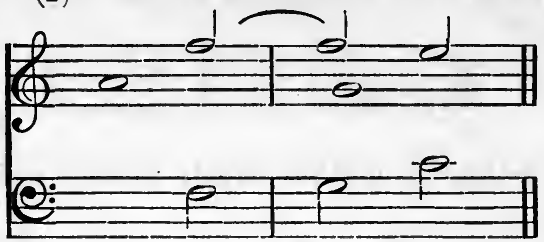

served to form a habit of mind which caused men to use such unessential notes as in (I) in a way that showed their judgement of the fitness of things. No one with any sense of harmonic propriety, would tolerate :-

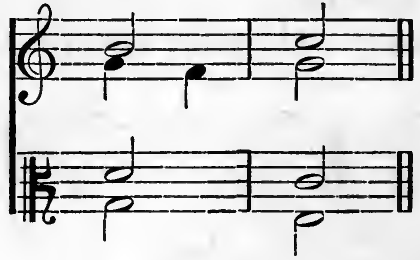

In two and three parts, at any rate, the harmonic interval of the second should not be quitted by similar motion. It is open to the objection that the discord is sounded against its resolution at the distance of a second, and causes unnecessary congestion-

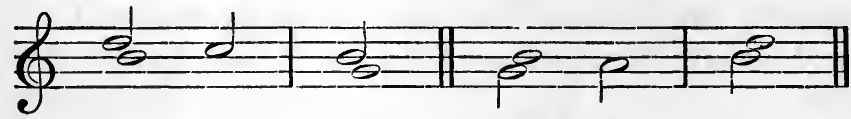

It is quite true that such things may be found in Palestrina, but they are best avoided.

i? 3 The chief points to notice at present are therefore :-

A. If the non-essential note is felt to be a portion of the modern essential discord, it should be treated as such, both in melodic progression, and harmonic accompaniment.

B. Unessential notes used between different positions of the same chord, should be common to the scale of the chord used, and that of the Tonic, except in the case of the subdominant- 


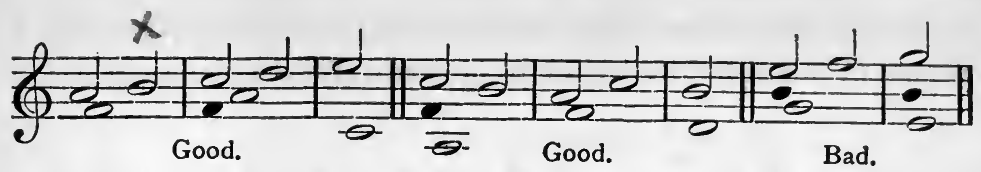

C. An unessential note used between different chords should be common to the scale of the first chord and that of the Tonic, unless such note form with the C.F. some good modern harmonic link with the next chord-

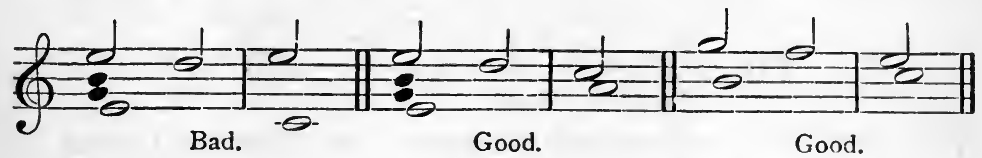

Note. Passing notes used in connexion with the first inversions of dissonant triads do not fall under any of the above considerations.

7. Consecutives are not allowed:-

(a) between the first minims of two consecutive bars-

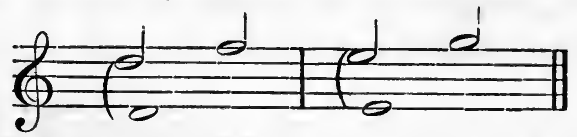

(b) between the second minim of one bar, and the first of the next-

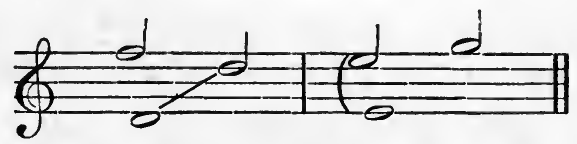

They are allowed between the second minims of each bar, if both notes in the Second Species are not the highest or lowest in the two bars-
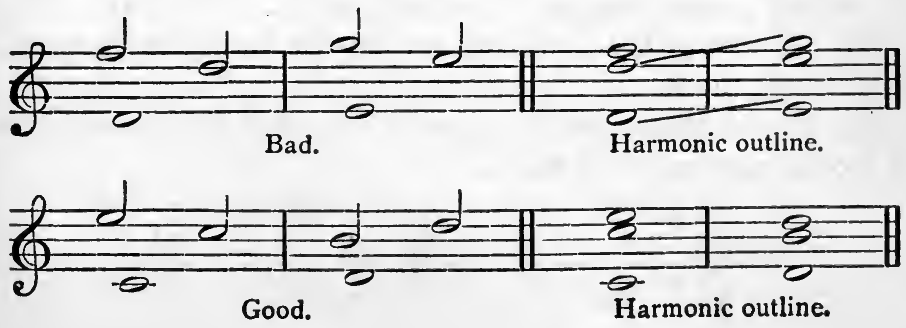
The distance between the first minim of one bar and the second minim of the next is too great to give any effect of consecutives-

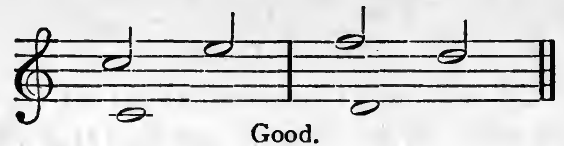

When either of the fifths is felt to be unessential no bad effect is produced-

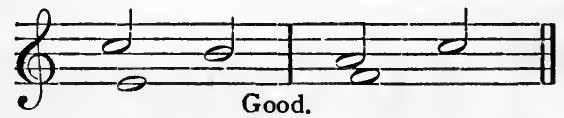

Nor can consecutives occur between the first and third of three harmonies -

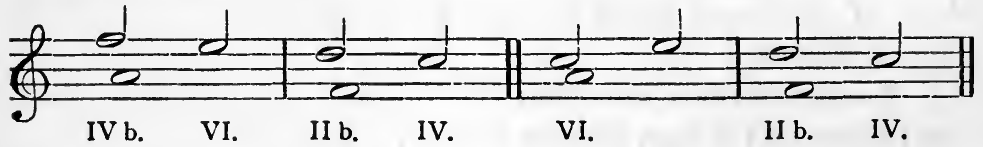

Exposed consecutives are forbidden under the same conditions as in First Species-

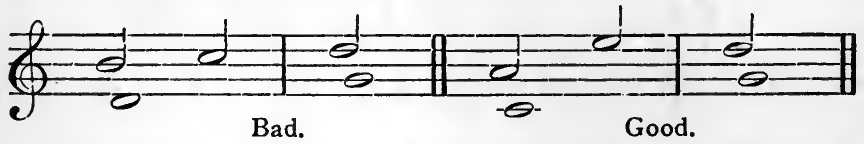

8. The following does not cause a false relation of the tritone, as one of the notes forming it is felt to be unessential.

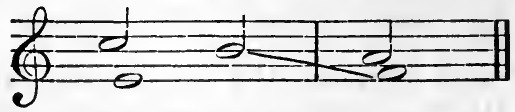

9. The unison may be used in the first and last bars, and occasionally elsewhere as the second minim in the bar.

Io. No note of the Counterpoint may be immediately repeated at the same pitch.

II. The leading note must not be doubled either on the first or second minim of the bar-

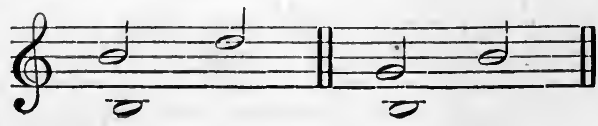


12. A note may not be chromatically altered in the same bar-

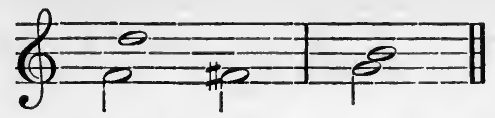

13. In this Species it is well to avoid the repetition of a melodic formula for more than two successive bars; a sequence having at least two bars as the limit of the formula is always good. In a more complicated exercise the student should use his own discretion.

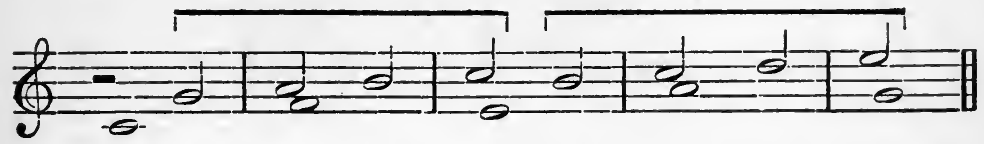

14. The Minor Key.

The application of the principles expounded in the previous chapter may be thus summarized :

(I) (a) The minor seventh of the scale may be used as a harmony note in proceeding from tonic to sub-mediant-

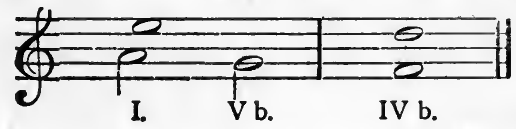

(b) It may of course be freely used as an unessential note-

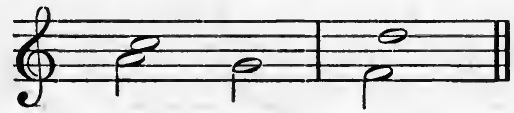

(c) It may be used in modulating to the relative major-

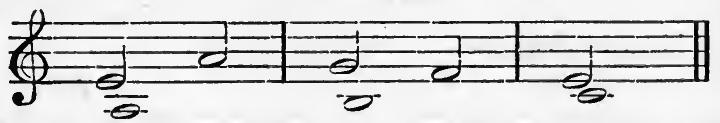

(2) (a) The major sixth of the scale may be used in the following idiom-

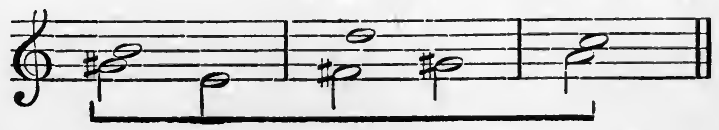


(b) In the Cadence-

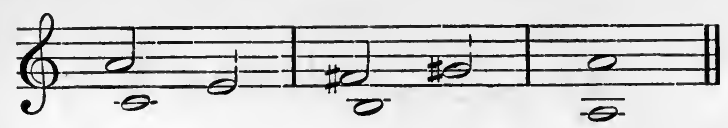

In all these cases the mental effect is that $F \#$ is an unessential note; nevertheless it forms a concord with the C.F.

(3) The major sixth, minor sixth and major seventh of the scale may all be used as discords-

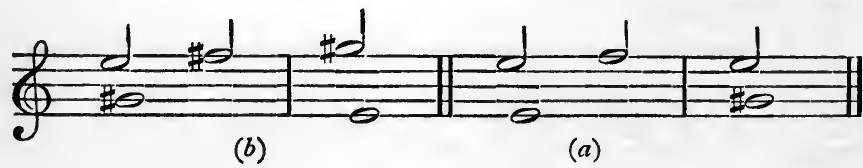

(a) Note that $\mathrm{F} \#$ would be incorrect; for there is no dominant major ninth in the minor key. At $(b)$ the $F \sharp$ is felt to be a true passing note.

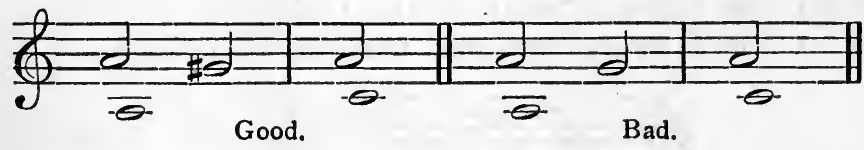

I5. The Cadence.

(a) The Major Mode-

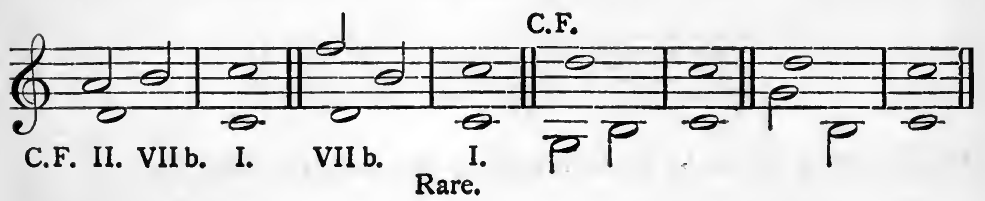

(b) The Minor Mode-

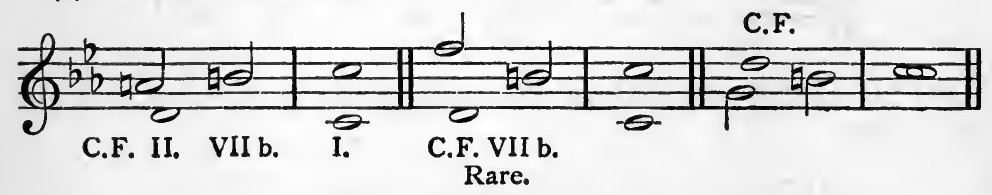


Examples in Second Species.

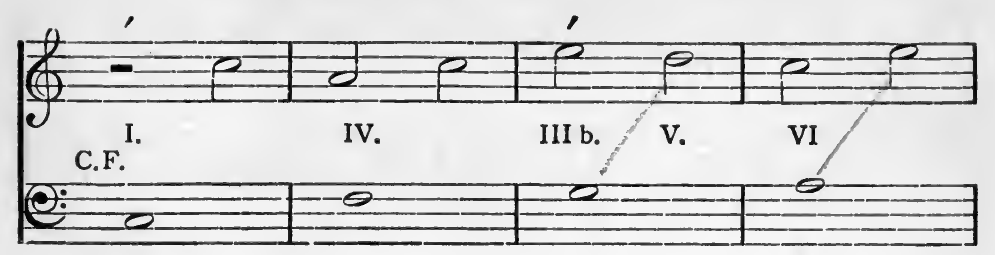

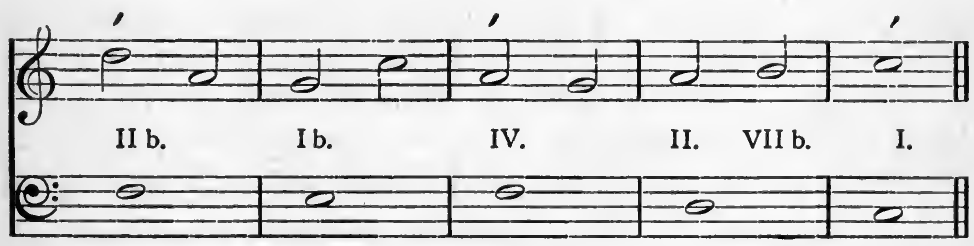

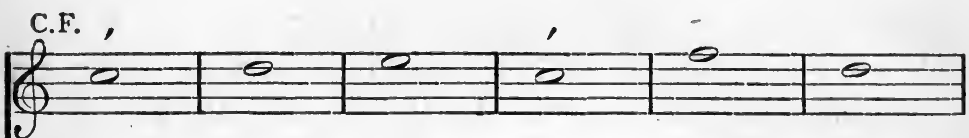
I.
V b. V. I.
VI.
II b. II. V.

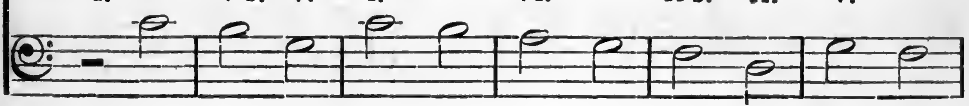

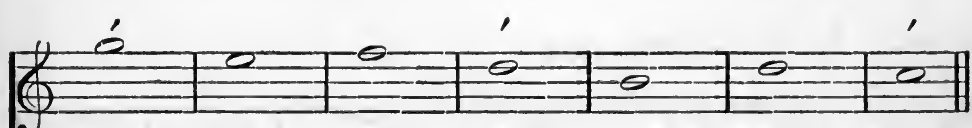

Ib. I. IV b. II b. II. V. V. Vb.

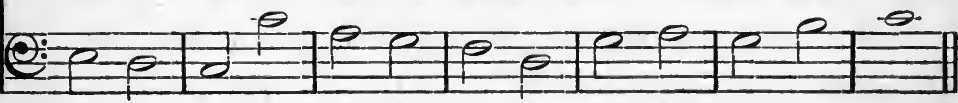

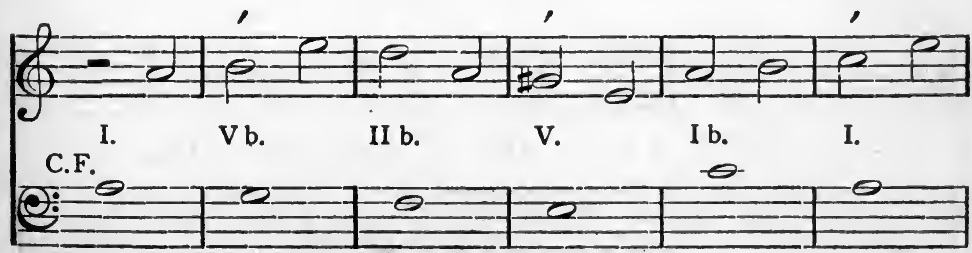


C major.

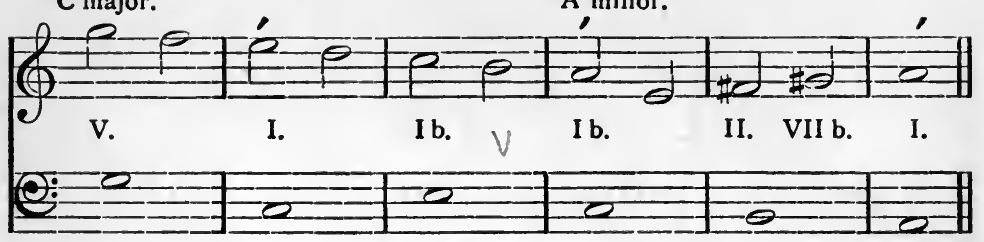

C.F.

\begin{tabular}{|c|c|c|c|c|c|c|}
\hline I. & II b. V. Ib. & VI b. IV. VII b. Vb. V.
\end{tabular}

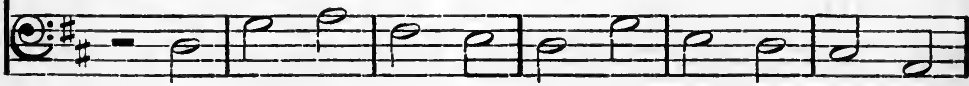

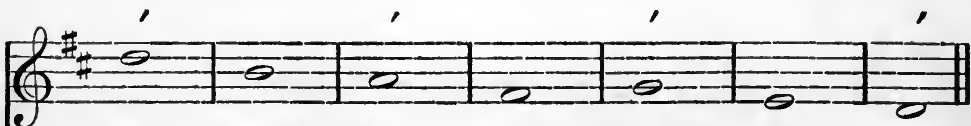

VI. VIb. II. I b. III b. I. IV b. IV. V. Vb. I.

20:-

2

First Mus. Bac. Cantab., May, 1904.

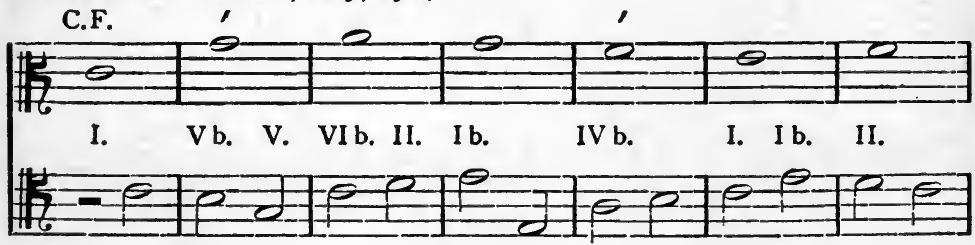

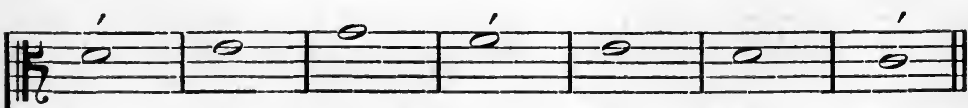

Vb. III b. I. Vb. I. II. Ib. V. Vb. I.

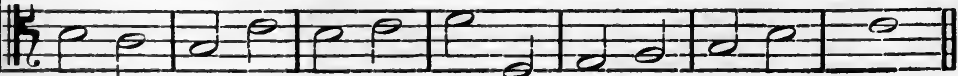


C.F.
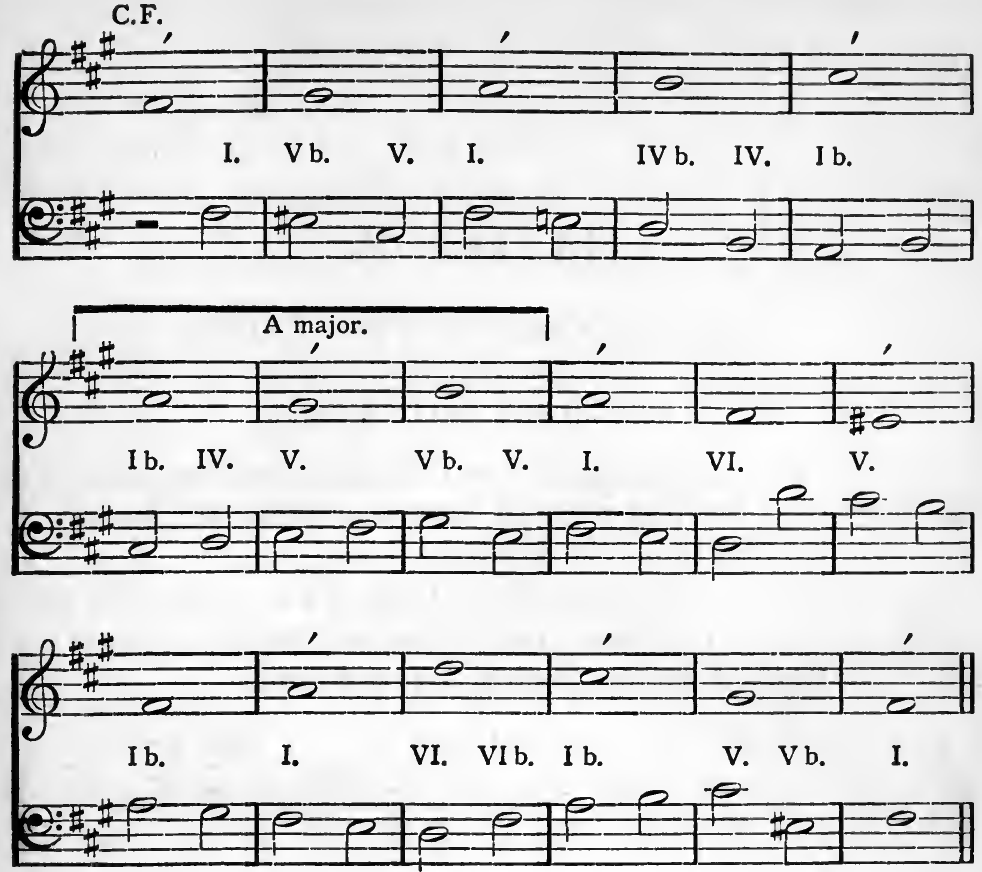


\section{CHAPTER IV \\ THIRD SPECIES IN TWO PARTS}

Four Notes against One.

I. THE Counterpoint begins on the second crotchet of the bar, and except in the first and last bars, four notes are written in the Counterpoint to one in the C.F.

2. The rules in First and Second Species as to the first note in the bar remain in force; in the Cadence the last two notes of the Counterpoint are the leading note and tonic-

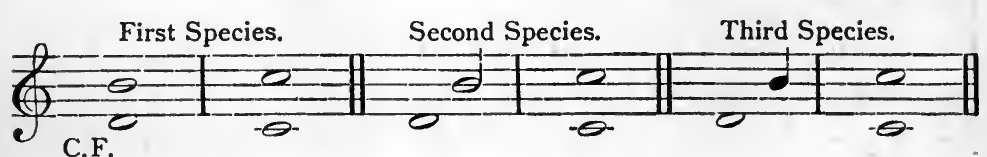

C.F.

3. If the second and third, or third and fourth crotchets are discordant with the C.F. the part must proceed in the same direction by step to the next concord. If the next step will not produce a concord, the passage must be rearranged-

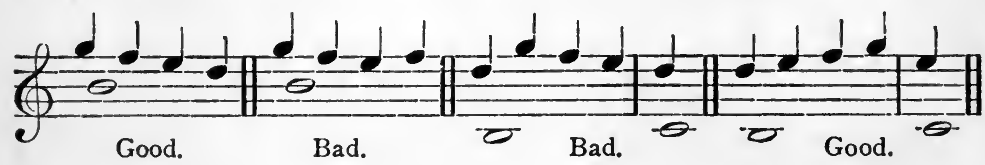

4. After using a scalic passage, it is inelegant to leap to an accented note in the same direction-

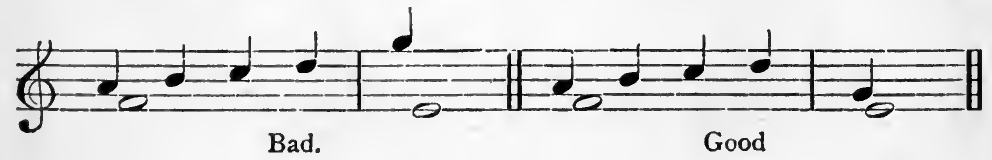




\section{Changing Notes.}

Much ingenuity was exercised by the writers of the Polyphonic Period in the matter of the various uses of unessential notes. One particular idiom, known as the use of the Nota Cambiata, became quite characteristic. Dufay (fourteenth century) wrote thus :-

Mass, Se la face ay pale (Kyrie).

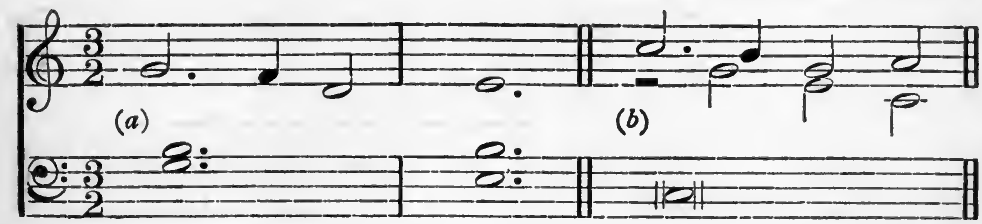

In $(a) \mathrm{F}$ is a passing note between $\mathrm{G}$ and $\mathrm{E}$, leaping a third to the other side of $\mathrm{E}$, and then proceeding to it by step upwards. This formula is seen in nearly all the works of the period; particular mention may be made of Josquin's Stabat Mater, Palestrina's Missa Papae Marcelli, and Gibbons's Hosanna. A few examples may be of interest :-

(a)

Martinus Leopolita, Mass, Paschalis (K'yrie).
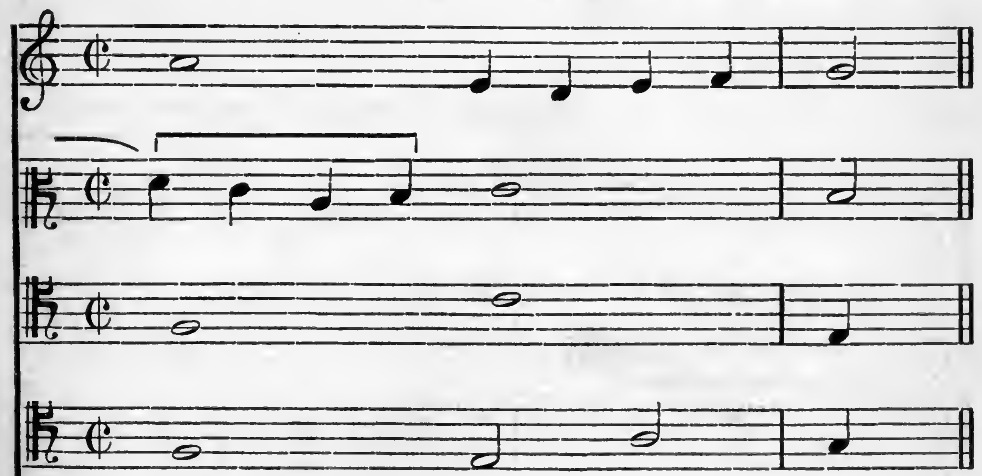

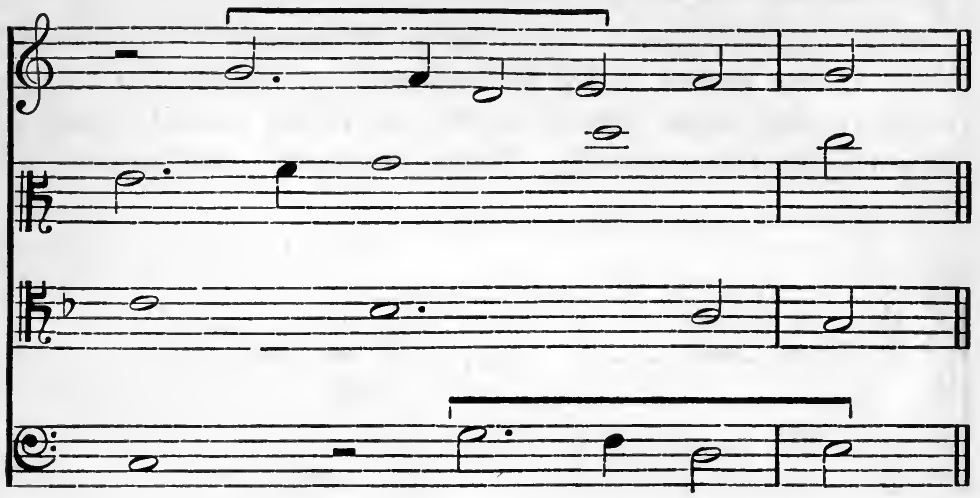

(c) Palestrina, $O$ admirabile commercium.
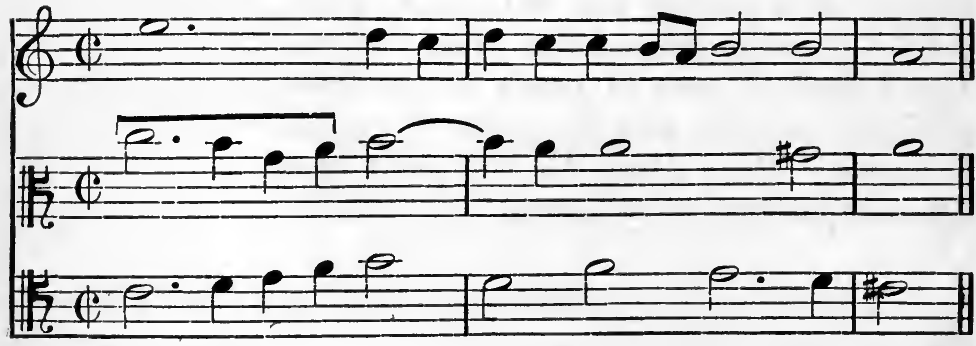

(d)

Palestrina, Benedictus, Missa Papae Marcelli.

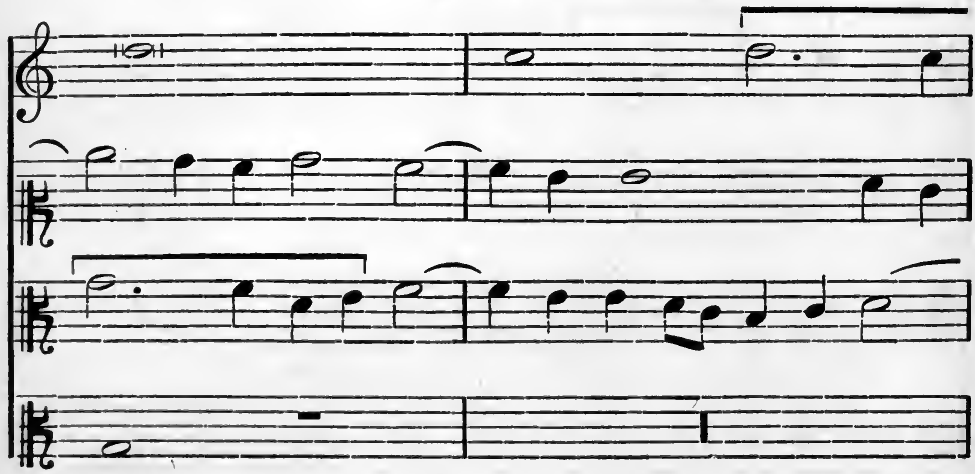




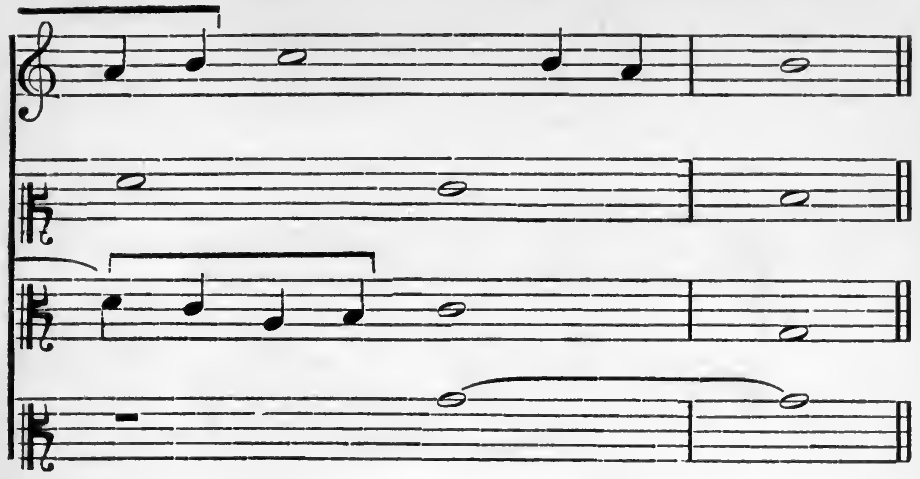

(e)

Palestrina, Ego sum panis vivus.

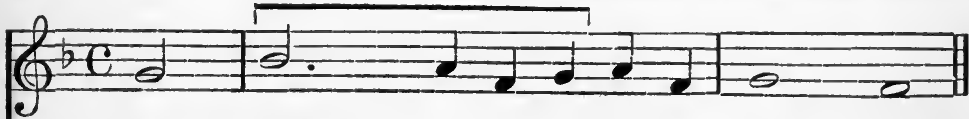

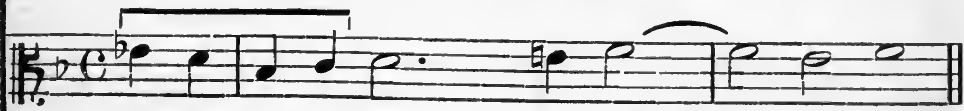

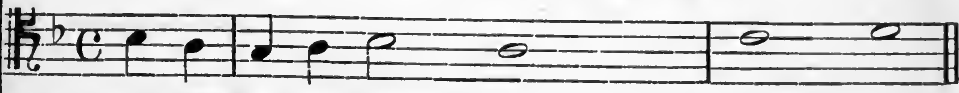

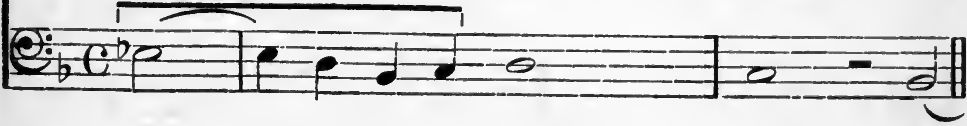
(f)

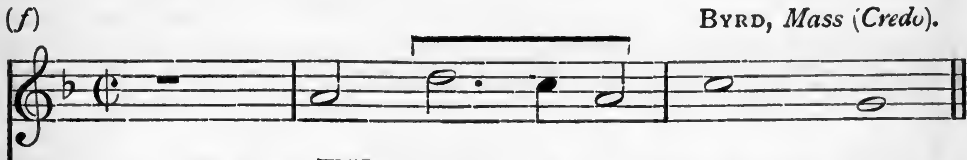

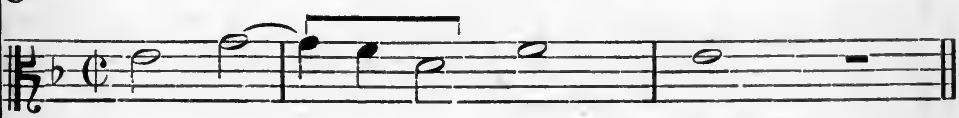

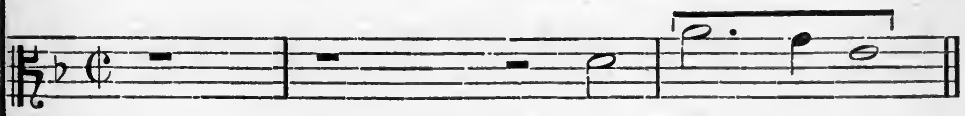

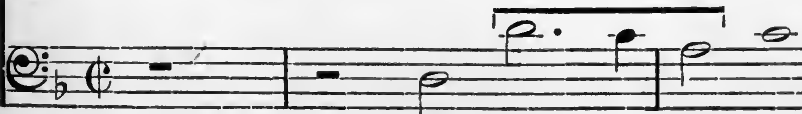



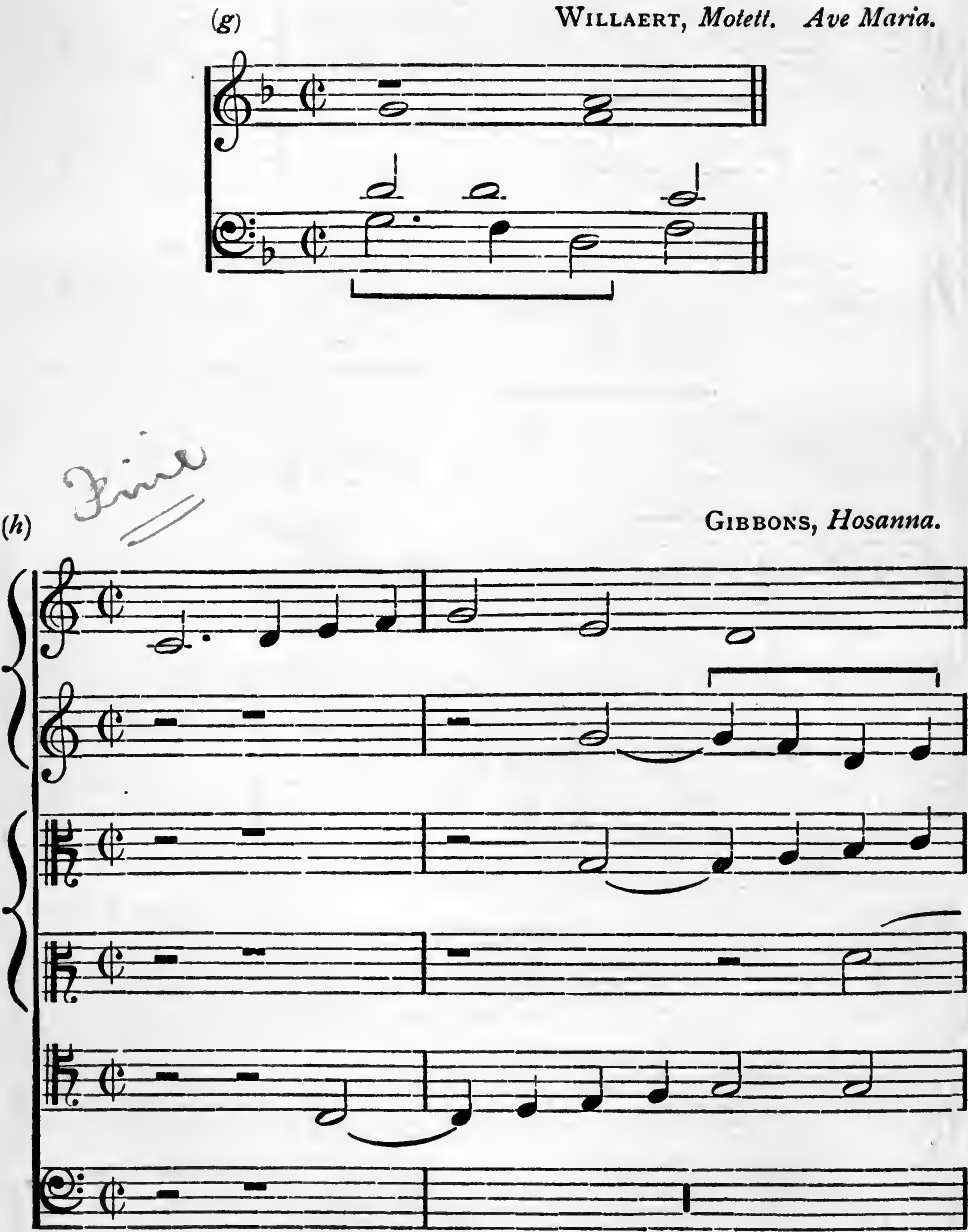


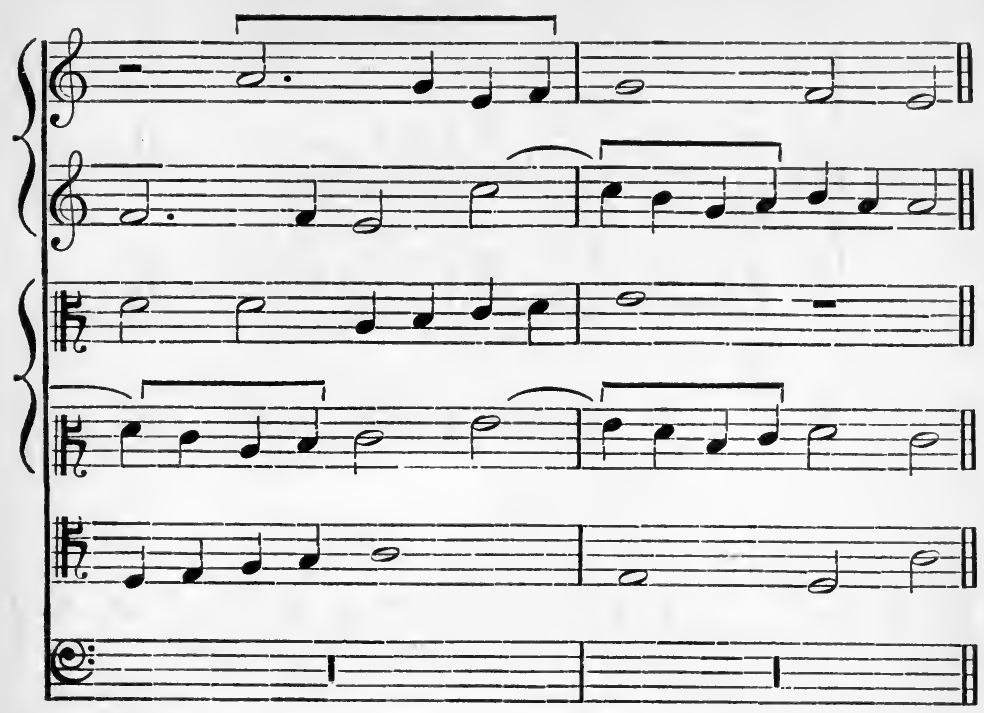

In $(f)$ and $(g)$, through constant use, the real significance of the Nota Cambiata has been forgotten. An interesting seventeenth-century example is added-

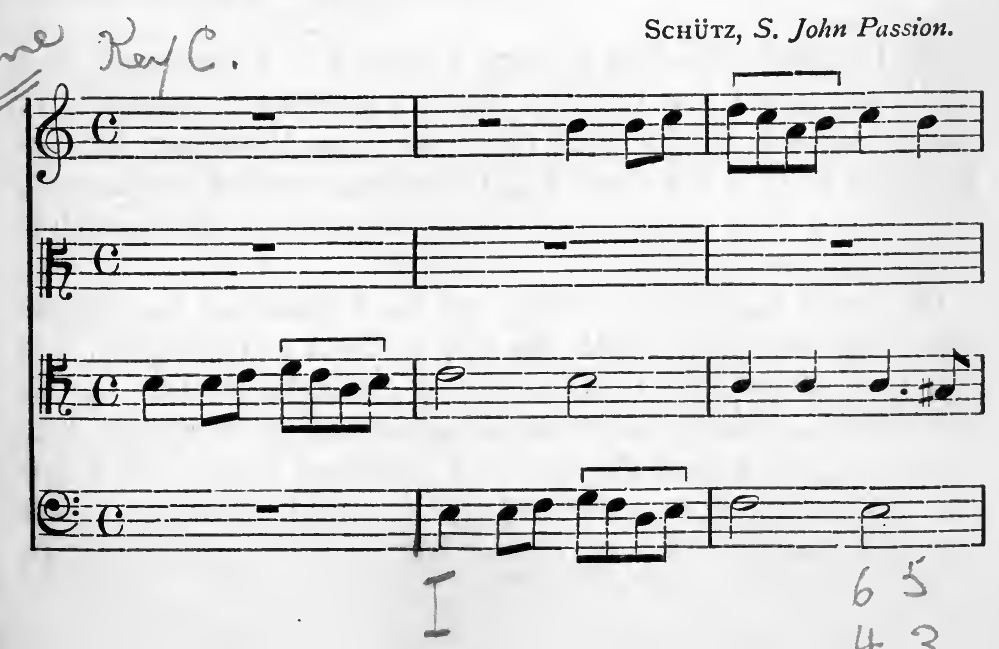




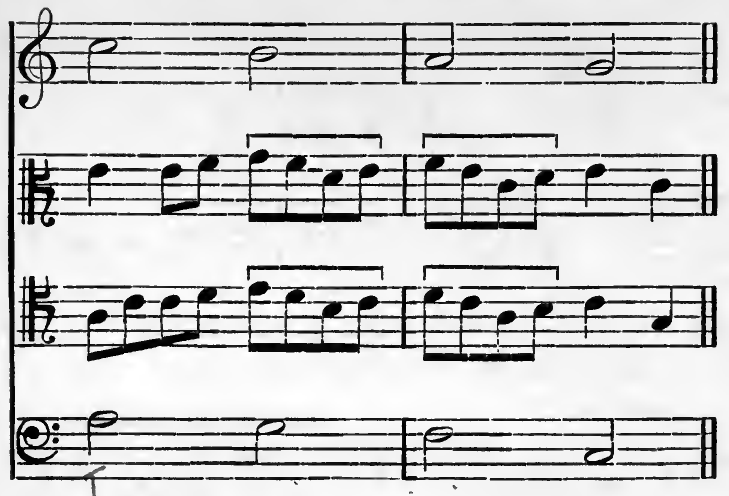

Consider the following cases :-
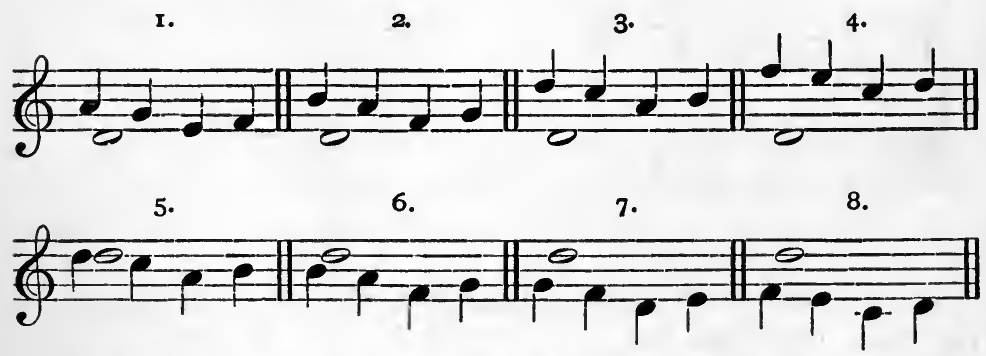

(a) In I, 4, 5 and 8 the leap is from a discord to another discord.

(b) In 3 and 6 the leap is from a discord to a concord. And in all these the last crotchet forms a concord with the C.F. These are all examples of the true Nota Cambiata, for the principle in all is the same, e.g., the second crotchet is a discord, and leaps a third to the other side of the concord on to which it finally resolves.

(c) But in 2 and 7 the second and third crotchets are concordant with the C.F., while the last crotchet is discordant. It has been stated that these are also examples of the use of the Nota Cambiata, but in consideration of the examples that we have quoted from Palestrina in reference to the use of two harmonies in a bar in Second Species, and bearing in mind that the harmony may be changed on the second crotchet in 
the Third Species, it seems probable that some such explanation as the following should be sought :-

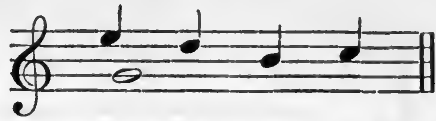

$\mathrm{E}$ is concordant with the C.F., so is $\mathrm{D}$, and so is $\mathrm{B}$, that is, the harmony has been changed on the second crotchet. Having got so far, the composer would feel that he was using a familiar idiom, and instinctively write the fourth crotchet as above, not perforce of rule, but by habit of mind. For the true Nota Cambiata requires the fourth crotchet to be concordant with the C.F., and support to this is given by the following example from Cherubini, who disallowed the use of the Nota Cambiata :-

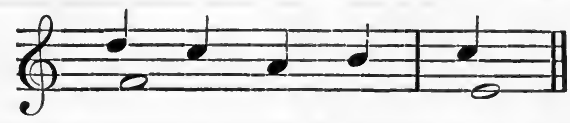

The following examples from Palestrina and others will show that in consequence of the frequency of the idiom, composers lost sight of its original use. We may therefore say that if the combination on the third or fourth crotchet is concordant, the passage may stand.

(a) Original use.

Palestrina, Kyrie, Missa Papae Marcelli.

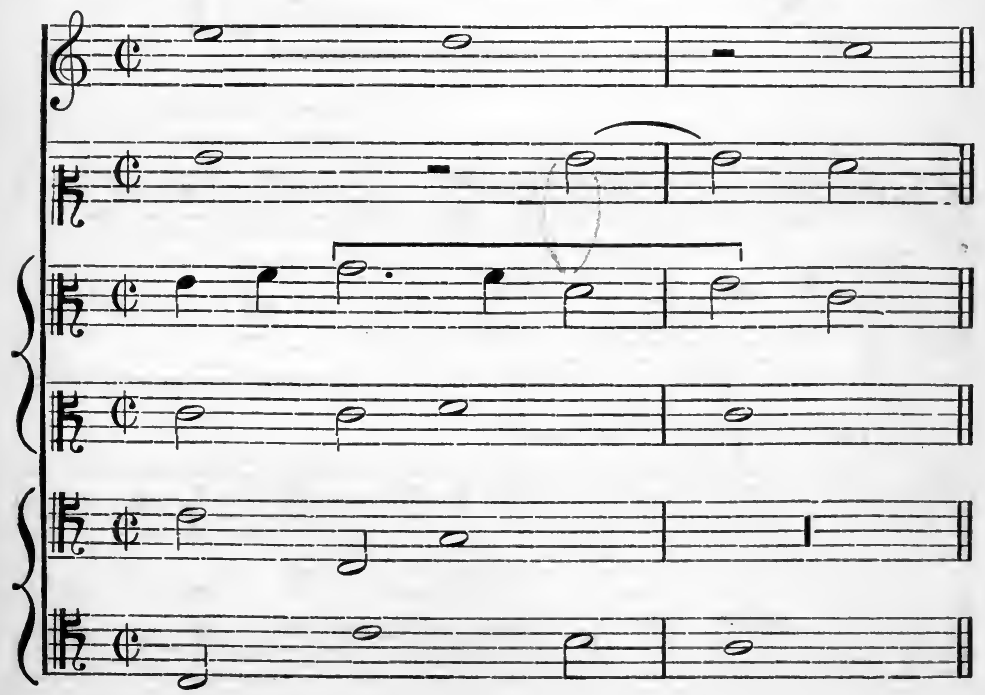




\section{THE ART OF COUNTERPOINT}

(b) As a Melodic idiom.

Busnois (d. $\mathrm{r}_{480}$ ), Je suis venut.

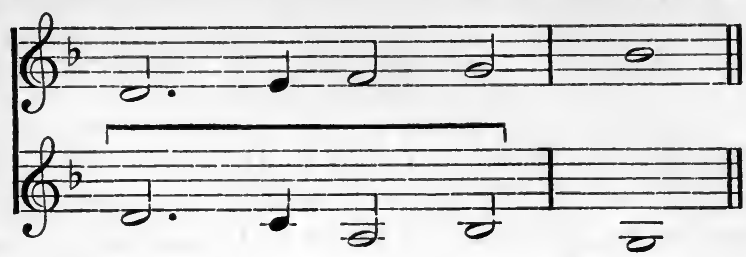

Oreghem, Mass, Cuiusvis Toni.

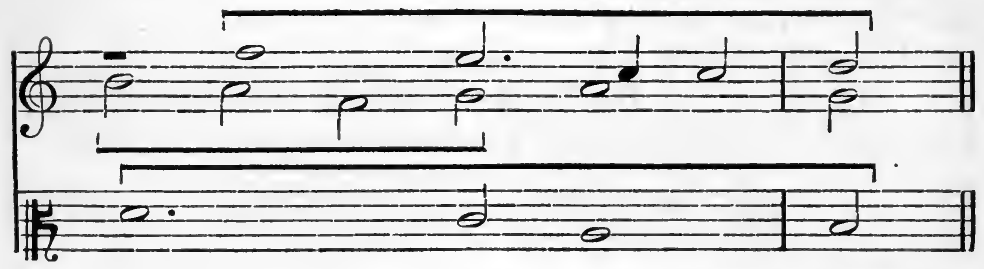

Palestrina, Gloria, Missa Papae Marcelli.

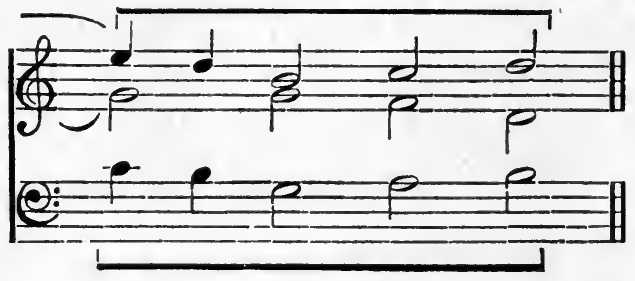

Palestrina, Credo, Missa Brevis.

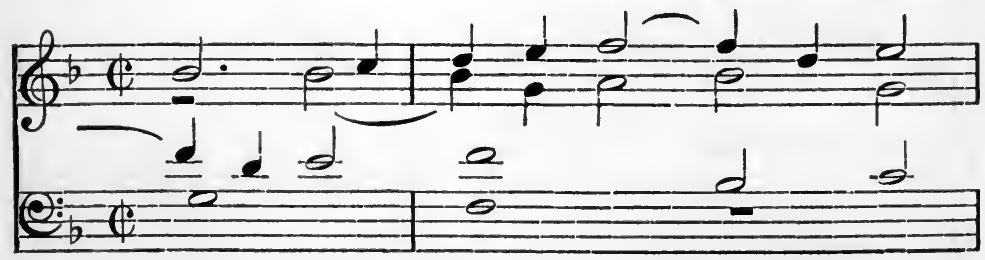

$\Lambda$

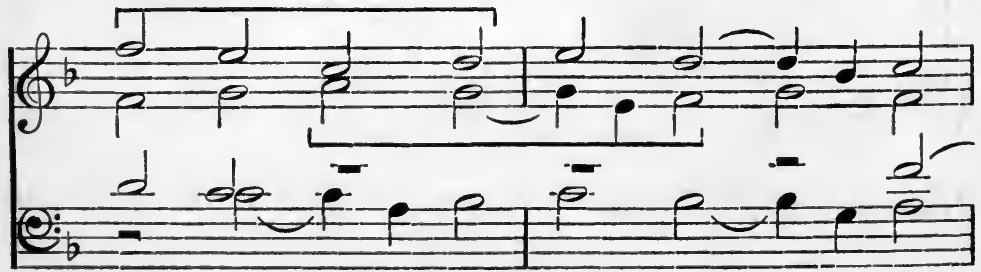




\section{B}
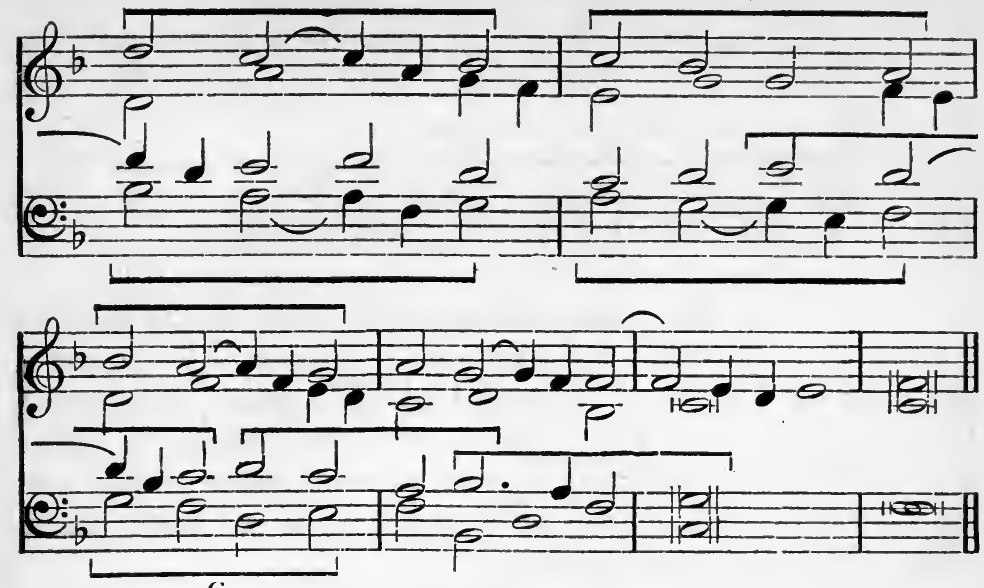

C

It will be remembered that in Chapter III (4) it was stated that the following was inelegant:-

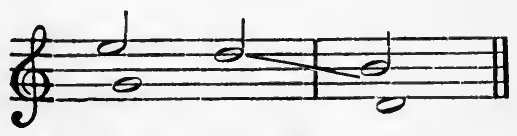

Now here we have three examples of it, A, B, C. But this is due to the melodic curve of the original formula-

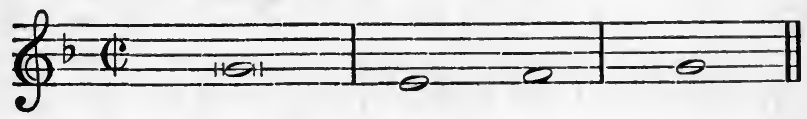

and in the majority of cases the phrasing is-

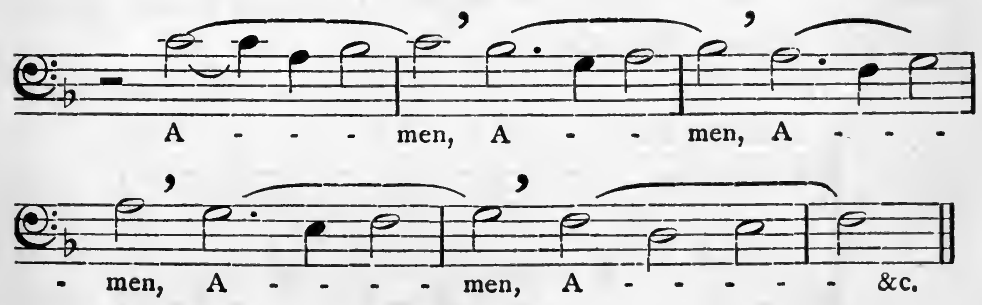

It is true that A and B are phrased- 
A

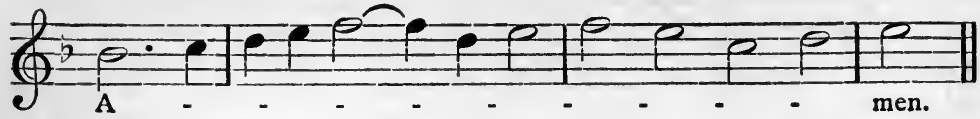

B

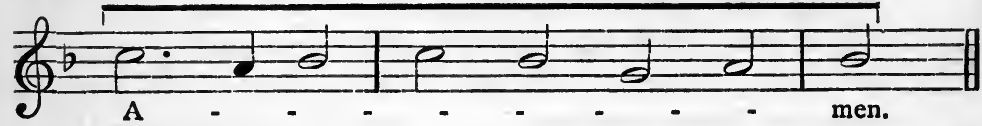

It will be seen that either the melodic curve of the original point or the phrasing makes these justifiable. For general cases the rule given in Chapter III (4) holds good. It is further to be remembered that the constant changes of harmony render the above quite satisfactory in effect: any attempts of this sort with a semibreve C.F. will be found to be harmonically crude, unless the C.F. leap an octave-

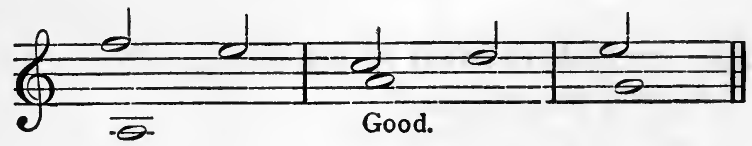

(c)

Palestrina, Sanctus, Iste Confessor.

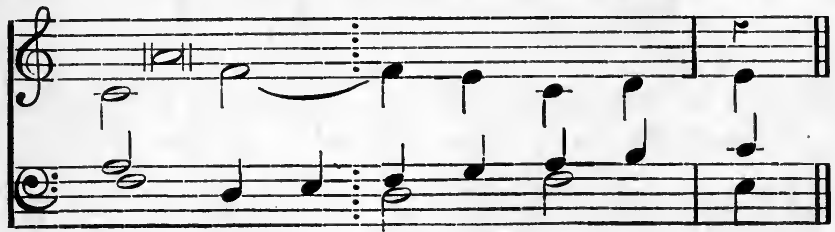

Here we have the Third Species idiom, with the first crotchet tied : both the second and third crotchets may be considered as unessential, or it might be argued that the fourth minim produced a change of harmony.

Palestrina, Ego sum panis.

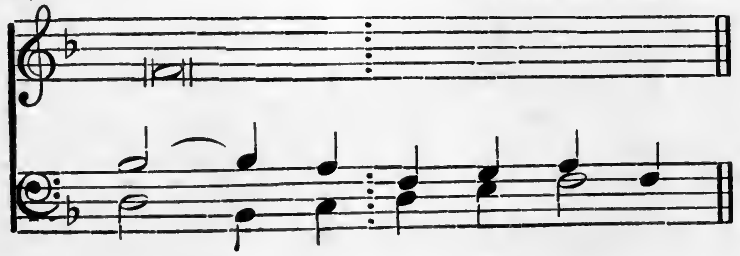


Here the idiom is divided between what corresponds in Scholastic Counterpoint to the second half of one bar and the first half of the next.

The above will serve to show some of the various uses of the Nota Cambiata. It has been said with great force that the device in its origin was 'the result of artistic feeling, and not of intellectual calculation'. Its various uses cannot always be defended by the rules of Discant, which are arbitrary; but they are always justifiable on aesthetic grounds.

No valid argument can be brought against an idiom which was very characteristic of the age, and which should be justified by its elegance alone. Here we have the origin of what we term Changing Notes. Theorists mention the following variants of the original formula :-

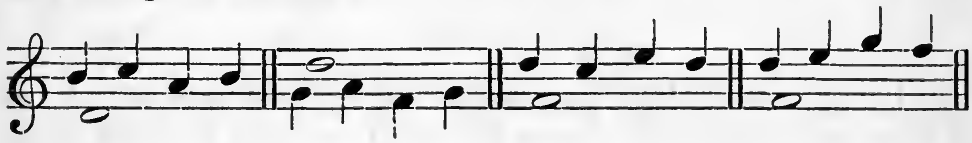

The first two only are recommended, and they should be used with careful discretion. No examples should contain, as a rule, more than two illustrations of their use.

The following is not a use of changing notes, but a change of harmony :-

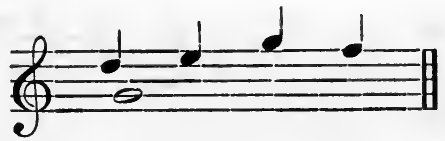

\section{Unessential Notes.}

The remarks made on the use of unessential notes in Second Species apply equally in Third Species, for the following are identical in outline :-

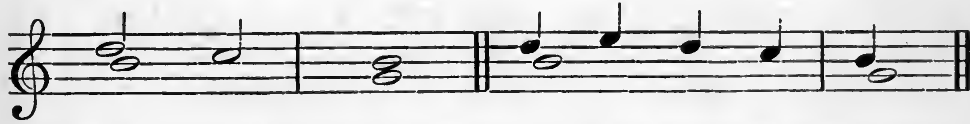

It will therefore be unnecessary to repeat what has already been said. But the Third Species has as a special feature the use of one or two passing notes in a bar between different notes of the 
same harmony. As our considerations in the Second Species covered the progression from the end of one bar to the beginning of the next, our remarks here will be mainly limited to the filling up of the distance between the first and fourth crotchets of every bar. The observance of the rules given in the previous chapter will prevent unmusical writing. The chief point to bear in mind is that, presupposing one harmony in a bar, unessential notes should be common to the scale of the chord used and that of the Tonic, unless such notes are felt to be parts of a modern essential discord, and in that case they must be treated as such-

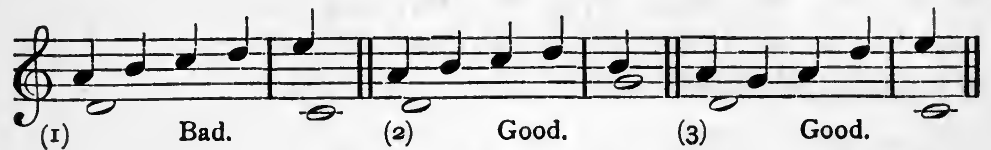

In (I) the mind does not accept the second crotchet B as a change of harmony. In (2) the third crotchet $C$ is felt to be the minor seventh with the resolution deferred.

The criticism depends on : $(a)$ context, $(b)$ mental effect of the harmony.

The following cases should be noted :-
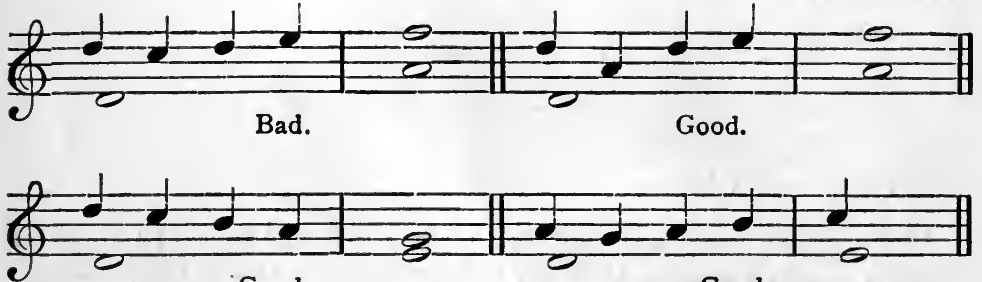

Good.

Good.

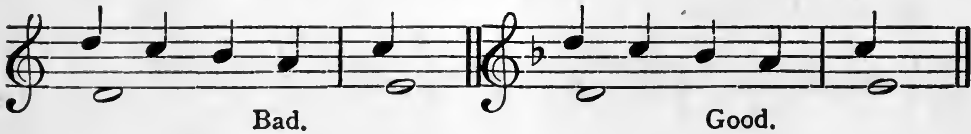

As regards the use of unessential notes over a minor chordal centre, the criticism should be based on the melodic form of the scale. 


\section{THIRD SPECIES IN TWO PARTS}

The following is objectionable :-

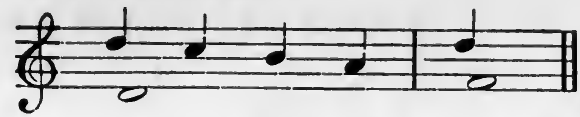

Because the mental effect of the first bar is that it is wholly built upon the triad of $\mathrm{D}$ minor as a centre, its correction would be-

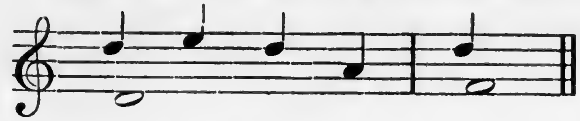

But the next example is good:-

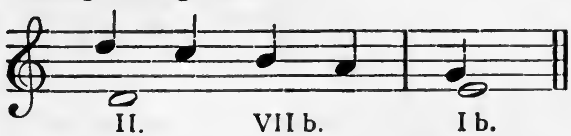

Note the mental effect as indicated by the figuring.

A few more examples may be helpful :-
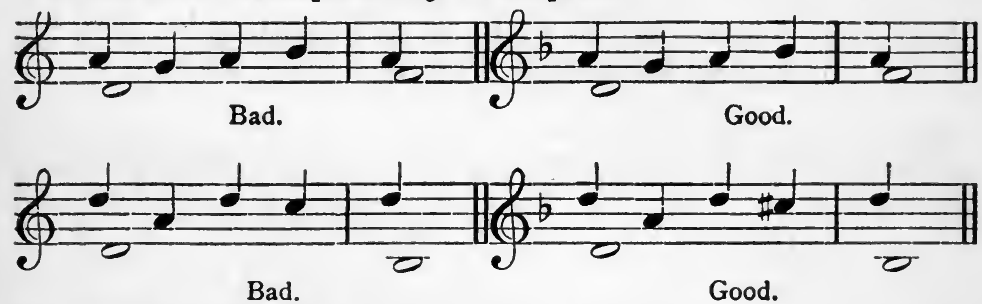

Note the following :-

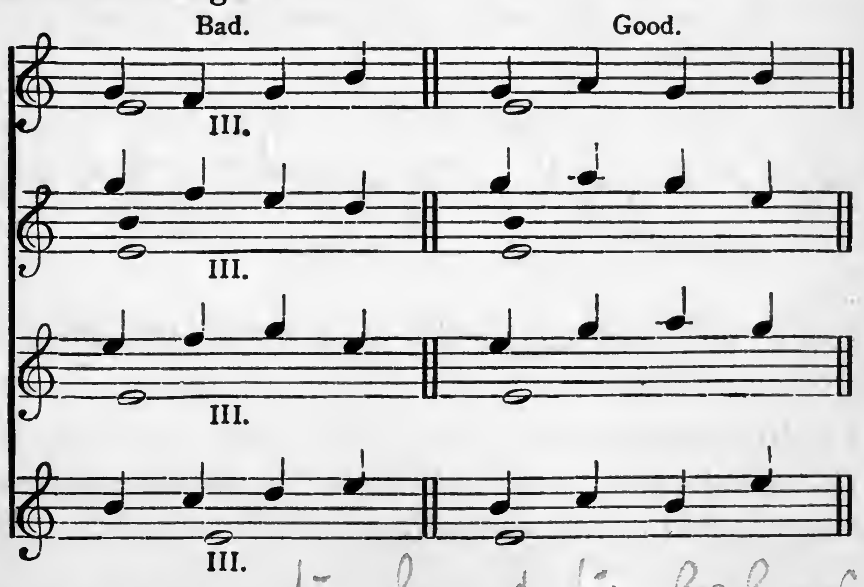




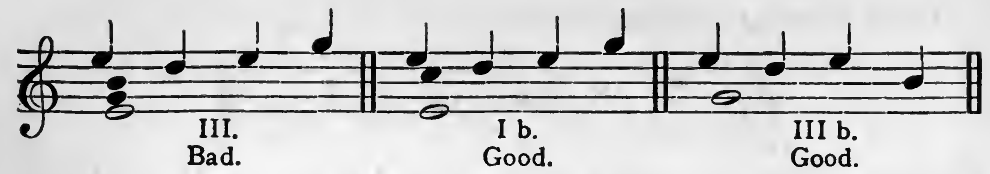

The last is good because of the perfect concord at the second crotchet.

With the subdominant as chordal centre the following are tolerated through common usage :-

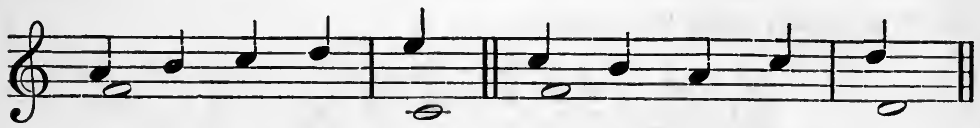

but in more complicated situations such a procedure will require care.

With the dominant as centre the only note likely to cause trouble is the minor seventh. In ascending it may be used, if it finally falls like an ornamental resolution of the seventh-

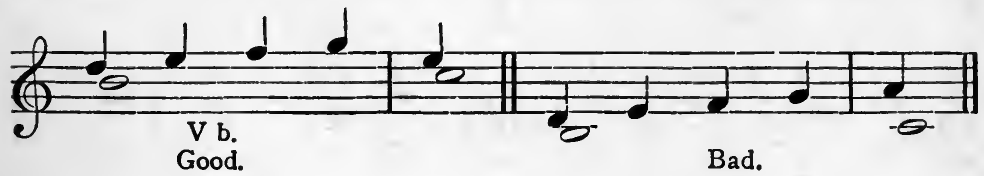

Of course in a descending scale passage it is quite good.

When the submediant is the centre the minor seventh is again the cause of difficulty.

(a)

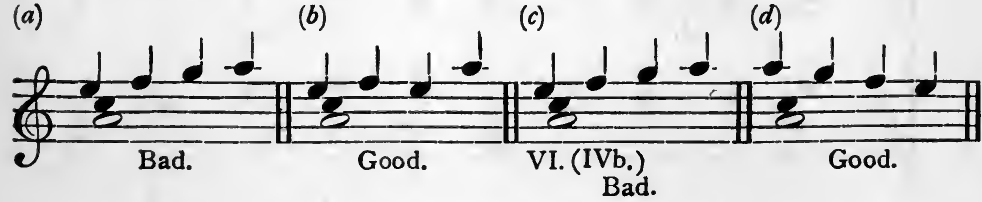

In $(c)$ it would be impossible to say that the change of harmony was felt.

\section{The Minor Mode.}

The melodic form of the scale is used in writing horizontally, the harmonic in writing vertically- 
C minor.

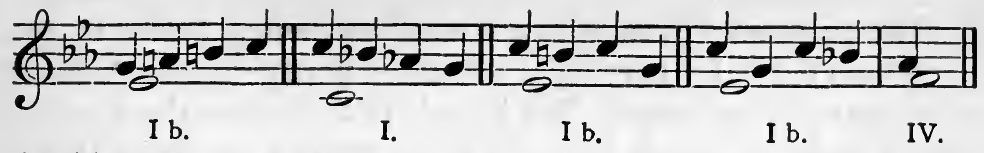

Avoid such things as :-

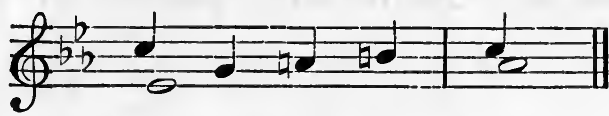

The reason of the bad effect is that A natural, and A flat, though causing no false relation (for A natural is unessential) are in too close juxtaposition. Further, the $B$ natural with the $E$ flat, considering the next progression, has to modern ears the effect of being a dominant minor thirteenth on $\mathrm{E}$ flat, and again the $\mathrm{A}$ natural is the cause of the crude effect. Note in IV. the following is good:-

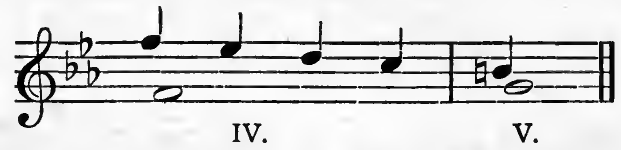

The reason may be found by applying the harmonic criticism.

The following should be carefully compared :-

C minor.

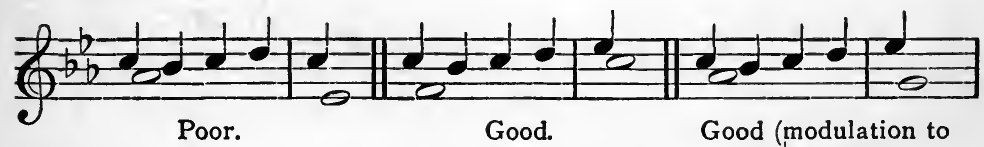

Eb major).

Two notes of the same name chromatically altered may not follow one another consecutively-

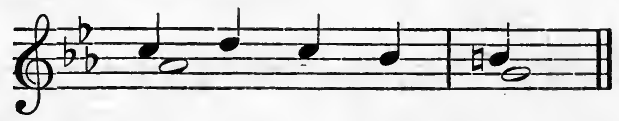

To a person of genuine musical instinct the foregoing remarks may seem superfluous. But a great many students are in the habit of working Counterpoint mechanically, and it is. hoped that the above considerations will help them towards a really intelligent use of unessential notes. 


\section{Consecutives.}

The rules as to consecutives must be limited partly by the view taken of the harmonic structure, partly by the relative complexity of the score. We have here to discuss the simplest cases, those in which four notes are written to one. It will be observed that the following produce an objectionable effect :-

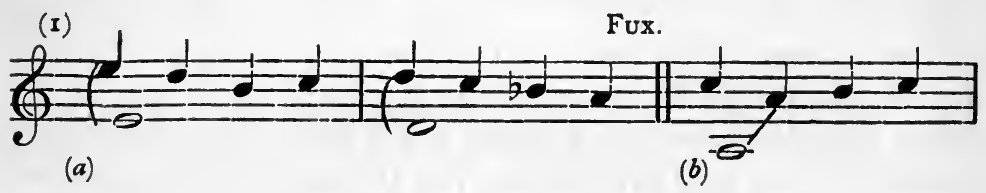

FÉtis.

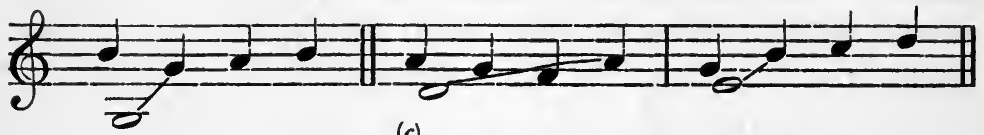

(c)
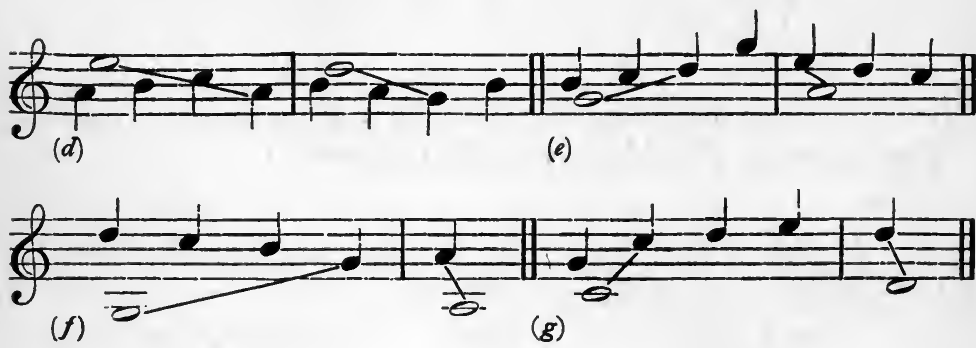

But no exception can be taken to:-

(2)

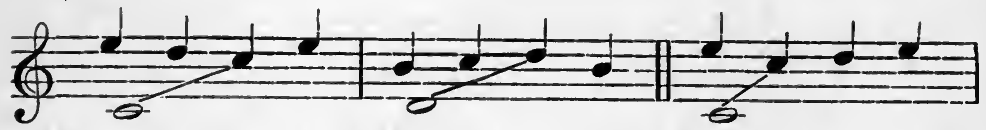

(a)

(b)

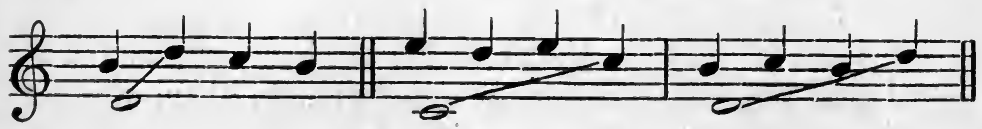

(c) 


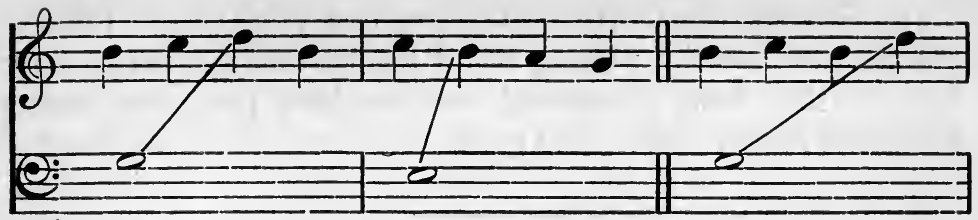

(d)

(e)

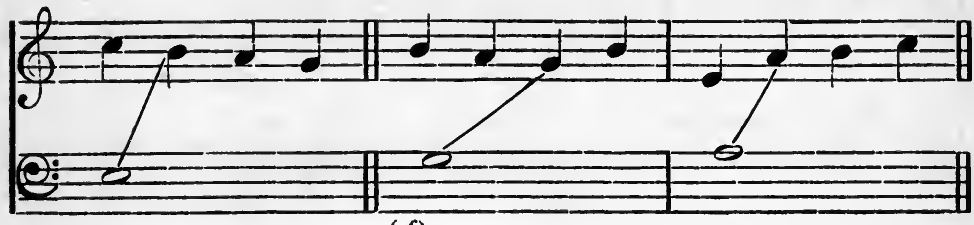

$(f)$

The good or bad effect does not depend entirely on the distance between the consecutives. The rules here codified refer to a score in which First and Third Species only are combined. Modifications, in cases of more complex movement, will be discussed as occasion arises.

In the following classification the use of only one harmony in each bar is presupposed, and it is also necessary that both the crotchets involved are harmony notes.

Case I. In which one or both of the crotchets involved fall on an accented part of the bar, e. g., the first or third crotchets.

Consecutive fifths or octaves are forbidden

(A) Between the first crotchets of successive bars (see above I $a$ ).

(B) Between the second crotchet of one bar and the first of the next, if the former be taken by leap ( $\mathrm{I} g$ ).

(C) Between the third crotchet of one bar and the first of the next (I $e$ ).

(D) Between the third crotchets of successive bars, or the third crotchet of one bar and the second of the next, unless the placing of all the harmony notes of each bar in the form of vertical combinations produces correct harmonic progression. See below ( $3 a$ and $b$ ).

(E) Between the fourth crotchet of one bar and the first of the next (I $f$ ). 
(F) Between the fourth crotchet of one bar and the third of the next, unless the placing of all the harmony notes of each bar in the form of vertical combinations produces correct harmonic progression. See (3c).

(3)
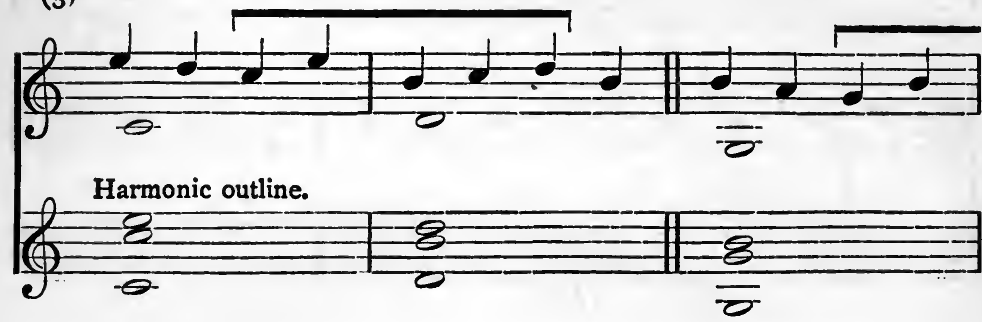

(a)

(b)

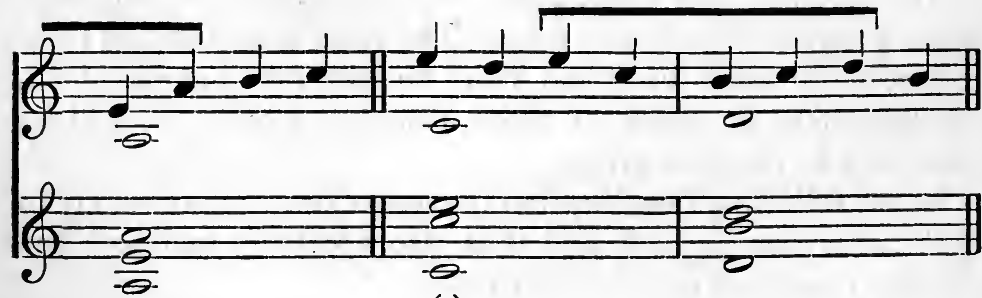

(c)

All good.

Case II. In which neither of the crotchets involved falls on an accented part of the bar.

Consecutive fifths and octaves are forbidden, unless the placing of all the harmony notes of each bar in the form of vertical combinations produces correct harmonic progression

(A) Between the second crotchets of successive bars.

(B) Between the fourth crotchet of one bar and the second of the next.

(C) Between the fourth crotchets of successive bars.

The distance of four crotchets between two consecutives saves the bad effect. So they cannot occur between :-

(a) The first of one bar and the second, third, or fourth of the next.

(b) The second of one bar and the third or fourth of the next. 
(c) The third of one bar and the fourth of the next.

In Third Species it may be taken as a general rule that the harmony is only changed on the first and third crotchets (although it is not wrong to change it on the second or fourth), so that any fifth appearing on the second or fourth crotchets, if approached and quitted by step, is not to be regarded as a harmony note, though concordant with the C.F. Thus $2(d)$ and $2(e)$ are both good.

It is of course obvious that consecutives cannot occur between the first and third of three harmonies ; but in the simple combination of First and Third Species it is not always easy to discriminate, unless the Third Species approach the new harmony by disjunct movement-
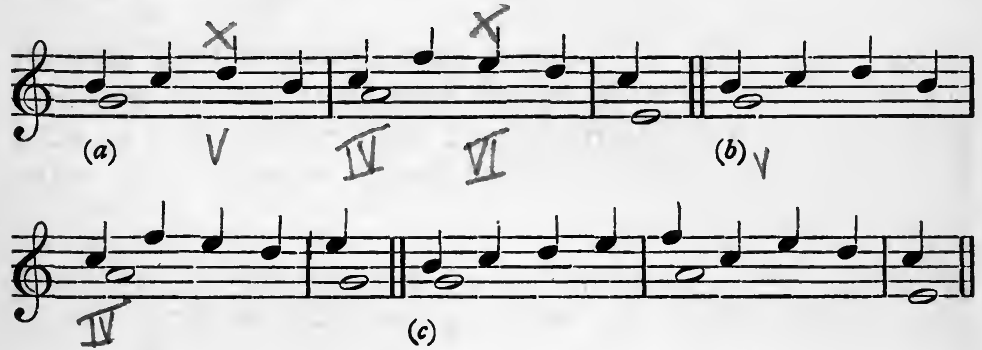

Now in $(a)$ the mental effect is that all the second bar is IV b. ; therefore the $E$ on the third crotchet is unessential. $(b)$ is an open point. Some theorists argue that if a sixth is followed by a fifth above the C.F. the latter does not produce the effect of change of harmony. The question seems to depend upon

(I) The relative value of the notes.

(2) The general harmonic rhythm.

(3) The position of the notes in reference to accent.

(4) Chiefly, on the way the fifth is followed.

(c) shows a clear change of harmony on the second half of the second bar. In all the above cases, however, the fifths are unobjectionable, for in $(a)$ and $(b)$ the second fifth is either separated from the first fifth by an, intermediate harmony or else is unessential. It may be objected by some that this is a complicated and laborious statement of the case. But any one 
who takes the trouble to grasp the principle involved will never be in doubt as to the good or bad effect of a passage in reference to consecutives. The principle has three aspects: (I) the relative prominence of the notes; (2) the harmonic outline; (3) the relative distance of the consecutives.

8. The leap of a sixth (major or minor) should be avoided in notes of such short time value as crotchets; the latter is the less objectionable.

9. The extremities of a melodic passage should not form the tritone-

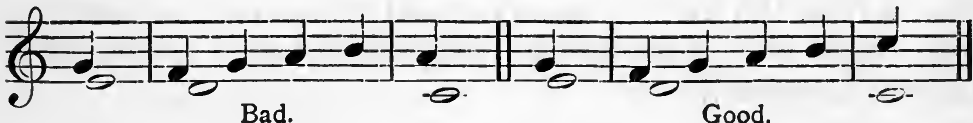

10. There is a great confusion as to the use of what we term the fifth of the root in the bass. Such an expression, however, has no place in Counterpoint, and we need only bear in mind the proper standpoint. The following examples will make matters clear :-
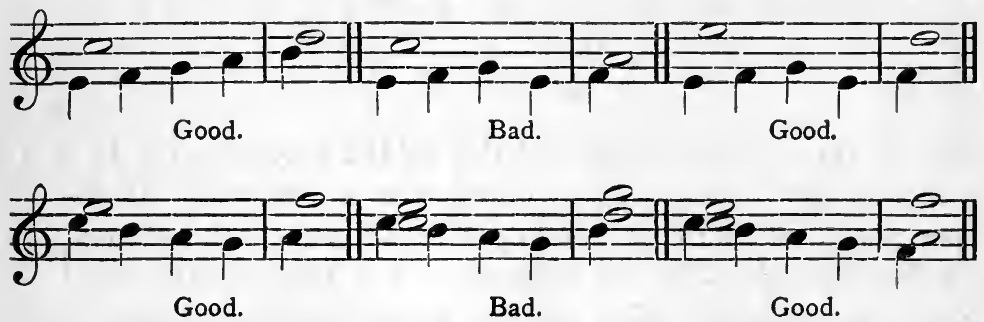

The following should be avoided as it gives on the fourth crotchet an impression of a ${ }_{4}^{6}$ wrongly quitted.

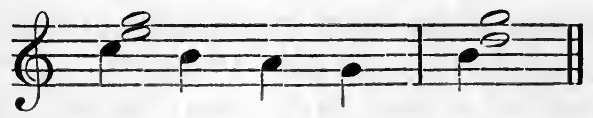

Not good.

If the lower part forms a fourth with the higher part it must of course be treated as a discord, and be approached and quitted conjunctly. The same reasoning applies to the dissonant fifth. This introduces an important point. It is quite true to say that 
a diminished triad in root position may not be used; then, some one will say, surely the following is incorrect :-

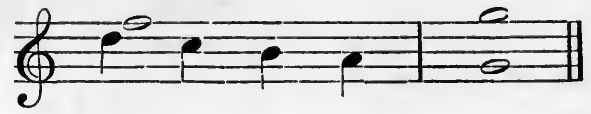

And surely a ${ }_{4}^{6}$ is being used on the last crotchet. Time after time this difficulty has arisen in the minds of students owing to a confusion of principles.

The contrapuntal analysis is:-the first crotchet consists of D F a concord; the second and third crotchets are passing notes, approached and quitted by conjunct degrees. The writers of the Polyphonic Period did not regard the combination on the third crotchet as a dissonant triad, or that on the fourth crotchet as an implied second inversion of the chord used at the beginning of the bar. $\mathrm{B}$ to $\mathrm{F}$ and $\mathrm{A}$ to $\mathrm{D}$ were simply treated as discords.

II. Avoid disjunct movement throughout a bar.

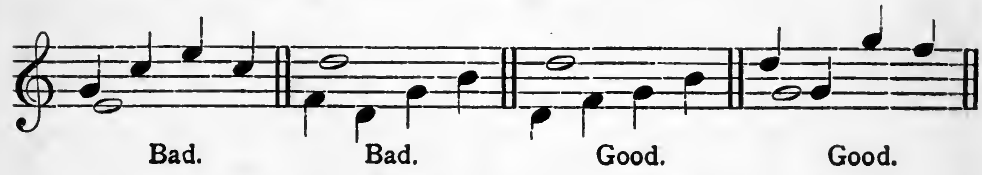

12. The unison is allowed under the same conditions as in Second Species.

13. Never run into the unison: it is permissible in a case of emergency to run through it-

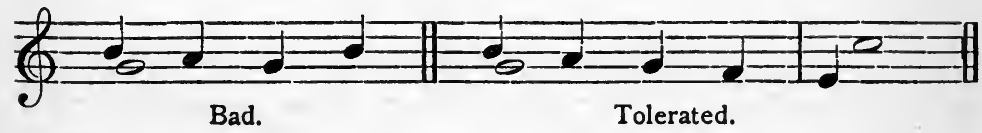

14. The employment of the ninth and major seventh as auxiliary notes on the third crotchet is liable to lead to pernicious results in actual composition; especially when the Third Species is below the C.F.-- 


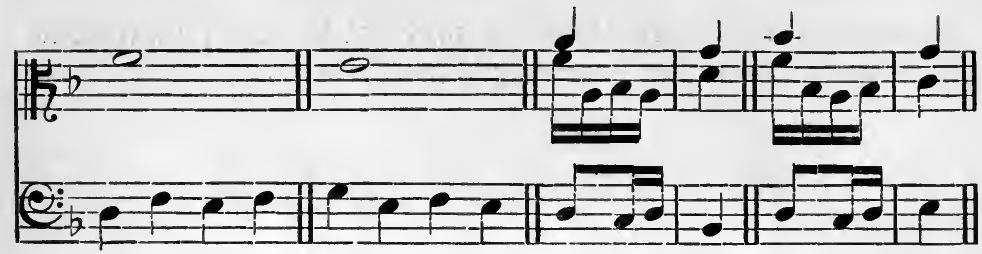

It has been said by some that auxiliary notes should never be used on the third crotchet of the bar-

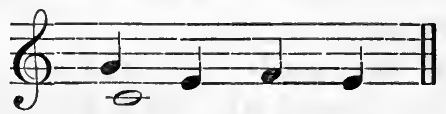

and it is noteworthy that it is Palestrina's practice to use either a true passing note or a harmony note on the third crotchet. But it is not forbidden to use auxiliary notes on the third crotchet ; indeed, in Combined Counterpoint, their use, together with other notes, often produces most satisfactory effects.

I5. The following are the usual cadences:-

(I) Major Mode.
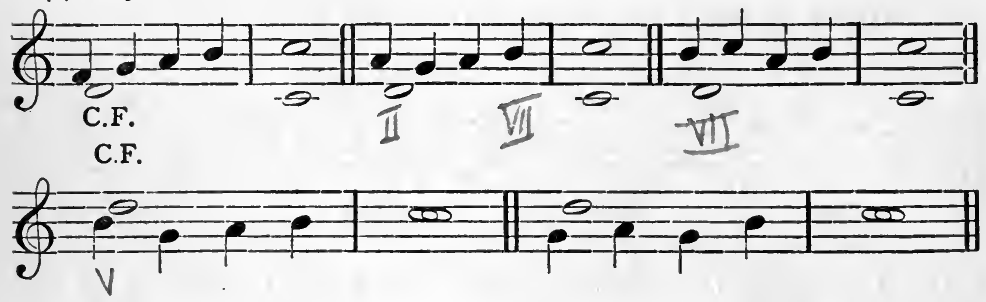

C.F.

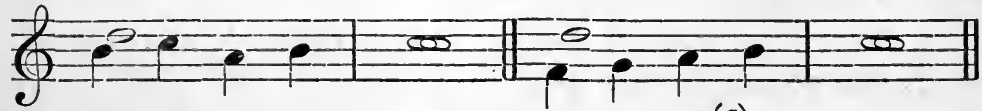

(2) Minor Mode.

(a)

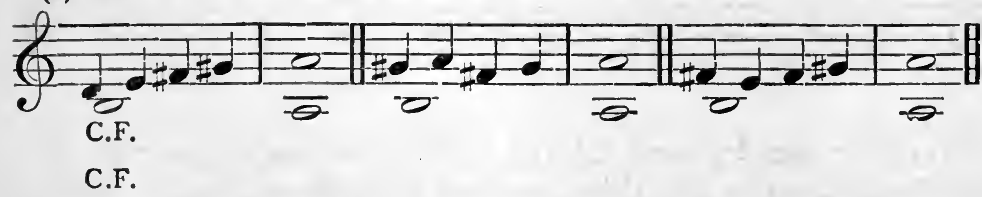

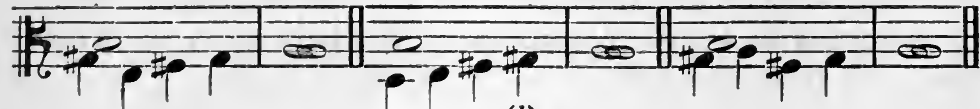

(b) 
$(a)$ and $(b)$ are only available as the completion of the scaleC.F.

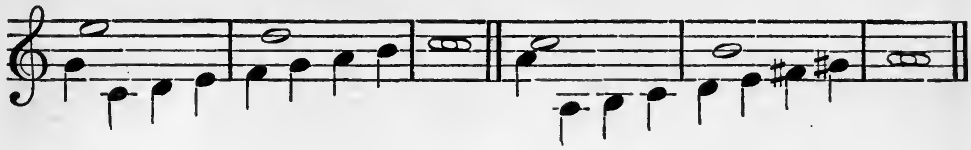

It is unnecessary to add the figuring to the following examples :-

First Mus. B. Cantab., May, I903.
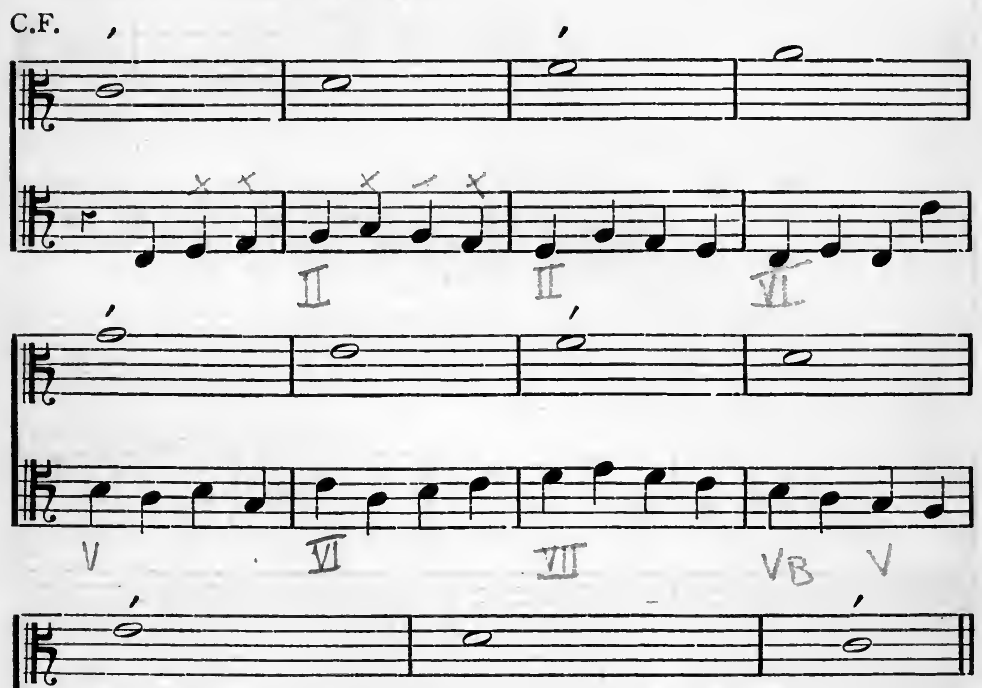

$+\frac{1}{1+6}$

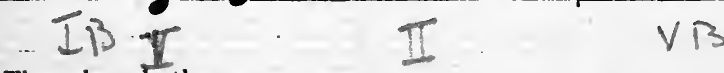

Three-bar rhythm.

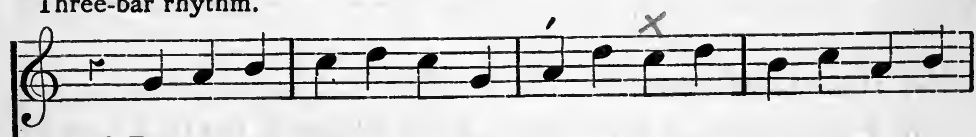

C.F.

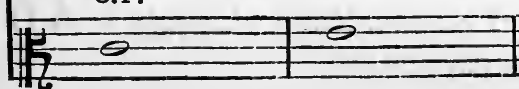




\section{THE ART OF COUNTERPOINT}
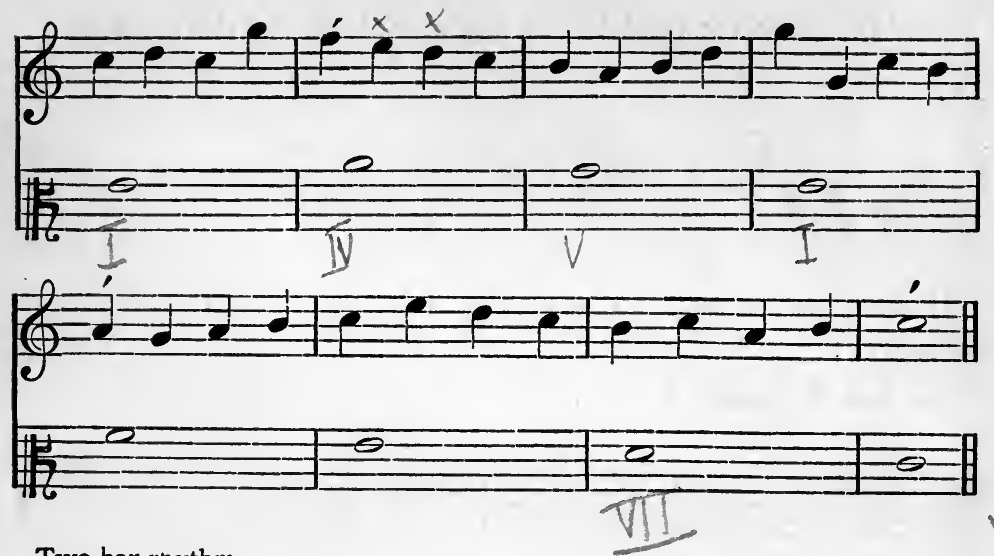

Two-bar rnythm.

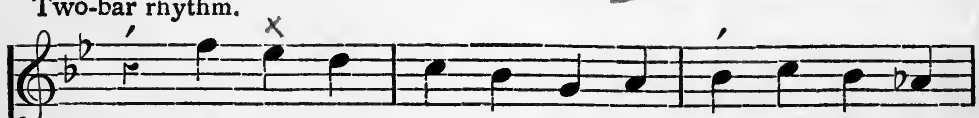

C.F.

(a)

(b)
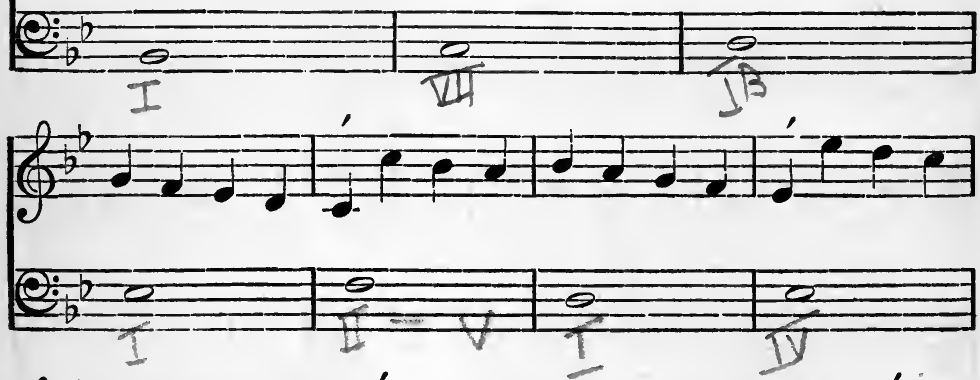

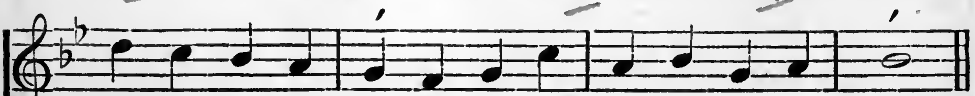
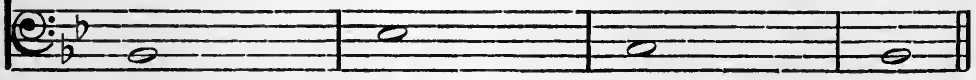

(a) Nota Cambiata.

(b) The somewhat hard effect of the tritone $\mathrm{E}$ flat to A may be softened by the introduction of an accidental : though as $\mathrm{A}$ is unessential, the matter is of no importance. 
C.F.

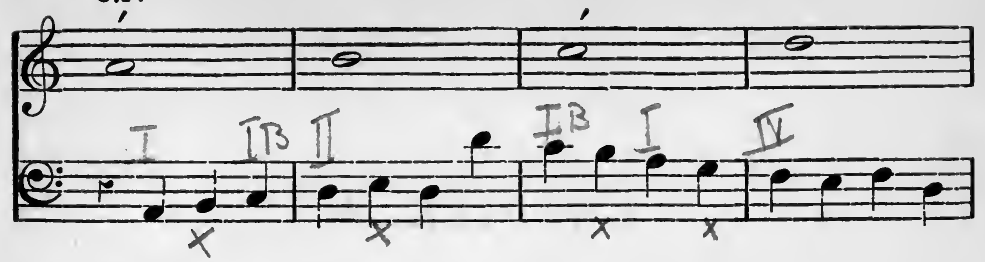

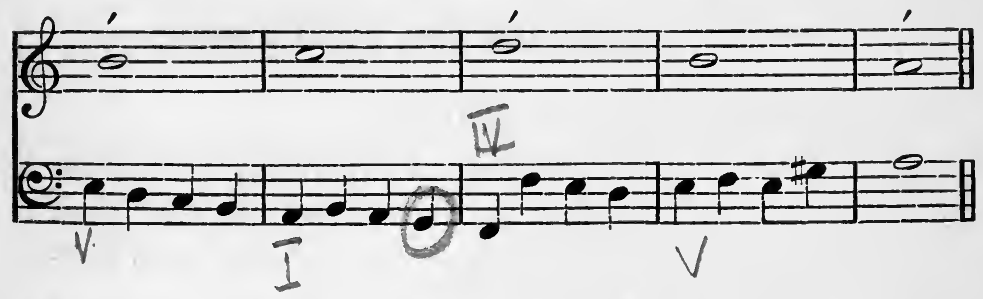

:

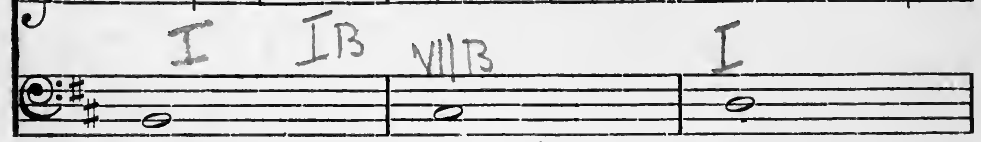

C.F.

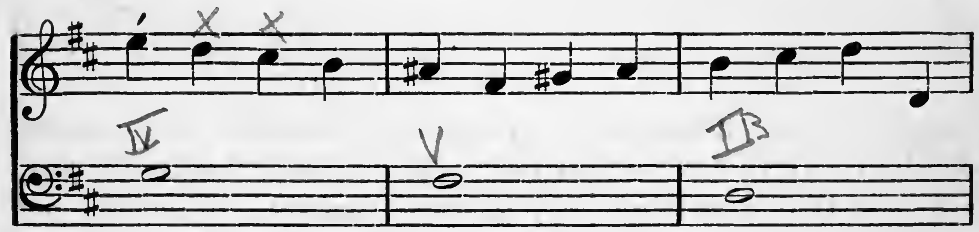

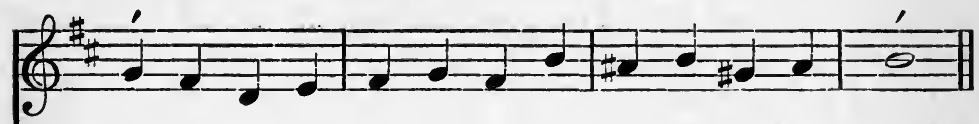

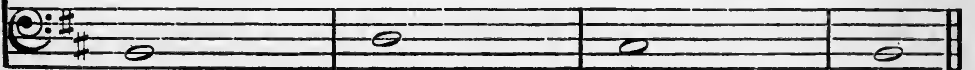

VI IB III I 


\section{CHAPTER V}

\section{FOURTH SPECIES IN TWO PARTS}

\section{Syncopation.}

I. ONE of the most obvious ways of obtaining variety of effect, besides the use of passing notes, is to withhold the movement of one part while the other proceeds one step forward, and thereby comes to another implied harmony.

(a) If the part that is thus arrested in its motion produce dissonance on the first half of the bar, this is relieved by its moving one step downwards, and thus merging into consonance again-

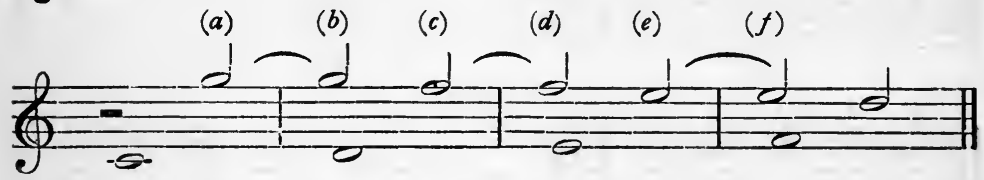

Here we have examples of syncopated discords. The second minim of every bar must of course be concordant with the C.F. as it is the 'resolution' $(c)$ of the 'discord' $(b)$ which was 'prepared' at $(a)$. The resolution $(c)$ forms the preparation of a further discord $(d)$. The combinations $a b, c d$, ef form what are termed 'suspensions'.

(b) If the first minim of the bar is concordant with the C.F. it is free to leap or move by step to another concord upwards or downwards, under certain restrictions stated below-

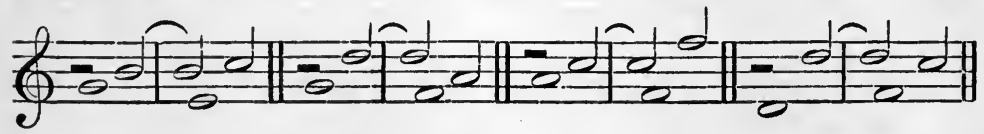

These are termed 'syncopated concords'. 
2. It is true, as far as uncombined Counterpoint is concerned, to say that Syncopation is a simple variant of First Species, in that the accent in the Counterpoint is displaced-

Fourth Species.

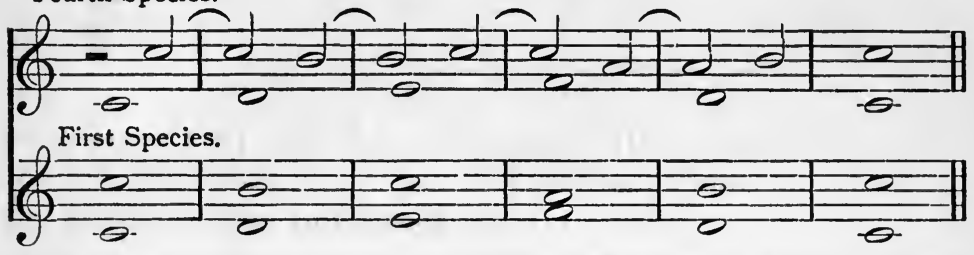

This view requires modification when we come to treat of Combined Counterpoint ; but for the present it forms a safe test as to faulty harmonic progression-

Fourth Species.

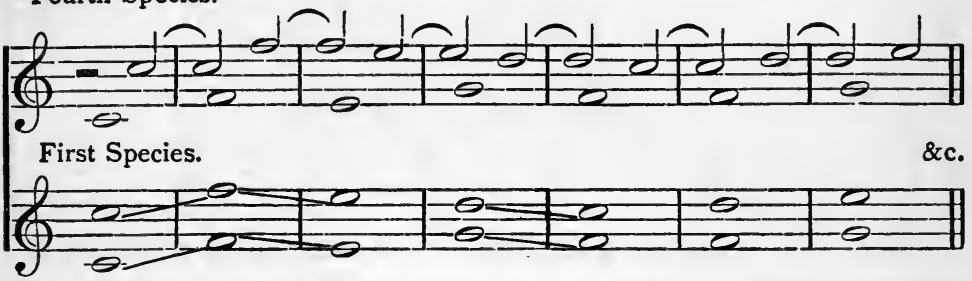

This test, as will be shown later, is not formed on the practice of Palestrina. Modern conditions, where rhythm and harmonic substructure are salient features, do not always admit of the same principles of criticism as those of the Polyphonic Period. At the same time theorists have wrongly applied the test of First Species as regards consecutives to all cases of syncopation. This matter will require careful discussion (see pp. I68-I 72).

But for the present the following rule holds good :-Consecutives between the second beats of successive bars are incorrect, whether direct or exposed-
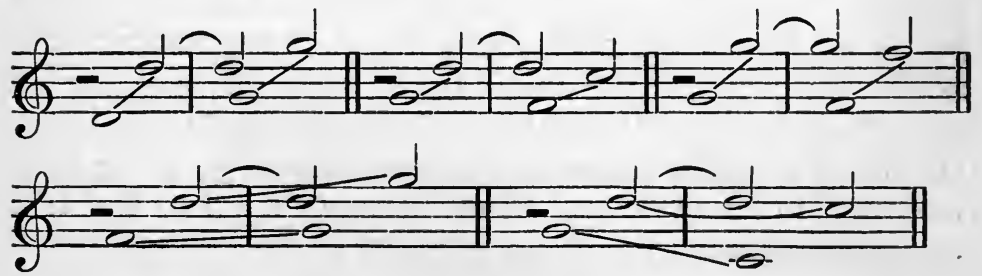
3. The Counterpoint begins on the second minim of the first bar, and must form a perfect concord with the C.F.-

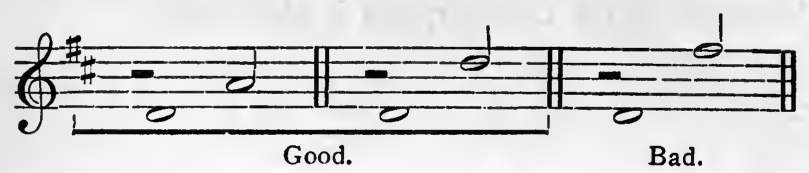

In the last bar, in all Species, the C.F. and its Counterpoint consist of notes of equal value.

4. Let us now take the consonances, and experiment in the formation of syncopated discords.

(A) Above the C.F.-

Consonances.

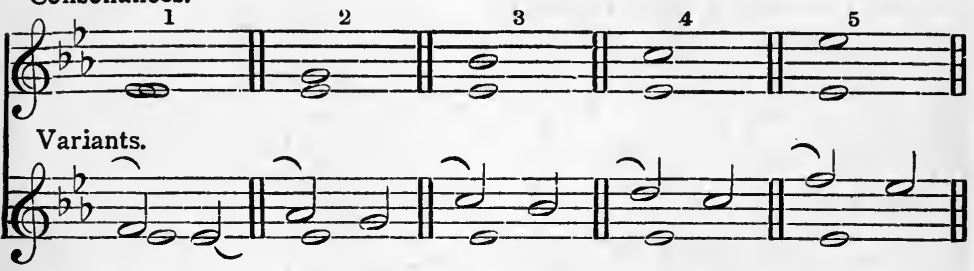

(I) is now considered too harsh and congested to be of any service; though found in Palestrina and Fux.

(2) (4) and (5) form what we know as the suspensions-

$$
4-3 ; 7-6 ; 9-8 \text {. }
$$

(3) is not, strictly speaking, a suspension at all ; it is merely a case of movement from concord to concord, 6 to 5 , and is termed a syncopated concord.

(B) Now experiment with syncopations below the C.F.-

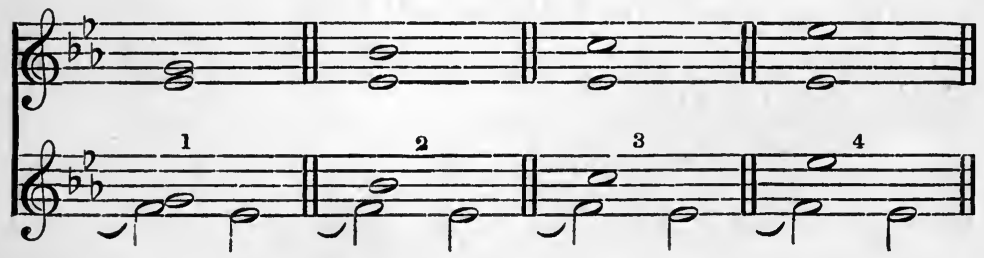

(I) and (2) are what we term the suspensions $2-3 ; 4-5 ;(4)$ is too harsh to be available. Some theorists allow it, and it is occasionally found- 


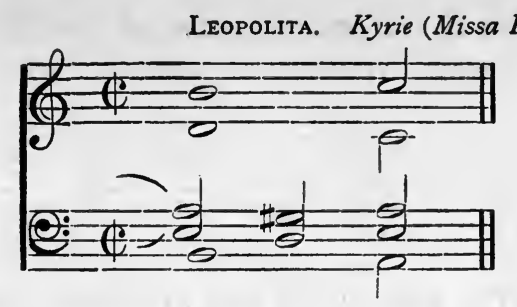

This is smoother than any other $7-8$, for obvious harmonic reasons, e.g. from a modern point of view both $E$ and $D$ are essential. There is also an example in Tye's Mass, Euge Bone (Agnus Dei)-

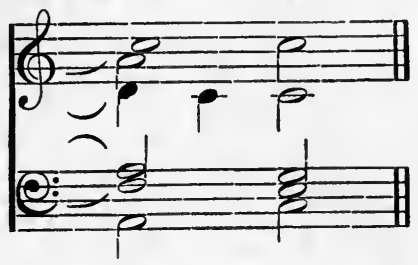

But these are isolated examples, and quite exceptional.

(3) is subject to the same criticism as (3) above, that is, we have here a syncopated concord.

5. The implied harmony of the suspensions is as follows:-

(a) The 9-8 may be used above any note except the leading note, and implies the harmony ${ }_{3}^{5}$ or ${ }_{3}^{6}$ -

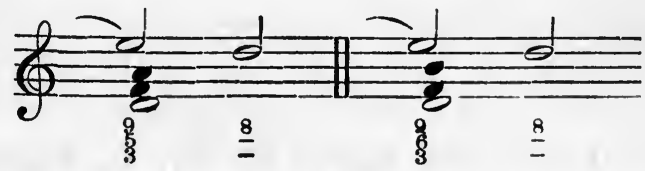

(b) The 7-6 may be used over any degree of the scale, and implies the accompaniment of the third.

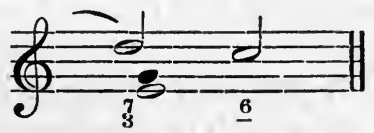

(c) The 4-3 is not used over the second or third degrees of the minor scale nor on the leading note in both modes, because 
it always implies the accompaniment of the fifth, and implied forbidden triads would result-

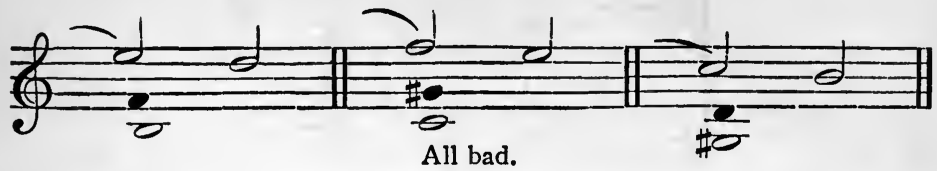

But in two parts, just as in the previous Species, a strict adherence to these restrictions is hardly necessary, especially if it causes the Species to be broken, and a bar of Second Species interpolated. Palestrina generally uses the perfect fourth in syncopation. He does, however, use the augmented fourth-

Feria vi, In Parasceve.

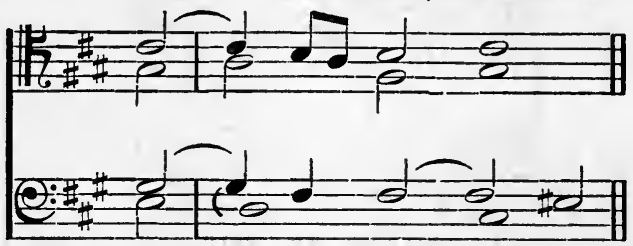

We need not therefore hesitate to write it, if the harmony be smooth.

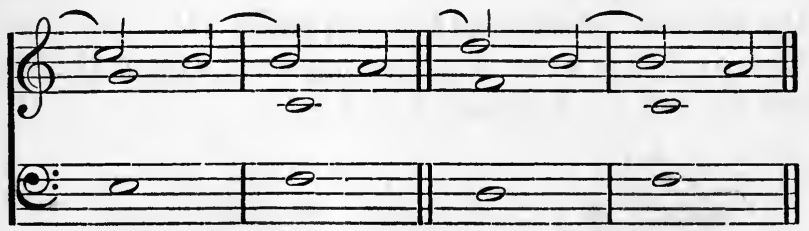

Good.

Ügly.

It is not at first quite easy to see why the fourth was not allowed to proceed to the third accompanied by the sixth only as a true harmony note. But from a study of Palestrina it would seem that the accompaniment of the sixth was restricted to two special cases.

(a) Where the sixth proceeds to the fifth, as the fourth proceeds to the third. The sixth is usually approached conjunctly, or treated as a syncopation, and it is felt to be in the nature of a passing or auxiliary note- 
Gloria, Iste Confessor.

Sicut cervus.

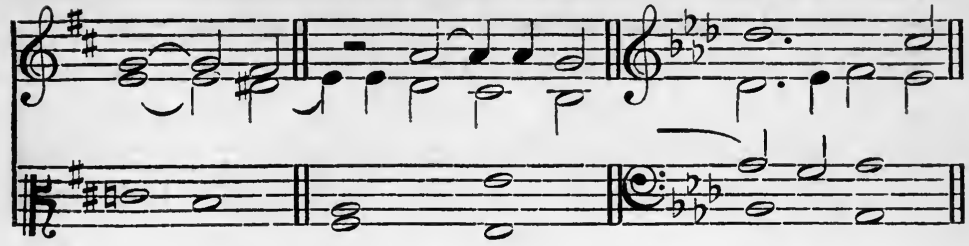

( $\beta$ ) Over a pedal, where the sixth becomes the real bass note for the time being-

Benedictus. Missa Brevis.

Gloria, Iste Confessor.

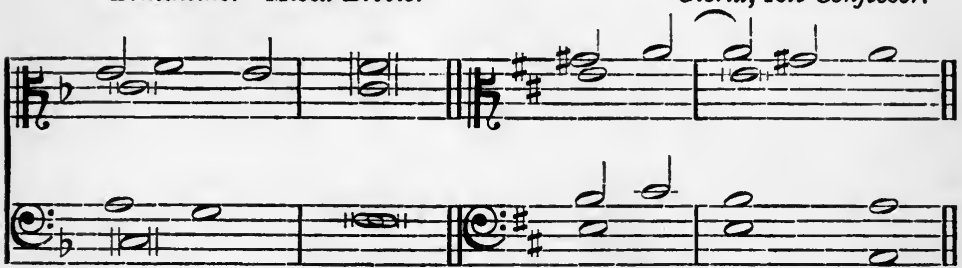

These idiomatic uses formed in men the habit of regarding the sixth as a passing note, so that they never kept the sixth stationary as the fourth proceeded to the third. And this is of course the ultimate origin of our ${ }_{4}^{6}{ }_{3}^{5}$.

(d) The $6-5$ is accompanied by the third

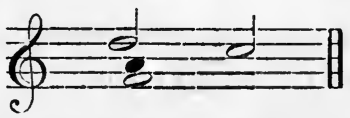

and may either imply two harmonies in a bar or one.

The 5-6 is not what moderns term a retardation, but the use of two concords, the first being syncopated-

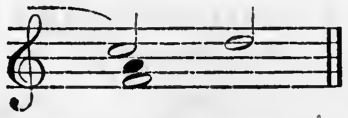

No upward resolving discord is allowed.

From an absolute point of view there is no possible objection to upward resolving discords. But we are here learning the first steps in the employment of dissonance, as a means of variety, not the later development of its possibilities. To sum 
up, the note that prepares the discord (the second minim of the bar) must be concordant with the C.F. and the discord is resolved by moving one step downwards-

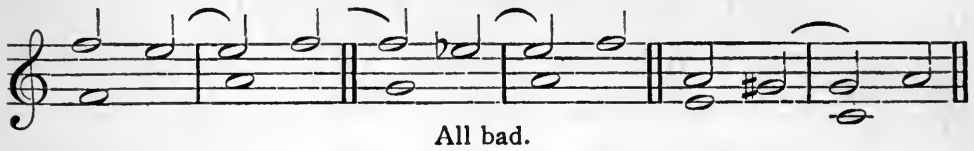

Below the C.F. the implied harmony is as follows :-

(a) The $2-3$

(b) the $4-5$

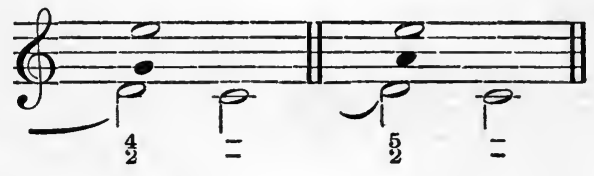

(c) the 5-6
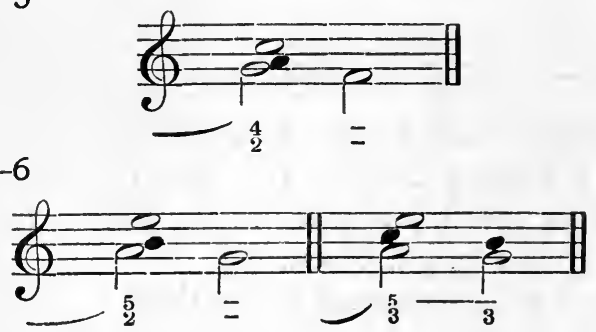

It is better not to use the diminished fifth as a suspended discord, on account of the harsh effect.

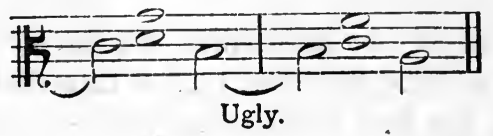

(d) the 6-5 (e.g. two harmonies). This may not be used as a retardation-

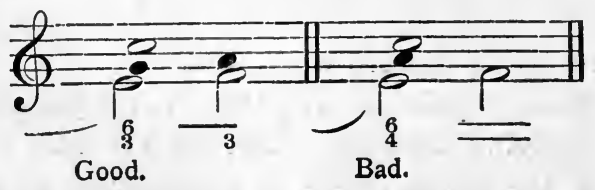

6. It has been pointed out that the second minim of the bar 
must always be concordant with the C.F. The following are obviously incorrect :-

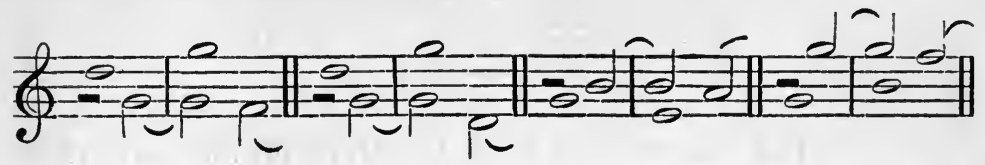

7. The False Relation of the Tritone may be found by reducing the score to First Species-

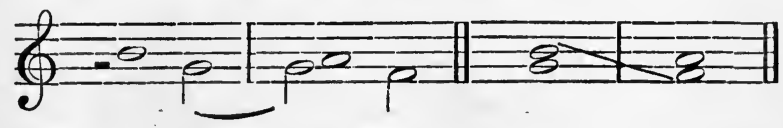

8. Two harmonies may of course be used in any bar. The first minim of the bar is often felt to be a repetition of the last minim of the previous bar with a new harmony-

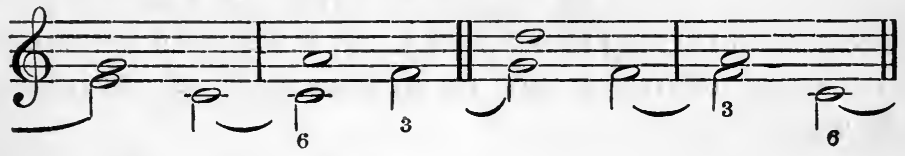

9. Avoid melodic repetition-

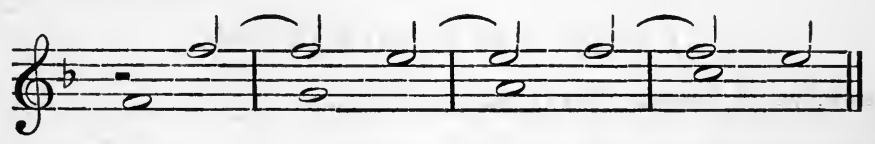

ro. The syncopation should not be broken, unless it involves melodic monotony, or unsound harmonic progression; in these cases a single bar of the Second Species may be interpolated-
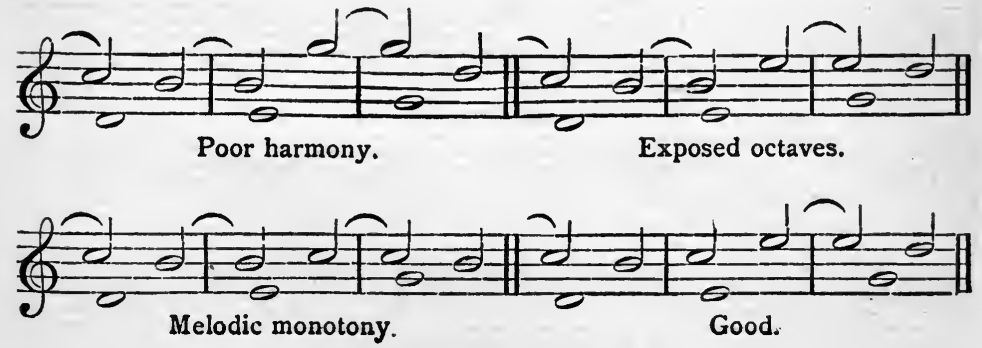
I I. The unison is allowed on the second beat of the bar-

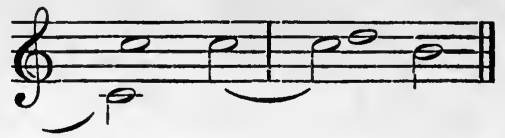

12. The last note but one of the Counterpoint must always be the leading note.

13. The Cadences are as follows:-

C.F.

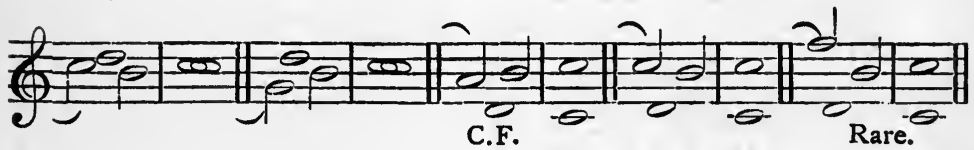

If it is impossible to use syncopation in the Cadence, the Second Species may be employed. The last Cadence given above does not belong to the period. It is a modern innovation, and may be tolerated only in circumstances of exceptional difficulty.

\section{Examples in Fourth Species.}

First Mus. B. Cantab., May, 1904.

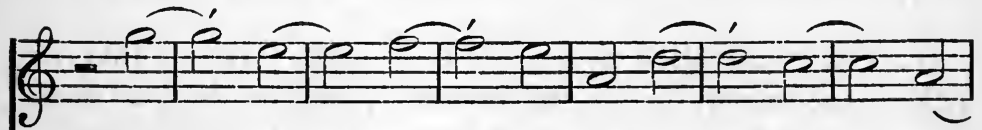

C.F.

(a)

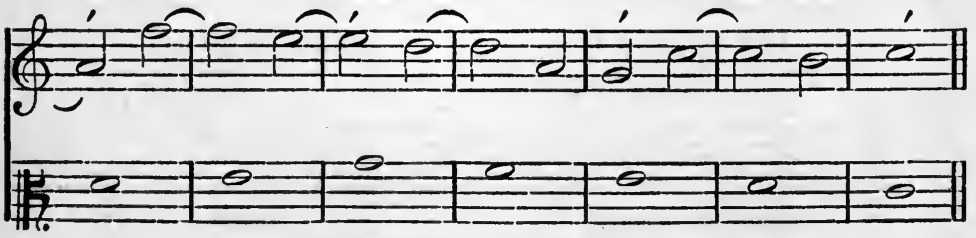

(a) Broken to avoid monotony. 
C.F.

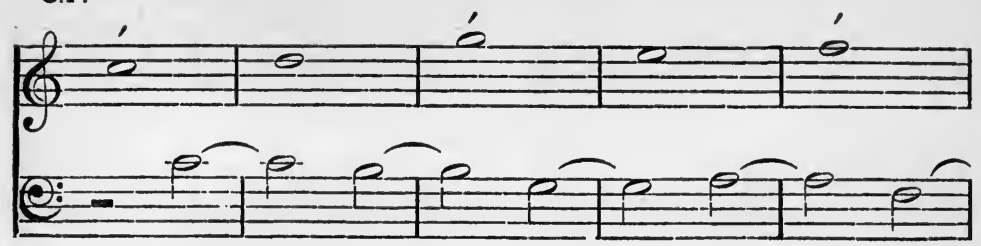

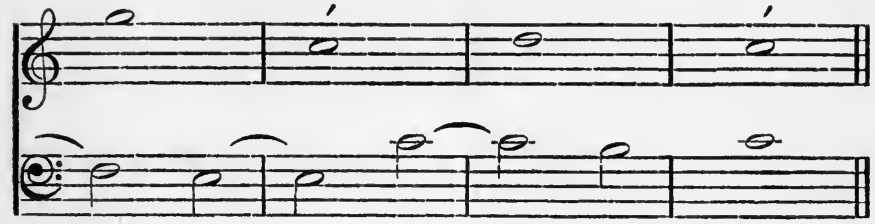

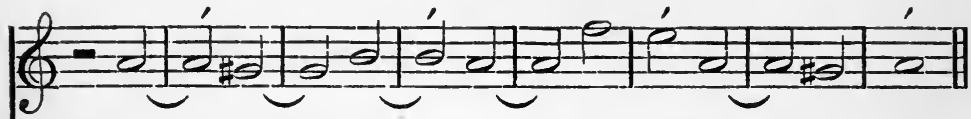

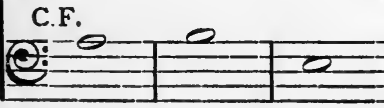

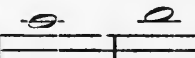

$T^{2} T^{2}$

C.F.

(1)

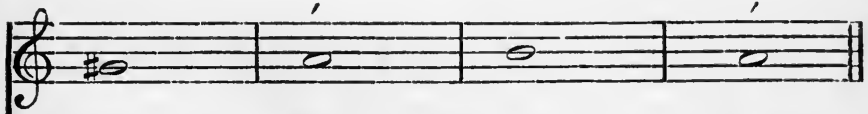

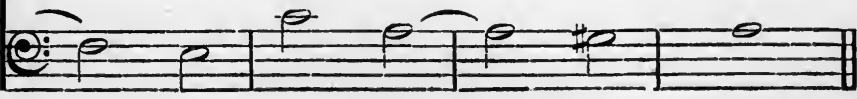






\section{CHAPTER VI}

\section{FIFTH SPECIES IN TWO PARTS}

\section{Florid Counterpoint.}

I. FLORID Counterpoint may be said to consist of the synthetical use of the technique of the four previous Species, together with a few variants of them, serving the purpose of more ornate texture, or improvement of melodic flow, and im. parting to the whole artistic variety.

2. The following are the devices used to form variants of the four Species :-

(a) The combination of two Species-
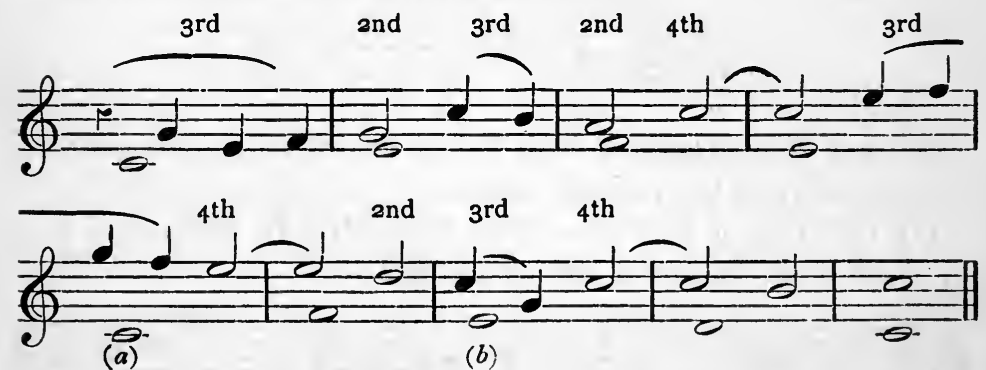

This analysis is in reference to melodic movement, e. g.

Minims, Second Species.

Crotchets, Third Species.

Tied minims, Fourth Species.

Whenever a minim on the second half of the bar is preceded by notes shorter than itself, it must be tied into the next bar; see $(a)$ and $(b)$ above. The only exception allowed is in the penultimate bar. It is true that cases may be found in Palestrina's works where this principle is disregarded. But in the large majority of cases the procedure mentioned obtains, and therefore may be taken as a fixed idiom. 
There is, however, one particular case that requires passing notice. In Palestrina's Missa Papae Marcelli the Nota Cambiata is extensively used, and the idiom constantly takes this form-

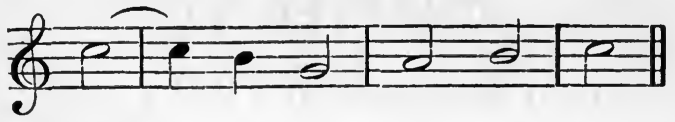

Such a use, however, is better avoided, not because it is a licence, but because changing notes, or a change of harmony on the second crotchet, should in any case be sparingly used. It may be asked, Why is any such rule necessary? The reason is that such a halt as-

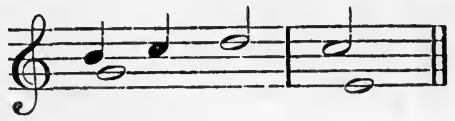

would be purposeless, unless employed to introduce syncopation-

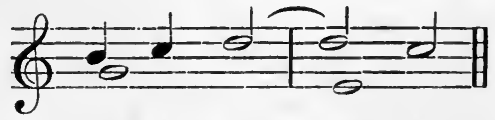

The case above, quoted from Palestrina, is of course justified by the nature of the melodic idiom.

(b) The use of ties other than those already explained, e.g. in which both notes tied are not minims-
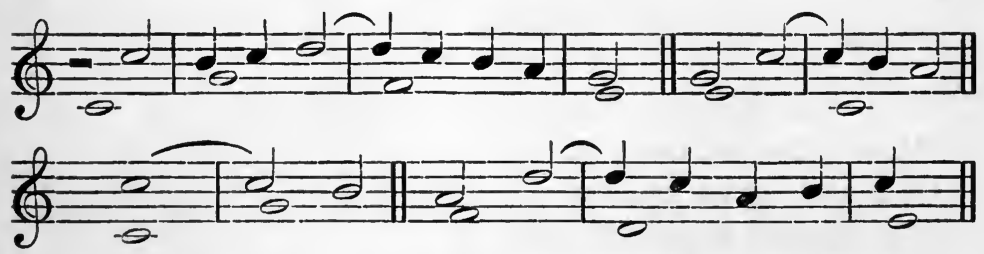

Benedictus, Missa Papae Marcelli.

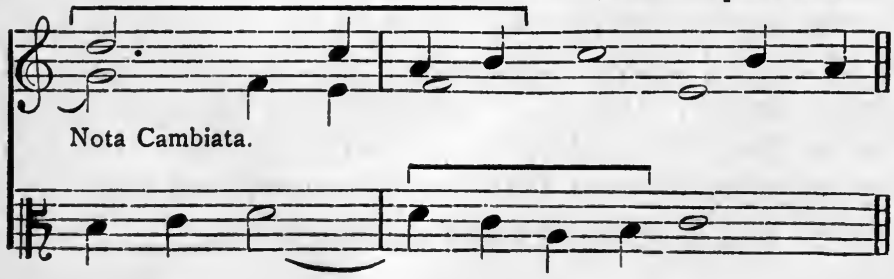


A tied semibreve should not be used in two-part writing, as the Counterpoint is thereby caused to lack interest.

The second of two tied notes must always be either of equal length with the first, or half the value of the first, and no note shorter than a minim should be tied into the next bar.

(a)

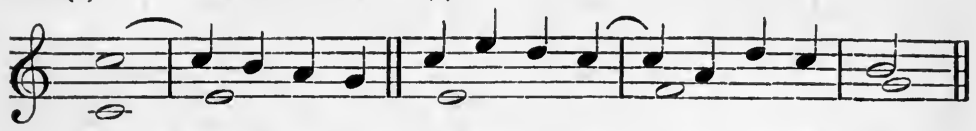

Both very rare : and never to be used except in six or more parts.

In $(b)$ two crotchets are tied together; such a procedure is so rare that it had better be discarded. It cannot be regarded as a variant of any Species, for the syncopation is introduced after its proper place. The second and third crotchets of the bar may never be tied, forming internal syncopation.

Morley in his Plaine and Easie Introduction (1597) gives the following as an order:-

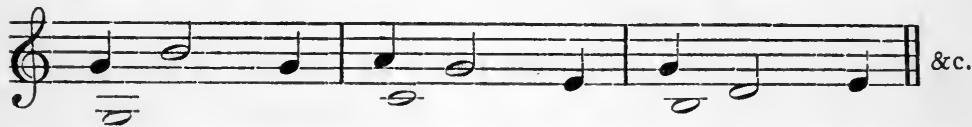

but it has not survived; see Kyrie, Aeterna Christi Munera (Palestrina) for an example-

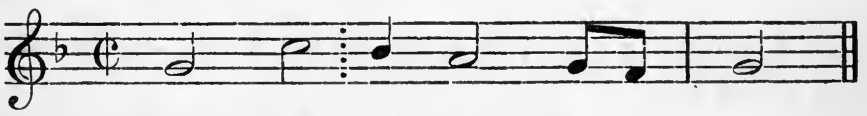

(c) The use of quavers.

Two quavers may be introduced in place of the second or fourth crotchets, and must be approached and quitted conjunctly. It is injudicious to introduce more than one group in any part in any bar, as the Counterpoint thereby loses dignity-

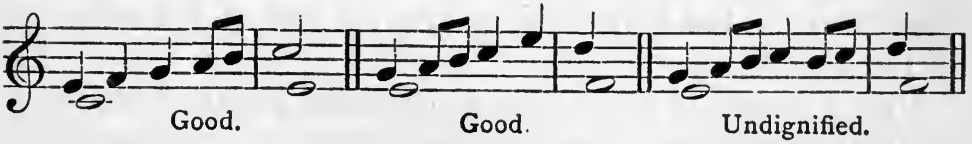

There are in Palestrina isolated examples of the use of four 
quavers in succession. The rarity of such a procedure justifies its exclusion from the regular technique of the period-

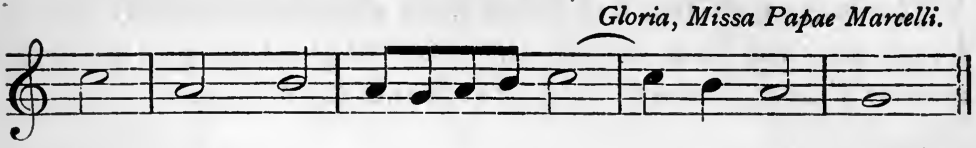

The following uses of quavers, involving melodic repetition, are bad:-

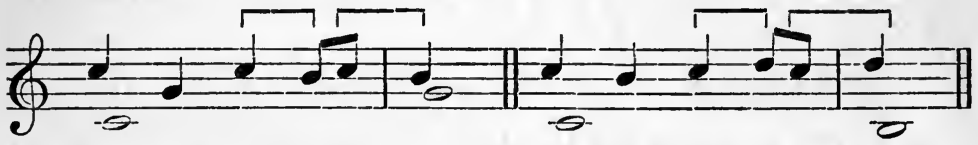

The following are good:-

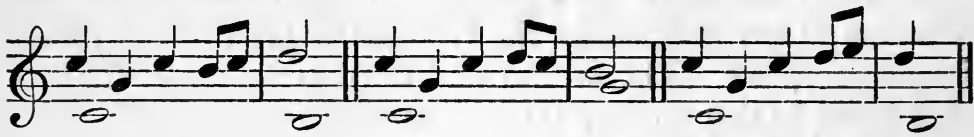

A study of Palestrina will show that quavers should be used sparingly.

(d) By the use of dots.

Their use between any two notes of one bar in two parts is not practicable-

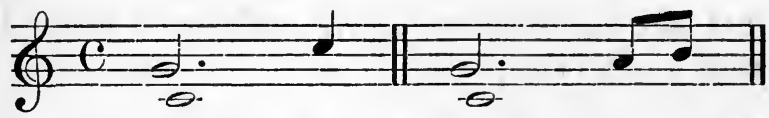

A dotted minim, in only two parts, causes a halt on the second accent of the bar.

Dotted crotchets, involving the use of a single quaver

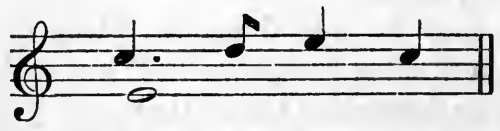

are forbidden, not because they are wrong from an absolute point of view, but because they form no part of the technique of the period.

(e) By the use of ornamental forms of the Fourth Species.

The following are ornamental resolutions of syncopated discords taken from Palestrina's works :- 


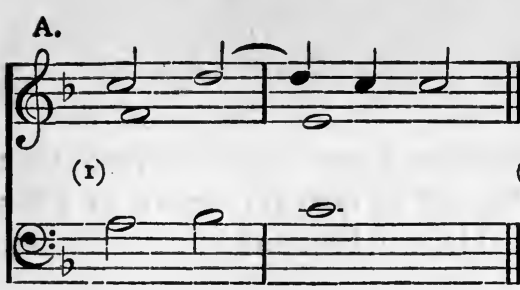

(2)
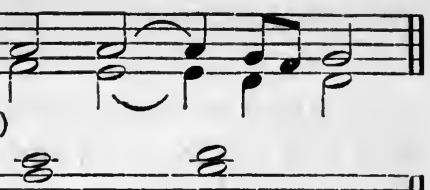

Aeterna Christi Munera.

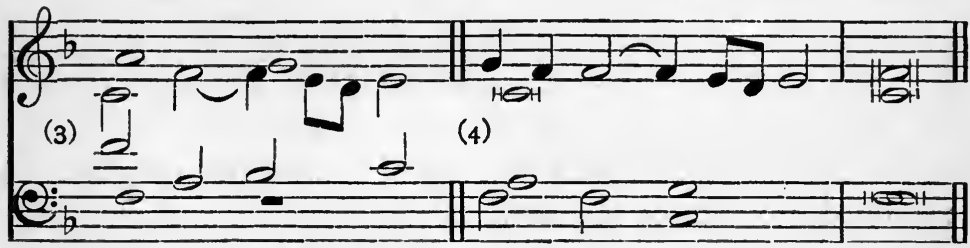

These are all examples of anticipation of resolution, and may be catalogued thus :-

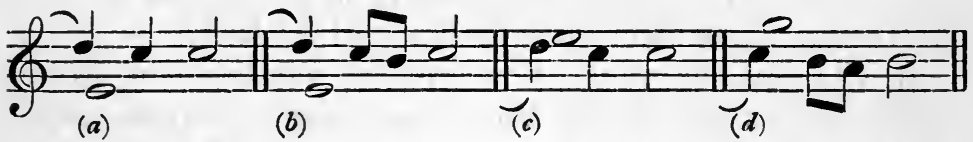

$(a)$ and $\rfloor(c)$ are not used in Scholastic Counterpoint: for $(b)$ and (d) can always be employed instead, thus avoiding any infringement of the rule, that, except in the First Species, no note should be immediately repeated at the same pitch.

B.

Credo, Missa Brevis.

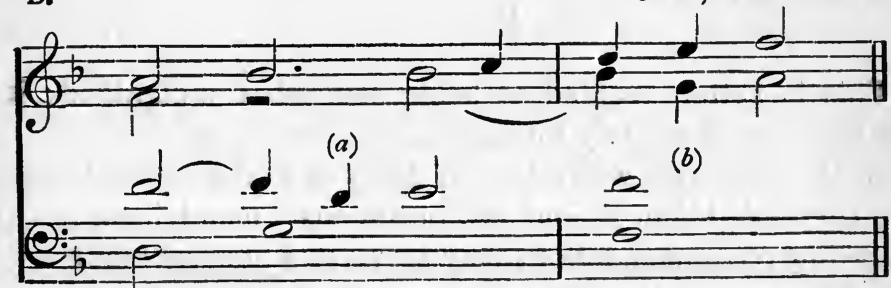

At $(a)$ the tenor leaps to a note consonant with the bass before resolving. At $(b)$ the alto leaps to a note dissonant with the bass before resolving.

These are really modifications of the use of the Nota Cambiata, and may be catalogued thus :- 


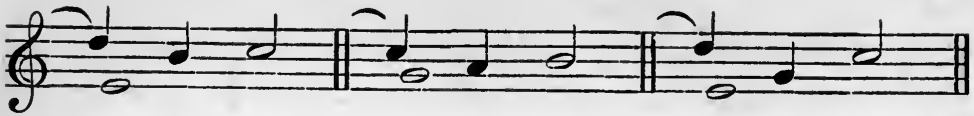

The leap or conjunct movement to a note higher in pitch than the note of resolution and the use of quavers higher in pitch than such note, though permissible, will be found to be extremely rare in practice-

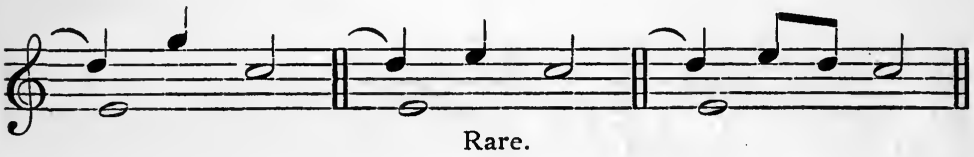

In every case the syncopated discord must be resolved on the third crotchet of the bar.

3. Each Species is of course governed by its own rules; as a general rule, a change of harmony should be made only on the second half of the bar, and rarely on the second or fourth crotchets. Some theorists entirely forbid the latter.

4. The first bar of the Counterpoint may begin in Second, Third, or Fourth Species. No part may begin with quavers, but two of these may replace the last crotchet in the Third Species-
(a) - $p$;
(b) $-\mathrm{r}$;
(c) $P \rho P$ P
(d) PPPPP|;

Many theorists give the following as a start-

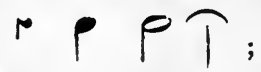

This is better avoided as being somewhat undignified; it is not however absolutely forbidden.

5. No absolute law can be laid down as to the extent to which any rhythmic idiom should be continuously maintained, but the following recommendations may serve as a general guide :-

(a) Not more than eight crotchets should be used in succession.

(b) Not more than three untied minims should be used in succession.

In two-part work the Second Species should be sparingly 
employed; it is better to tie the second minim of any bar to the first note of the next.

(c) In two parts the First Species should only be used in the last bar.

(d) Aim at 'the greatest amount of variety consistent with true dignity of style' (Rockstro).

6. The cadences are the same as in the Second, Third, and Fourth Species, with or without ornamental resolutions-
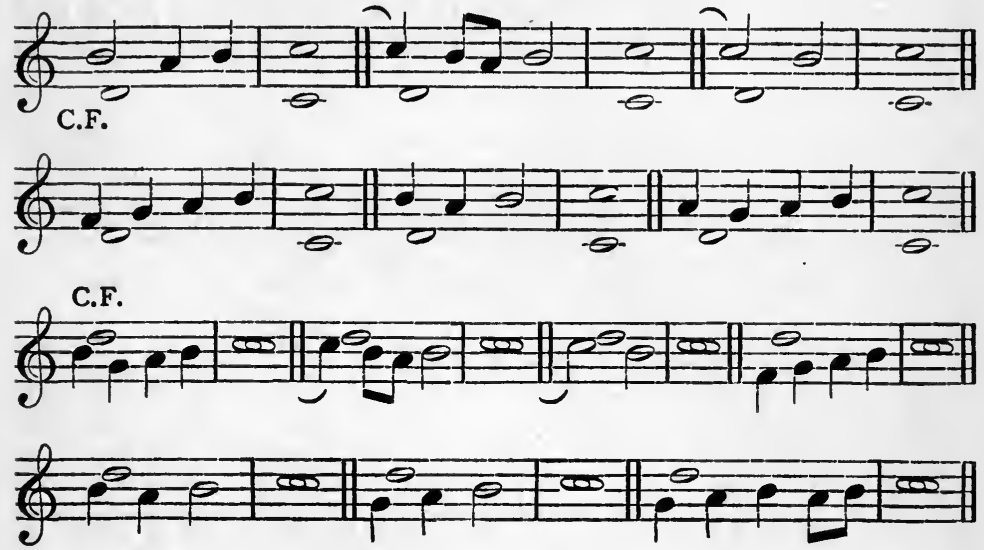

7. Avoid over-elaboration,

8. Avoid, if possible, allowing a part to return to the same point of repose in two successive phrases-

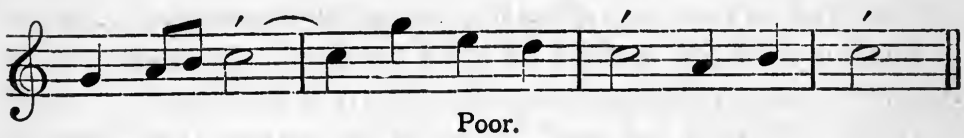

9. One theorist remarks that a passing note may only be used on the third crotchet in unmixed Third Species. This is hardly in accordance with practice.

Such a rule may be good in only two parts.

But with the addition of another part forming a concord with the dissonance such a procedure is found- 


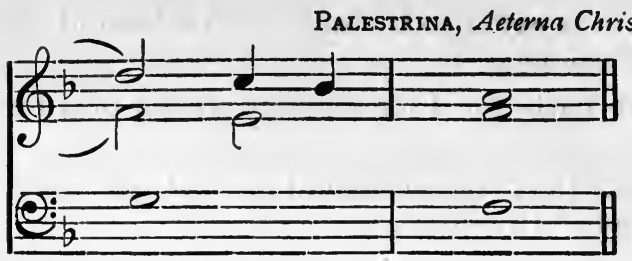

ro. The remarks made in the chapters on Second and Third Species in reference to the mechanical use of passing notes of course apply equally well here. A few examples of good and bad progressions are here given; the latter are generally due to over-elaboration-
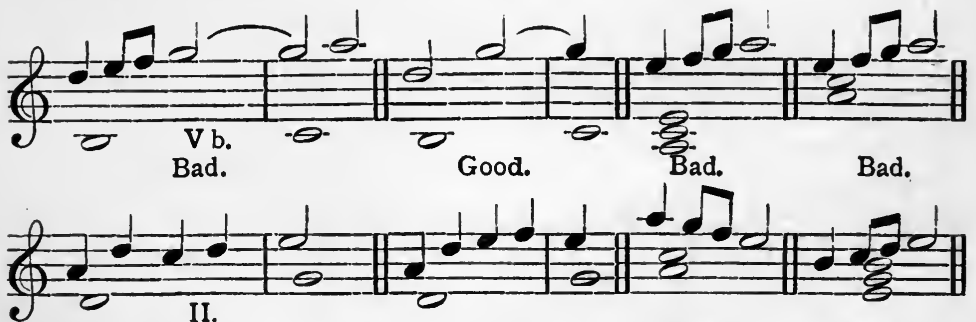
Bad.

Good.

Good.

Bad.

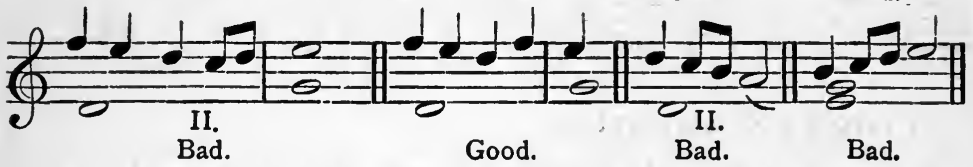

Everything depends upon the definition of the harmony, and the mental impression. In none of the above cases can it be fairly argued that any one bar gives the mental impression of a change of harmony on the second or third crotchet. Criticisms as to harmonic effect must be based on modern principles.

In the following, the mind receives the impression of a change of harmony, and the effect is therefore perfectly satisfactory.

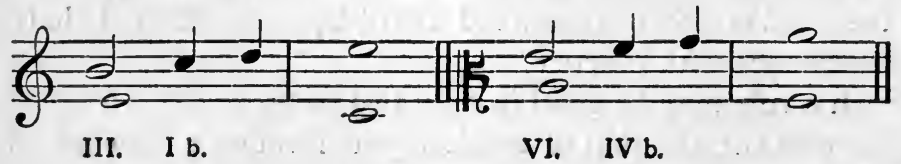


Ir. The ornamental resolution of a suspended discord must never cause consecutives between essential harmonies.

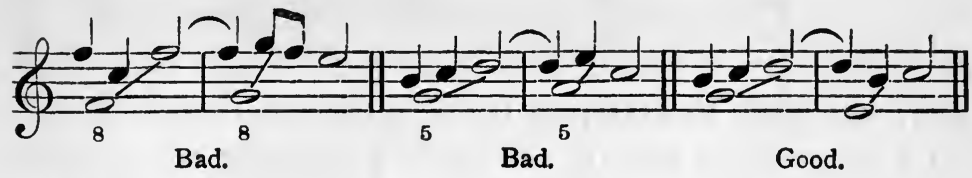

On a Study of Palestrina.

Now that the student is conversant with the five orders of Counterpoint, he should begin to study Palestrina for himself. There are two points of view, and they must be kept quite distinct :-

(I) The horizontal view. Every bar of the score corresponds to two bars of Scholastic Counterpoint, that is, in reference to melodic rhythm.

Thus

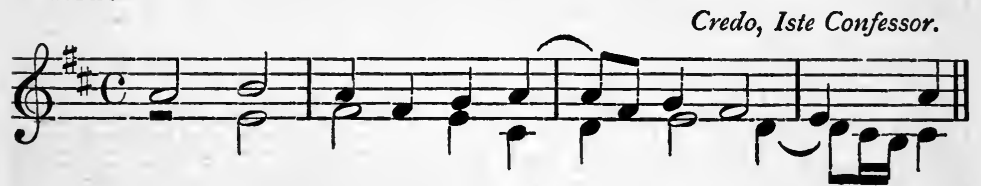

in notes of the time value of Scholastic Counterpoint is-

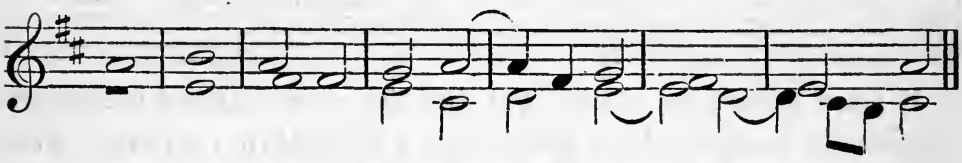

Palestrina commences the Kyrie of the same Mass thus

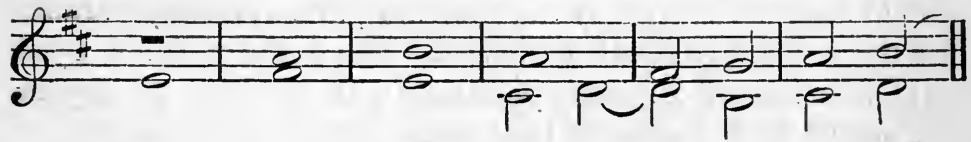

that is, the first note of the Counterpoint is a semibreve, and forms with the C.F. an imperfect concord. This is a frequent case in Palestrina, the reason being that the imitation justifies it; but the student is advised to conform to the ordinary procedure. Again 
Kyrie, Aeterna Christi Munera.

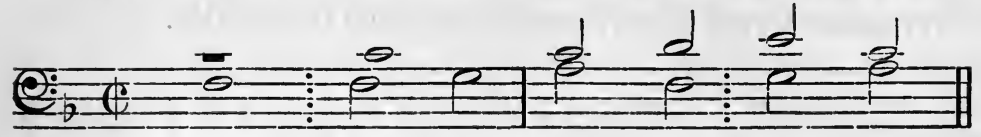

Here the dotted line inserted indicates the scholastic analysis.

(2) The vertical view, in reference to harmonic combination. The following is placed in its actual form, and in that of the scholastic analysis. That is, we here analyse not according to melodic idiom, but according to the 'species' used, the standard of measurement of course constantly varying-

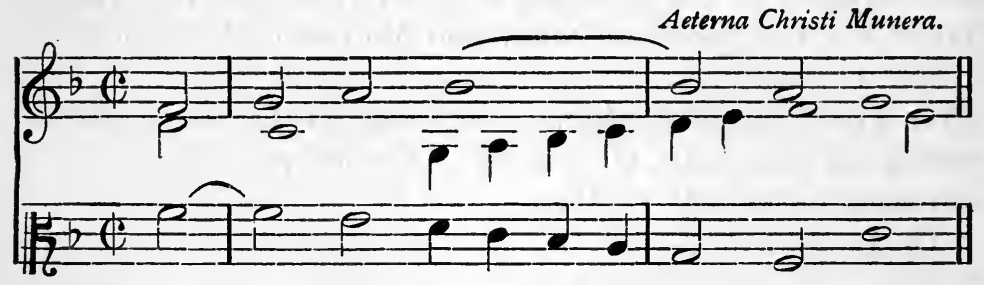

Scholastic Analysis.

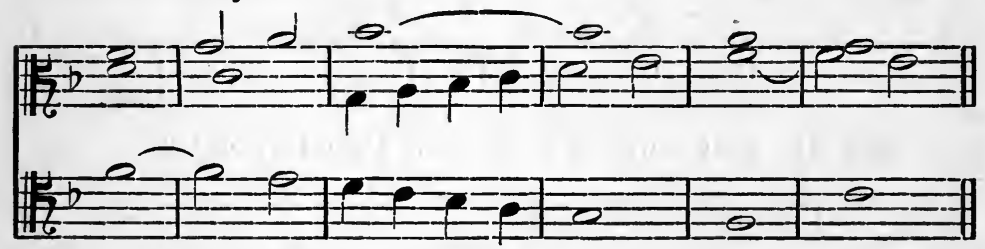

It need hardly be pointed out that the notation used in modern editions is a convenient adaptation for modern readers. Bars came into use at the beginning of the sixteenth century, to help men to read the scores, by keeping the parts vertically parallel, and not to mark rhythmic divisions. The works of Tallis, Byrd, and Gibbons were originally published without bars; the rhythm was shown by the value of the notes.

\section{On the use of two chords in a bar.}

In this connexion, it may be explained upon what authority it is stated that two chords in a bar may be freely used. The horizontal system is absolutely elastic in this respect. There is 
no definite rule that a change of harmony shall or shall not take place at any particular point in the bar: such a question never concerned the writers of the Polyphonic Period, since their music was unrhythmic, and all their attention was concentrated upon the melodic elegance of their parts.

The harmonic principle is quite simple: concords may be approached and quitted by leap, discords must be approached and quitted by step. Prepared discords have nothing to do with the essential harmony.

Some of the most beautiful harmonic effects of the period are obtained by a process of combined conjunct and contrary motion, involving the use of two harmonies. It may be asked if one change of harmony in the bar be allowed, why not two or three? Such a latitude would permit unnecessarily disjunct movement between notes of short time value, and would foster a style inconsistent with the spirit of contrapuntal writing. The writers of the period knew by instinct how far they might go in this respect: further, it is to be remembered that every scholastic bar contains two accents. Palestrina's scores are generally so simple in texture that any more frequent change is rare, and this largely accounts for the dignity of his style. It will therefore be seen that the restriction to one harmony in a bar and the use of more than two harmonies in a bar are equally undesirable, for the fundamental principles of music remain good for all time.

It has been said that bars of Scholastic Counterpoint contain two accents, - and $u$. Consider the rhythmic structure of the following:-

Third Mus. B. Oxon., May, rgo3.

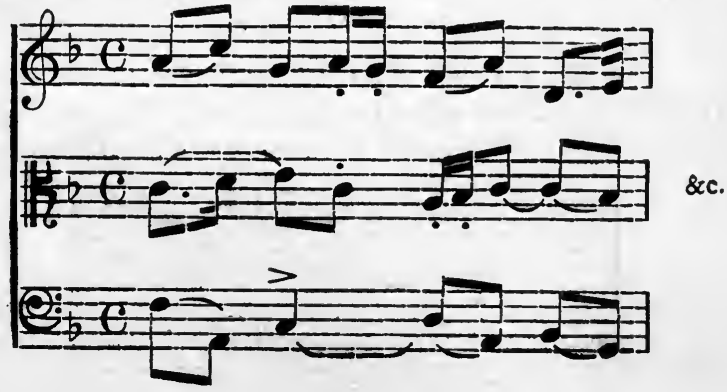


It will be felt that the time signature is misleading in reference to the accents, for the bar clearly contains four strong and four weak accents. Putting this section into bars of Scholastic Counterpoint we obtain :-

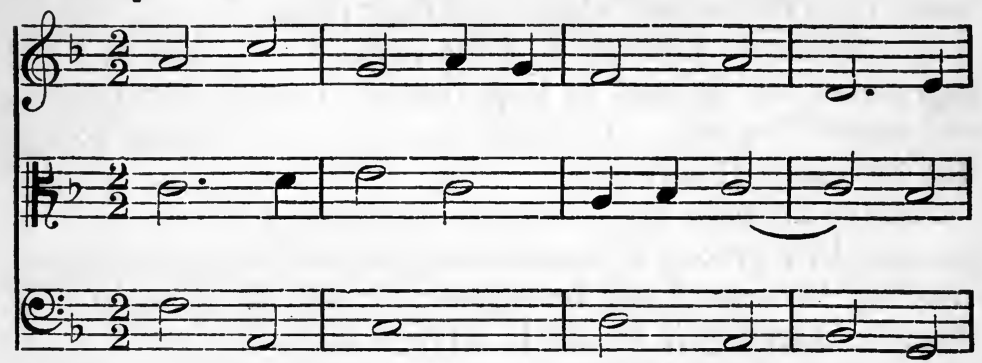

That is, we have here a piece of perfectly strict Counterpoint with the framework, in the form of a C.F., omitted.

Implied C.F.

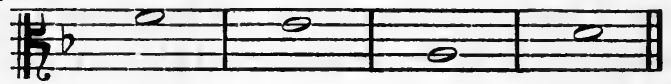

It is not of course implied that modern harmony is Counterpoint in notes of different time value. But it is maintained that Scholastic Counterpoint and modern composition are identical in principle. The C.F. in notes of uniform length supplies the framework which guides the student in the proper path as regards dignity of style, and method of decoration. Remove the C.F. and the frame remains in a preconceived harmonic basis : this of course, in actual composition, is a mental process, cultivated by the proper study of Counterpoint. But, it may be asked, why should not each accent be regarded as a bar of Counterpoint? The melodic view of the question supplies the answer. The rules which regulate the melodic idiom of bars of Counterpoint are those which regulate half bars of Palestrina.

For instance, the following is incorrect Counterpoint :-

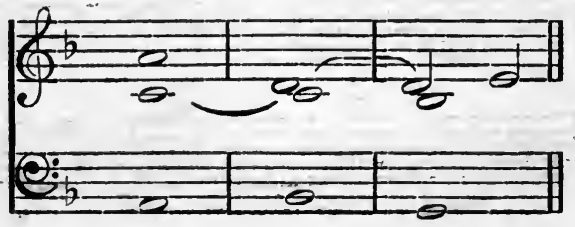


In this example a bar of First Species is made to contain a suspension, which is resolved on the first beat of the next bar.

It will thus be seen that the principles here expounded are not arbitrary: they are founded on a practice, which, because it is true, forms the foundation upon which all succeeding progress has been made. And one of the chief reasons why so many students seem utterly unable to write smooth harmonic progressions is to be found in the fact that they have studied a false system of Counterpoint. The modern German system and the English Macfarren school are not those that moulded the styles of Bach, Haydn or Mozart. Any one who studies the church music of our own Wesleys must at once recognize the influences of historic Counterpoint clearly engraved on every page. It may be added that, while limiting the change of essential harmony to one variation in a bar, the principle does not involve that the harmony must be changed once in every bar; but it does imply that it is never wrong to change it. Unessential combinations may produce what are now classified as chords, but they do not affect the impression of harmonic rhythm.

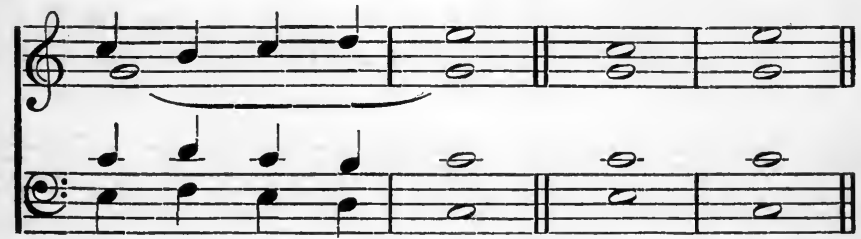

Chordal centres.

It is necessary to regard all Scholastic Florid Counterpoint as containing two accents in each bar : thus the time signature ${ }_{2}^{2}$ is used. The student may be left to mark the implied harmonies for himself.

Examples.

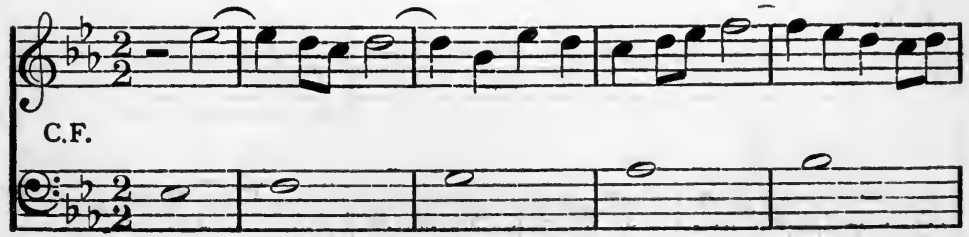




\section{Ioo THE ART OF COUNTERPOINT}

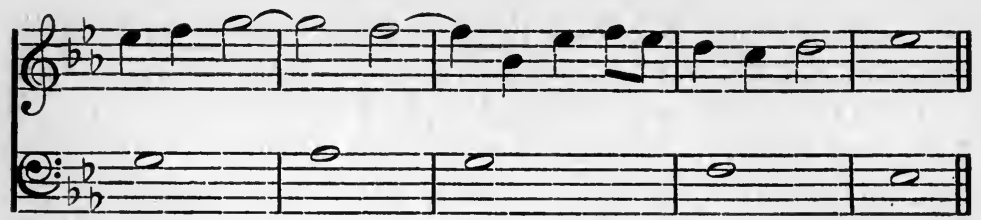

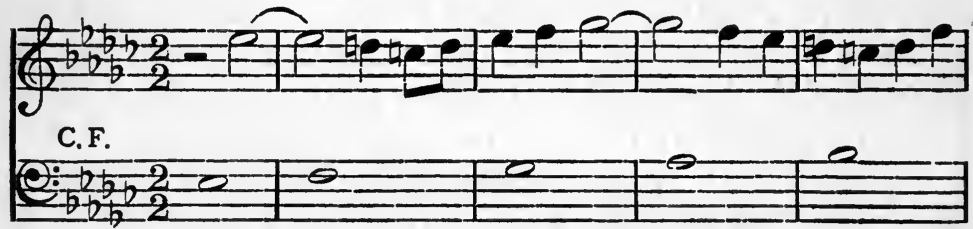

(2)

年

C.F.

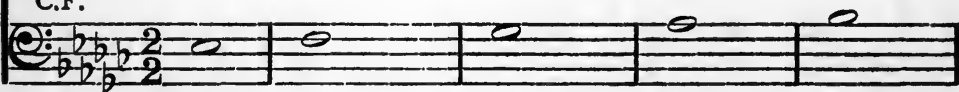

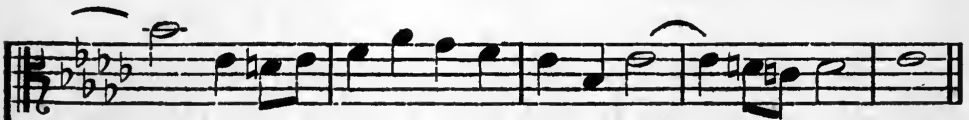

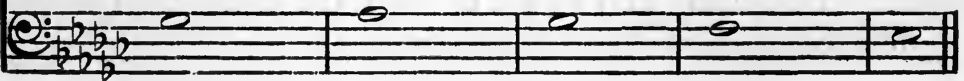

C.F.

$H=\frac{6}{2}$ 


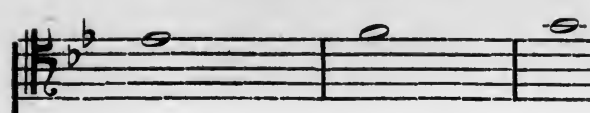

(a)
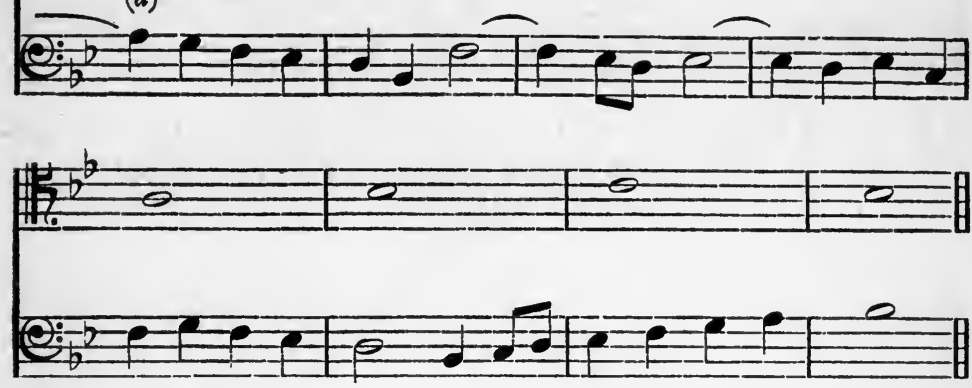

(a) Not a suspension resolved on the second, crotchet, but a change of harmony.

$$
\text { C.F. }
$$

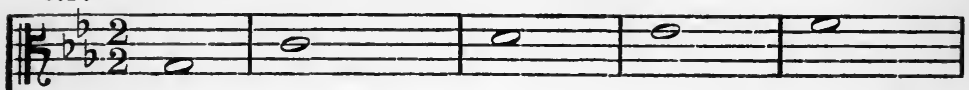

(b)
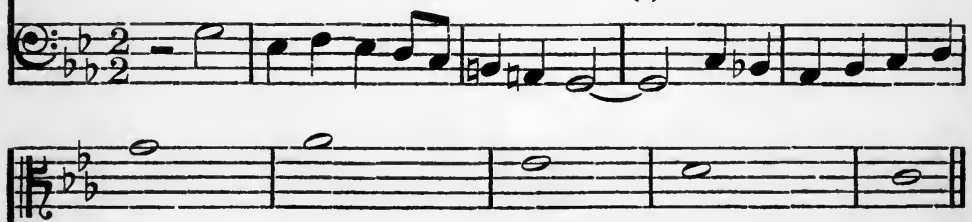

(b)

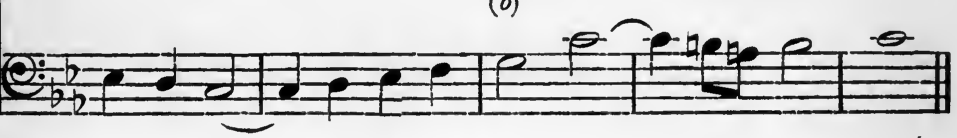

The same, according to strict harmonic principlesC.F.

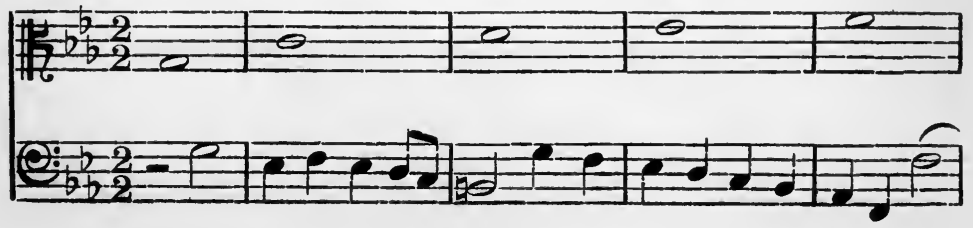




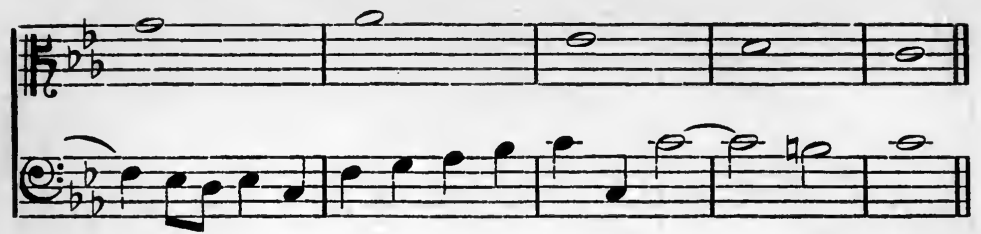

Either of these versions is correct Counterpoint ; the student will have no difficulty in seeing why the Macfarren school would condemn the former version at $(b)$. 


\section{CHAPTER VII \\ COUNTERPOINT IN TRIPLE TIME \\ Second Species.}

I. IN a system of notation now obsolete, a semibreve might be considered equal to three minims : this value of the semibreve was termed the Greater Prolation, and was denoted by the use of a circle, thus :-

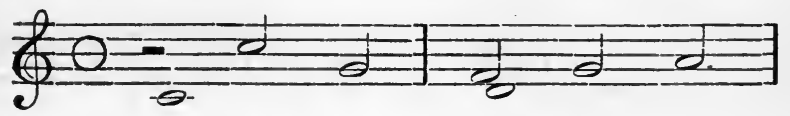

Its modern equivalent is of course ${ }_{2}^{3}$ with a dot added to the semibreve-

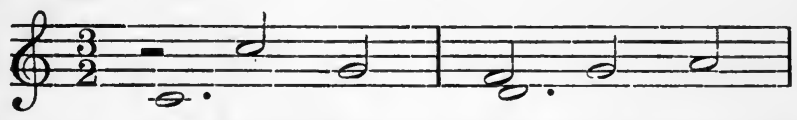

2. The Counterpoint begins on the second minim of the bar, and must form with the C.F. a perfect concord.

3. The first minim of each succeeding bar must be in concord with the C.F.

4. All discords must be approached and quitted conjunctly, except when the idiom of the Nota Cambiata is employed.

When the Counterpoint moves one step downward at the beginning of the bar, the leap of a third in the same direction, provided that the next note proceeds to the intermediate sound, is quite in accordance with contrapuntal practice-

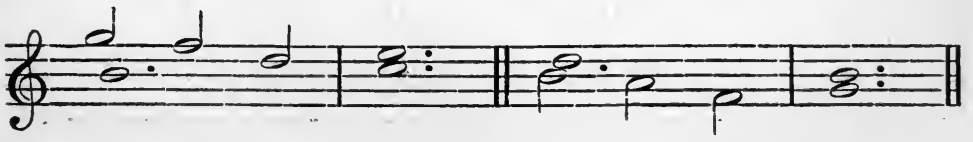


5. A change of harmony in the bar is always allowable-

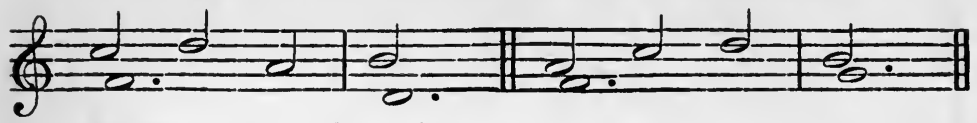

.6 Never run into the unison-

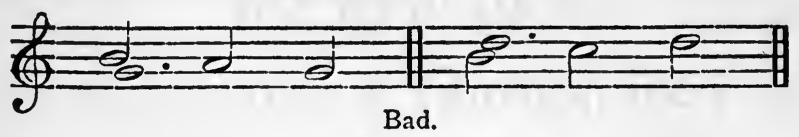

7. Two leaps in the same direction are unjustifiable-

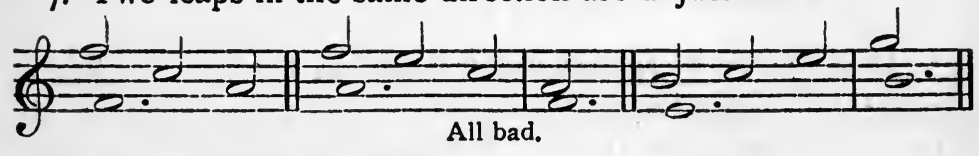

The Cadences are as follows:-
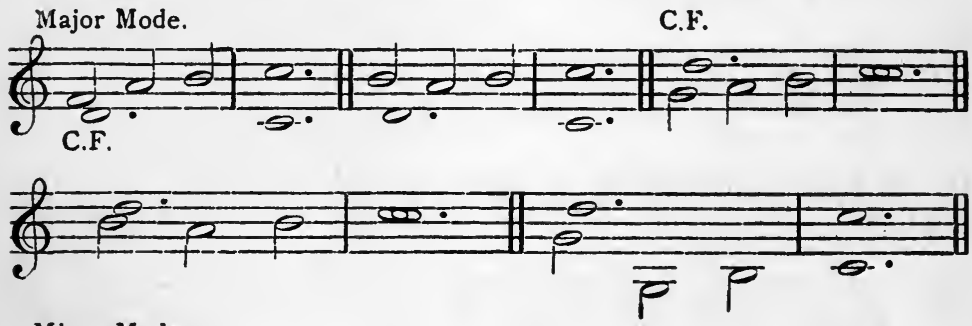

Minor Mode.

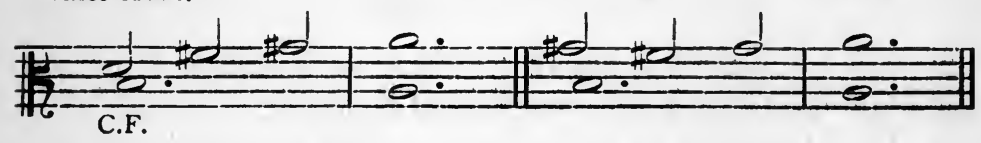

C.F.

Examples.

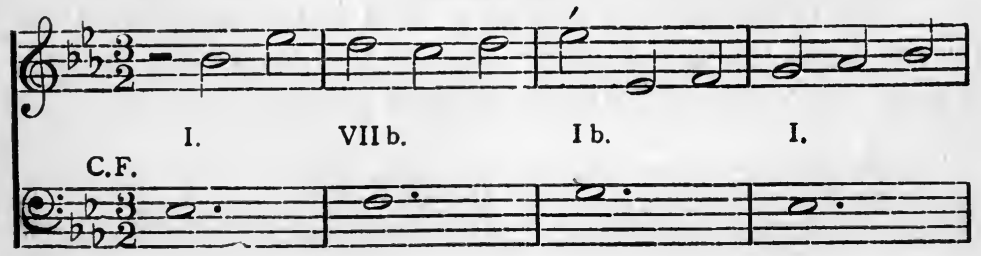




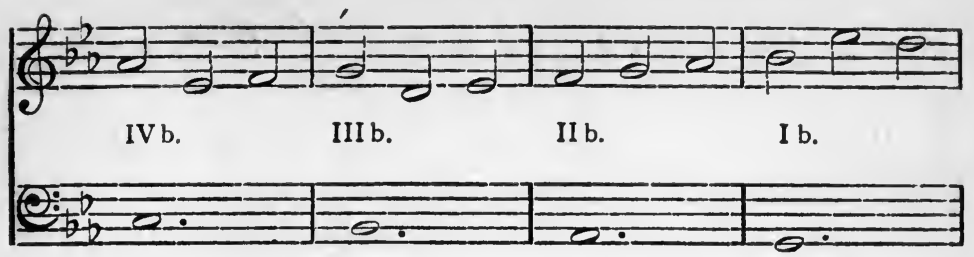

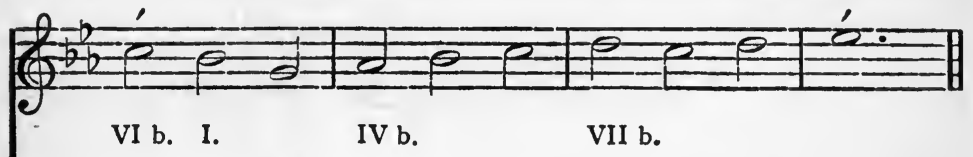

(2:0.0.0.

C.F.

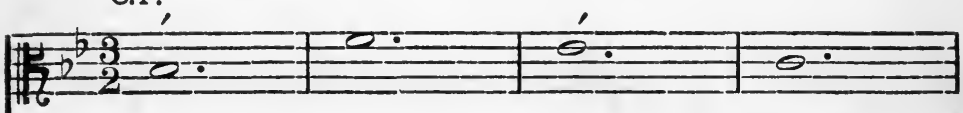

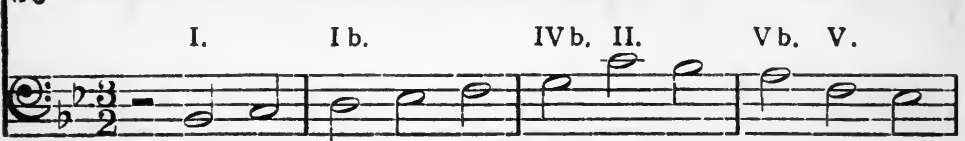

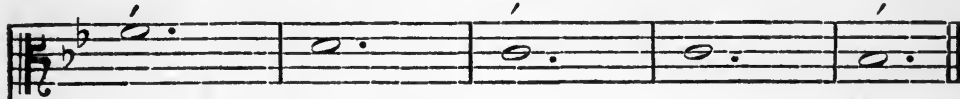

Ib. Vb. I. Ib. II b. Vb.

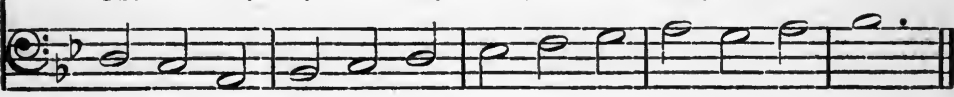

Third Species.

No additional remarks are necessary. The following examples will serve to show the method of procedure :-

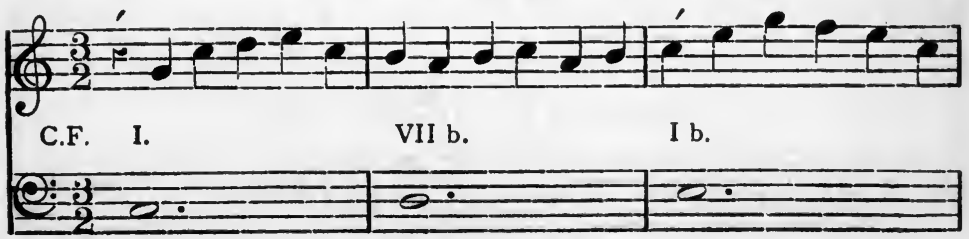




\section{6 \\ THE ART OF COUNTERPOINT}
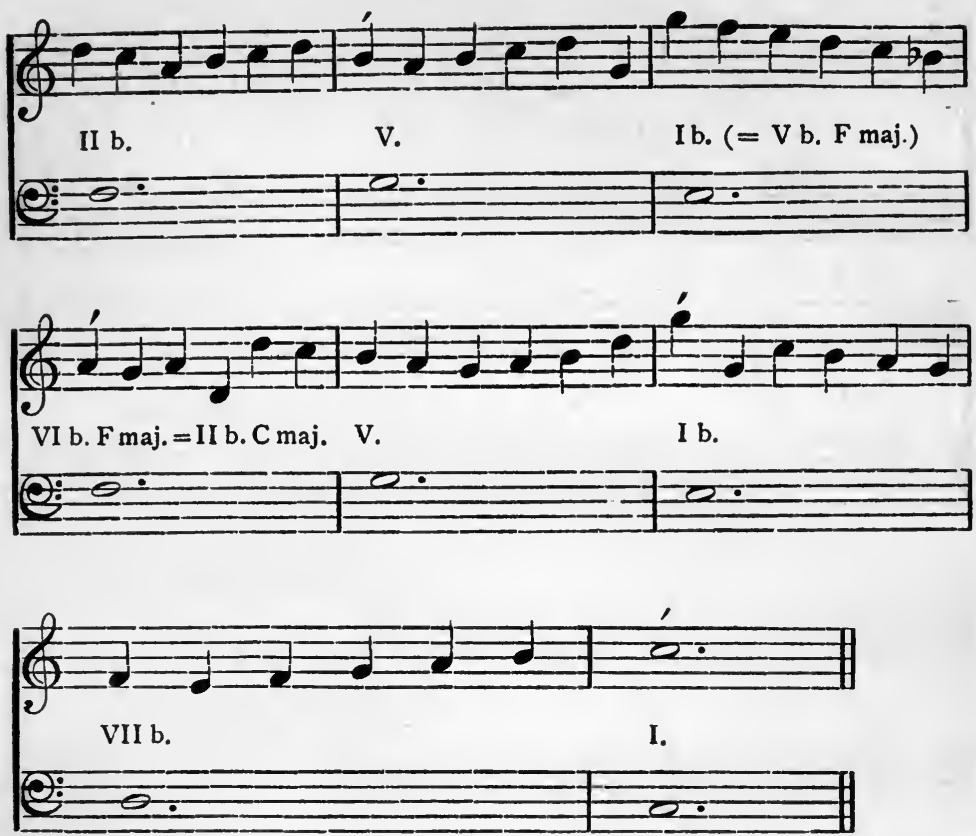

C.F.
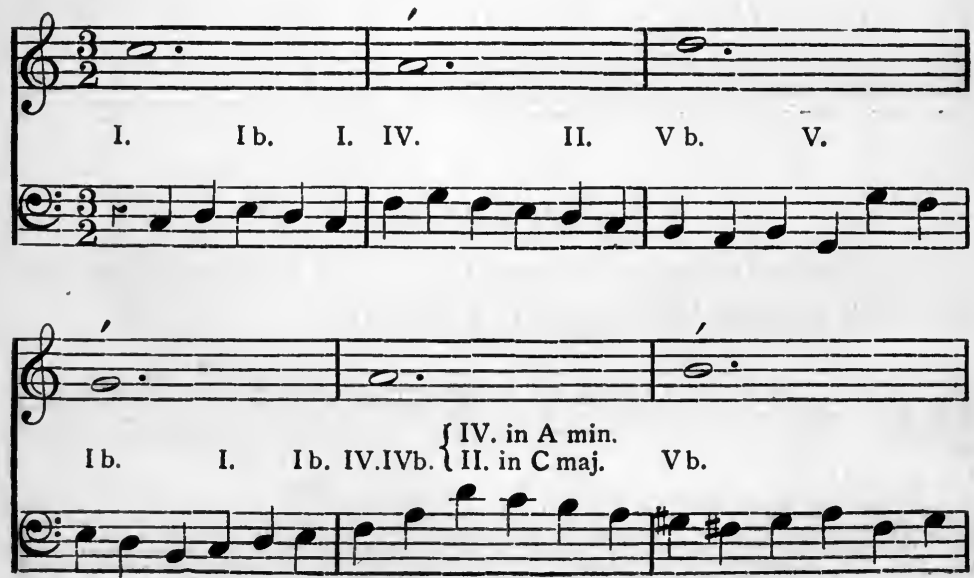


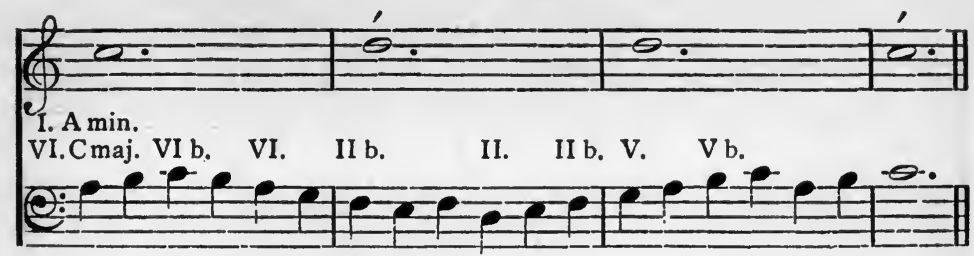

Fourth Species.

I. The second note in each bar of the Counterpoint may be either a harmony note, or a passing note. The third note must be a harmony note, corresponding to the second in duple time.

2. A discord of suspension may be ornamentally resolved as in Florid Counterpoint, the actual resolution taking place on the third minim. It is equally good to resolve the discord on the second minim.

3. The following is of course a case of exposed consecutives :-

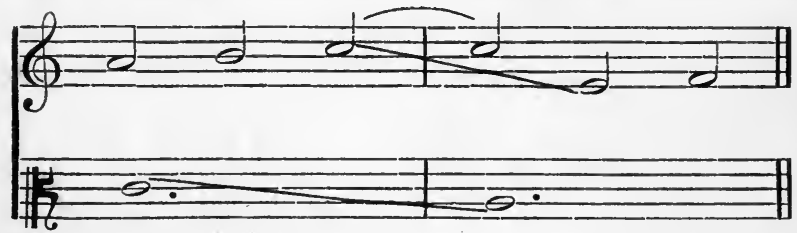

But the interpolation of another minim prevents the objectionable effect-

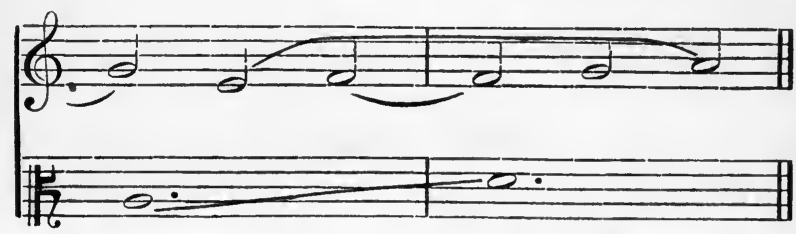

EXAMPLES.

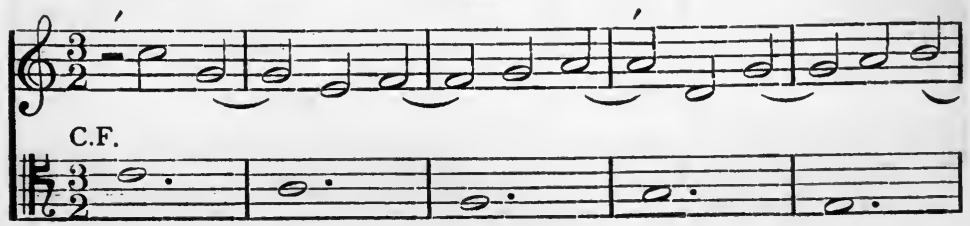


(a)

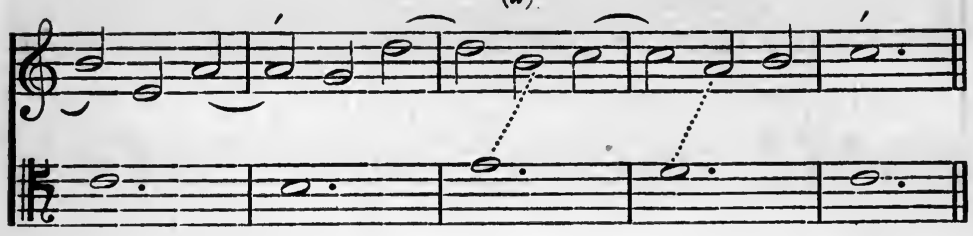

(a) not consecutives, as the notes involved are mentally unessential.

C.F.
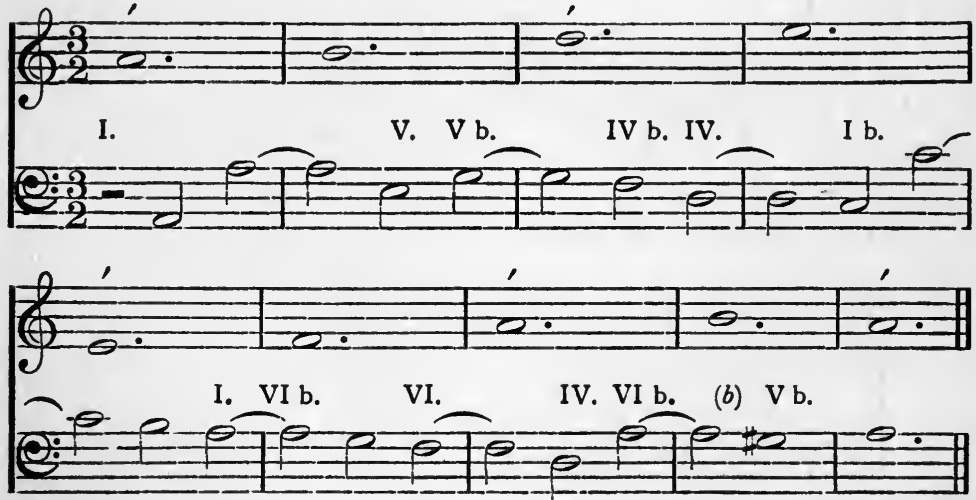

(b) a possible ending.

Fifth Species.

No further rules are necessary.

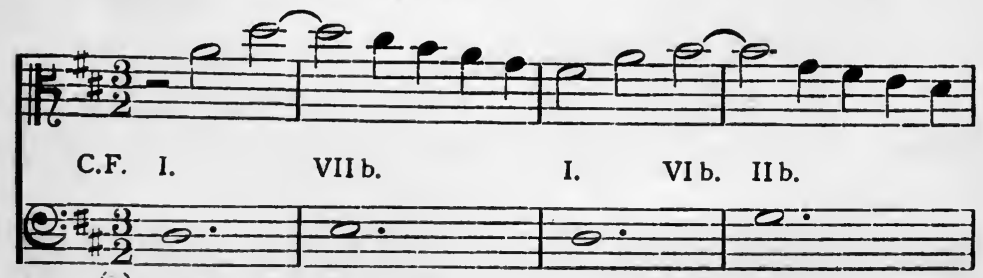

(a) $-v v-v v$ 


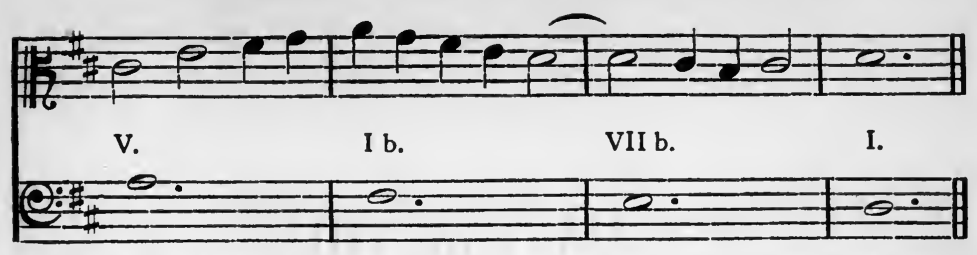

(a) It is better to regard this as containing three accents in each bar.

C.F.
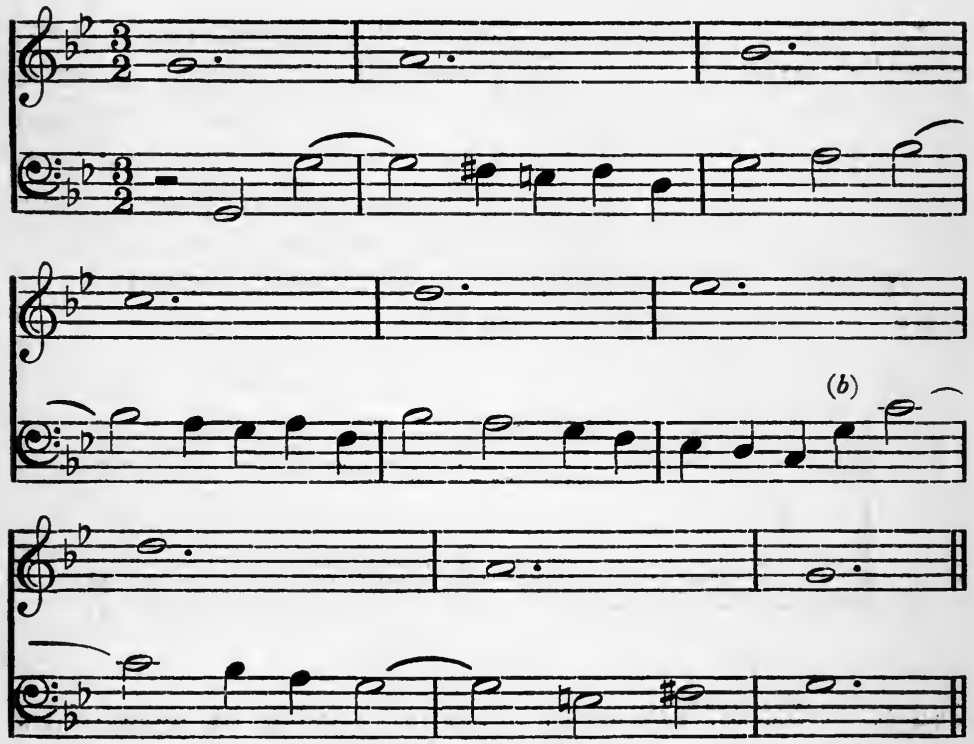

The figuring is not inserted here: it will be unnecessary to add it further.

(b) $\mathrm{G}$ to $\mathrm{E}^{b}$ is a concord : mentally an implied ${ }_{4}^{6}$. From such cases our rules for the treatment of the ${ }_{4}^{6}$ are evolved. 


\section{CHAPTER VIII}

\section{COUNTERPOINT IN THREE PARTS}

I. With the addition of a second Counterpoint to the C.F. a complete triad is possible, and harmonic progression ceases to be mentally ambiguous. In fact, this step in the development of polyphony led to the vertical consideration of combined parts, and finally to the establishment of the modern scalic system. For instance, in the treatment of the cadence, it was found well to accompany the penultimate note of the C.F. not only by the sixth above, but also by the fifth below-

Dorian Mode.

Ionian Mode.

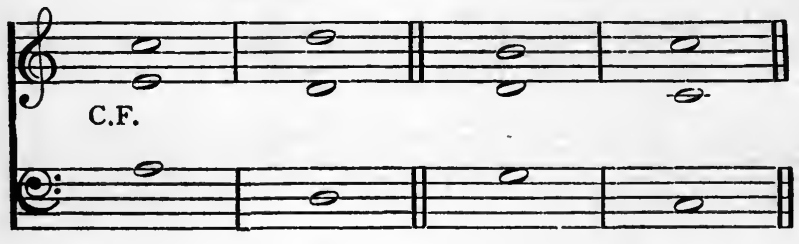

(a)

(b)

But the effect of the minor third (a), created by most of the modes, was felt to be objectionable, insomuch as the Ionic cadence sounded so satisfactory. And so in the system of Musica Ficta a sharp was added to the C, to make it like the Ionian cadence, forming the upward-tending leading note, and producing a satisfactory effect of completeness. This progression of the bass from V. to I. has become stereotyped as the perfect cadence, and up to a short time ago was the universal method of concluding a movement. Other experiments have been made by modern writers with varying success.

It has been shown how men experimented with combined 
sounds in three parts; the fourth being rejected, the following combinations were left:-

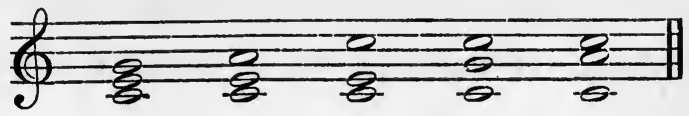

The fourth was allowed between two upper parts. This is the basis of our scheme of common chords with their first inversions. The ${ }_{4}^{6}$ would come to be catalogued after such a process as-

Palestrina, Missa Aeterna Christi Munera.

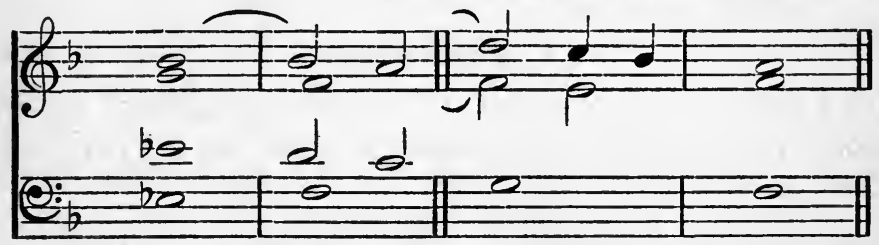

2. The majority of the rules of two-part Counterpoint hold good for the extreme parts in all simple Counterpoint.

3. Wherever possible the chord should be complete on the first beat of the bar ; if in the last bar it is necessary to omit the third, the fifth had better be omitted also.

4. The part-writing should be compact; the different voices should be nearly equidistant in the matter of interval ; if a wide gap is necessary, it should occur between the two lowest parts. No two upper parts should as a rule be more than an octave apart.

5. When incomplete harmony is made necessary by the exigencies of melodic flow, the root is to be doubled in preference to the fifth or third, the fifth in preference to the third. It is not forbidden to double the major third, though it should be done with cautious discretion.

6. Except in the first and last bars, and occasionally in the use of Fourth Species, the unison should be avoided on the first beat of the bar.

- 7. Both the perfect and augmented fourth are allowed as harmonic intervals between any two upper parts ; but not between the bass and any upper part- 

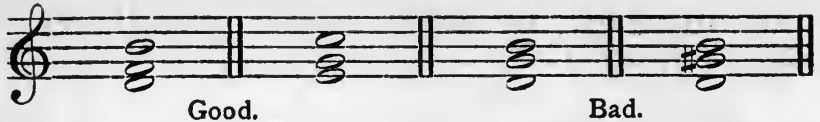

The following combinations are of course possible in the minor mode :-
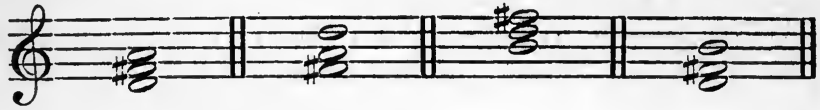

8.

Consecutives.

(a) Exposed consecutives are usually disallowed between extreme parts, except at the cadence where the top part moves by step-
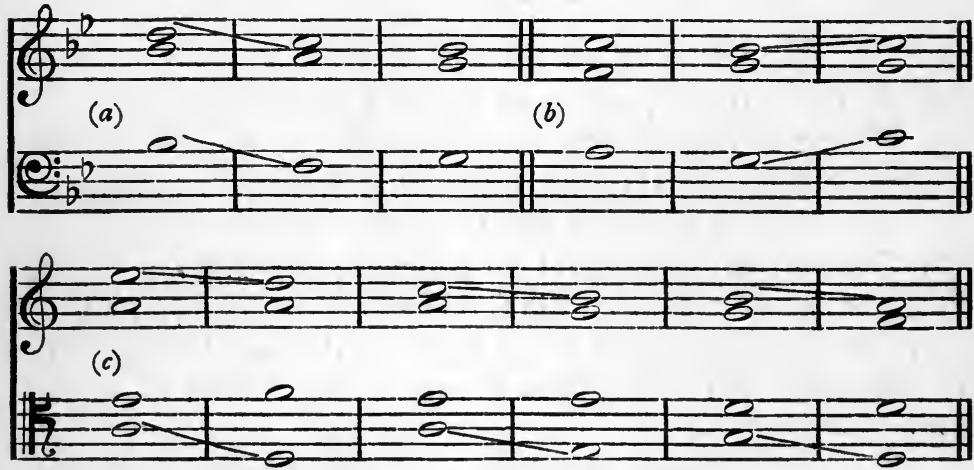

Many theorists consider all these to be bad.

$(a)$ is found in Palestrina's $O$ admirabile commercium-

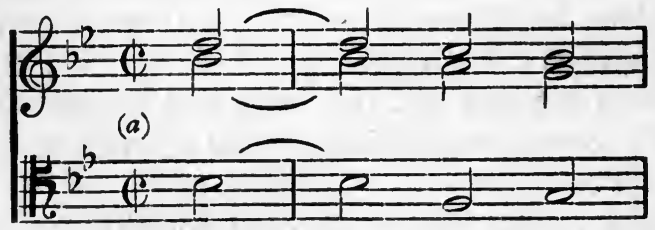

(b) is found in the Kyrie of the Missa Brevis, and (c) is found in the Stabat Mater- 


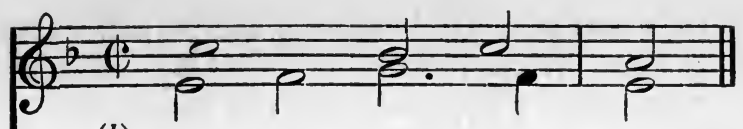

(b)
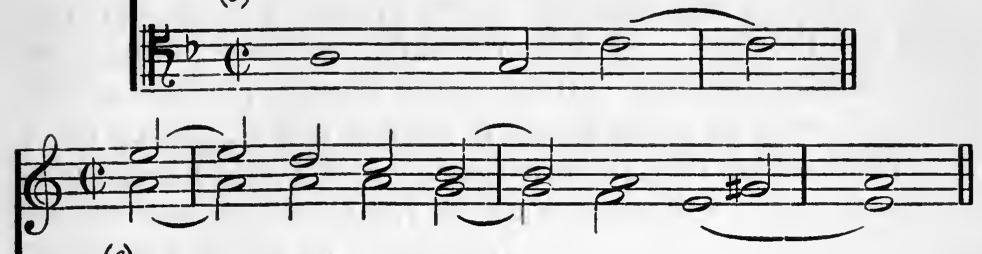

(c)

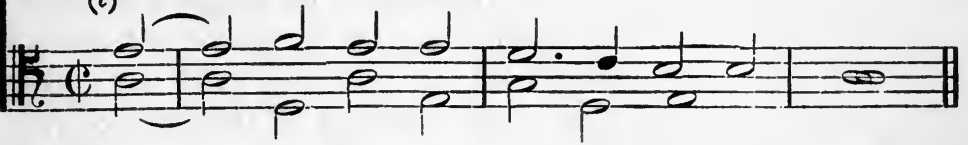

These are by no means isolated instances. Now it is quite true that cautious discretion has to be used in accepting Palestrina's procedure as absolute in reference to consecutives. For instance, moderns would not tolerate the first two of the following; opinion is divided as to the third.

Credo, Missa Brevis. Kyrie, Aet. Ch. Mun.

Credo, Ibid.

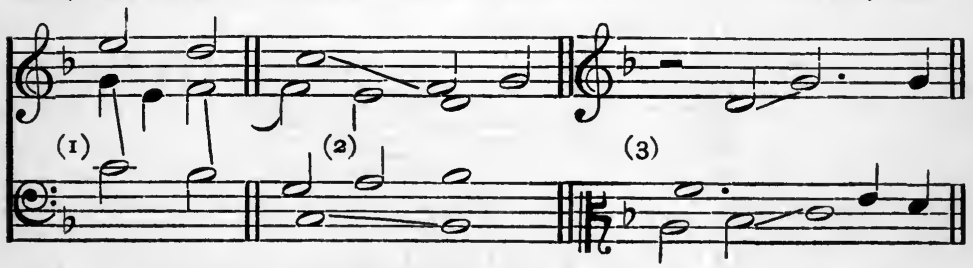

But in modern harmony all broad-minded critics agree in allowing exposed consecutives between the extreme parts, if the top part move by step; and one often finds this in Palestrina. That is, his procedure in this case is accepted as absolute. It is inconceivable that there can be any benefit in prohibiting what was certainly a common procedure in the sixteenth century, and which still holds good. It is obvious that there are many things which may not be done in Strict Counterpoint which are perfectly good in modern harmony ; the reason is not that they were ever wrong from an absolute point of view, but that they 
did not form a part of the technique of the period. The use of retardations is an obvious illustration. And if we see a procedure common to both the Polyphonic and the Harmonic Periods, it is inconceivable why theorists should prohibit it in the one, and allow it in the other. If it is argued that exposed consecutives are forbidden between extreme parts for the, purpose of discipline, it may be replied that if we can find no means other than those which impose restrictions which are of no practical value, and only serve to make theory and practice irreconcilable, such discipline, if at all necessary, should be sought elsewhere. There is no point in making difficulties for the sake of intellectual exercise, if the restrictions imposed have not even the saving grace of historic tradition; indeed, things of this nature bring the study of Counterpoint into ridicule. What is right must be right, whether it be a technical exercise or a piece of composition. But Counterpoint is Counterpoint, and whatever limitations are made must be in accordance with the practice of the time. This is the only valid reason for restrictions of any kind in the sphere of technique.

Consecutives (exposed) between different positions of the same chord are entirely unobjectionable-

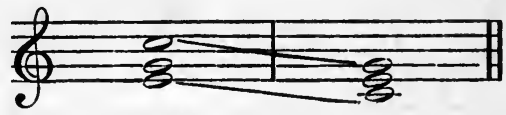

Good.

It is necessary to warn the student against an excessive use of exposed consecutives between the extreme parts, but he need not hesitate to write them if by so doing he can improve both the melodic and harmonic progression.

As a general rule they should be confined to those progressions in which the roots rise or fall a fourth or a fifth. In all cases one of the chords should be in the root position. The student should exercise his musical instinct in deciding upon the effect of progressions of this kind. 


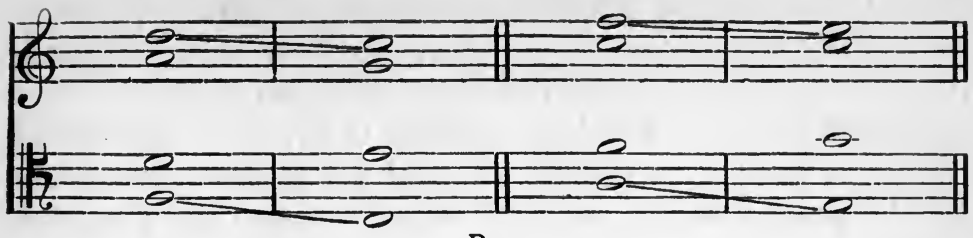

Although Palestrina writes-

Poor.

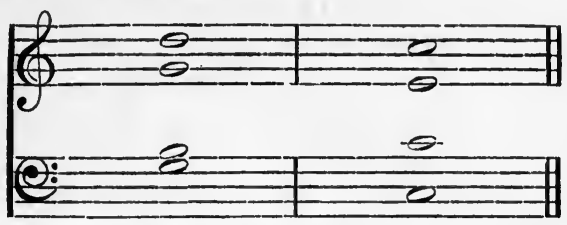

the following is not to be considered as a part of the technique of the period-

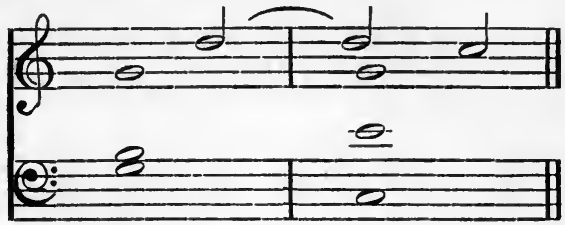

but the next examples are entirely unobjectionable because of the use of a syncopated concord in each case-

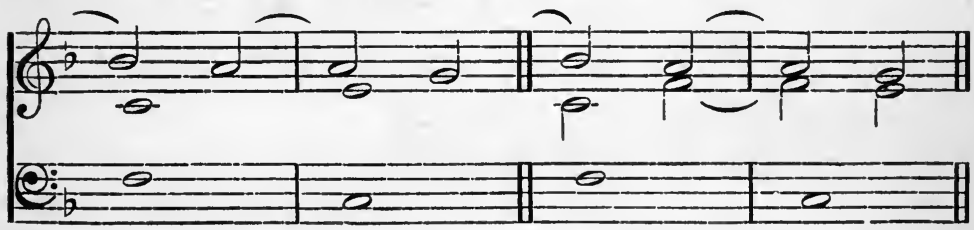

The fact that the effect of the following is good is no guarantee that it will be equally effective in purely contrapuntal music :-

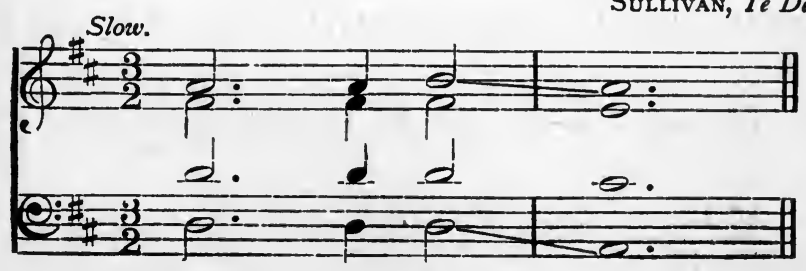

Sullivan, $T e$ Deum. 
Indeed, many of the exposed consecutives, which are perfectly good in music conceived in a modern spirit, are objectionable in the more severe style of the old contrapuntal school. The languishing effect of the above example is foreign to the style of Palestrina and his contemporaries, and the student must therefore be guided in such matters by his sense of the fitness of things.

$(\beta)$ Exposed consecutives are allowed between a mean and an extreme part, if the upper part move by step-

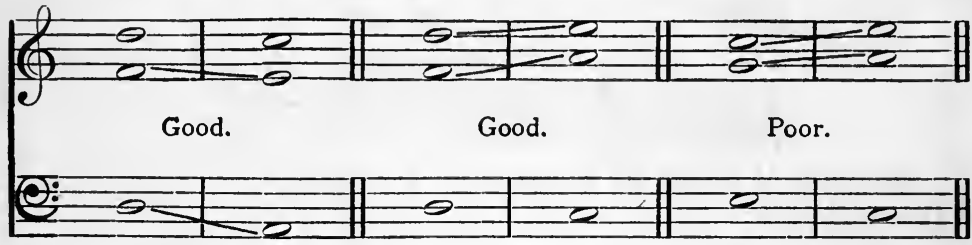

The interpolation of a passing note does not justify exposed consecutives which would otherwise be condemned :-

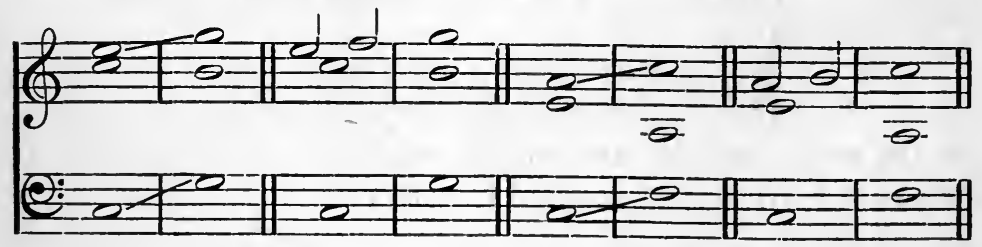

Bad.

Bad.

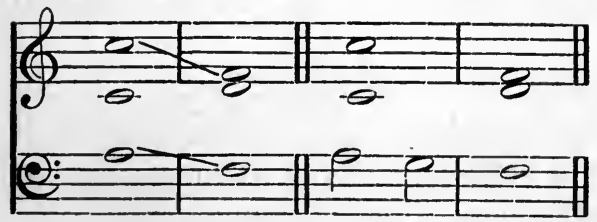

Bad.

If the unessential note be auxiliary, the effect is equally bad.

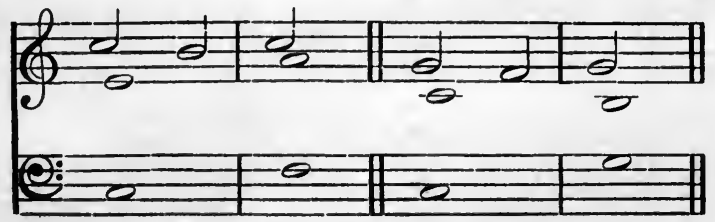

Bad.

Bad. 
That is to say, the principle only applies when all the notes involved are essential.

$(\gamma)$ One modern writer says that consecutive fifths are not allowed, if one of them be diminished -

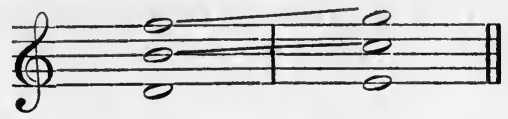

But there is no valid objection to them between two upper parts, if the chords are VII $b$. and I b.

(ס) Progression to the unison by similar motion is always bad-

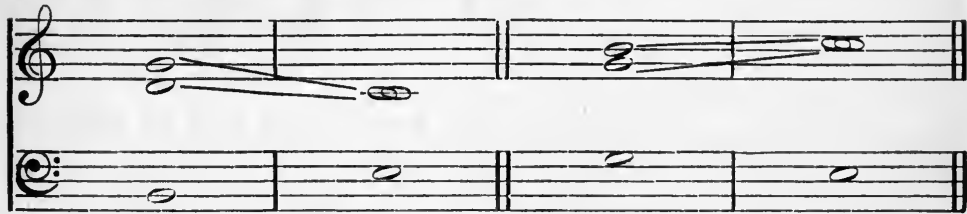

( $\epsilon$ In the time of Palestrina consecutive fifths and octaves were prohibited only between the same parts.

Thus we find the following:-

Credo, Iste Confessor.

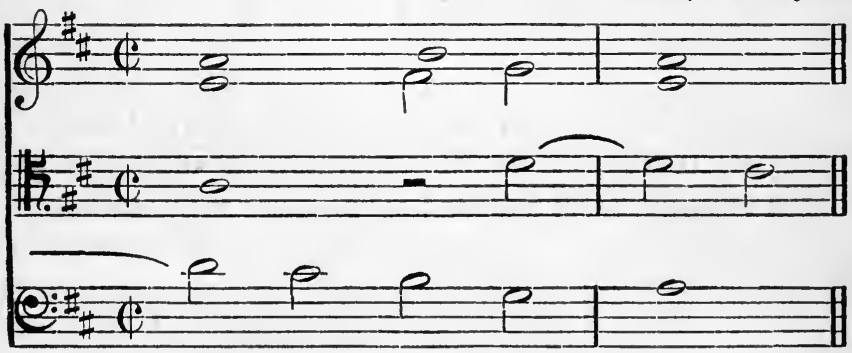

Now, in unaccompanied vocal music it is possible to follow the contour of each part. But under any other conditions the above will have the effect of

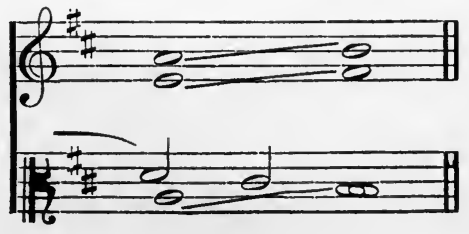


so that it is advisable to discard this principle, at any rate in less than six parts.

9. The parts may cross freely, but in the First Species it is better that this procedure be restricted to upper parts.

ro. Tritone. This is forbidden between the extreme parts by conjunct movement. Except the following:-

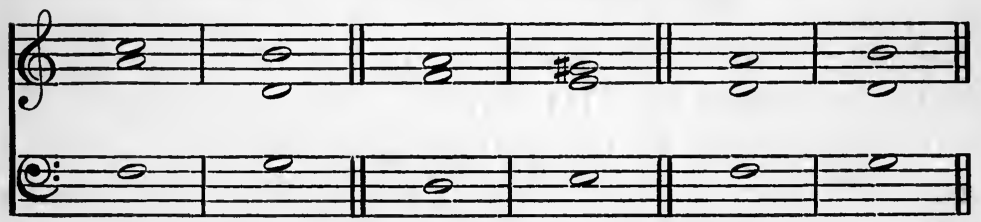

All good.

It is allowed between a mean and an extreme part by contrary motion-

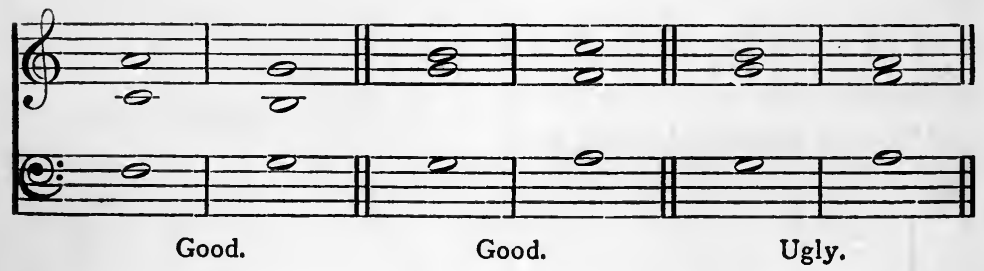

But it is hardly wise to lay down any hard and fast rule : much depends upon the context, and the student should rely more upon his musical sense than the mechanicai application of rule. 


\section{CHAPTER IX}

THE FIRST AND SECOND SPECIES IN ONE OF THREE PARTS

I. IT is one of the canons of elementary Harmony that if there be a note common to two consecutive chords, it should be kept in the same part; it is one of the canons of Counterpoint that, unless involving bad balance of parts or exposed consecutives, the movement of each part should be varied as much as possible-

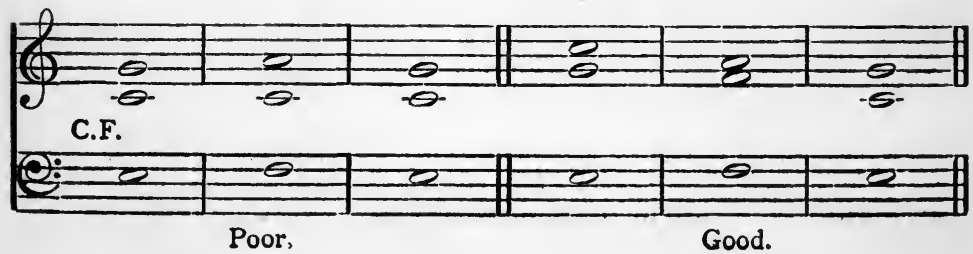

2. Never double the leading note-

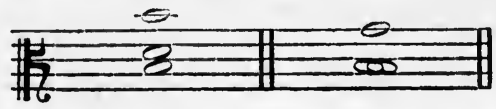

3. In the first bar the lowest part should always have the root-

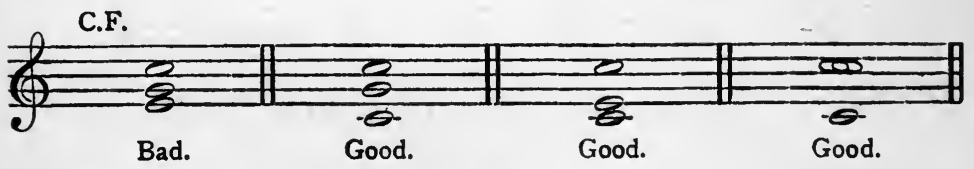

The following are the cadences:- 

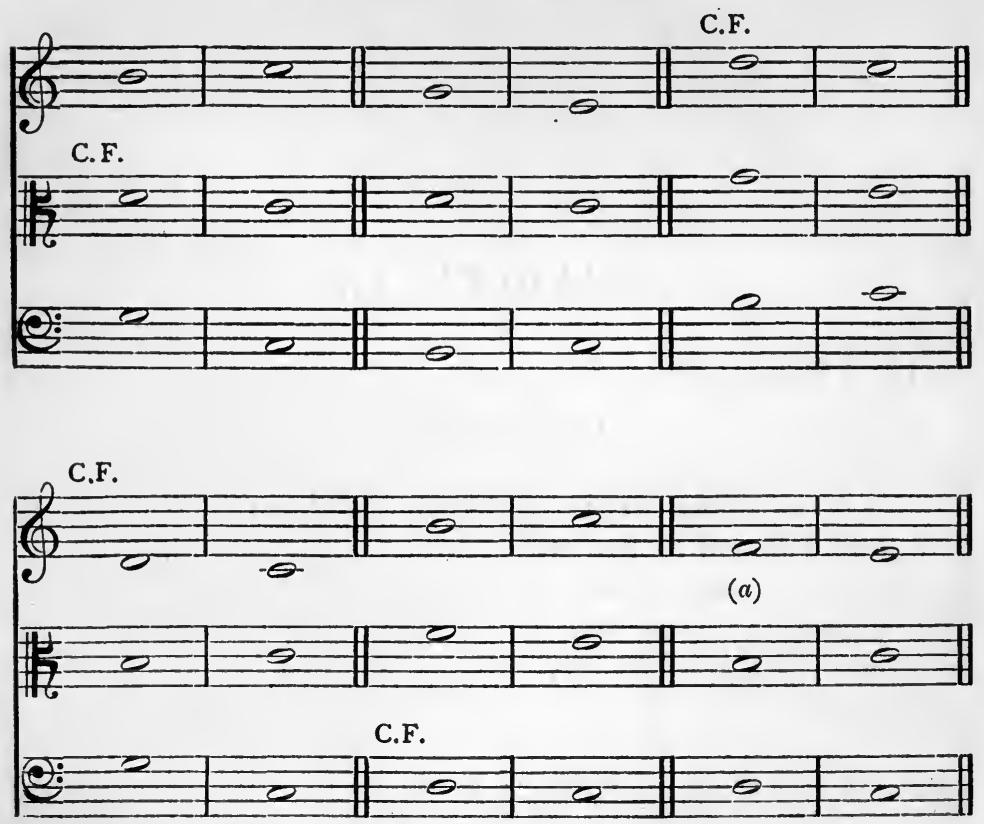

-(a) - The following is quite correct :-

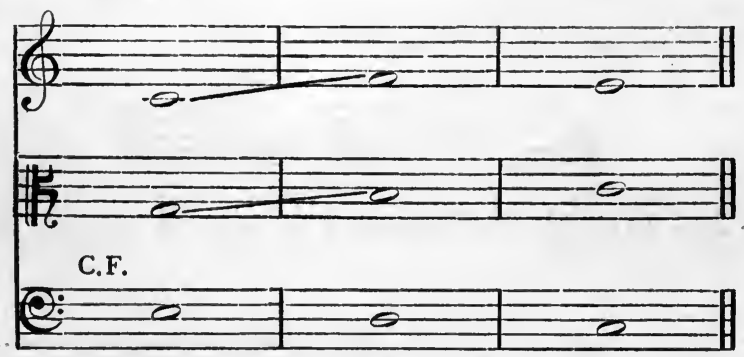

that is, the exposed diminished fifth is open to no objection.

Examples :- 
First Mus. B. Cantab., May, 1903.

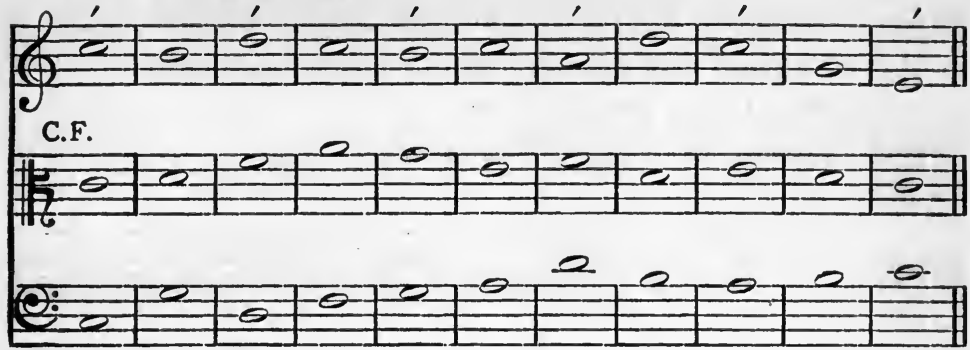
C.F.

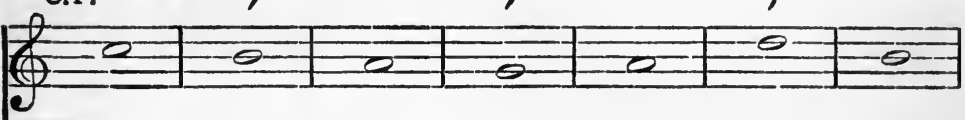

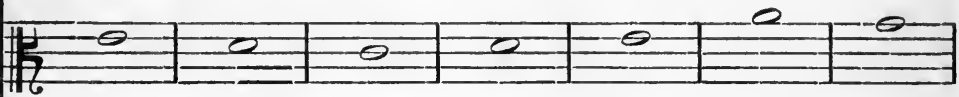

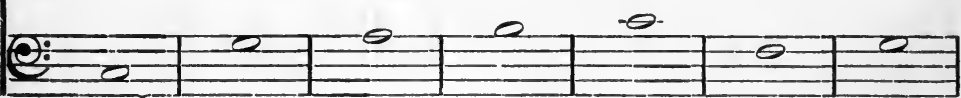

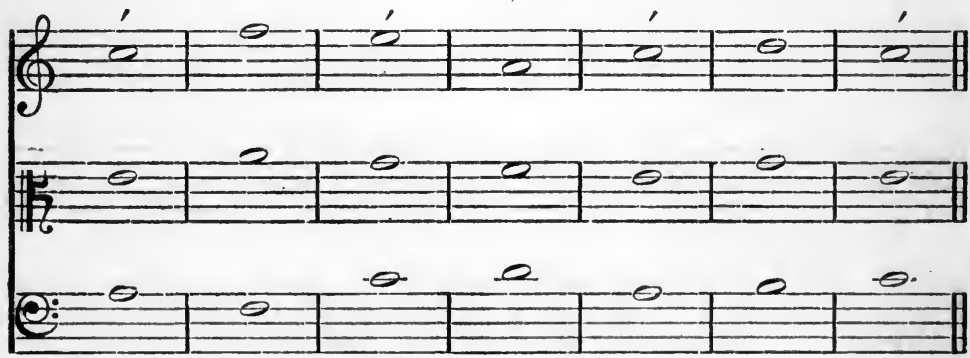

An example in practical form-

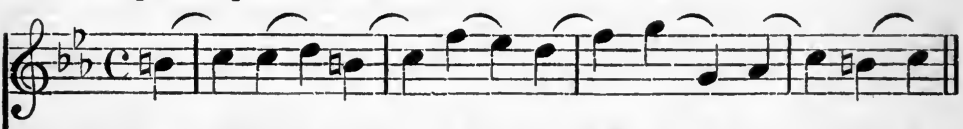

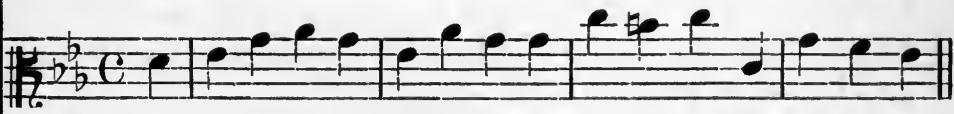
C.F.

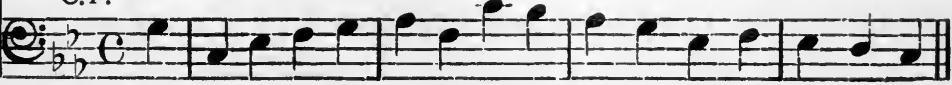
$V$ IIBIBV V V $T V V_{-1} W_{B} V=N I B V \| B$ 
Second Species in one of Three Parts.

I. The unison may be used on the second minim of the bar, if it secures a better melodic flow.

2. The cadences are as follows :C.F.

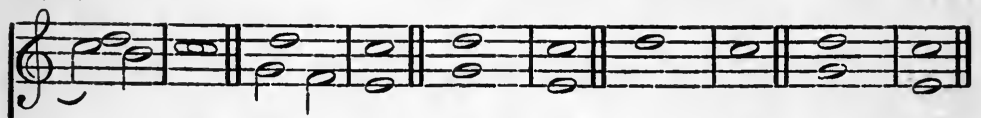
0.0.

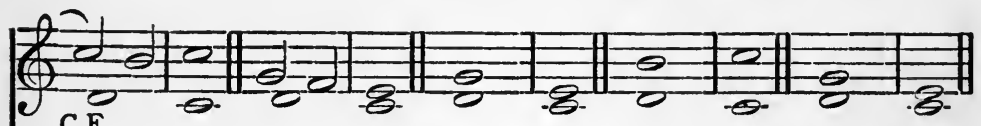
C.F.
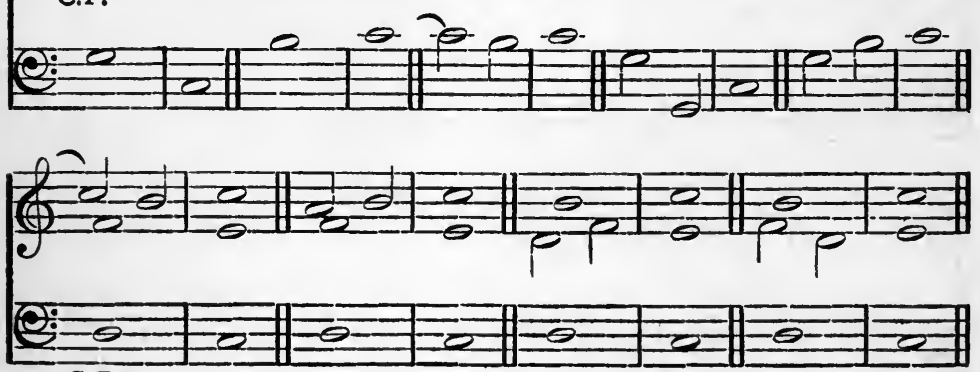

C.F.

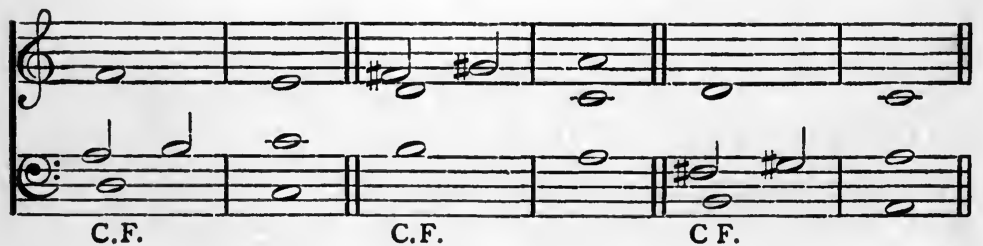

Observe that the last two notes in some part are invariably the Leading Note and Tonic. This is the case in every Species.

3. The use of passing notes :-

(a) The effect of the fourth against the third is very harsh when the third is an inner part and the fourth is above- 


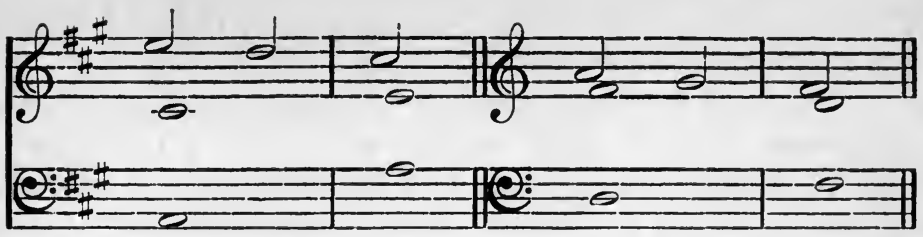

(b) Wherever possible let the second minim form with the holding notes either a modern essential discord, or a good passing chord -

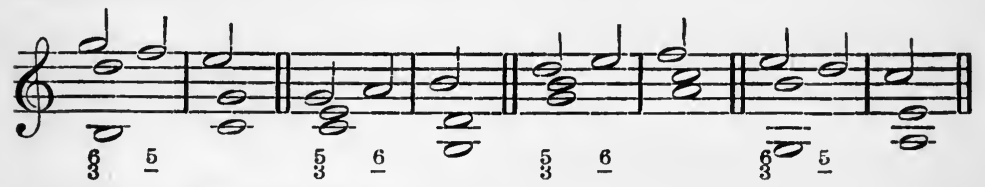

(c) Note the following:-

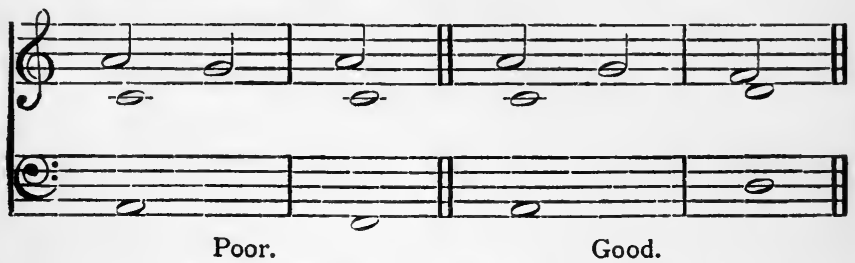

4. No two notes next each other in alphabetical order may proceed to an octave by similar motion (Macfarren, Harmony)-

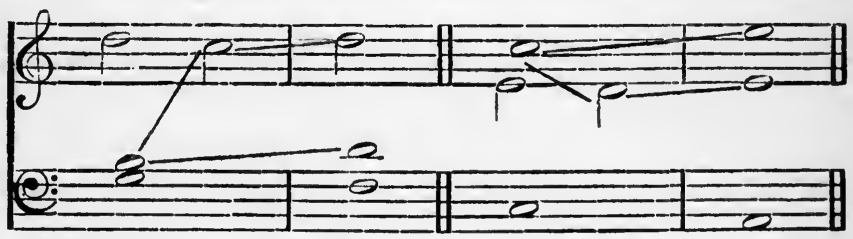

5. The first note of the Counterpoint must be a perfect concord-
C.F.
C.F.

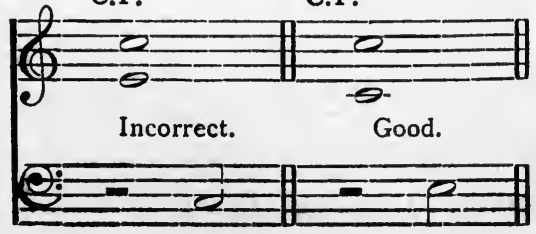


Theorists differ on this point: the above rule is based on practice, not theory (see page 95).

6. The rules as to consecutives between the C.F. and a part in Second Species apply equally well when a third part is added. The leap of a fourth does not take away the objectionable effect, even if the Second Species be a mean part-

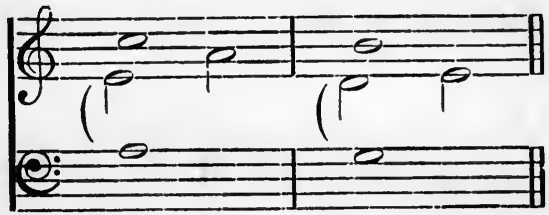

Bad.

The earlier theorists allowed this : but, as it has been pointed out, the arguments which support Palestrina's procedure have ceased to have significance under modern conditions.

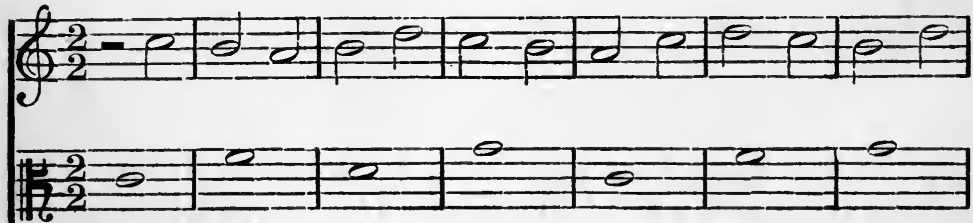

C.F.

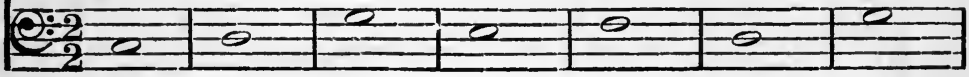

(a)

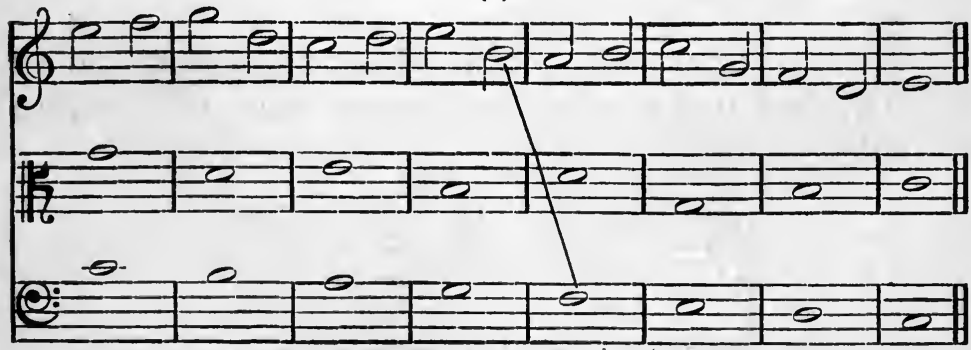

(a) the sequence justifies the tritone. 


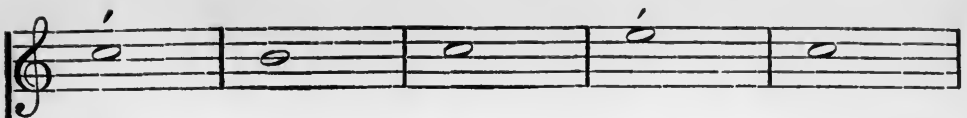

C.F.

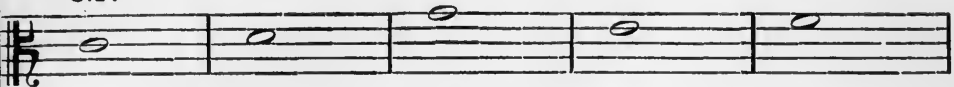

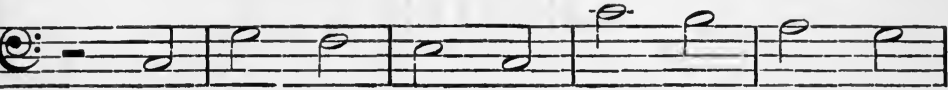

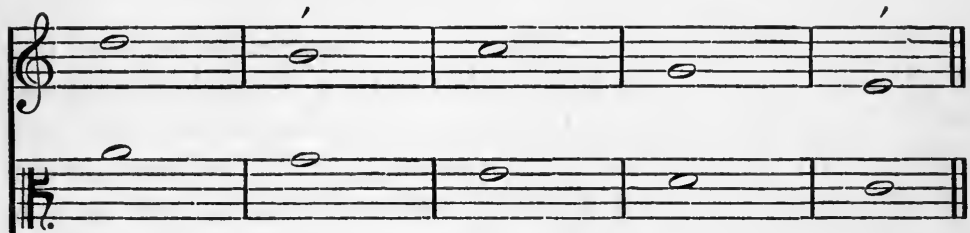

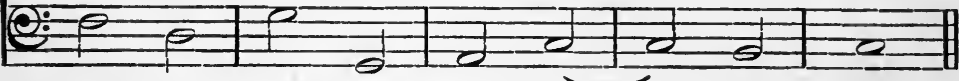

An example in practical form-

C.F.

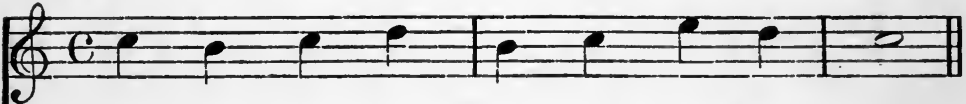

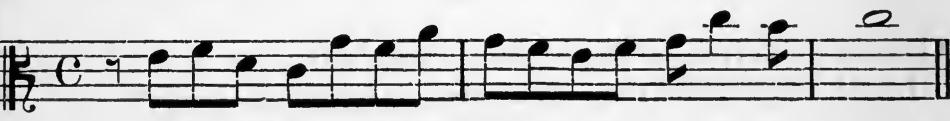

Oi:

or

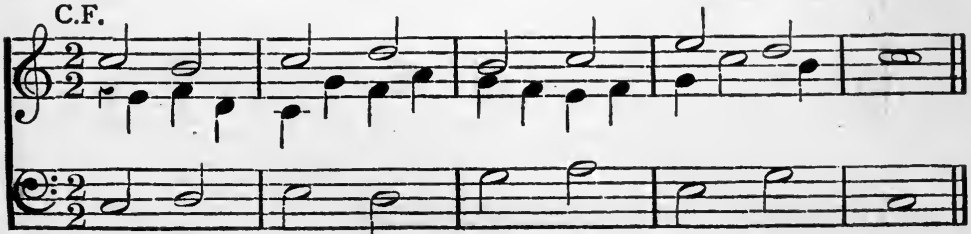




\section{CHAPTER X}

\section{THE THIRD SPECIES IN ONE OF THREE PARTS}

I. GREATER licence in reference to consecutives between the First and Third Species is allowable, in that except in the case of consecutives occurring on the first beats of successive bars (one harmony in each bar being presupposed), they may appear on the second, third, or fourth beats of successive bars, even though the reduction of the score to vertical combinations of essential notes should not bear the test-

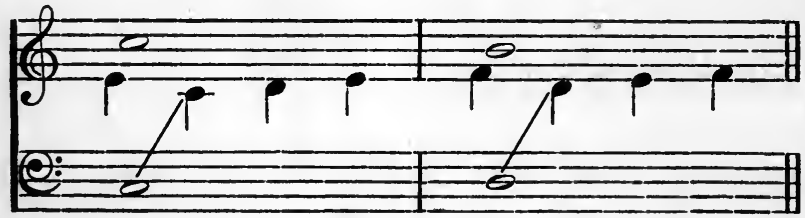

Good.

The ear perceives no unpleasant effect here.

In all other cases the rules given in two parts apply.

2. The leading note may be doubled in the Third Species, if it is in the middle of a scalic passage, or a part of an arpeggio-

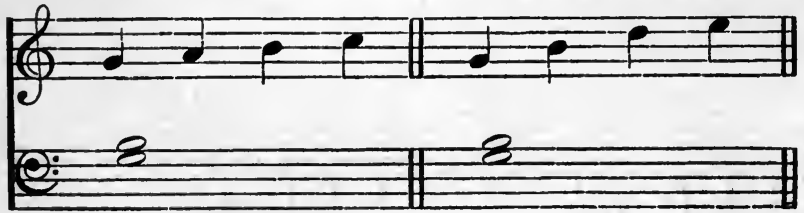

Good.

Good.

3. The cadences are generally as follows:- 
C.F.
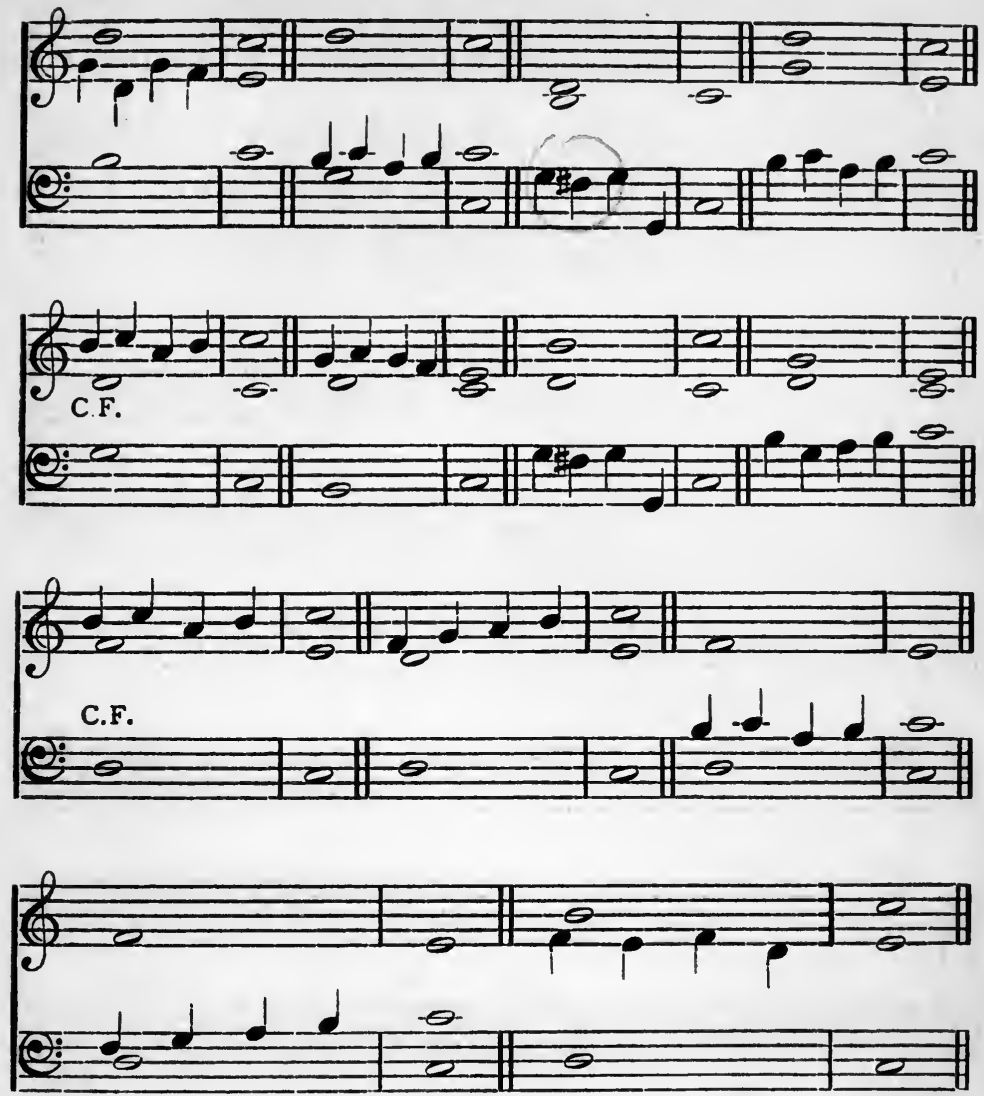

C.F.

4. The previous recommendations in reference to passing notes should be carefully followed. Observe however-

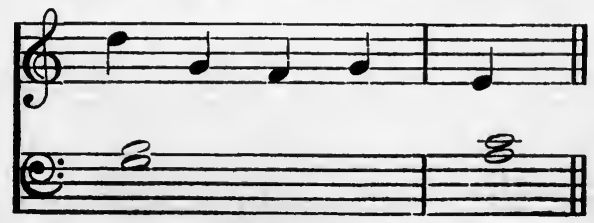

Good. 
Here we have the effect of a deferred resolution of the seventh.

Compare the rule in harmony textbooks for the treatment of auxiliary notes.

The fact is, under the modern harmonic system, a great many notes, which are theoretically unessential, must be treated as if they were, as indeed they are mentally, essential. The early writers used them artistically by instinct.

Examples :-

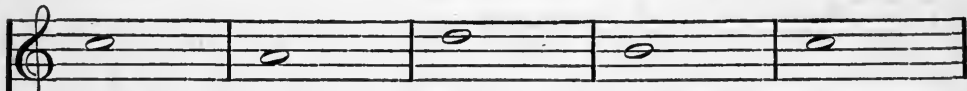

C.F.
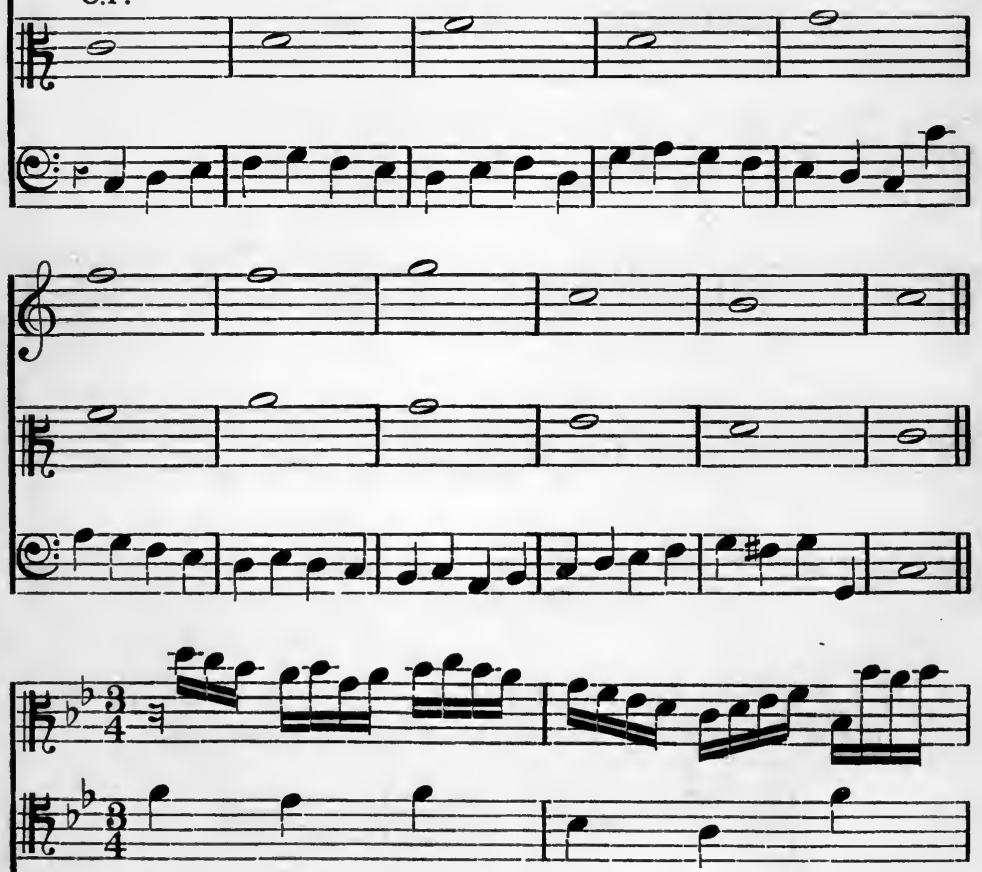

C.F.

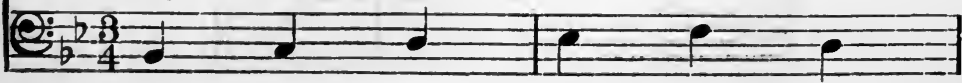



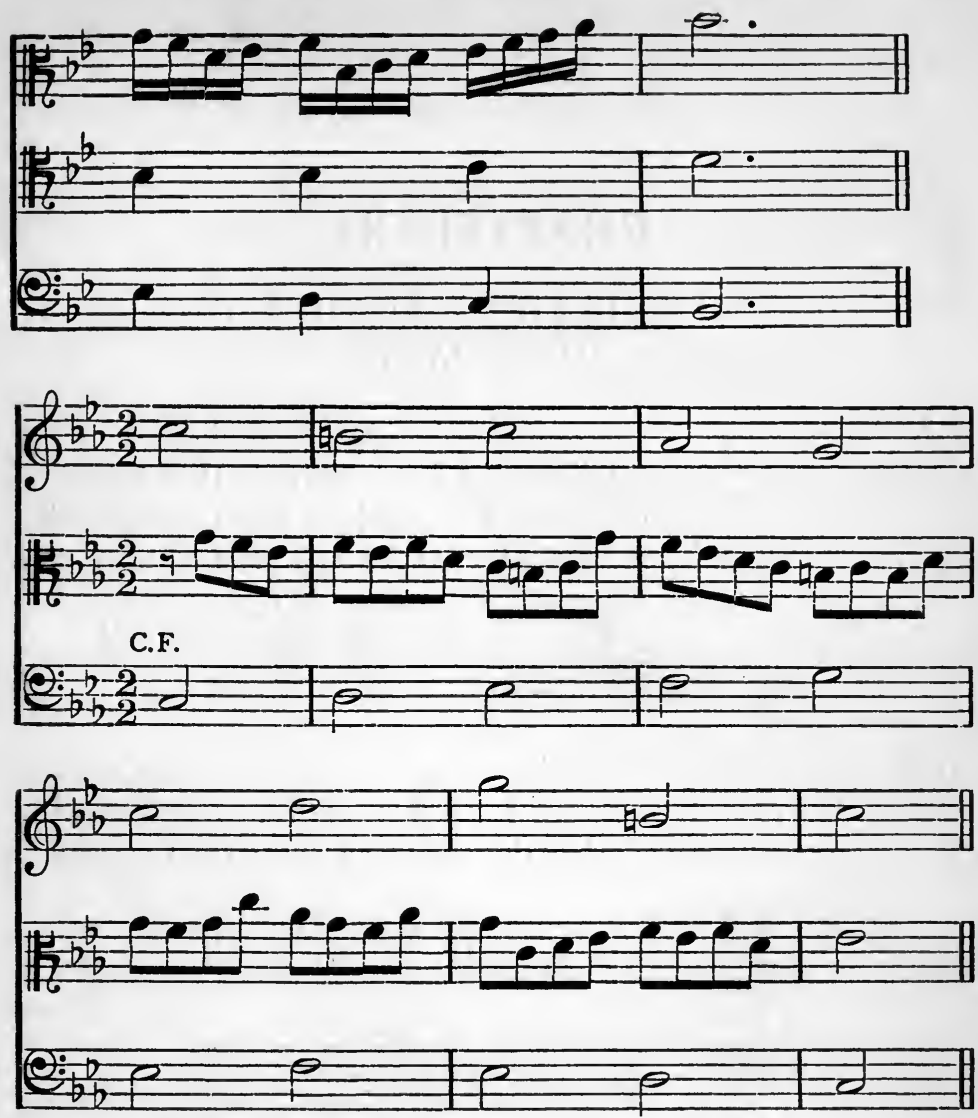

The student will at once see that the only difference between these examples is in the time signature. They are all absolutely strict examples of Third Species Counterpoint, merely put in various times to illustrate the practical uses of Counterpoint. 


\section{CHAPTER XI}

\section{THE FOURTH AND FIFTH SPECIES IN ONE OF THREE PARTS}

I. THE accompaniment of the various syncopated discords has already been adequately discussed in Chapter V. It is, however, necessary to remember that the $9-8$, besides being accompanied by the third, may be accompanied by the fifth or sixth also-

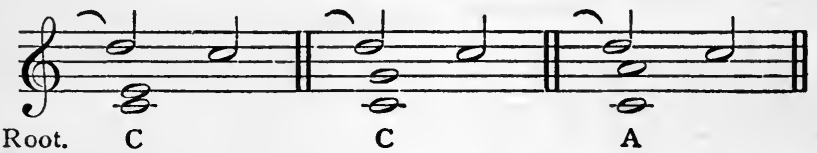

The reason is of course obvious, e.g. we have here the suspension of the root or third of a common chord. Similarly, the 7-6 may be accompanied by the octave as well as by the third-

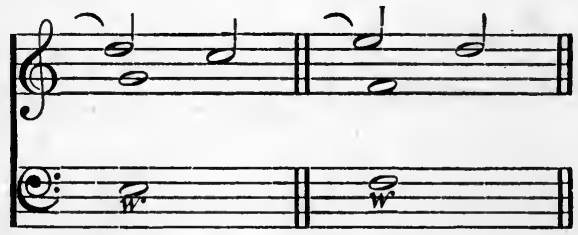

and the $4-3$ by the octave as well as the fifth-

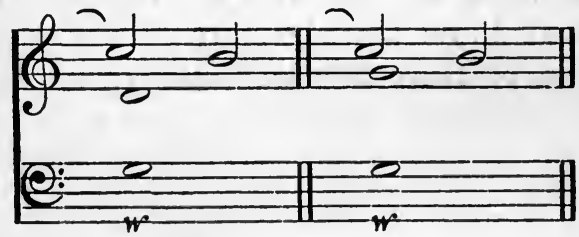

This may be summarized as follows:--Any portion of the chord, e.g. the root, third or fifth, may be doubled except that note which is suspended. Thus the following are faulty:- 


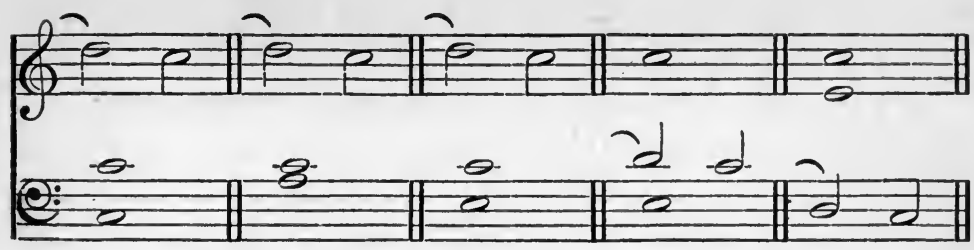

2. Cherubini and Rockstro give the following as examples of the correct accompaniment of suspended discords :-

Cherubini.

ROCKSTRO.

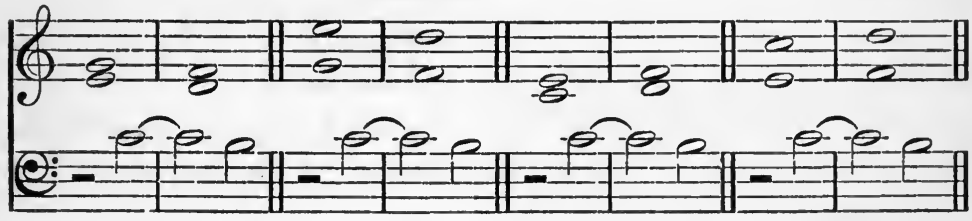

But all these are impossible as they stand, for the combination on the second minim is a dissonant triad in each case.

3. Consecutives must never occur on the second beats of two or more successive bars-

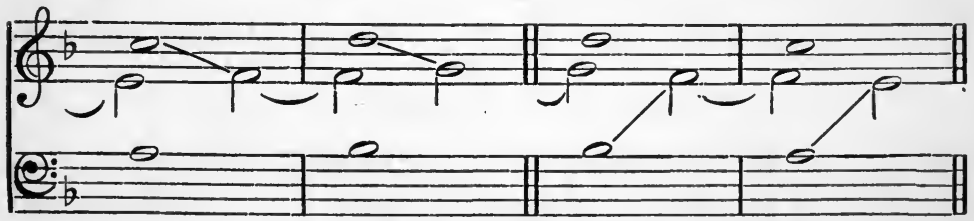

Such things were constantly done in the Polyphonic Period, but with no definite indication of change of harmony owing to the absence of other moving parts, and with no indication of tempo, they should be avoided.

The following is of course allowable:-

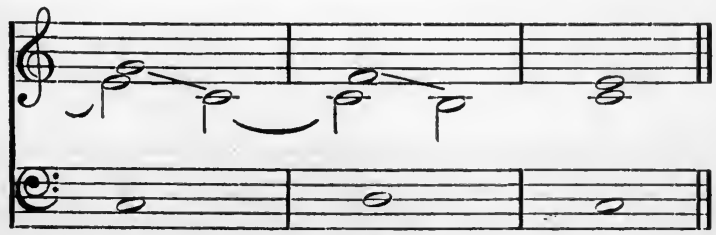

K 2 
Exposed consecutives are allowed under the conditions mentioned in First Species.

4. As to the accompaniment of syncopated concords, the following examples will make things clear:-

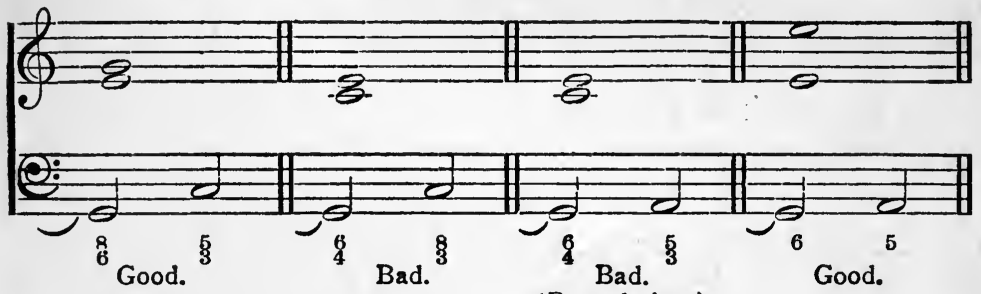

(Retardation.)

5. When the Fourth Species is in the bass, the upper parts may form a fourth, because the second minim represents the real harmony note-

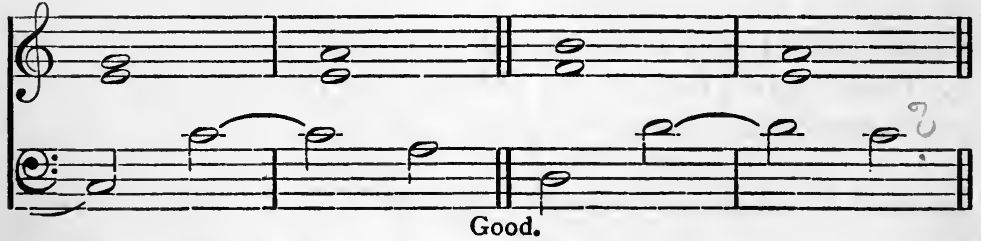

6. The best cadences are as follows :-

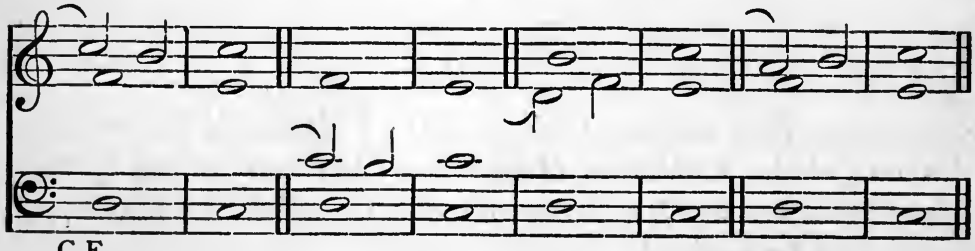

C.F.

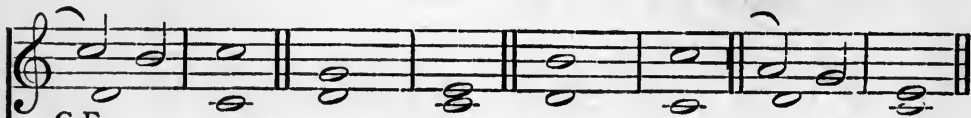

C.F.

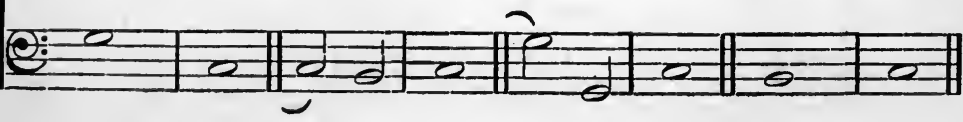


C.F.

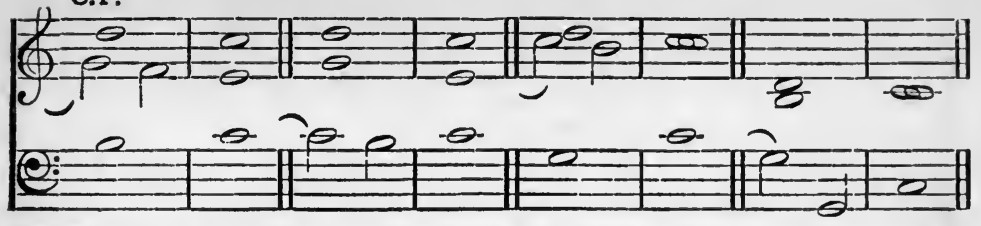

The leading note may be doubled, if it is a syncopated concord-

(a)

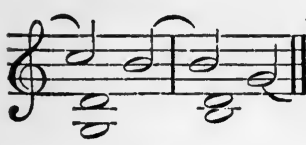

Good. (b)

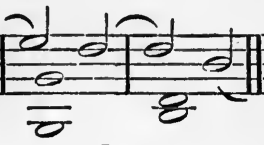

Bad. (a)

(b)

\section{Pedal Point.}

Sometimes the lowest part contains the same note for two or more bars: it then becomes a pedal point, and the next part above it is to be considered as the real bass.

The dominant and tonic are the only notes that can be thus used, and only in approaching the cadence-

Palestrina, Aeterna Christi Munera.

(c)

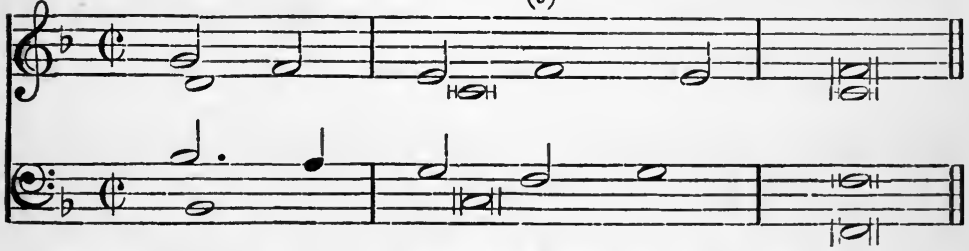

From a modern point of view the fourth (c) prepares a discord of suspension : but from a contrapuntal point of view the tenor is the real bass on the second minim, the bass again becoming essential on the third minim. A few examples are added-

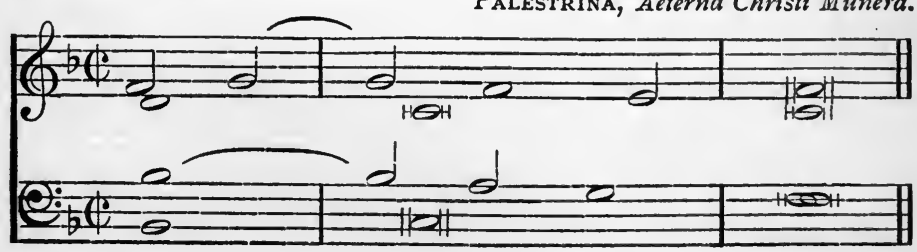




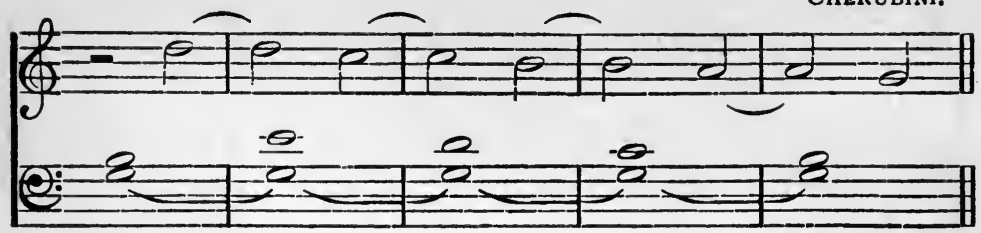

Cherubini.

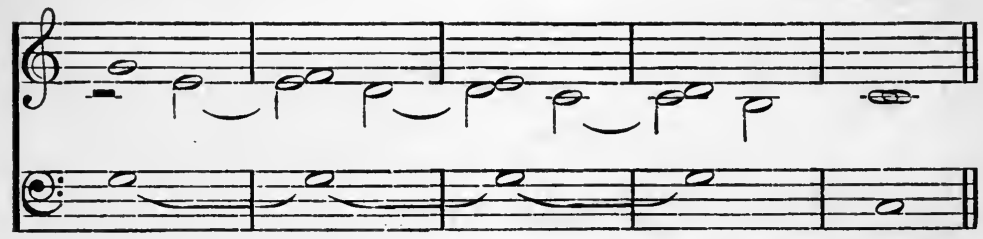

Cherubini, while allowing that Palestrina uses the discord of the fourth without preparation in order that it may become its own preparation, makes the stipulation that the first discord be prepared by a concord, and the last discord be resolved by another concord.

The use of the pedal point in Scholastic Counterpoint is rare, but the following is an illustration of the sort of case in which it may be introduced with advantage-

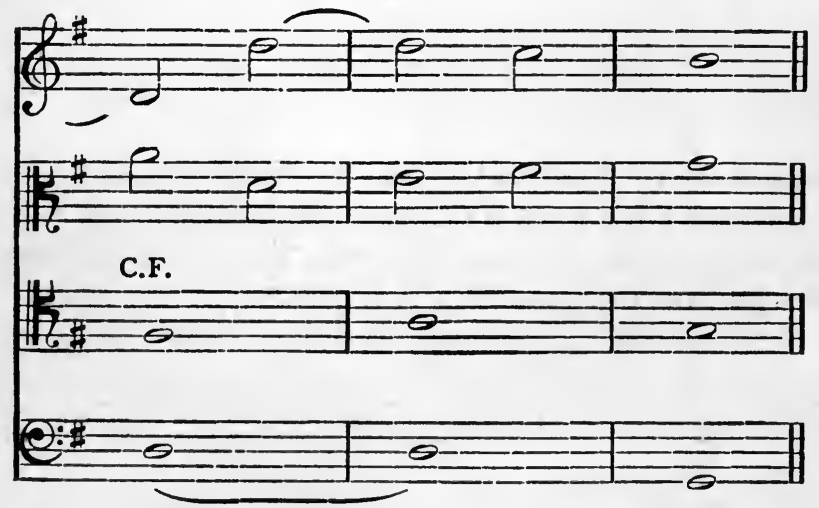

Examples :- 


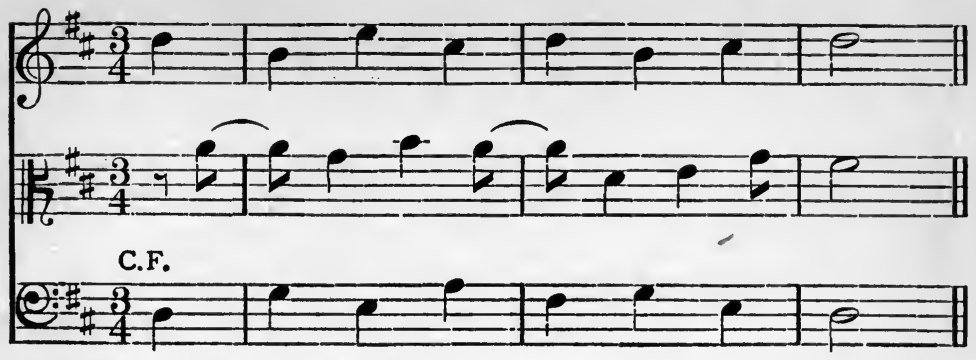

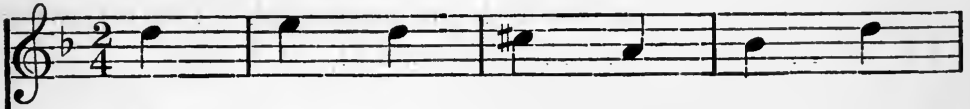
C.R

$\cdots \cdots$

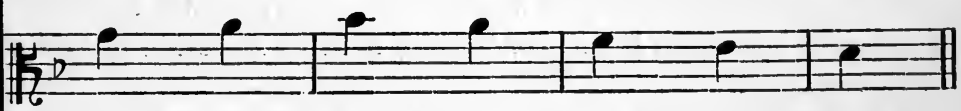
: एव की C.F. C.r. ए; 


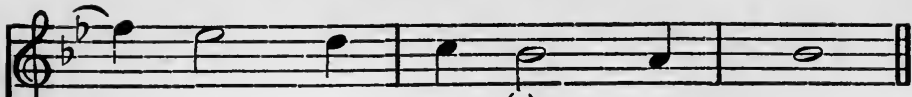

(a)
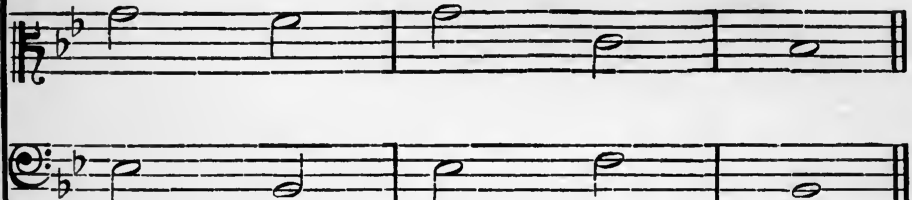

(a) It is hardly necessary to point out that some theorists wrongly regard such a concord as unessential. The following is sufficient to refute any such argument:-

Palestrina.

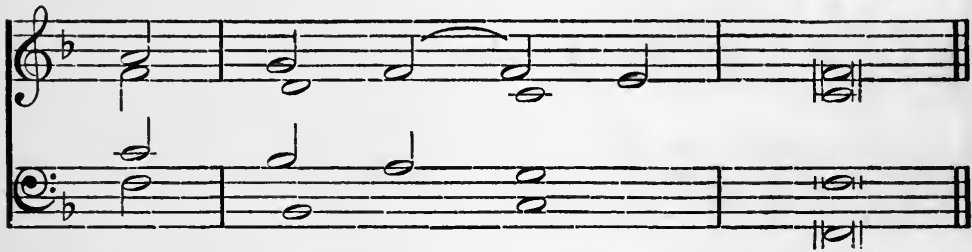

The examples given with the above time signatures will show the purposes of uncombined Counterpoint, and serve to illustrate the fact that bar lines do little more than help the eye to keep things clear. It is the accents that really mould the music, and it will be shown later how time signatures are often deceiving in this respect.

The ordinary form in which problems are given implies two accents in the bar thus-

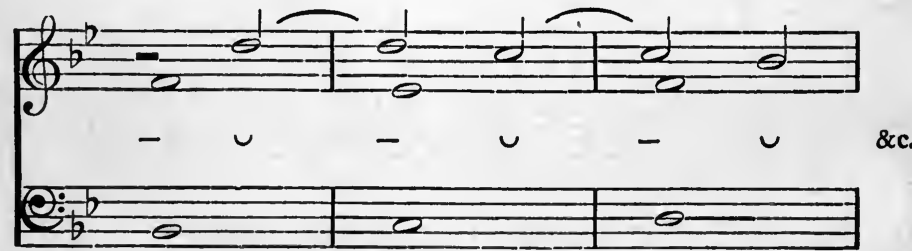

This analysis is equally satisfactory: our purpose has been to show the various uses of the scholastic process.

In future we shall regard each bar as containing two accents $-\cup\left(\begin{array}{l}2 \\ 2\end{array}\right)$. 
The Fifth Species in one of Three Parts.

Too frequent syncopation is unadvisable. The rules with regard to rhythmic and melodic variety already given in two parts must be most carefully followed. No further rules are needed in addition to those in the introductory chapter on threepart Counterpoint. The occasional use of an untied minim on the second half of the bar in uncombined Counterpoint is effective if it has been preceded by another minim.

\section{Examples.}
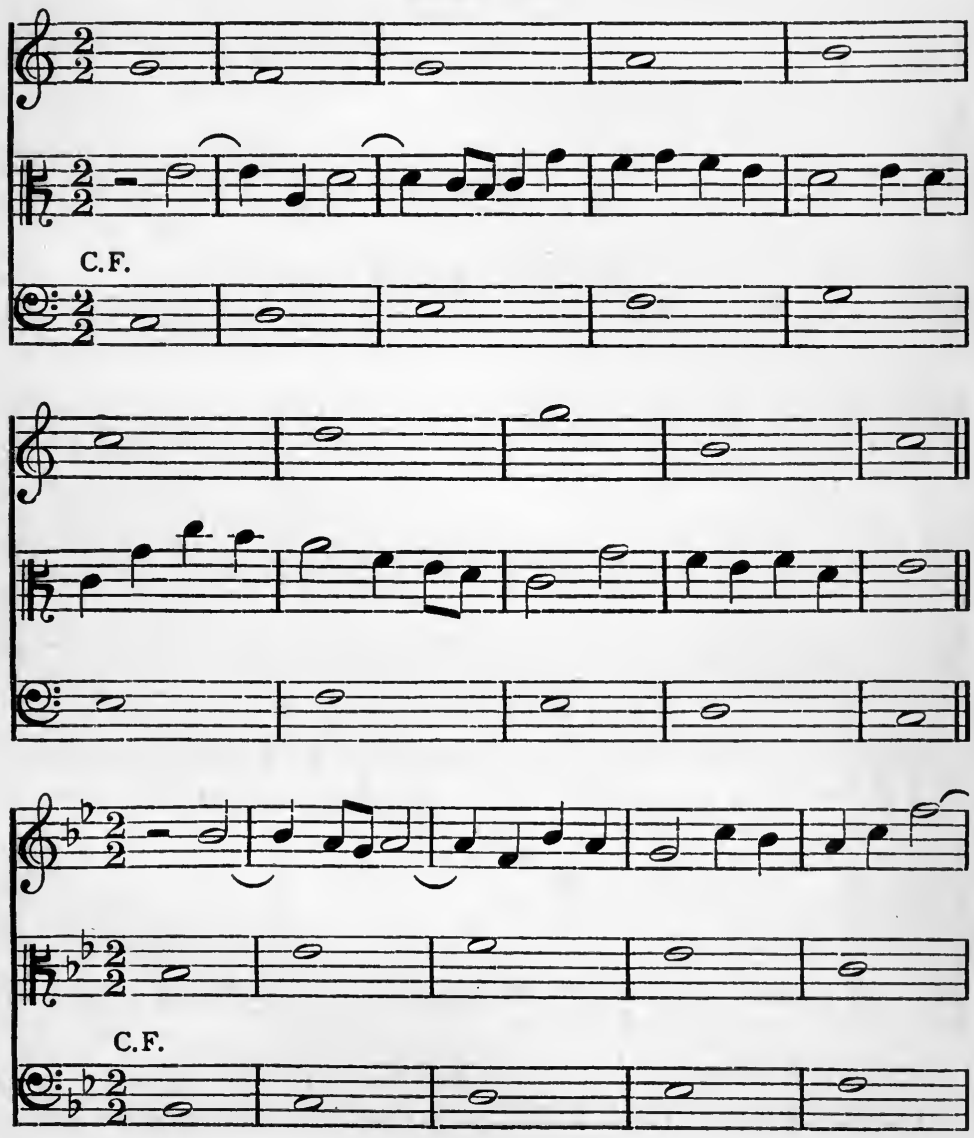


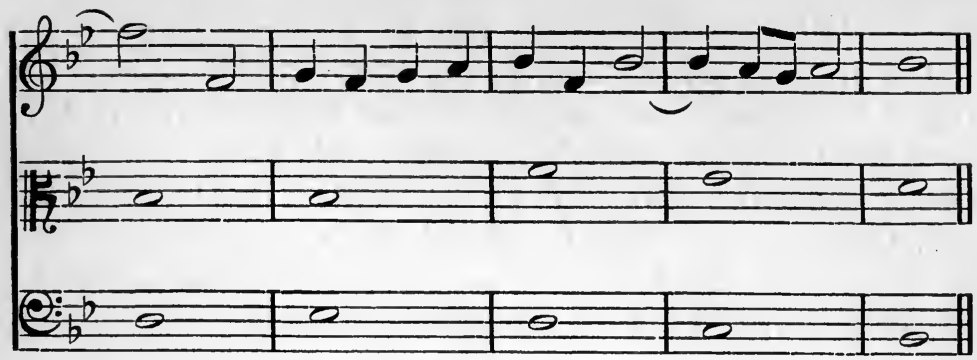
C.F.
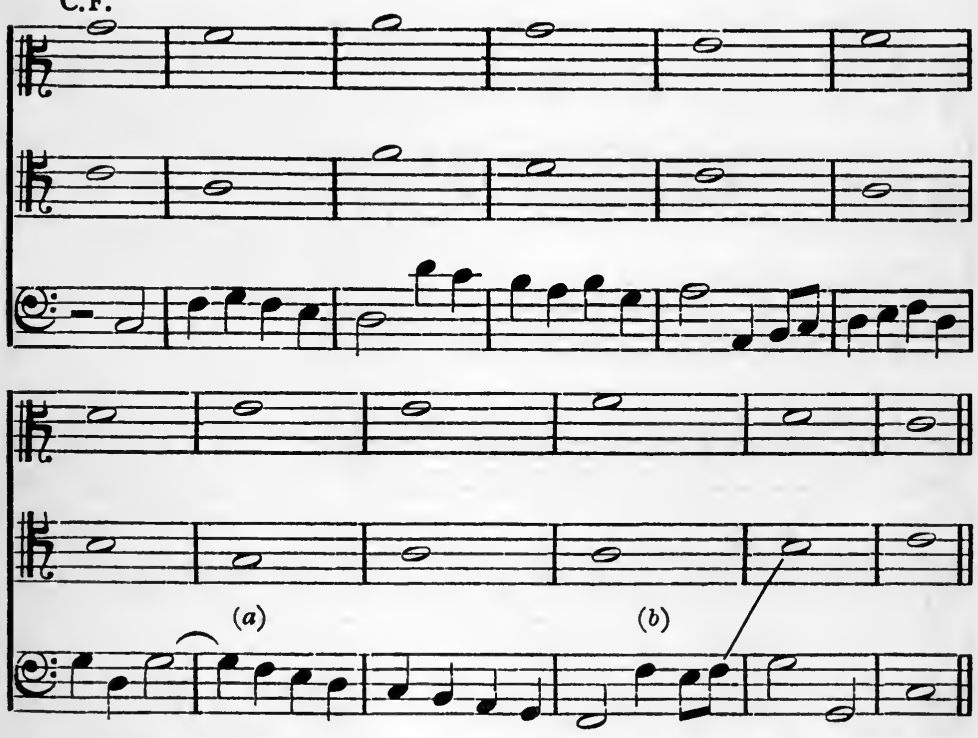

(a) The ambiguity of the harmony makes $\mathrm{F}$ unobjectionable : if a B had been present the effect would have been bad. Note

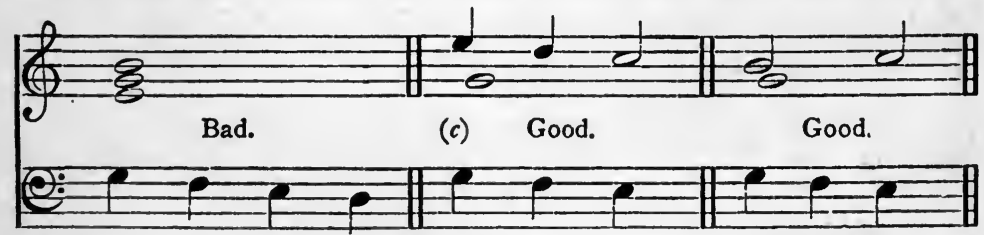

The mental effect of $(c)$ is that it is a thirteenth on G.

(b) The effect of the tritone is entirely unobjectionable. 


\section{CHAPTER XII}

\section{COMBINED COUNTERPOINT IN THREE PARTS}

WhEN more than one of the added parts are in some species other than the first, we have what is termed Combined Counterpoint.

This is a branch of the subject that has received very inadequate treatment at the hands of theorists. The earlier writers merely gave a few examples of Combined Counterpoint with little or no comment, while the modern explanation of it, as seen in textbooks of the Macfarren school, is not only inconsistent with practice, but imposes unnecessary limitations, and by so doing actually encourages a system of writing which is productive of far-reaching evil. But it must be admitted that the whole of the blame cannot be laid at the door of the Macfarren school, and in order to explain the position clearly it is necessary to consider musical decoration in some detail.

It has been previously insisted that what is considered correct Scholastic Counterpoint should not be capable of condemnation under harmonic conditions. In reading the compositions of the Polyphonic Period a modern can easily see what is correct relatively or absolutely. We have seen that Palestrina's procedure in the matter of consecutives cannot be generally followed under modern conditions. We have also seen that the constant running into the unison is only tolerable under the conditions of the time. The adoption of the modern scalic system has brought with it points of view which were nonexistent in the sixteenth century, except as matters of artistic feeling, both in reference to choice of harmony and melodic progression. We have now to discuss in a more detailed way the question of harmonic propriety. The great principle to be 
kept in view is, that if Counterpoint is to be a training in the art of combining melodies, it is useless to teach a procedure as being correct Counterpoint, but bad Harmony. The antiquarian student may, if he choose, learn to write exactly as Palestrina did under the modal system ; but this is not Scholastic Counterpoint, though the latter is based upon it. Scholastic Counterpoint is the adaptation of the principles of Palestrina to modern conditions; that is, no enlargement of his harmonic or melodic resource is made, for what is good in this sphere remains so for all time, though the range of possibilities in this direction may be indefinitely enlarged. But in questions which involve relative criticism, that is when a particular procedure may be correct under conditions which have now ceased to exist, theory must bring such cases into line with modern thought.

The work of the early Polyphonic school may be said to consist of experiments in combinations of sounds resulting in what we term common chords and their first inversions, a few suspensions and prepared discords, and passing notes linking concord with concord; that is, we have in the work up to the time of Palestrina the foundation of diatonic Harmony. Then, under the leadership of Monteverde came the enlargement of the harmonic scheme.

Discords came to be used without preparation, and combinations of essential and non-essential notes which were pleasing in effect came to be catalogued as chords, and root theories were devised. Thus in Palestrina we have

Missa Aeterna Christi Munera.

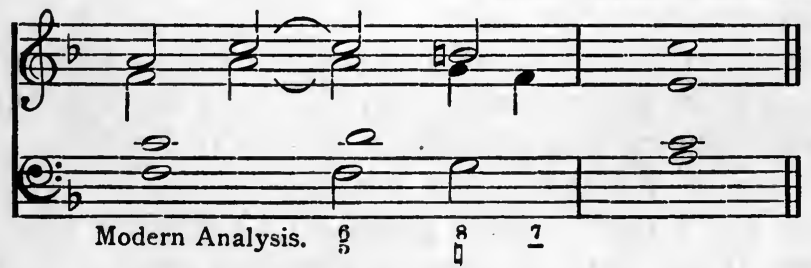

According to modern classification the combination on the third minim is a dominant eleventh or secondary seventh, and the last combination of the bar is a dominant seventh. 
The process then is one of transference of notes which were regarded as non-essential to definite positions in relation to the fundamental harmony. This, while not lessening the means of decoration within the limits of diatonic resource, does, of course, tend to restrict both melodic and harmonic progression. This will be best understood by means of illustrations :-

Fix.

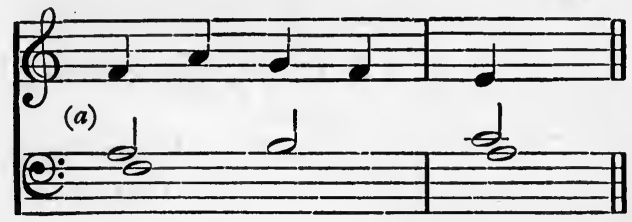

MACFARREN.

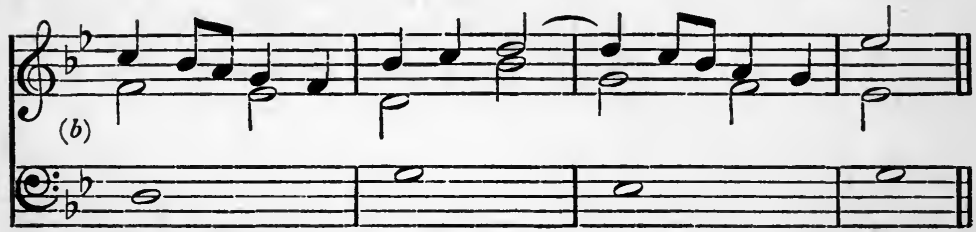

Now these two examples are defended on the following grounds:- 'Two passing or auxiliary notes may be written at the same moment with good effect, provided that they are consonant with one another.' It may be contended that each part taken separately is correct Counterpoint, and that the combinations keep the above rule, but no amount of argument prevents the modern ear from accepting

(a)

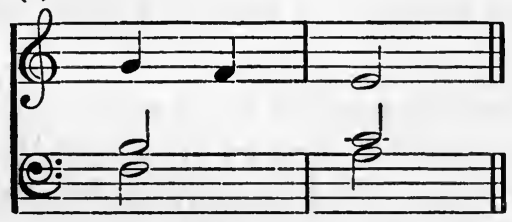

as a ${\underset{2}{4}}_{2}^{6}$ on $\mathrm{F}$, with the seventh doubled on the second crotchet, and the bass unresolved. 
In the second case quoted

(b)

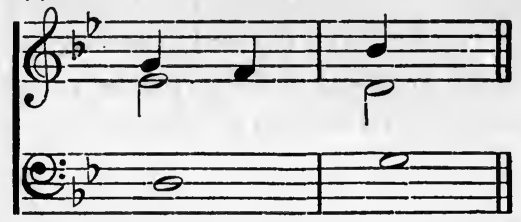

the bass (D) is felt to be a pedal wrongly quitted; in any case the harmony is obscure and confused.

No one would tolerate such things in a harmony exercise ; the position therefore is illogical. It is true that such a case as (b) is corrected by a modern rule :- ' In combinations of the Second and Third Species, a note of the latter, if dissonant with a passing note of the former, must not be taken or quitted by skip, even though it be consonant with the implied harmony.' But such a rule allows of

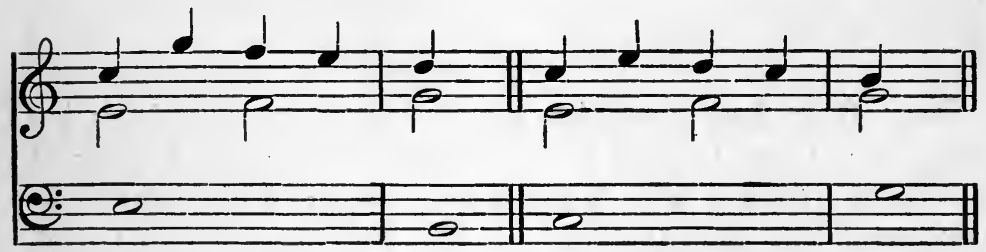

which are no better than $(b)$.

Now the chief rules of the Macfarren system, in reference to Combined Counterpoint, are

r. 'The lowest moving part must be a good bass to the others above it.'

2. 'All parts moving together at the same instant (i.e. all notes which are struck together) must be in concord,' except when two moving parts are proceeding by conjunct and contrary motion; in this case they may strike a discord.

The following is a typical student's application of these rules :- 

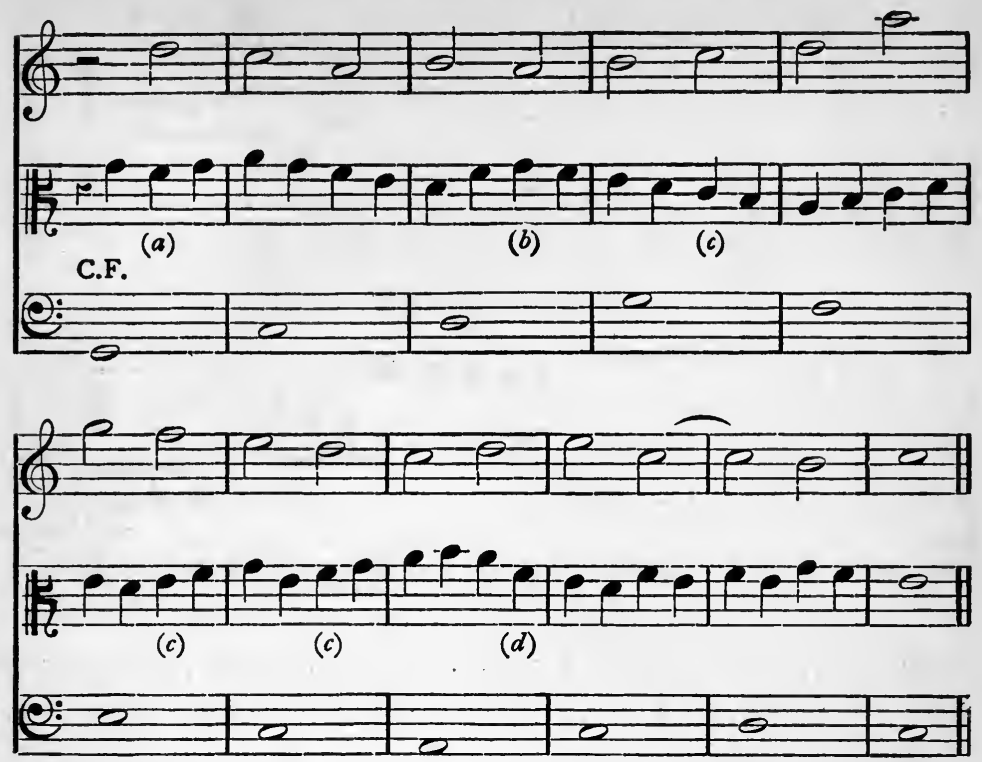

The mental effects to be noticed are

(a) Unresolved seventh.

(b) Crude harmony.

(c) Obscure harmony.

(d) ${ }_{4}^{6}$ quitted by leap.

If such writing as the above be the result of a study of Strict Counterpoint, the sooner it is given up the better.

The results of this system are seen in a few extracts from students' attempts at composition :-

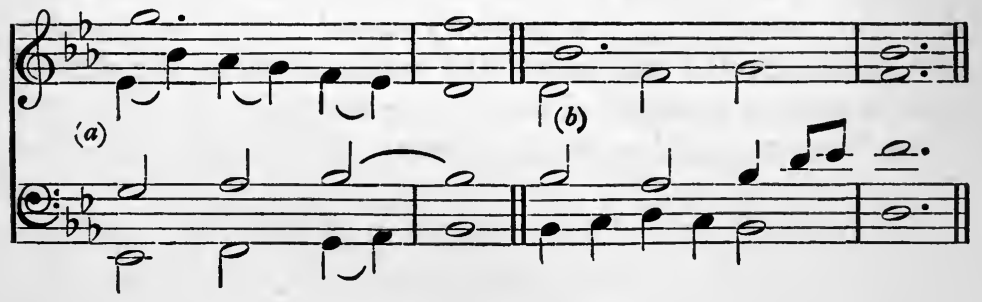




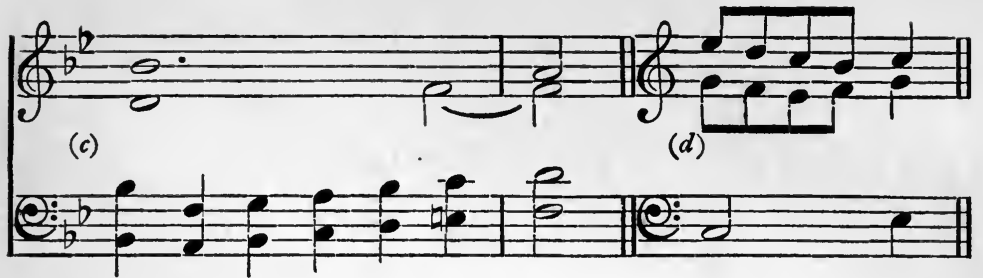

(e)

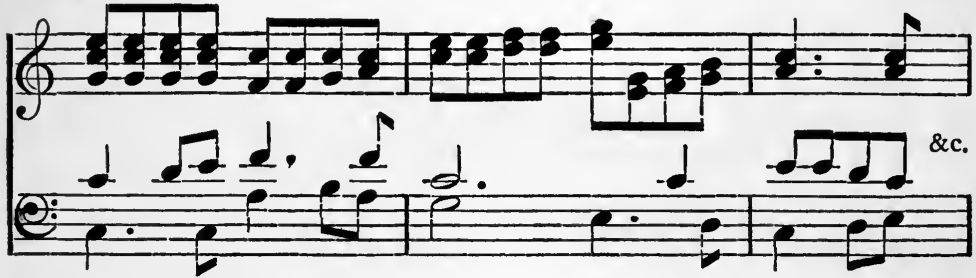

These are only a few of the many examples of such crudity that are to be seen every day in students' work. There is evidently something wrong somewhere.

The fact is, it is not true to say that all that is necessary is that all notes struck together must be in concord, except under the condition mentioned. This will be dealt with in its place. Sufficient has been said to prove the pernicious influence of the rule.

J. S. Bach stands midway between the instinctive harmonic purity and simplicity of Palestrina, and the calculated harmonic purity and complexity of the modern diatonic school.

Judging of Bach's work from an absolute point of view, his deficiency lies in his indifference to the harmonic effect of his decoration; for though his chordal centres and seams are quite clear and satisfactory, the harmony caused by the combination of various essential and unessential notes decorating the chordal centres is quite a matter of indifference to him.

An illustration will make this clear- 


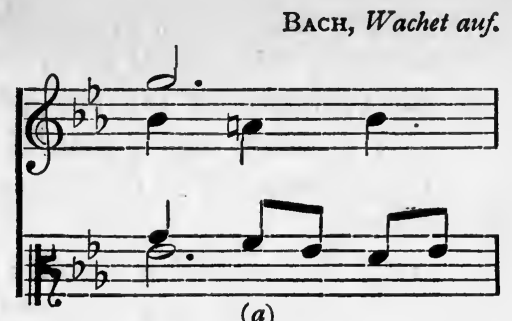

(a)

(a) the effect here is that we have a supertonic ninth, with the seventh in the bass, the tenor clashing into and reinforcing it, and the resolution wanting. Bach intends the chord of $E$ flat as the prevailing harmony. In the modern diatonic school, in the best examples, there is an absence of this confusion of harmonic and contrapuntal principle. Harmony and Counterpoint are so perfectly balanced that good Counterpoint forms irreproachable Harmony, and good Harmony forms interesting Counterpoint. And so with the clearness of harmony in every detail, the way was prepared for the adoption of the chromatic scale as the unit, and the regarding of 'all notes as equally related'. The Romantic movement shows tendencies in this direction, finding of course its culmination in Wagner, Dvorák, Elgar, and Strauss.

The origin of the much debated chord of the augmented sixth shows in embryo the principle of Elgar. The following table shows how the chord was formed:-
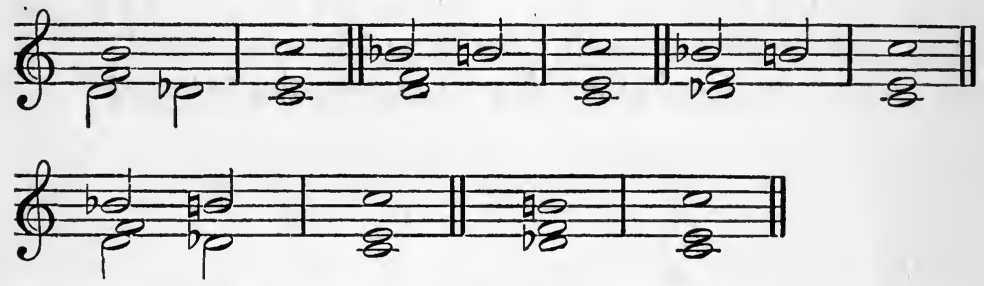

And under the present system it is probable that many combinations, formed in the same way, will be in time catalogued as chords. The present modern school is at the same stage of evolution in chromaticism as Bach was in the diatonic system; resultant harmony being a secondary consideration, while the 
chordal centres themselves exhibit new progressions. With the problems and possibilities of this school we are not concerned; our knowledge of the evolution of the art would point to a process similar to that through which diatonic harmony has gone, a process of the elimination of the unessential, and the enlargement of the essential.

It will have been seen that attention must be paid to vertical considerations, and unless this is done students form a habit of mind which has disastrous effects on practical work; and it cannot be too strongly urged that teachers should not condone in Counterpoint what they know to be bad Harmony. As time goes on, composers are finding new harmonic progressions, and it must not be supposed that the recommendations given in this treatise in reference to this point are in any way absolute.

The laws of harmony, as at present stated, require that $(a)$ should be resolved as at (b) if the bass move downwards by any interval other than an octave

(or)

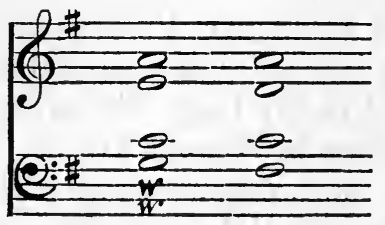

(a)

(b)

and that the bass note of a ${ }_{4}^{6}$ should not be quitted by leap, unless proceeding to another position of the same chord.

There is nothing in the rules of Counterpoint to forbid

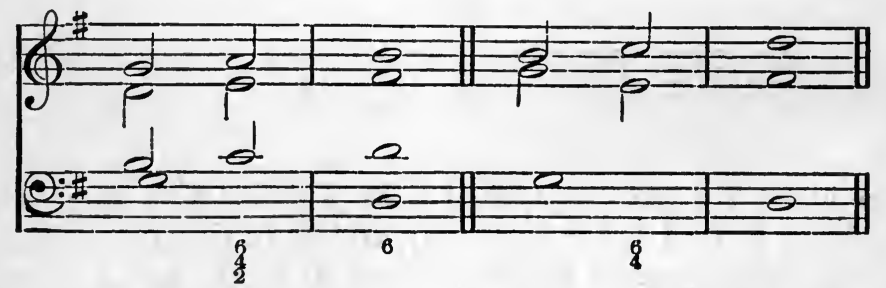

Suppose these discords were to appear on the first half of a contrapuntal bar, the following would be the procedure :- 


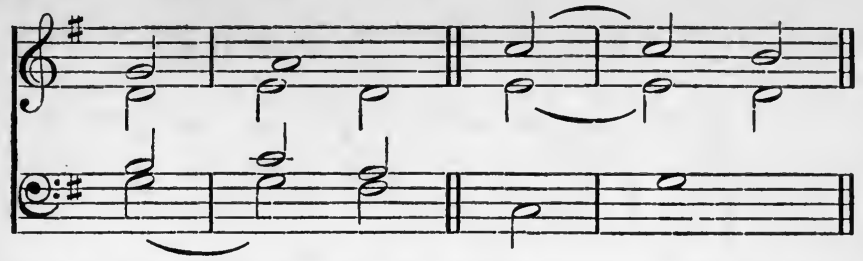

Thus it will be seen that contrapuntal theory makes a clear distinction between the two cases, and herein lies the basis of conflict between harmonic and contrapuntal principles.

The above combinations occur in a part song by Sir C. H. H. Parry, 'If I had but two little wings.'

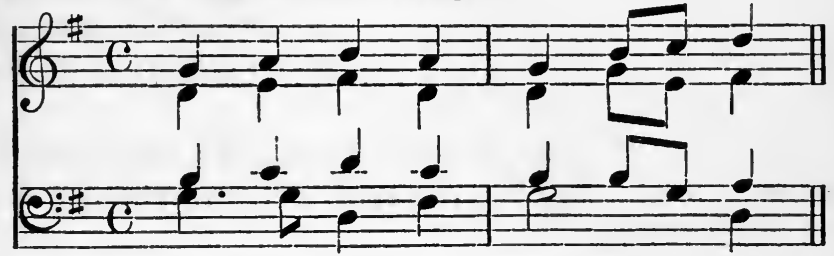

A consideration of the grounds of their justification may prove helpful to students. It may be asked with reason how it is that the ear accepts the first two of the following examples as satisfactory, and rejects the third as crude-

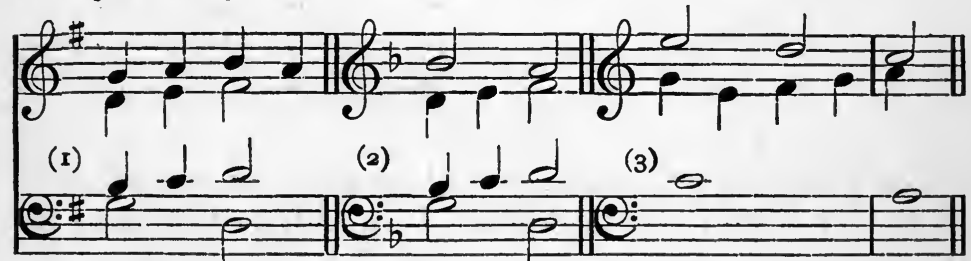

In the first place, it is of course obvious that the question of accent plays an important part in the matter. The following are undoubtedly bad :-

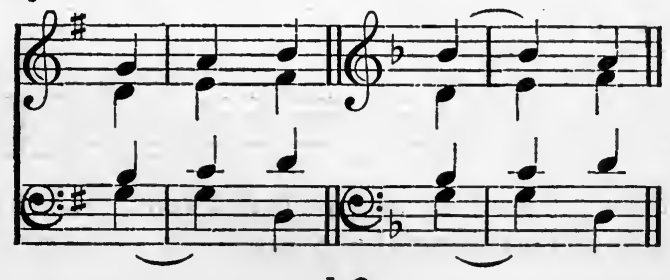

L 2 
But this is not the only consideration, for both the following are unsatisfactory :-

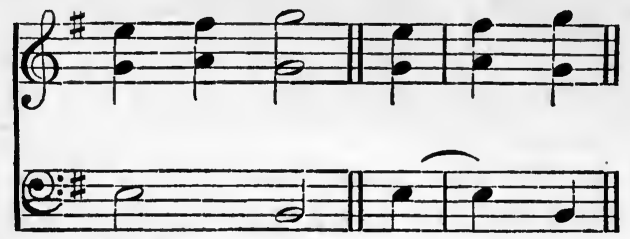

It may at once be said that the effect will be good if the parts concerned proceed in simultaneous and parallel movement, and if the two contrapuntally essential combinations connected are satisfactory as a harmonic progression, when the intermediate sounds, which of course must proceed by conjunct degrees, are omitted.

In such cases the contrapuntally unessential combinations on the weak parts of the bar do not produce the mental impression of a new harmony.

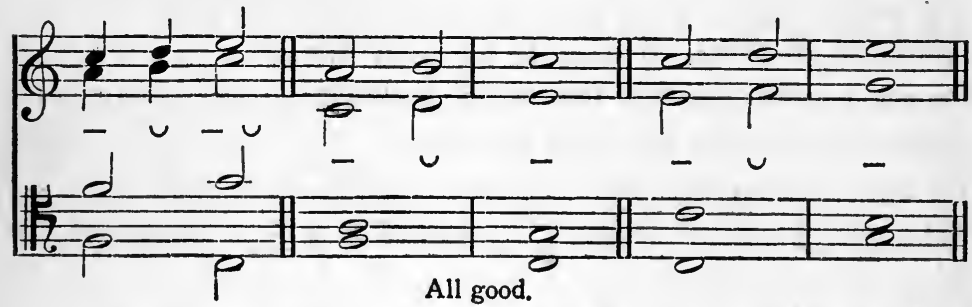

But when the two parts involved take two unessential notes by similar motion and then proceed by contrary motion to the next centre-

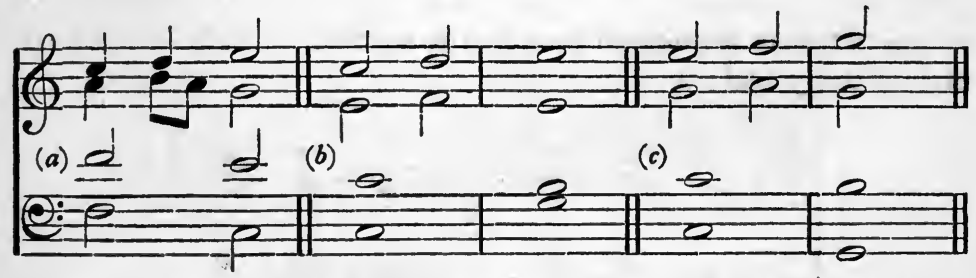

or when they proceed in notes of different time value after having struck two unessential notes- 
COMBINED COUNTERPOINT IN THREE PARTS I49

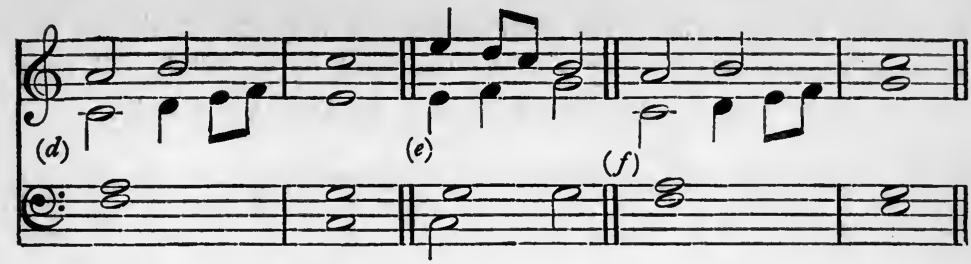

the mind at once loses the impression of the original essential combination, and receives the following confused effects :-
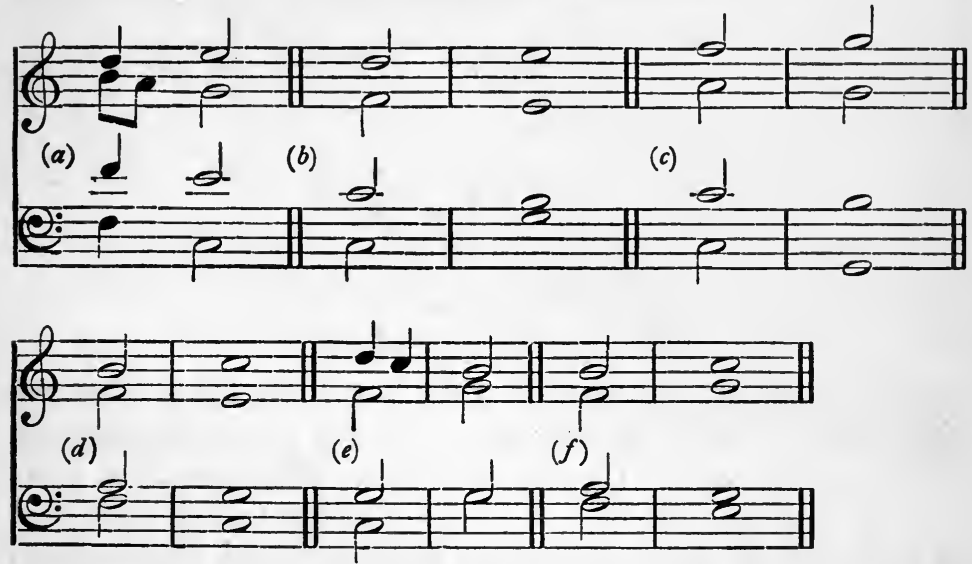

There are cases in which combinations of this nature are satisfactory in effect; they will be dealt with under their proper headings.

A want of attention to matters of this sort is the cause of the following crudities seen in students' composition-

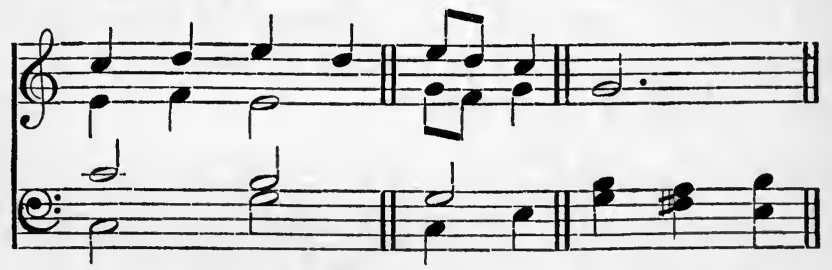

The following rule will form a safeguard against such things :Whenever two parts move in parallel thirds or sixths by 
conjunct degrees (the first combination being essential and the second unessential), they should proceed in parallels till they again reach essential harmony, unless the bass be a pedal-

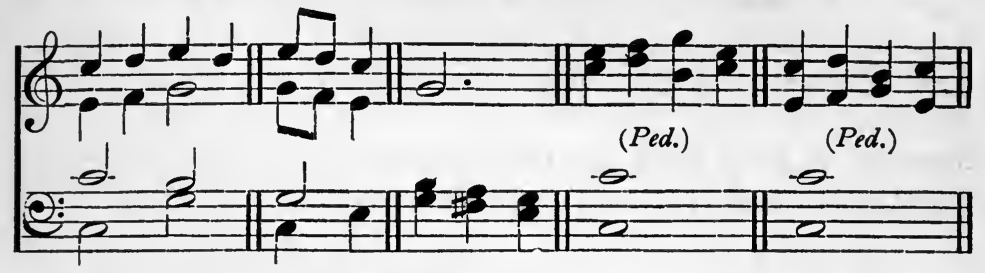

It may be thought that the above statements place us in a dilemma, for we have yet to justify-

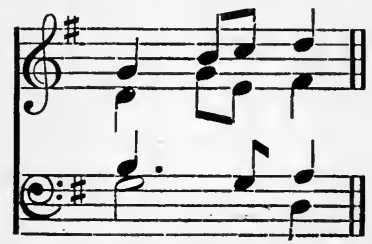

which apparently contradicts what has been said. But the above happens to be a case which forms a very rare exception to the general rule. The considerations which justify it, apart from its intrinsic effectiveness, belong rather to harmonic than to strict contrapuntal conditions. For the mental impression is that the centres are the chords of $G$ and $D$ major. The note $E$, being unessential to the harmony of the chord of $G$, would hardly be written under strict Scholastic conditions, though its presence can be explained by principles of concordance. The fact is that $G$, instead of proceeding to $F \#$ direct, first leaps a third to the other side of it in the manner of a changing note. This can be conclusively proved by a comparison with the following, which is unsatisfactory :-

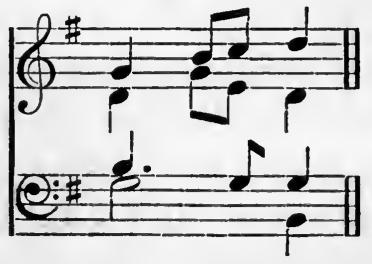


This is one of the most important questions raised by a study of Combined Counterpoint, and the matter has been dealt with in detail, so that the student may see the exact purport, and also the limitations from an absolute point of view, of the following recommendations. They are intended to serve as safeguards against some very prevalent faults in students' work, and it is necessary to insist on their being observed until the student has a grasp of harmonic effect sufficient to enable him to find out artistic exceptions such as the case quoted. This opens out the whole question of complex movement, and it cannot be too strongly emphasized that indifference to questions of this sort is one of the most fruitful sources of crude writing.

Artistic instinct often revolts against what is merely mechanically accurate, and laws which allow of unmusical application can scarcely be considered authoritative. For instance, the following are not condemned by any formulated rules; they are actually an application of the rules of Combined Counterpoint as stated in some modern treatises:-

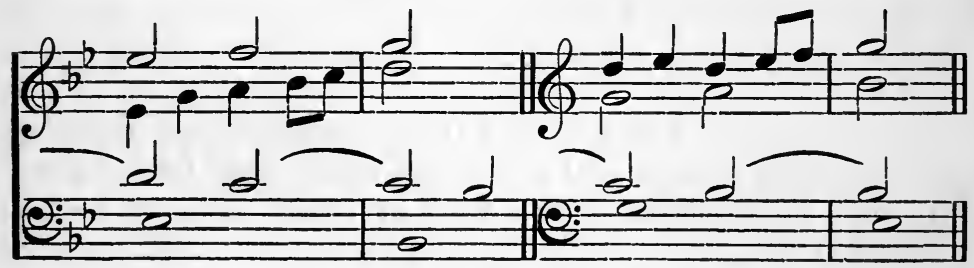

It is inconceivable that any one could write such things under any pretext whatever: but, owing to a mechanical system of Counterpoint, students are driven to believe that crudities of this nature are some of the necessary evils of Combined Counterpoint. The maintenance of the conjunct nature of the Second Species is the crucial point in such cases. Apart from any questions of historic authority, the restriction to the use of one chord in a bar either compels such discords as the above, or a Second Species that can only be regarded as artificial. 
The Combination of Second and Third Species.

I. Each part enters after a rest of the time value of the notes of its Species. It is of course obvious that all three notes struck on the first beat of the bar must be concordant.

2. The chief difficulties of the combination lie in the maintenance of clear, good harmony, and the conjunct motion of the Second Species. The following are the methods of securing this :-

(A) If the second minim of the bar be taken by leap, it is often possible to make the movement to the first minim of the next bar conjunct-

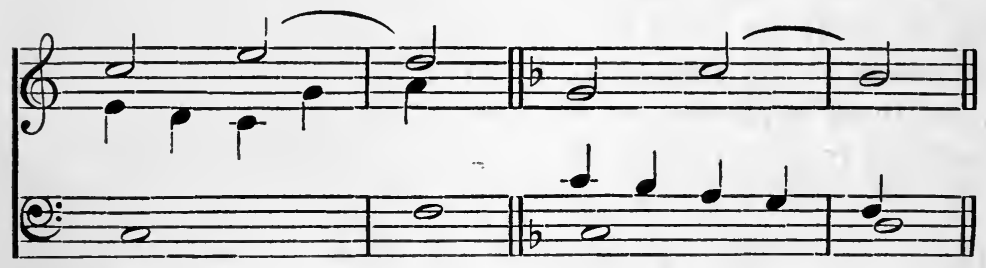

(B) If the second minim be a true passing note, it, together with all other moving and stationary parts, must form a good harmonic progression on the third or fourth crotchet to the next bar-

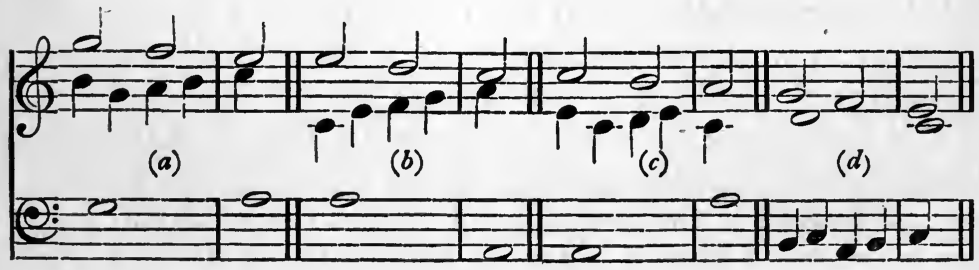

The mental effects of the above are as follows:-

(a) dominant major ninth resolving on the third, the seventh also resolving one step downwards ;

(b) a passing ${ }_{4}^{6}$; 
(c) the dominant eleventh (root E), or the dominant seventh (root E) on a pedal bass;

(d) the dominant ninth (root $\mathrm{G}$ ).

Note.-In each case, the Third Species keeps the rules relating to the use of one harmony in a bar.

(C) A change of harmony may be used-

Missa Brevis.
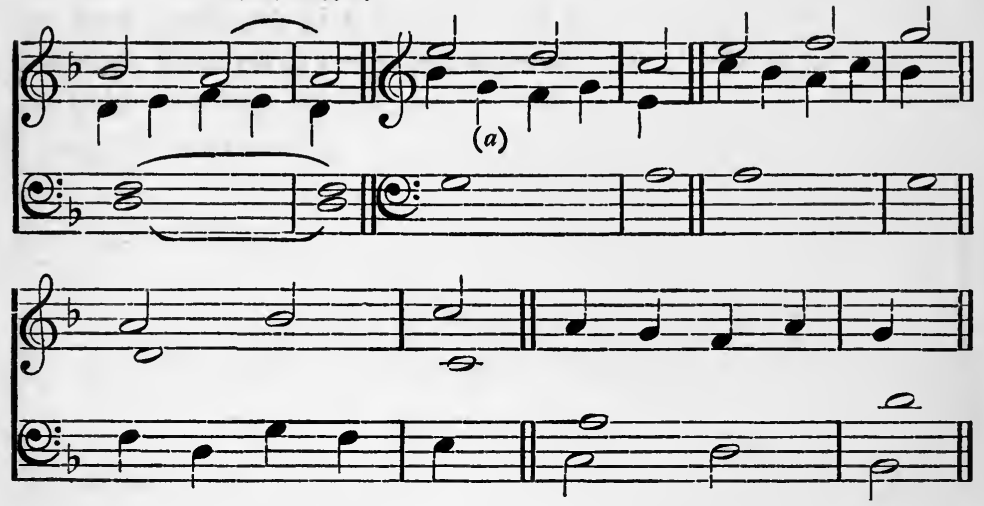

Iste Confessor.

Ibid.

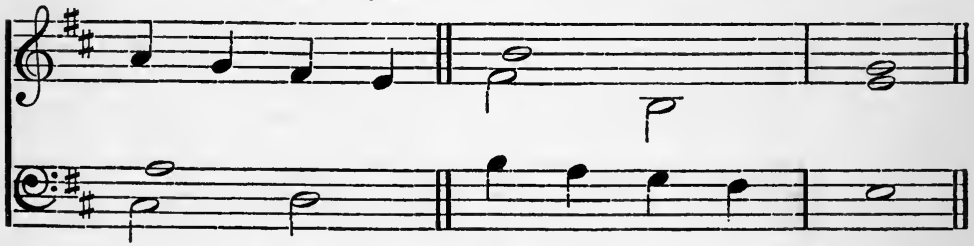

(a) this auxiliary note on the third crotchet of the Third Species is quite correct under such conditions.

3. In all these cases the notes struck together have been consonant. Under what conditions may they be dissonant?

A. (I) If the Third Species be discordant with the Second Species on the third crotchet, the minim being a true passing note, the third crotchet should generally be approached and quitted by step; and all the parts must on the third or fourth crotchet form some good harmonic progression to the next bar- 


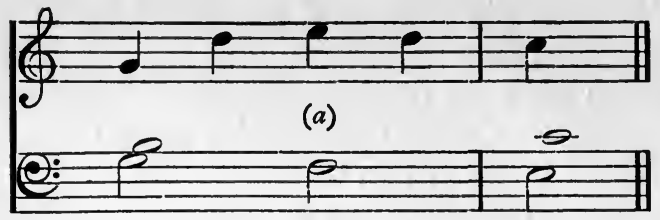

(a) is a dominant thirteenth (root G).

If, however, the note in crotchets that is dissonant with the unessential minim is a harmony note, there is no valid objection to its being either approached or quitted by leap, provided the above limitation in reference to harmonic propriety be kept-

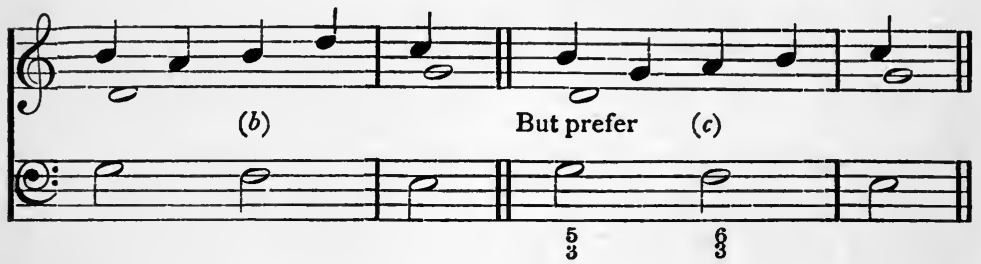

In $(b)$ the movement of the Third Species is governed by the laws of the prevailing harmony of the bar.

(2) Sometimes, by striking a discord by conjunct and contrary motion, we may get two harmonies in the bar; here we see the origin of the appoggiatura-

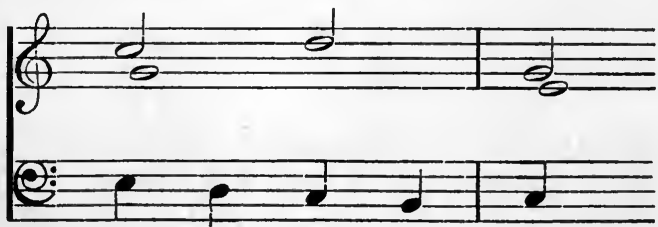

'Of these collisions', says Rockstro, 'the greatest of the masters took no notice whatever. Provided their florid parts moved well with the bass, they cared nothing for the crashes which took place between them.' Undue licence in this respect is not recommended, but the use of occasional discords of this nature makes really melodious and musical Counterpoint a possibility. 
B. If the second minim is consonant with the C.F. the third crotchet may form with it a discord, if the part in crotchets does not on the fourth beat run into the same sound as that of the Second Species-

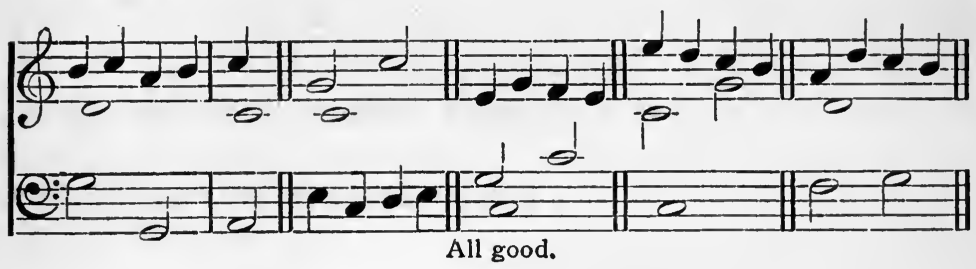

The following examples from Palestrina will be of interest:Credo, Missa Brevis. $\quad O$ admirabile commercium. Adoramus $T e$ Christe.

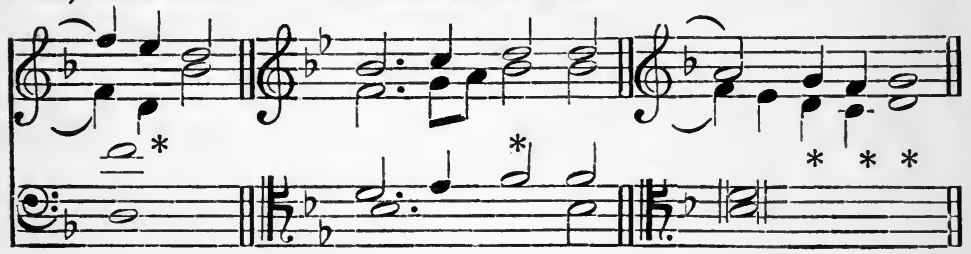

Sicut cervus.

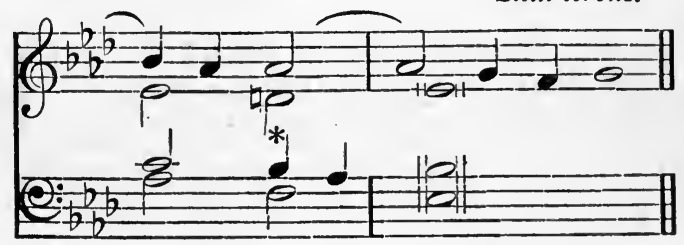

Sanctus, Missa Brevis.

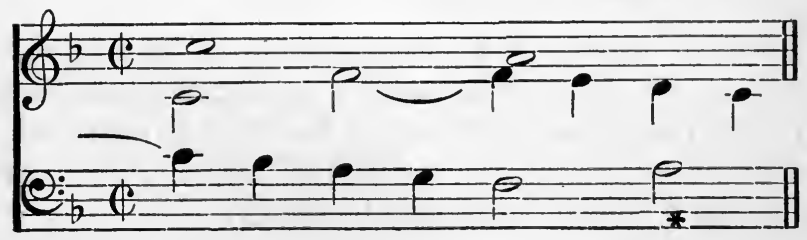




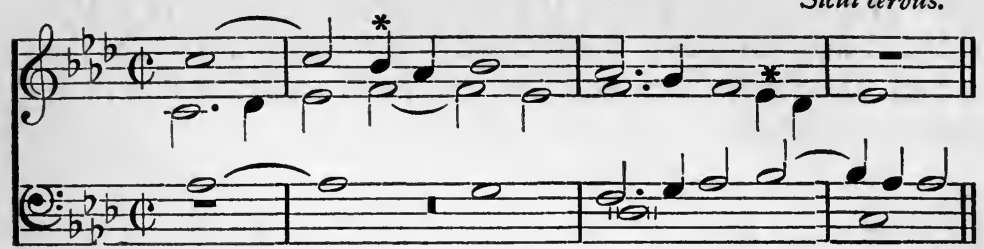

These are only a few of the many examples to be found in the scores of Palestrina.

We find in his works a great many examples of the bare essential fourth-

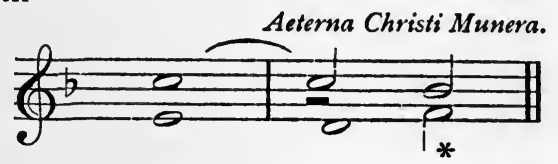

Under modern conditions the bare essential fourth is not considered good, but if one of the notes forming the fourth be an unessential note, there is no objection-

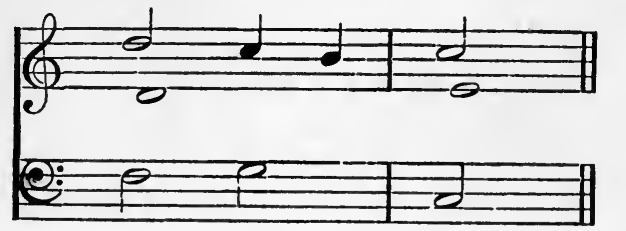

Palestrina uses the fourth in both ways, and it is also found in the works of other composers of the period. Men were obviously trying to gain some liberty for the fourth, and the first step was to strike it bare according to ancient use, but with the third or root sounding beneath it-

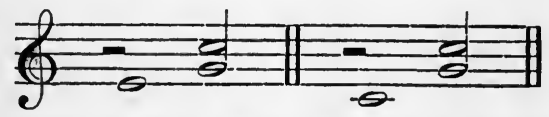

The effect of this is not pleasing, and for this reason it is better avoided ; but its appearance is important in the history of the evolution of the art, as showing the dawn in men's minds of the harmonic or vertical aspect of things. In cases of emergency, however, the essential fourth may be struck. 
Consecutives.

(a) Consecutives cannot occur between the first and third of three combinations-

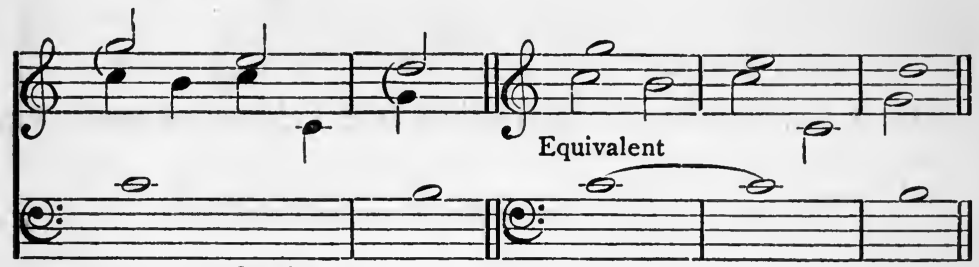

Good.

(b) Consecutives cannot occur with a change of harmony intervening-

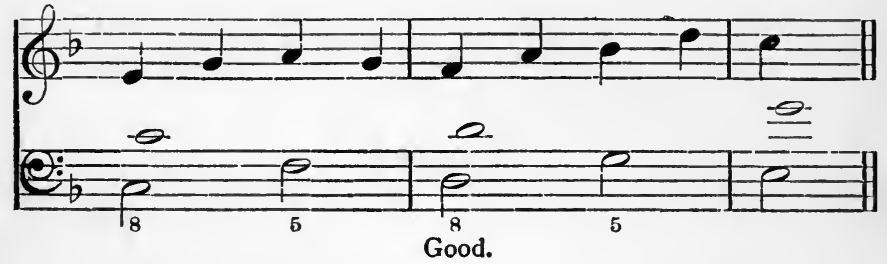

(c) Avoid the progression 9 to 8,7 to 8 between any two parts.

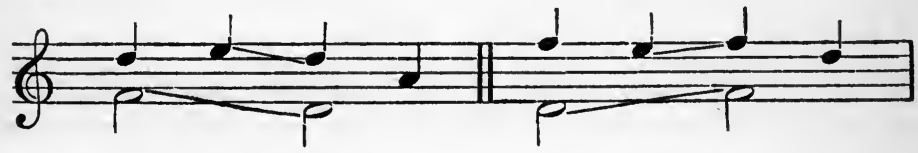

5. Avoid, if possible, combining the discord of the fourth against its resolution, the third, in a descending Third Species passage when the third is in a mean part. It may often be impossible to observe this recommendation, and the student must use his discretion. It is necessary, however, to point out that this is one of the most harsh of such dissonances. The sounding of the ninth against the root in this manner is quite free from objection. 


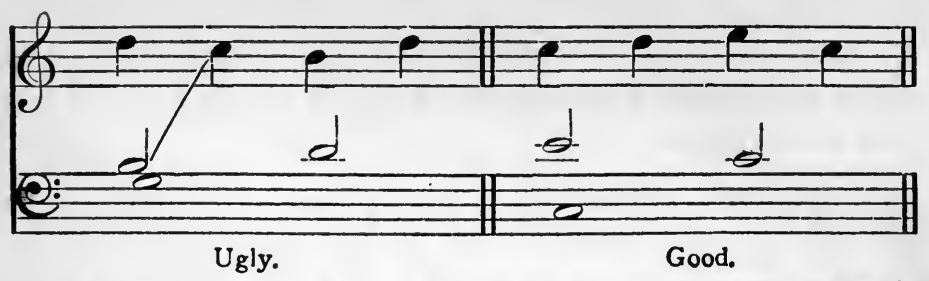

6. It was pointed out in treating of the Second Species that the following was inelegant :-

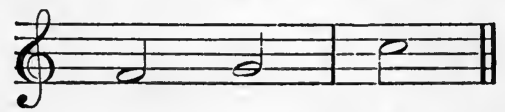
but in the bass, especially in the cadence, it is quite good-

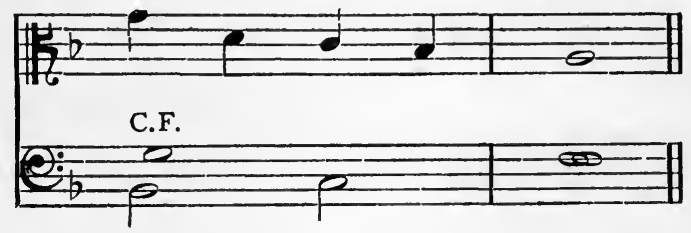

Examples.

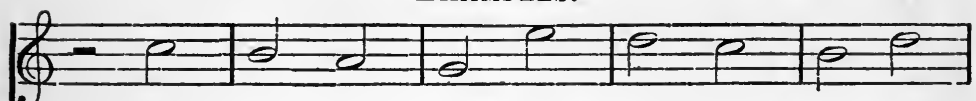

(a)

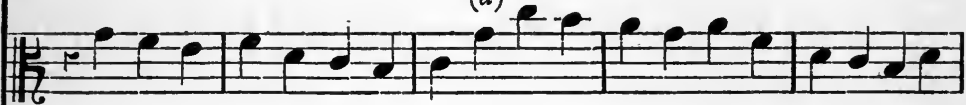
C.F.

\begin{tabular}{|l|l|l|l|}
\hline$:-0$ & 0 & 0 & 0 \\
\hline
\end{tabular}

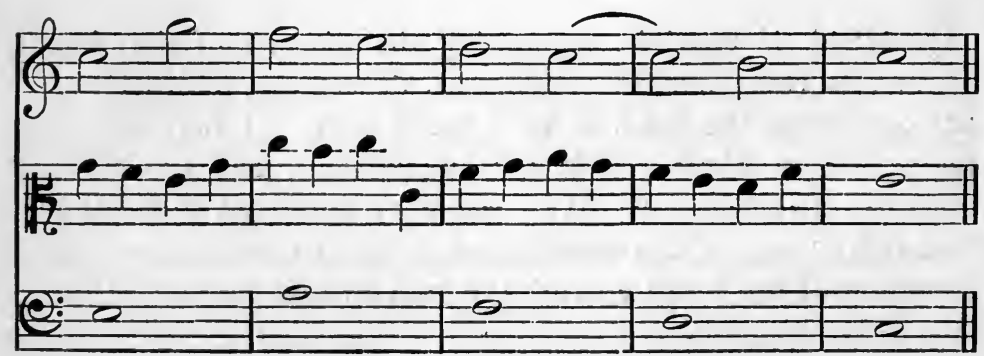


(a) There is really no objection to the leap of a major sixth in minims between factors of the same root: modern vocalists find no difficulty in its execution-

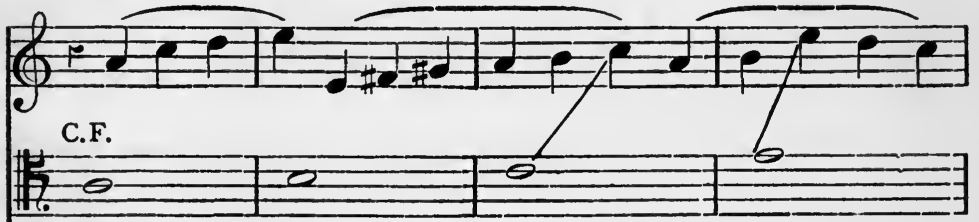

(c)
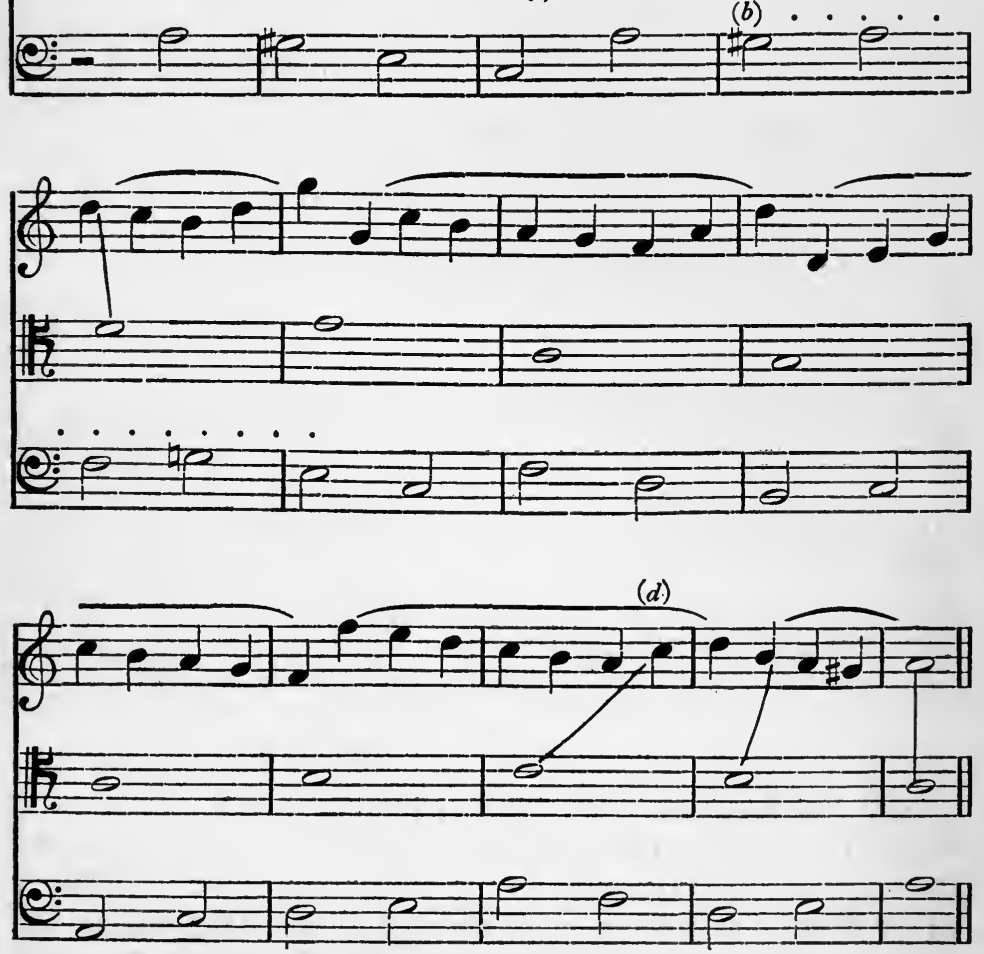

(b) The maintenance of the same melodic formula in two or more successive bars of Second Species is often quite effective- 


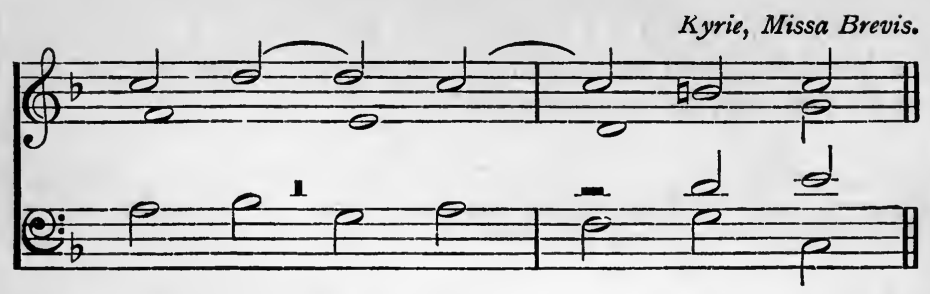

(c) The addition of a part in Second Species gives a clear effect of duple time: the criticism as to consecutives may be based on the Second Species principle, except when they occur between the first crotchets of each bar. It will be seen that the rules as to consecutives in Combined Counterpoint follow in the main those laid down in general terms by Cherubini. But it has been thought better in this treatise to give the matter more detailed consideration, so as to help the student to grasp the principles of criticism.

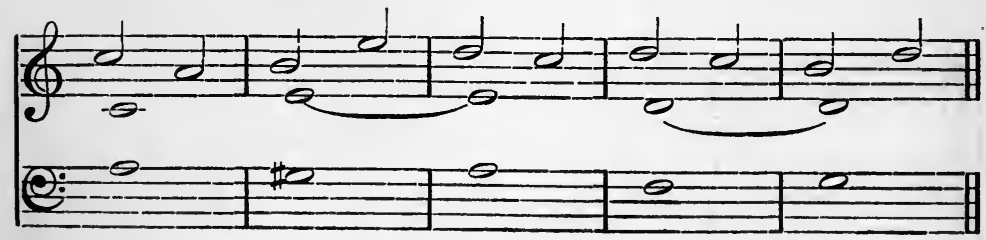

(d) equivalent to

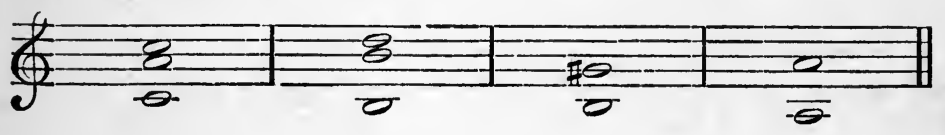

\section{Second and Fourth Species.}

I. It has been pointed out that no two parts should enter together, except several in the First Species. The Second Species therefore may enter on the first minim of the first bar, or either Species may enter on the second minim of the second bar.

2. As in the previous combination, one of the chief difficulties is the maintenance of conjunct movement in the Second Species. The following are examples of various ways in which this may be accomplished :- 
COMBINED COUNTERPOINT IN THREE PARTS I6I
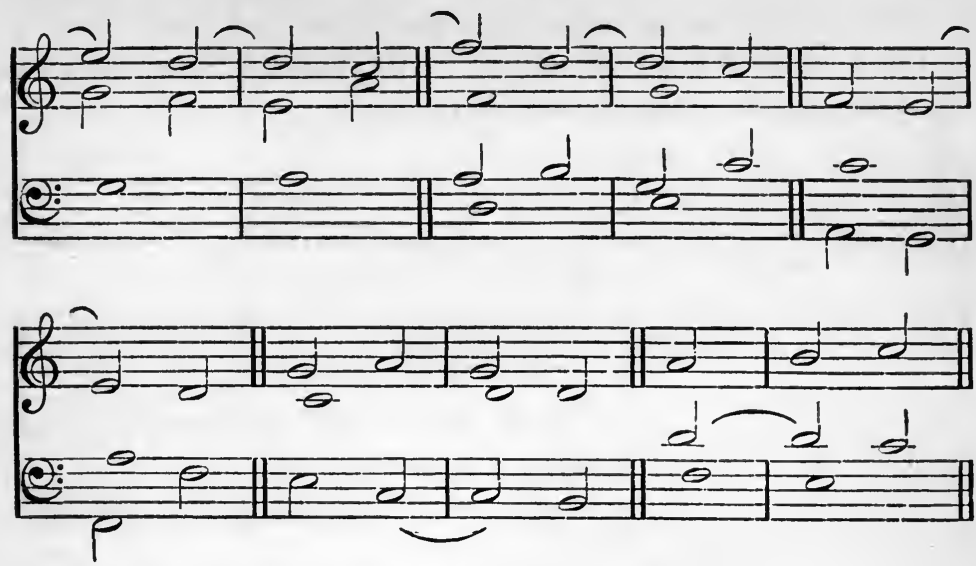

The last will be explained in the next paragraph.

The following examples are not recommended :-

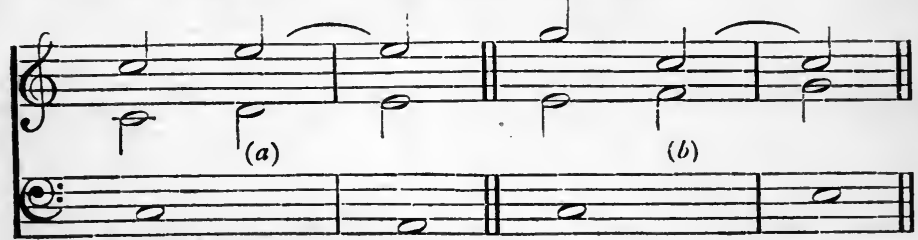

(a) forms obscure and crude harmony,

(b) forms a ${ }_{4}^{6}$ quitted by leap. The above, in which the harmonies of the two bars involved are deducible from the same root, are the least objectionable instances of such a procedure.

3.

Prepared Discords.

The scores of Palestrina exhibit a very elegant variation of the original use of syncopated discords.

In this original use the accompanying parts remain stationary, while the discord resolves into concordance with them.

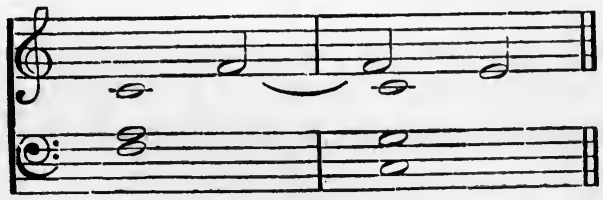


There are three variants of this procedure.

(a) in which the second minim of the Second Species moves to a different note of the same harmony-

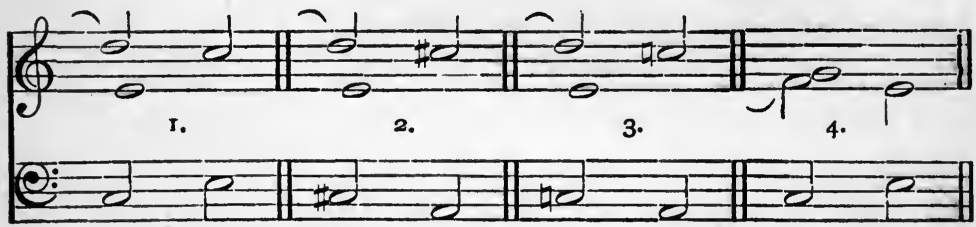

(2) is liable to be very harsh and is not recommended, but (3) with the minor third is much smoother.

(b) in which the second minim of the Second Species produces a change of harmony-

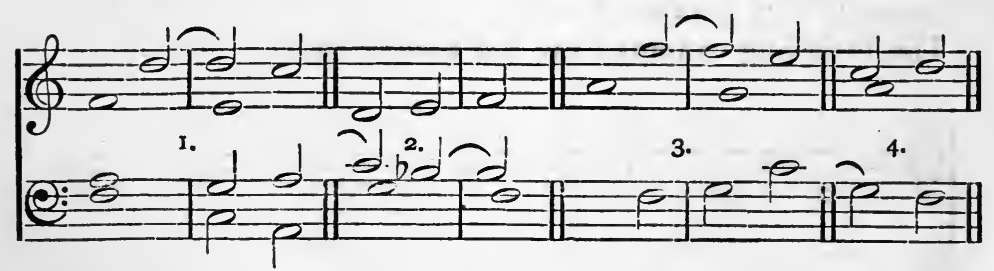

Observe in (3) the origin of our chord of the dominant seventh.

Note that in all the above cases in $(a)$ and $(b)$ the first minims could have remained as semibreves while the discord resolved, and the resultant combination would in each case have been concordant, e.g.-

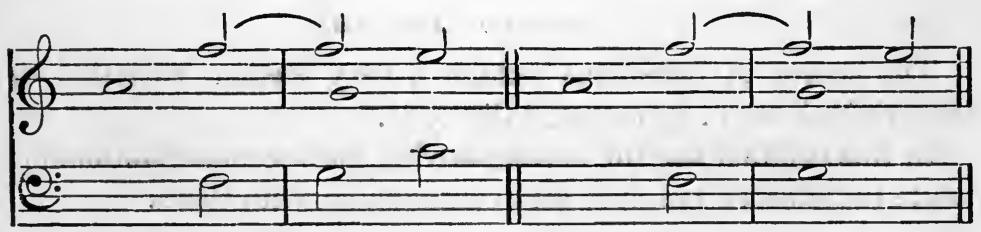

(c) In which the first minim of the Second Species, though concordant with the C.F. could not have remained stationary while the discord resolved. - It usually proceeds by step into concordance- 
COMBINED COUNTERPOINT IN THREE PARTS I63

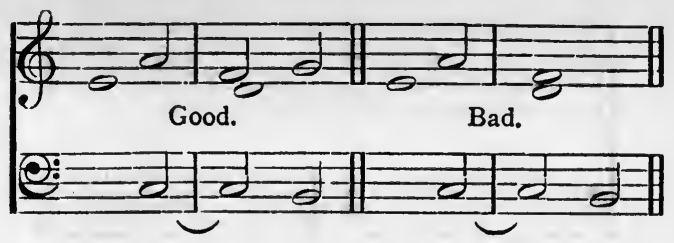

The following are examples of these prepared discords :-

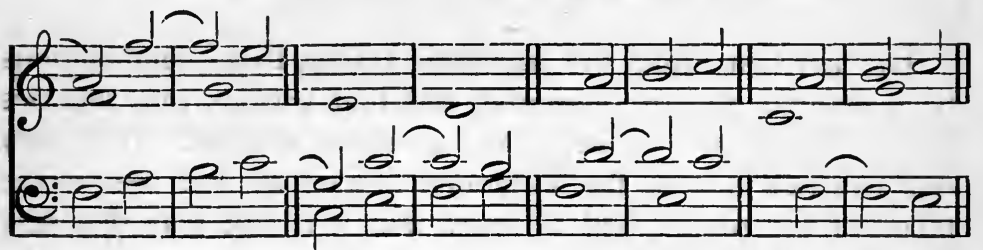

Missa Brevis,

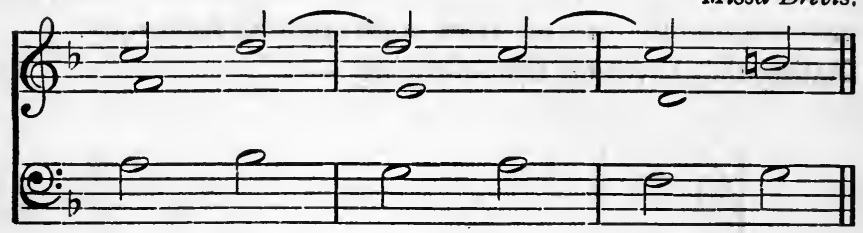

Students often find a difficulty in understanding these combinations, because they confuse the harmonic with the contrapuntal standpoint. It should be borne in mind that any classification of the vertical combinations we call chords is entirely foreign to the horizontal system, and that no prepared discord bears any relation to the essential harmony, that is, it demands no consideration except that it move one step downward into consonance.

Ignoring the discord, the following is the contrapuntal analysis :-

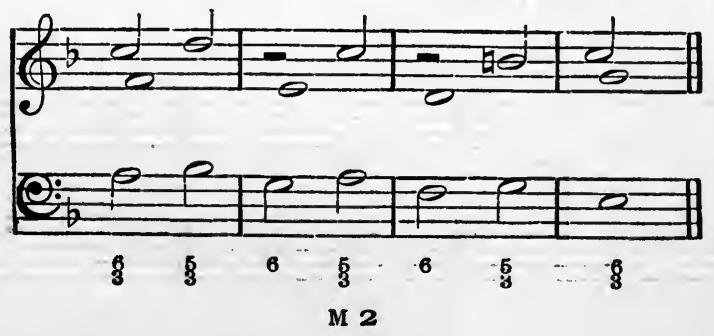




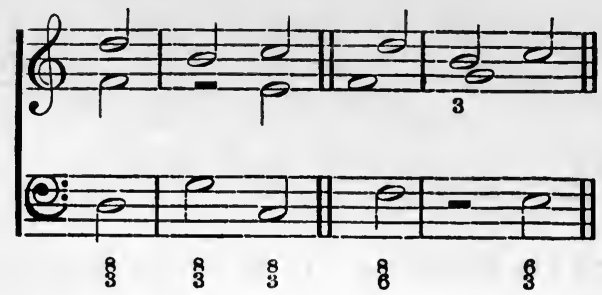

The fact that under the harmonic system these combinations have merged into the essential, and that various root theories have been devised is beside the question at issue. It is only our province to state the horizontal system as it is seen in the music of the period, and to show that it is still true that only common chords and their first inversions are used as the essential fundamentals of strict contrapuntal technique.

Distinguish between the following :-

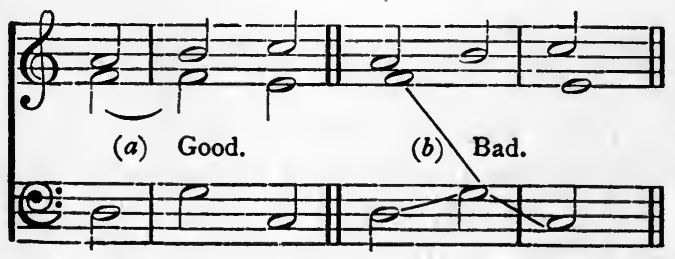

in the second case $(b)$ the second minim in the bass $(G)$ is discordant with the alto $(F)$ and incorrectly approaches and quits this discordance by leap.

4. The extreme parts may not approach a fifth, nor may any two parts approach an octave, by similar motion, when one of them is resolving a discord:-

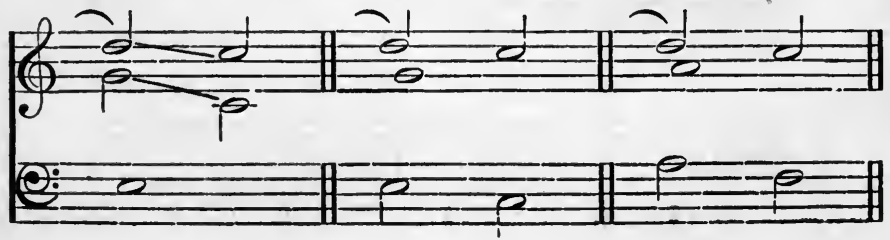




\section{COMBINED COUNTERPOINT IN THREE PARTS 165}

Palestrina avoids this as follows:-

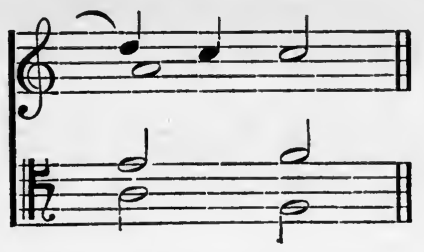

In combining the Second and Fifth Species, the following are therefore good :-

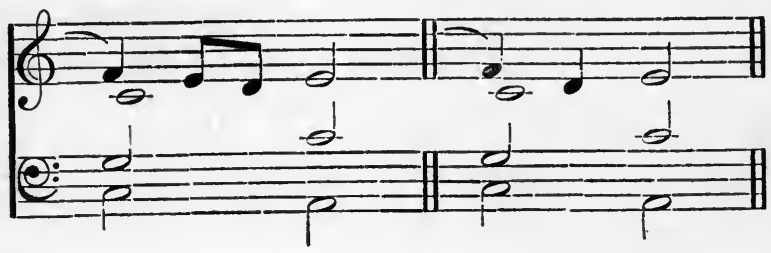

The ninth should never be prepared by the octave :-

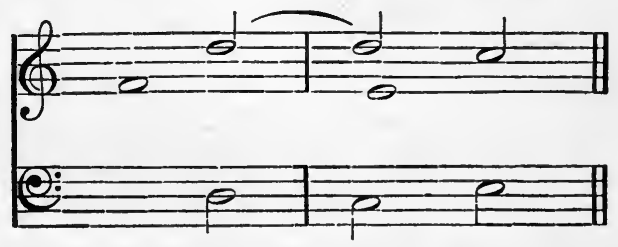

5. The fourth may be accompanied by the sixth, if the latter proceed to the fifth as the fourth proceeds to the third-

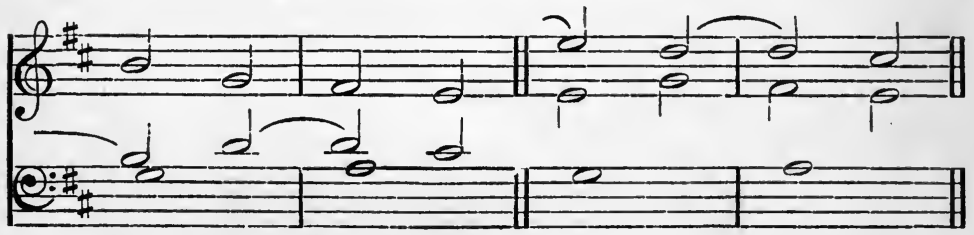




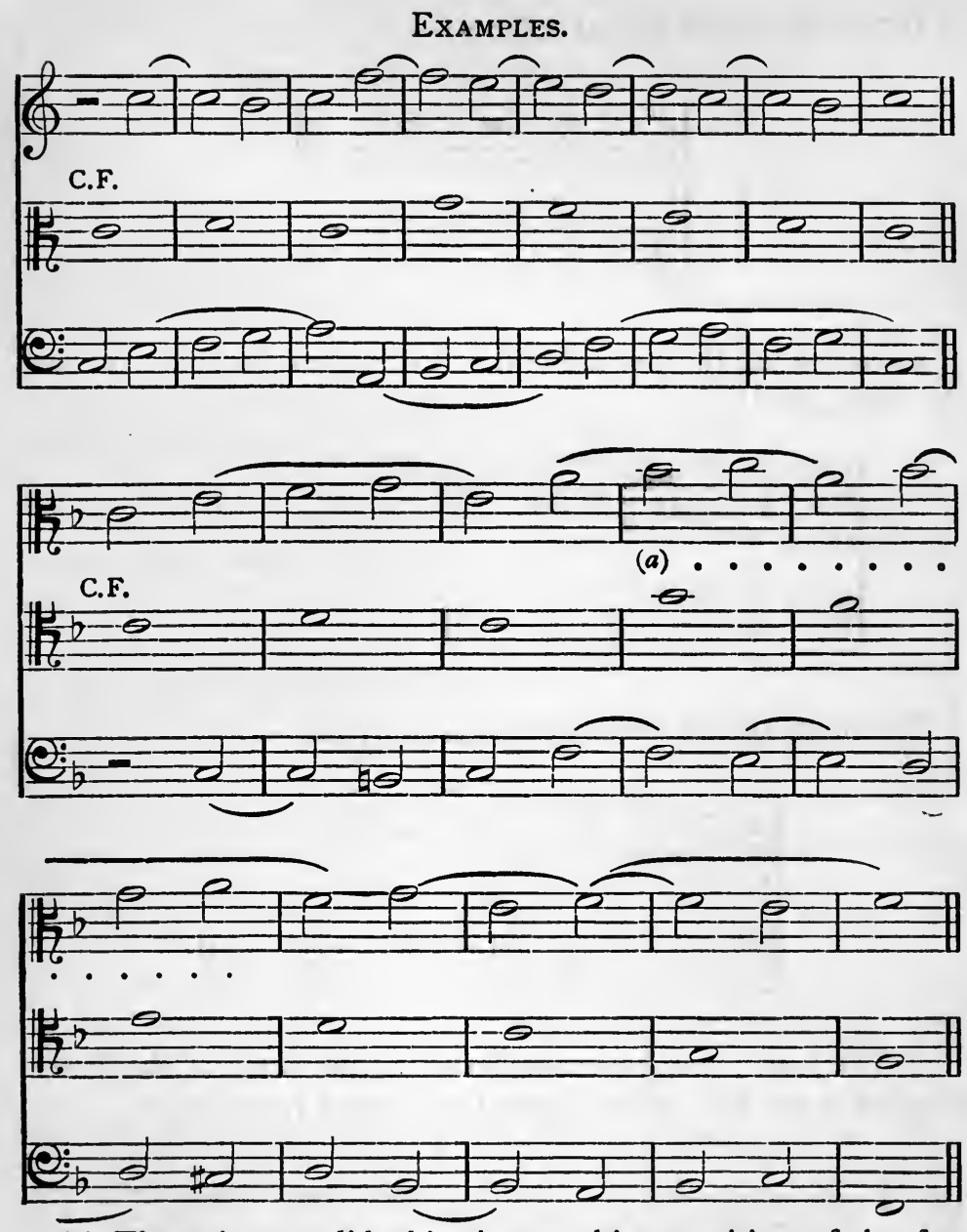

(a) There is no valid objection to this repetition of the formula. Note

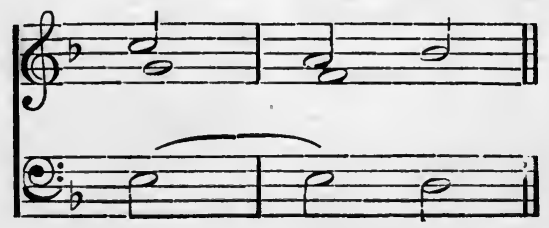


COMBINED COUNTERPOINT IN THREE PARTS 167 is not to be criticized as

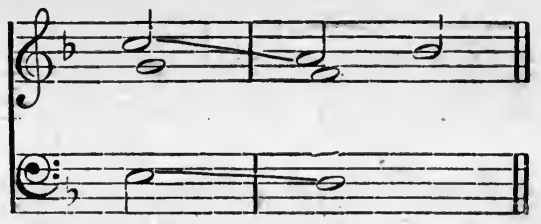

for no exposed fifth occurs.

\section{Third and Fourth Species.}

I. It is sometimes possible, just as in the combination of the Second and Third Species, to obtain the effect of a passing chord on the third or fourth crotchet-

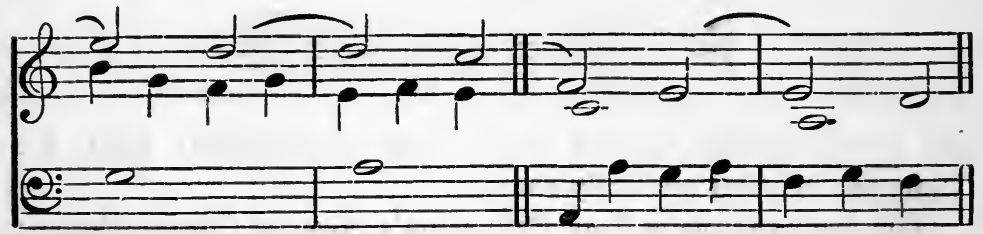

2. The harmony may be changed on the third crotchet-

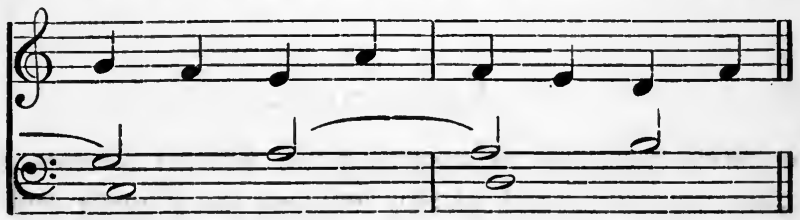

3. No two parts should approach an octave by similar motion, when one of them is resolving a discord-

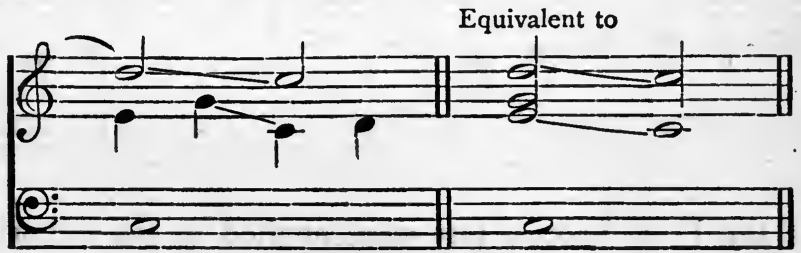

Poor. 
4. Prepared discords may be used-

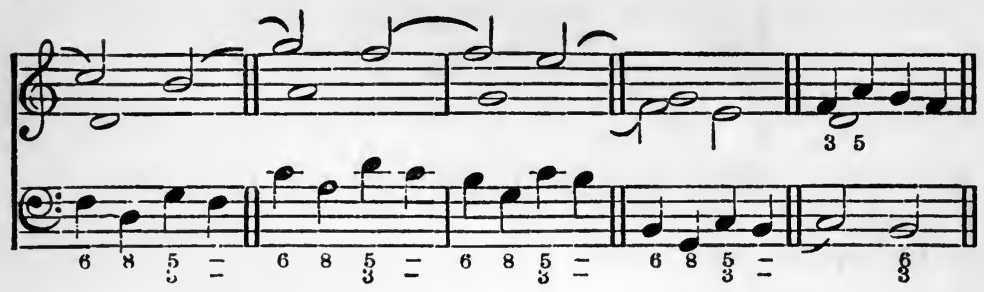

Note when the Third Species leaps, it is concordant with the C.F.-

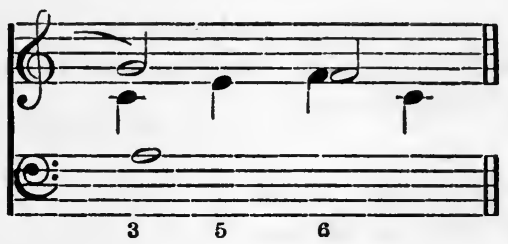

By regarding the discord in its true contrapuntal light, the analysis becomes perfectly easy.

The last example is from Palestrina's Aeterna Christi Munera, and is a 76 treated in the manner of a prepared discord.

$5 \cdot$

Consecutives.

The usual test, that of reducing the Fourth Species to the First does not hold good, except between the Fourth and First Species (in extreme parts). The following is obviously correct :-

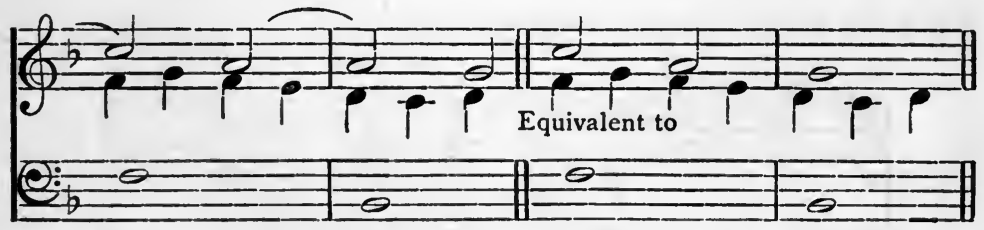

but, if the First Species test were applied to the following, it would have to be condemned- 
COMBINED COUNTERPOINT IN THREE PARTS I69

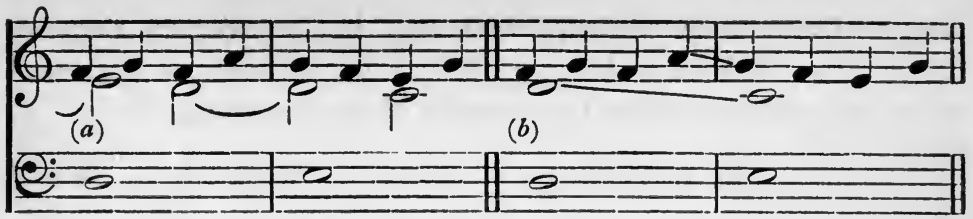

But in the original form (a) no fifth actually occurs, and the progression is perfectly correct.

With the addition of another part, moving in crotchets, another view of syncopated concords in reference to consecutives comes into use.

Examine the following :-

Palestrina, Credo, Missa Brevis.

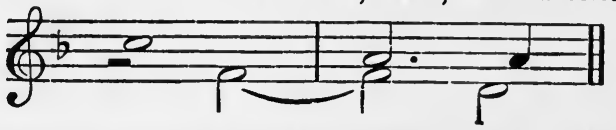

Now, if it is true that the Fourth is always a variant of the First Species, its equivalent in that Species in the present case is

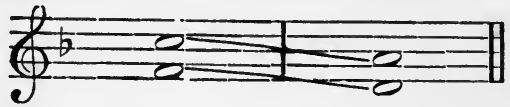

Palestrina did not consider consecutive fifths objectionable between the first and third of three concords, if the first two were syncopated-

Agnus Dei, Missa Brevis.

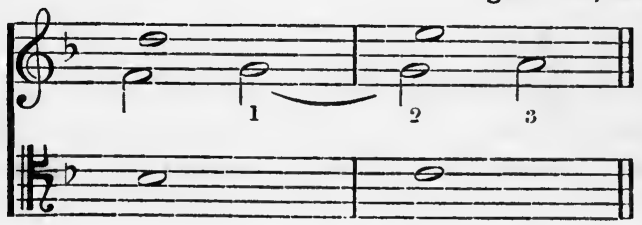

nor if the first were a completion of a previous syncopation-

Credo, Missa Brevis.

Ego sum panis.

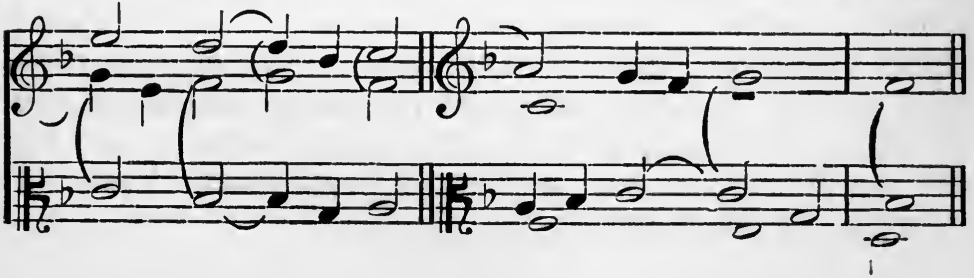


Even without these syncopations, that is, in ordinary Second Species, we find he had no hesitation in writing consecutives, if the intervening concord proceeded in contrary motion-

\section{Credo, Iste Confessor.}

Confirma hoc Deus.

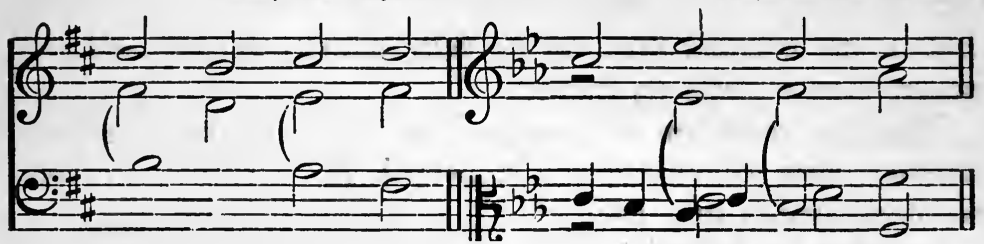

He also wrote

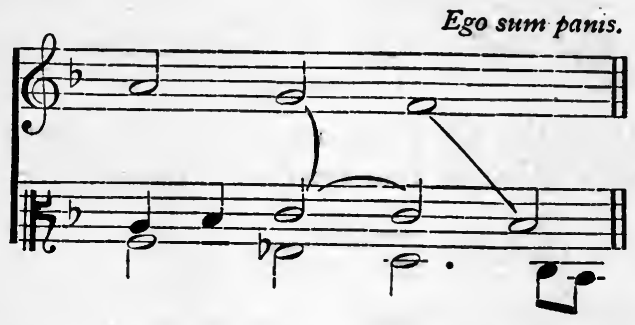

Fux considered the interval of the fourth the smallest allowable between the consecutives-

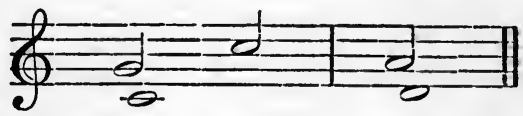

But modern conditions, where rhythm and harmonic substructure are salient features, do not allow of such arguments holding good. However, the above examples from Palestrina distinctly point to one fact, namely, that in the case of what are termed syncopated concords the test as to consecutives should not be exclusively limited to a reduction of the score to plain First Species. It is more often a case where the Second Species principles should supply the test, the second minim of one bar and the first of the next bearing the same sound. Take, for example, the following :- 


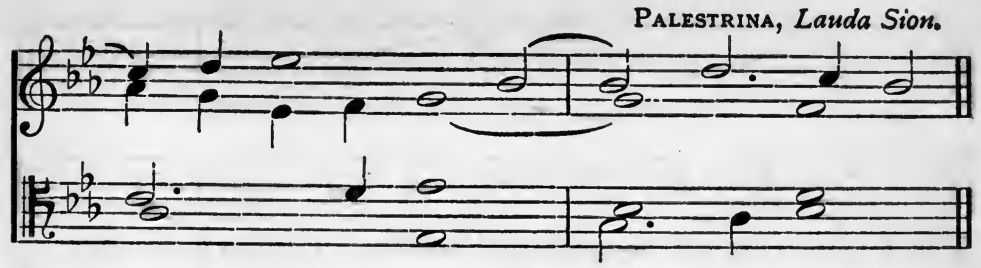

By the rules of Counterpoint, as stated, the extreme parts are equivalent to

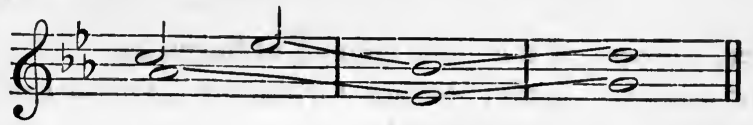

and moderns would rightly object to this in extreme parts. But to illustrate Palestrina's point of view, put this in the form of Second Species-

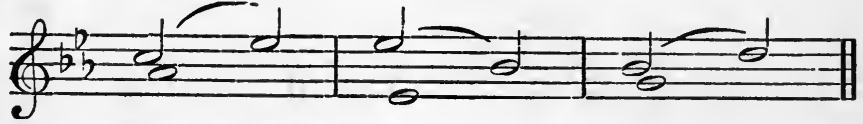

and the outline becomes-

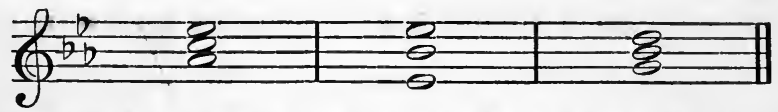

The fact is, the ear often accepts the first minim of the bar not as a note in place of the second, but as a distinctly separate harmony note. It is not to be supposed that in plain two-part work this test is allowable, for the harmony is of necessity so bare and often so indefinite that the First Species test should be strictly maintained; nor is the above test always practicable in more complex situations. But it will be felt that it is really impossible to condemn such cases as the following on any logical or aesthetic grounds :-

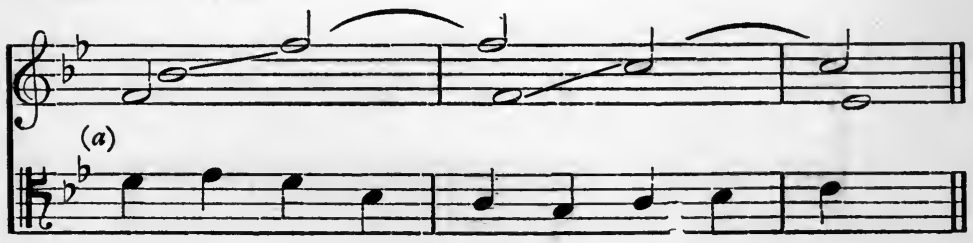




\section{THE ART OF COUNTERPOINT}

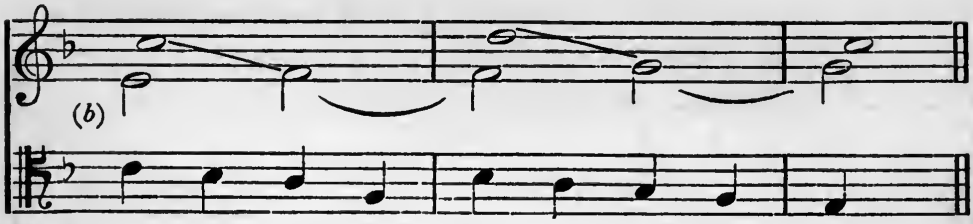

The former is good, because the harmonic outline is correct-

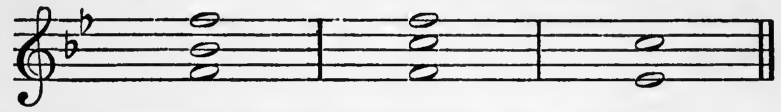

the latter, because of the clear change of harmony.

6. Consecutive fourths are not forbidden between the upper parts, when the bass is suspended-

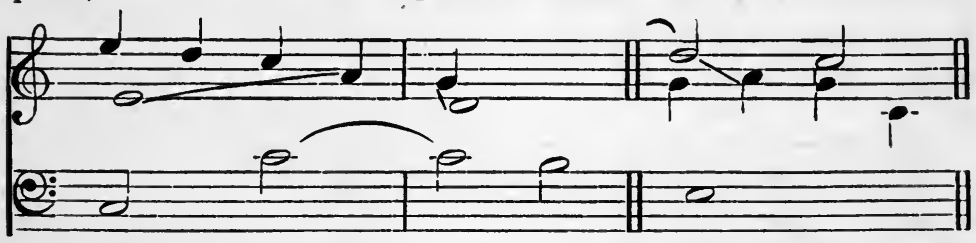

Good.

Bad.

7. The suspension should not be sounded against its resolution-

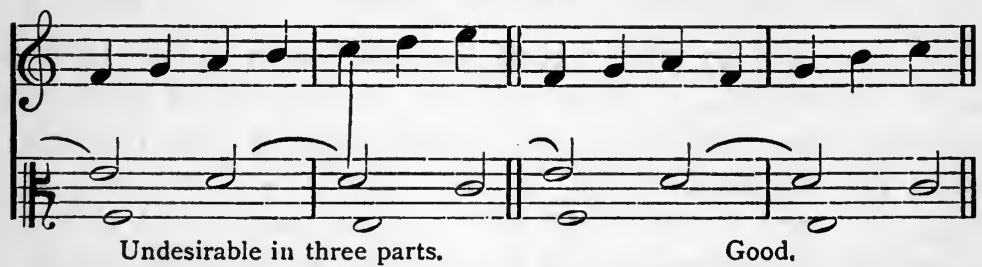

8. Some theorists regard the doubling of the leading note as an unpardonable crime. As Palestrina knew of no such harmonic restriction, he wrote

Aeterna Christi Munera.

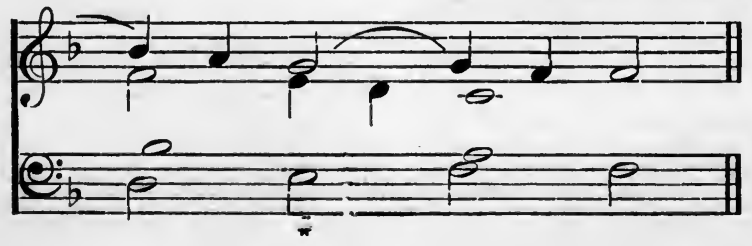


However, most men agree that the doubling is unobjectionable when it occurs in the middle of a scalic or arpeggio passage, or when the leading note is syncopated :-

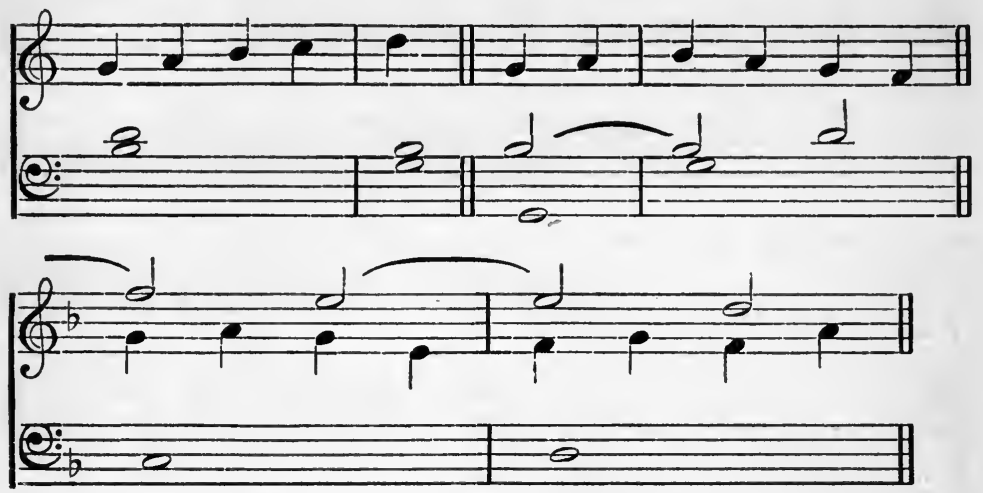

Students should base their judgement on effect, and not on the question as to whether the letter of the law has been kept.

The doubling of the leading note may be unobjectionable under other conditions, but these will be dealt with as occasion arises.

EXAMPLES.

First Mus. B. Oxon., Nov., 19or.

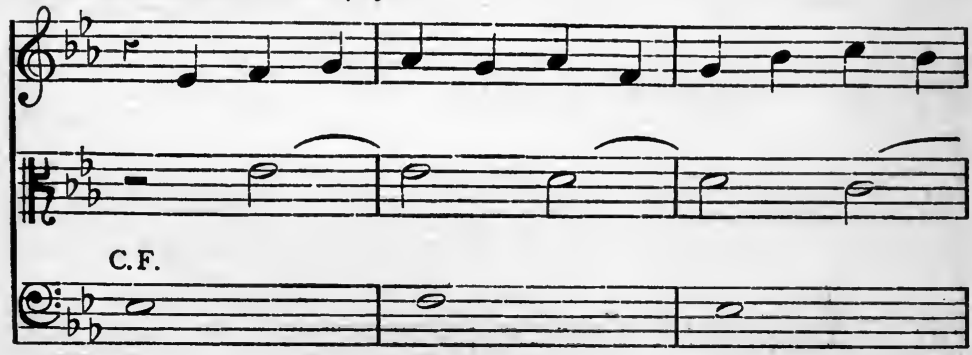




\section{I74 THE ART OF COUNTERPOINT}

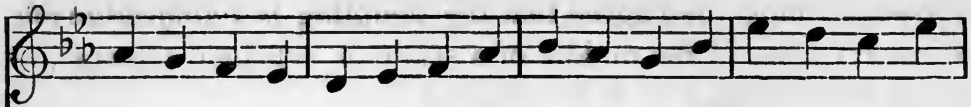
रिक कि $\left(3: \frac{b}{b}-2-0=0\right.$

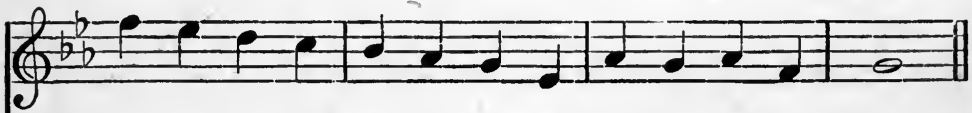
?

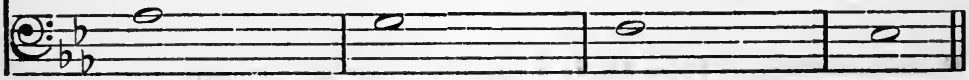

First Mus. B. Oxon., May, 1903.

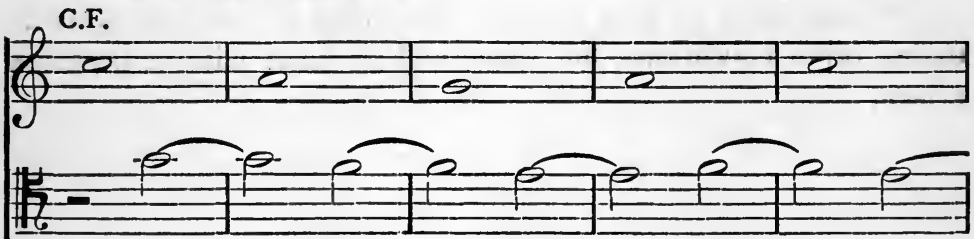

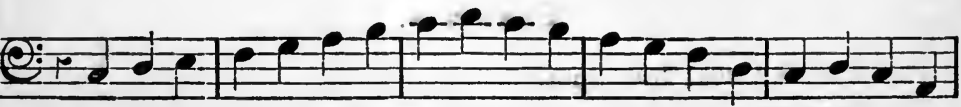

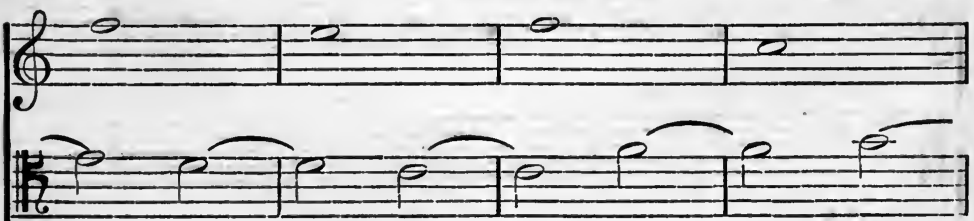


COMBINED COUNTERPOINT IN THREE PARTS 175



\section{I76 THE ART OF COUNTERPOINT}

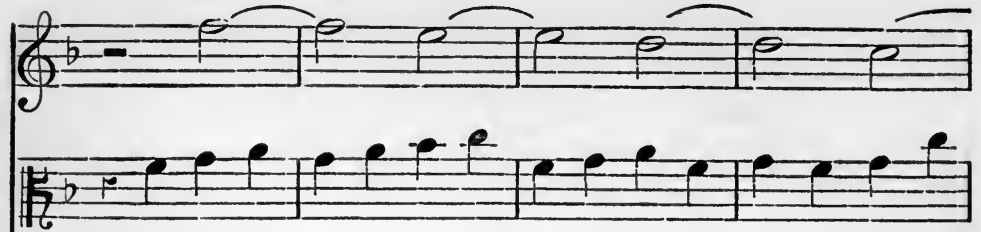

C.F.

$1 \frac{+16-5}{6-5}$

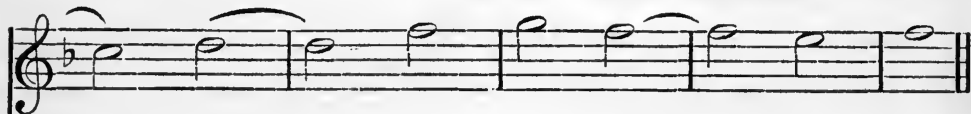

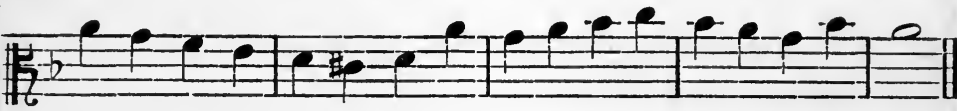

$+\frac{1}{2}$

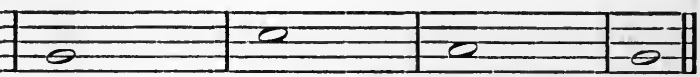

It is excellent practice to take a C.F. in Second Species, and add parts to it in Third and Fourth Species, thus-

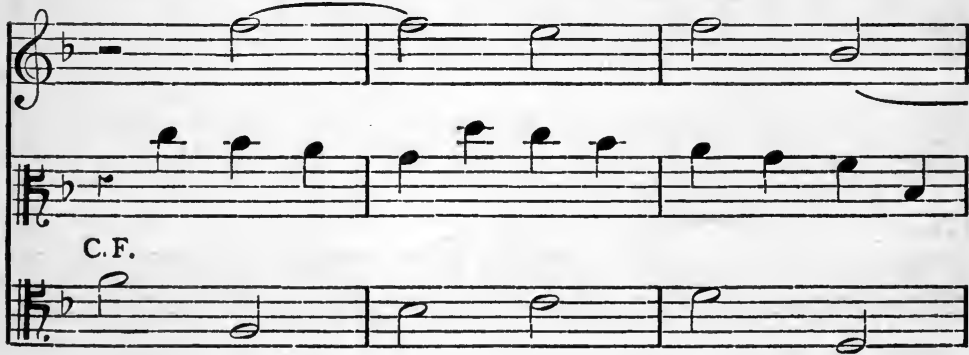

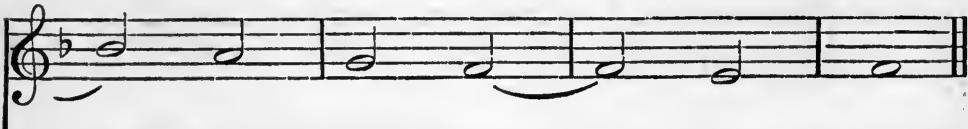

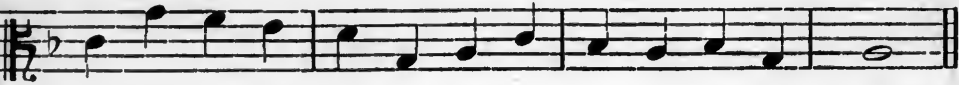


This is a valuable preliminary study in the art of adding florid parts to a chorale.

The combinations of Second and Fifth, Third and Fifth, and Fourth and Fifth Species call for no further comment. The following illustrations of various points will however be useful :-

(a) Second and Fifth Species: the use of two harmonies in a bar, and prepared discords-

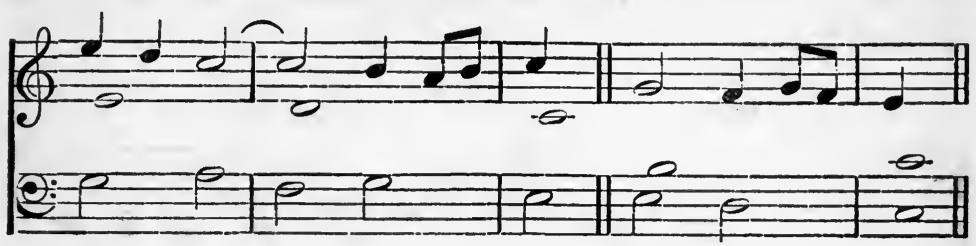

(b) Third and Fifth Species : the striking of discords will be quite good, if the harmonic sub-structure be kept clearly in view, and the seams between the chordal centres be entirely satisfactory-

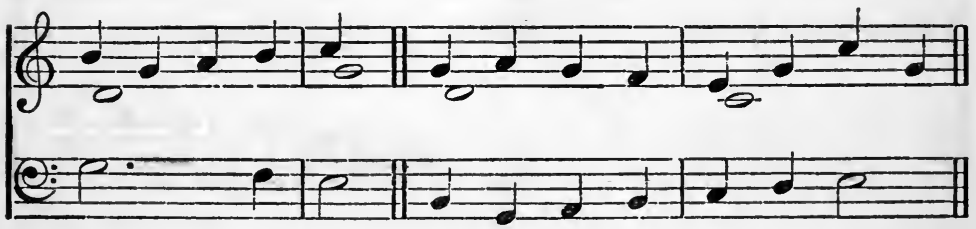

(c) Fourth and Fifth Species: the florid part need not be concordant with the C.F. on the third crotchet, so long as the harmonic progression of all the parts is good-

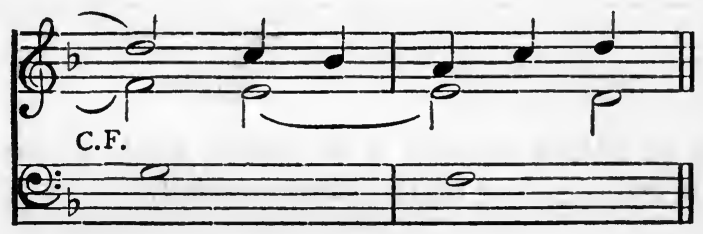

Double suspensions are possible, though unadvisable as a general rule- 


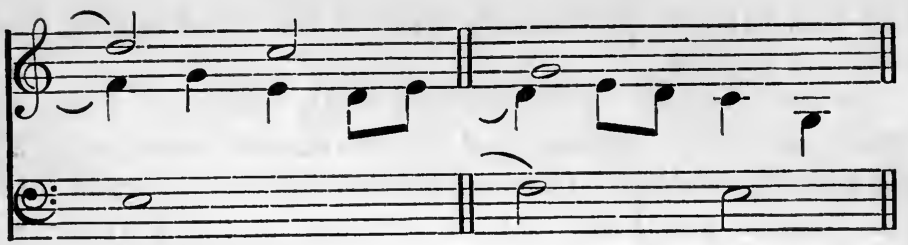

Prepared discords may be accompanied in an ornamental fashion, thus :-
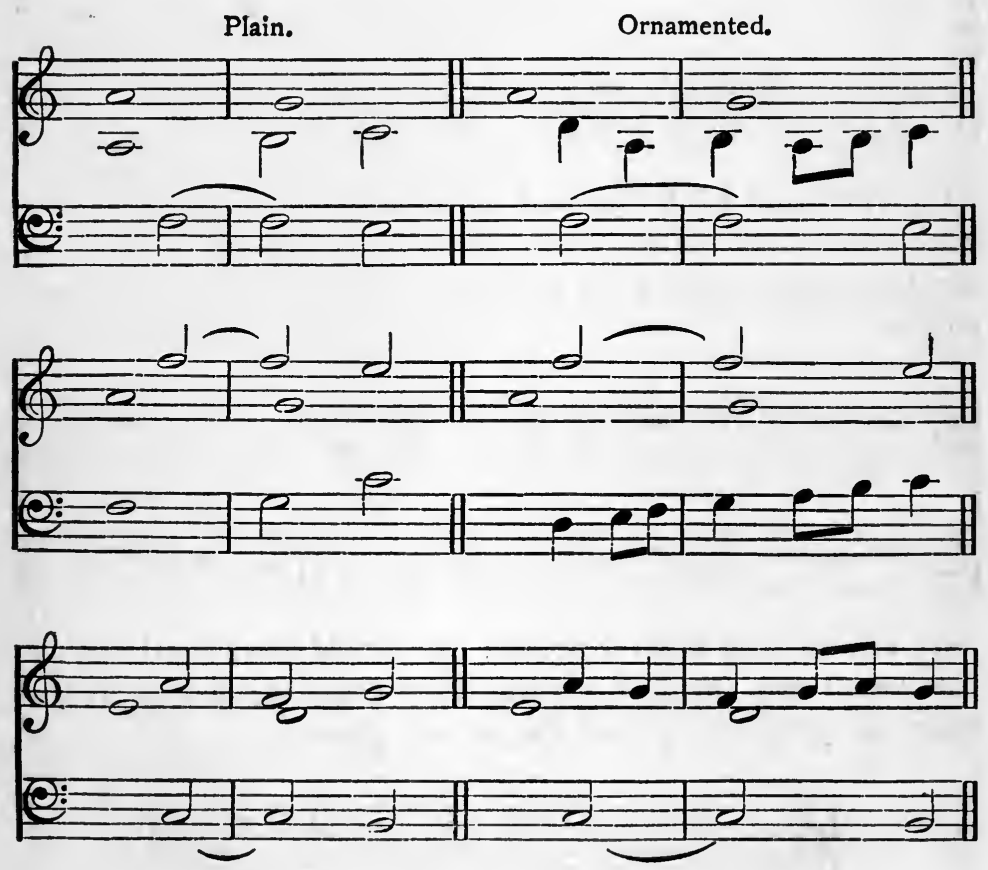

The use of dotted minims is of course freely allowed, if the third part strike a note on the third crotchet. 
Fourth and Fifth Species in Triple Time.
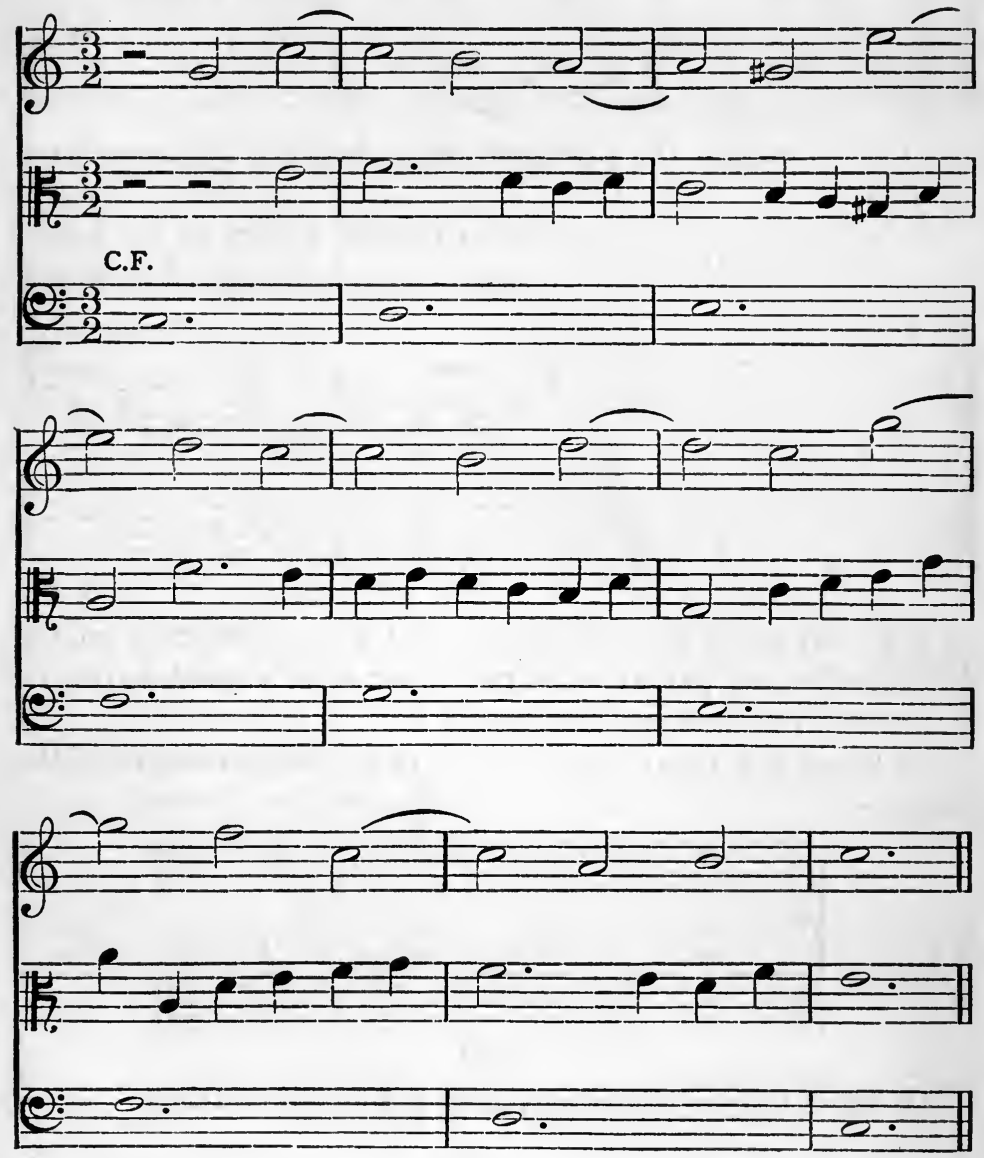

Two Parts in Florid Counterpoint.

I. The use of two quavers combined with a crotchet requires care- 


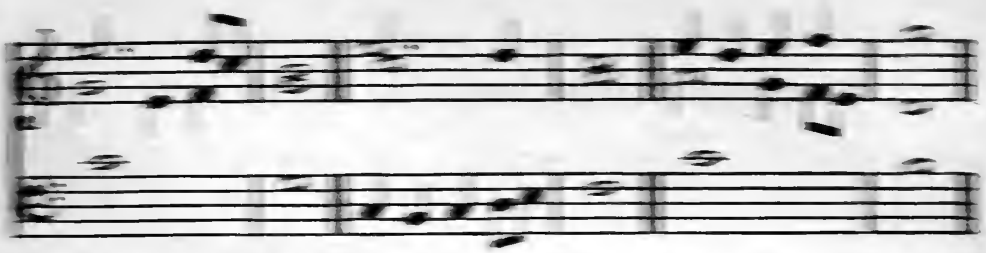

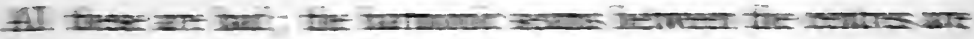
3i lace

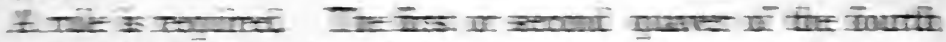

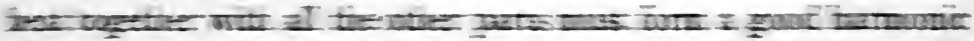

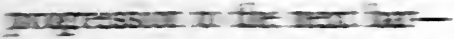
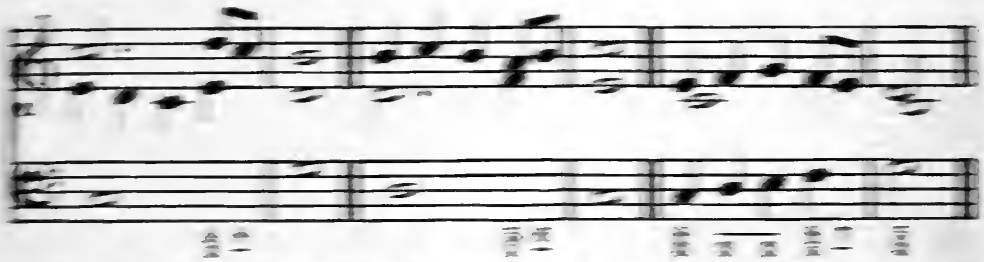

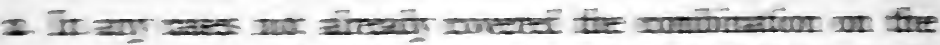

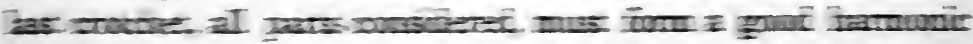

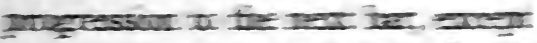

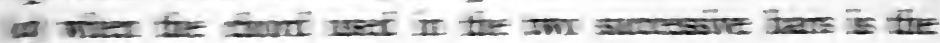
seme-

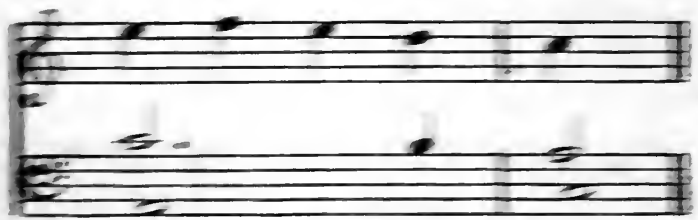

Inove

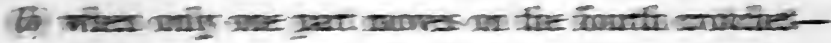

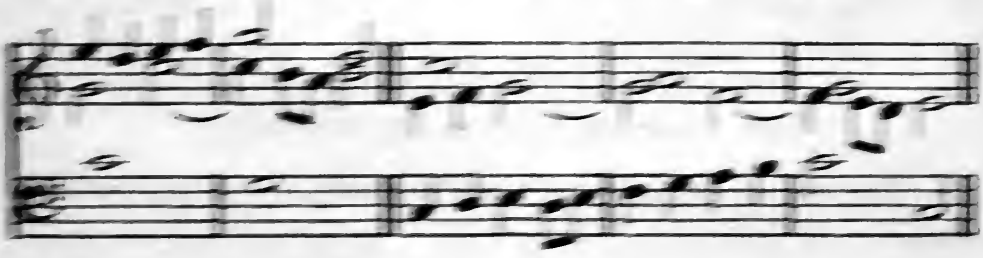




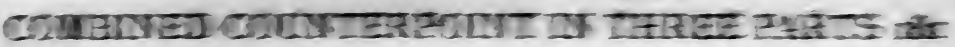

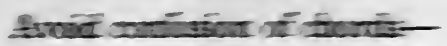

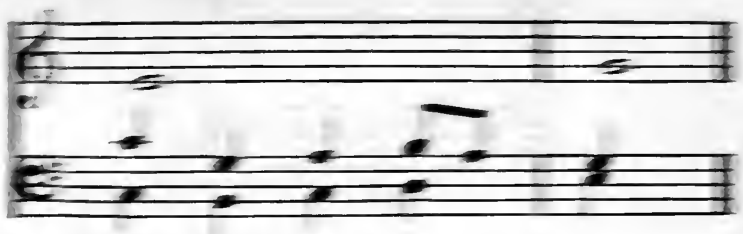

Iar

Ileer are bres ane- jeermetina:-

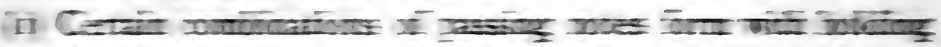

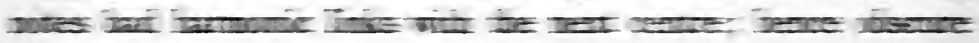
Iancito-

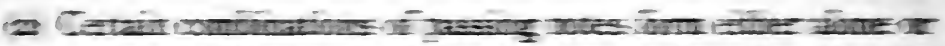

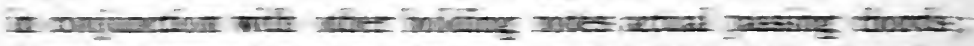

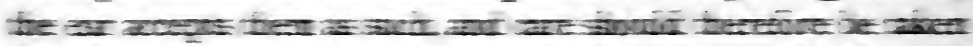

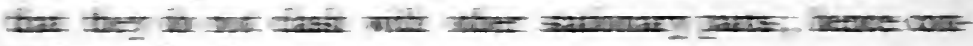

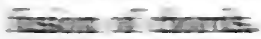

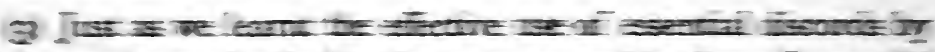

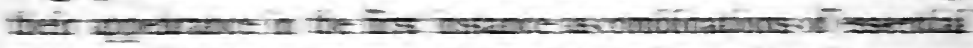

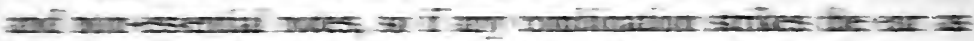

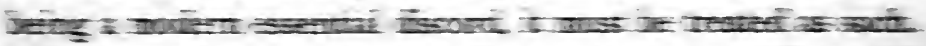

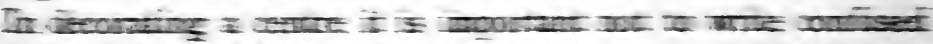

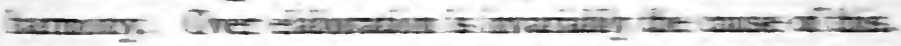

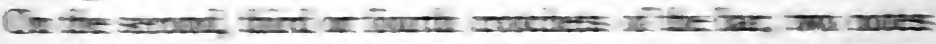

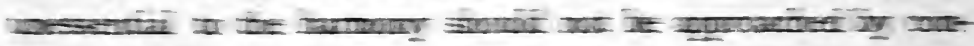
Int

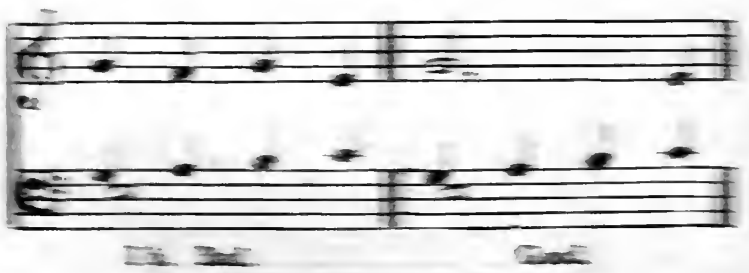

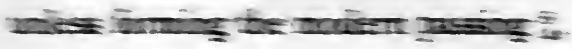




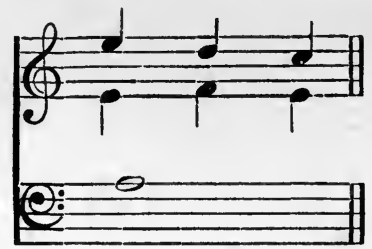

Good.

or a good implied modern harmonic progression on a pedal.

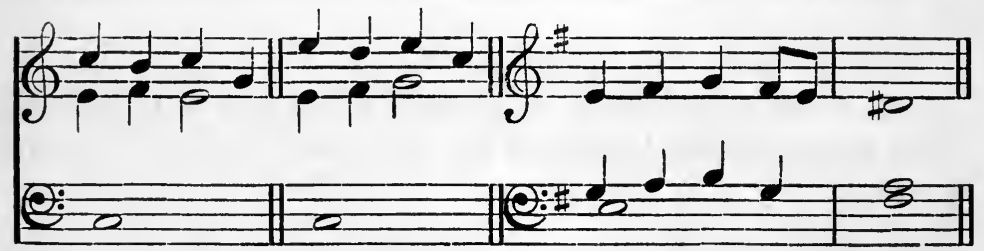

Good.

Gcod.

Good.

The discussion on the excerpt from 'If I had but two little wings' will explain the following.

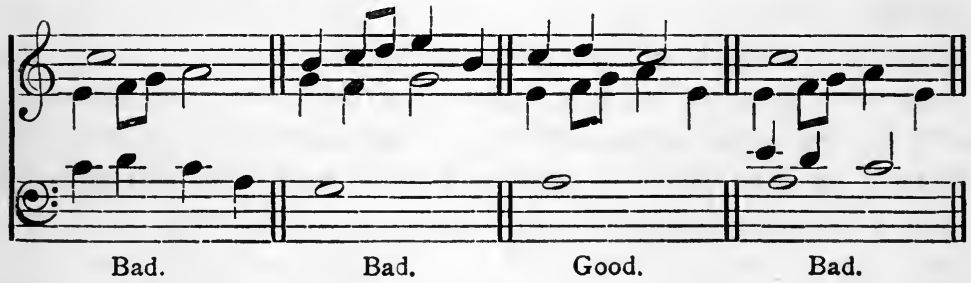

Such examples are due to the law of concordance with the lowest moving part, which ignores the effect of the particular combinations struck together in conjunction with stationary parts.

Nor should the parts, after striking an unessential combination proceed in notes of different time value,

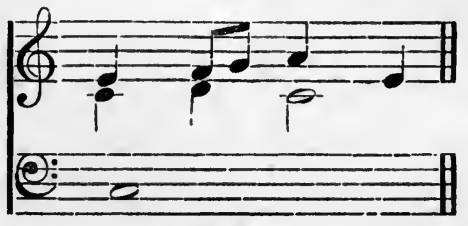

Bad. 
COMBINED COUNTERPOINT IN THREE PARTS 183 unless the bass forms a satisfactory pedal,

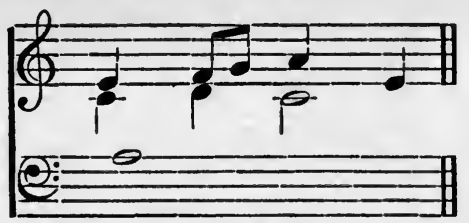

Good.

or when the C.F. is in an upper part, unless the harmony be satisfactory from a modern point of view.

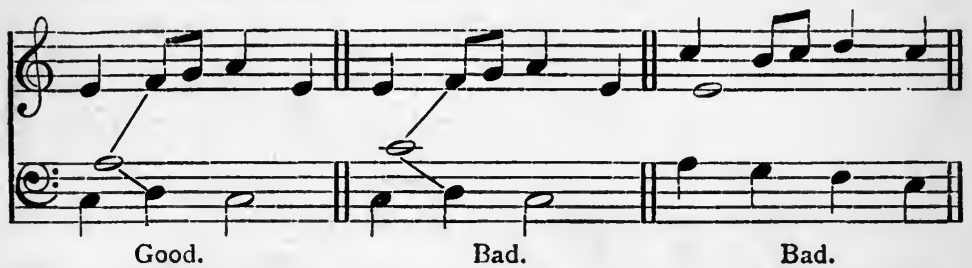

It is well to repeat what has been stated previously, that conjunct and parallel movement is good, provided that the unessential notes are used properly.

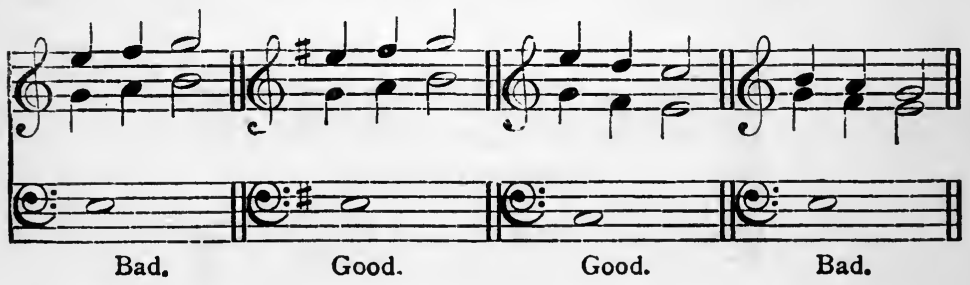

Some of the following examples are given in four parts, to avoid further reference to the subject-

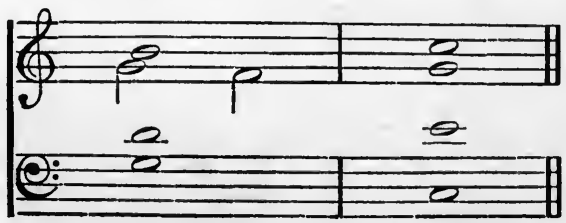

Unresolved seventh. 


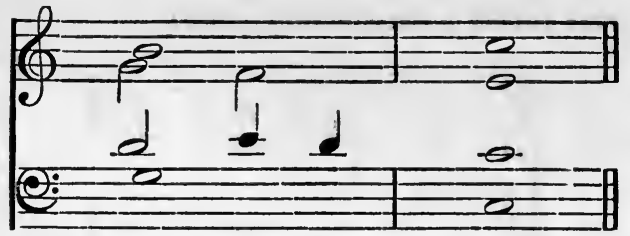

The thirteenth should not be sounded below the seventh.
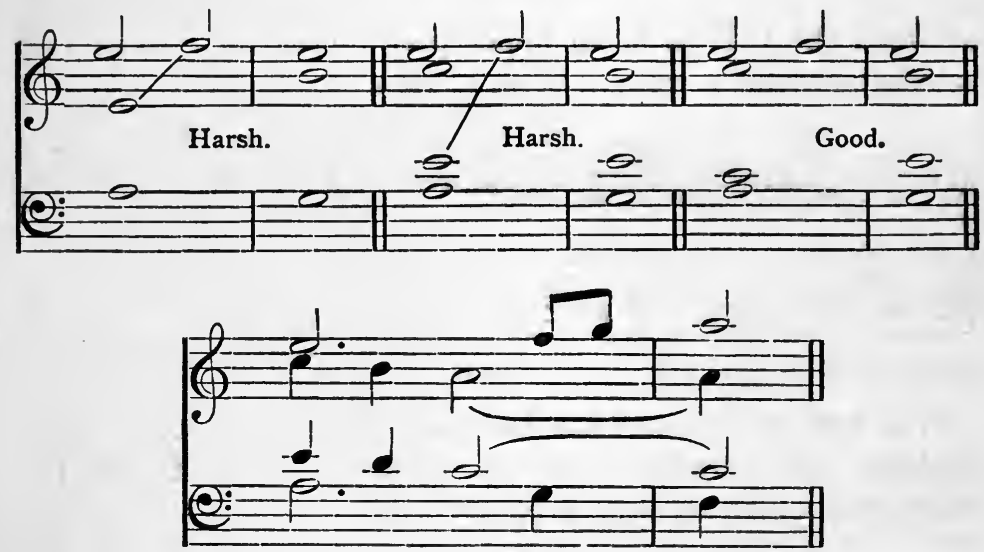

Bad seam.

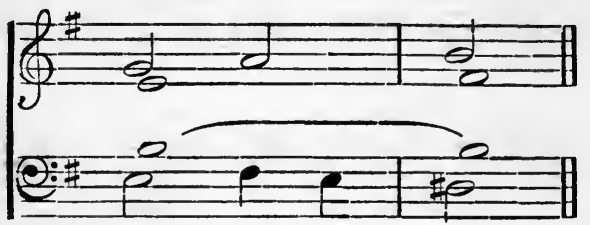

Bad seam.

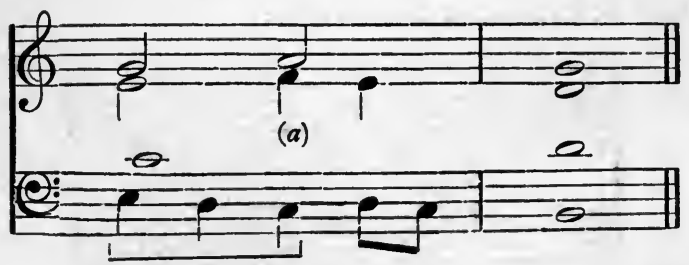

(a) ${ }_{4}^{6}$ approached by leap from an inversion of another chord. 
As the passing note (D) is unessential, it will be seen that the movement of the bass from a ${ }_{3}^{6}$ on $\mathrm{E}$ to $\mathrm{a}{ }_{4}^{6}$ on $\mathrm{C}$ is a violation of the spirit of the law. When it is said that a ${ }_{4}^{6}$ may not be approached by leap from the inversion of another chord, the interpolation of a passing note in order to make the bass conjunct, does not remove the bad harmonic effect which is the cause of the rule. Examples of this sort are to be seen every day: and it is very difficult to undo the mischief, when the rules in textbooks make such things possible.

It is impossible to write good Counterpoint without a thorough grasp of the principles of harmonic progression. It is really deplorable that the majority of students who have been through some harmony textbook are utterly unable to write four bars of plain chords in any intelligible way. To take for granted that so long as the Counterpoint is technically correct, the question of the spontaneity of the harmony does not matter, is undoubtedly the wrong view. This is a question which should be dealt with in elementary harmony, rather than in a textbook on Counterpoint. As soon as a student has learnt the use of the common chord and its inversions, he should compose simple phrases of four and eight bars, and he should not be allowed to proceed further till his choice of harmony is smooth and natural. When he has learnt the use of the chord of the dominant seventh and elementary modulation, he should write hymn-tunes, with no decoration whatever, that is, simple plain chord progressions, with the phrases properly balanced, and with a due consideration of cadential effect. Unless these foundations are sound, it is impossible to make any progress : and any one who attempts to proceed will meet with disaster at every turn.

Many theorists of the present day argue that such a system as has been propounded in this treatise is not Strict Counterpoint, and indeed some go so far as to say that Strict Counterpoint has no connexion with the technique of any period. If this is so, it is unfair that it should be based upon, and at the same time confused with, the practice of the sixteenth century. It is also difficult to find any excuse for a hybrid system which consists of 
a set of arbitrary rules drawn up from misconceptions in the following directions :-

I. It is taken for granted that Strict Counterpoint has no historical or practical status : but that it is merely a mechanical contrivance for the exercise of the intellect in overcoming diffculties. This is a view which is absolutely inconsistent with facts. Any one who has read the various early treatises must at once admit this.

2. In attempting to bring the teaching of the earlier treatises into line with modern conditions, the true significance of the terms 'common chords and their first inversions' in reference to the fundamentals of contrapuntal technique has either been misunderstood or ignored.

3. It has been wrongly assumed that the horizontal system implied that the lowest moving part was the real bass, and that so long as all notes struck together formed a part of a common chord in root position or in the first inversion, the writing was correct.

4. It has also been laid down by some that no consecutives may be nearer than the inclusive distance of six crotchets. Thus, no matter what the Species, or the harmony, consecutives must never appear between any two parts on the first, second, third or fourth beats of successive bars. Such teaching is due to the inadequacy of textbooks, and the application of mechanical criticism to cases which are not really relevant.

These views have been exploded partly by reference to practice, partly by an appeal to musical intelligence and common sense. Such a system as the above is non-existent except in the imagination of a school of theorists, and its arbitrary formation is not only in hopeless collision with the practice of any period, but is in direct violation of artistic instinct and logical reasoning. It is small wonder that from time to time there are agitations for the abolition of Strict Counterpoint; such a system has only obtained the ridicule it deserves.

We cannot do better than quote the words of the late W. S. Rockstro in Grove. After stating that the term 'Strict Counter- 
point' is applied to a method which forbids the direct percussion of a Fundamental Dissonance, and the term 'Free Counterpoint' to one which permits it, he goes on to say: 'The laws of Strict Counterpoint are not open, like those of Harmony, to scientific discussion; for Counterpoint is not a Science but an Art. It is true that its most important rules, when tested by the principles of Natural Science, are found to coincide with them, in all essential particulars; and to this circumstance alone are they indebted for their unassailable position, and promise of future security. Their mathematical accuracy fails, however, to account for their universal acceptance as a code of artistic regulations. Their authority for this rests solely upon the praxis of the Great Masters of the Polyphonic Schools; which praxis was, from first to last, purely empirical. The refined taste, and true musical instinct, of Josquin Desprès, Willaert, Byrd, Tallis, Palestrina and their contemporaries, rebelled against the hideous combinations demanded by the rules of Diaphonia and Organum, and substituted for them the purest and most harmonious pro. gressions that Art, aided by a cultivated ear, could produce; but, in their search for these, they were guided by no acoustic theory. They simply wrote what they felt : and because the instinct of true genius can never err, that which they felt was uniformly good and true and logical, and based unconsciously upon a foundation firm enough to stand the test of modern mathematical analysis.'

It only remains to say that as modern conditions bring new light upon certain aspects of the art, the present treatise aims at modifying the system in so far as procedures which were relatively correct cease to have any place under modern conditions; as, for example, the treatment of unessential notes, and the criticism of consecutives. But those procedures which do not involve any violation of modern methods are left intact. By this means the student is enabled to look at harmonic resource from two distinct points of view, the horizontal and the vertical, and he is thus taught how Counterpoint merges into and becomes the basis of Harmony. It is a system which can be directly applied in modern work; and he student who builds up his 
decoration of diatonic harmony on such principles, is the one who is best equipped to enter upon the field of chromatic resource.

\section{EXAMPLES.}

First Mus. B. Oxon., Nov., Igoo.
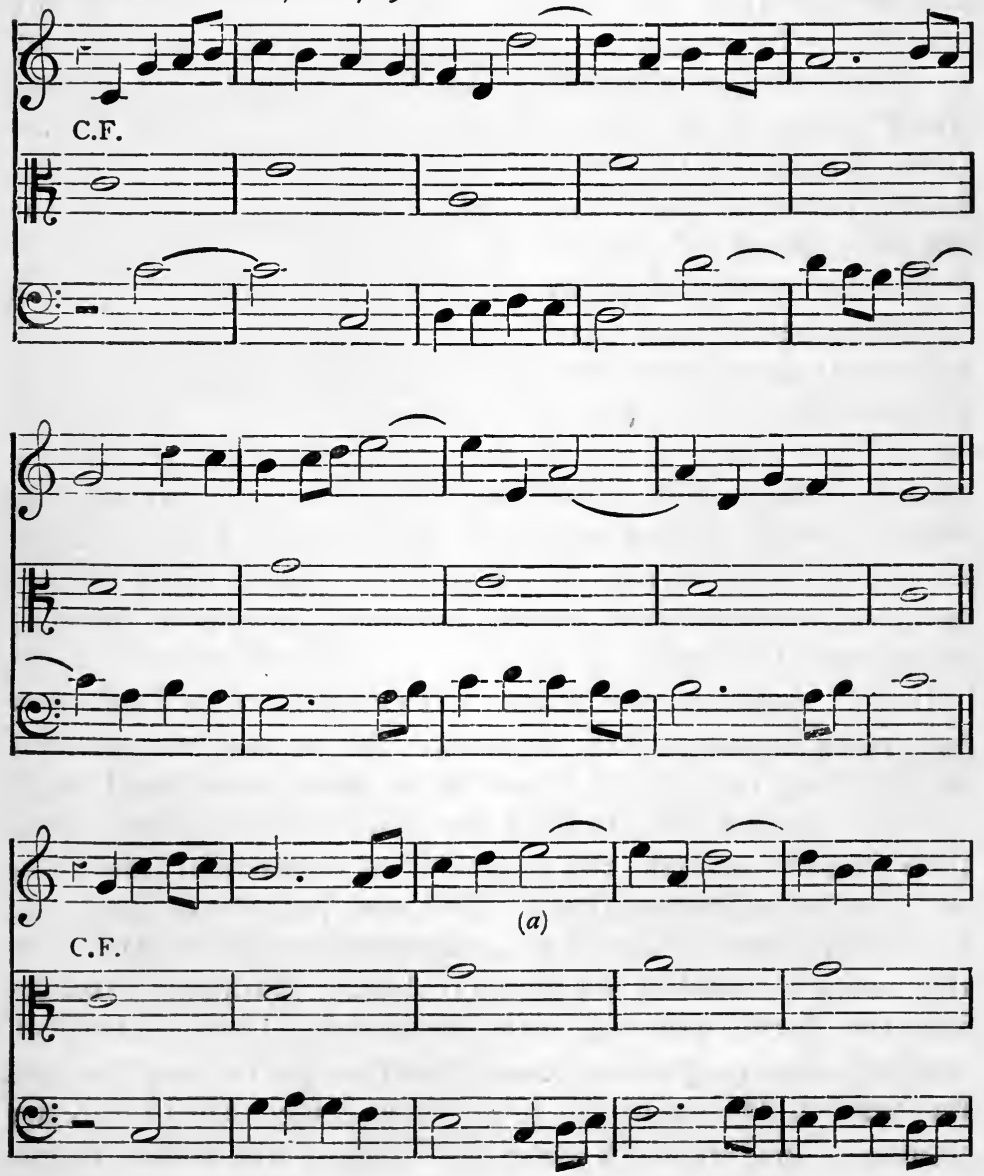
COMBINED COUNTERPOINT IN THREE PARTS I89

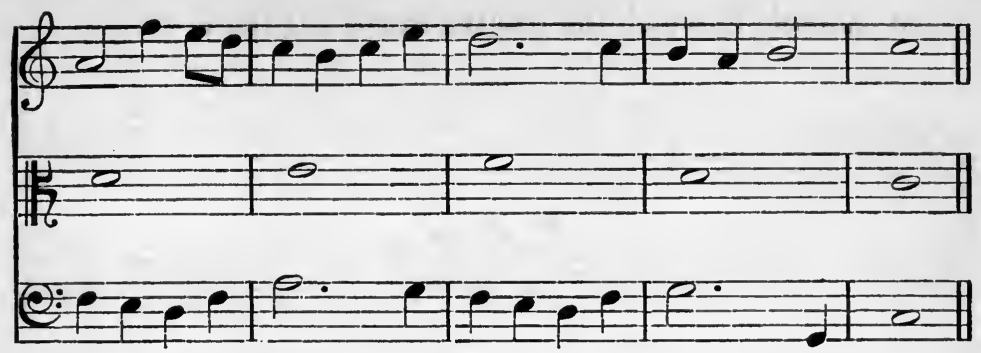

(1)

Canon at the Seventh.

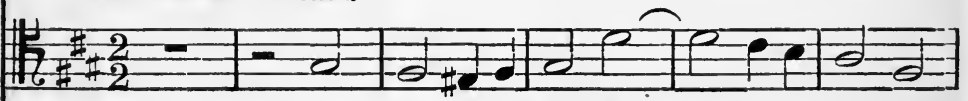

C.F.

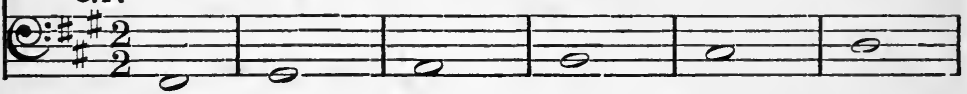

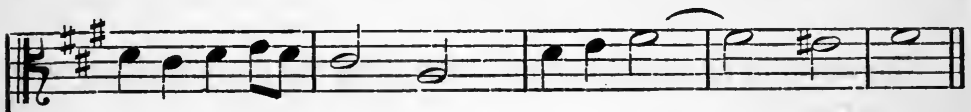

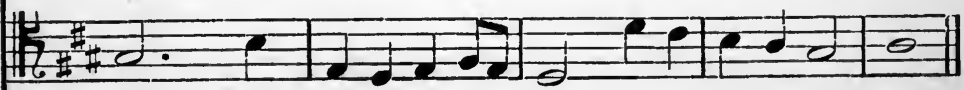

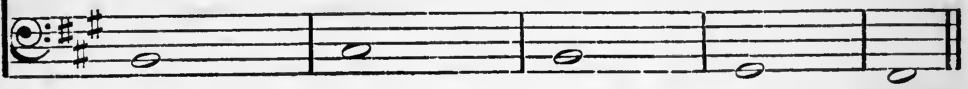

(a) Avoid-

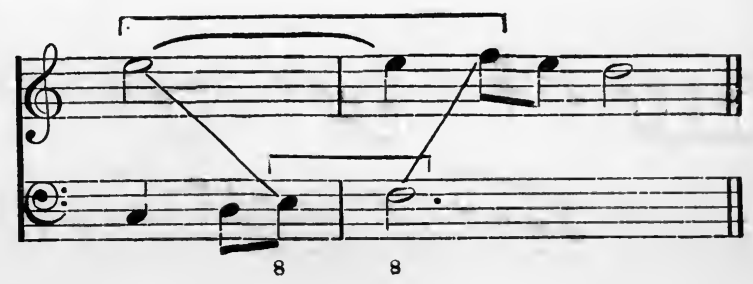




\section{THE ART OF COUNTERPOINT}

An example in which the Fourth Species is the C.F.C.F.
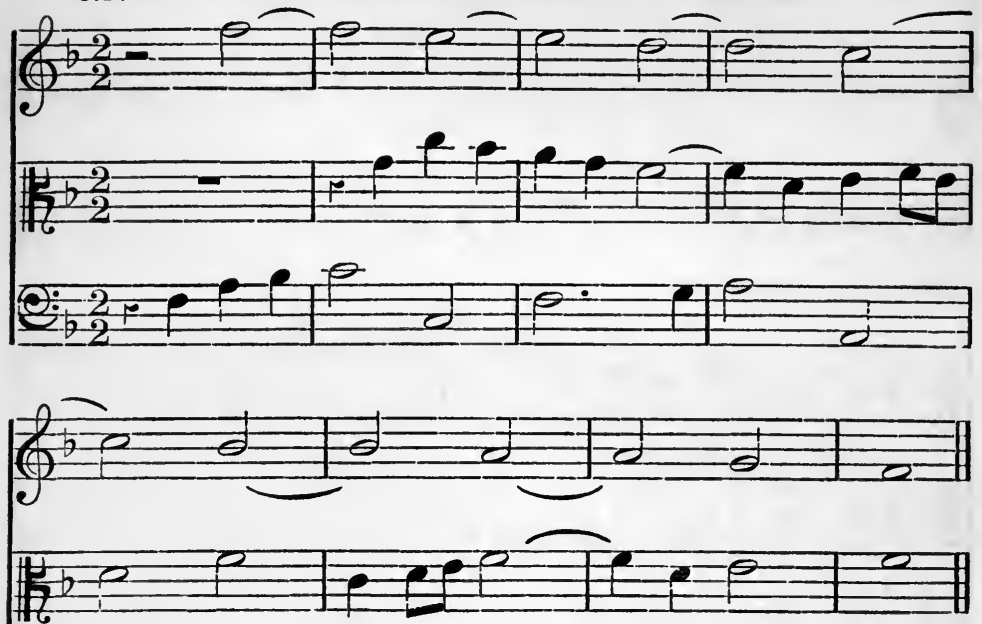

(a)

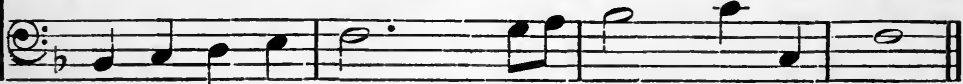

The same in practical terms, illustrating the aims of Scholastic Counterpoint-
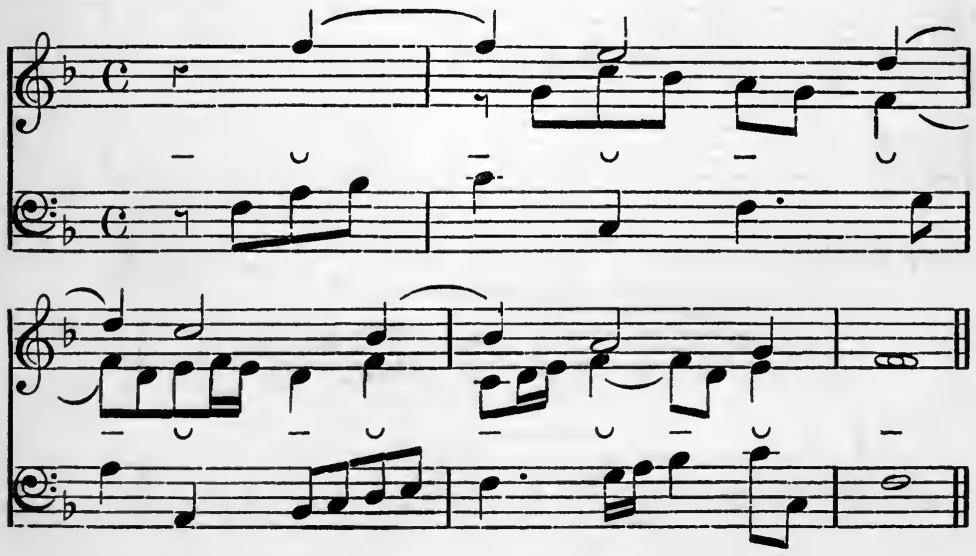
(a) The last four bars might have been arranged so as to illustrate the origin of the cadential six-four.

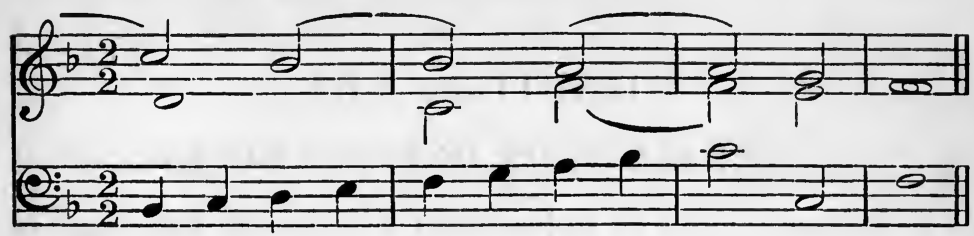

in practical terms :-

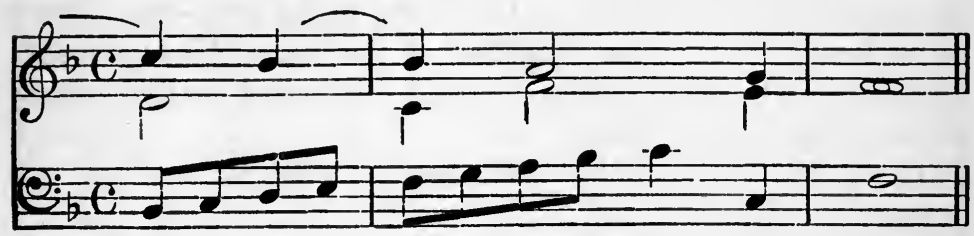




\section{CHAPTER XIII \\ COUNTERPOINT IN FOUR PARTS}

r. THERE is little to add to what has already been said in reference to three-part writing. The addition of a fourth part of course causes one note of the chord to be doubled. There is no law forbidding the doubling of the major third; but this procedure is likely to produce a thick effect, and for that reason its use requires discretion.

The following examples will serve to illustrate one or two points :-

(I)

(2)

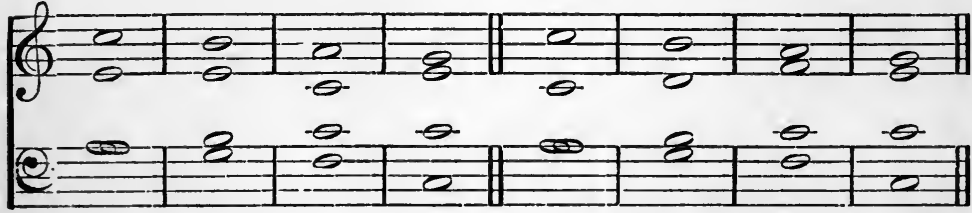

(a) Between bars 2 and 3 of the first example there are exposed fifths between the alto and the bass-

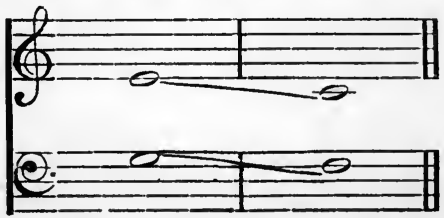

These are freely allowed between any but the extreme parts, whether the higher part move by step or not.

(b) The doubled leading note in the second bar of each example is entirely unobjectionable.

Some teachers condemn every doubled leading note, irrespective of the context, simply because some textbook says the leading note must not be doubled. 
There are of course many cases in which it is open to objection, for instance-

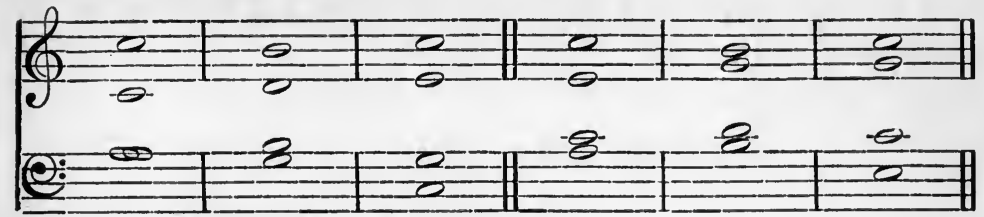

Bad.

In the above cases the leading note is felt to have a fixed melodic progression, and to be a predominant feature of the cadential idiom; its doubling encroaches upon this position, and causes an unsatisfactory effect.

(c) Between bars 2 and 3 of each example we see the false relation of the tritone between the extreme parts in similar motion. But the effect is quite good.

Compare the opening of Palestrina's Stabat Mater-

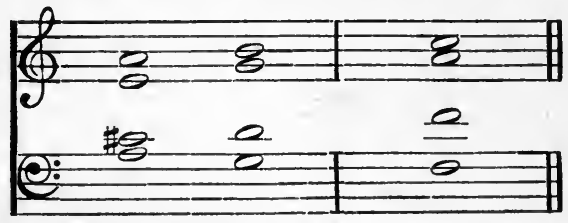

(d) Between bars 3 and 4 of each example the extreme parts approach a perfect concord by similar motion; both chords are what we term Primary Triads, and the top part moves by step. It has been pointed out before that such cases are common in Palestrina, and that theorists are unnecessarily severe in this respect.

3. A common way of avoiding the interval of the diminished fifth between the bass and an upper part was to flatten the lower note a semitone, thus-

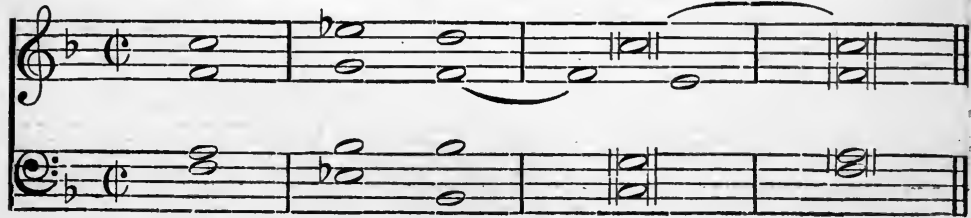


This progression has come to be regarded as quite an idiom of ecclesiastical music, and may be introduced with discretion ; not for the purpose of shirking a difficulty, but to enhance the harmonic effect.

Section A. Uncombined Counterpoint.

I. First, Second, and Third Species.

First Mus. B. Oxon., Nov., Igo3.

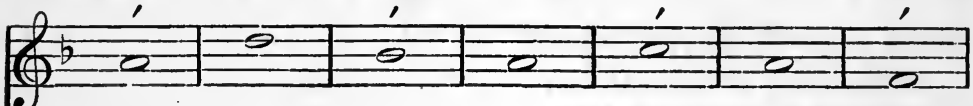

C.F.
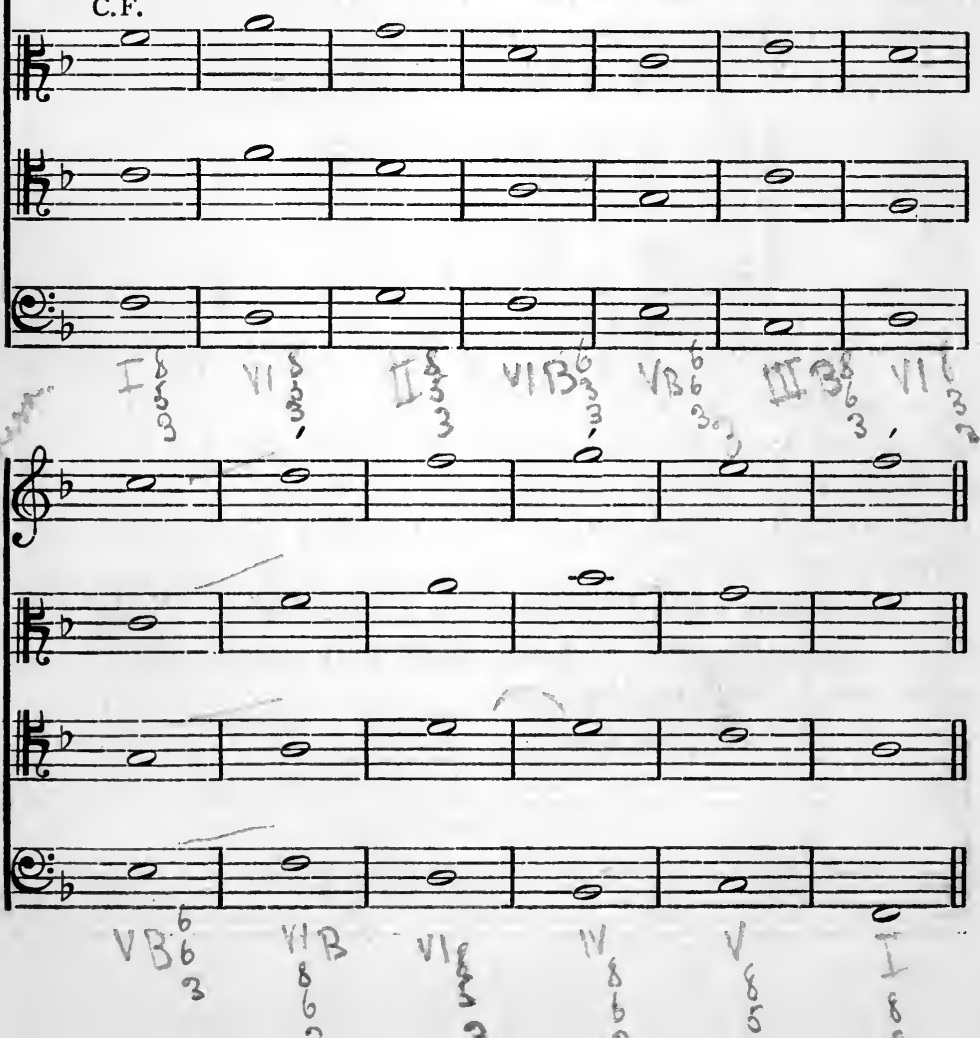


\section{COUNTERPOINT IN FOUR PARTS}

First Mus. B. Oxon., Nov., rgot.

\begin{tabular}{l} 
First Mus. B. Oxon., Nov., rgot- \\
\hline
\end{tabular}

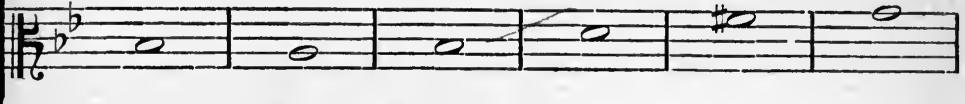

स:

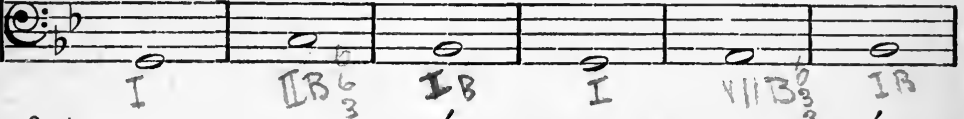

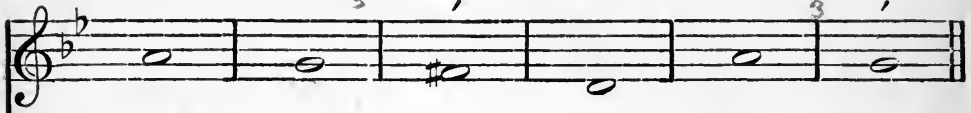

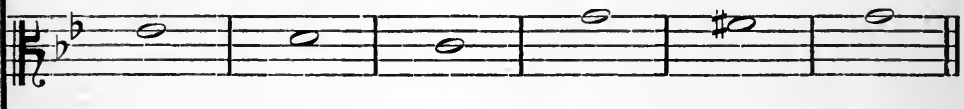

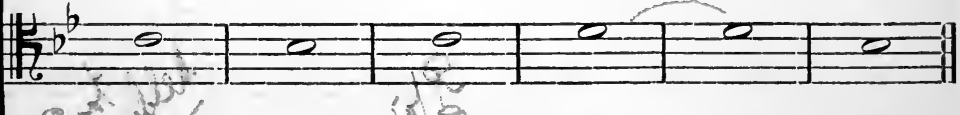

(2)

- क म

$\because$ T0

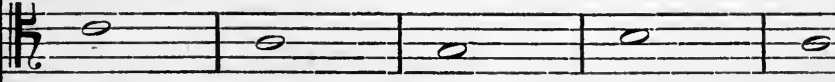

C.F.

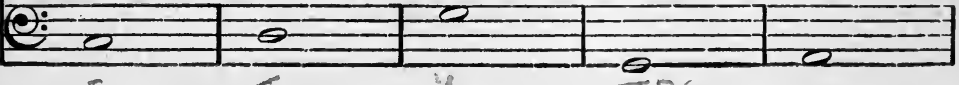


I96 THE ART OF COUNTERPOINT

10

00 C.F.

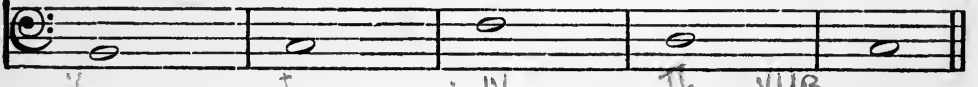
C.F. $\&^{\circ} \dot{0}=\dot{0}=0$

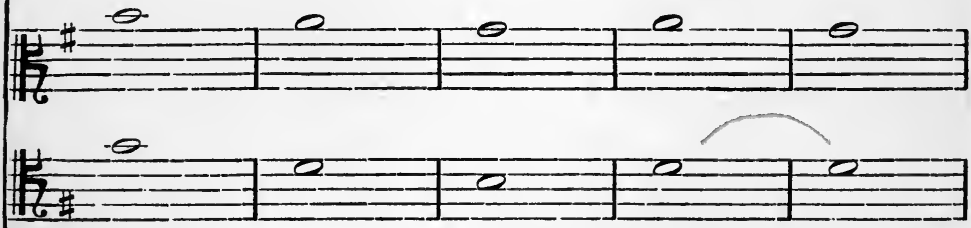

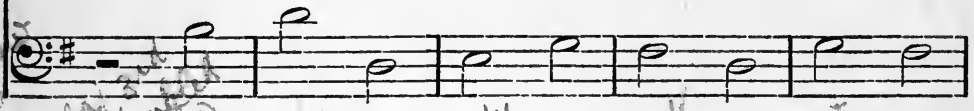
- तर $\frac{1}{20}=0$

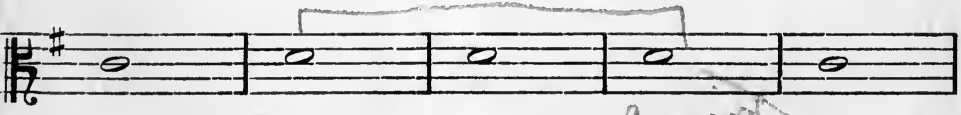

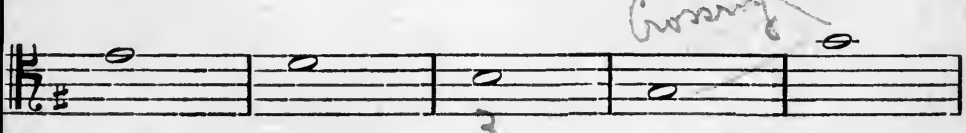
(a)

Q:18 ? 


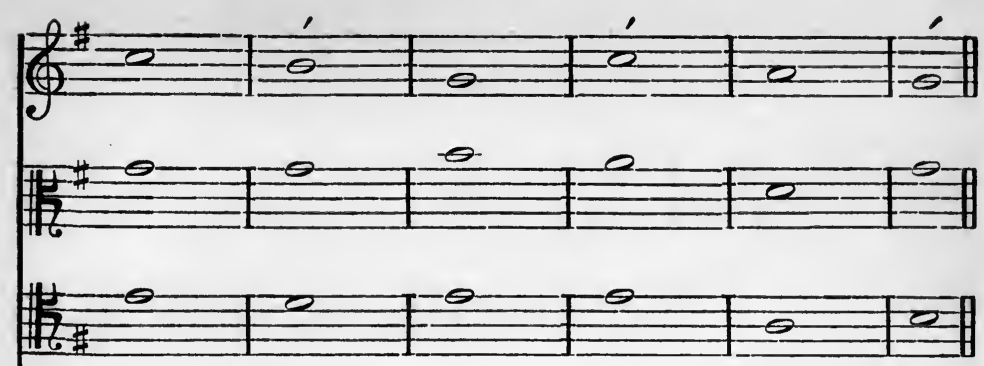

(a)

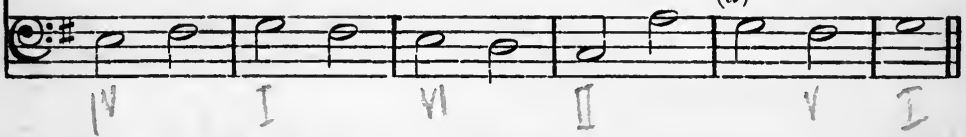

(a) The use of the discord on the first half of the bar makes a much smoother part: in such a case, however, the previous and succeeding notes must be concordant, and the procedure must be sparingly employed, for it does not strictly belong to the period. It should never be requisitioned in Combined Counterpoint.

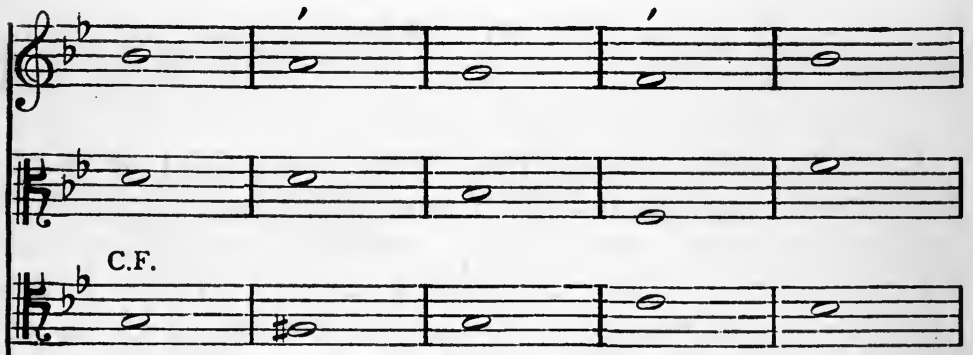

(b)

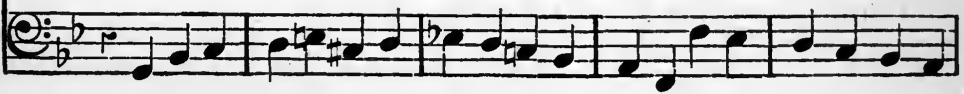

(b) As the complexity of the score increases, the rules as to consecutives between First and Third Species are rightly relaxed. 

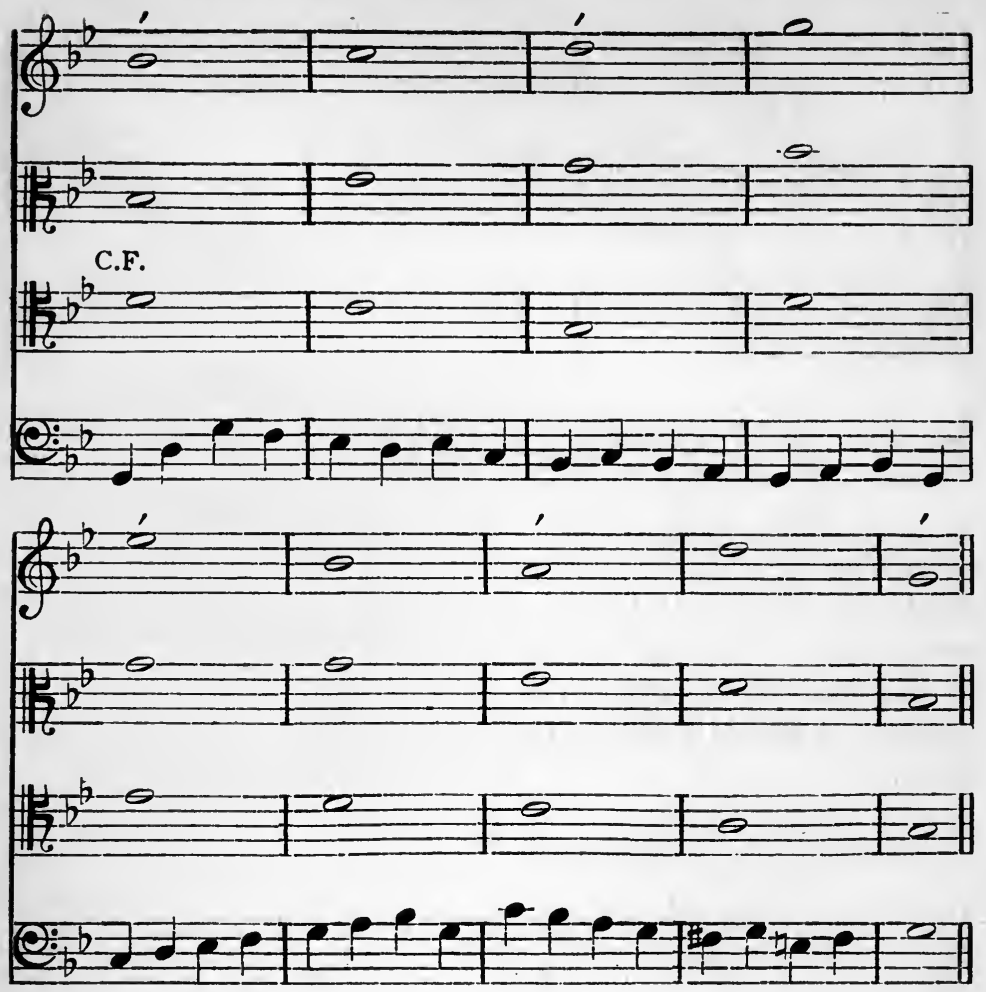

Cherubini allows consecutives between the third or fourth crotchets of one bar, and the second of the next (e.g. one or both of the notes involved being unaccented) in two parts with no consideration as to the harmonic equivalent. However, this consideration may generally be ignored in Combined Counterpoint, if one of the parts involved be a mean. Artistic perception and intelligence are required rather than rules.

\section{Fourth and Fifth Species.}

4. In the Fourth Species suspended discords are accompanied as follows :- 
(a) Above the C.F.

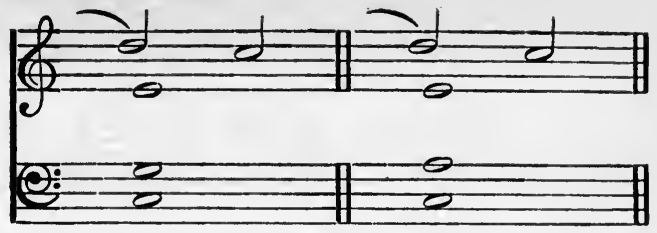

98 by ${ }_{3}^{5}$ or either. $\quad 3_{3}^{6}$ or either.

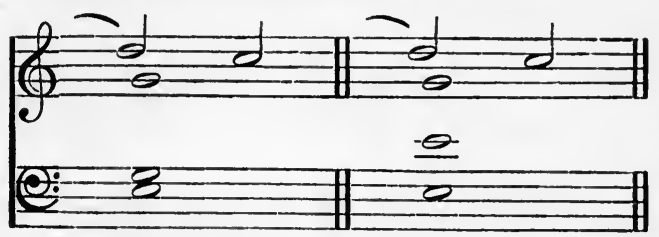

76 by ${ }_{3}^{8}$ or either.

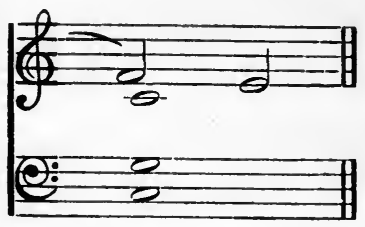

43 by ${ }_{5}^{8}$ or either.

(b) Below the C.F.

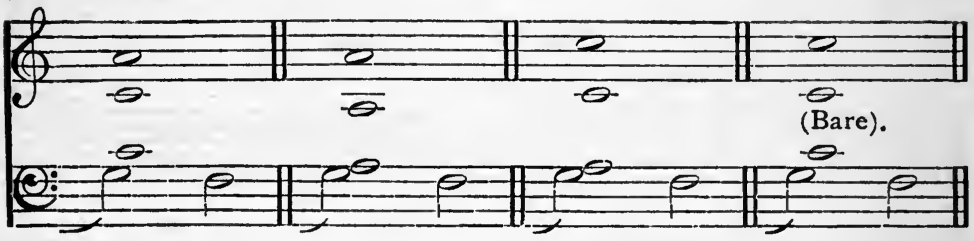

${ }_{2}^{4}=$ by 4 or 2 .

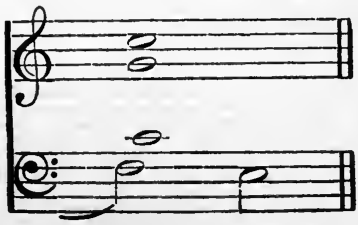

$\frac{5}{2}=$ by 5 or 2 . 
200 THE ART OF COUNTERPOINT

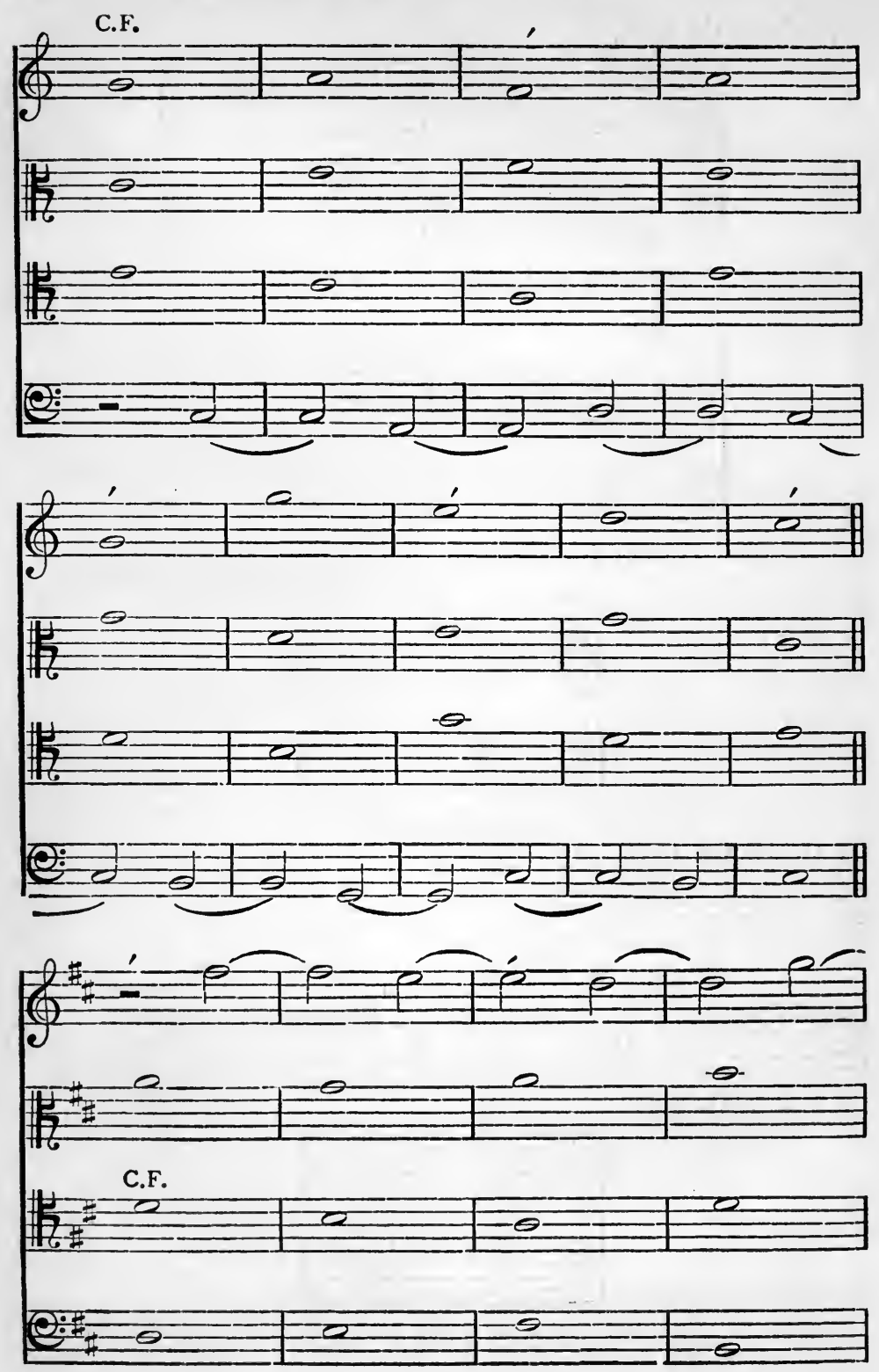




\section{COUNTERPOINT IN FOUR PARTS 201}

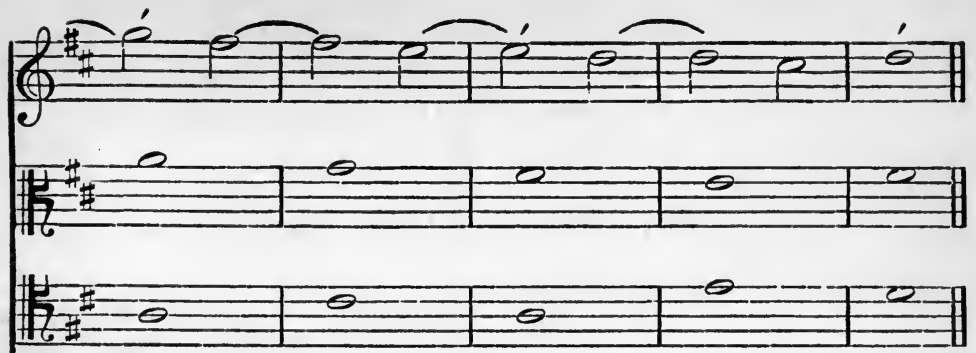

(a)

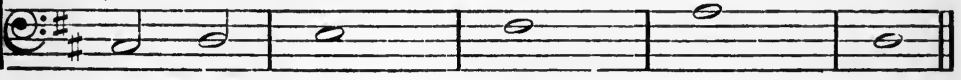

(a) A bar of Second Species may be interpolated, to improve or vary the harmony, or to avoid bad disjunct movement.

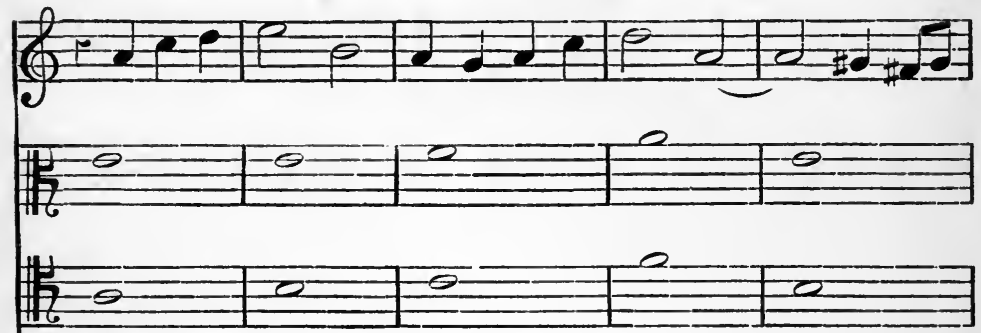

C.F.

000000

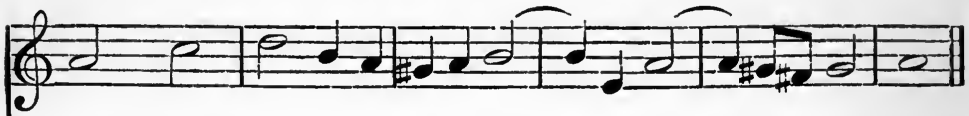

To lo to

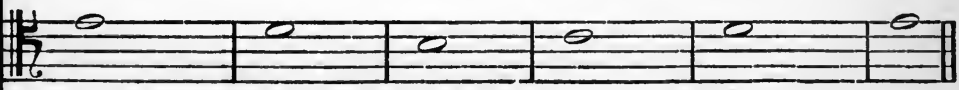

\begin{tabular}{|l|l|l|l|l|l|}
\hline $0 ; 0$ & 0 & 0 & 0 & 0 & 0 \\
\hline
\end{tabular} 
Section B. Various Combinations of Species in Four PARTs.

First, Second, and Third Species.

First Mus. B. Oxon., May, 1904.

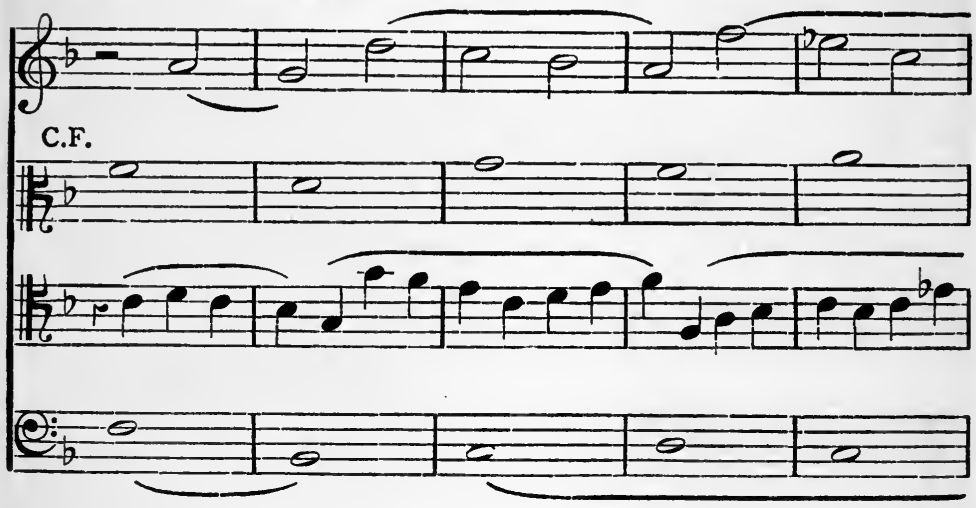

का का?

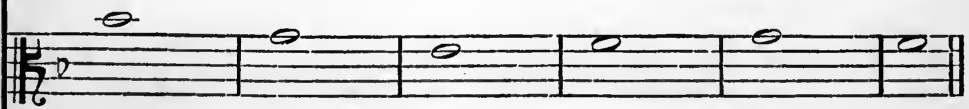

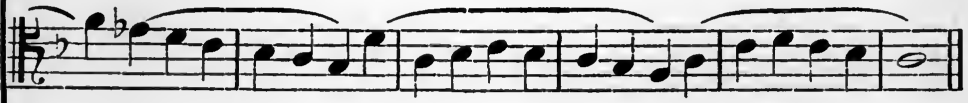

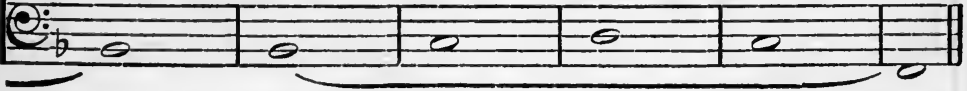




\section{First, Third, and Fourth Species.}

First Mus. B. Oxon., Nov., 1900.

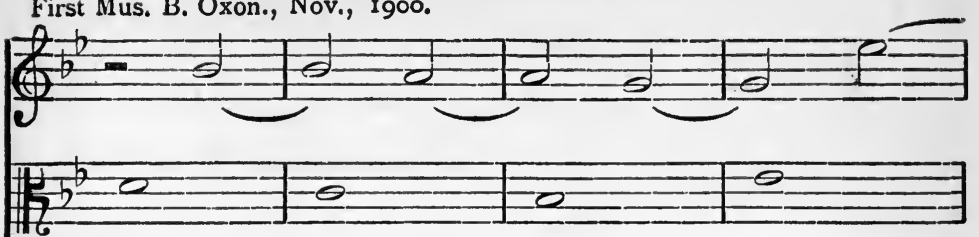

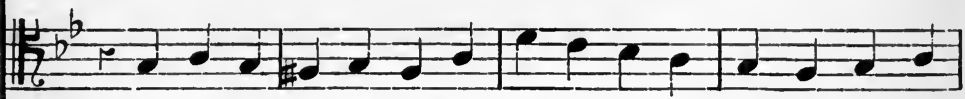

C.F.

$e_{i}^{i} \frac{b}{b}-0=0$

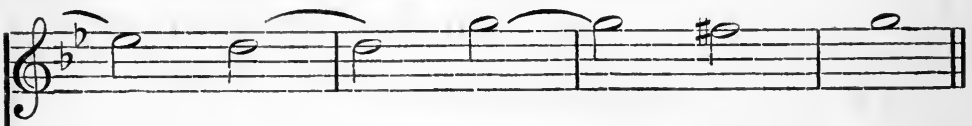

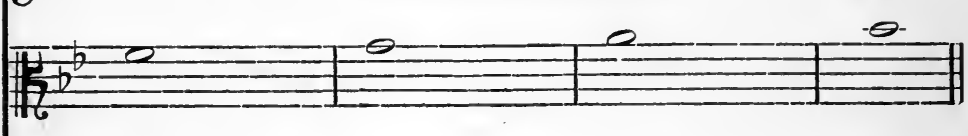

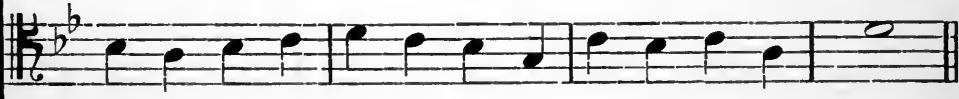

$\theta_{i b}^{b-\theta}=970$

First Mus. B. Oxon., May, rgo4.

C.F.

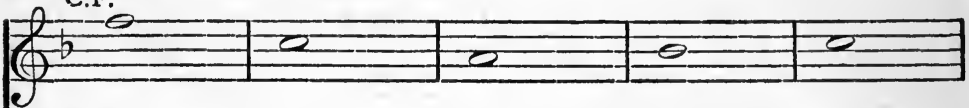

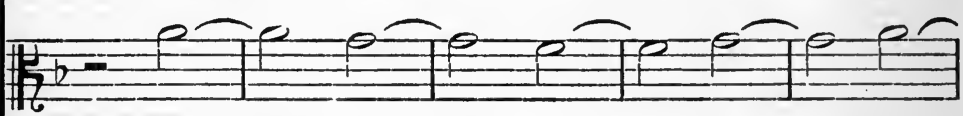

进安

$0+0-0$

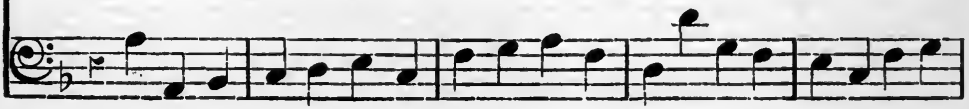


C.F.
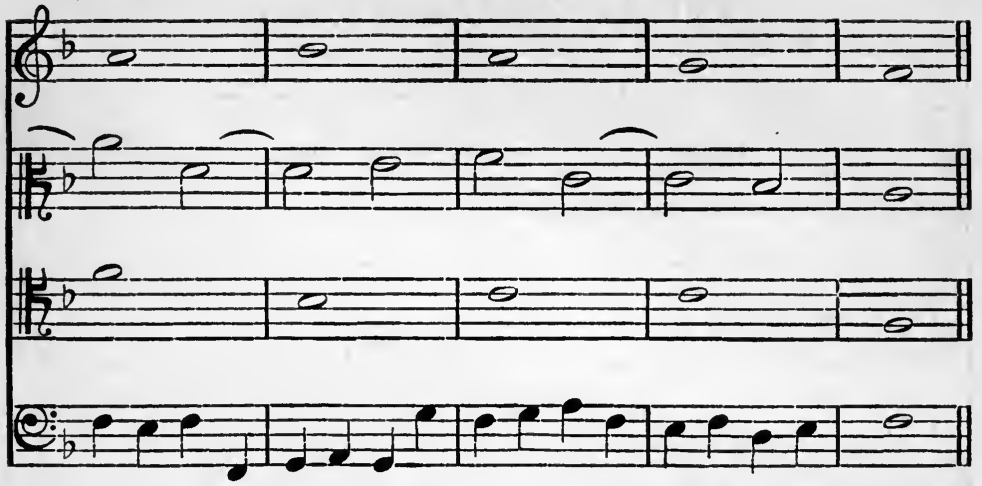

Double Suspensions may be used in Combined Counterpoint in four parts, the additional part, by its florid movement, preventing any halting effect.

(a) Examples of double suspensions-

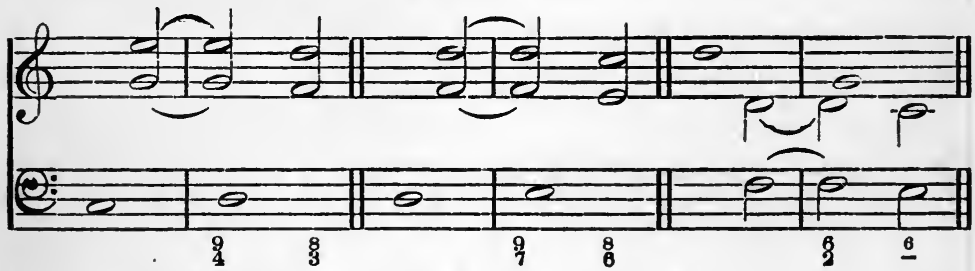

(b) With a florid part added-
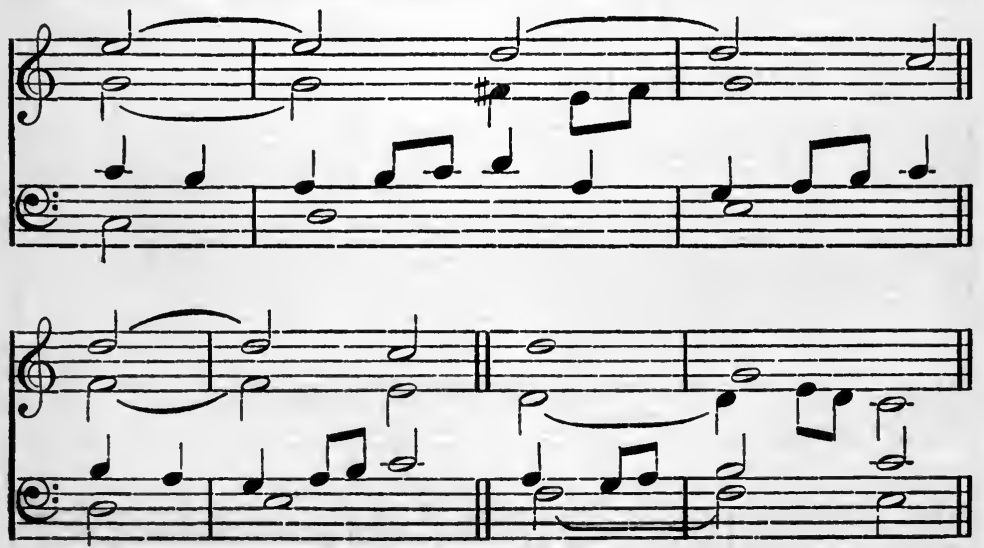
(c) Prepared discords and syncopated concords with Third Species-

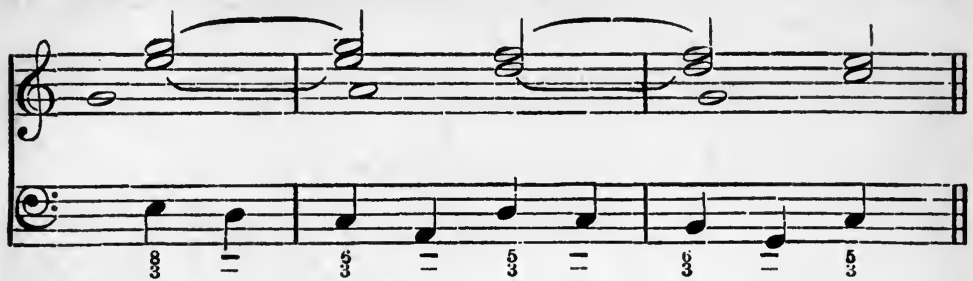

The following examples of prepared discords will be readily understood. They are taken from Cherubini-
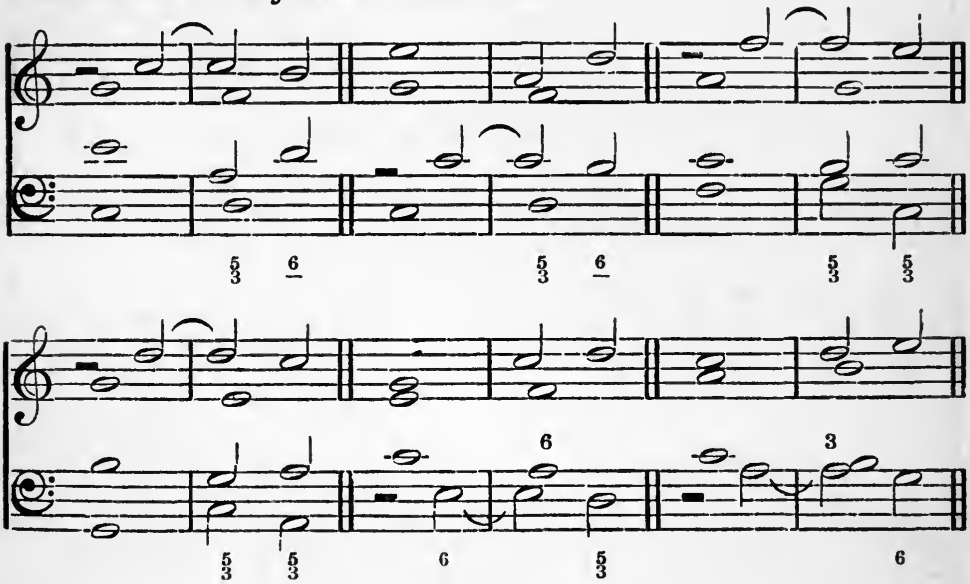

Palestrina.

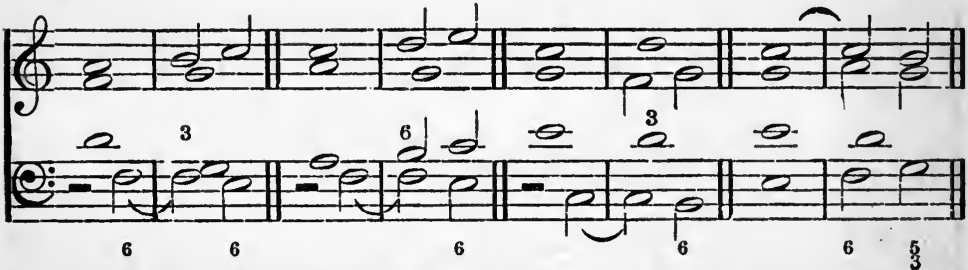

Here we see the origin of our modern fundamental discords. It will be observed that all the notes except the suspended discord are concordant on the first minim of the bar; and that all the parts are concordant on the second minim of the bar. Ornamental forms are of course quite common- 


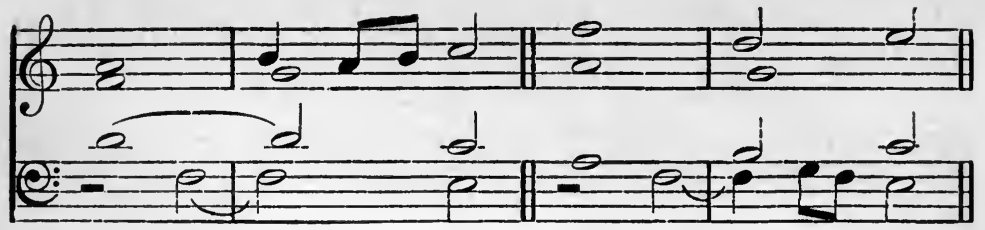

Suspensions and syncopated concords may be combined-

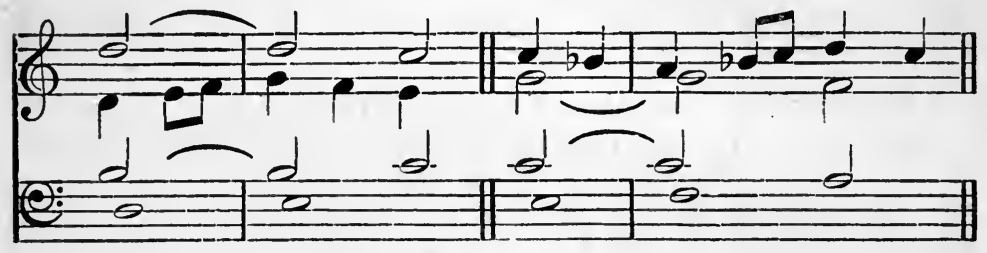

First, Second, Third, and Fourth Species.
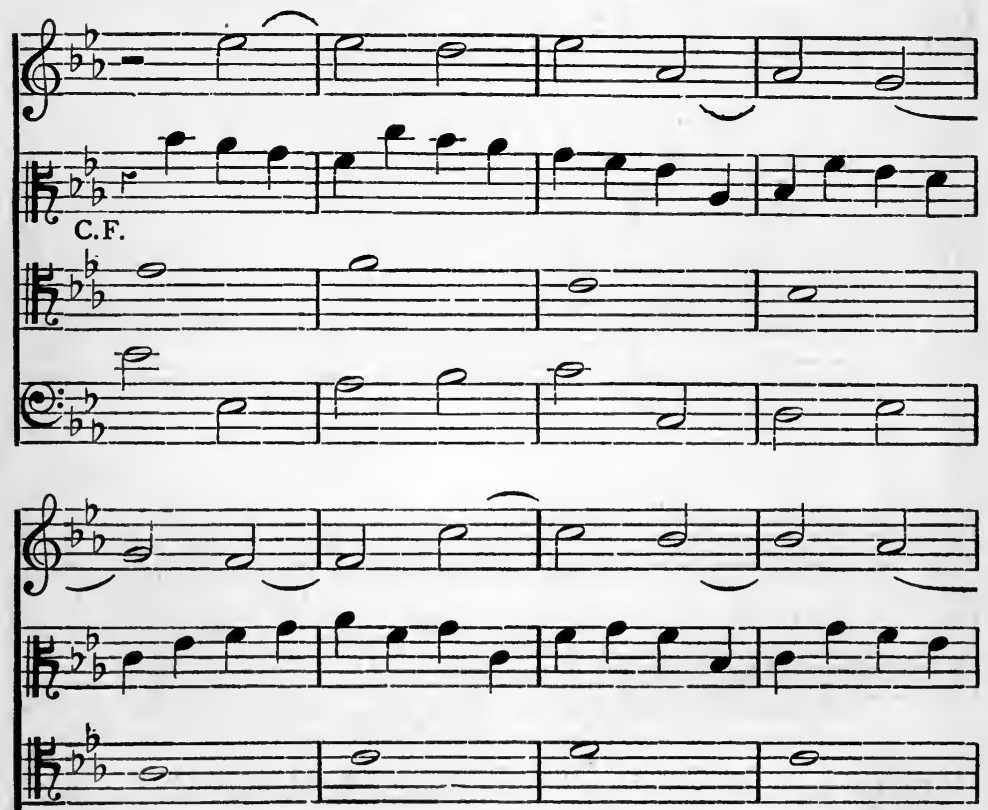

(a)

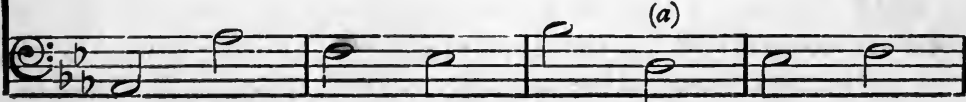




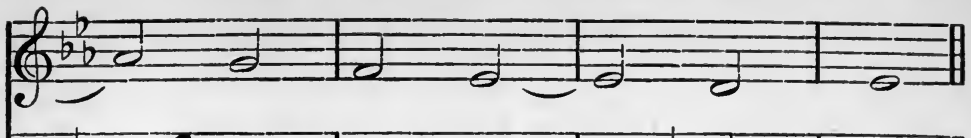

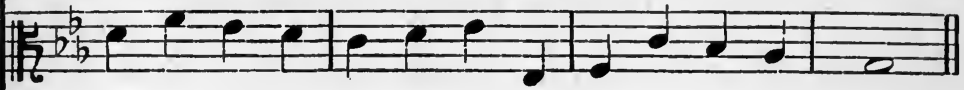
$t \frac{b}{6} \frac{b}{6}=$

O:-

(a) The doubled leading note is unobjectionable.

First, Second, Third, and Fourth Species.

Third Mus. B. Oxon., May, 1905.

$8=-2=0$

C.F.

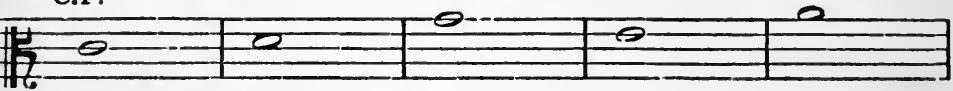

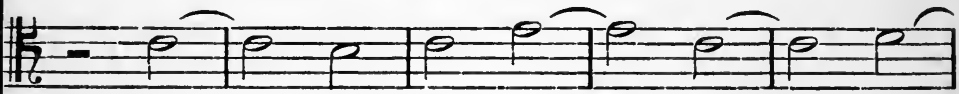

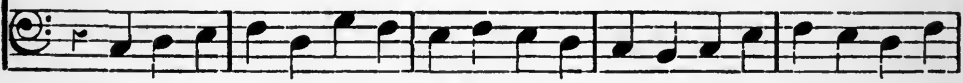

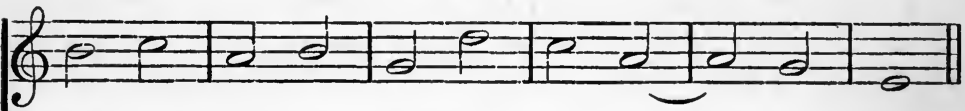
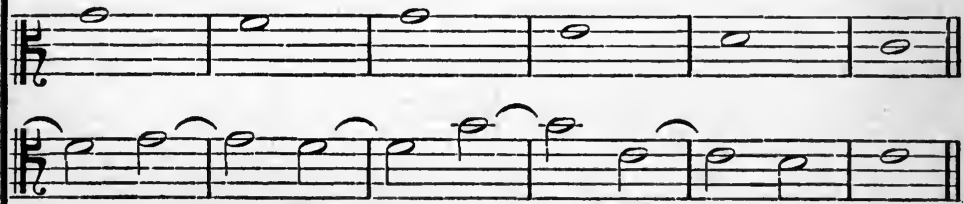

(b)

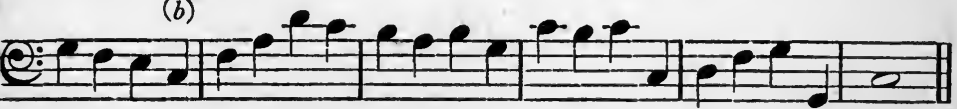

(b) Three leaps in the same direction are here unobjectionable. 


\section{Three Parts in Florid Counterpoint.}

I. Distinguish between combinations which are felt as

(a) passing notes, causing harmonic obscurity;

(b) passing chords;

often such possible combinations have to be discarded, and the score simplified: sometimes the addition of another note makes the effect satisfactory--

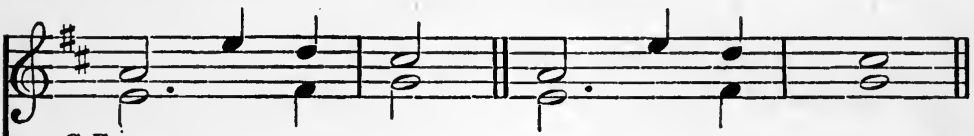

C.F.

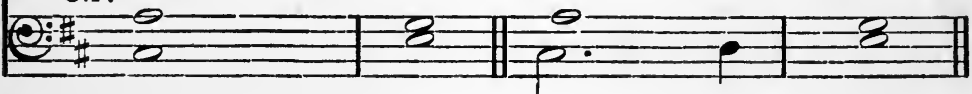

Bad.

Good.

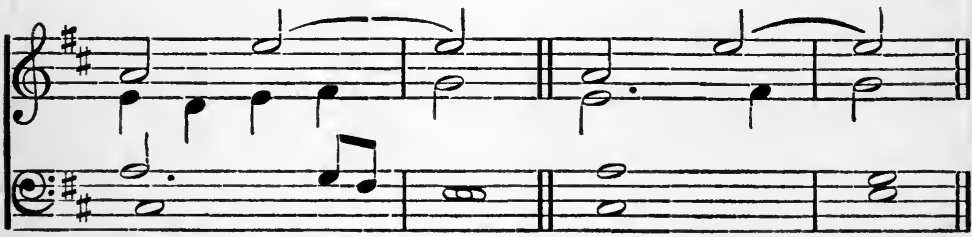

C.F. Bad.

2. Just as, in three parts, the bare fourth was allowed as a harmonic interval between two upper parts, so the following is legitimate in four parts:-

Palestrina. $O$ admirabile commercium.

BYRD. Gradual. Beata Virgo.

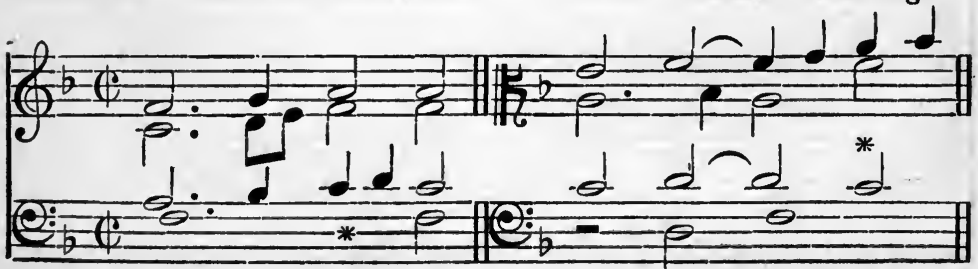

Some theorists are accustomed to argue as follows :- 


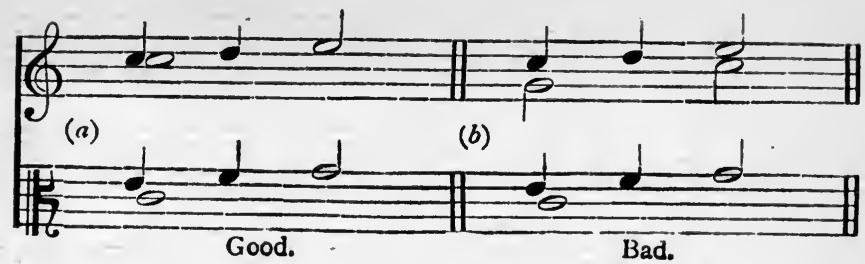

It is surely time that broader views should prevail, even at the expense of a doubtful theory. The mental effect of both is identical : Palestrina employs both; we therefore follow practice, not theory. It will be observed that in $(b)$ a second is quitted by similar motion-

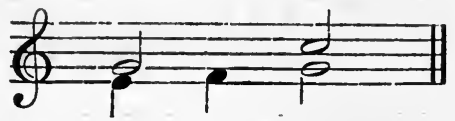

In this case, the bass is felt to be a pedal, and the $\mathrm{F}$ and $\mathrm{G}$ the root and seventh of a passing dominant seventh; the effect therefore is good.

3. Occasional combinations of groups of quavers may be employed.

4. The score should be kept as simple and as clear as possible. Movement should never be made merely for the sake of doing something; nor should any possible move be made, irrespective of questions of melodic interest, and of variety of rhythm.

First Mus. B. Dunelm, rgo3.

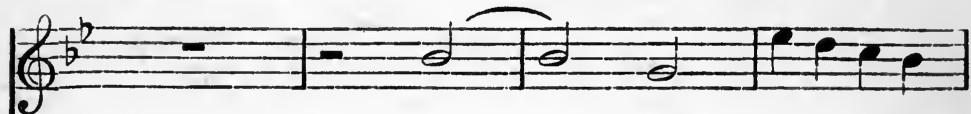

C.F.
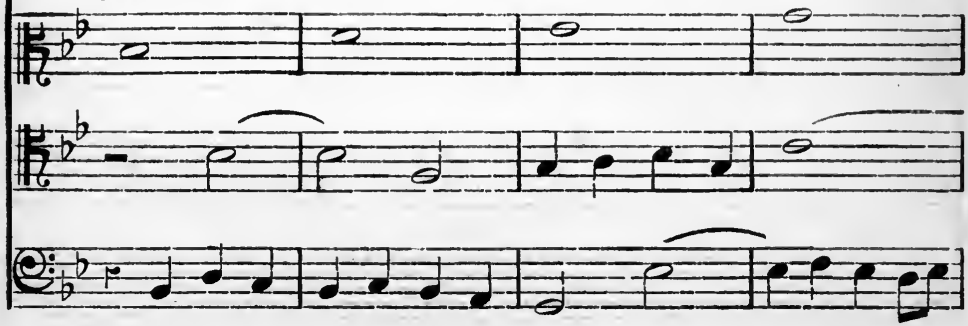

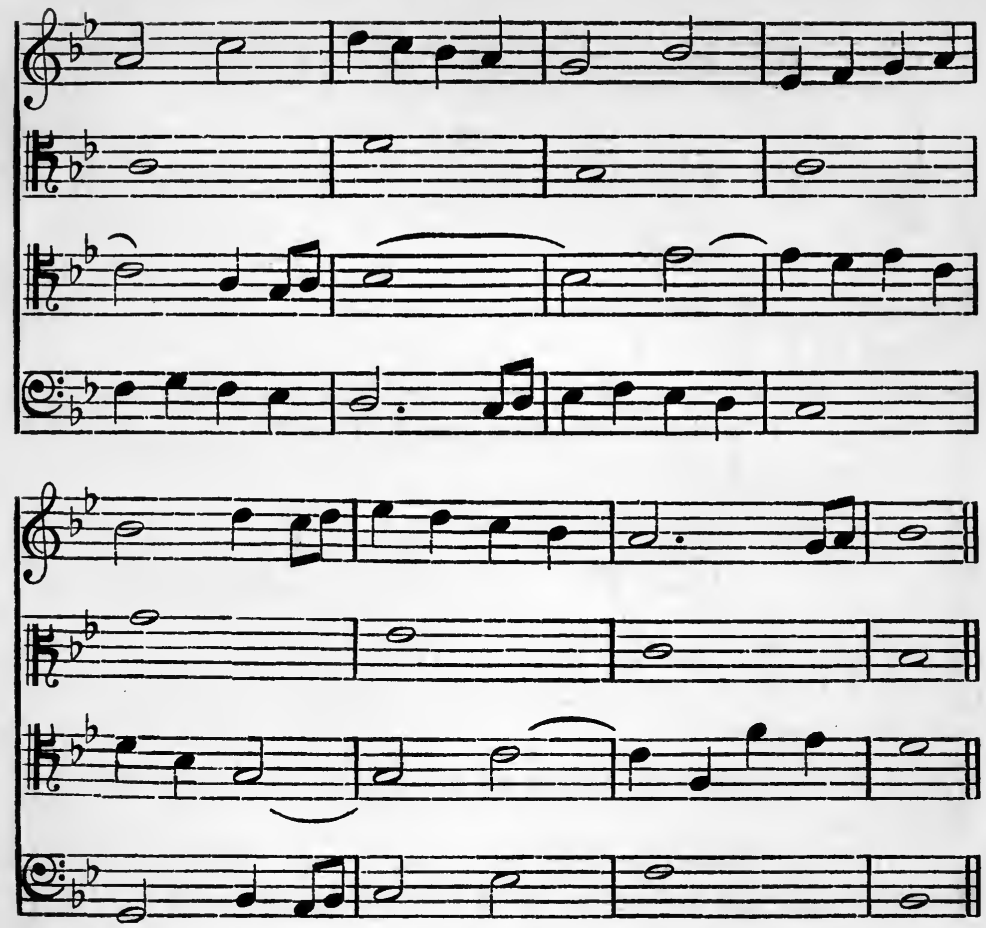

An example in which the contour of the C.F. encourages modulations:-

First Mus. B. Oxon., Nov., Igor.

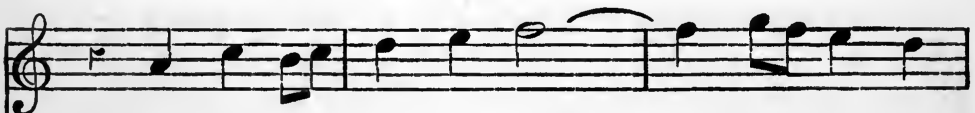

C.F.

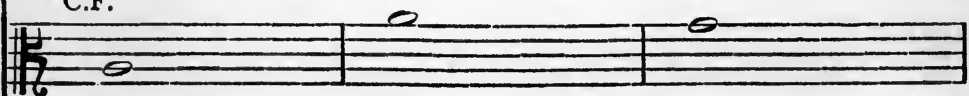

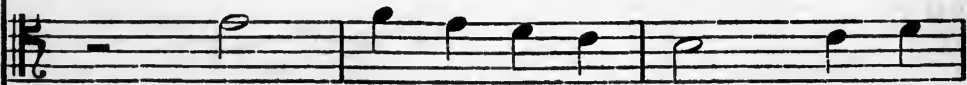




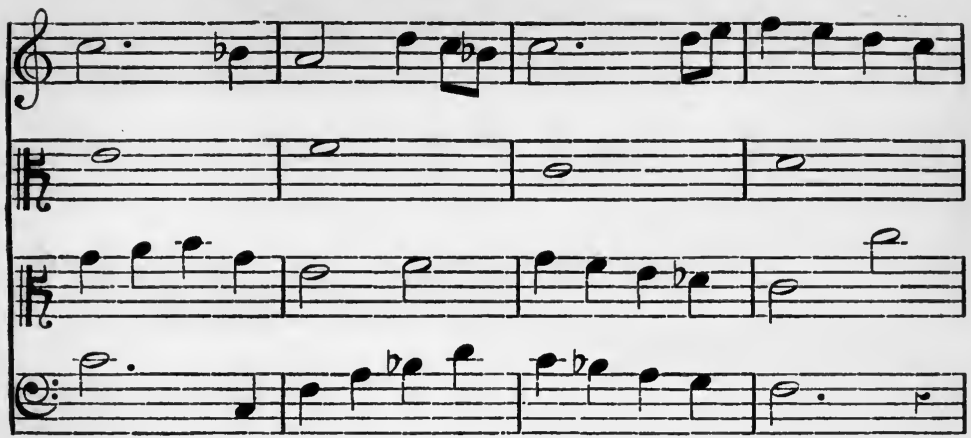

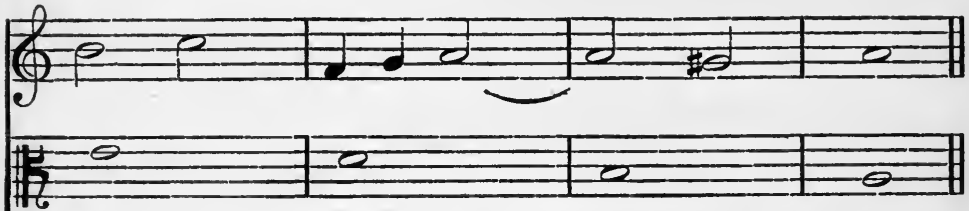
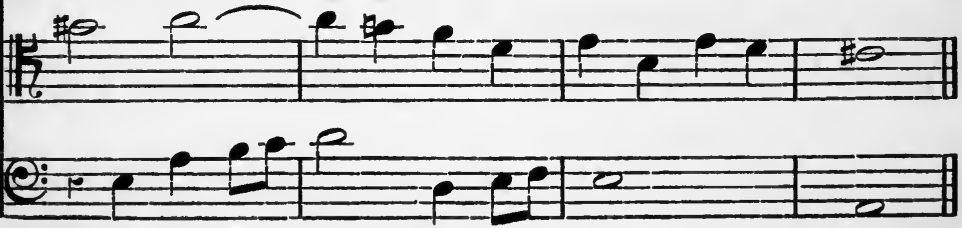

First Mus. B. Oxon., May, I904.

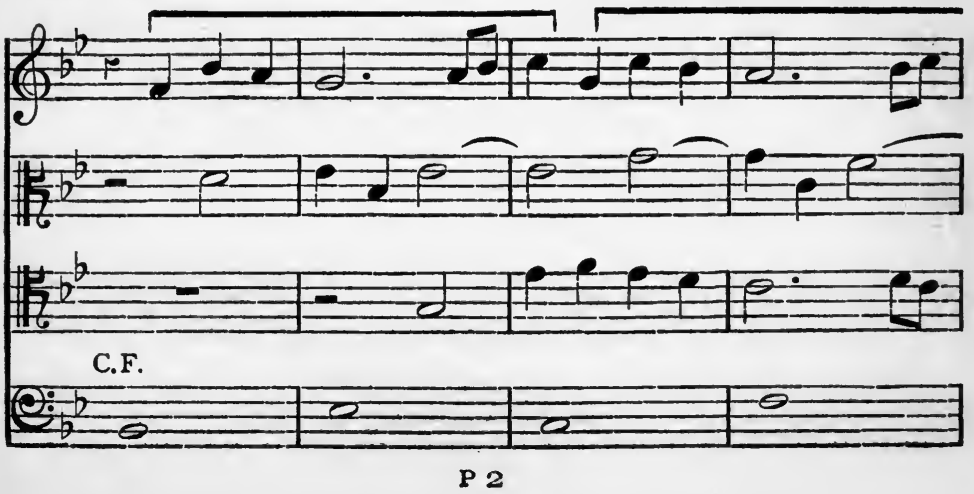




\section{THE ART OF COUNTERPOINT}
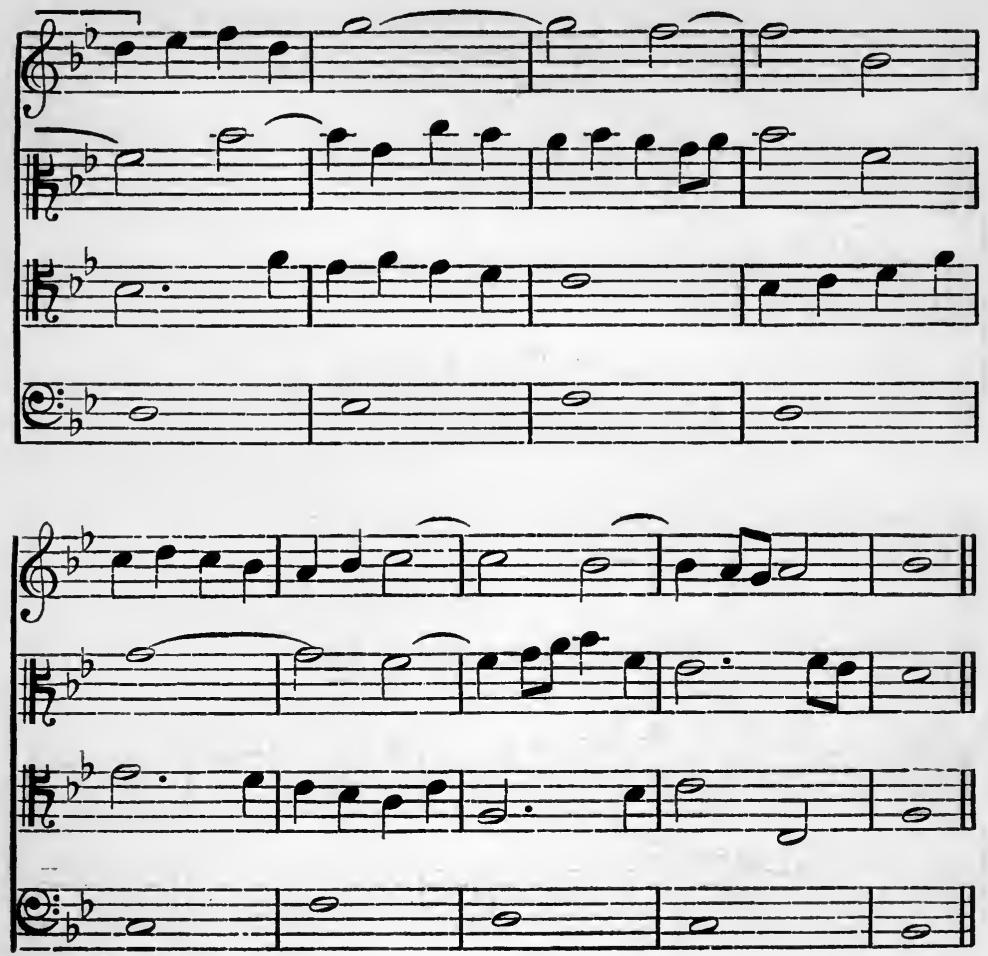

First Mus. B. Oxon., Nov. 1904.

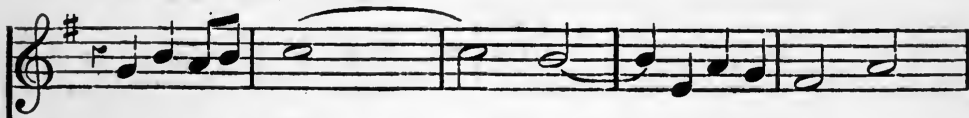
F= \$ C.F. \begin{tabular}{l|l|l|l|}
\hline & 0 & 2 & 2 \\
\hline
\end{tabular} 

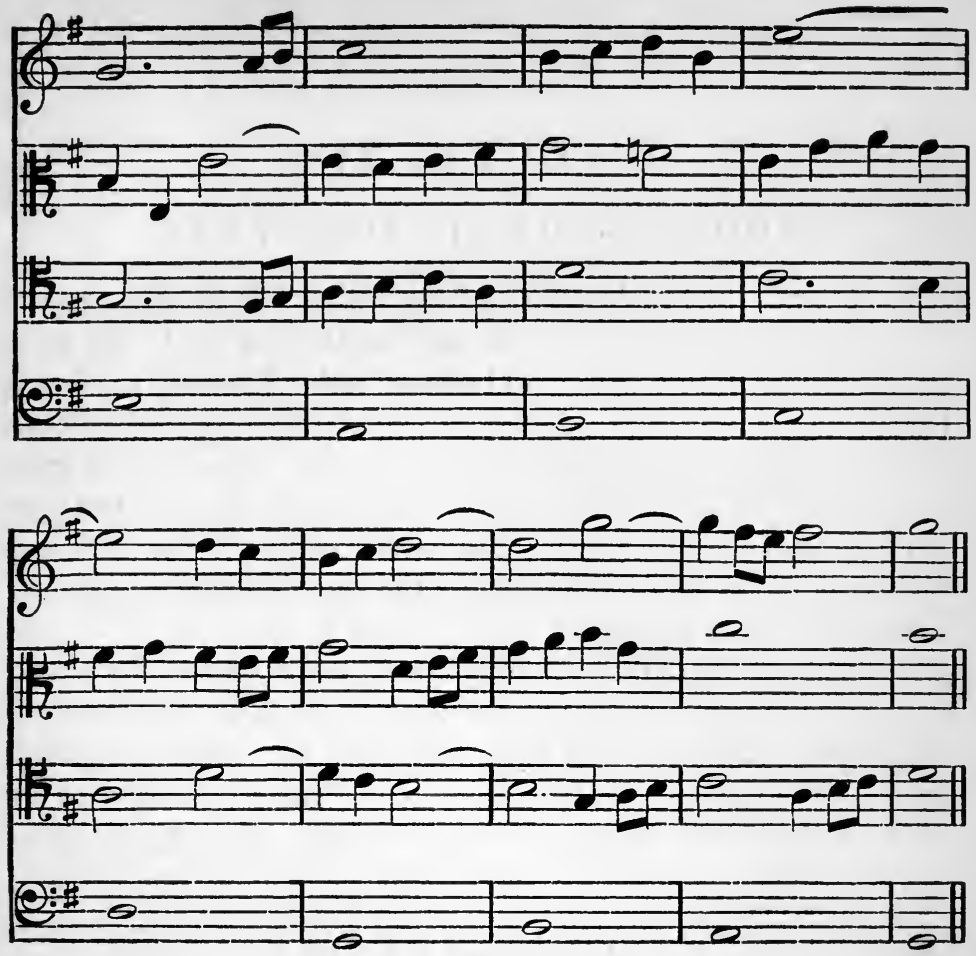


\section{CHAPTER XIV \\ COUNTERPOINT IN FIVE PARTS}

I. HARDLy any relaxation of the rules previously given is necessary in five parts, and in any number of parts the strict idioms of Counterpoint should be observed. There is never any need to approach or to quit quavers by leap, or to use dotted crotchets. Simplicity and clearness are the essentials of good style, and no single part must suffer from a lack of melodic variety.

In difficult combinations, the strict letter of the law may occasionally be ignored, provided the end justifies the means. The following are cases:

(a) A suspended discord may be sounded against its resolution, except at the interval of a second, by contrary and conjunct movement. But the part that anticipates the resolution should be in the nature of a scalic passage. The anticipation of the third of the chord is the most objectionable of such extensions of the rule.

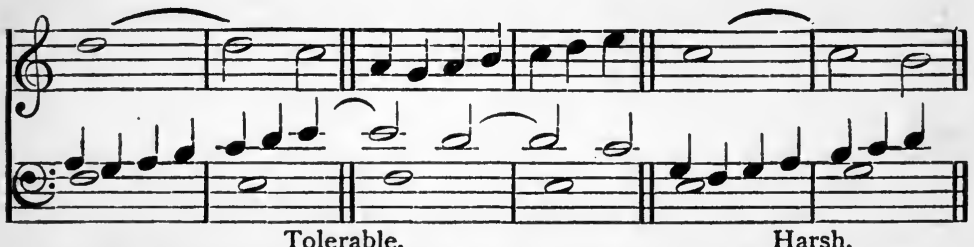

Such a procedure is often most useful in difficult combined Species.

The following are obviously bad:-

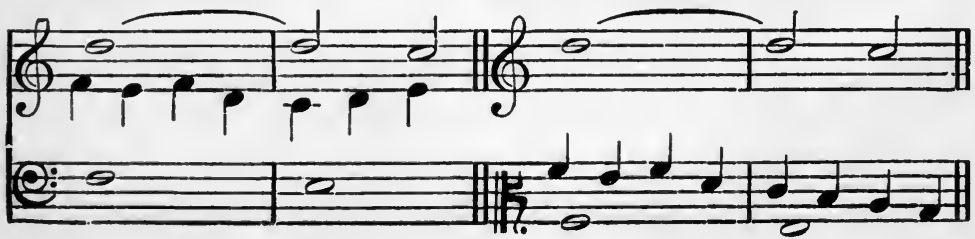




\section{COUNTERPOINT IN FIVE PARTS}

In other words, the progression of the suspension must not be heard in diminution in another part, as in the above examples.

Students often find it difficult to avoid fifths in such a case as the following:-

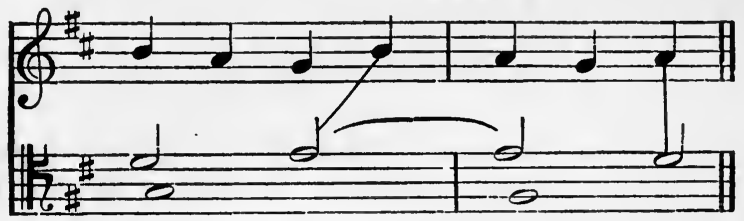

There are three ways out of this difficulty:-
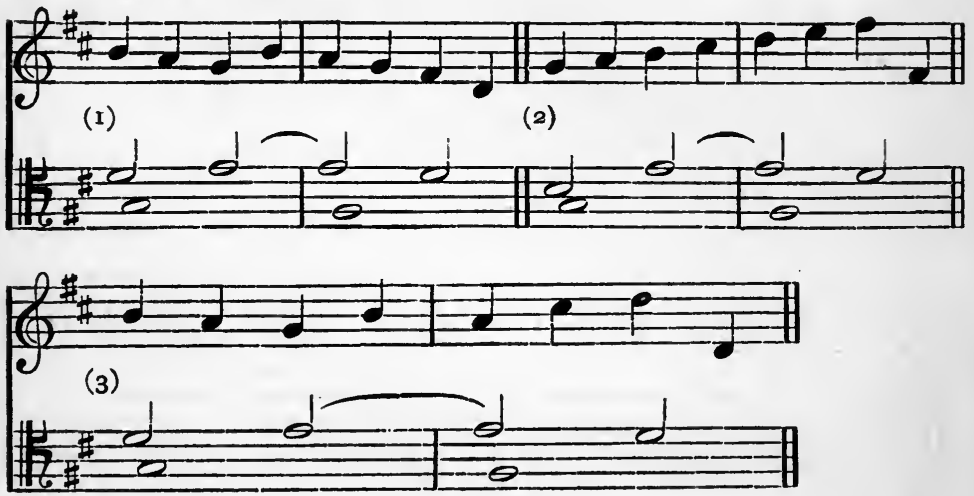

In (I) the fifths never occur;

(2) has just been explained ;

(3) has been explained under the heading of Combined Counterpoint in three parts.

It is of course obvious that octaves cannot be avoided in this way, see p. 165, without causing other faults.

It may be urged by some that the permission of the licence (a) is inconsistent, as it is hardly in keeping with sixteenthcentury Counterpoint. But this is in reality only a legitimate extension of what we see in Palestrina occasionally- 
Gloria. Missa Brevis.

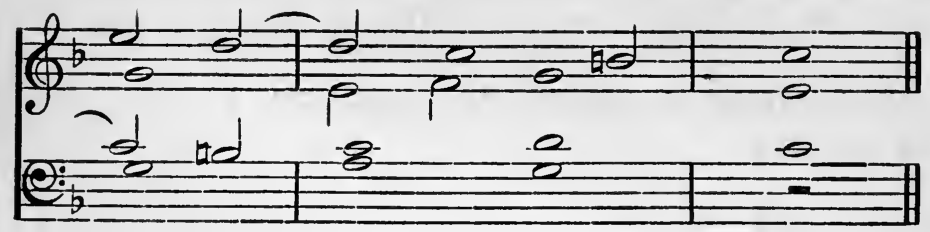

(b) The leap of a major sixth, if the value of the former of the notes forming the interval be at least a minim, may be tolerated.

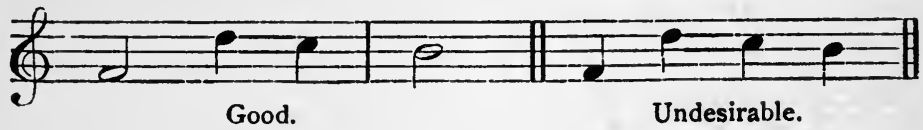

Five parts in First Species.

Third Mus. B. Oxon., Nov., Igo2.

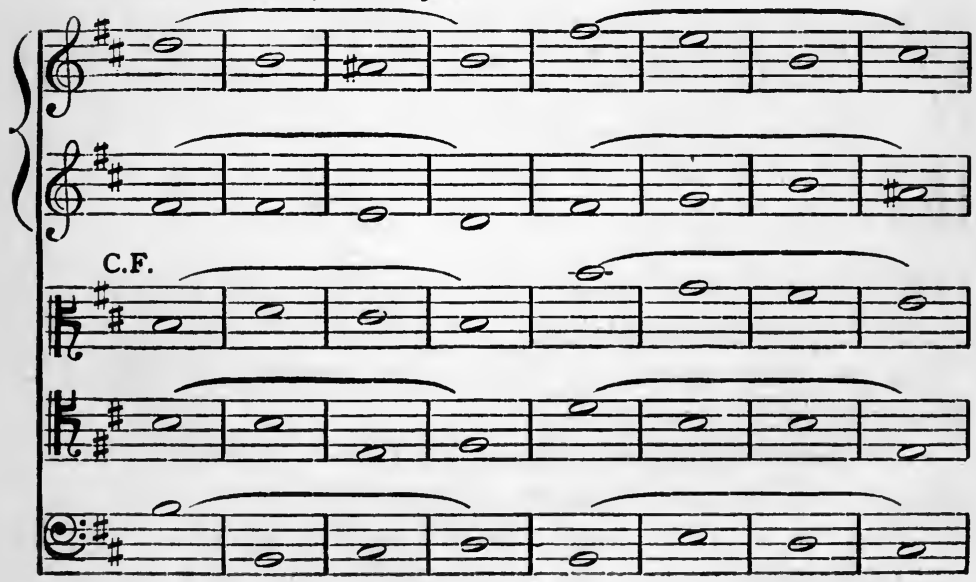




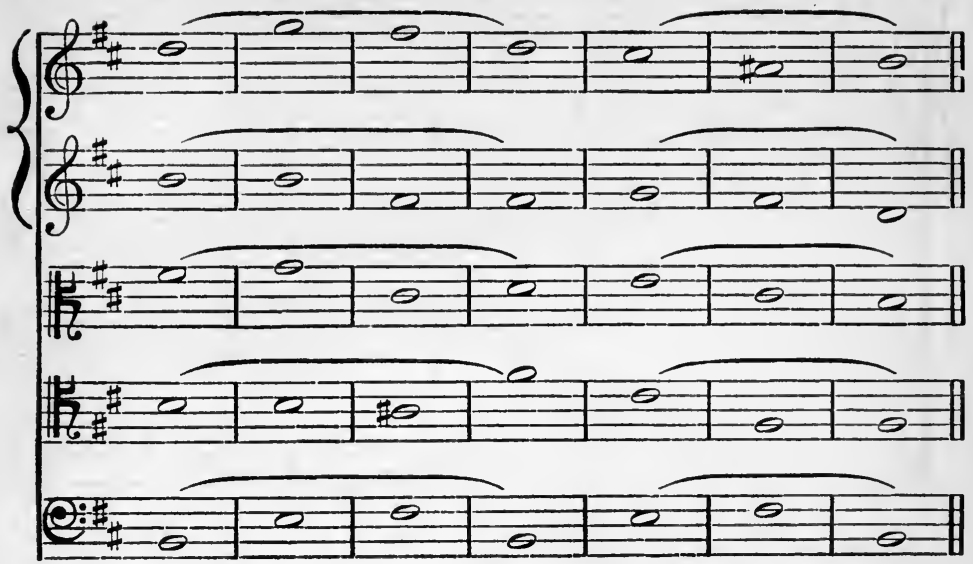

Third Mus. B. Oxon., May, r9o4.

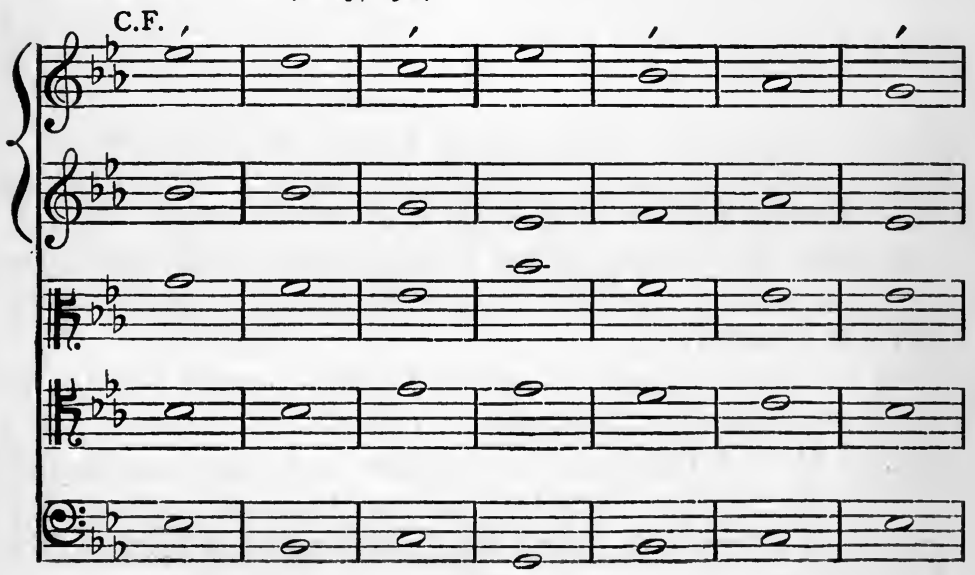


C.F.

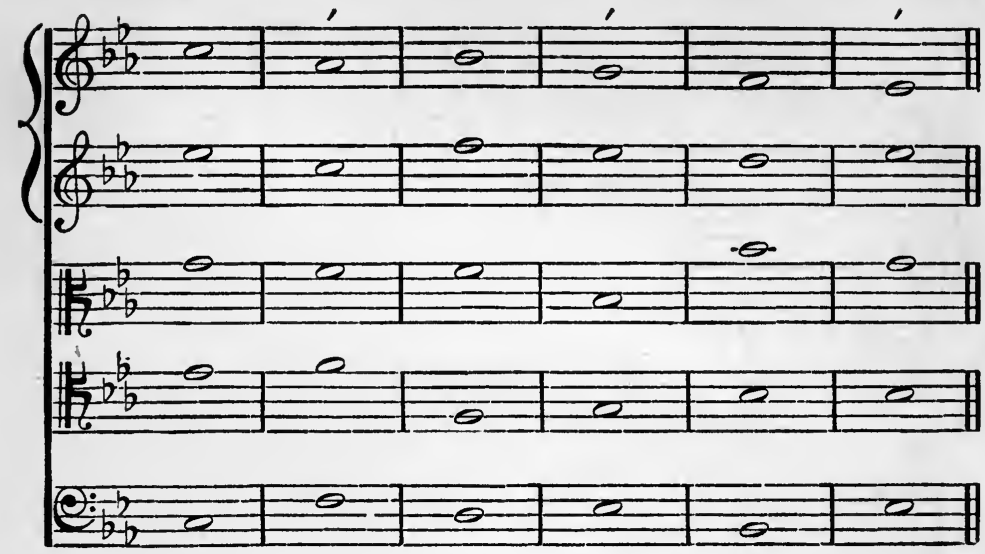

2. All the Species combined.
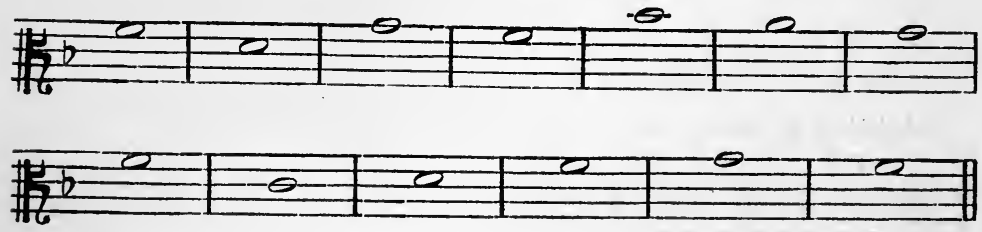

To the above C.F. add a First Treble in Fifth Species, a Second Treble in Fourth Species, a Tenor in Third Species, and a Bass in Second Species (Mus. D. Oxon., I903).

So long as the bass is not in semibreves, very interesting combinations are possible. It is obvious that a bass in semibreves is a limitation.

As a preliminary study it would be well to write the Fourth and Second Species first, not as being final, but as giving a general idea of the harmonic outline, and adding any little sections in the other parts that seem at the time worth noting. The chief difficulty of Combined Counterpoint is to make the Second Species adequately characteristic (i. e. conjunct). 
Preliminary Sketch.
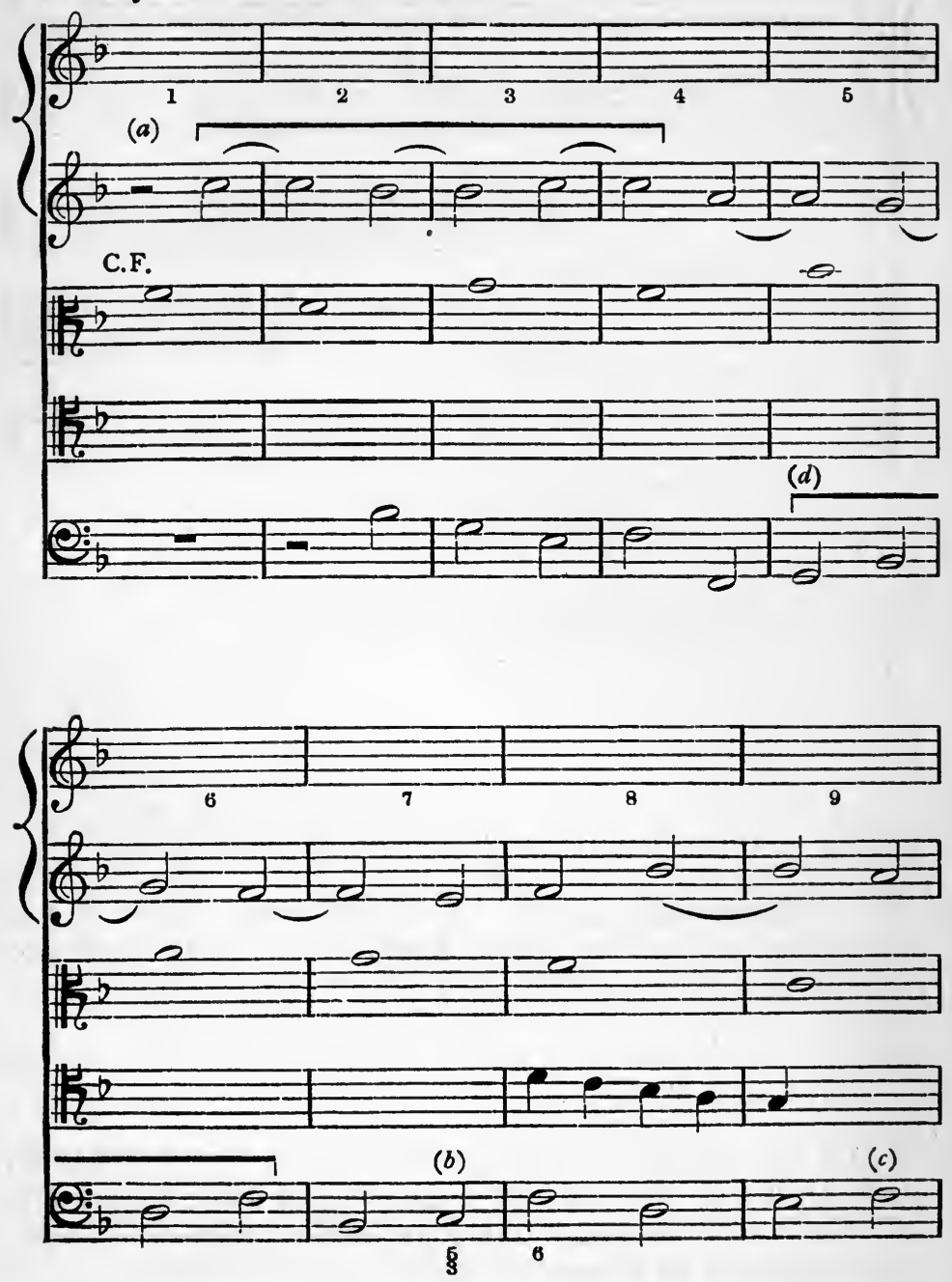
THE ART OF COUNTERPOINT

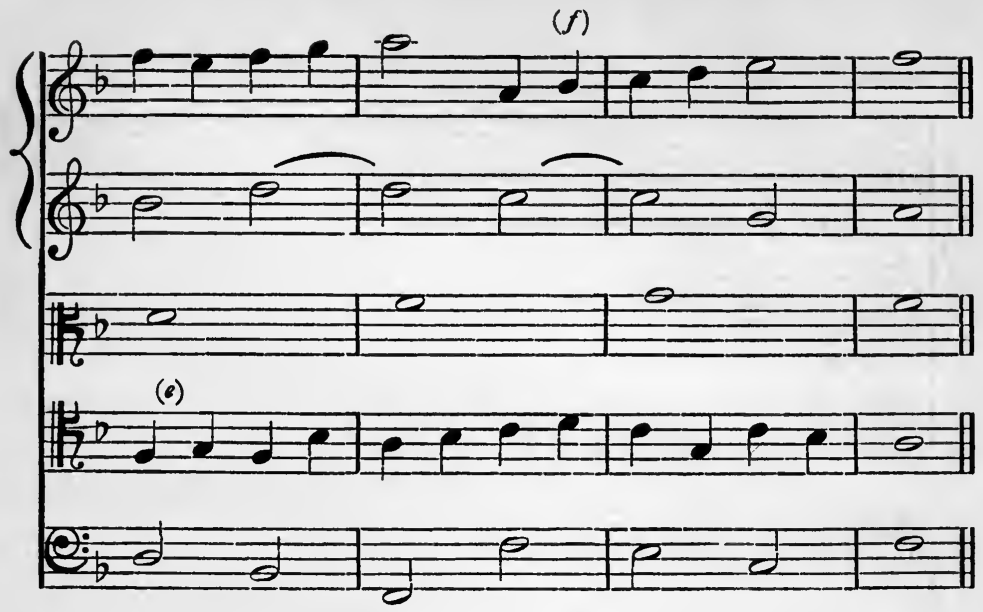

Now there are certain flaws in this :

(a) Is dull.

(b) The progression sounds forced and unnatural ; there is no objection to the use of $\mathrm{V}$. and $\mathrm{I}$. if the effect of finality is avoided.

(c) Is also open to a similar objection; the music seems to be making for the key of $\mathrm{B}$ flat Major but never reaches it.

(d) Compare page $35(5)$. In difficult combinations of this nature, such a progression is allowable, if the harmonic effect be entirely satisfactory.

(e) The combination might be better: EG, G, and Bb demand resolution on $\mathrm{I}$. or $\mathrm{VI}$.

$(f)$ The combination on the fourth crotchet causes confusion, for $\mathrm{F}, \mathrm{D}, \mathrm{F}$ and $\mathrm{Bb}$ clearly have the effect of being a passing ${ }_{4}^{6}$, and the $\mathrm{C}$ in the second treble jars against this. A simpler procedure must be adopted.

In fitting the parts, one or two more points deserve attention. Suppose bars 5 and 6 stood thus:- 


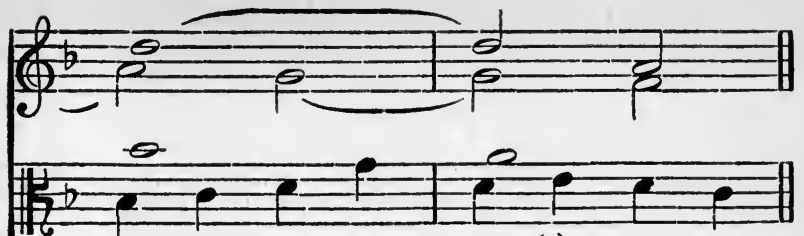

(a)

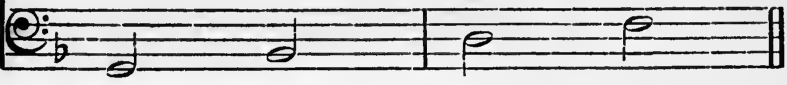

The $\mathrm{E}$ at $(a)$ sounds disturbing; it is no doubt due to the fact that the previous chord (being G minor and not followed by the dominant of $\mathrm{F}$ major) leaves in the mind an impression that $\mathrm{E}$. is foreign to the key. It is true no modulation has been made, but the context seems to encourage the idea that a change of tonal centre would be natural. Had the key been $\mathrm{G}$ minor, with the $F$ sharp, we should have written $E$ flat.

On the other hand, the following is entirely good--

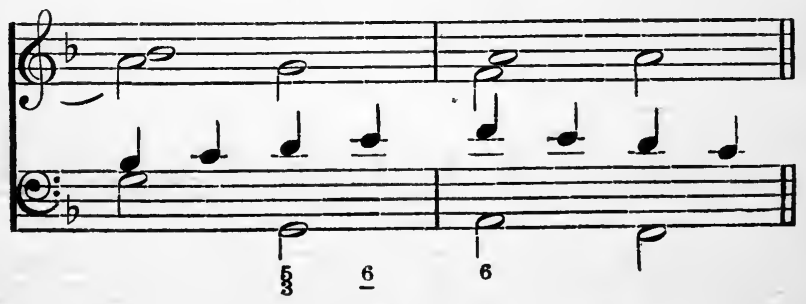

because on the fourth crotchet we get a passing chord clearly defining the tonality. This serves to illustrate the fact that bad or good effect depends largely on the context, not upon the mere mechanical application of this or that rule.

Care must be taken to give the Third Species adequate variety in compass; the parts may of course cross.

It is not necessary to keep the Fourth Species entirely unbroken, though it is nearly always possible to do so; in the present example it was broken at bars 7 and 8 to produce smoother harmony, for as the chord of D minor was used at bar 6 it had to be avoided at bar 8 . 
The following is the problem worked out: a few comments are necessary.

\section{Mus. D. Oxon., rgo3.}

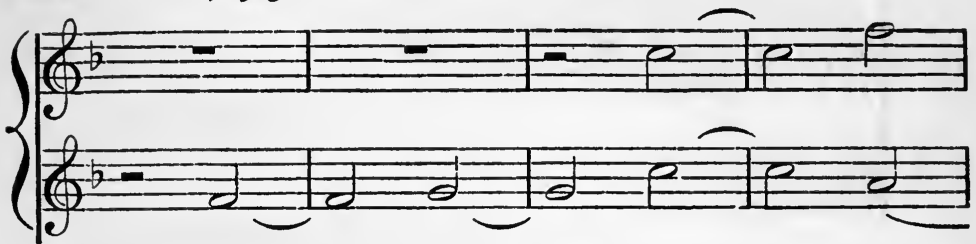

C.F.

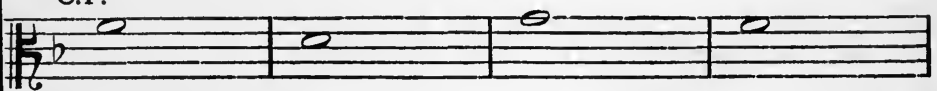

(a)

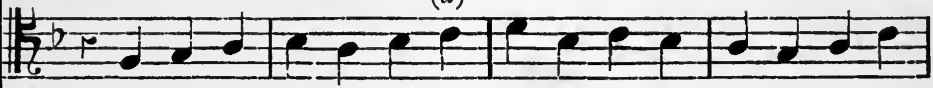
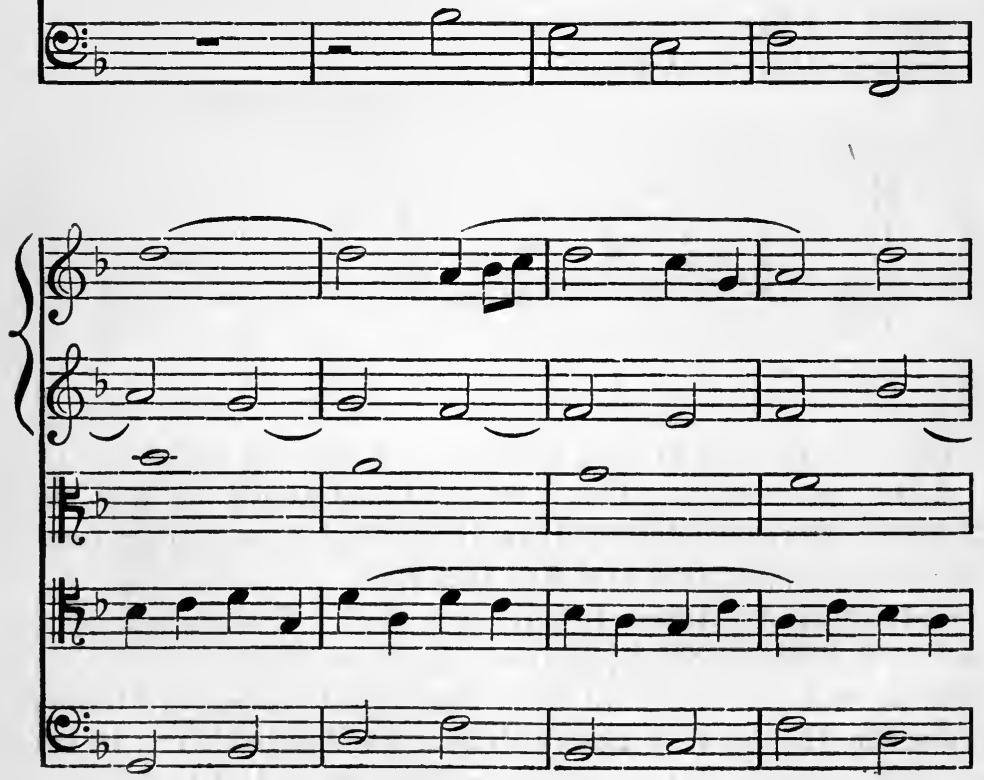


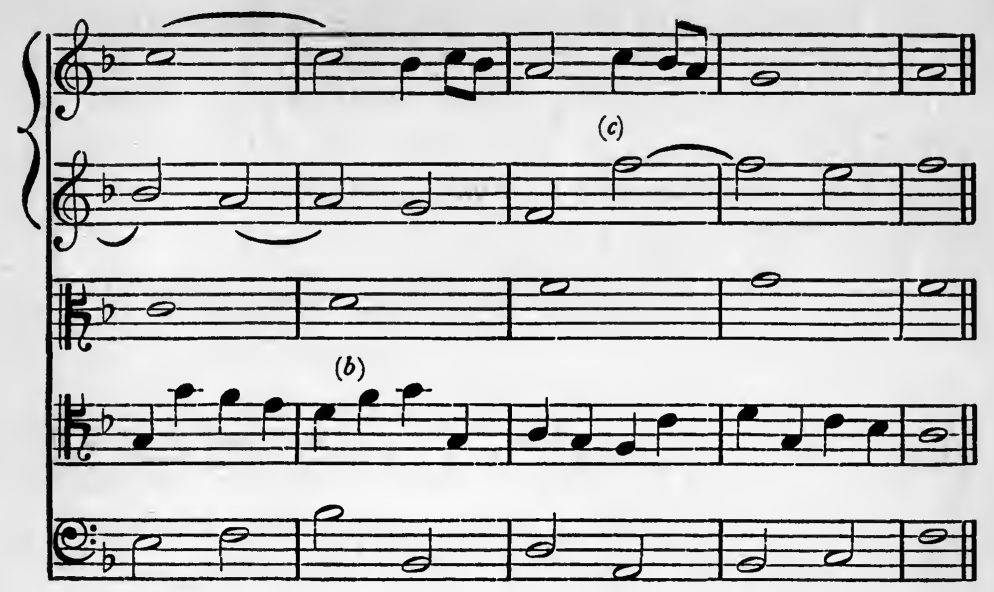

(a) The Alto and Tenor quit a second by similar motion; in difficult combinations this is allowable. The recommendation is only made to teach students to write as purely as possible.

(b) Here the F is concordant with the C.F. and the Bass : see p. I68. This is quite in accordance with Palestrina's procedure. (c) Variety is given to the Second Treble by crossing the parts, and the First Treble is also prevented from going up to A. It is well to learn to write within a moderate compass.

(d) The Fifth Species is simple in texture: any attempt at over elaboration will lead to harmonic confusion.

It is of course impossible to give examples of all combinations; the following, set in November, 1905, is a difficult problem.

To the following C.F. (see below) add a First Treble in Third Species, a Second Treble in First Species, an Alto in Second, and a Bass in Fourth Species. Space will not allow a full discussion of this problem. The student should consult the chapter on Combined Counterpoint in three parts, and study the various combinations.

The insertion of an additional part in First Species makes the working difficult: for if two harmonies are to be used in any bar, the semibreve must be common to both; at $(a)$ the First Species has been broken, not of necessity, but because the use 
of two harmonies was felt to be desirable, in order to keep up the Third Species figure. The diminished fifth is twice used $(b)$.

The Bass starts above the Tenor; if it had started below, we should have arrived at an ugly suspension in bar 3 , or else it would have been necessary to break the Species.

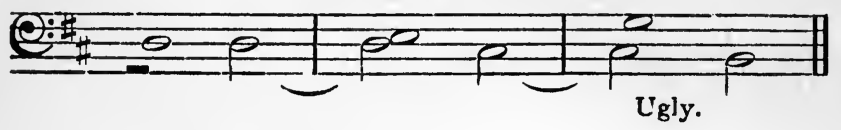

Third Mus. B. Oxon., Nov., 1905.
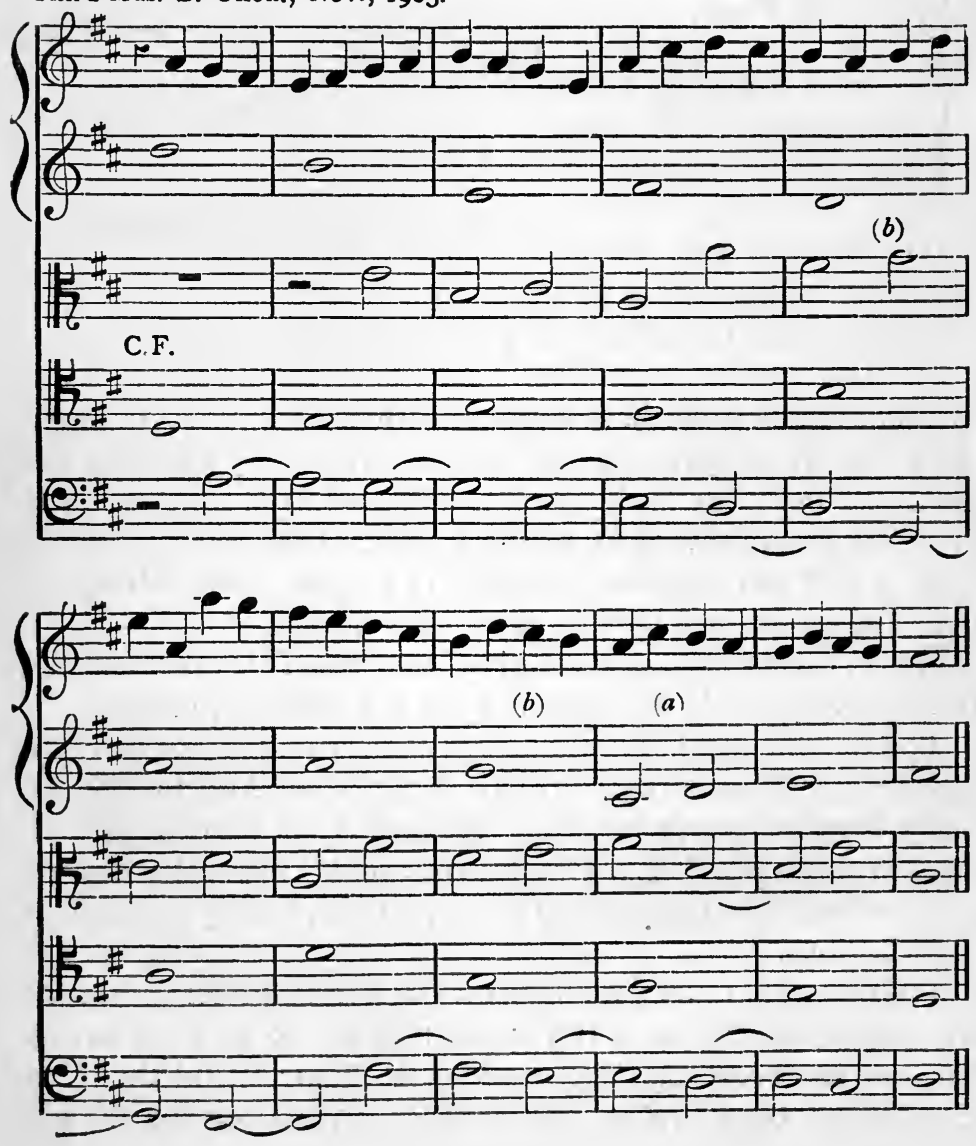
Four of the parts in Florid Counterpoint :-

Third Mus. B. Oxon., Nov., rgo3.
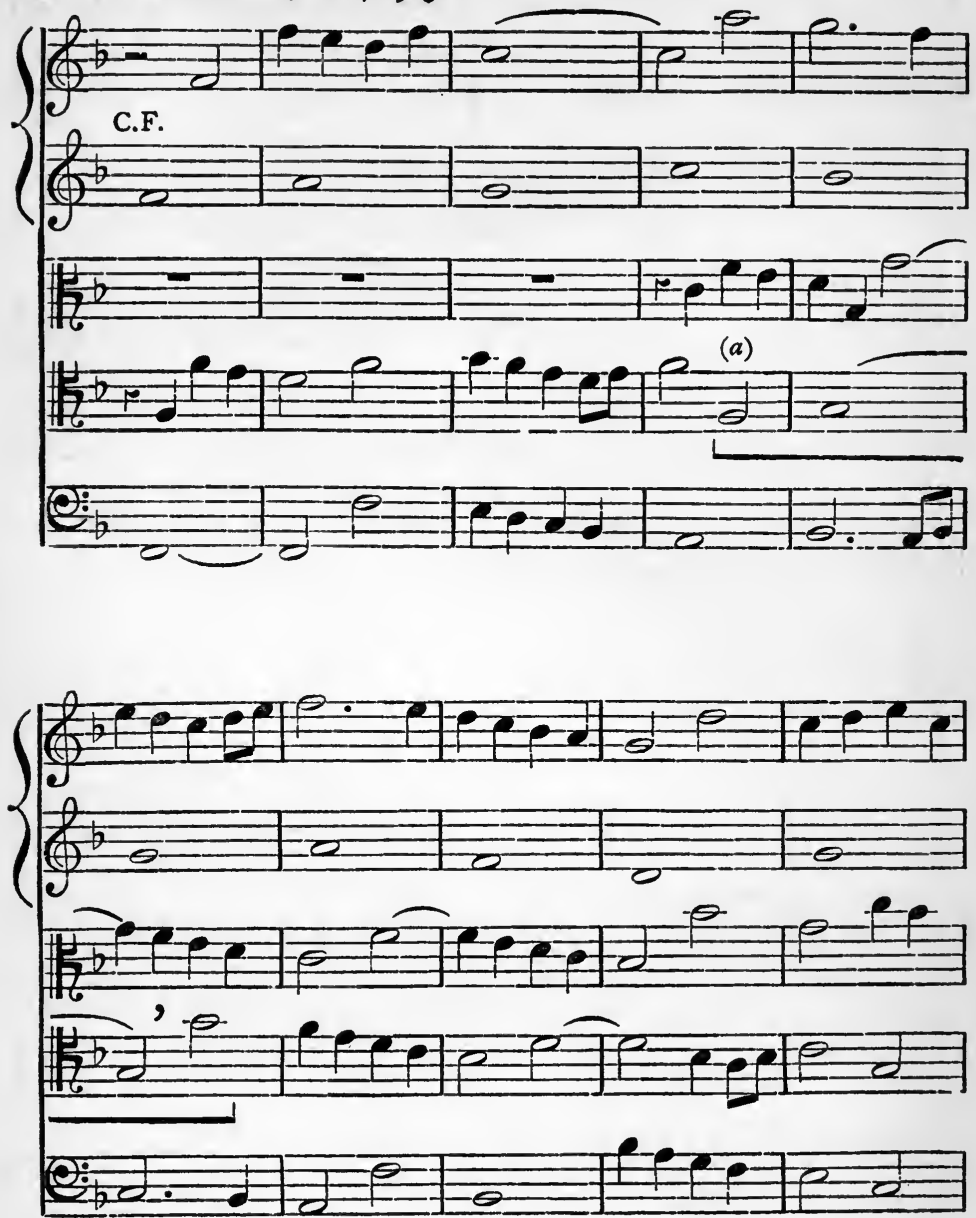

(a) See p. 16 (4). 


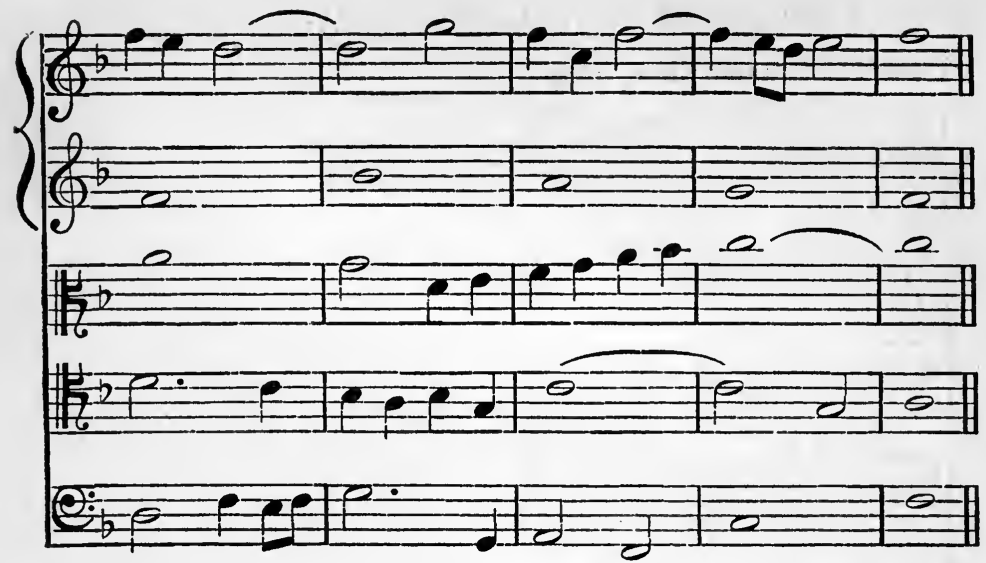

No comments are necessary: care has been taken that the harmonic seams are satisfactory. 


\section{CHAPTER XV}

\section{COUNTERPOINT IN SIX, SEVEN, AND EIGHT PARTS}

I. THE following are permissible relaxations of the rules :-

(a) In six parts, consecutive octaves by contrary motion may be used.

In more than six parts, consecutive octaves and fifths by contrary motion may be used.

Palestrina used consecutive fifths by contrary motion, even in four parts-

\section{Alma Redemptoris.}

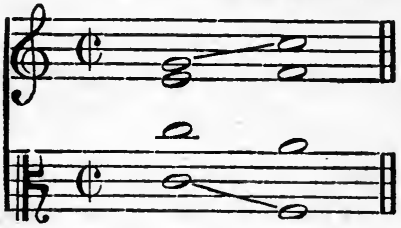

and the writers of the period considered them far less objectionable than consecutive octaves, which rarely occur in less than six parts. Moderns, however, seem to have reversed this decision.

(b) Palestrina's procedure as seen in $8(e)$ Chapter VIII in reference to consecutives produced by different parts may be followed.

(c) The doubling of the leading note at the unison, which merely reinforces the sound, is open to no objection.

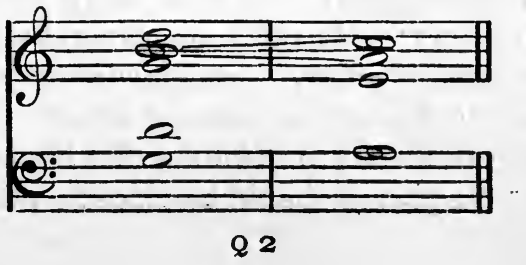


In such progressions as the following, the doubling of the leading note at another pitch is quite good. The reason is that the mind does not accept it here as demanding its cadential treatment-

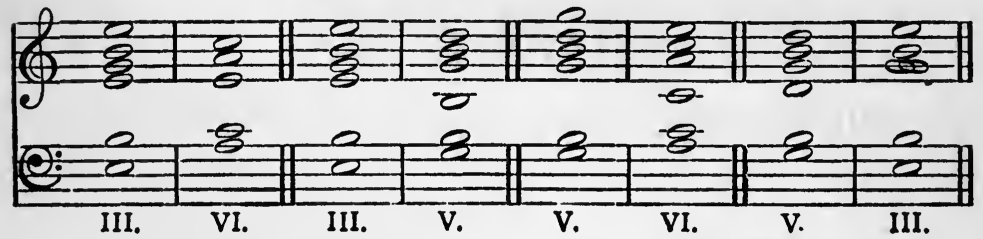

If the key-signature of these examples were $G$ major, no theorist would object to them. And this points to the reason that their effect is perfectly good. In such cases the leading note is accompanied in a way different from that which was the origin of this well-known rule, e. g.-

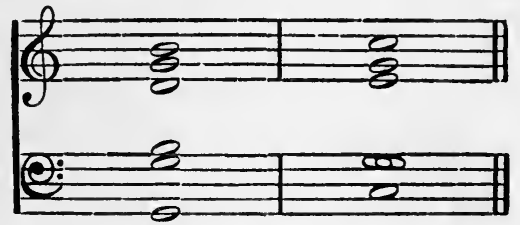

It is really only in such a case that the doubling of the leading note is bad: and even such a case as the above is often adversely criticized more from prejudice than from judgement of effect. For though in four parts

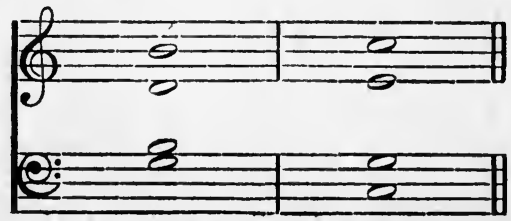

such a progression is unjustifiable, in six or eight parts, the effect is often quite good; and it is really the effect and not the rule that matters; only rules are generally supposed to be based on judgement of effect. Academic pedantry is sometimes allowed to stand in the way of common sense.

2. It is hardly necessary to point out that if a part should rest, the last note of the phrase should be placed on the first or third 
beat of the bar ; and that a part should re-enter with some point of imitation.

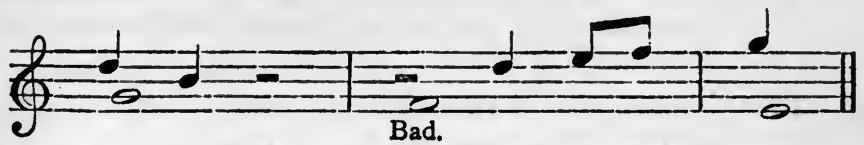

A part should never rest for the purpose of avoiding a difficulty, but only to bring into prominence a 'point' or to afford artistic relief.

After a rest, no two parts should enter together. Neither should one part stop before another enters.

The phrases should always dovetail-

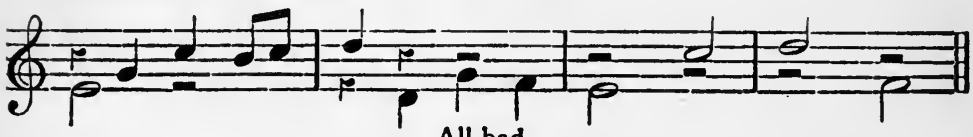

All bad.

Unless careful attention be paid to this, a piece of six-part work becomes merely five-part Counterpoint, with one part distributed between two voices.

3. In cases of difficulty, the unison may be approached by similar motion.

4. The leap of a minor sixth may occasionally occur in crotchets.

5. In florid work, it is better that some of the parts should begin imitatively.

6. The simpler the style, the better the Counterpoint. There is no absolute rule that no two successive bars in any part should be in the same rhythm. The maintenance of one Species unvaried for more than two bars might be open to objection as florid work. But the First Species may be thus used in approaching the Cadence; and it may be used with discretion for two consecutive bars in the course of a problem. The overelaboration of the score leads to three disastrous evils :-

(a) confusion of harmony;

(b) obscurity of harmony ;

(c) congestion of harmony.

The student must guard against these, and he must be particularly careful that his harmonic seams are good. He must form 
the habit of keeping in mind every part, and he may find it useful to figure the following examples according to modern principles.

7. Counterpoint in eight parts may be written for two Choirs of four parts each (S.A.T.B.). In the opening passages the Choirs should be treated in an antiphonal manner, but one should not stop before the other enters, that is, they should dovetail. When towards the close they sing together, the harmony given to each Choir should be complete and satisfactory in itself. In such cases the basses of each Choir often move as follows :-

Palestrina, Stabat Mater.

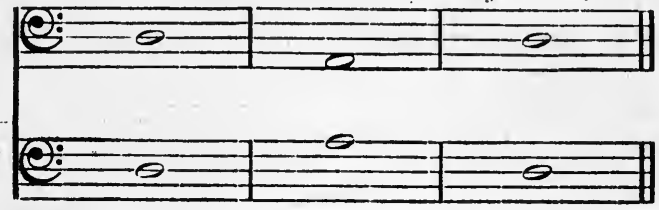

The student is recommended to read Palestrina's Stabat Mater as an example of Counterpoint in eight parts for a double Choir.

As examples of ordinary six and eight-part work, the following may be studied :-Palestrina's Lectio III, Sabbati Sancti (Tom. XXXI), Missa Papae Marcelli.

Five-part work :- $O$ admirabile commercium.

Four-part work:-Aeterna Christi Munera; Missa Brevis; Iste Confessor.

Also the following motetts:-Lauda Sion; Ego sum panis; Sicut cervus; Sitivit anima.

Modern examples of eight-part work should be studied. The following are recommended:-

(a) Eight-part Chorus : Parry, Blest pair of Sirens; Lloyd, (a) Song of Judgement, (b) The Righteous live; Harwood, Inclina Domine.

(b) Double Chorus :-Bach, (a) Be not afraid, (b) Sing ye to the Lord, (c) I wrestle and pray; Wesley, In exitu Israel; Lloyd, Art thou weary?; Mendelssohn, (a) When Israel came out of Egypt, (b) Why rage fiercely the heathen.

- 8. A few general remarks on Combined Counterpoint may be useful. 
(a) It is a sine qua non that the result should be a musical piece of work.

It is insufficient that the work should be merely technically correct; it must show in addition artistic perception in the contour of the parts, and in the choice of harmony. The rigid use of one chord in a bar will produce very crude results ; moreover, it is a serious limitation.

(b) The first thing to do is to see that the harmonic outline is perfectly smooth and natural ; the next thing is to ensure that the Second Species is tolerably conjunct and flowing.

(c) If the First Species be in the lowest part, it would be well to look out for opportunities of using in the Second Species-

(I) 5.6 .6 .5 .

(2) 8.7 .
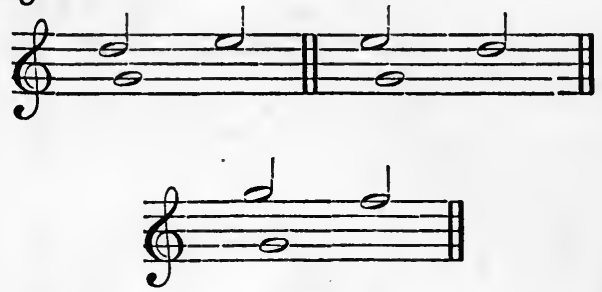

(d) If any of the other Species are in the bass, resource is not so limited. It would be well to turn back to the preliminary studies in three parts and examine the various possibilities, not only in change of harmony, but also in the use of prepared discords, noting specially those cases in which the Second Species is conjunct; for if the Second Species be good, the Third Species can easily be made good also, but the converse is not true.

The student should form the habit of comparing every possible combination of two parts, e.g. in a score of, say, six parts Compare Bass with each part above it.

$\begin{array}{lll}" \text { Second Tenor " } & \text { " } \\ " \text { Airst Tenor } & " \\ " \text { Alto } & \text { Second Treble with First Treble. }\end{array}$

This will enable him to be sure that his score is free from grammatical errors. 
(e) All the parts should seem to flow smoothly and spontaneously, and the Counterpoint should form irreproachable harmony. Summa ars celare artem.

Mus. D. Oxon., rgo3.
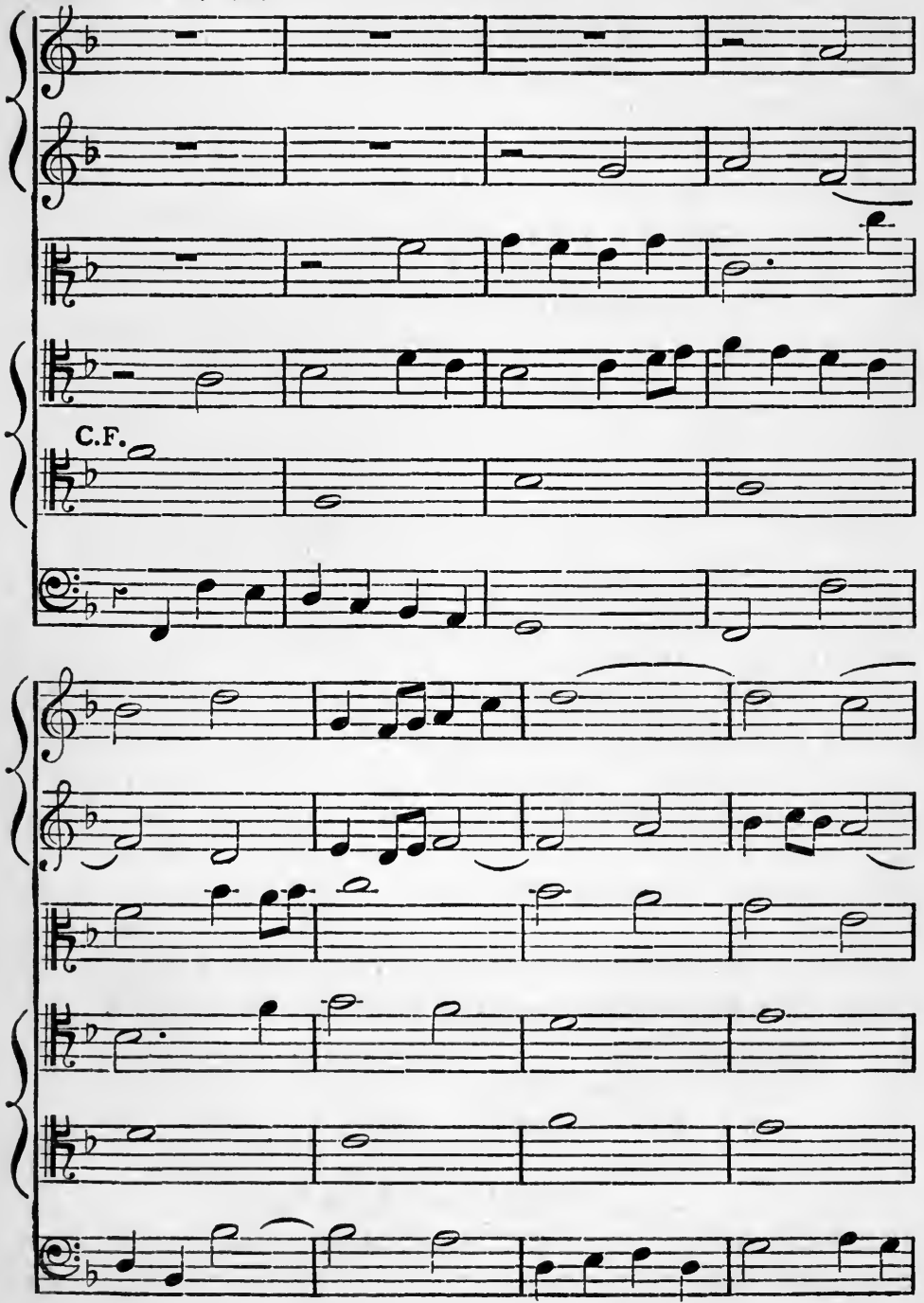


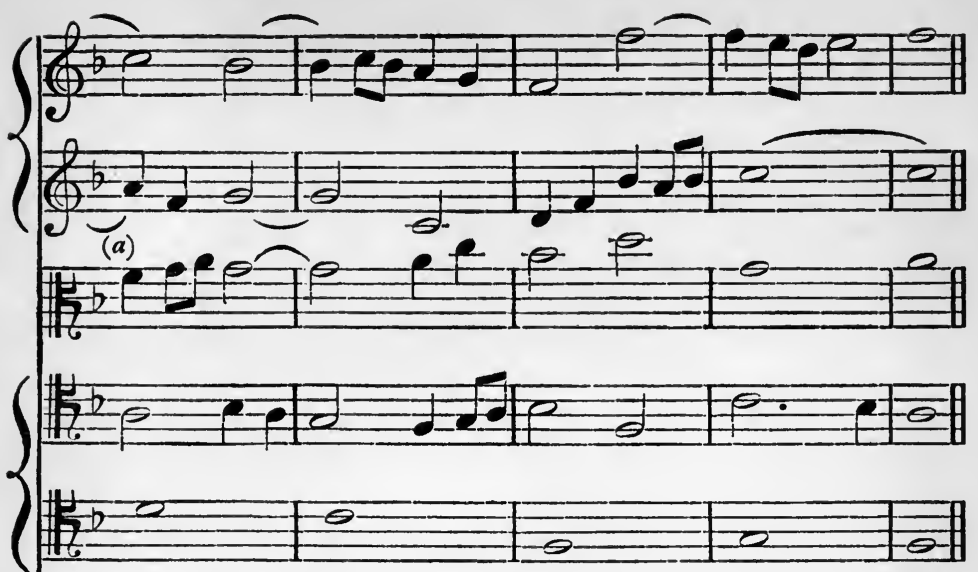

(a)
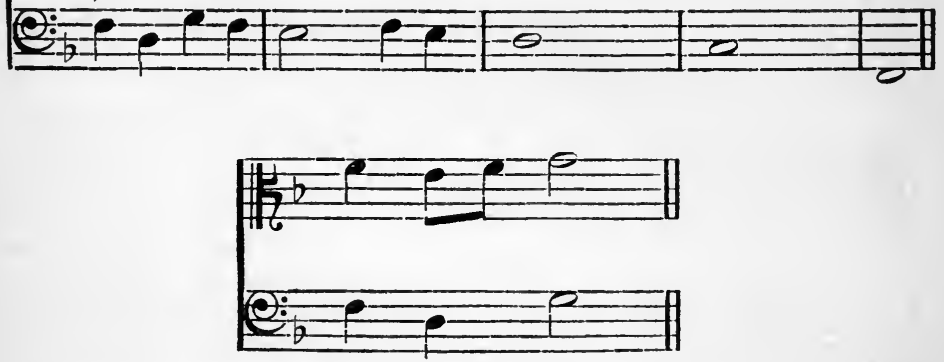

(a) is open to some objection: the alto is in outline

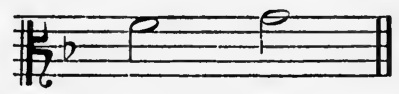

that is, a mere auxiliary note is hardly sufficient to prevent the effect of octaves in such short notes. But

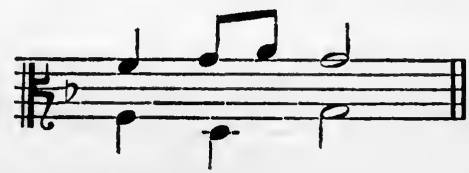

is quite free from objection. 


\section{4 \\ THE AR'T OF COUNTERPOINT}

Six parts. All the Species combinedC.F.

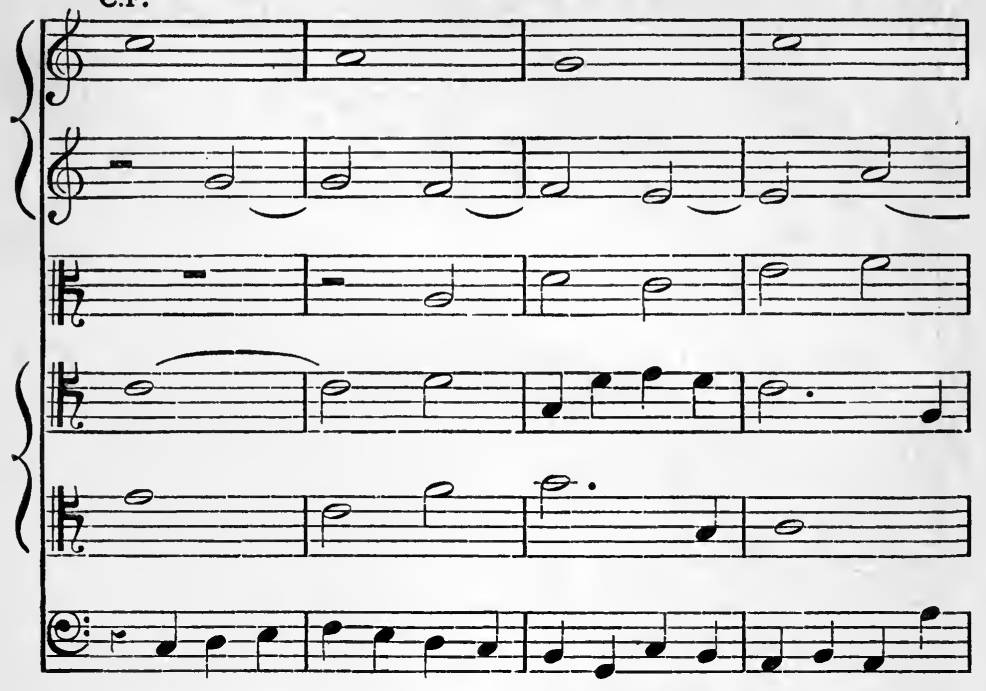

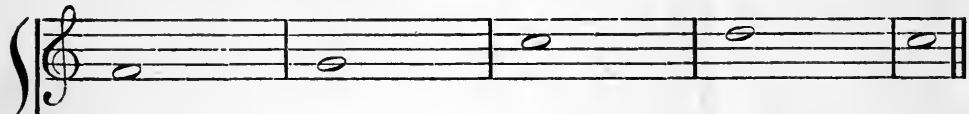

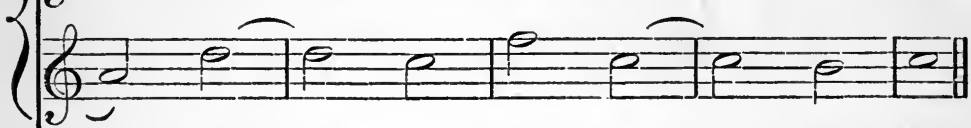

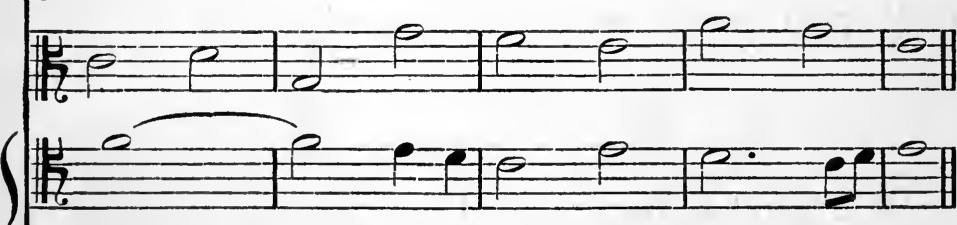
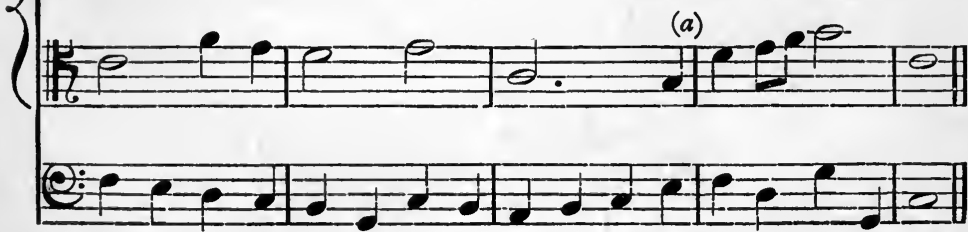

(a) A change of harmony on the fourth crotchet is occasionally allowable. 
Seven-part Florid-

Mus. D. Oxon., Igor.

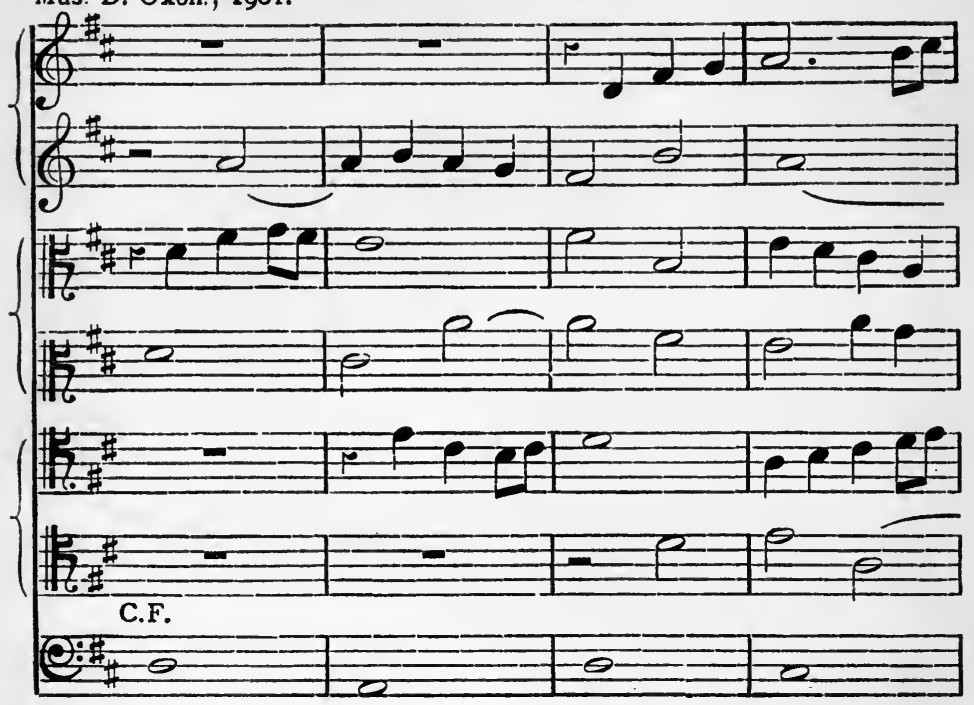

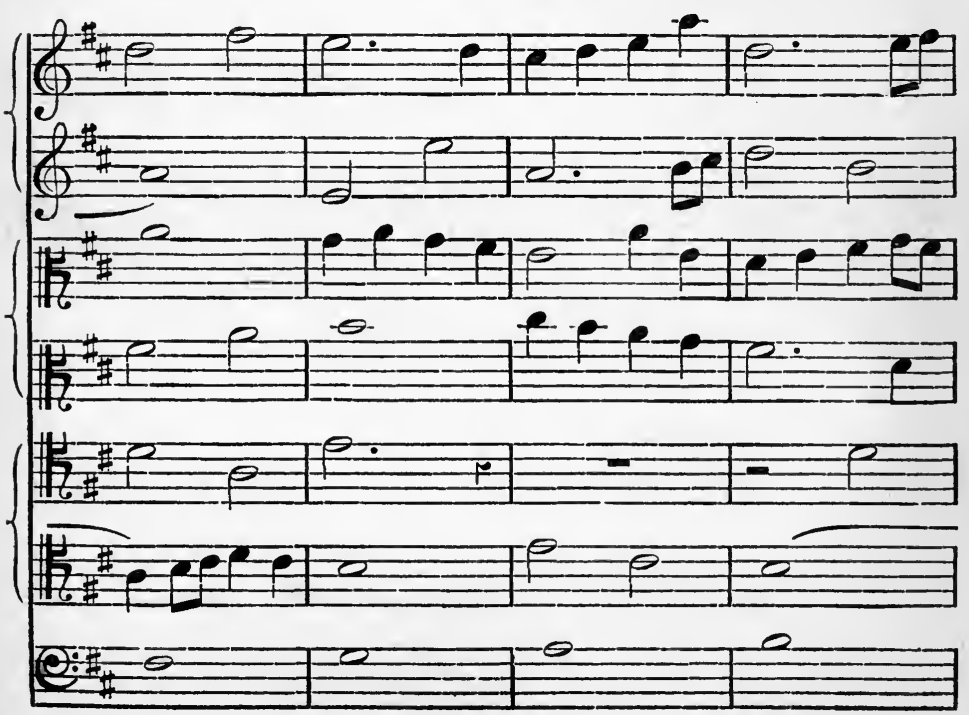




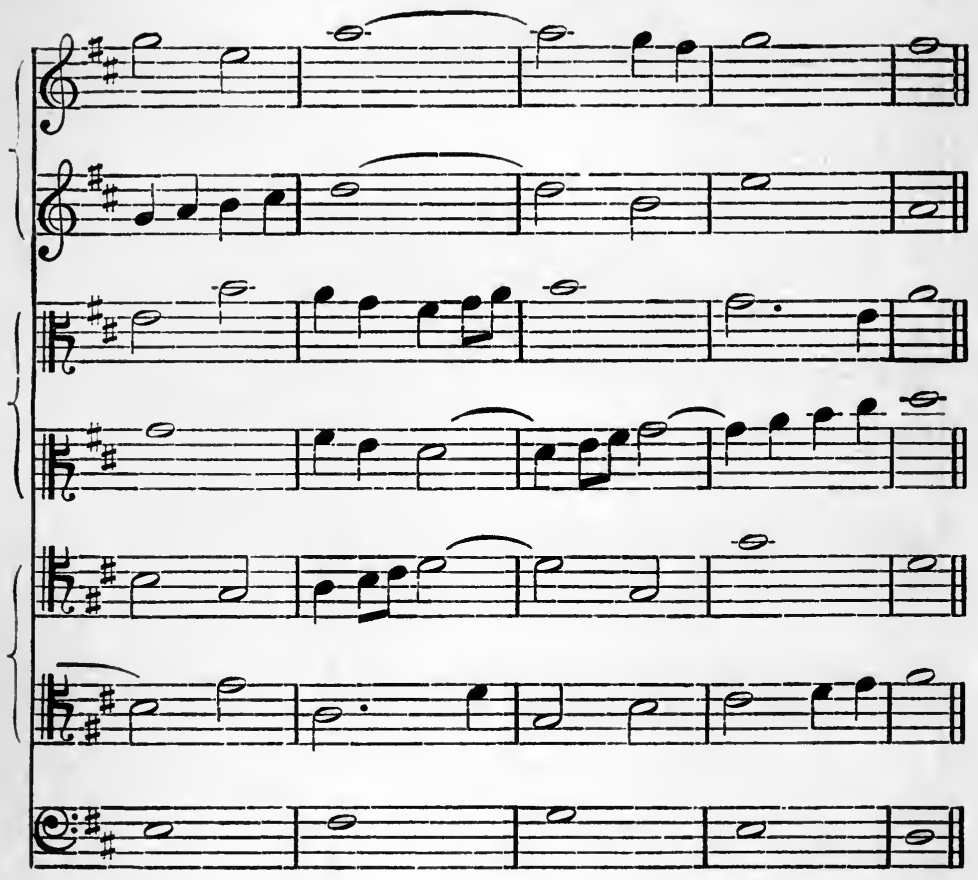


Eight parts in the First Species-

Mus. D. Oxon., I904.

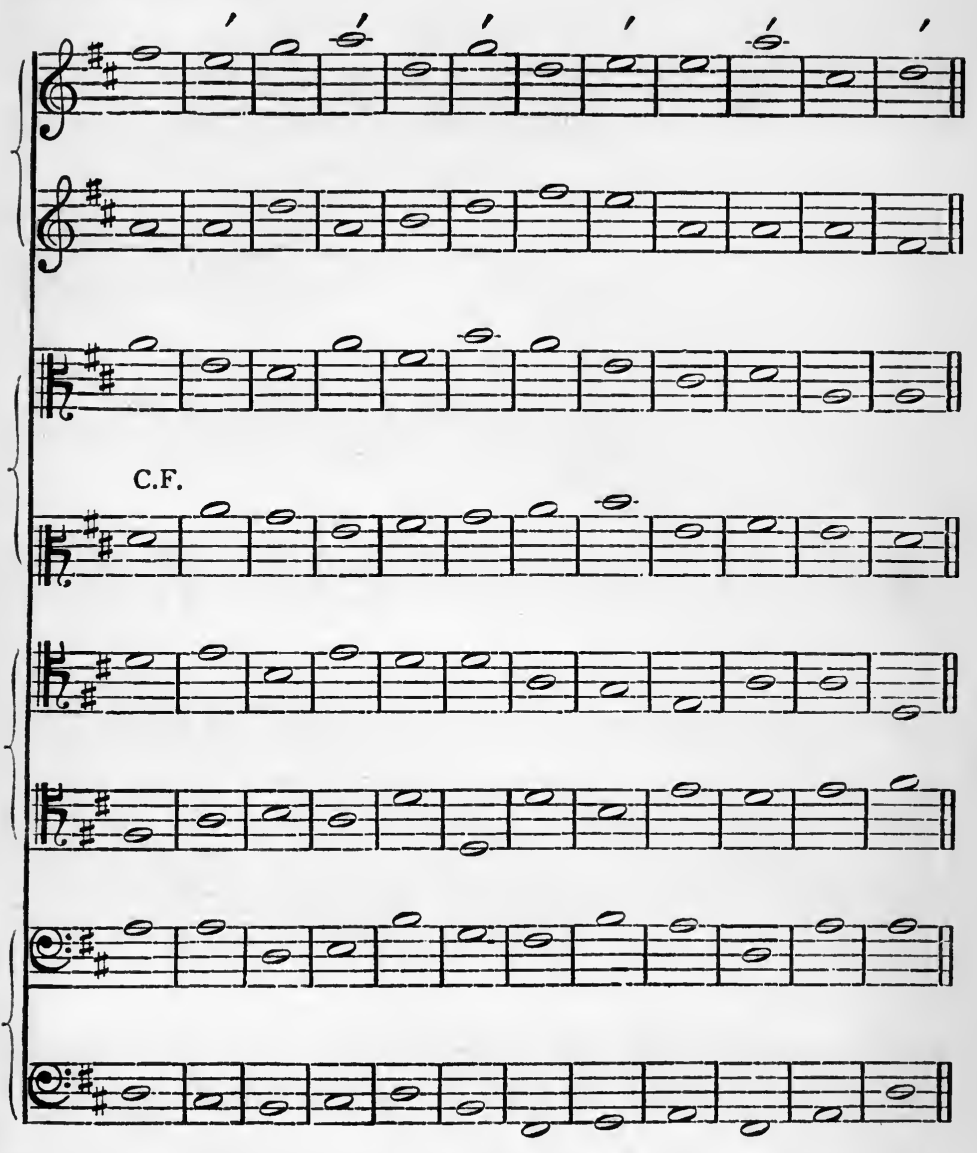


Eight-part Florid (alternate bar lines are omitted)—

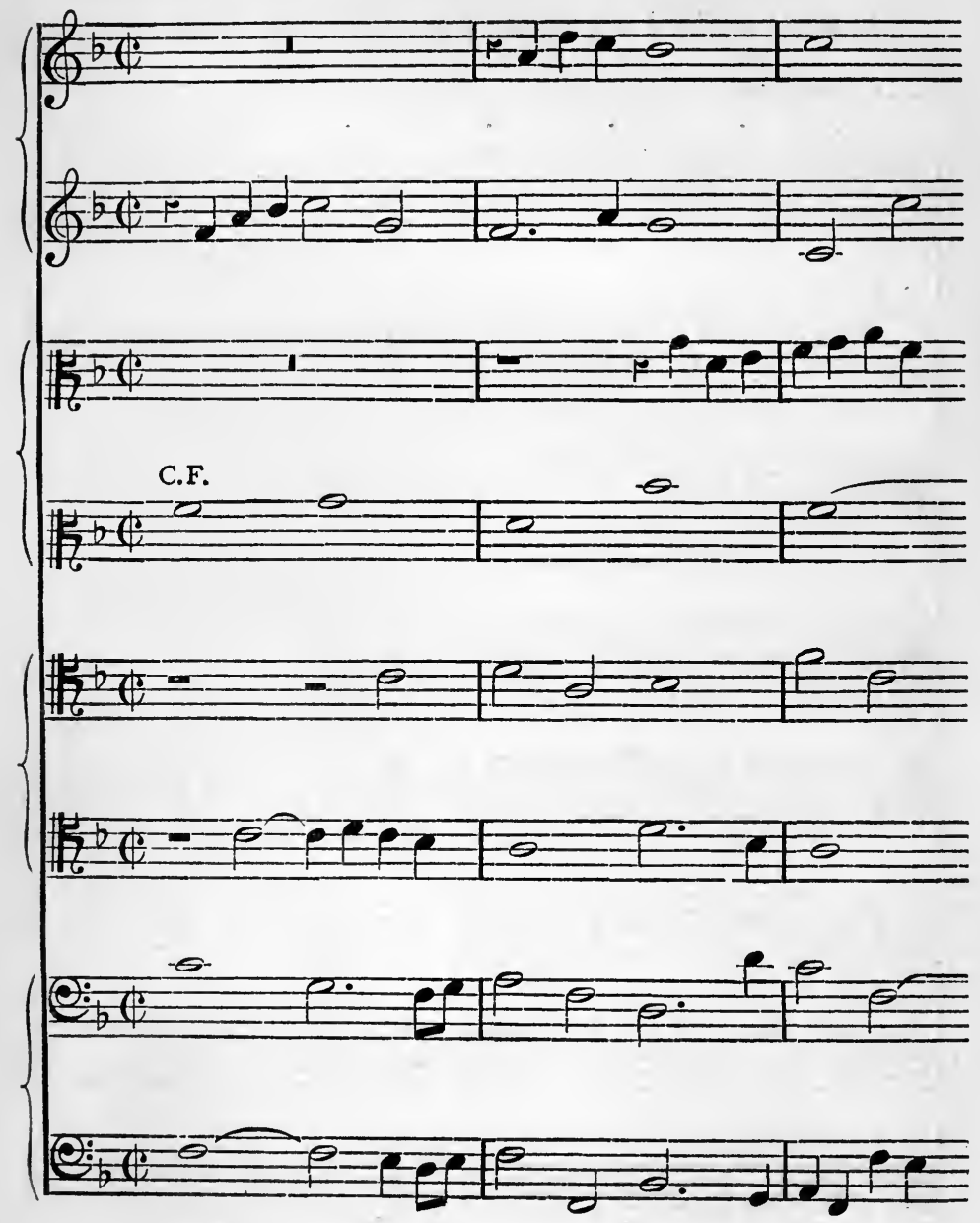

(a) In writing in alla cappella time a semibreve may be occasionally tied to a breve. This will be very rare in Scholastic Counterpoint, for the breve is of course equivalent to two tied 


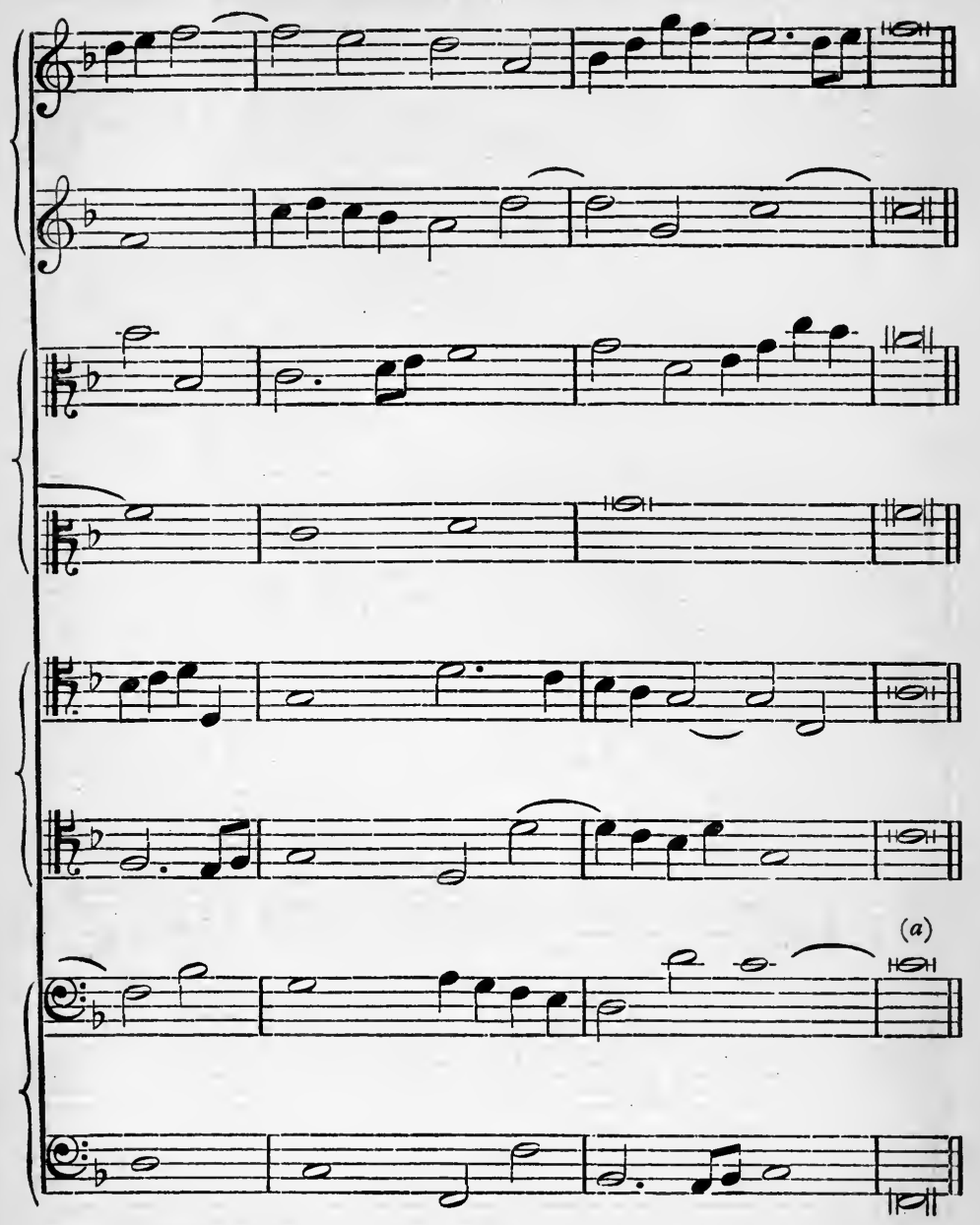

semibreves. But it will be seen that the case in question is in reality only o 0 , the final bar being of necessity double the length of an ordinary scholastic bar. 
Mus. D. Oxon., Nov., 1906. 'Introduce each part by some variation of the same figure. All the parts should have entered by the sixth bar.'

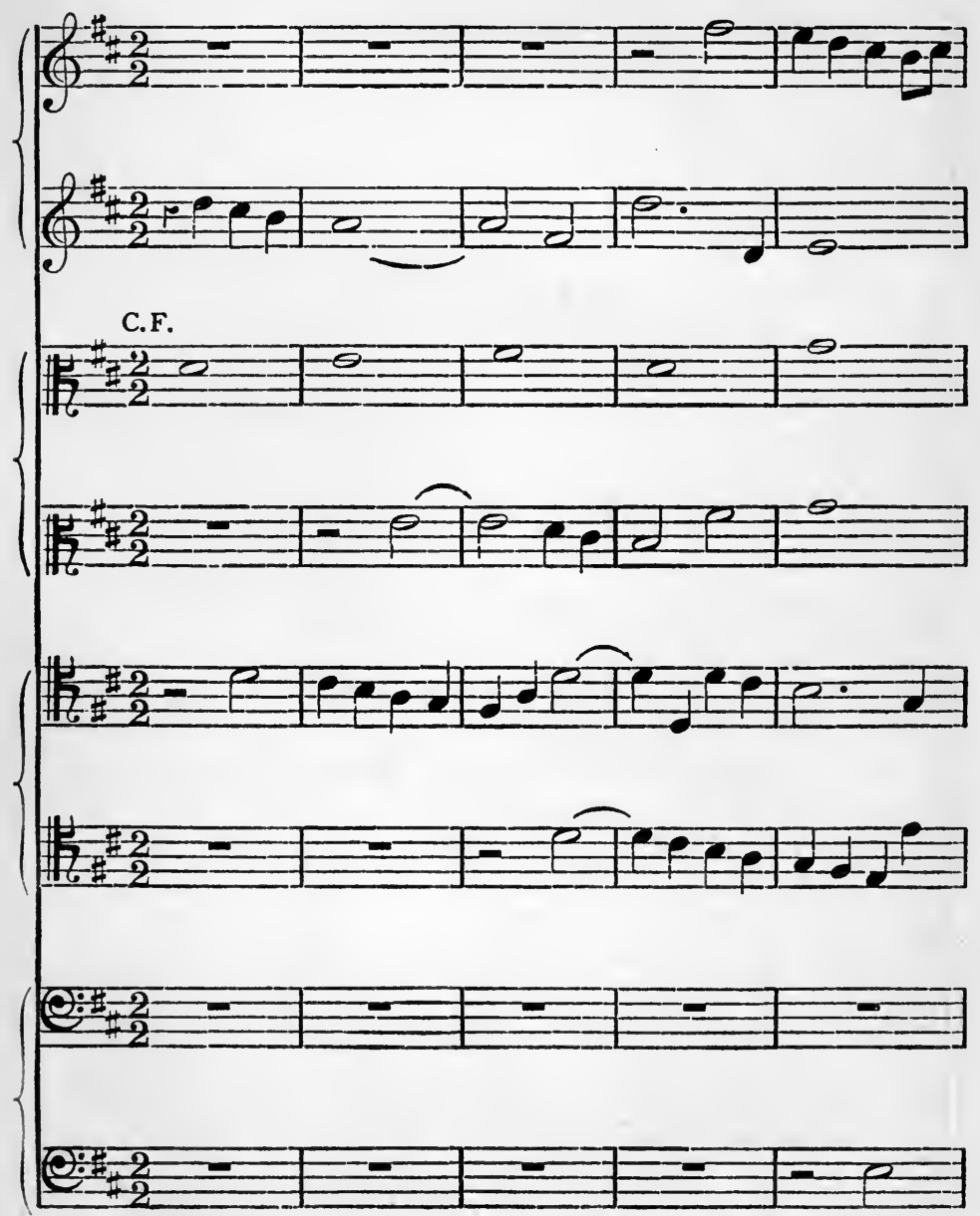




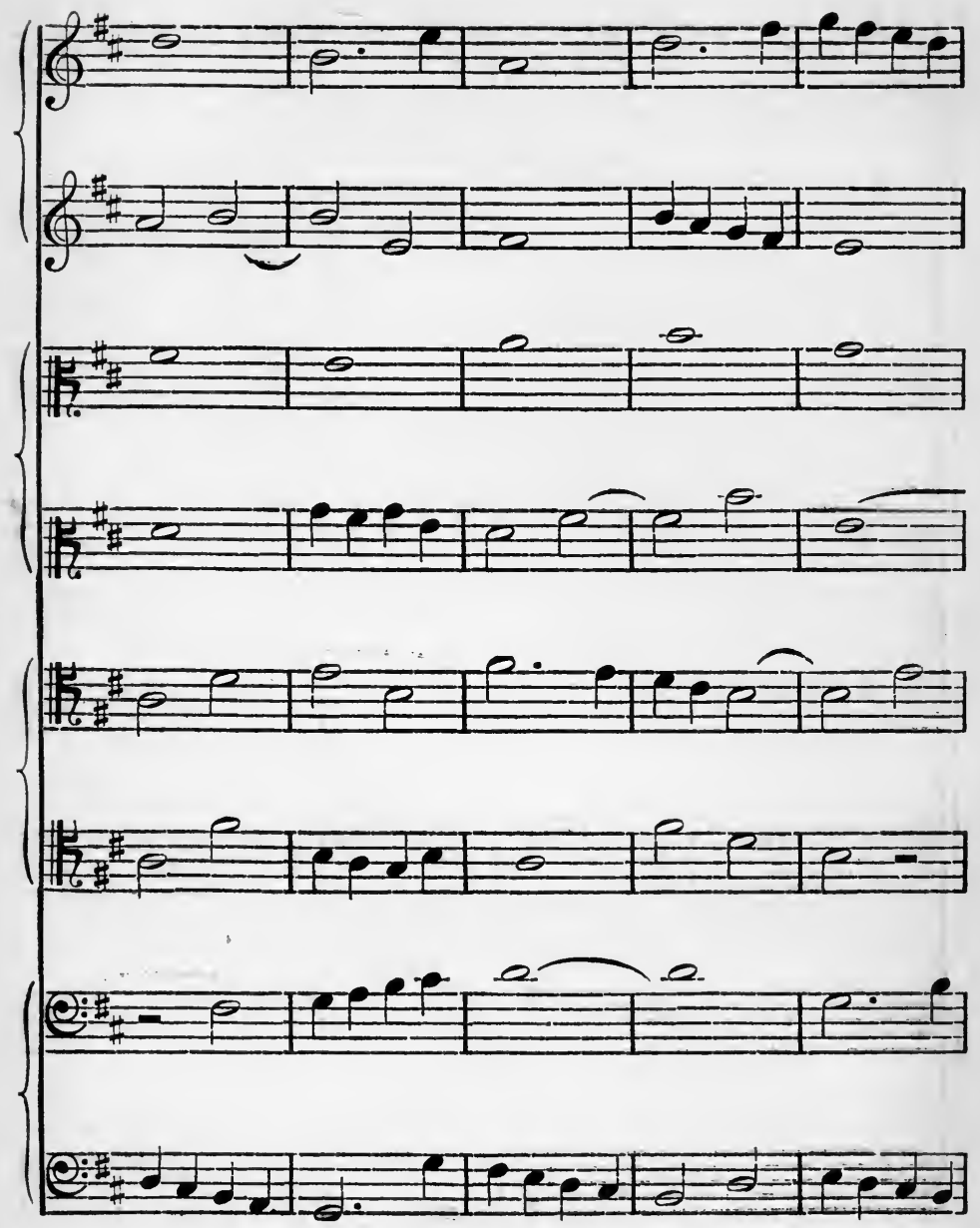




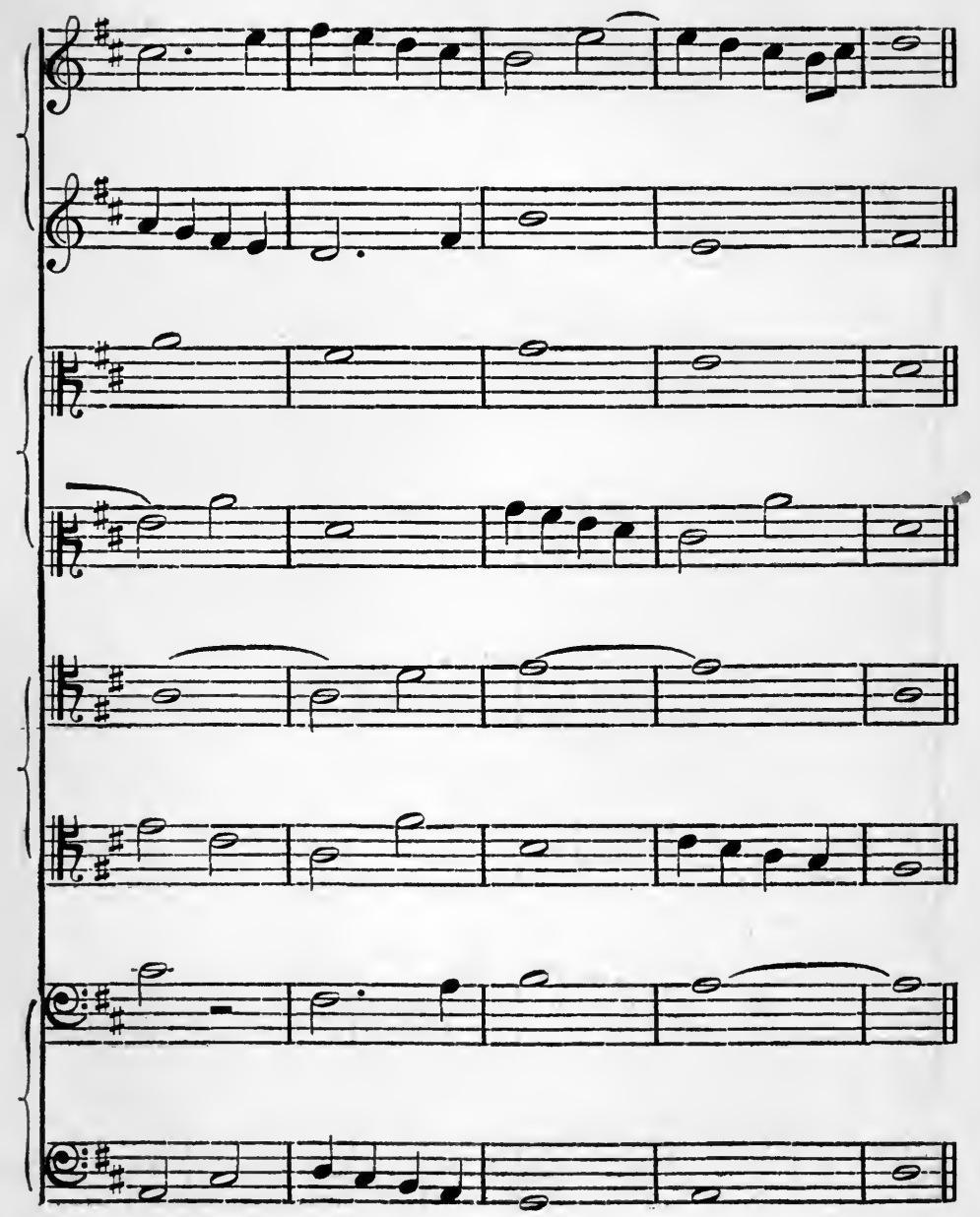


Mus. D. Oxon., 1899. 'Commence the following in three parts, adding one part every two bars until they amount to eight, including the C.F. Begin with First Species, and introduce the others successively, combining them as you please, but ending with Fifth Species in all parts.'

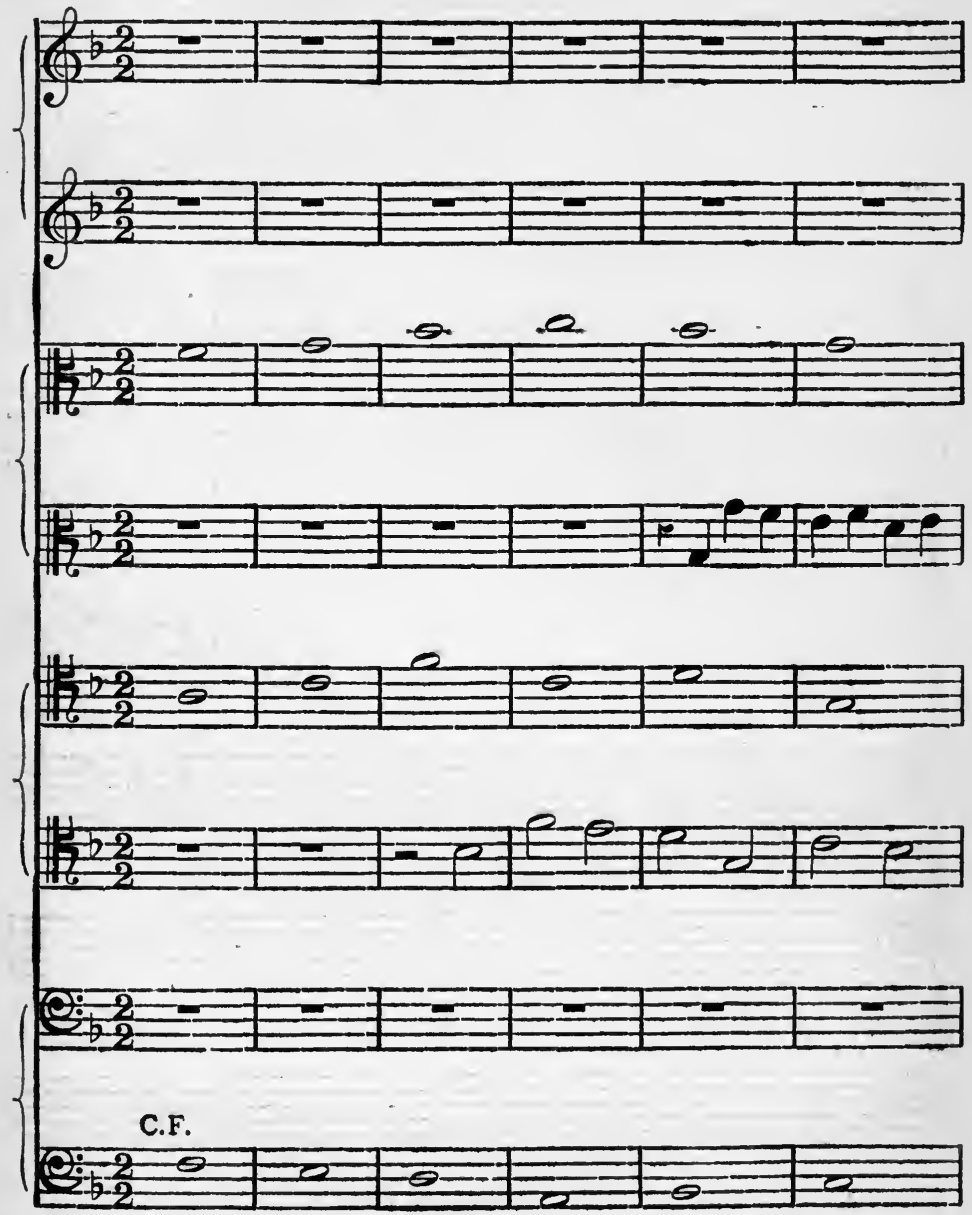




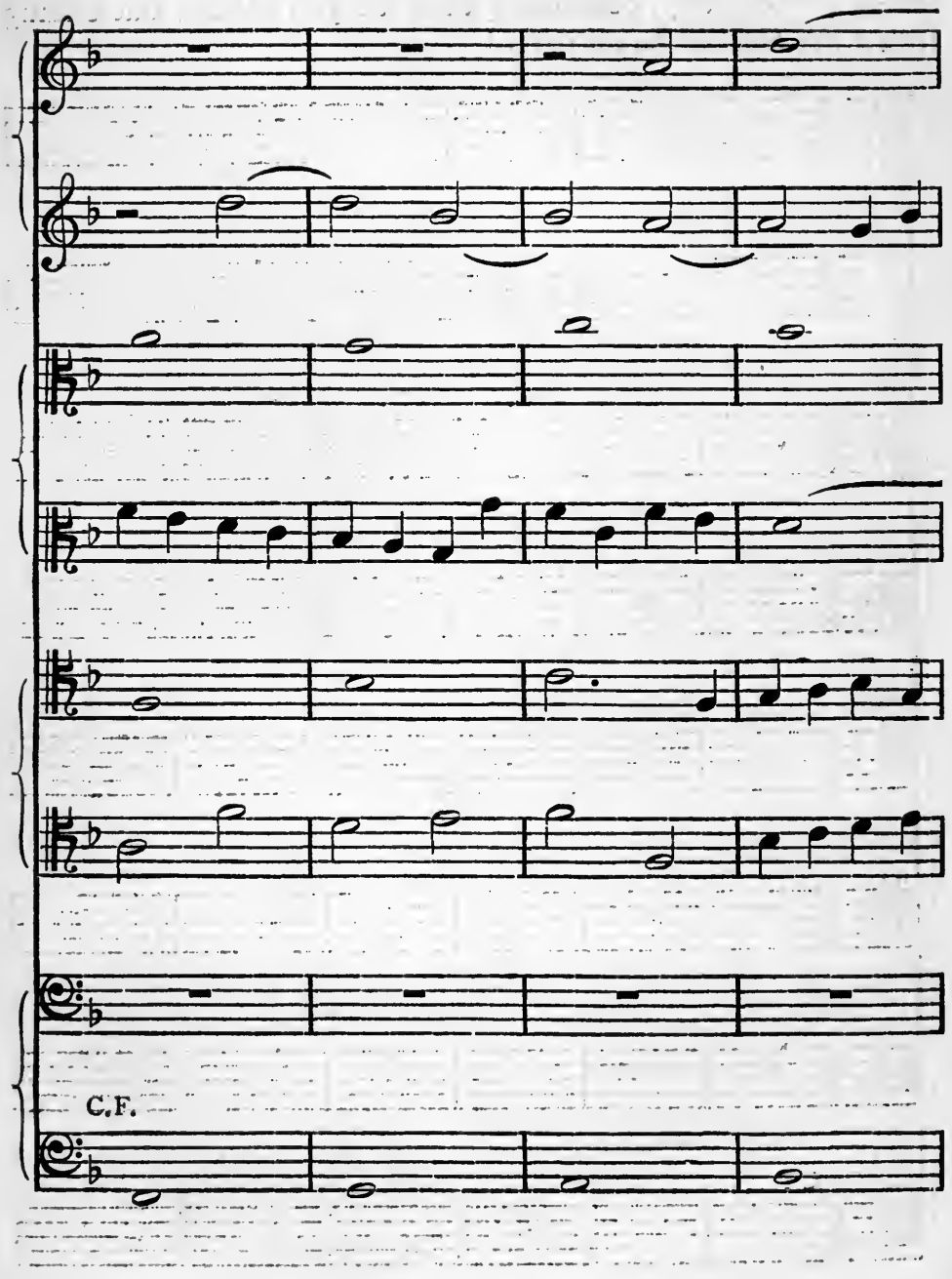




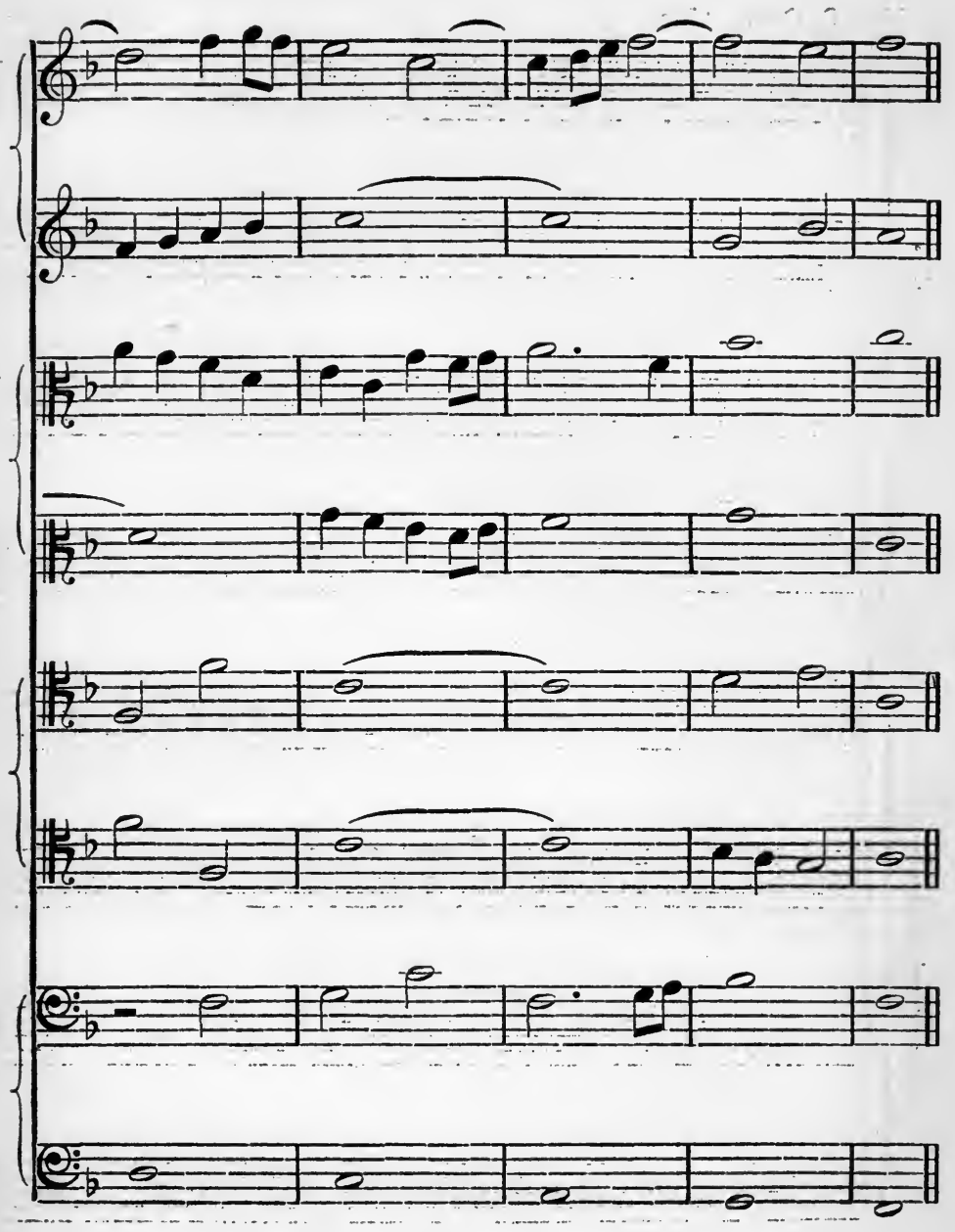


Mus. D. Oxon., 1899.

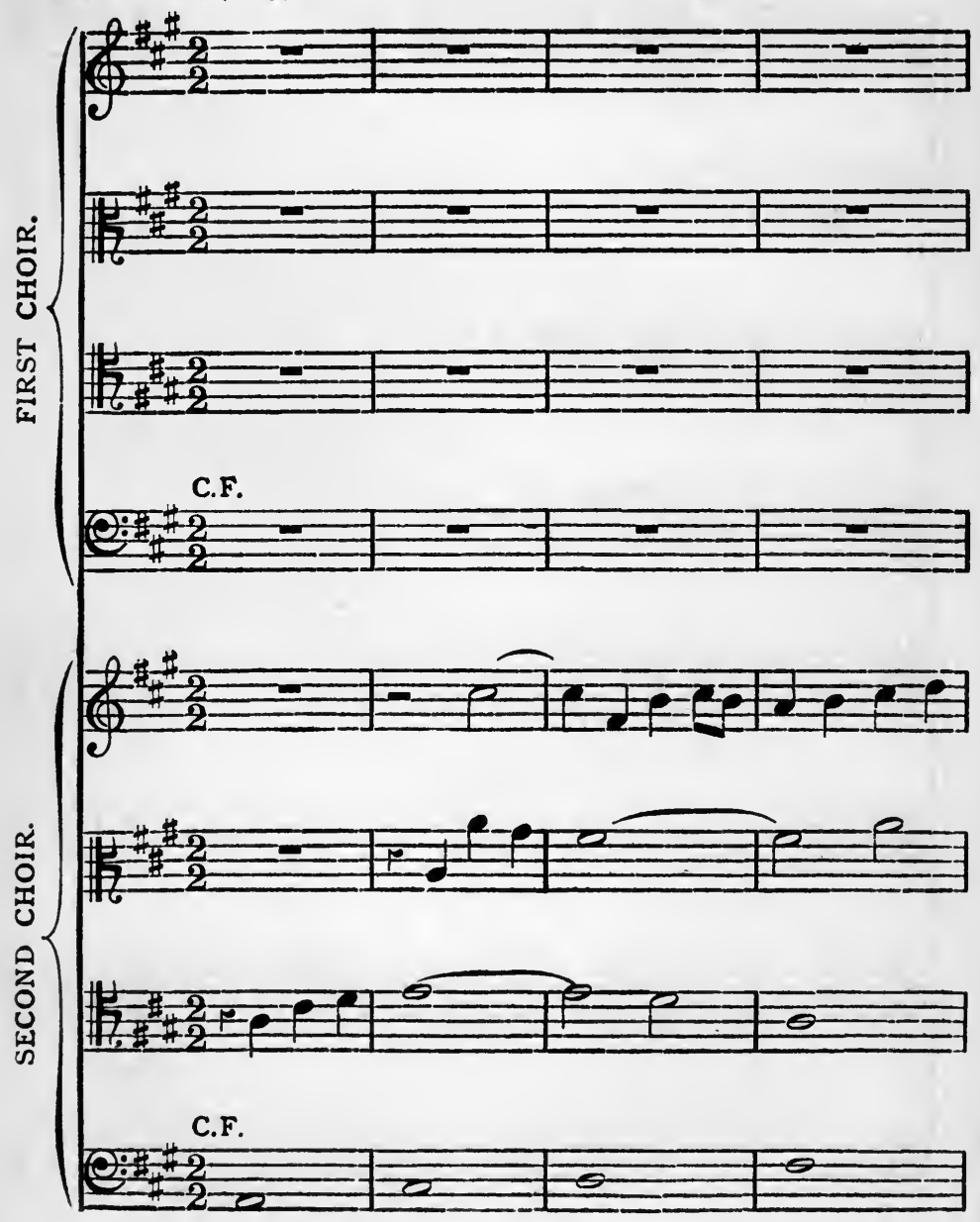




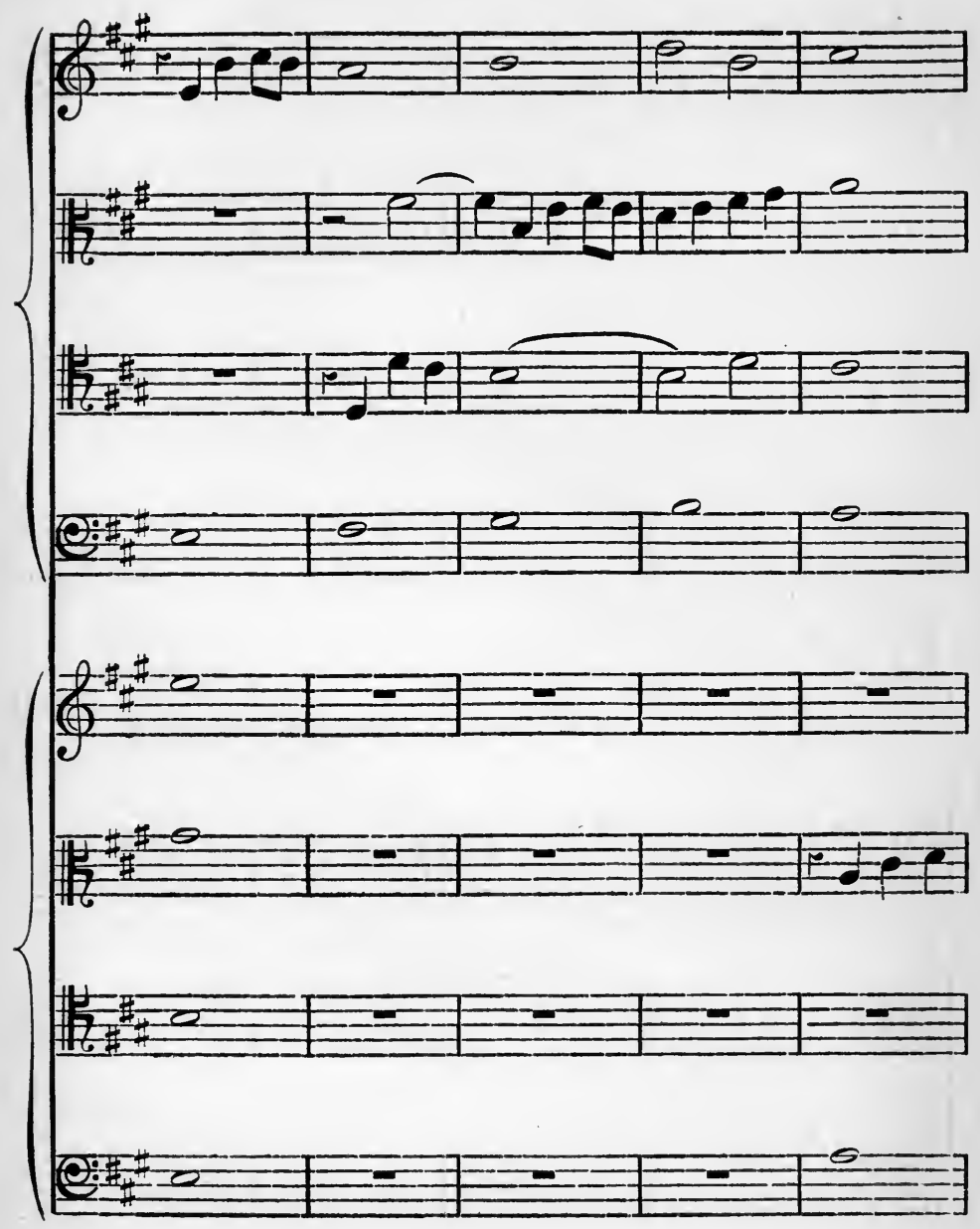




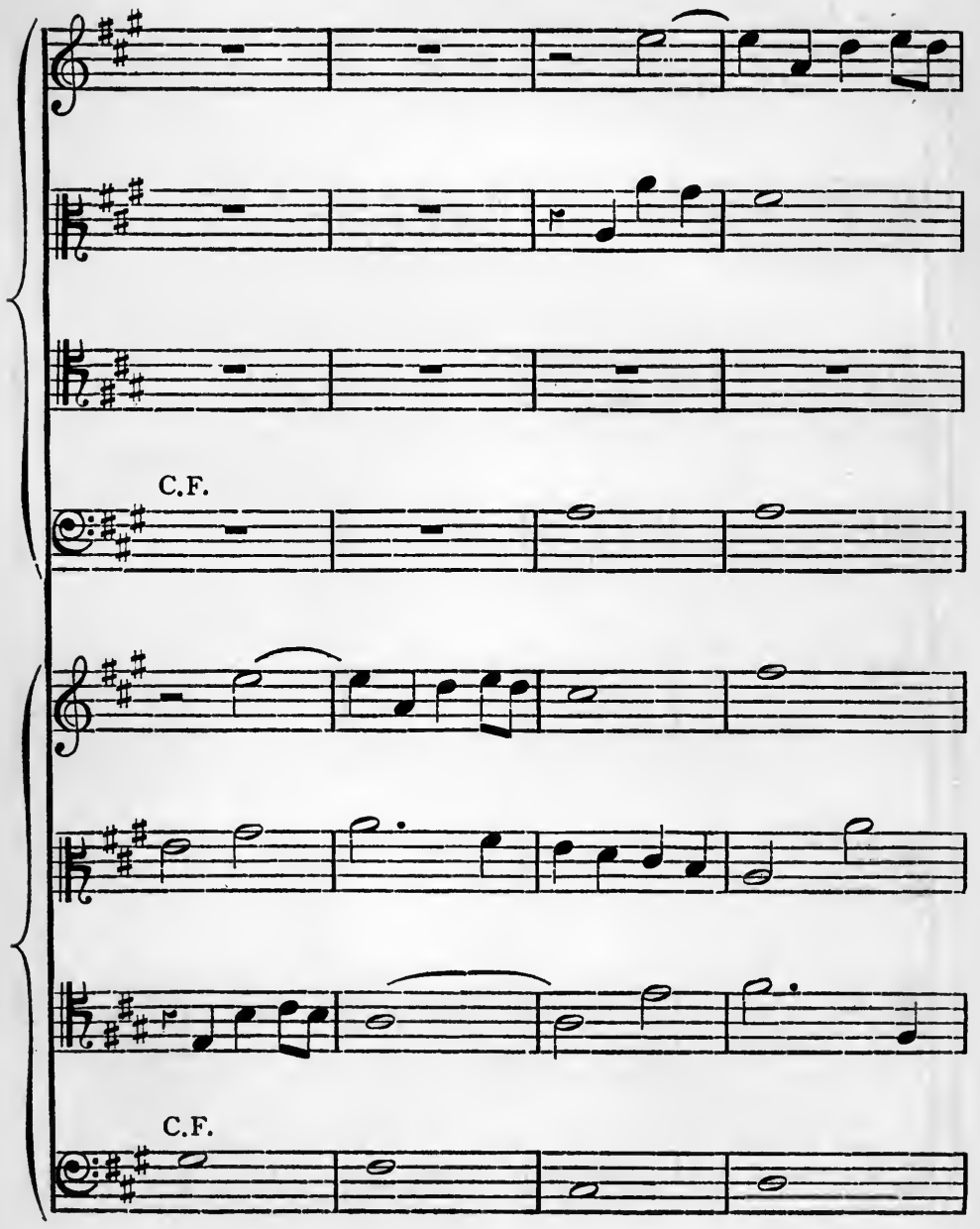




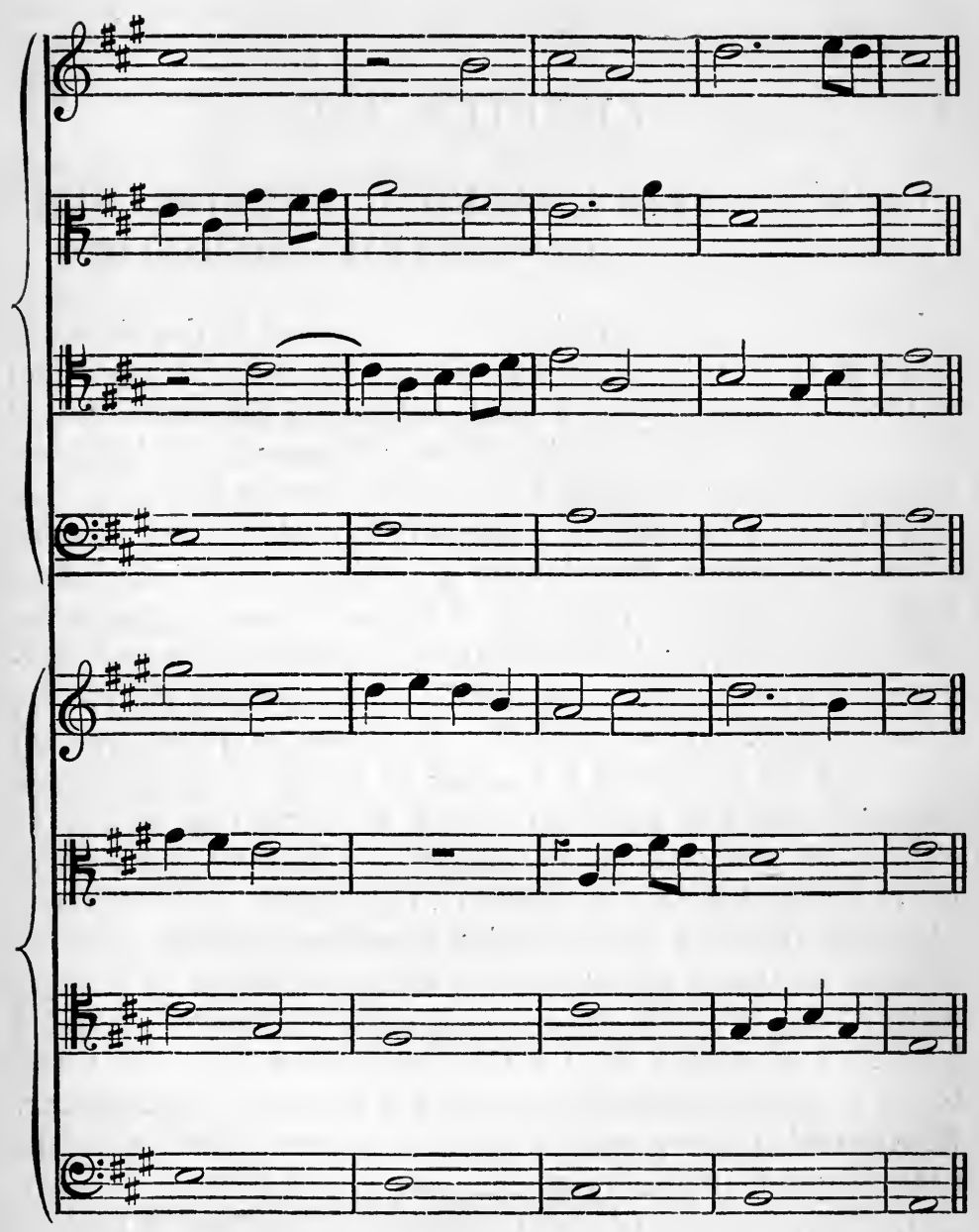




\section{CHAPTER XVI}

THE ELEMENTARY HARMONIC SUB-STRUCTURE OF MODERN CONTRAPUNTAL DECORATION

THE student of history knows that the end of the sixteenth century witnessed a complete change in the character and methods of musical art. The whole trend of the possibilities of the 'Nuove Musiche' led to the enlargement of harmonic resource. It is beyond the scope of this treatise to discuss this subject; but it has been thought well to deal with the material of the Polyphonic Period from a vertical standpoint, and by touching slightly upon the use of the unprepared seventh, the essential six-four, and dissonant triads unavailable in the Polyphonic Period, to cross the frontier that separates the old art from the new. It is hoped that a discussion of this will not only help the student in his choice of harmonies in Scholastic Counterpoint, but will also afford a preliminary course in elementary composition. A treatise on Harmony is a book of reference, and does not pretend to do more than catalogue harmonic resource in as concise a way as possible, and the student of musical theory will not learn to compose by working figured basses, which are arranged as studies under classified heads. The present chapter aims at teaching the student how to use the simplest harmonic resource in elementary composition. Between this chapter and the next it is assumed that the student has covered the following ground:-

(I) The principles of chord connexion, including all modern resource.

(2) The principles of harmonic and melodic form.

(3) Rhythm. 
(4) The means of modulation.

(5) Differentiation of style.

(6) The decoration of chordal centres.

It is true that the present chapter covers some of this ground, but only in an elementary way, and in so far as these principles apply to the formation of phrases and sentences. The elementary means of modulation are also discussed, because they come within the scope of the chapter.

The following chapter does not deal with the various points raised in considering harmonic decoration, but it aims at showing how the principles of Counterpoint may be applied in constructing such texture.

It is of the greatest importance that the student should obtain a firm grasp of the principles of elementary chord connexion. He should read the Church Music of Croft, Boyce, Attwood, and Goss, and later that of Walmisley, S. Wesley, S. S. Wesley, and Smart. He will find it specially valuable to assimilate the clear smooth progressions of Corelli, who was the first mature composer of violin music. In his Sonatas he gives as an accompaniment to the solo instrument a figured bass, and the student will there see in the clearest way the basis of all decorative work.

\section{Section I.}

The Uninverted Triads of the Major Key.

It is not possible to write more than six or seven uninverted triads in succession without producing a crude and artificial effect. We shall therefore limit ourselves in this section to the formation of phrases. But before we proceed to this we have to consider the very simplest fundamentals of harmonic progression -the principles of chord connexion, in other words the aesthetic basis of diatonic harmony.

(i) The Primary Triads, I. IV. V.

Any combination of these is good. But the use of the progression V. to IV. requires care; the following is the only satisfactory arrangement:- 


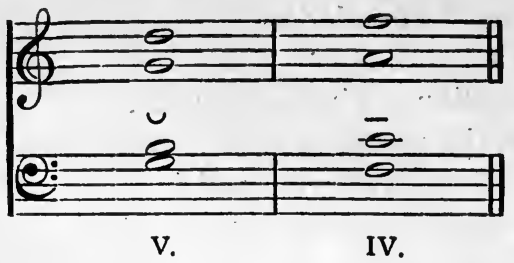

At the beginning of the first phrase of a sentence, we should choose primary in preference to secondary triads. Nothing gives a phrase a more unsatisfactory effect than a preponderance of secondary triads.

(ii) The Secondary Triads, II. III. VI. (VII.)

We omit VII. from the discussion, as it is impracticable.

In following one secondary triad by another the only pro. gressions that are bad are II. to III. and III. to II. ; in the other cases it will be seen that the roots are a fourth or a fifth apart.

(iii) If a primary triad be followed by a secondary triad, or vice versa, the following root progressions are satisfactory.

(a) Root rising or falling a fourth.

II. to V.

(b). Root falling a third.

I. to VI. ; III to I. ; IV. to II. ; V. to III. ; VI. to IV.

(c) Root rising a second.

III. to IV. should only be used in the following idiom.

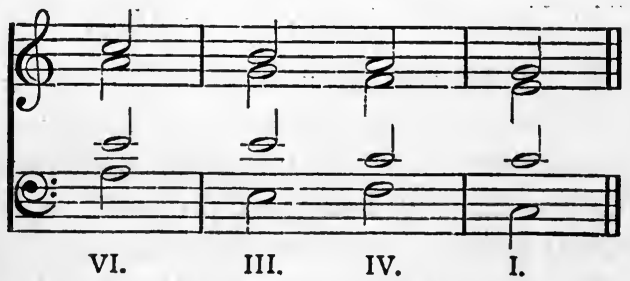

The following root progressions are weak.

(a) Root rising a third.

The progression is tolerable if the first chord occur on a strong accent.

(b) Root falling a second, except VI. to V. 


\section{HARMONIC SUB-STRUCTURE}

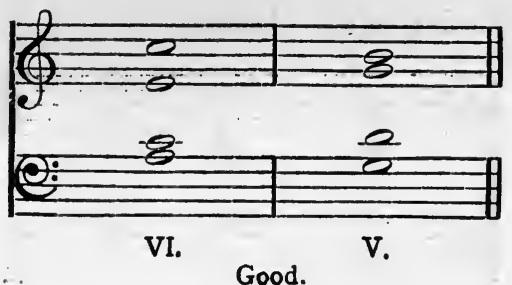

We have now to consider the formation of phrases from this material, and we shall discuss the progressions under two aspects, $(a)$ duple time, $(b)$ triple time. It has been observed that all decorated work may be reduced to two forms of rhythm, iambic or anapaestic. Each foot forms a motive, two motives form a section, and two sections form a phrase: that is, a phrase consists of four motives.

The Iambus.
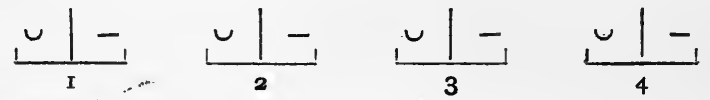

The Anapaest.
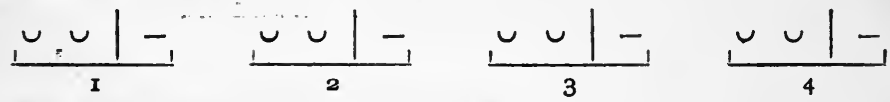

The first foot is often incomplete.
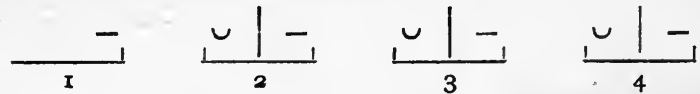

The last two accents, $u-$, form the Cadence, and we shall here only deal with the Perfect Cadence, V. to I.

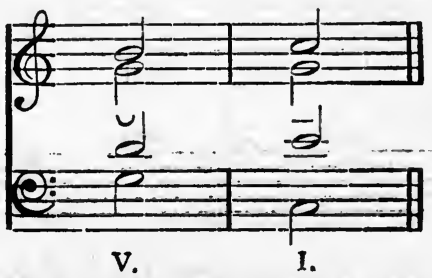

A chord should not as a rule be repeated in the relation of $\checkmark$ to - , except :- 


\section{THE ART OF COUNTERPOINT}

(a) at the beginning of the phrase.

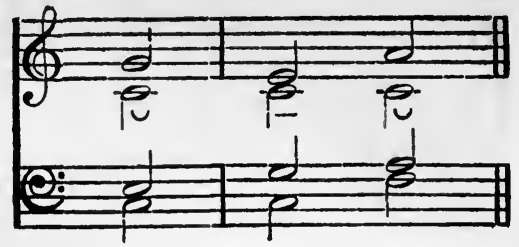

(b) when the previous strong accent has the same chord.

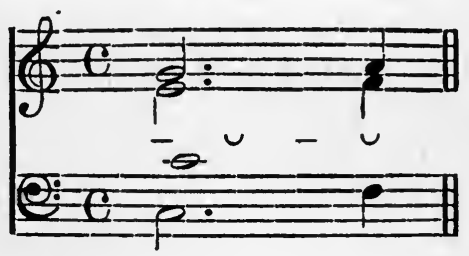

(c) for characteristic effects.

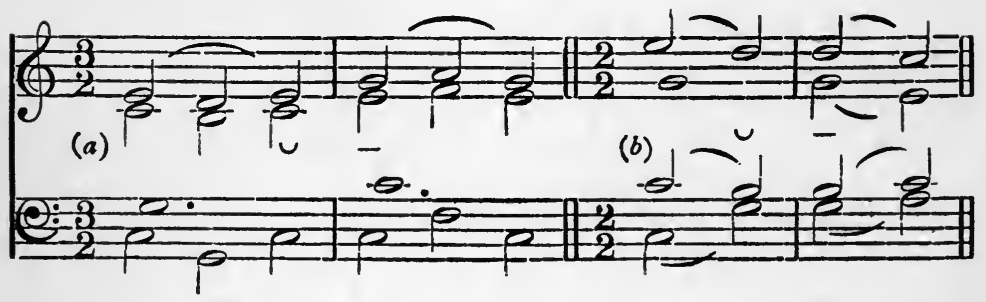

Examples of phrases.

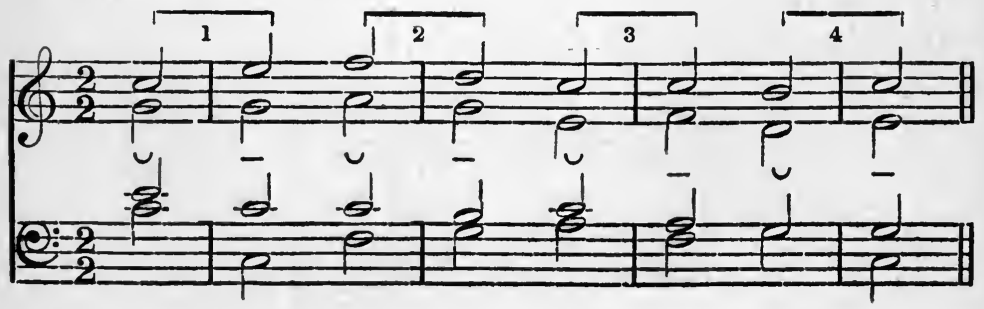

When the first foot is incomplete, the last foot often completes it by maintaining the final chord over the first half of the next foot, thus forming the caesura. 

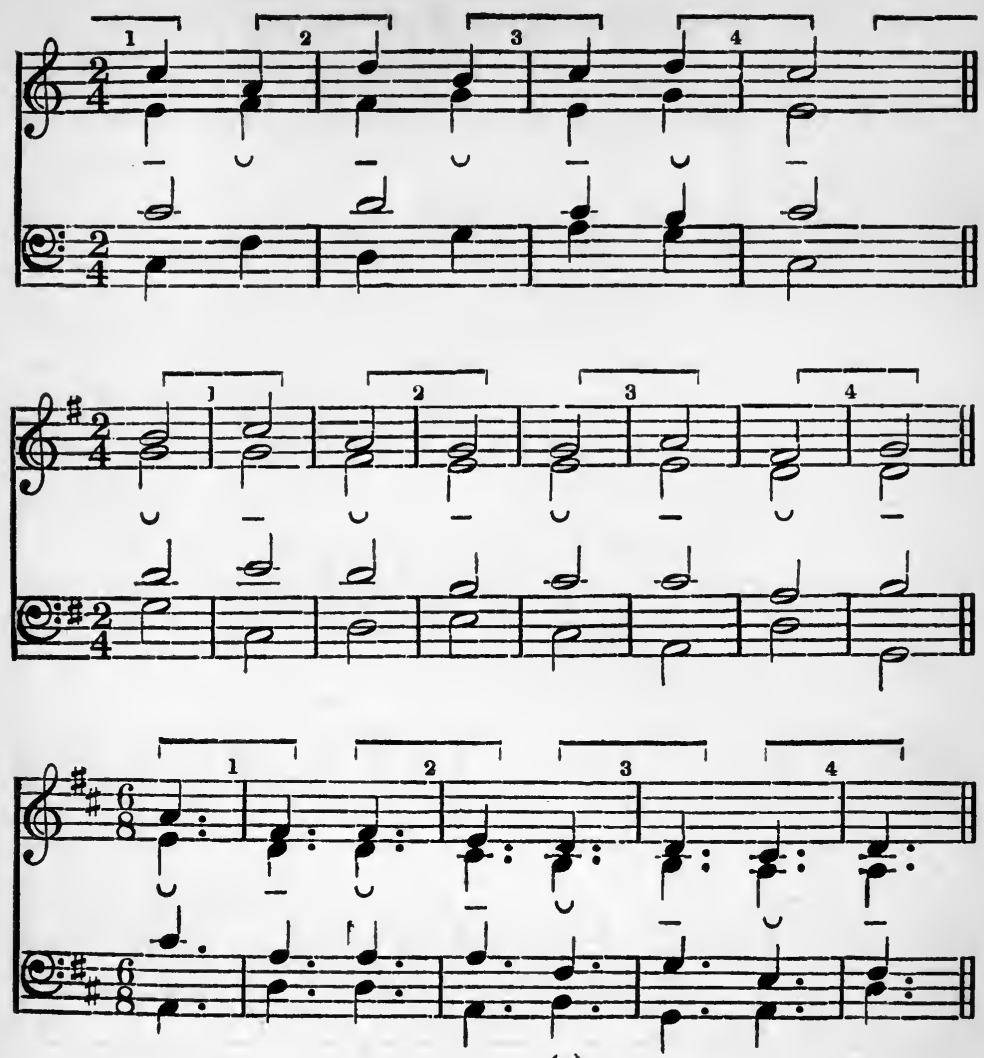

(a)

(a) Avoid anticipating the harmony of the Cadence just before it is approached.

The following would have been very poor :-

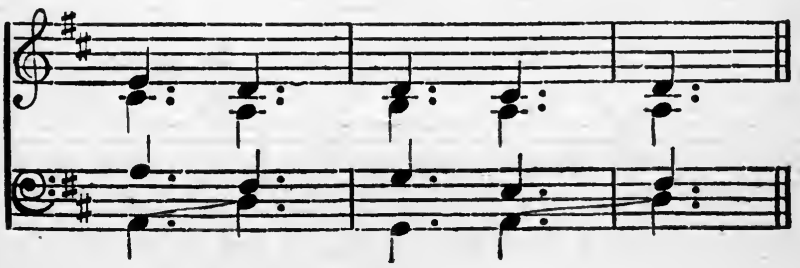




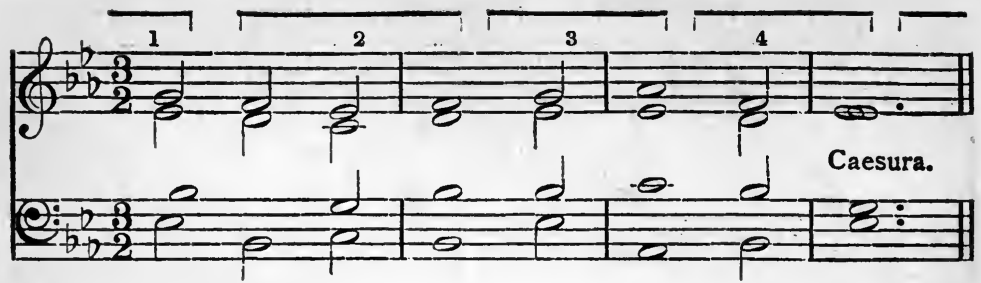

The above is equivalent to :-

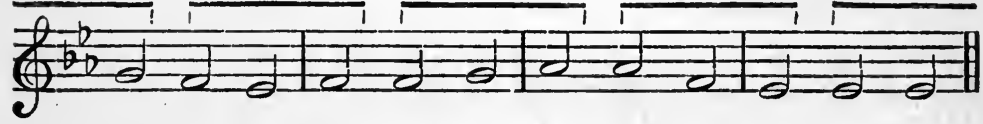
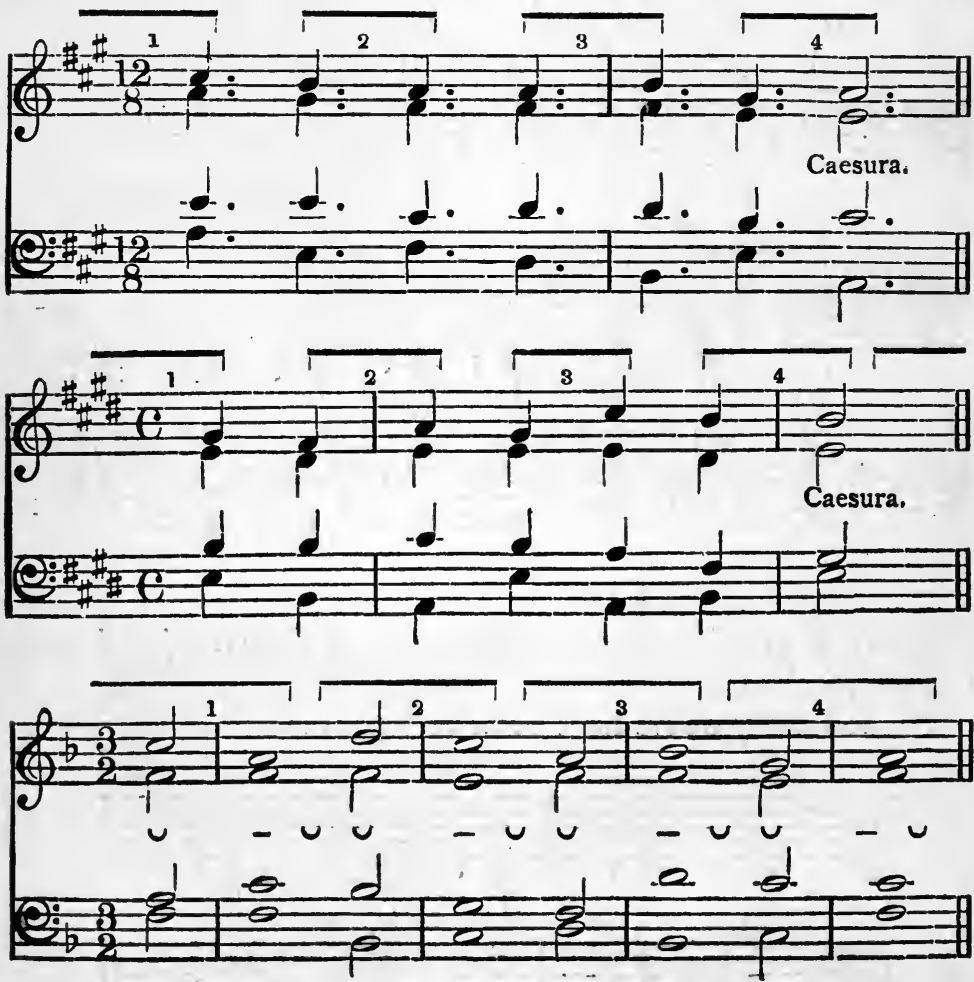

Except for strong rhythmic purposes, the same chord should 
not be used for the second and third accents of a bar of triple time, because it has the effect of a displacement of accent.

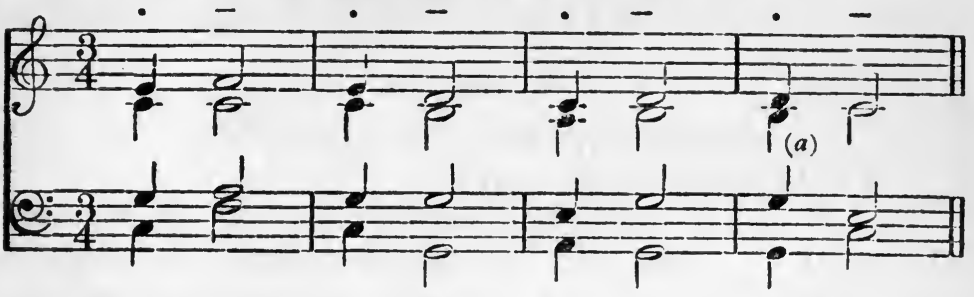

(a) Note that the first chord of the Cadence falls on the accented part of the bar; this produces what is termed a Feminine Cadence.
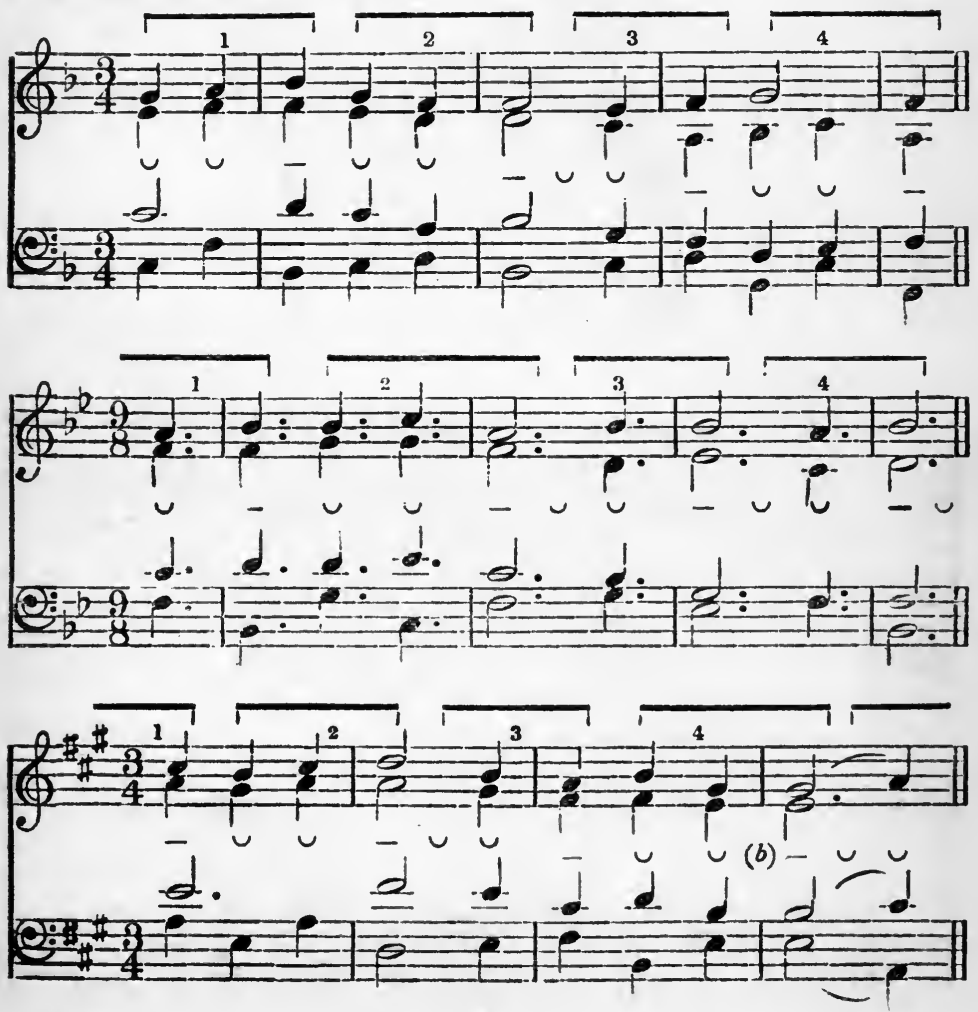
(b) Feminine Cadence. The two weak accents should be considered as the completion of the first foot. Bar 4 may be regarded as containing a suspension of the complete chord $\mathrm{V}$.

\section{Section II.}

Uninverted Triads of the Minor Key.

\section{The Harmonic Minor Scale.}

This is almost exclusively used for purposes of harmony. Omitting those triads which are not common chords we get:I. IV. V. VI.

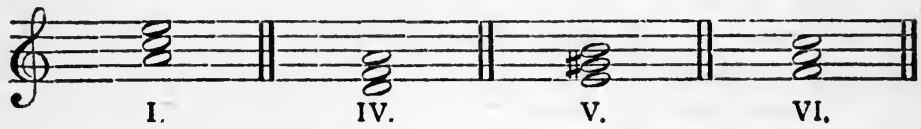

Any combination of these is good.

V. to IV. is best thus :-

Examples.
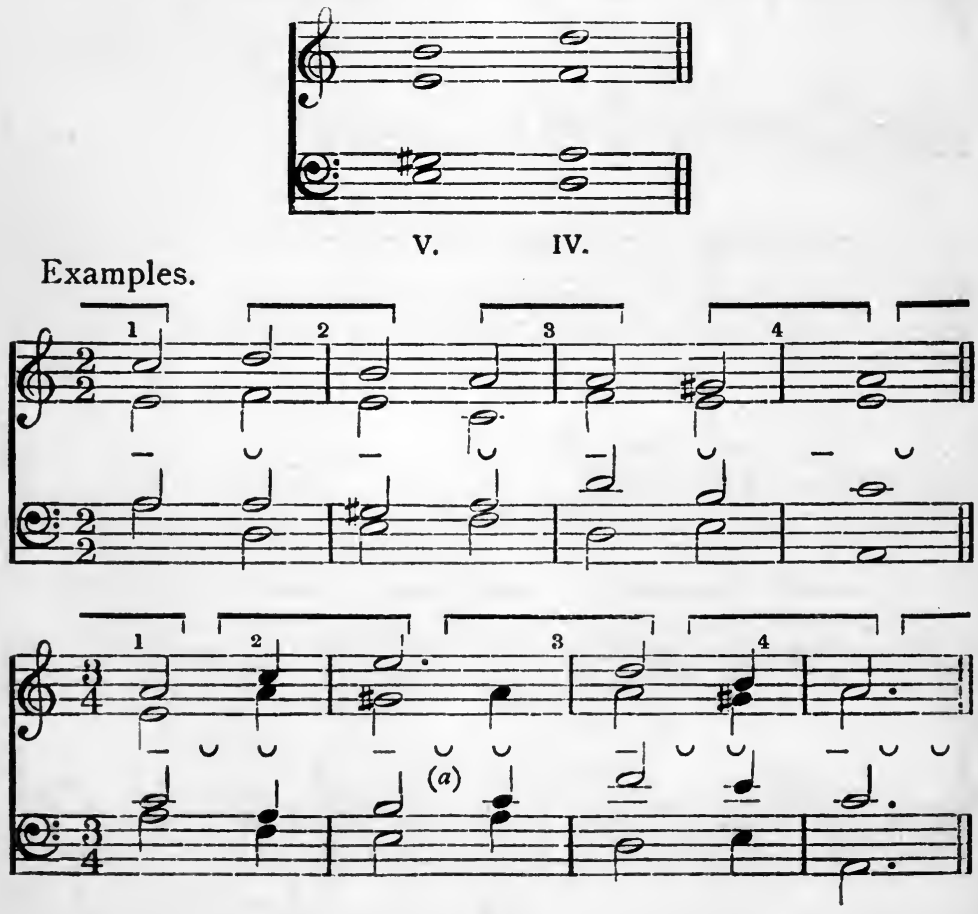
(a) The anticipation of the Cadence is here good, the different time values of the two chords removing the objectionable effect. Next, consider the triads II. III. and VII.

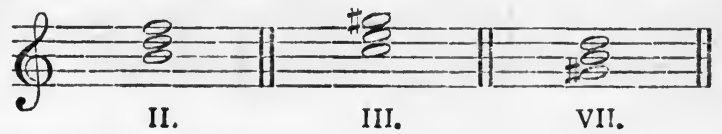

These should be rarely used.

(a) II. should be preceded by IV. or VI. and followed by V.

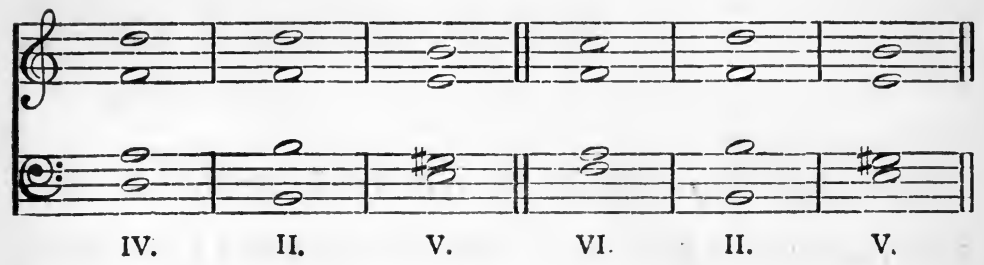

(b) III. should be preceded and followed by I. V. or VI.

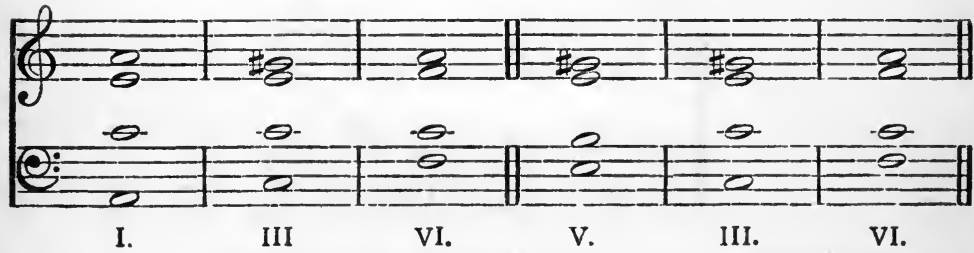

(c) VII. is impracticable.

II. and III. should be used with extreme caution, and should generally be avoided except in a sequence.

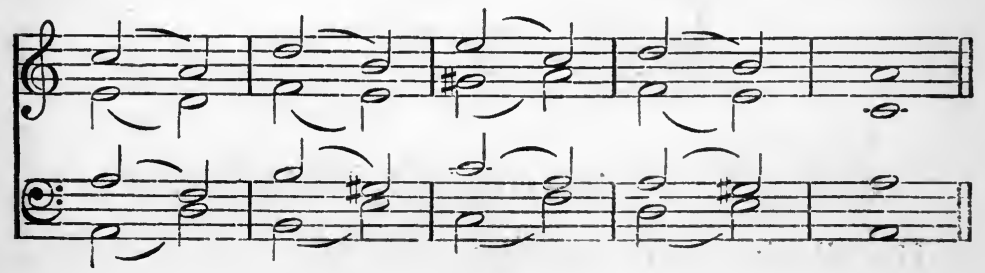




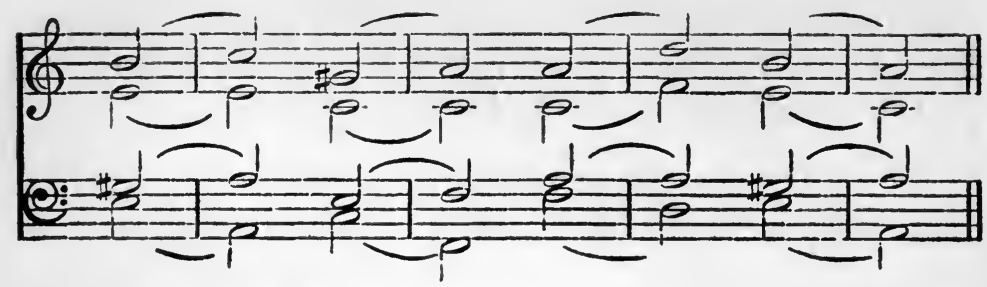

II. The Melodic Minor Scale.

This will give the following additional triads.

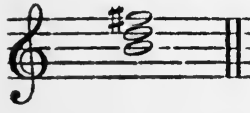

( $)$

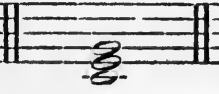

(a)

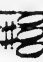

(3)

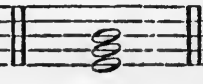

(4)

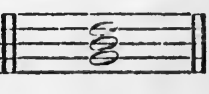

(5)

(I) 7 should be very rarely employed. It is best restricted to the case in which it is followed by V. and I., the melody being the last three notes of the ascending melodic minor scale.

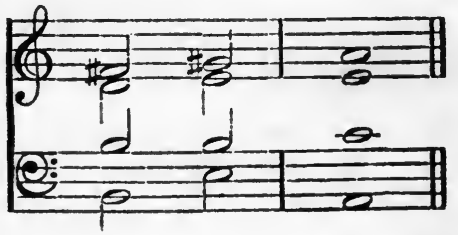

The following is less good, because unnecessary.

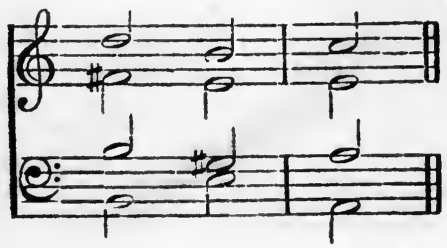

(3)

The following are the best cases of its use. 


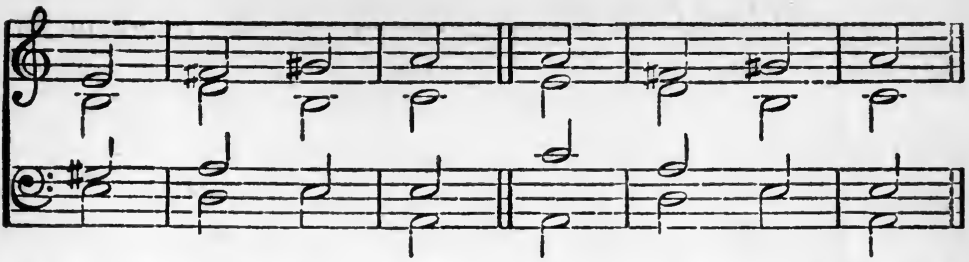

The use of these chords was common in the time of Handel and Bach, originating in the Dorian Mode: they should not be employed unless the major sixth occurs in the melody, and demands harmonization.

(2)

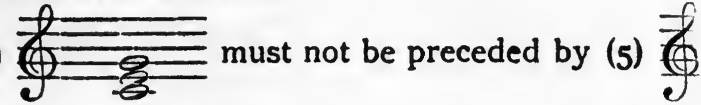

A minor.

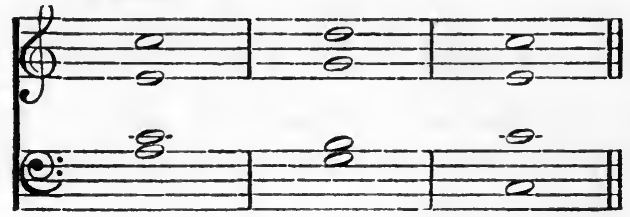

This produces a modulation to the key of $\mathrm{C}$ major. In order to confirm a modulation, the following conditions must be fulfilled :-

(a) The root of the second of the two chords must be a fourth higher than that of the first.

(b) The first chord must be a major common chord.

(c) The first chord must also contain a note foreign to the scale of the previous tonic: if it does not contain such note, the minor seventh must be added.

Thus the following is not a modulation to D minor, for the first of the two chords contains the minor third.

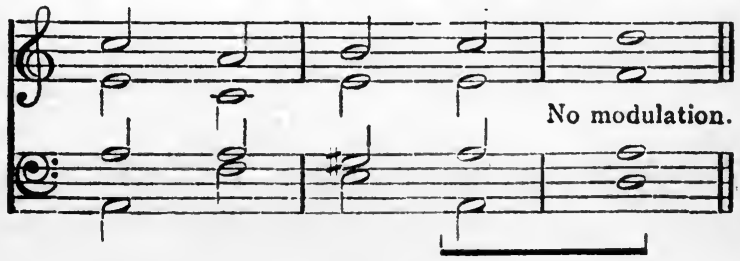


Neither is the following a modulation from $\mathrm{C}$ major to $\mathrm{F}$ major, for neither chord contains a note foreign to the scale of $\mathrm{C}$ major.

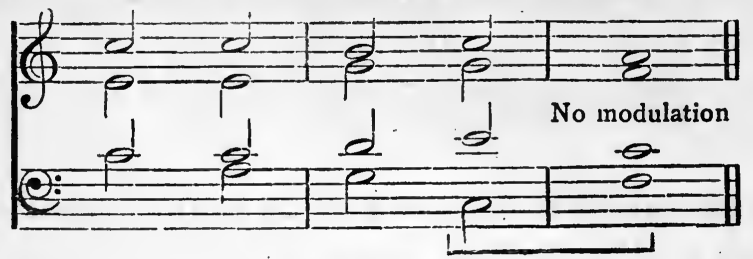

The following is an example of modulation.

$C$ maj. to $F$ maj.

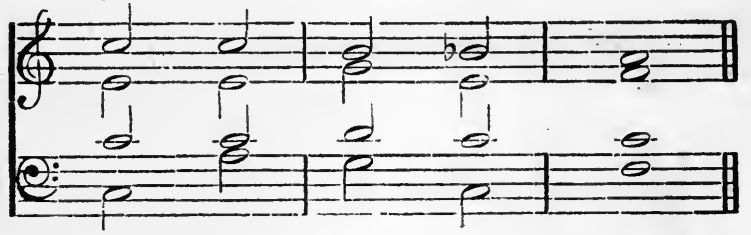

Of course, if an intervening chord be foreign to the key quitted, but diatonic in the key approached, the addition of the minor seventh is not necessary.

C maj. to F maj.

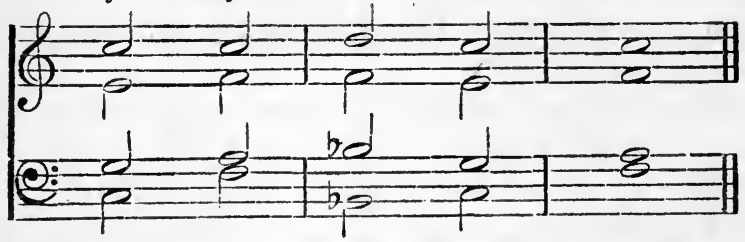

If it is desired to use the chord in question without inducing modulation, it should be preceded and followed by I. IV. or VI.

It is best preceded by I. or VI. and should not be immediately preceded or followed by $\mathrm{V}$.

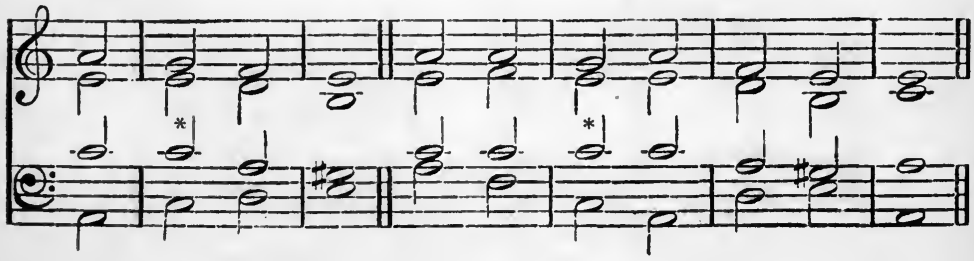




\section{HARMONIC SUB-STRUCTURE}

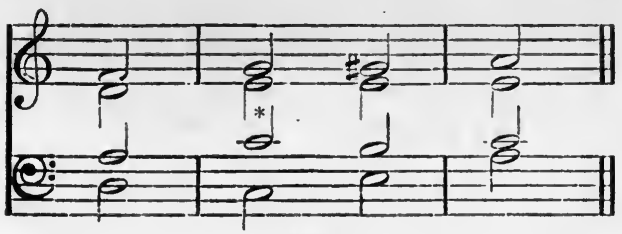

Bad.

As a matter of fact this chord should only be used in two cases:-

(a) in descending from the tonic to the dominant by conjunct degrees, this progression being in the highest part.

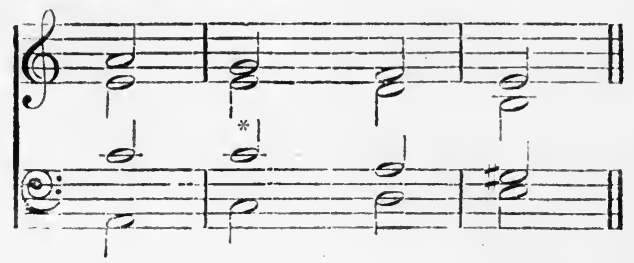

(b) When the minor seventh is preceded and followed by the tonic.

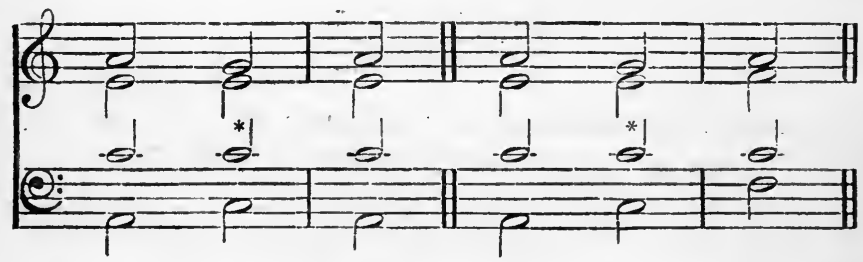

The following is possible, but not recommended.

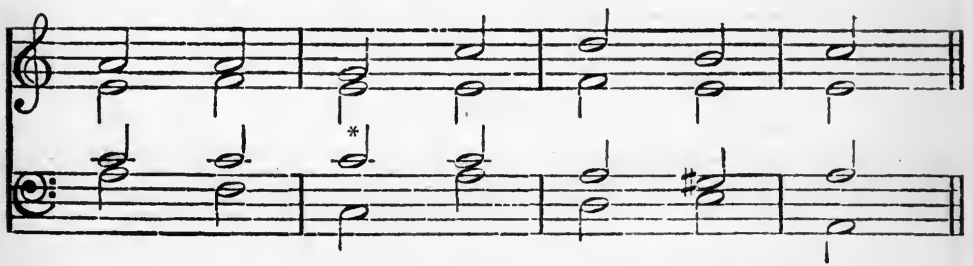

For the melody is modal, and should not be written thus, unless for special effect. 
(4) $\bar{\equiv}$ should be preceded by I. and followed by VI. It should not be preceded or followed by V. with the major third.

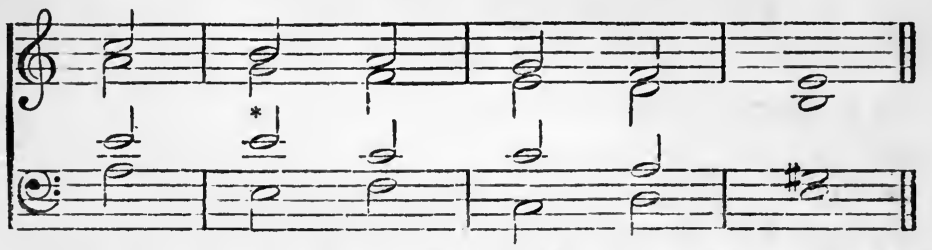

If $\mathrm{G}$ natural had not occurred in the melody, we should have preferred the following-

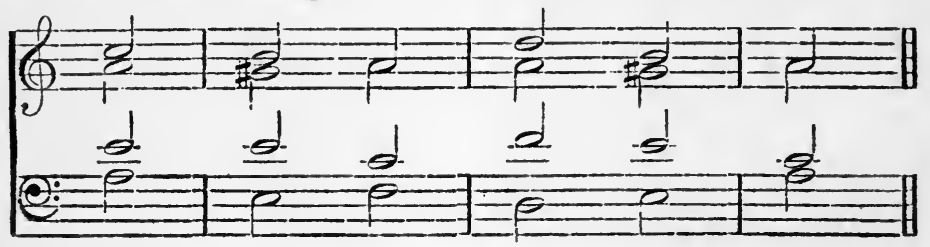

Lastly (5).

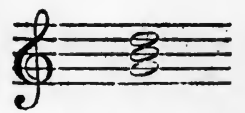

This should be preceded and followed by I. IV. or VI. but not by $\mathrm{V}$., causing the chromatic alteration of the G.

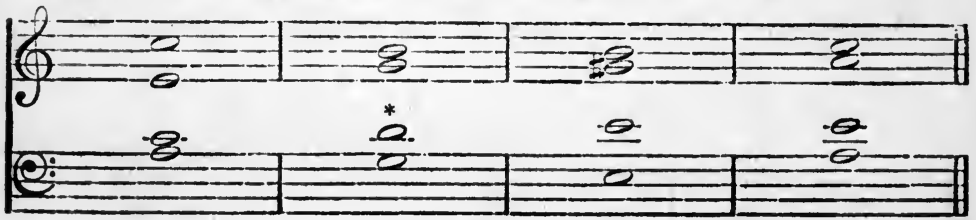

Bad.

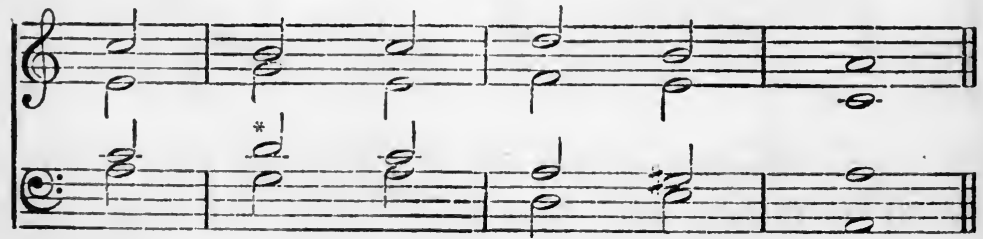




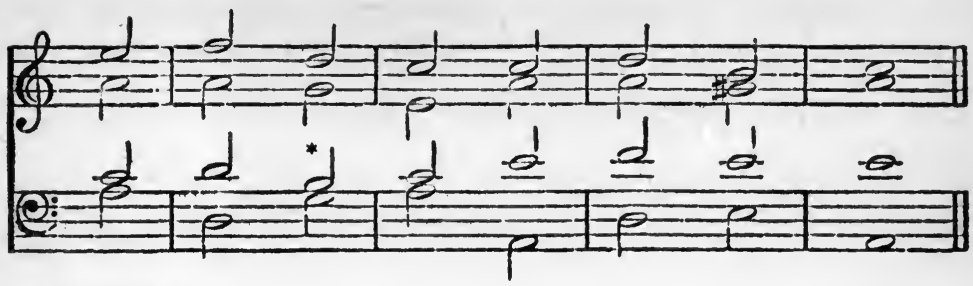

\section{Summary.}

(1) The characteristic common chords of the minor mode are I. IV. V. VI, derived from the harmonic minor scale.

(2) The minor common chord on 11. and the major common chord on IV. should only be used when the major sixth of the scale occurs in the highest part.

(3) The additional chords derived from the melodic minor scale should only be used in harmonizing the descending melodic minor scale, or in cases in which the note thus harmonized is in the nature of an auxiliary note. And in all these cases these chords must be preceded and followed by I. IV. V. and VI. except when the use of $\mathrm{V}$. causes the immediate chromatic alteration of one of the notes of the triad.

Unless these restrictions are observed, such progressions as the following, causing the tonality of the minor mode to be destroyed, will ensue.

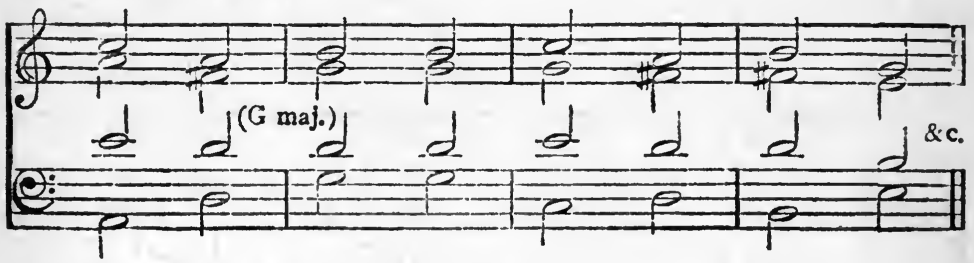

Examples.-Using I. IV. V. VI.

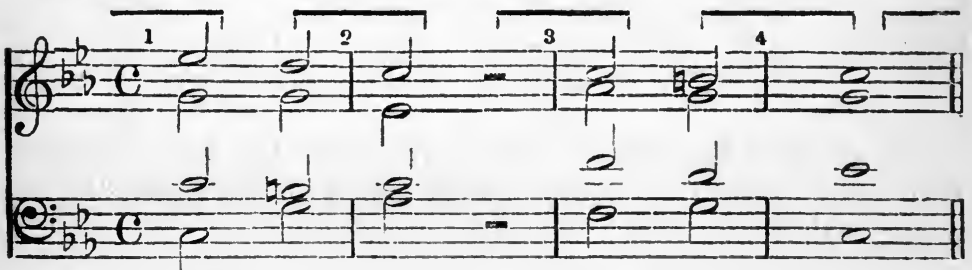




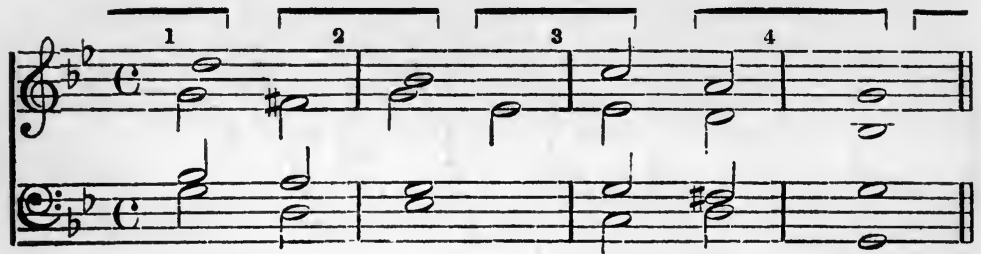

Introducing II. and III.
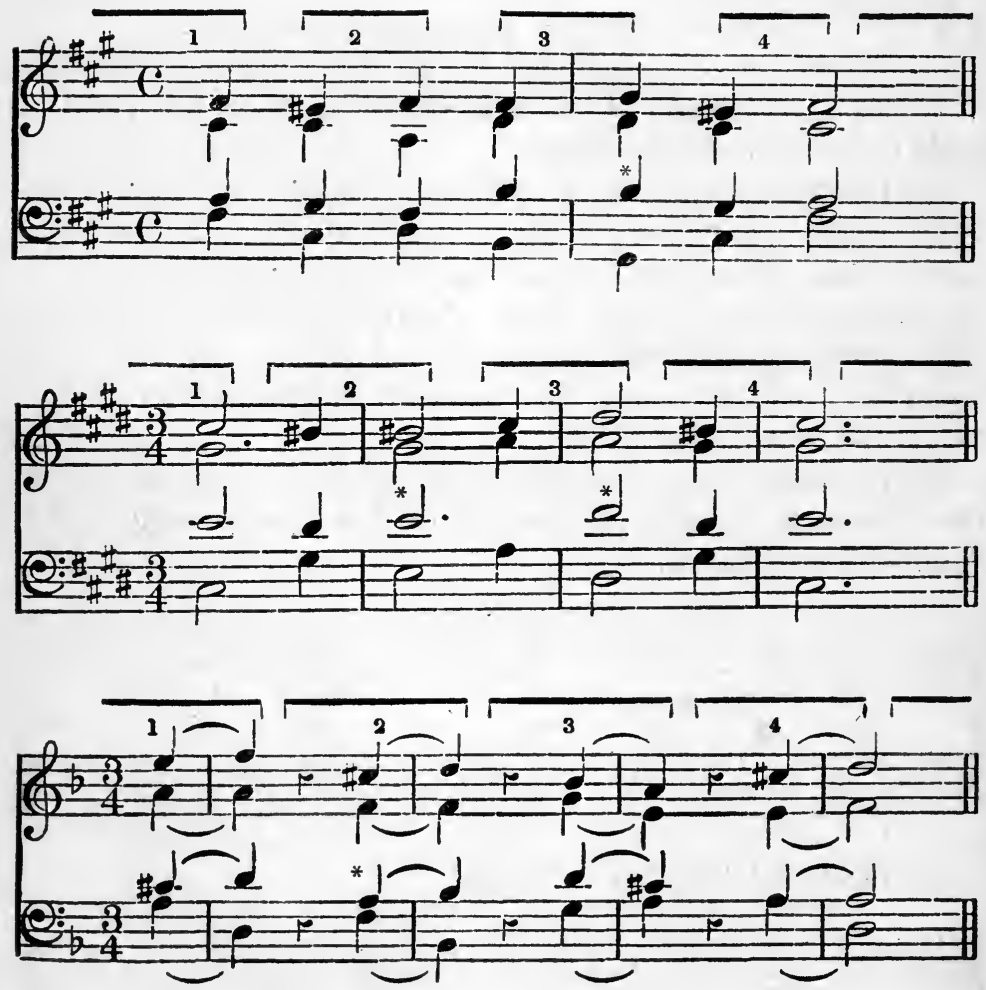

Introducing the minor common chord on the second degree of the scale, and the major common chord on the fourth degree of the scale. 

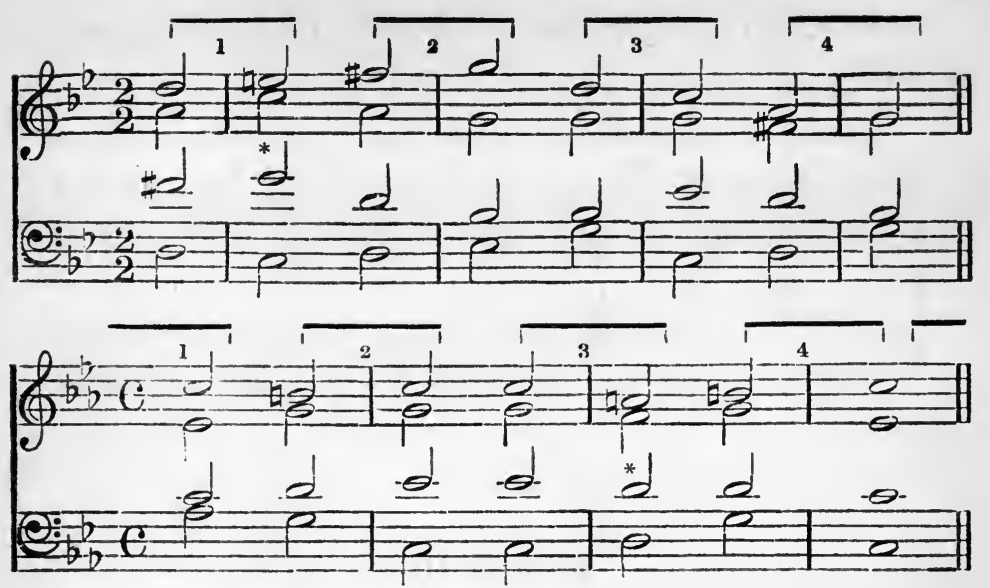

Introducing III. with the perfect fifth.

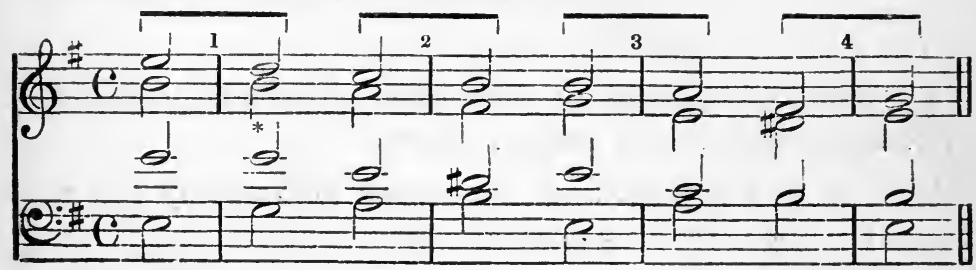

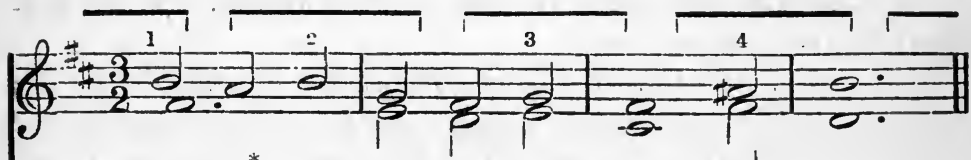

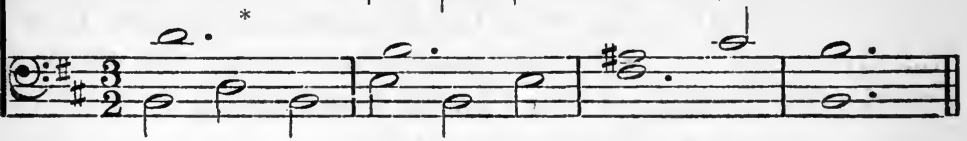
Introducing $\mathrm{V}$. with the minor third.

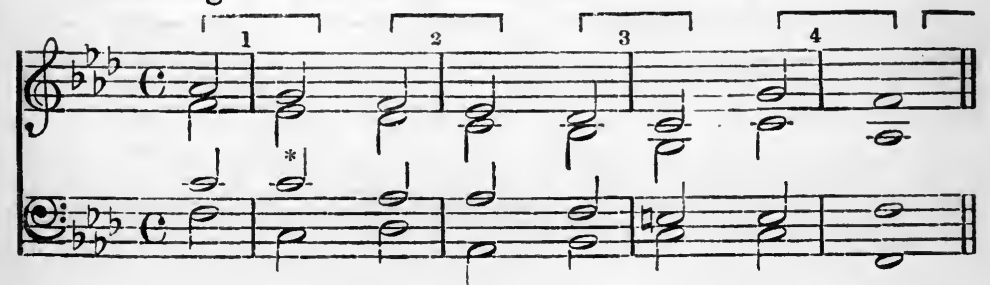


Introducing the major common chord on the minor seventh of the scale.

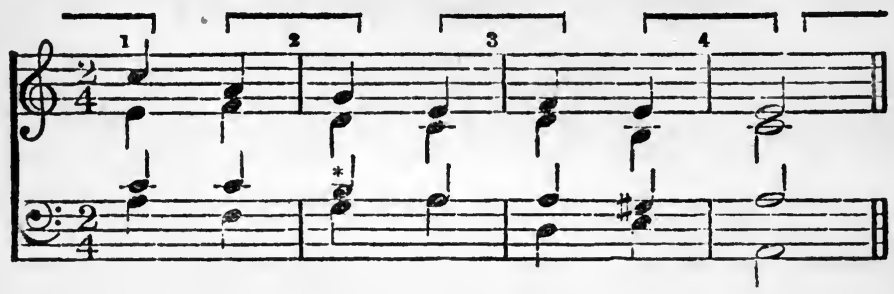

Section III.

First Inversions of the Triads of the Major Mode.

The possible combinations are:-

(I) The root position of one chord followed by the first inversion of the same or another chord.

(2) The first inversion of one chord followed by the root position of the same or another chord.

(3) The first inversion of one chord followed by the first inversion of another chord.

$\mathrm{V}$ b. to IV. and IV. to V b. are obviously impossible, as the harmony would be crude in effect. All the other combinations are possible, but they are not all of the same excellence. If two first inversions are used consecutively, it is better that the bass should move by step, or leap a fourth downwards.

Examples, using Primary Triads only.

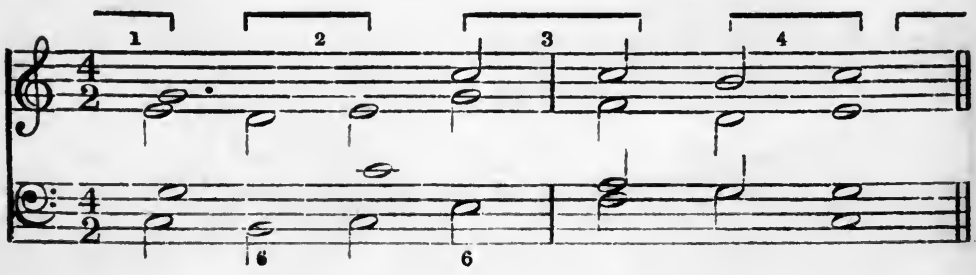



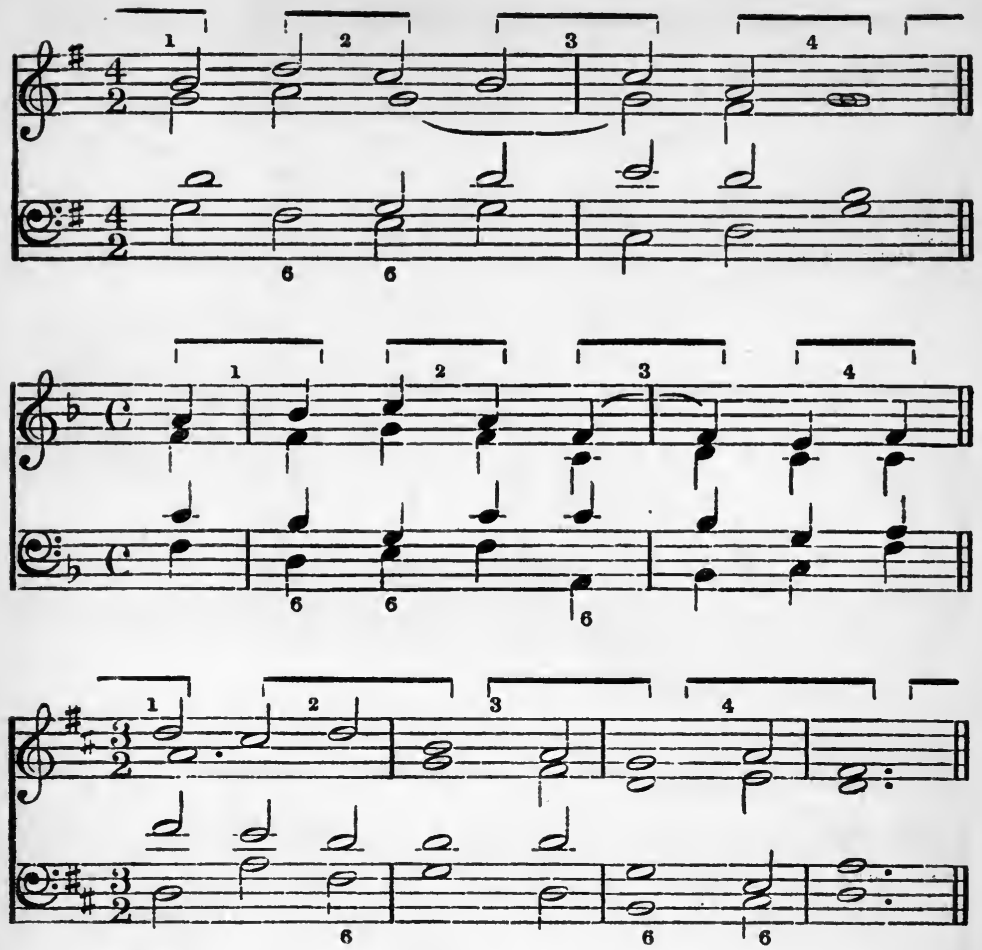

If we write two consecutive phrases, we form a sentence. Each phrase should end with a Cadence; at the end of the first phrase we can introduce the Half, the Deceptive, or the Plagal Cadence.

Examples consisting of eight feet.

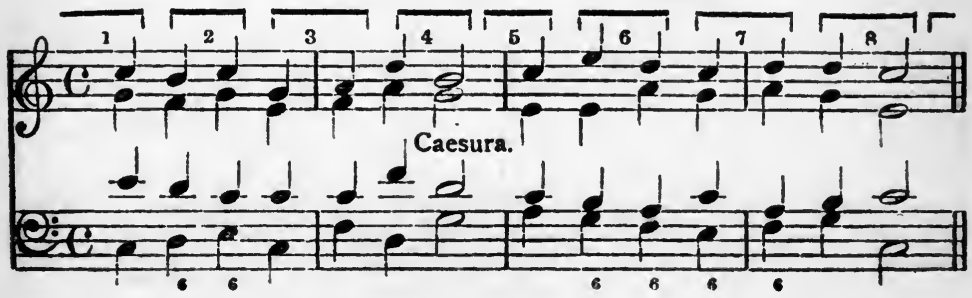



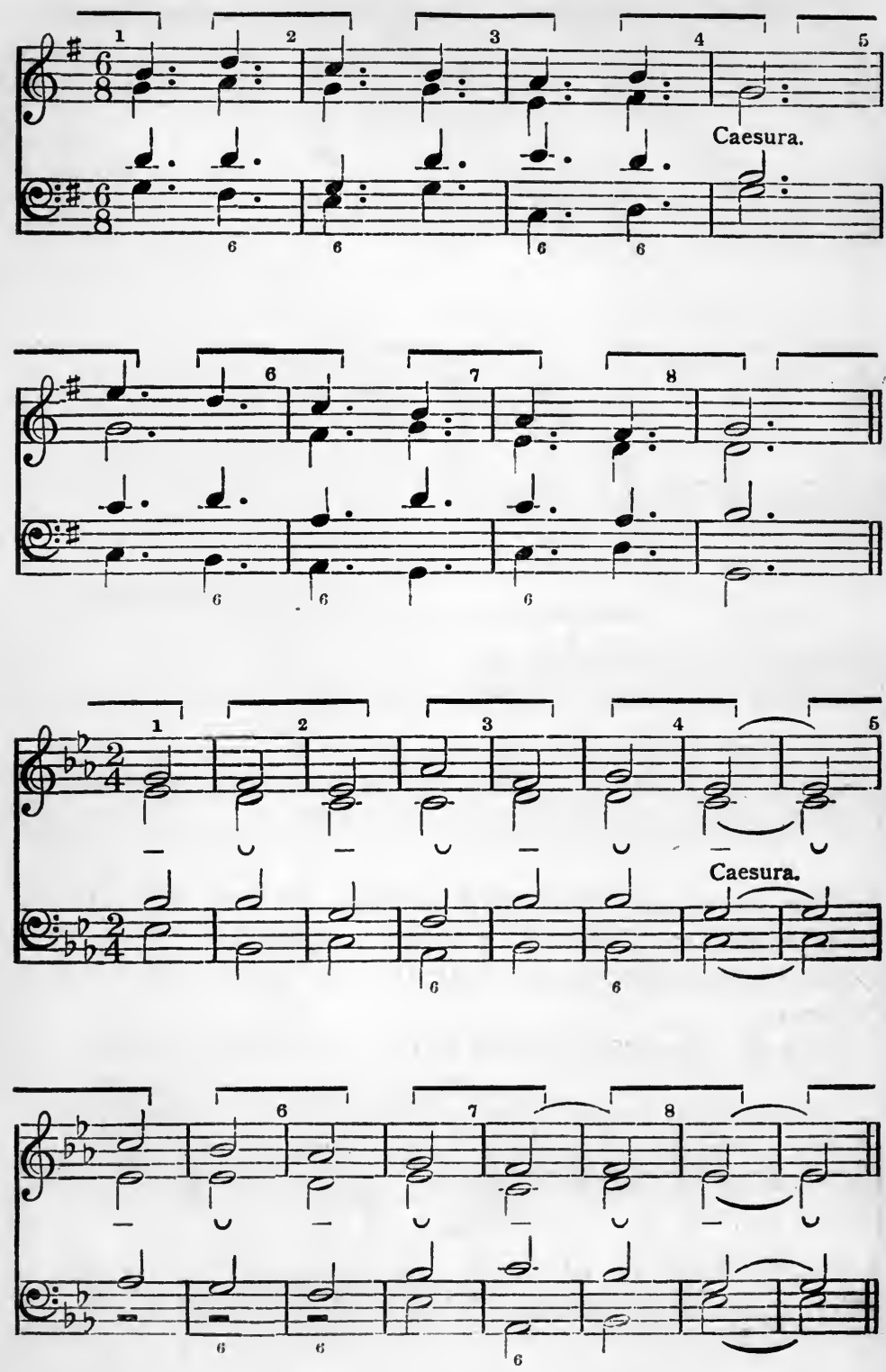

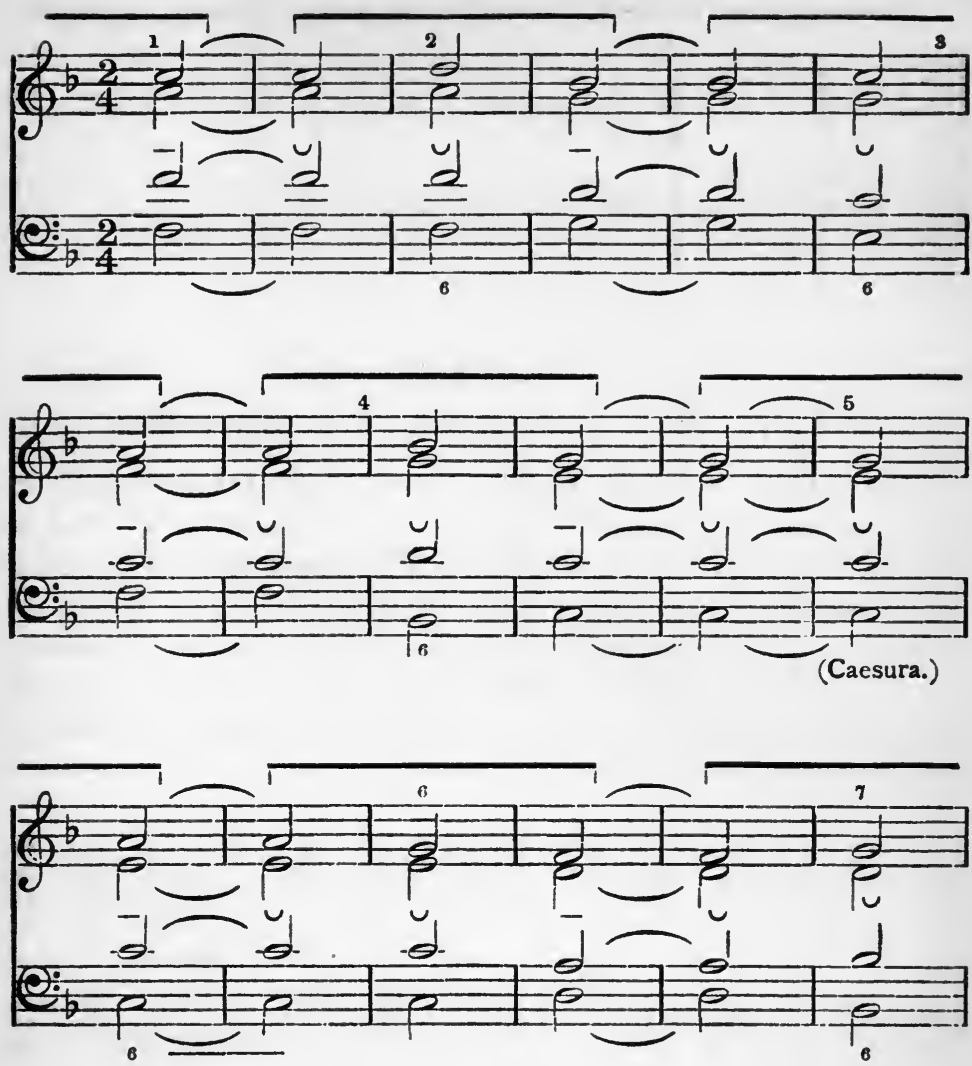

Completion of first foot.

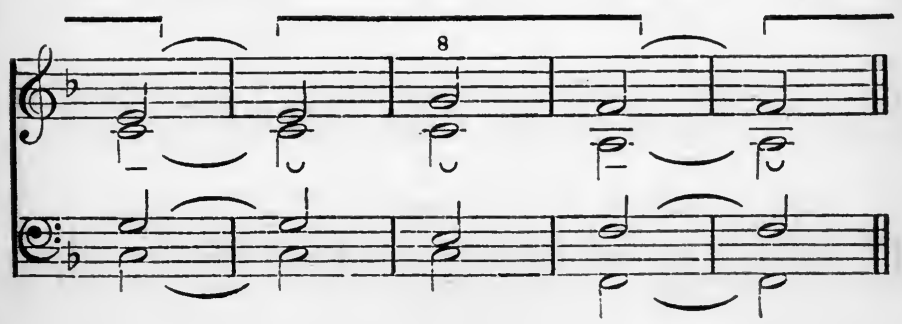




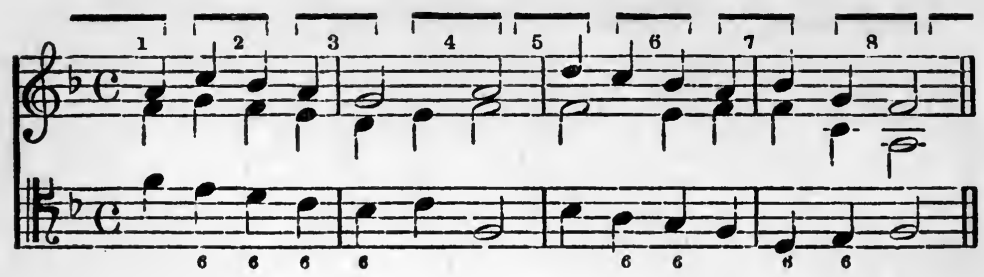

Section IV.

First Inversions of the Triads of the Minor Mode.

(r) Combinations of I. IV. V. VI. and their first inversions are good in any order except IV b. to $V$ b., $V$ b. to $I V b$., $V b$. to I b., Vb. to IV., Vb. to VI., VI. to Vb. All these involve bad melodic progression in the bass or poor harmony (cf. p. I4 (2) and the general observations in Section I. regarding root progressions). It should be remembered that a bass note should not be repeated in the relation of $\checkmark$ to - , unless the previous - has also borne the same bass note.

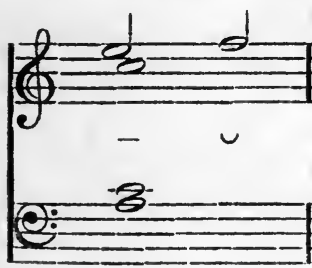

Good.

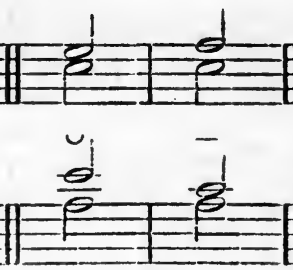

Bad.

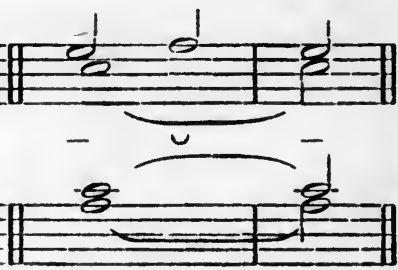

Good.

Further, such weak harmony as the following should be avoided.

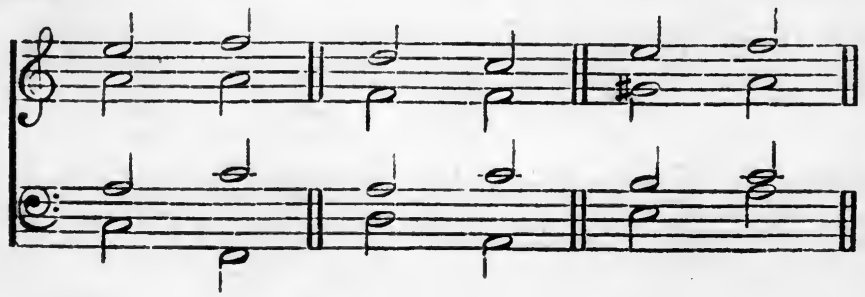


Examples.
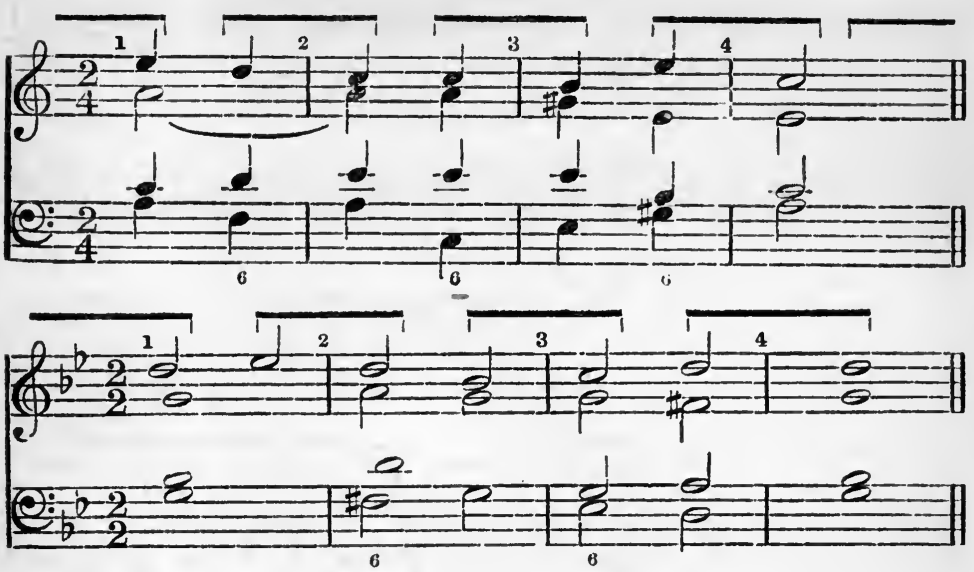

(2) Consideration of II b. IIIb. VIIb.

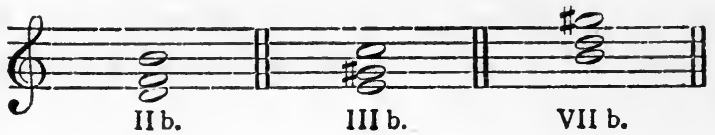

(a) II b. is best preceded or followed by I b. V. or IV. (- to u). Note the following weak effects.

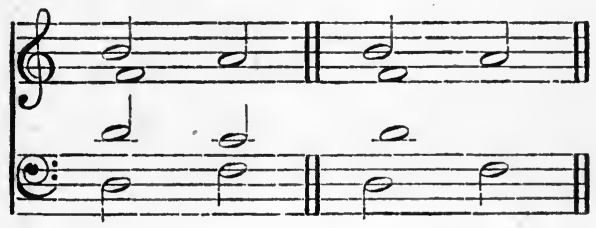

(b) III b. is best preceded or followed by I. I b. IV. or VI.

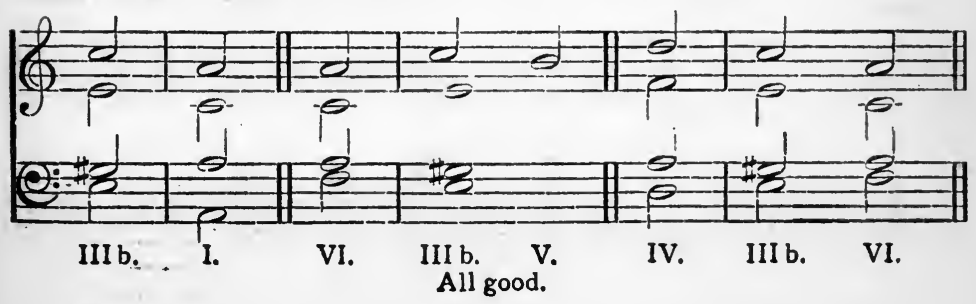


(c) VII b. is best preceded or followed by I. or I b.

Examples introducing triads and their first inversions derived from the harmonic minor scale.
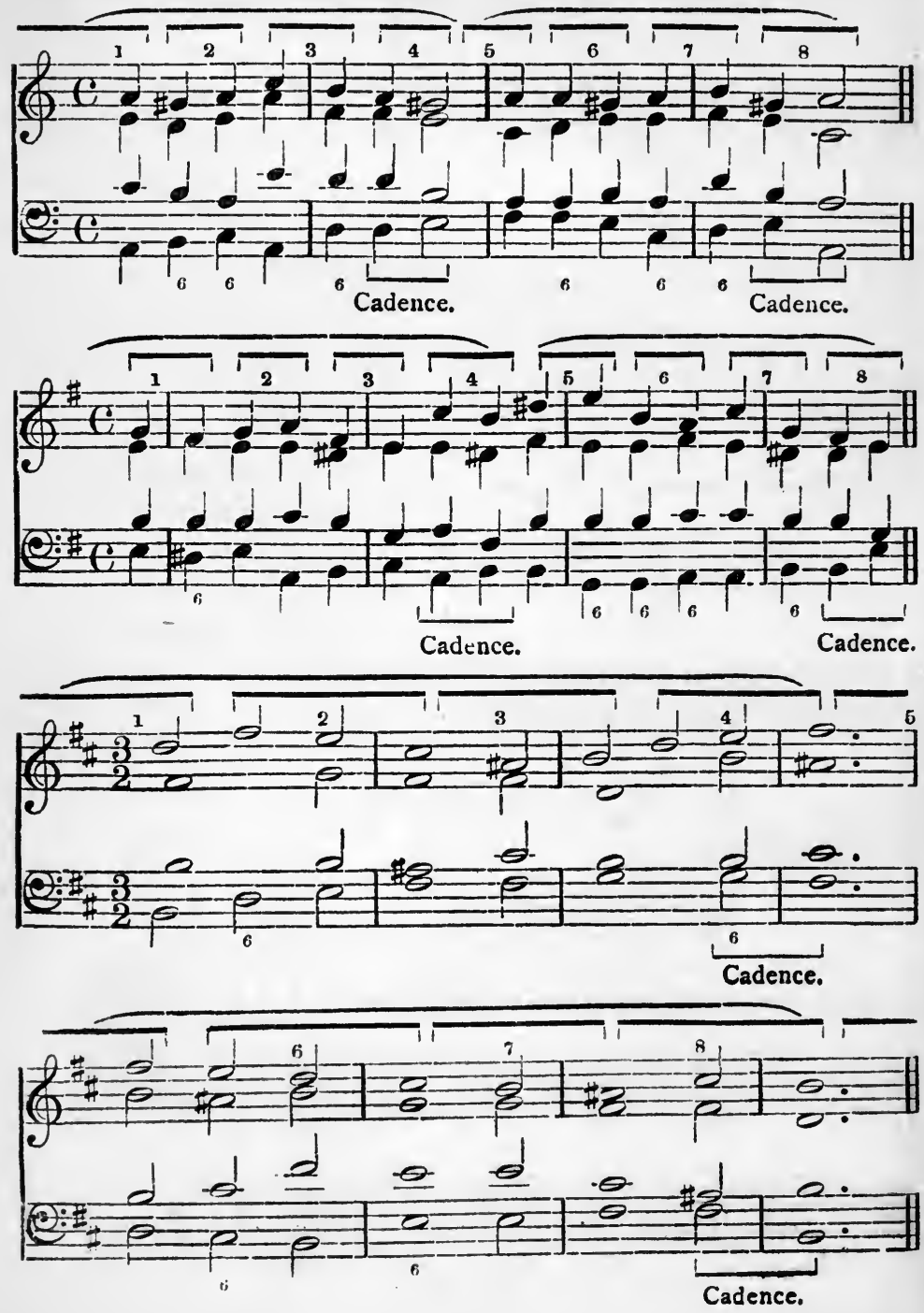

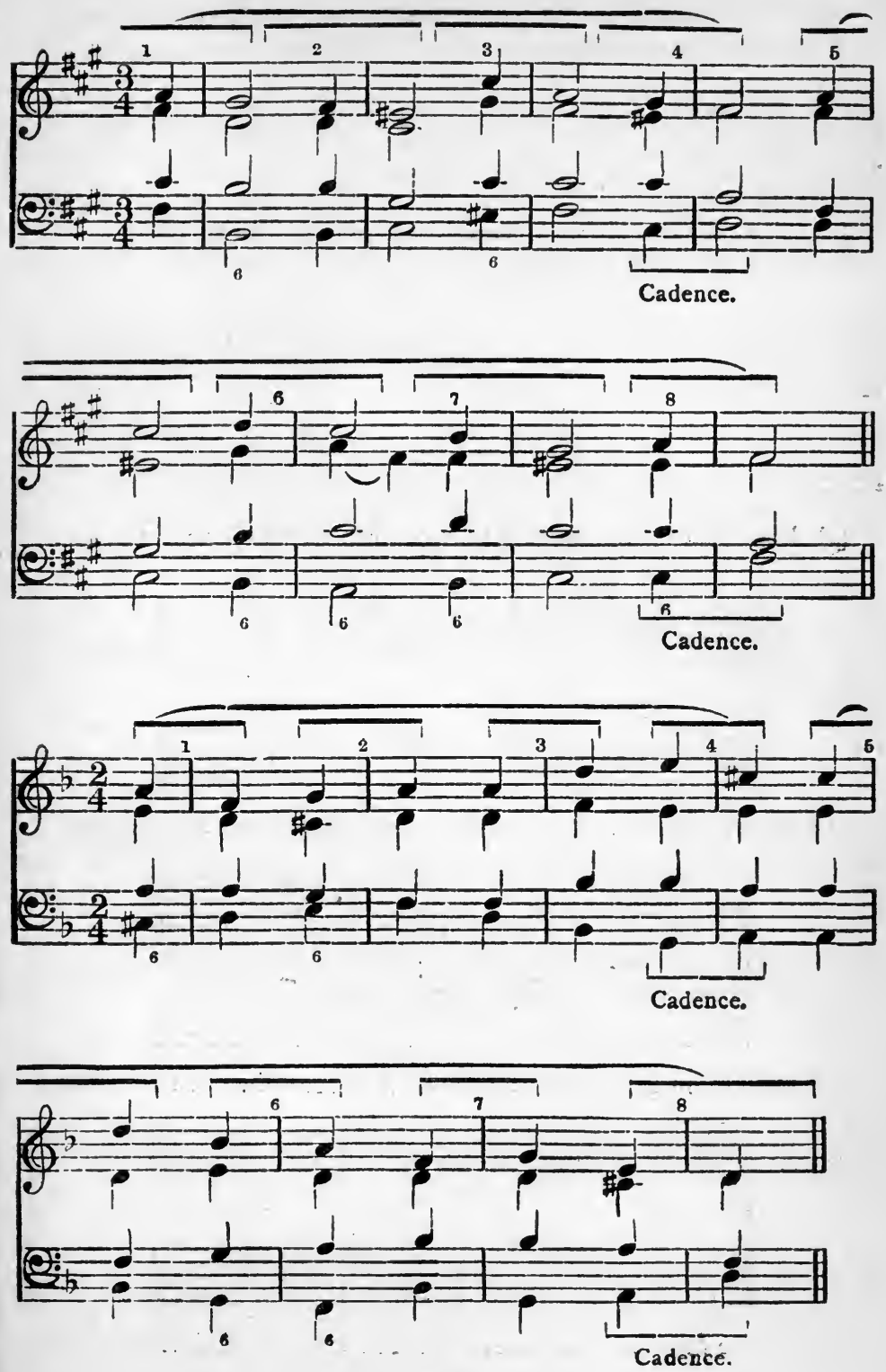
(3) The following first inversions derived from the melodic minor scale require notice.

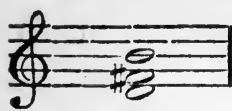

1.

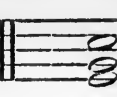

2.

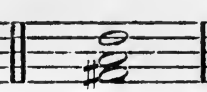

3.

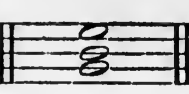

4.

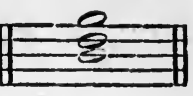

5.

(I) Should only be used when the melody demands it.

(2) May be used as follows:-

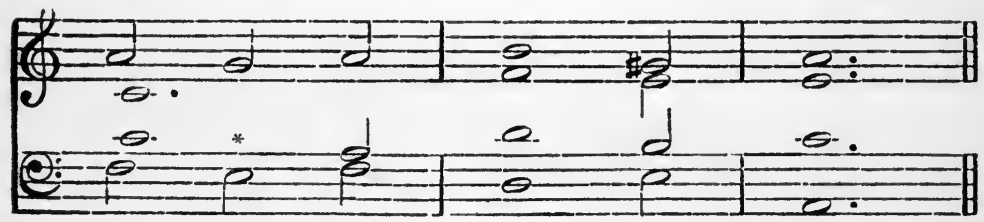

(3) May form a harmonization of an ascending scalic bass.

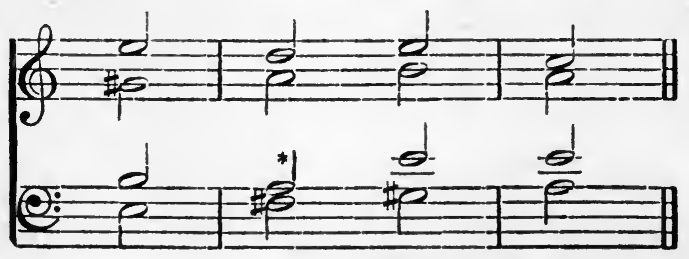

(4) May form a harmonization of a descending scalic bass.

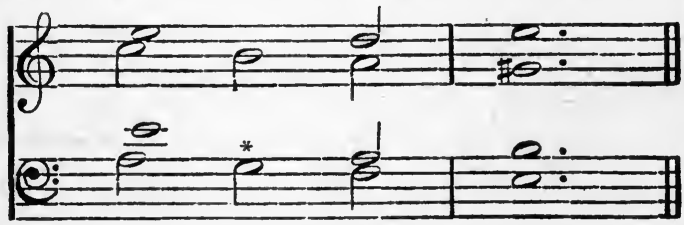

(5) May form a harmonization of a descending scalic treble.

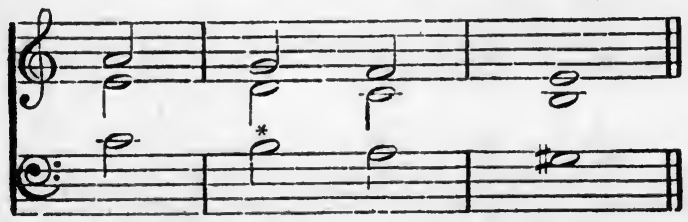

The above are the best uses of these chords, and students are 
recommended to confine themselves to them in the earlier stages of their work.

\section{EXAMPLES.}
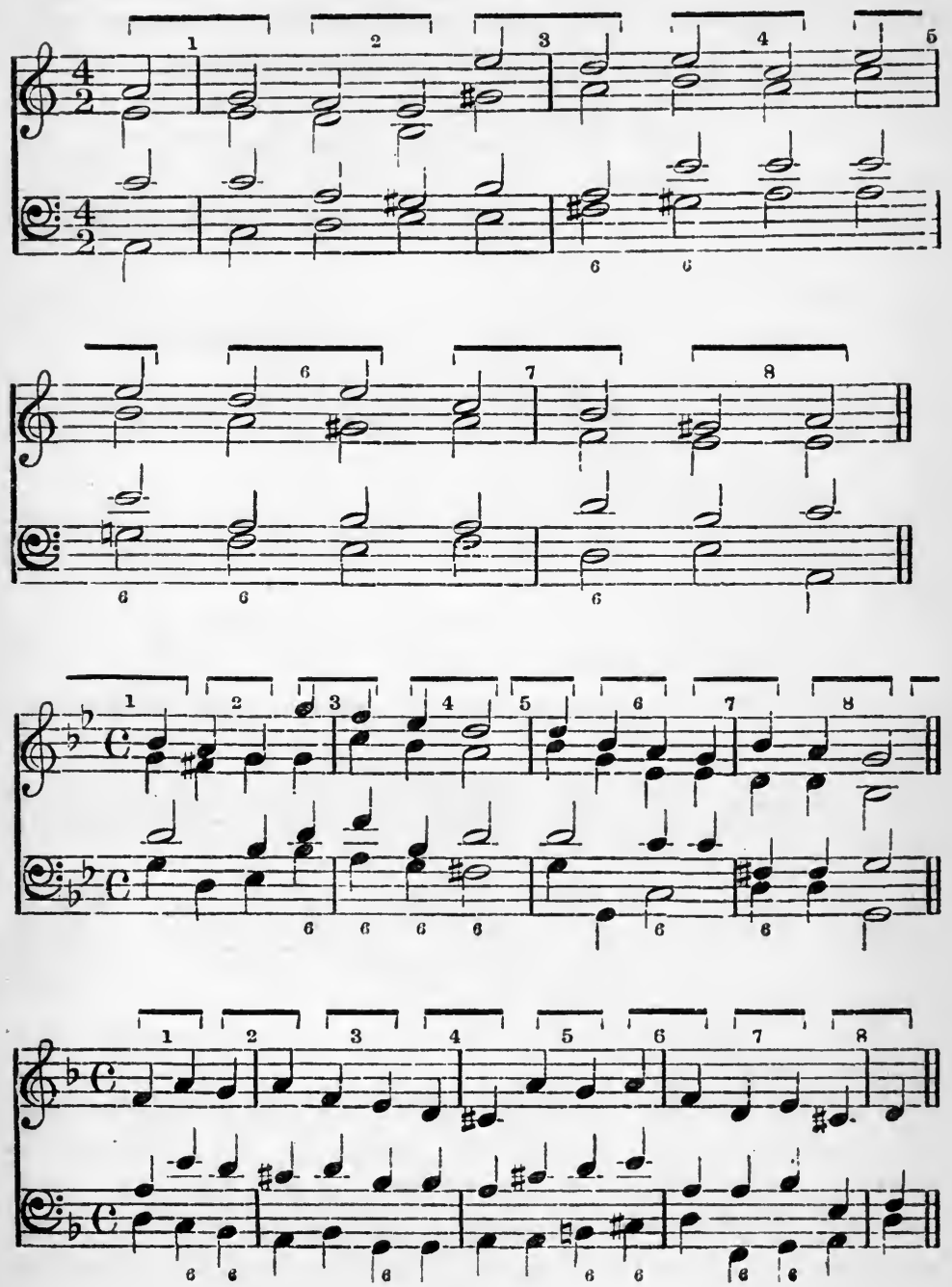


\section{Section V.}

The use of the Chord of the six-four.

It has been shown that the combination which moderns analyse as the chord of the six-four often occurs under strict contrapuntal conditions, either as a suspension, or as an unessential harmony on the weak accents of the bar. It is hardly necessary to say that as these uses are the foundations of modern procedure, they are by far the strongest.

(I) Examples which are legitimate under strict contrapuntal conditions.

(a) Suspensions.

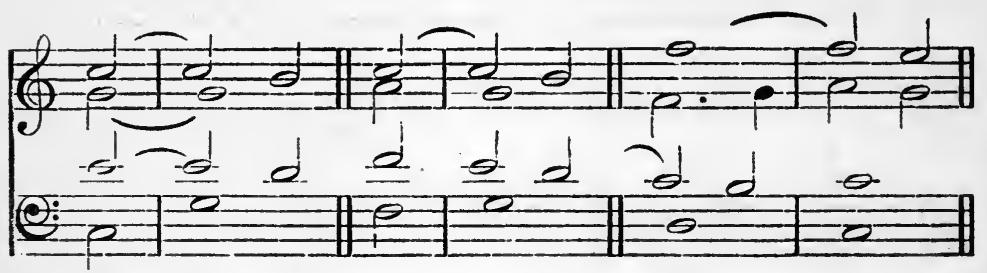

Here we have the origin of the cadential six-four.

It should only be used on I. and V.

It is obvious that the following are contrapuntally inaccurate.

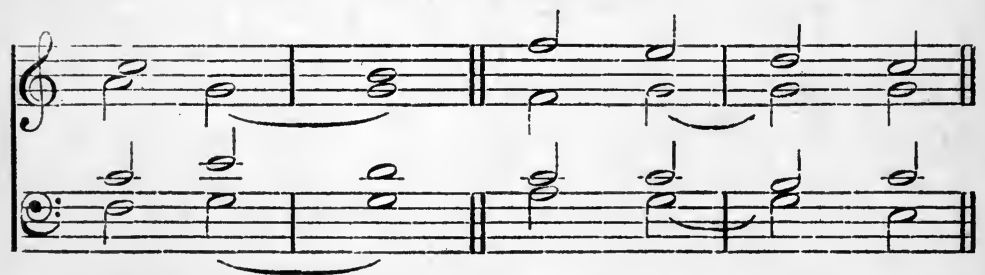

Hence we get the rule that the cadential six-four must occur on the strong accent.

This also introduces another important point :-repeated notes are not good in the bass in the relation of $v$ to -, unless the previous - has borne the same note (as has been previously stated) or unless such note be a suspension or a prepared discord. 

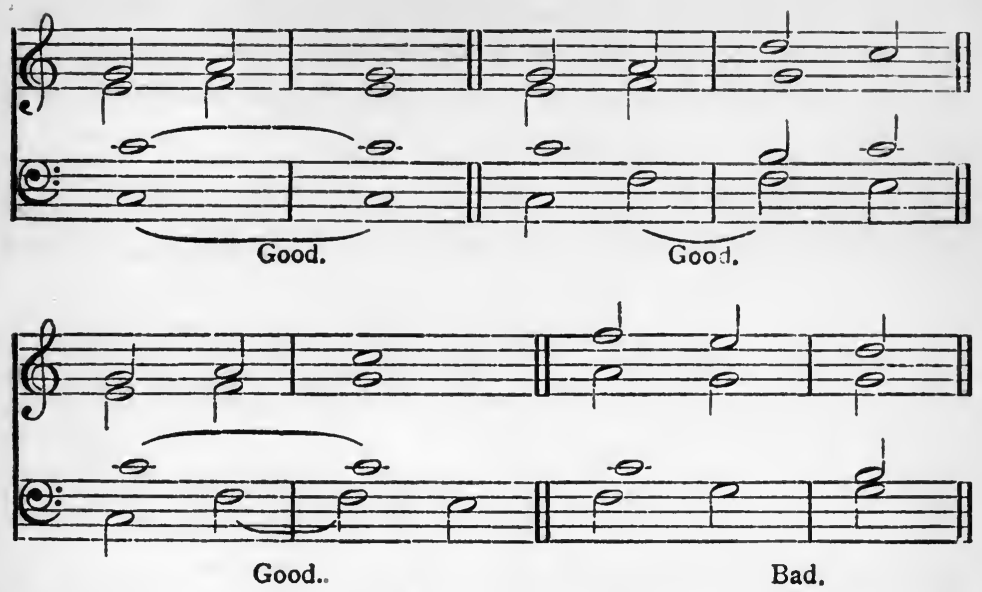

(b) Over a pedal, the lowest moving part being the contrapuntal bass for the moment.

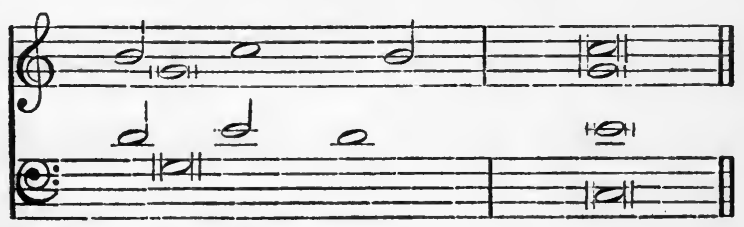

(c) As an unessential harmony.
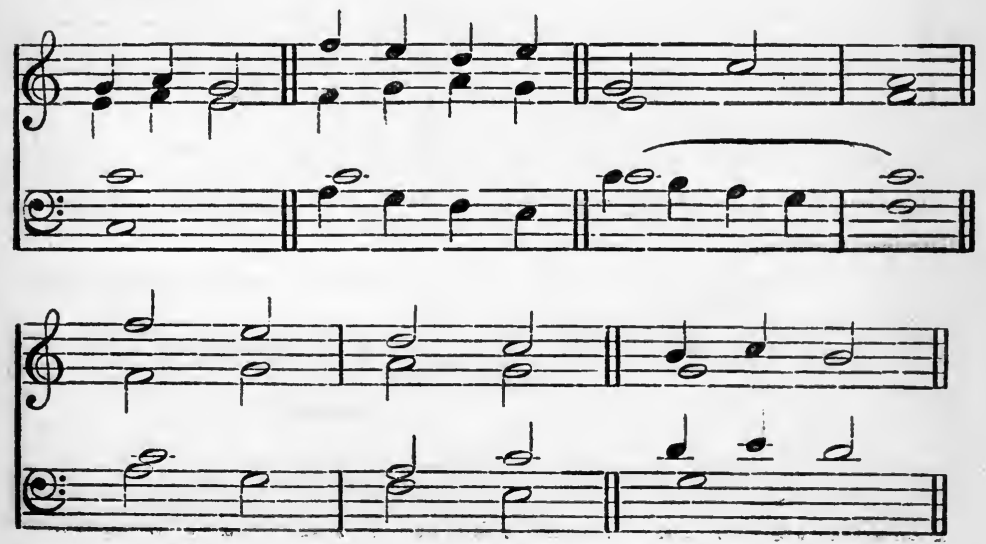
But what may be termed the auxiliary six-four should not be used on II. IV. and VII. for obvious harmonic reasons. If the contrapuntal criticism be applied to the following, it will at once be seen why the effects are crude.
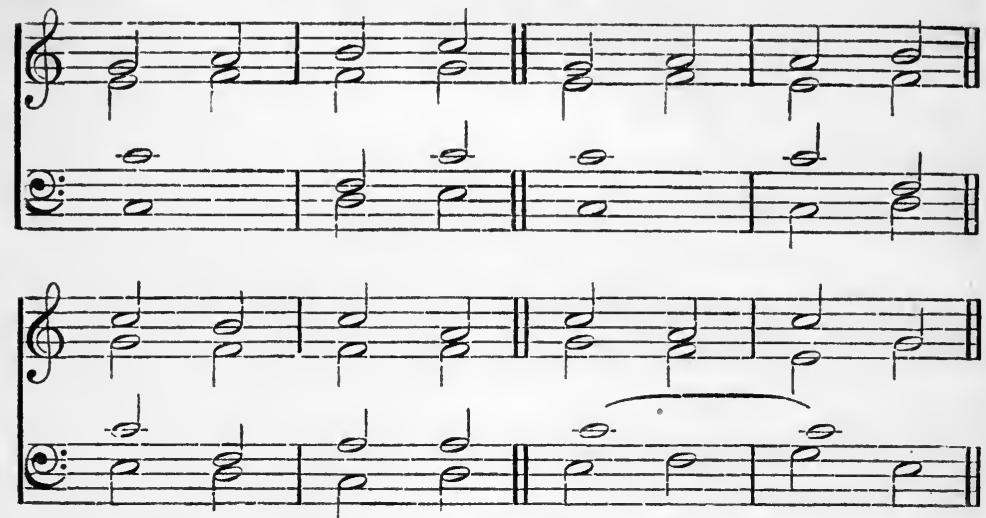

All weak.

(2) Examples in which the fourth is not prepared, though occurring on the strong accent.

From an aesthetic point of view, both of the following are equally satisfactory :-

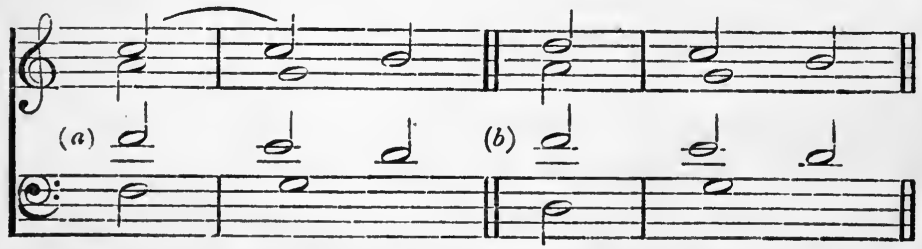

Formerly, the sixth and fourth in (b) were often written as appoggiaturas.

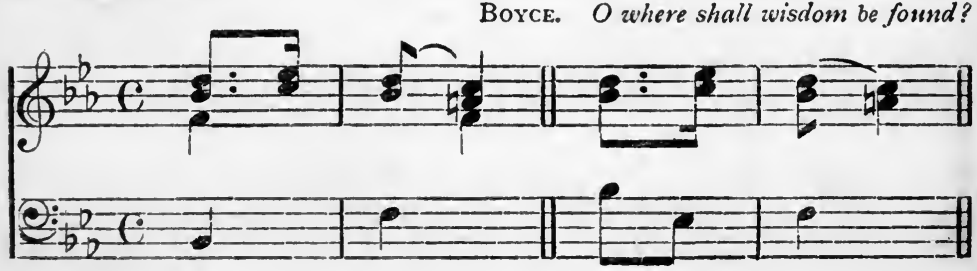


And this of course indicates the true nature of a cadential six-four.

Thus in scanning a Feminine Cadence formed in this particular way, the following is the explanation of the seeming irregularity.

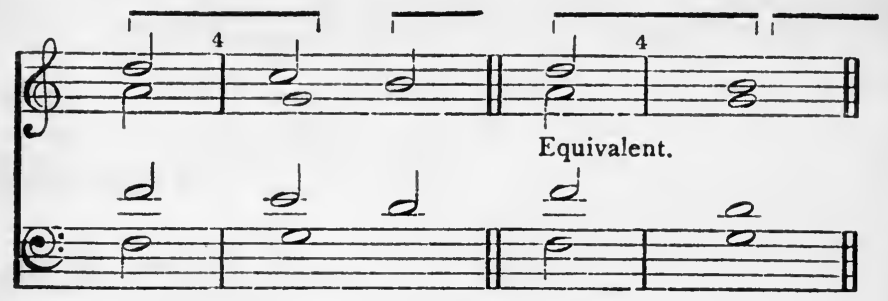

\section{EXAMPLES.}
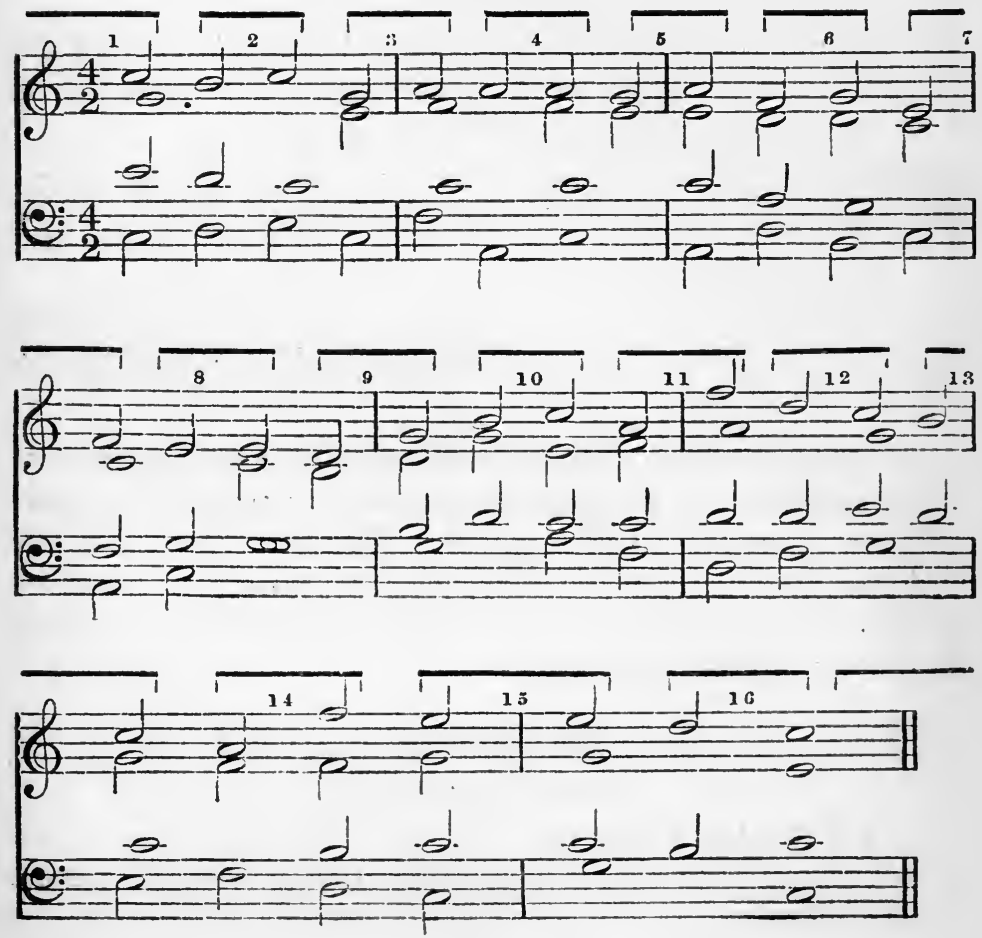

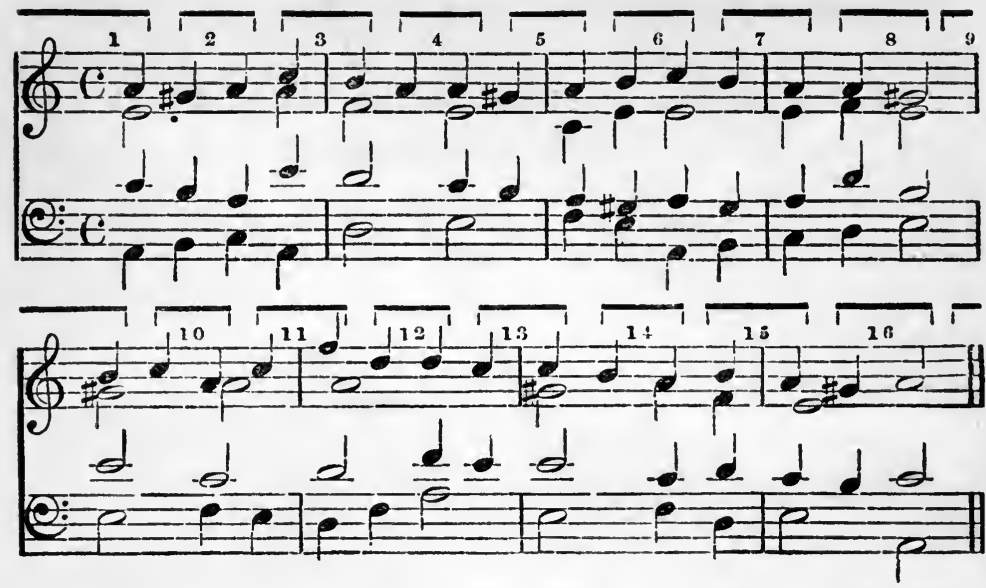

It is an essential of good style that the chords forming the Cadence should not be used in the same order immediately before the Cadence, causing harmonic redundancy.

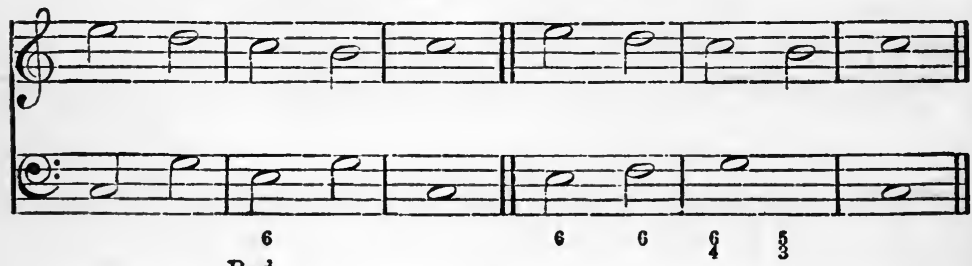

Bad.

This discussion has covered the elements of chord progression, or the connexion of chordal centres, and to a certain extent the question of proportion and balance. But matters of this nature lie beyond the scope of this work, and it remains to study the use of chords of the seventh before finally considering the modern decoration of chordal centres.

\section{Section VI.}

Chords of the seventh.

(a) The dominant seventh.

The student will easily analyse the following from a contrapuntal standpoint. 

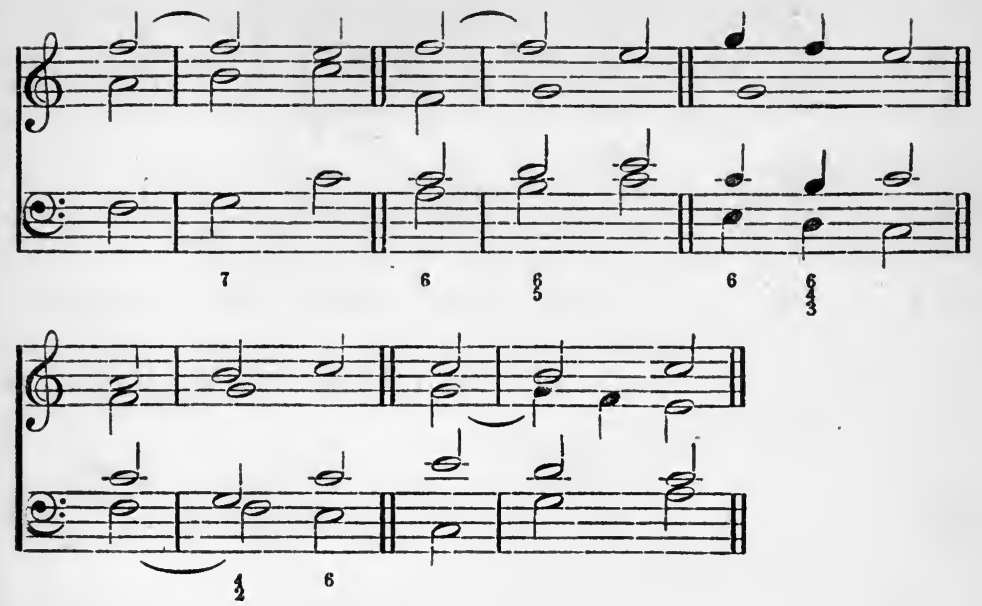

The second inversion ${ }_{3}^{6}$ is best used in cases where it is capable of contrapuntal analysis.

The other inversions follow the same rules as $\mathrm{V}$. with the seventh added: the seventh may or may not be prepared.

(b) Secondary sevenths.

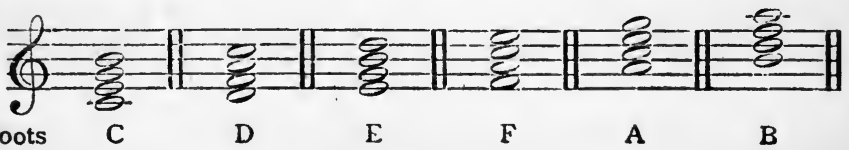

In using these-

(I) the seventh must be prepared.

(2) the root of the next chord must be a fourth higher than the root of the secondary seventh.

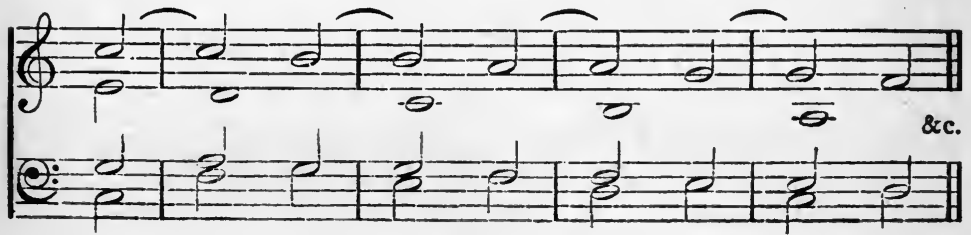




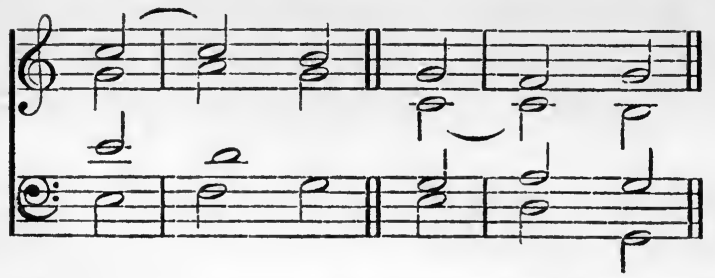

This technique is of course directly applied from contrapuntal methods.

The third of the first chord may remain to be the seventh of the next.

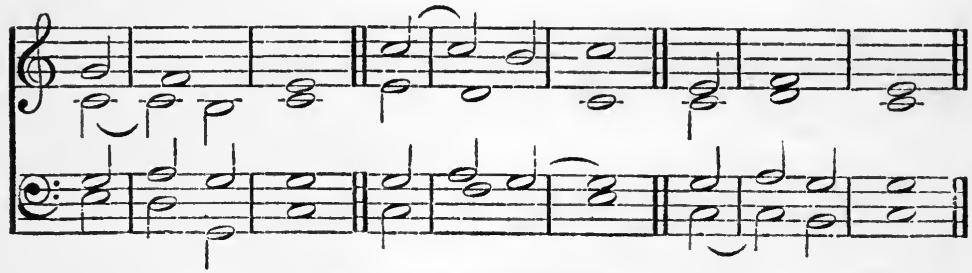

Examples. The dominant seventh.

(I) Prepared, or unessential (the preparation need not be on a weak accent).

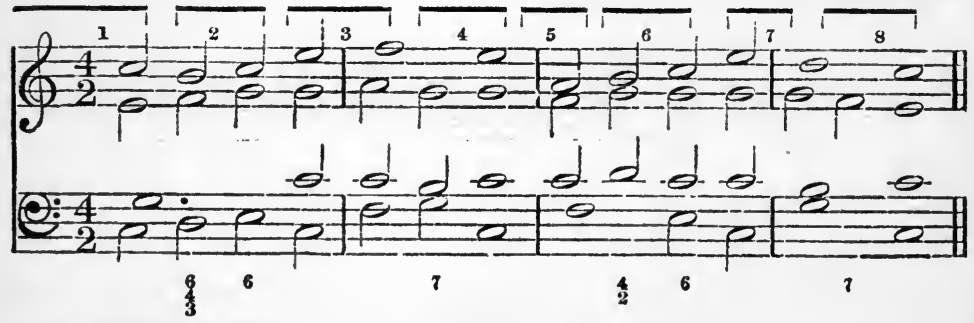

(2) Unprepared.

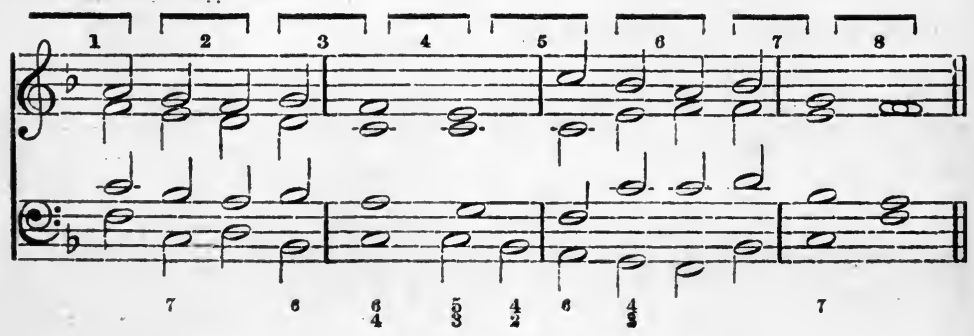




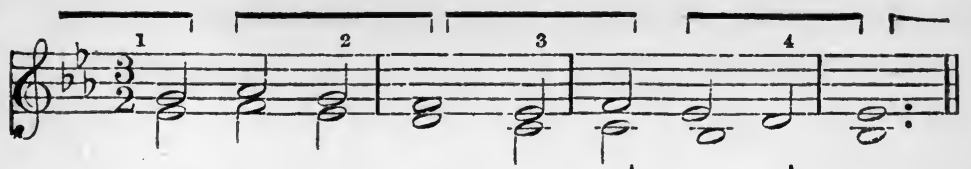

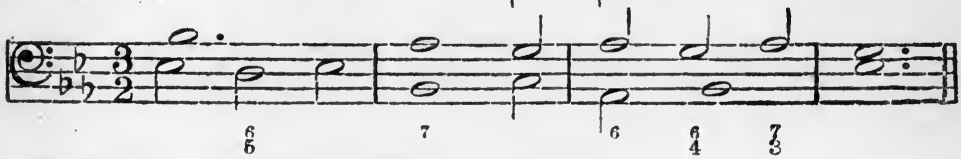

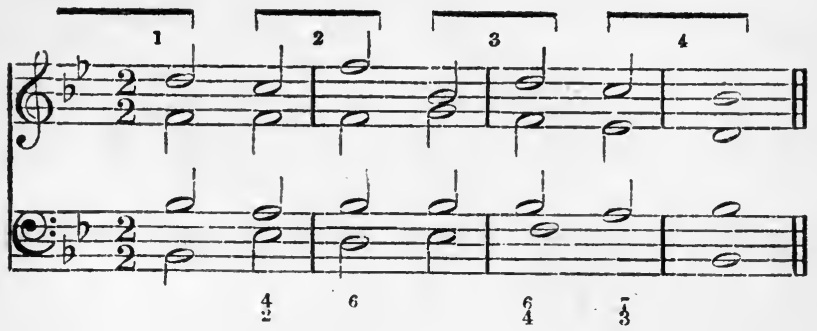

Note, in all cases where the seventh is unprepared, "it stands in place of the root.

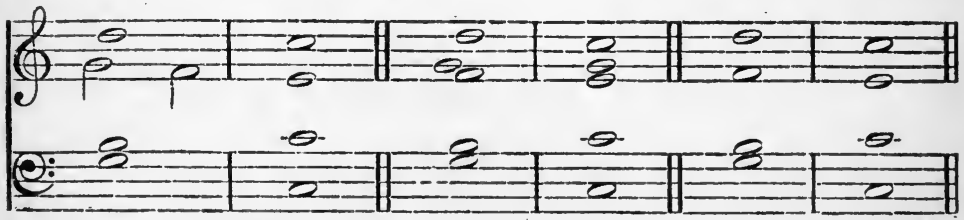

Secondary sevenths.

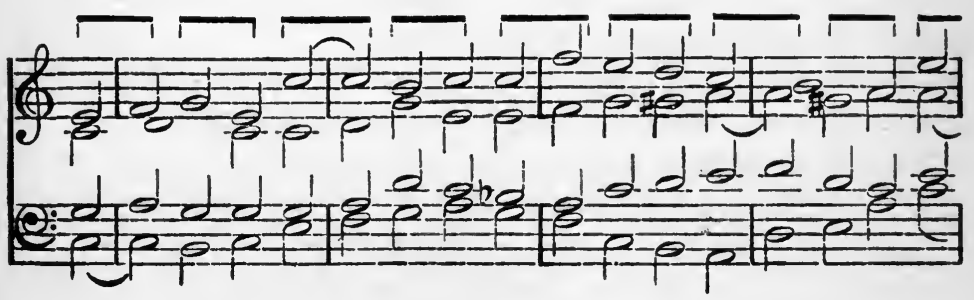



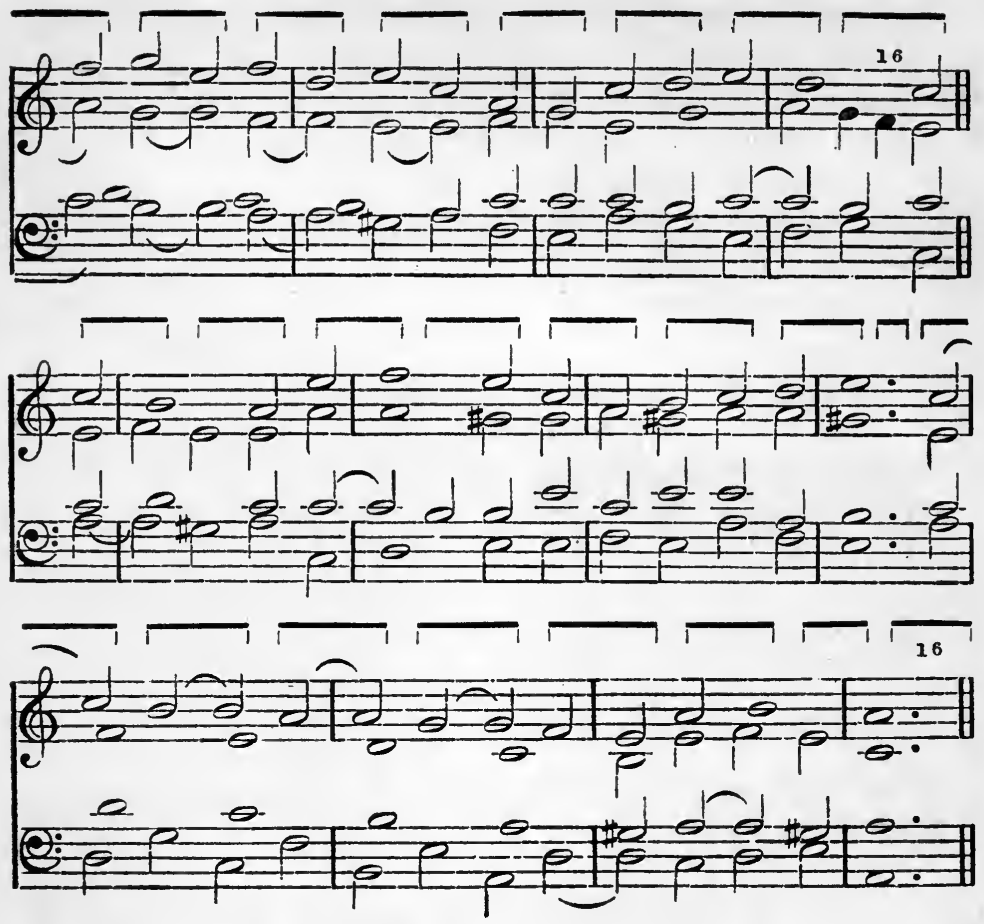

\section{Section VII.}

Key Distribution.

As a rule no sentence remains in the same key throughout. We have discussed the elementary principles of balance and proportion so far as the chordal centres themselves are concerned, and we have now to consider the principles which govern the use of variety of tonal centre.

We shall employ the form invariably used in short sentences-Binary, which consists of two equal halves.

Say, for example-bars I -8

$$
\begin{array}{r}
9-16 \\
\text { or } \begin{array}{r}
1-4 \\
5-8
\end{array}
\end{array}
$$


The first half establishes the key, and modulates, either directly or through some other key, to the complementary key, which should always be on the sharp side of the tonic, or at least not on the flat side; here we should have a full close in such key.

The second half begins, often with a reference to the opening bars of the first half, with frequent modulation and sub-phrases, leading gradually back to the tonic.

Nearly all the questions set in modulation should be worked on these lines. The plan then is-

First half. Tonic: modulating to the complementary key (with occasional subsidiary modulation).

Second half. Modulating back to the tonic.

In the following examples, the letters A, B, C, \&c., are used to indicate melodic formulae. The numerals added to the letters indicate sections. If a letter be repeated, it signifies that the formula "is repeated. This introduces the most important matter of melodic form. All that can be said here is that an observance of the principles of melodic form is an absolute necessity, if the student wishes to write intelligible music. How these principles are applied in decorated work will be seen in the next chapter.

A r.

A 2

B 3.

$\mathrm{C}_{4}$.

D 5.
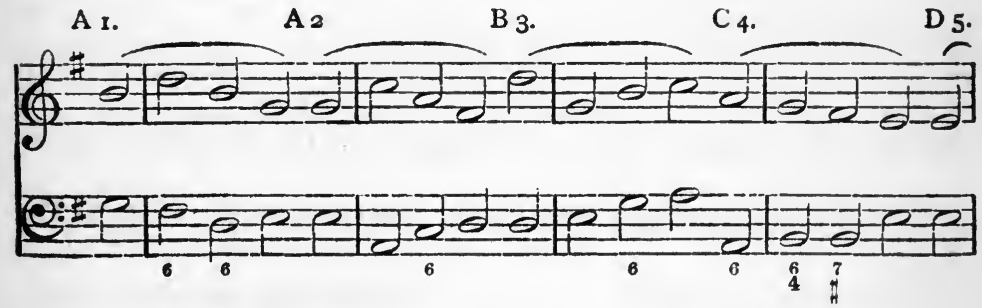

D 6.

A 7.

C 8.

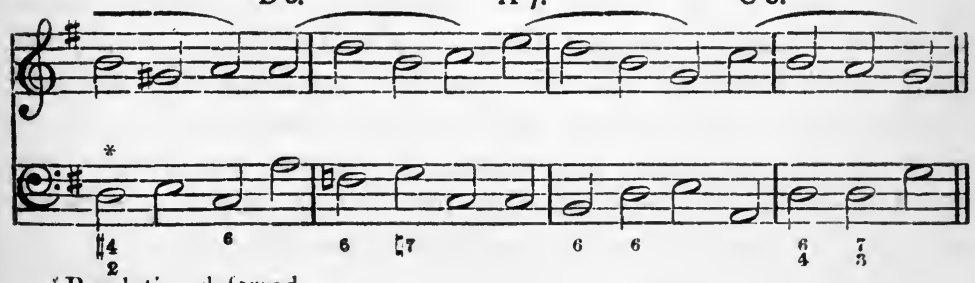

"Resolution cieferred. 
As.

A 2.

$\Lambda_{3}$.

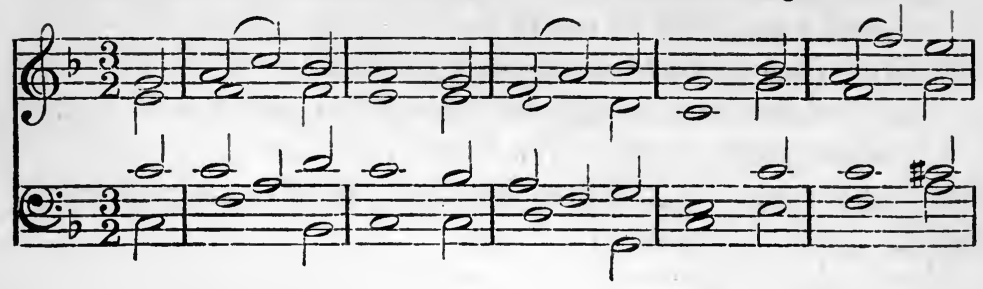

$A_{4}$.

B 5 .

B 6.

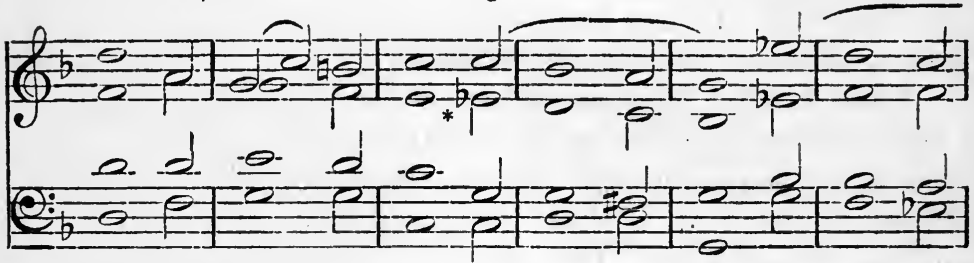

$\mathrm{C}_{7}$.

D 8.

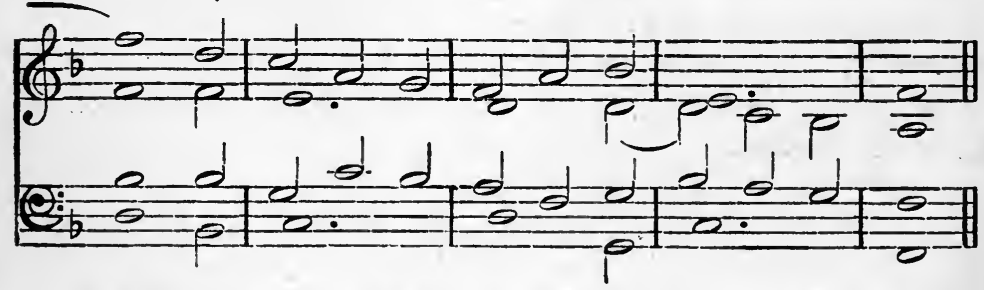

This at the end of one phrase and the beginning of the next is always good.

The repetitions at the start make variety desirable towards the close.

A consideration of the means or varying the rhythmic structure of the normal sentence is beyond the scope of this work. Nothing is more inartistic than the maintenance of a 'square and rigid' rhythm; but the power of avoiding this involves a knowledge of the normal structure, and it has been our aim in this treatise to foster this sense of balance and-proportion. Students who are interested in the matter will find it.worth while to study the following from this point of view :-

Mozart, Ave Verum. 
Church Music Society Reprints, No. I (Communion Introits by Vittoria and Pearsall).

Parry, 'God breaketh the battle' (Judith).

" 'Forward through the glimmering darkness' (War and Peace).

Further information will be found in Prout's Musical Form, chapter viii. 


\section{CHAPTER XVII}

\section{THE APPLICATION OF COUNTERPOINT AS A DECORATIVE PRINCIPLE}

IT is claimed that if Scholastic Counterpoint be studied on the lines indicated in this treatise, the student will have at his command a great deal of the technique of vertical decoration.

The following points must be clearly understood :-

(I) The C.F. ceases to be a fixed part of uniform length. It can be either the implied chordal centres, or a florid part based on these. In either case it must be remembered that every strong and weak accent is a chordal centre, and that we regard every group of accents $-v$, or $-v v$, as a bar of Counterpoint.

Time signatures are of no value in helping to find the centres. Thus the following:-

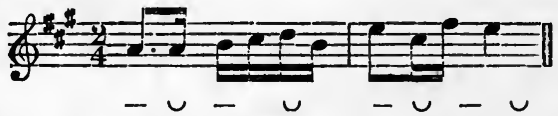

is really in ${ }_{8}^{4}$ time, that is every bar contains four accents, and therefore the scholastic analysis is :-

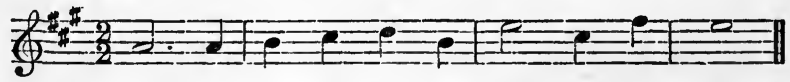

The time value of the centres is generally that of the first chord of the Cadences.

(2) The strict contrapuntal idiom gives place to the free modern style, both in the melodic rhythm, and in the treatment of unessential notes.

(3) The chordal centre may be any modern classified chord.

(4) Just as in Scholastic Counterpoint it would be said that the prevailing harmony in the following bar was that of the chord of $\mathrm{F}$ major, though from the modern point of view we use four distinct harmonies :- 


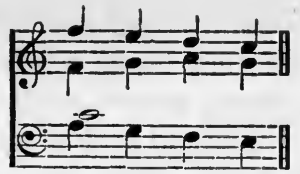

So the chordal centres of the following :-

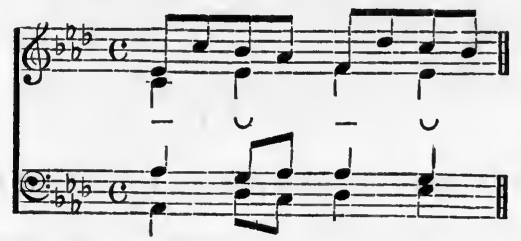

(a)

(b)

are :-

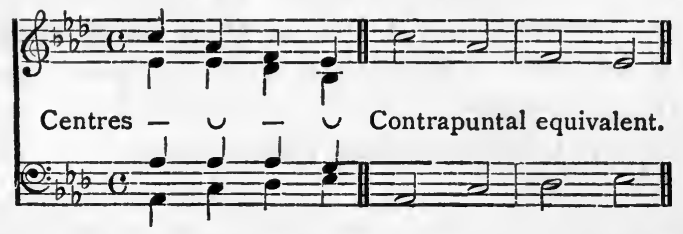

$(a)$ and $(b)$ may be regarded as harmonized accented passing notes, which, however, do not disturb the predominating effect of the centres. A few further examples will make things clear.
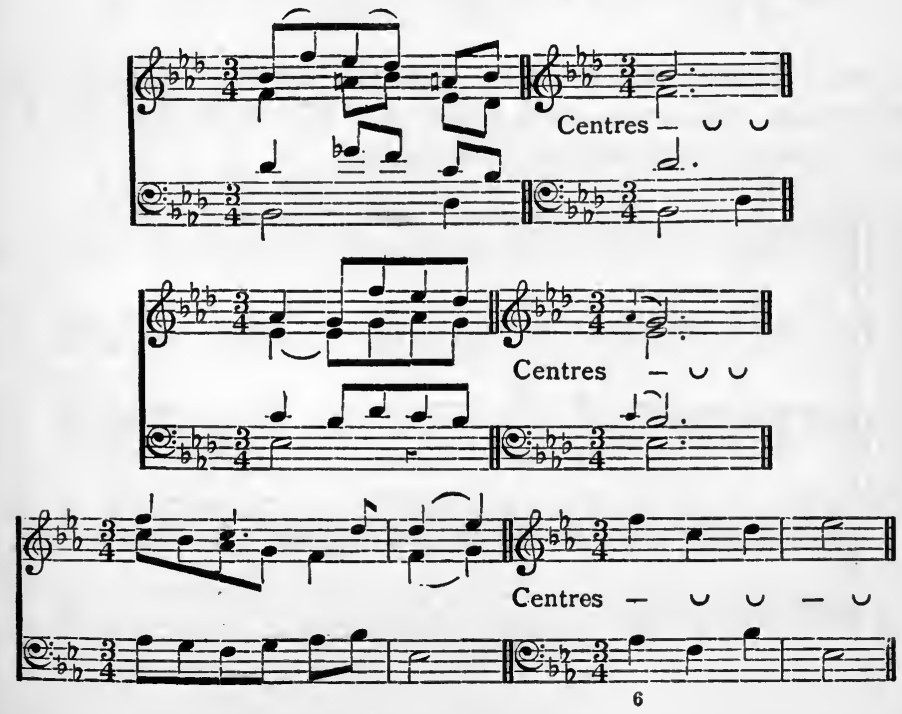
(5) The principles of the technique of Combined Counterpoint apply absolutely. There must be no crude, obscure, or confused harmony; and the harmonic seams must be satisfactory. The previous chapters have explained how to avoid the various pitfalls of elaborate texture, so we proceed at once to the working of problems.

\section{Section I.}

The adding of Florid parts to a given Bass.

We first take one of the simplest cases, that in which the bass consists of notes of uniform length. In the following problem (Ground Bass, Third Mus. B. Oxon., May, 1905), each bar contains three accents. Thus every bar represents one bar of Scholastic Counterpoint.

Add four parts to the following Ground Bass.

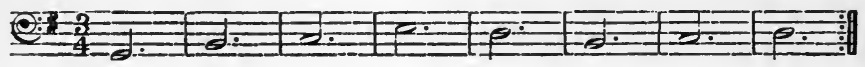

Scholastic equivalent.

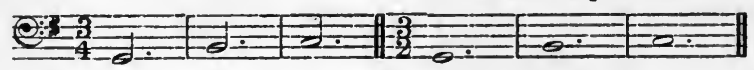

It must be remembered that accented passing notes, and all like modern resources, may be used. We write the first two bars in terms of Scholastic Counterpoint.

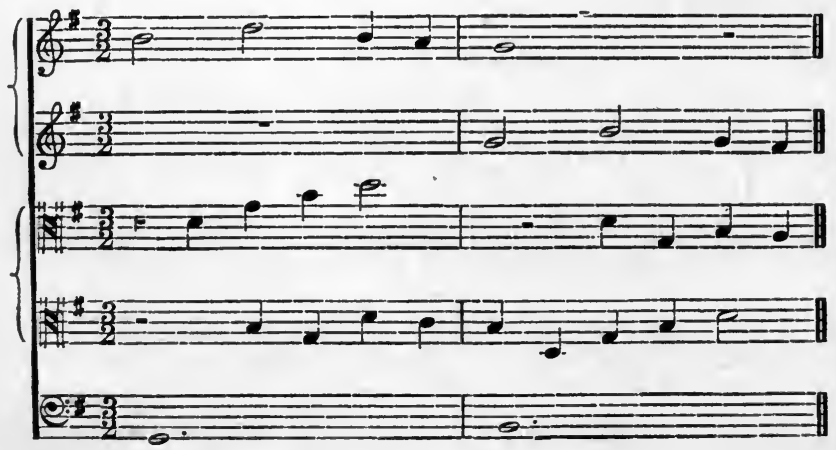


The following is the example worked out.

Ground Bass, Third Mus. B. Oxon., May, Igo5.
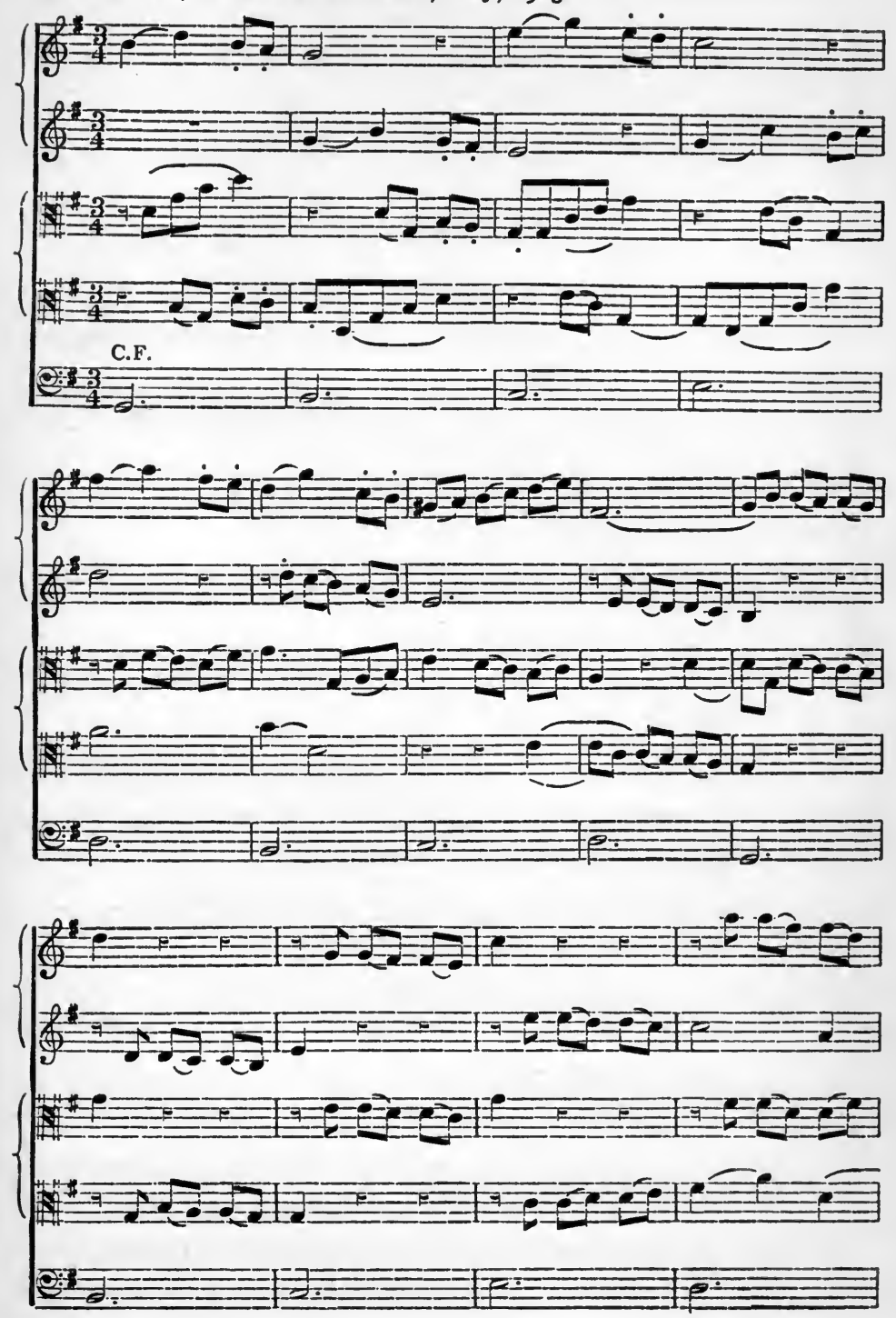

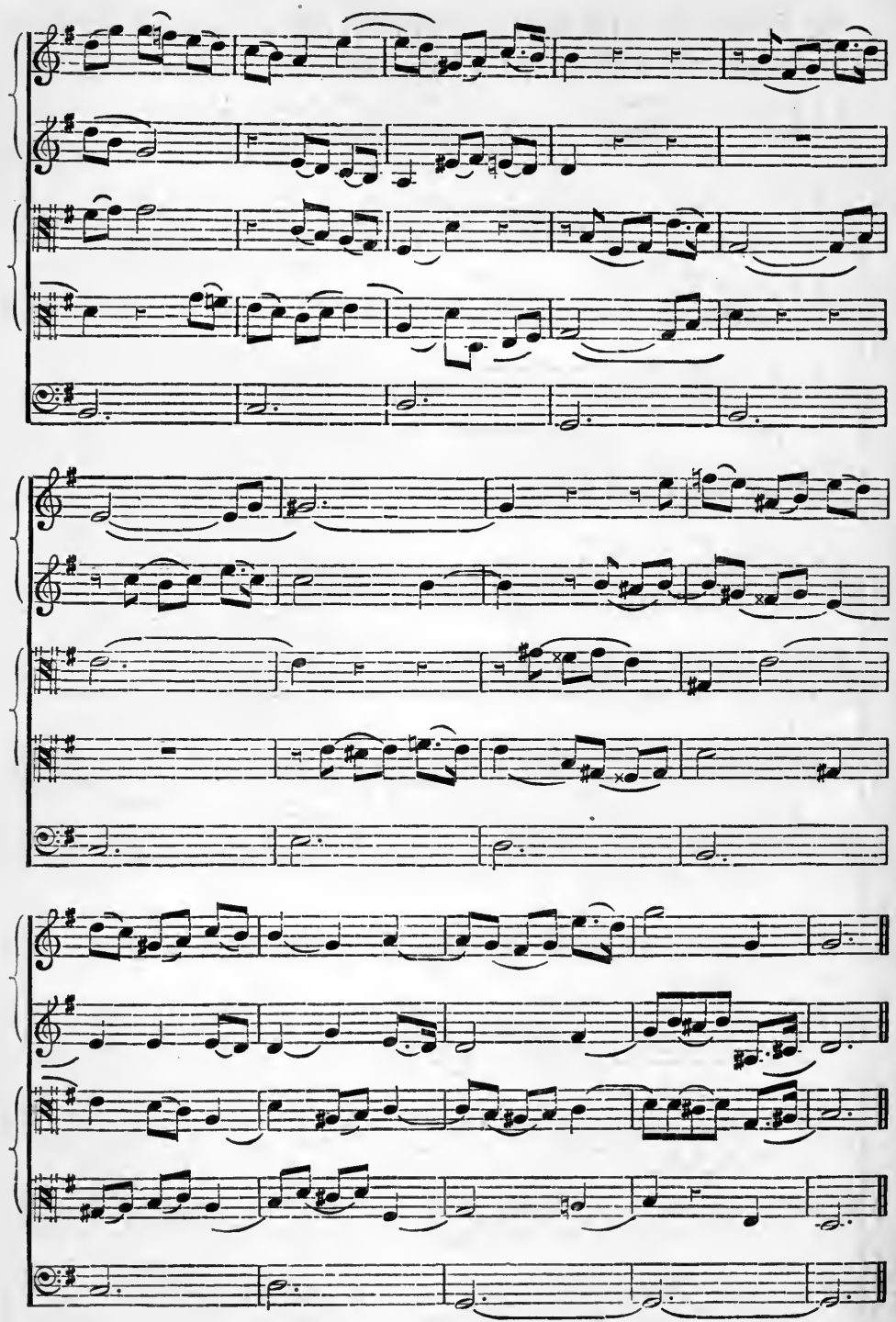

Students should read Purcell's examples of the Ground Bass. They should of course be acquainted with Bach's Passacaglia in 


\section{APPLIED COUNTERPOINT}

C minor for the organ. There is a most ingenious example in Stainer's Six Pieces for the organ (On a Bass.)

The following may also be studied :-

Bach, Crucifixus. Mass in B minor.

Handel, 'Envy, eldest born of Hell' (Saul).

Handel, 'O Baal, monarch of the skies' (Deborah).

Brahms, Finale. Variations on a Theme of Haydn.

Dr. C. Wood, 'On Time.'

Arensky, Basso Ostinato.

In the next example each minim is equivalent to one bar of Scholastic Counterpoint.

Add three parts above the following bass :-
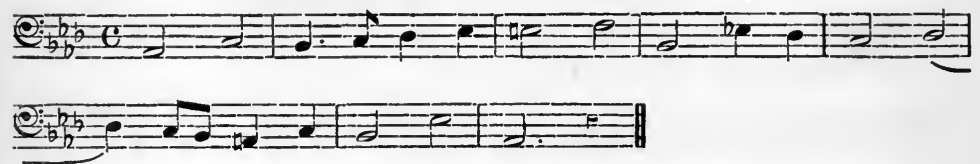

The question of melodic form cannot be discussed here, but the student will see the principle of thesis and antithesis, or statement and response. The letters serve to draw attention to the matter.

The student is recommended to write out some examples in scholastic form.

One bar is given in this way.

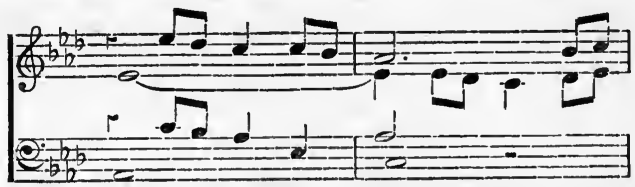

The following are the centres.

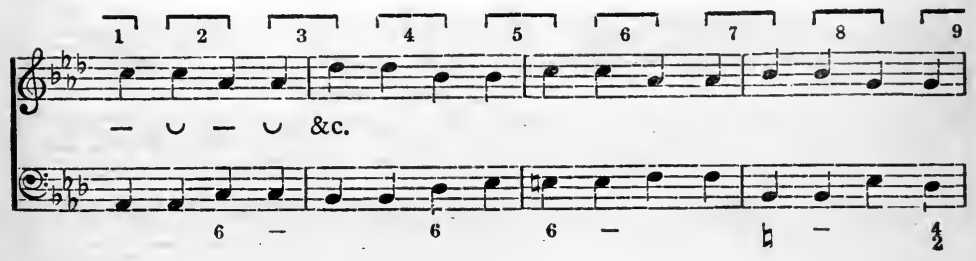




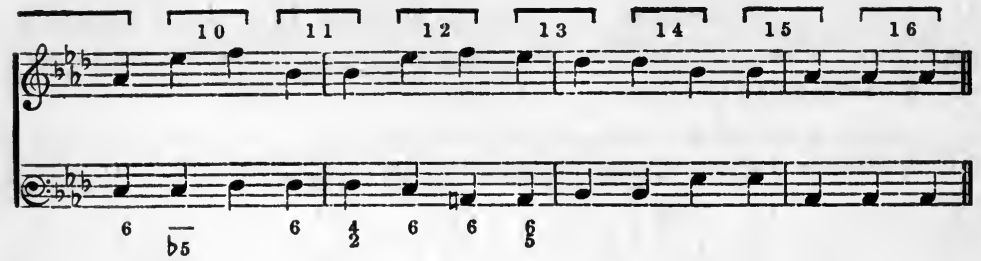

Equivalent in Scholastic Counterpoint.

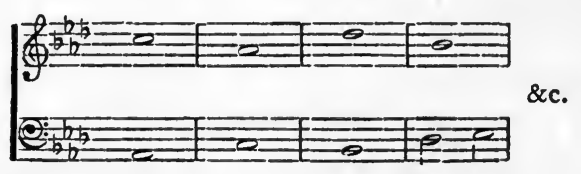

The example worked out:-

First Mus. B. Oxon., Nov., 1903.

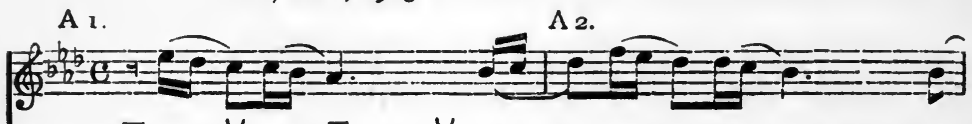

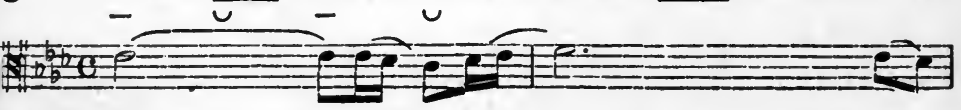

$4+\frac{b}{2}$ C.F.

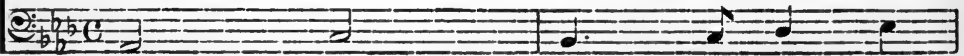

$\mathrm{B}_{3}$.

$\mathrm{B}_{4}$.

C 5 .

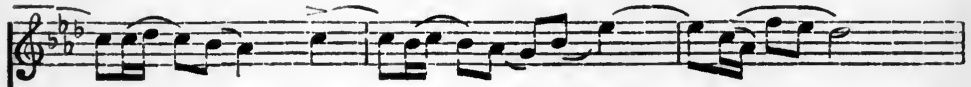

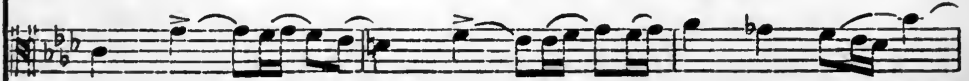

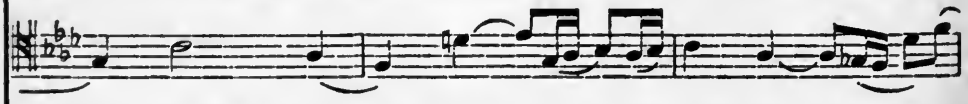

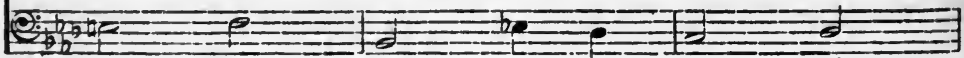




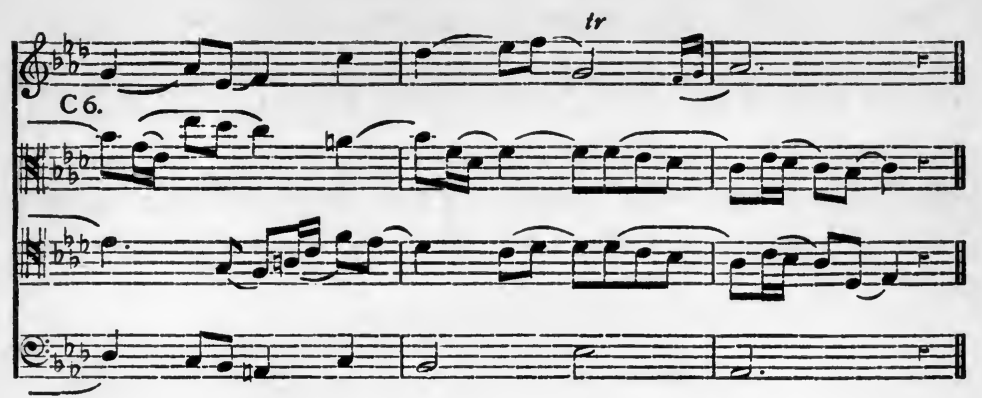

Section II.

The adding of parts to a given melody.

The given part must be reduced to chordal centres, by elimina. ting all unessential notes, then the synthetical process must be adopted, building up a florid texture from the centres.

The melody is first given with the centres indicated in the bass. The figuring only indicates the essential harmonies.

Third Mus. B. Oxon., May, 1905 .
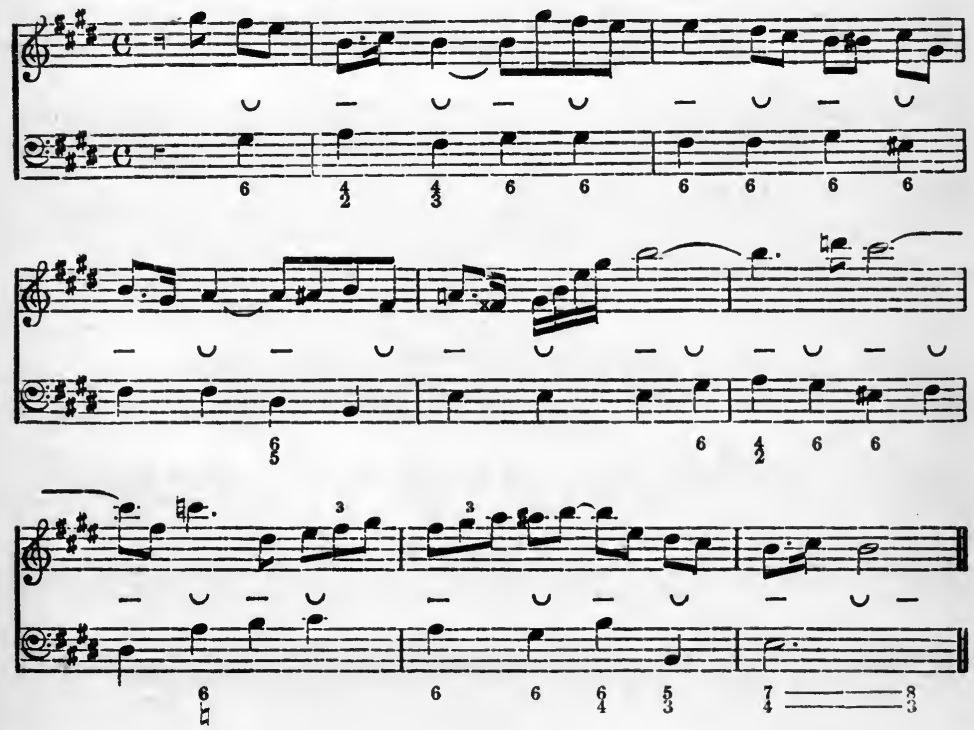
Each half bar represents a bar of Scholastic Counterpoint. Note that there will be frequent changes of harmony on the second half of the bar, and further, that passing notes may be harmonized as passing chords, for if the chordal centres have been correctly deduced, passing chords do not in any way disturb their effect. Two bars are given in the form of Scholastic Counterpoint.

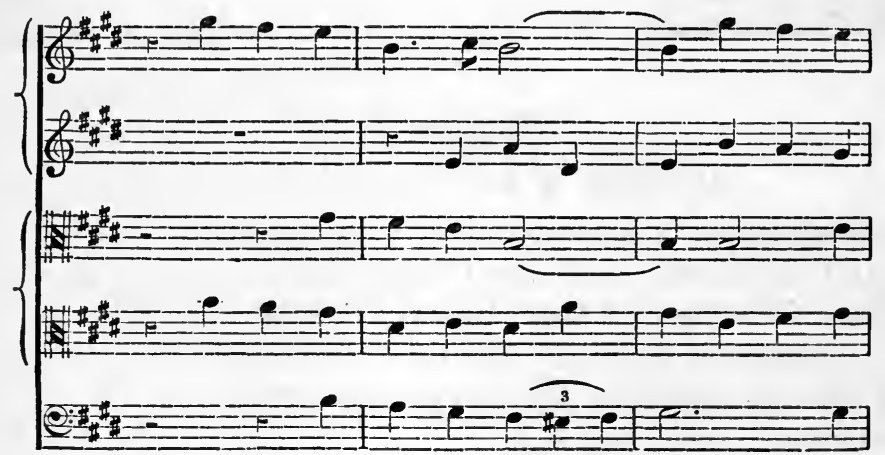

The example worked out:-

Third Mus. B. Oxon., May, rgo5.

Moderato. C.F.

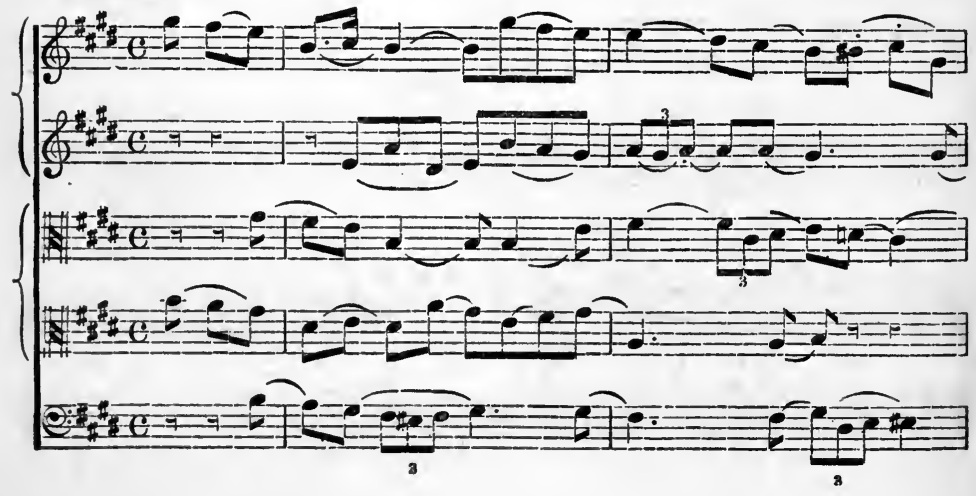



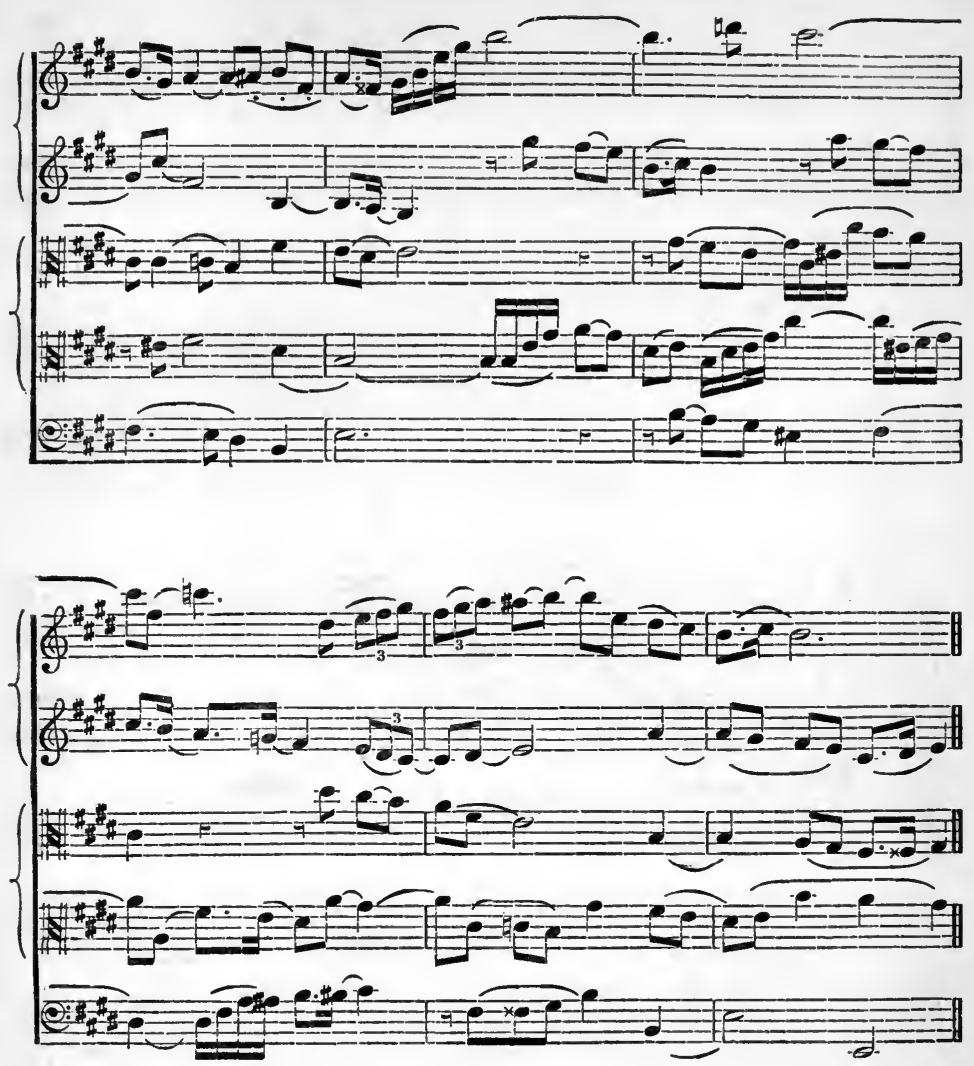

In the next example (Third Mus. B., Nov., 1905) the chordal centres are quavers, that is, each half bar represents a bar of Scholastic Counterpoint.

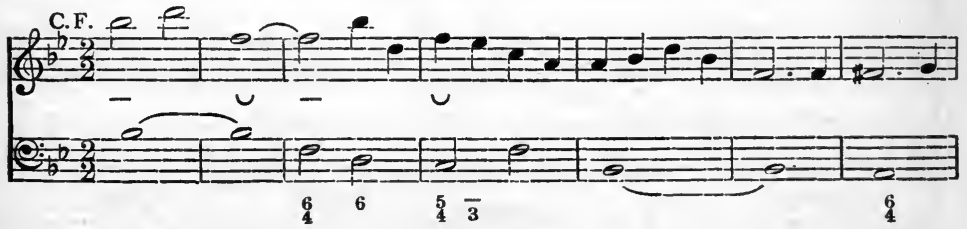


tone

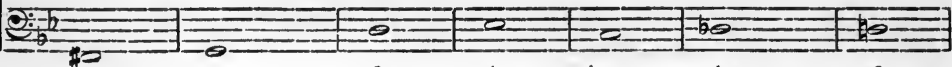

6

6

$b^{b} 6$

b5

$b_{5}^{6}$

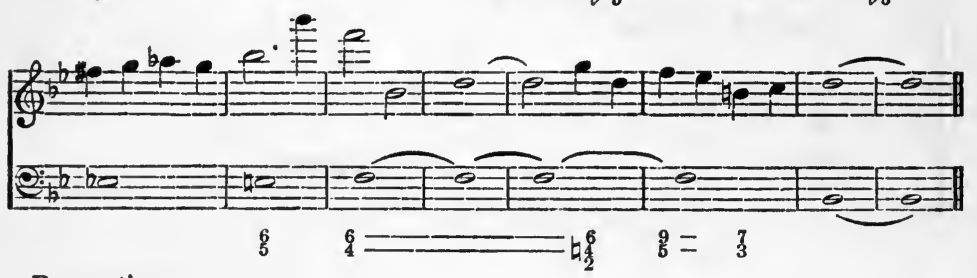

Decoration.

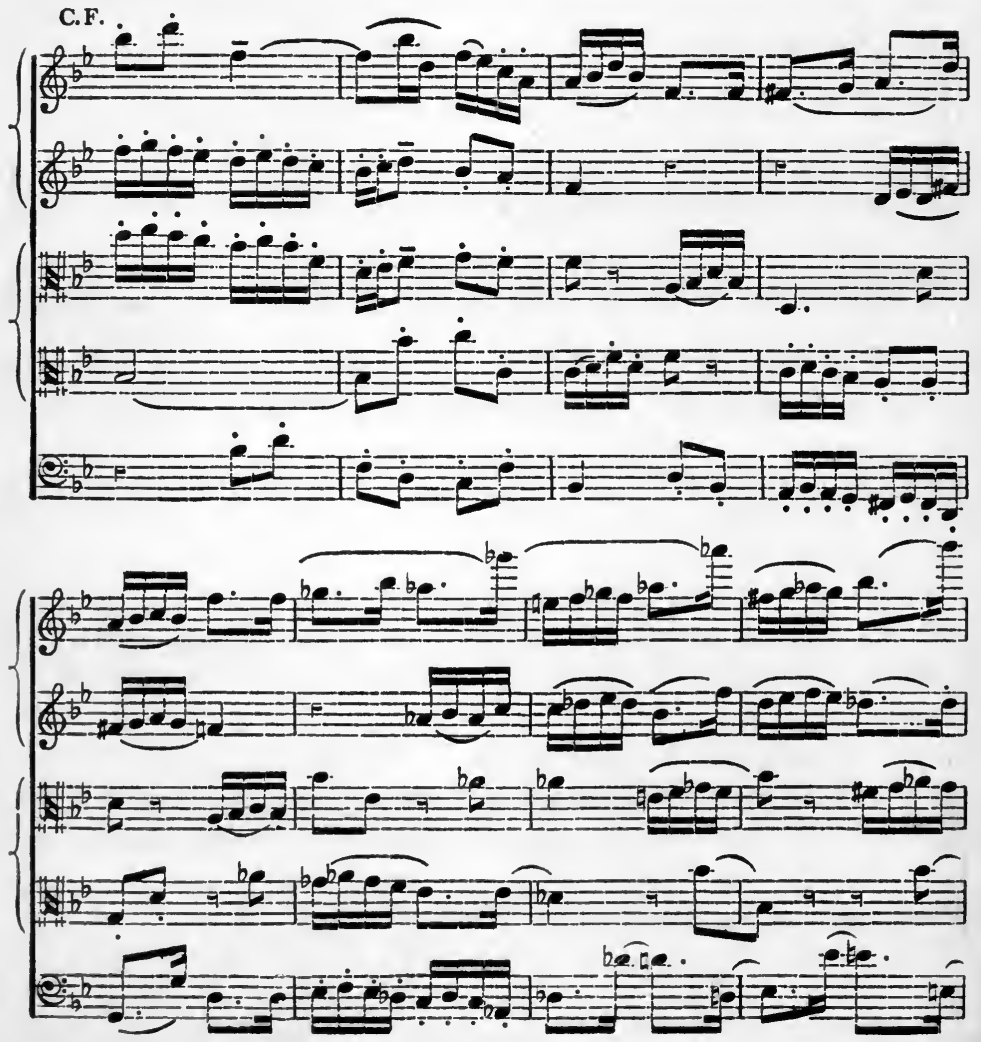




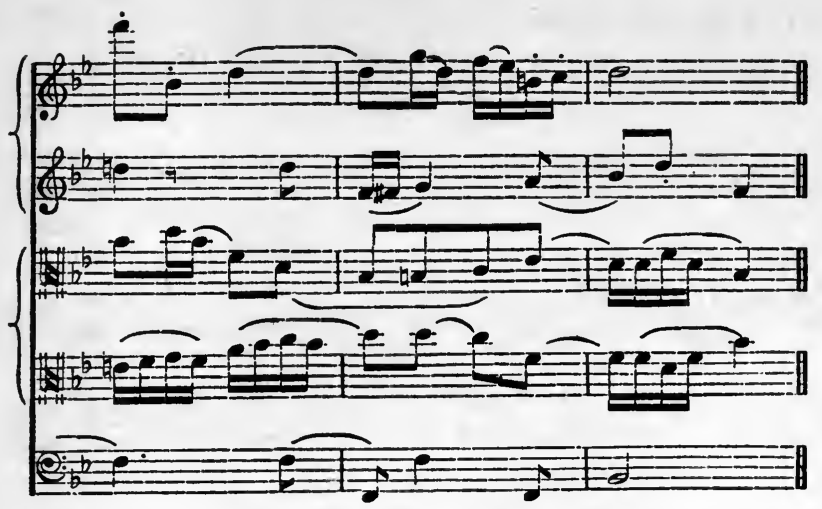

It would be possible to regard each bar as one bar of Scholastic Counterpoint (eight notes to one). In this case the analysis of the centres would be :-

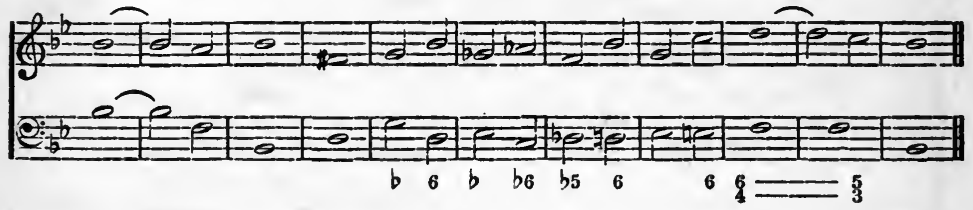

The Scholastic C.F. might have been expressed thus :-

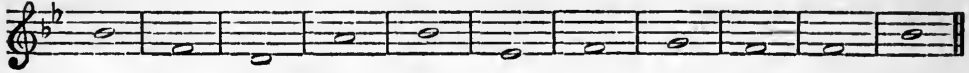

If the student will add this to the working of the problem he will see what is meant, and how the principle of two chords in a bar in Scholastic Counterpoint is also a principle of modern decorative work.

The following is a parallel case. The centres are not given, for it will be good practice for the student to work them out for himself. The analysis of four notes to one is again maintained here, so that the student may see clearly the connexion with Scholastic Counterpoint.

Each crotchet represents a bar of Scholastic Counterpoint. 
Third Mus. B. Oxon., Nov., 1904.
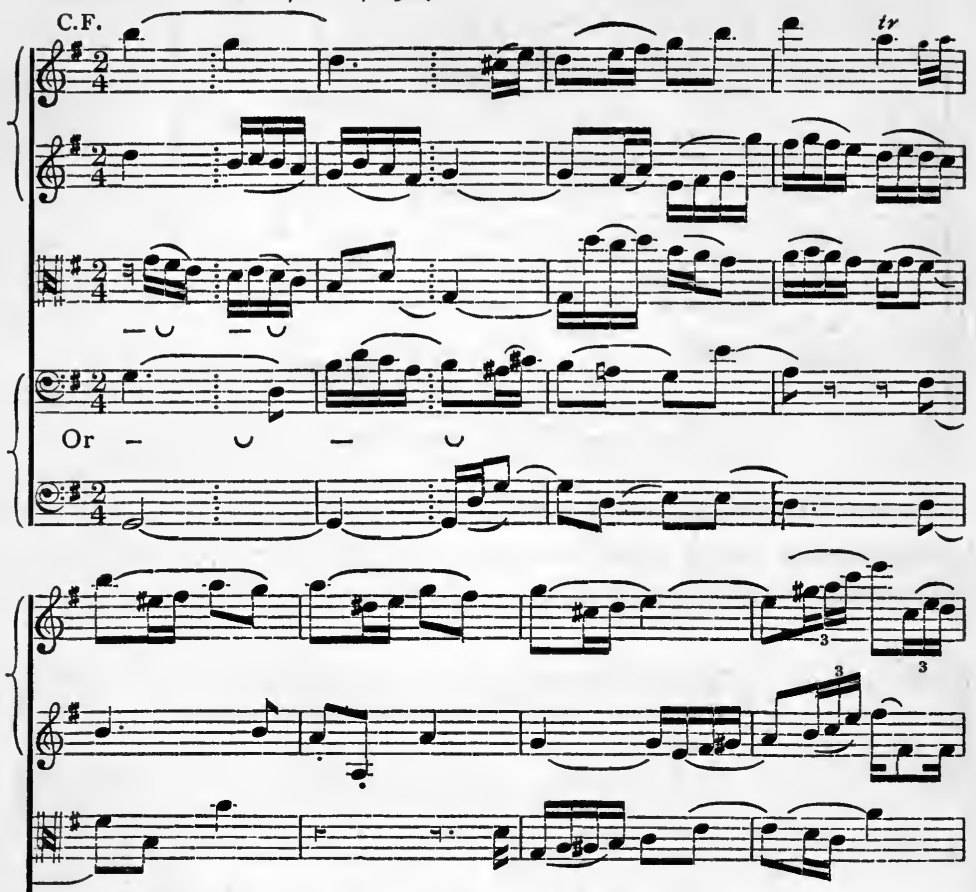

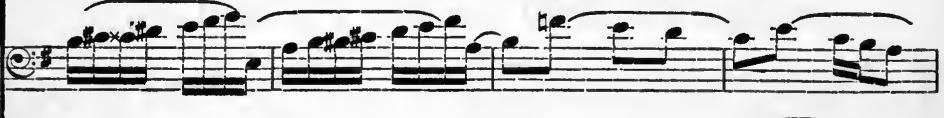

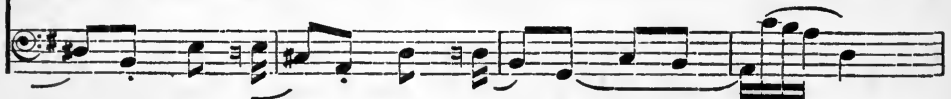

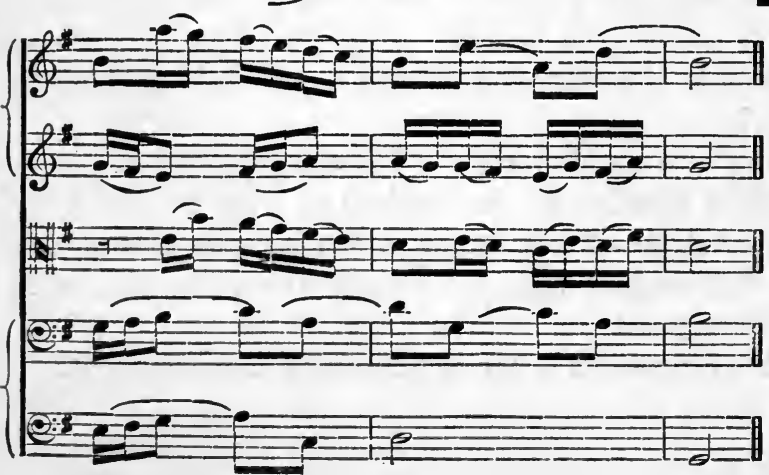




\section{APPLIED COUNTERPOINT}

Third Mus. B. Oxon., May, 1906.

(Centres are Crotchets.)

Adaoio. C.F.

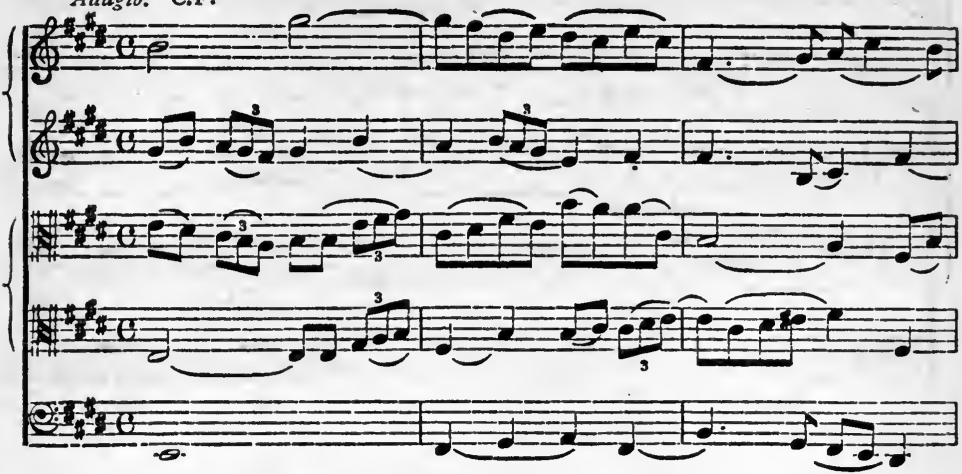

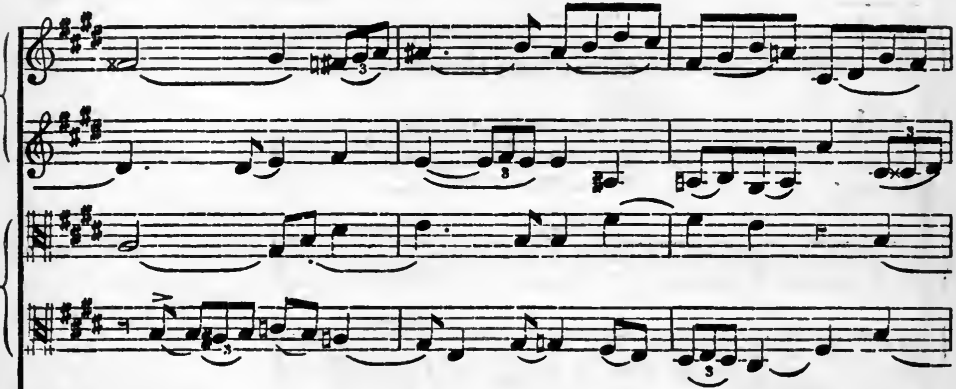

Fi.

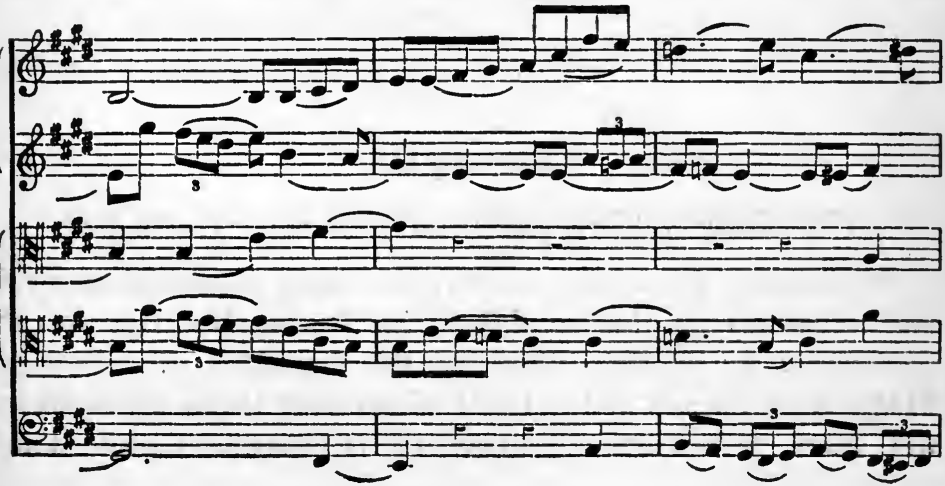



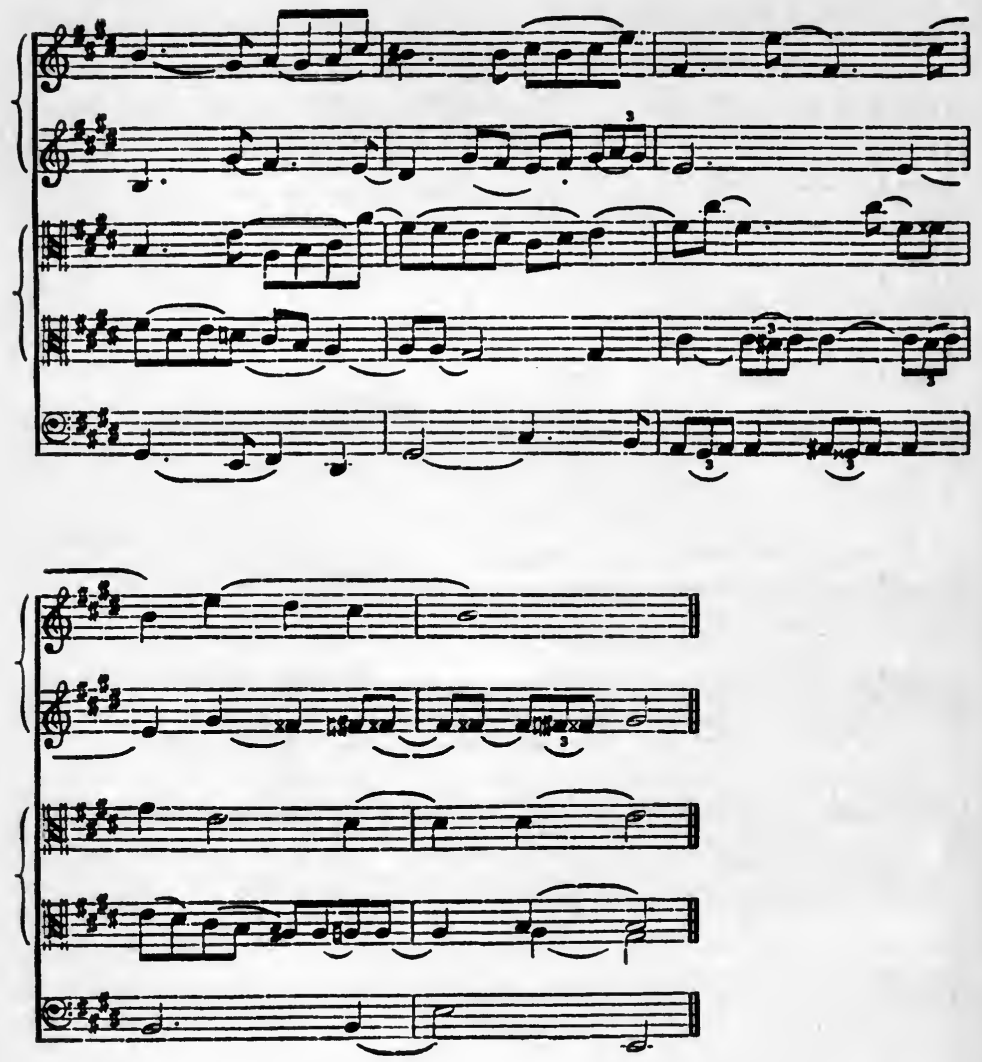

Section III.

The adding of Florid parts to a given inner Florid part.

Problems of this nature involve the same process of analysis and synthesis as that explained in Section II. But we are confronted by another difficulty, the formation of a melody. Many students are in the habit of working these problems on the same principles as those of Scholastic Counterpoint, 
forgetting that modern work demands modern principles, such as the systematic use of formula, the evolution of rhythmic phrases balancing one another, and so forth. In other words the C.F. is not a part that should be treated in the imitative manner of the Polyphonic Period, but as a component of a piece of modern texture.

To the following and Violin part, add parts for Ist Violin, Viola, and 'Cello.

Third Mus. B. Oxon., Nov., rgo5.
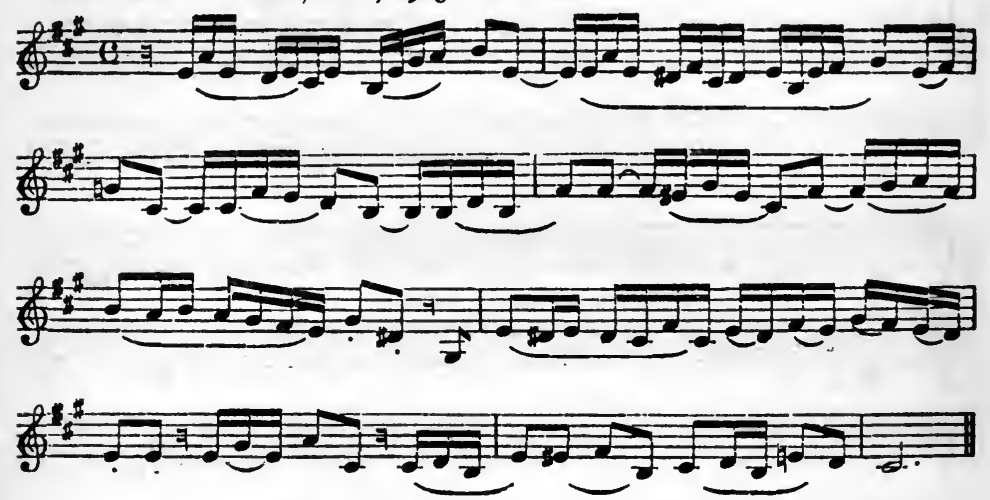

It is at once felt that the centres are quavers, that is, every crotchet corresponds to one bar of Scholastic Counterpoint. In order to make this principle perfectly plain, we give in the first line of the next example the C.F., in the second line, an evolved Scholastic C.F. (showing the application of the contrapuntal principle), in the third line, the centres.

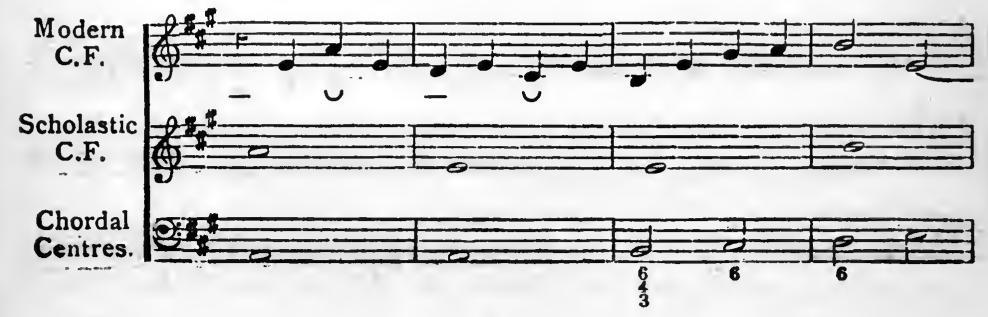




\section{THE ART OF COUNTERPOINT}

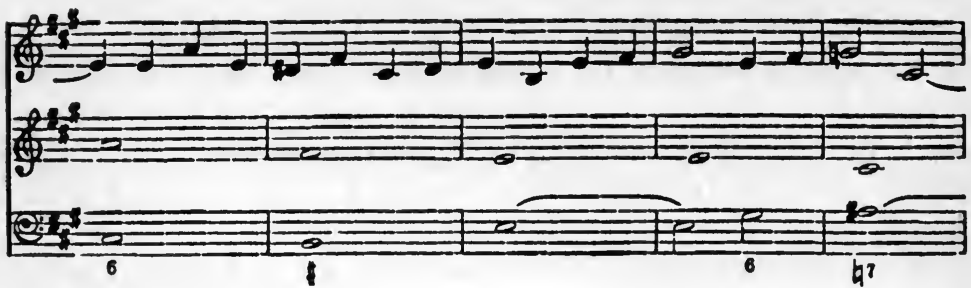

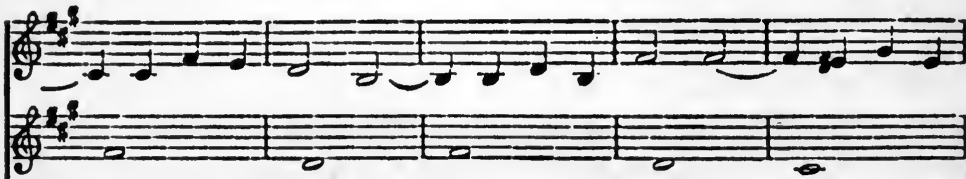

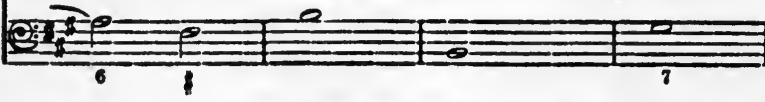

$2=$

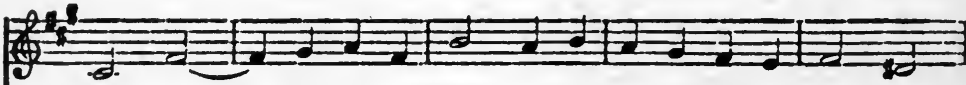

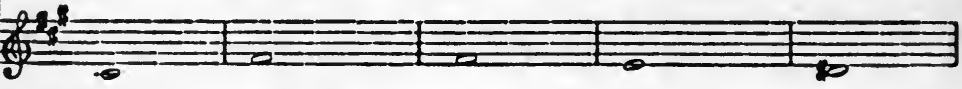

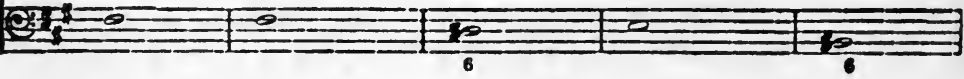

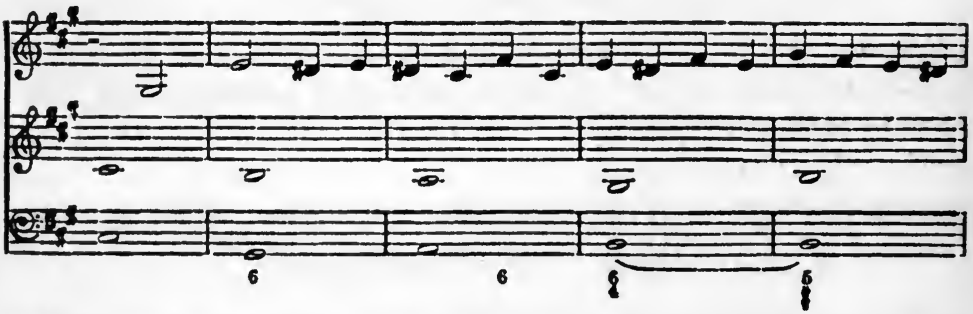

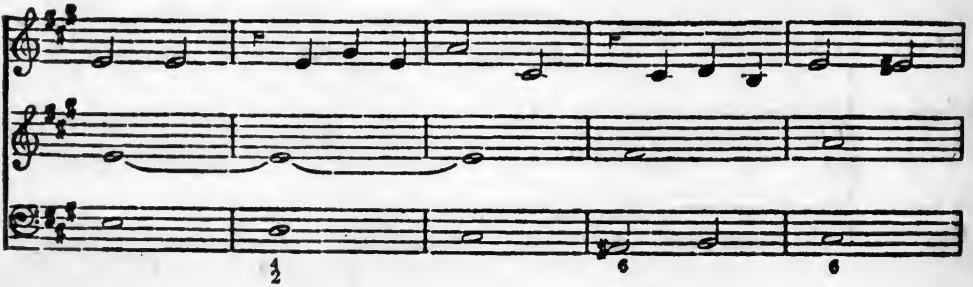




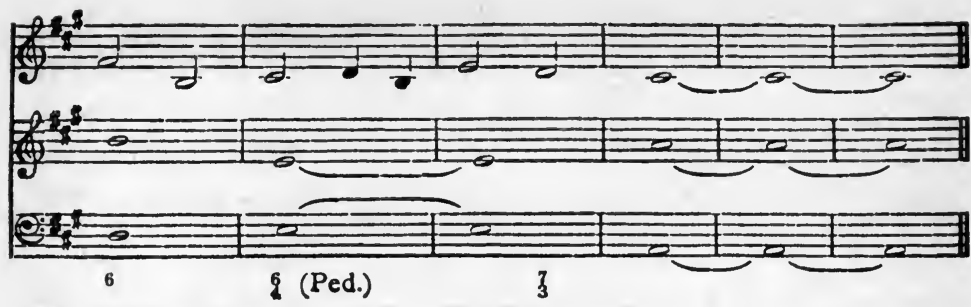

The student will see how historic Counterpoint is a preliminary study leading up to modern work. In modern work the C.F. is mentally implied. It is still true that it is never wrong to use two chords in a bar. The working of the problem :-

A A

$7^{8} \mathrm{~B}=\mathrm{E}$

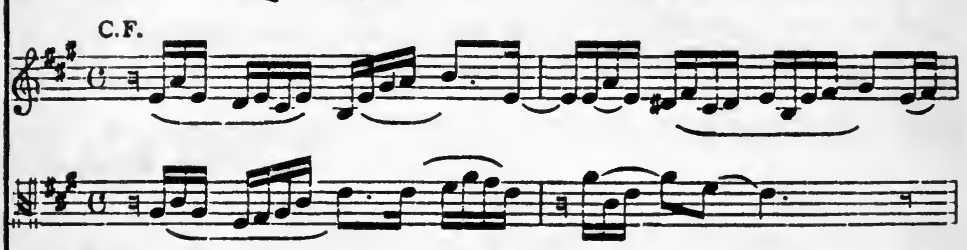

For

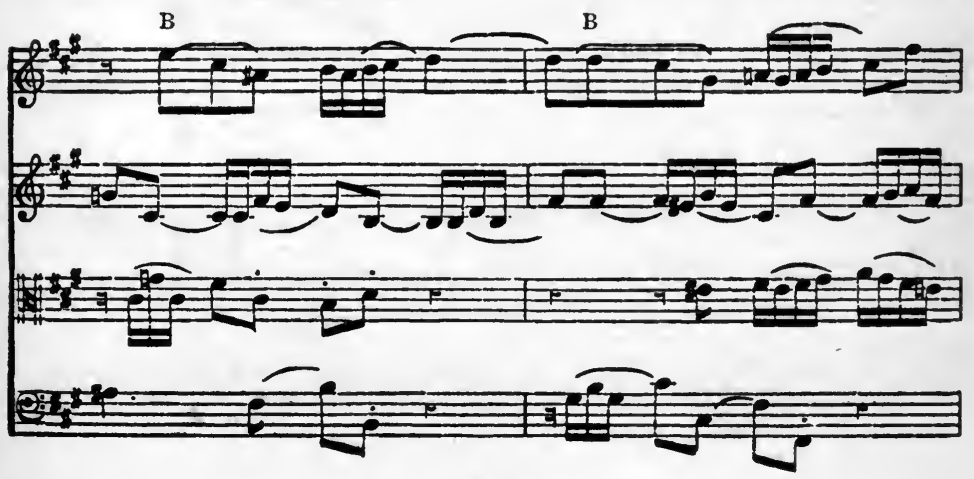



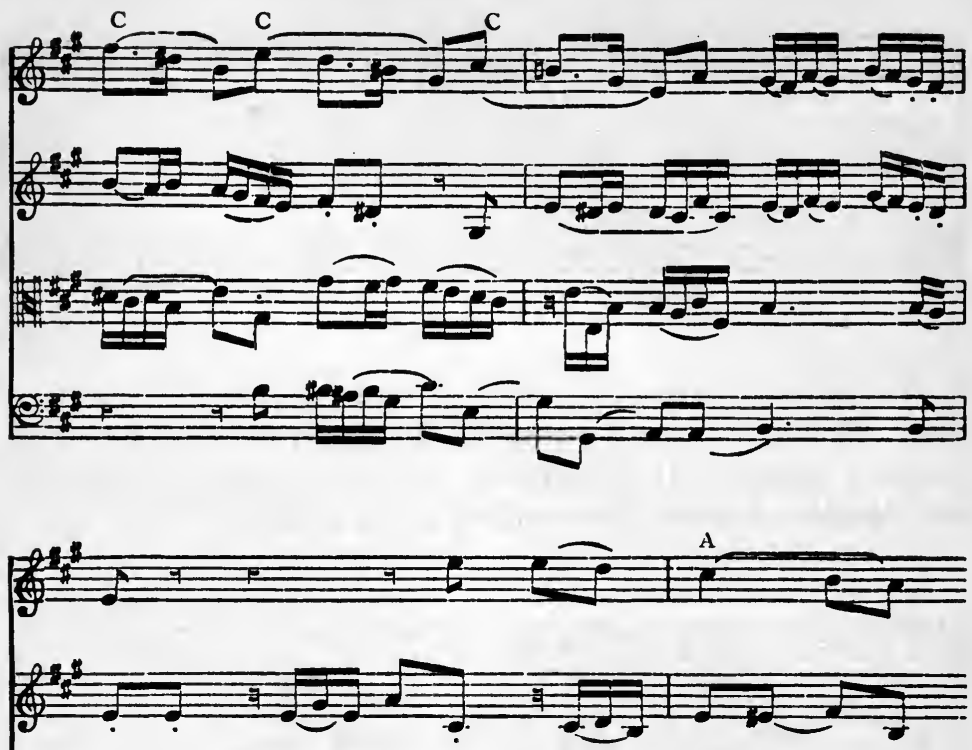

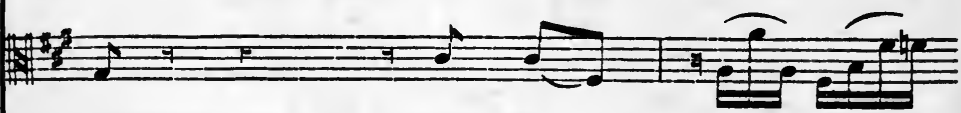

Q:

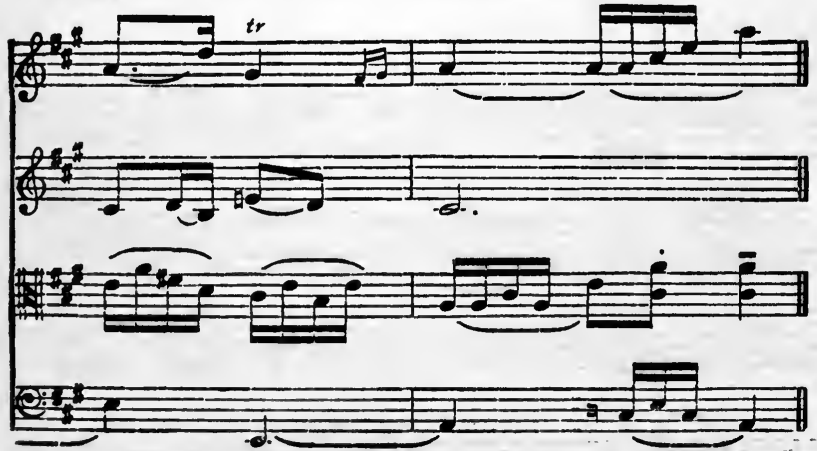




\section{Problem.}

To the following and Violin part add parts for Ist Violin, Viola and 'Cello.

Mus. D. Oxon., Nov., 1905 .
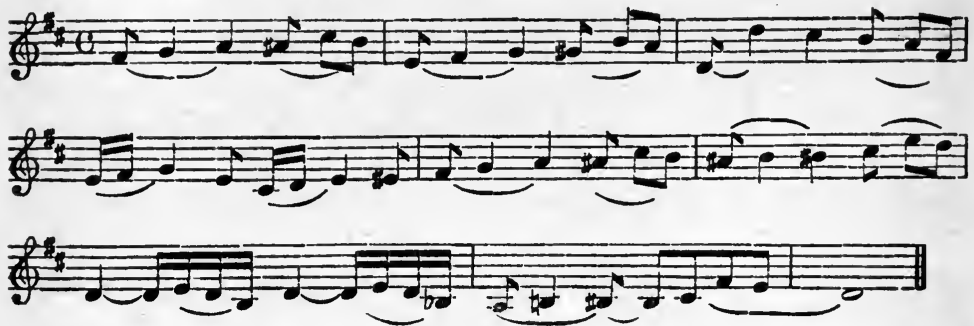

A grasp of the harmonic outline may be obtained by altering the time signature and the value of the notes thus:-

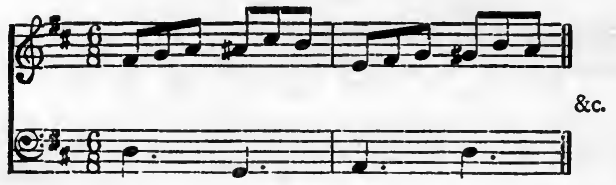

In perplexing cases, it is often helpful to eliminate what at first sight appear to be unessential notes, and then see if the remaining harmonic skeleton is satisfactory. If this is not the case, the probability is that the student has not chosen the right notes for his essential harmony.

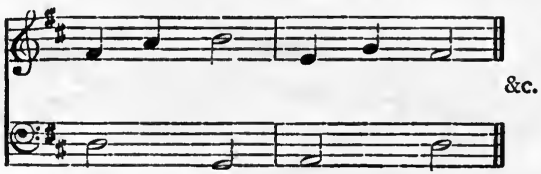

Help may also be obtained by writing unessential notes as ornaments.

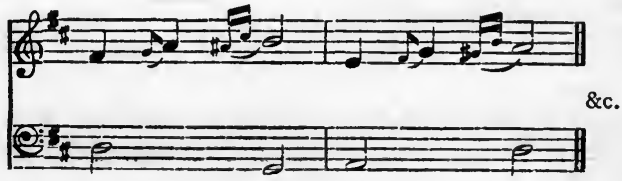


We now give the C.F. in Scholastic form with the chordal centres.
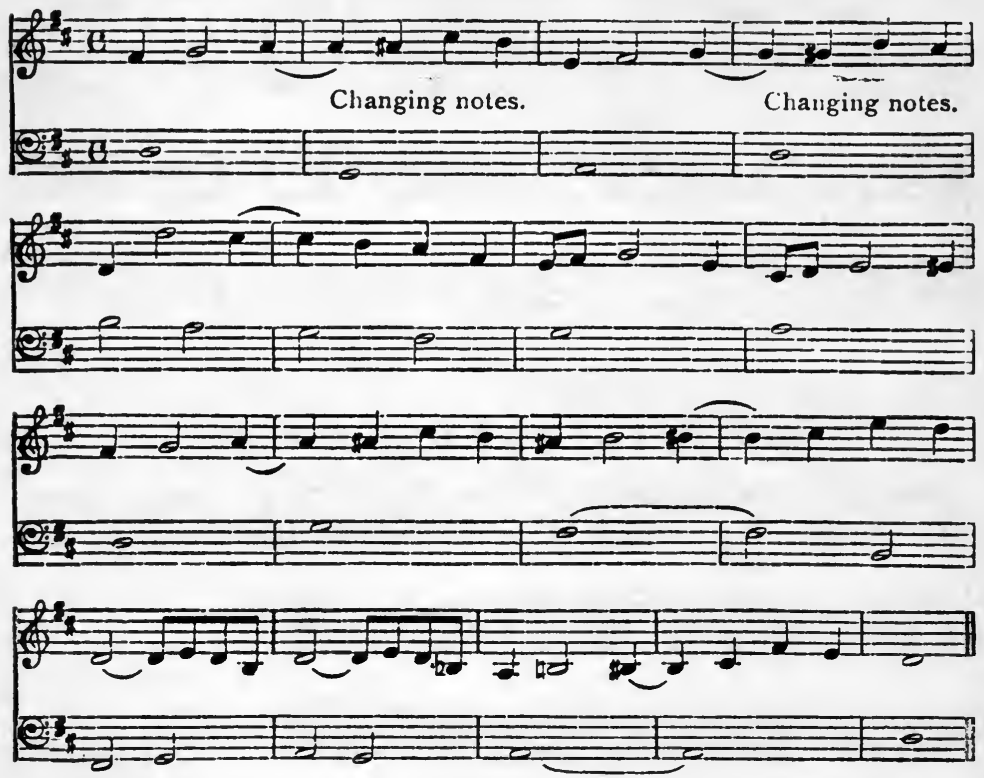

Here we have examples of internal syncopation. The student will remember that this is a species of Scholastic Counterpoint that has fallen into disuse.

The following working is modelled on a parallel case in Brahms's Variations on a Theme of Handel.

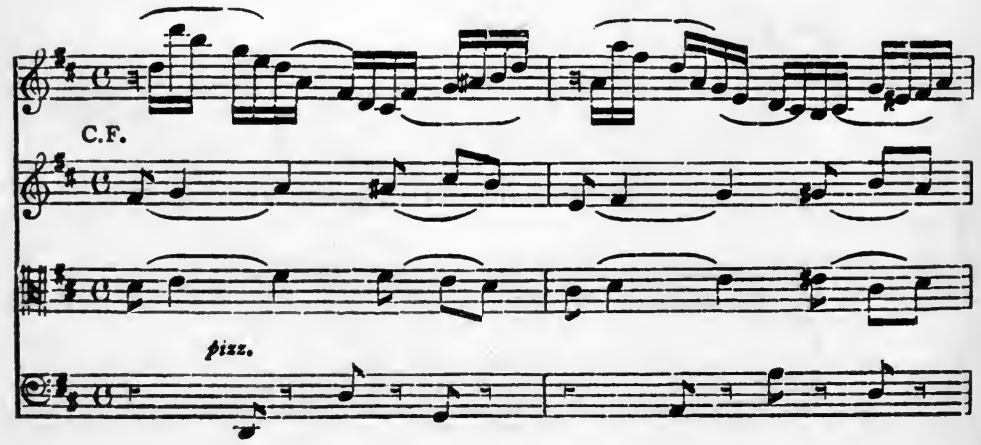




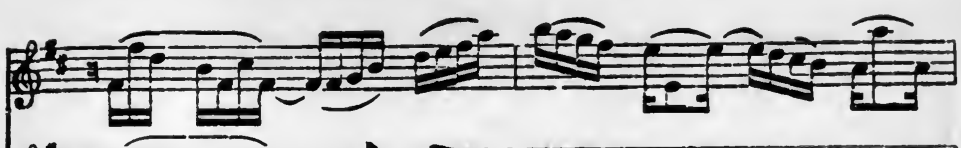

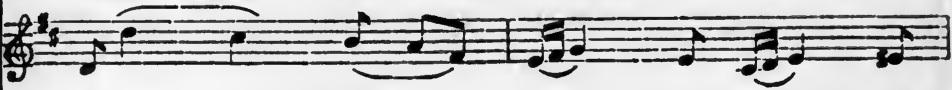

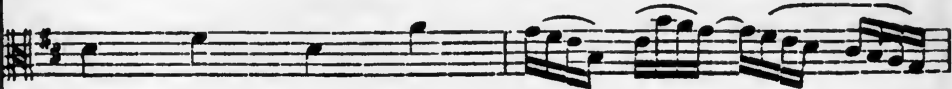
arco

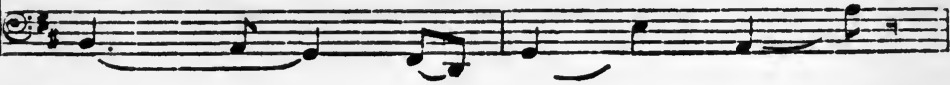
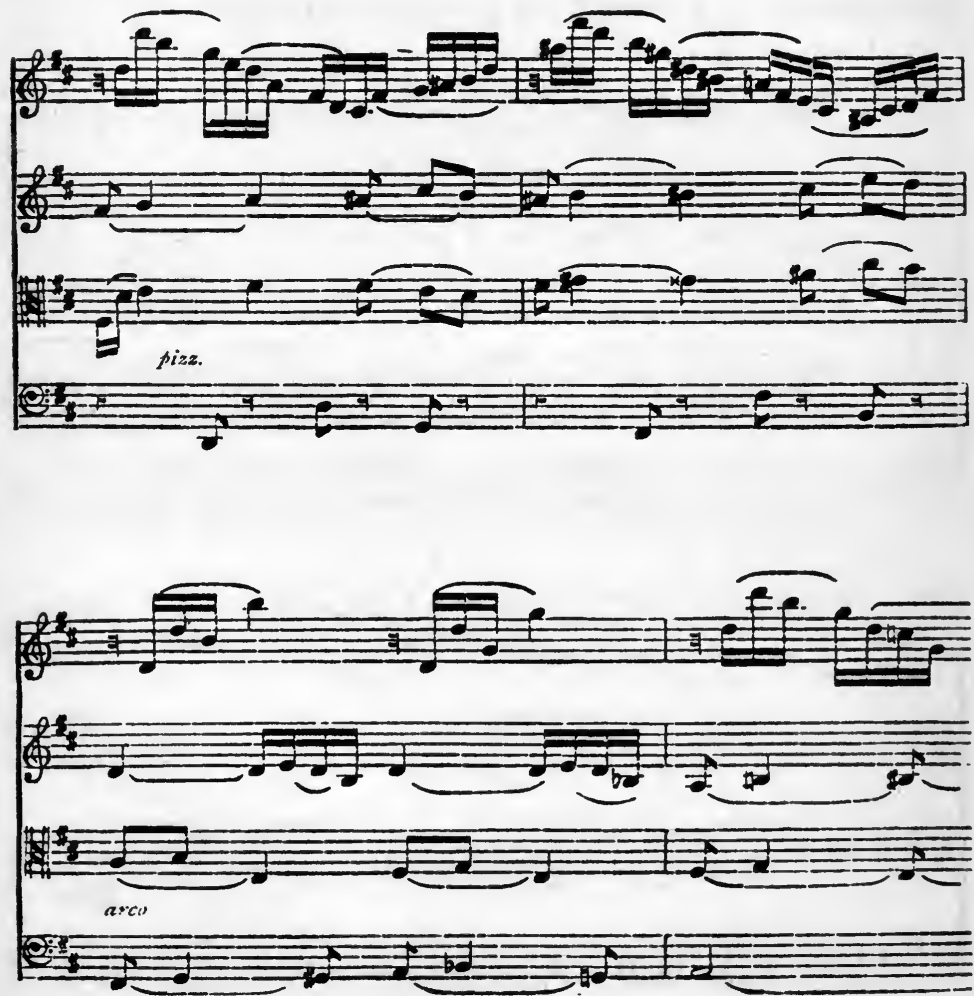


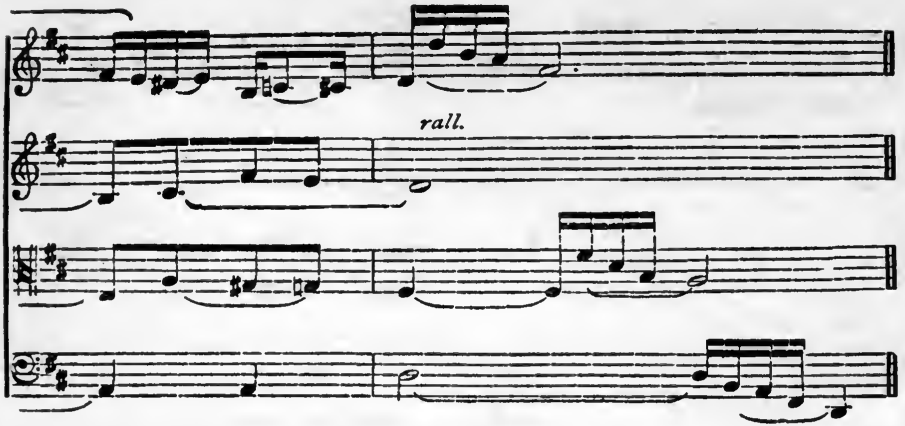

In the following problem, quavers are centres, that is each crotchet represents one bar of Scholastic Counterpoint: it is unnecessary to go through the preliminary stages.

Third Mus. B. Oxon., Nov., Igo4.

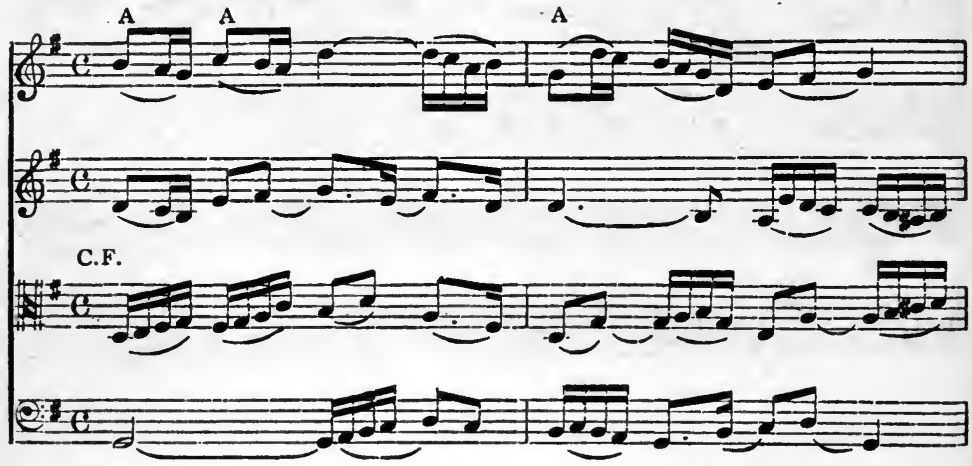

B

B

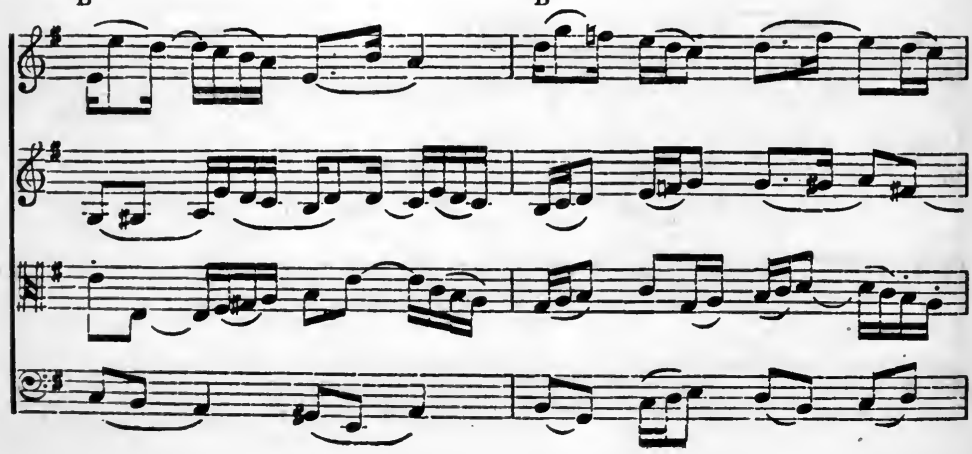




\section{APPLIED COUNTERPOINT}

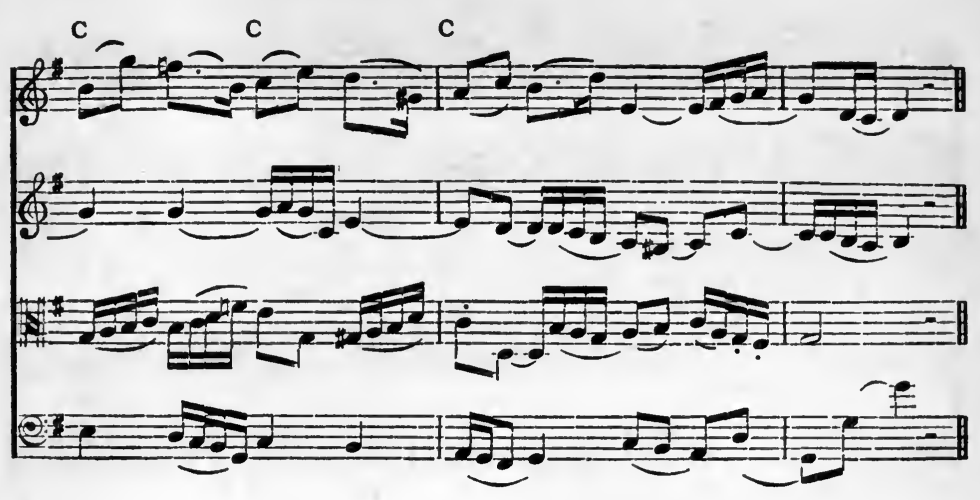

Problem.

Add parts for Ist and and Violins, Viola, and and 'Cello. Mus. D. Oxon., 1897.
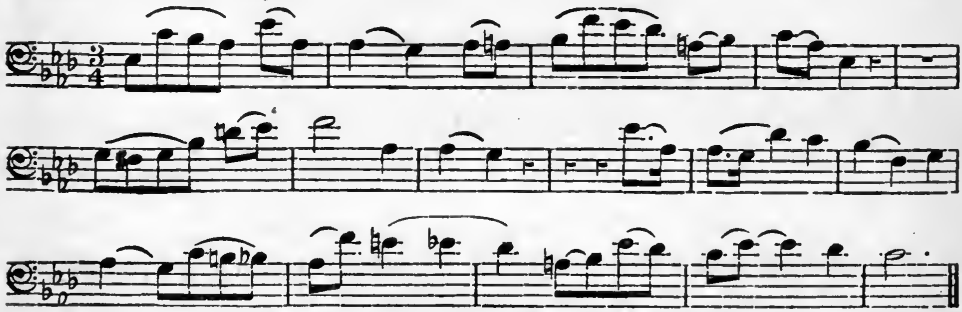

Write the C.F. with all unessential notes omitted (counting all discords higher than the seventh as unessential), and add a plain bass.

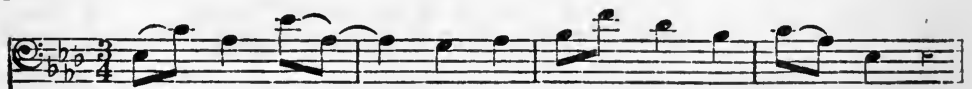

(4)
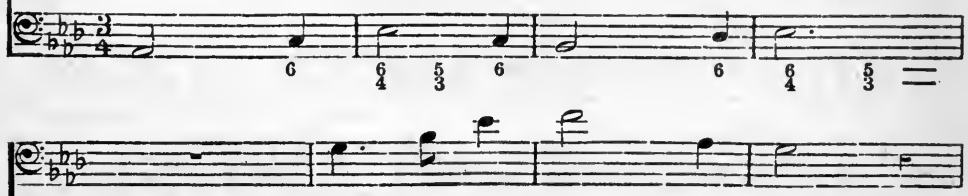

Harmony arranged to connect smoothly with next bar.

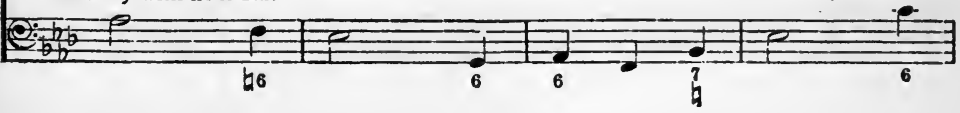



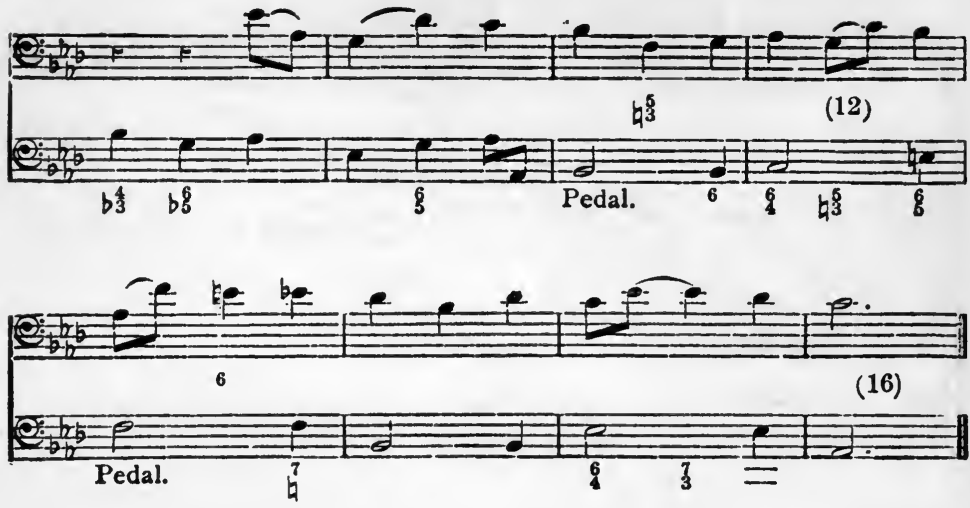

Now regard each crotchet as a chordal centre and evolve five free parts. In the working, the following abbreviations are used :-

(I) = accented passing note (appoggiatura).

(2) $=$ accented passing chord.

(3) = unaccented passing chord.

(4) = deferment of change of chord until the second half of the beat.

Notice that when the cadential ${ }_{4}^{6}$ is used (bars 2, 4, 12, 15) the sixth and fourth are really appoggiaturas of the fifth and third of the succeeding chord.

Andante. A

A (2) (1)

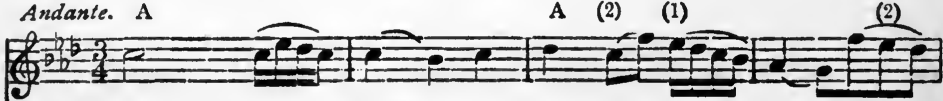

(3)
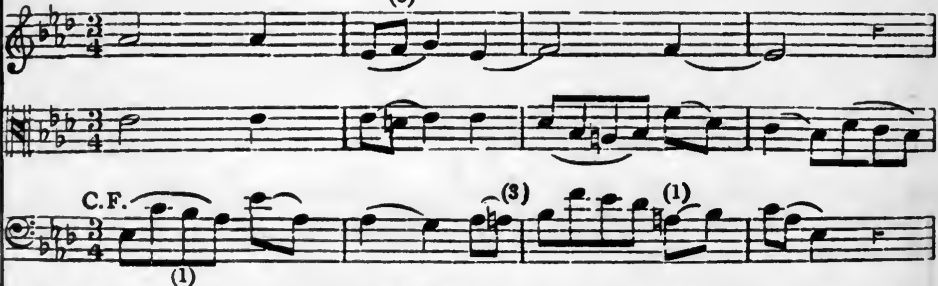

(1)

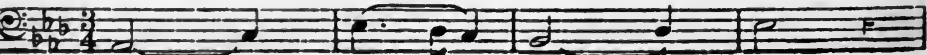




\section{APPLIED COUNTERPOINT}

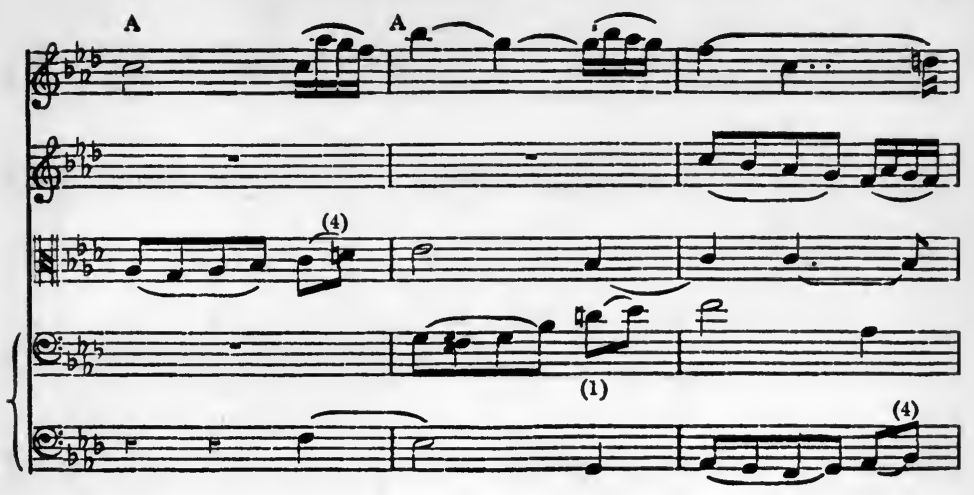

Evolved from A.

(f) $=1+2$
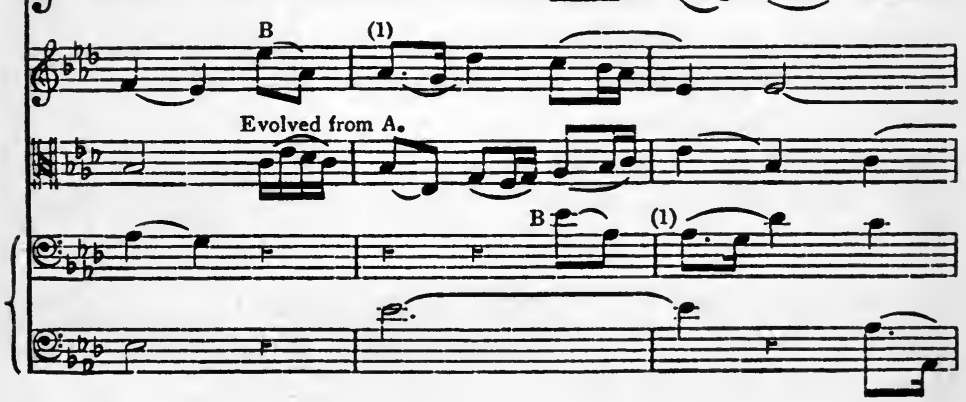

Part of A developed.

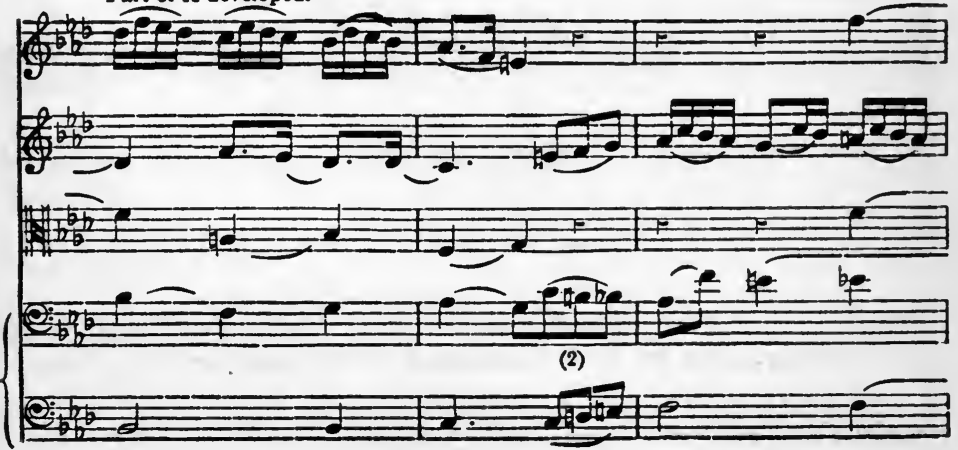




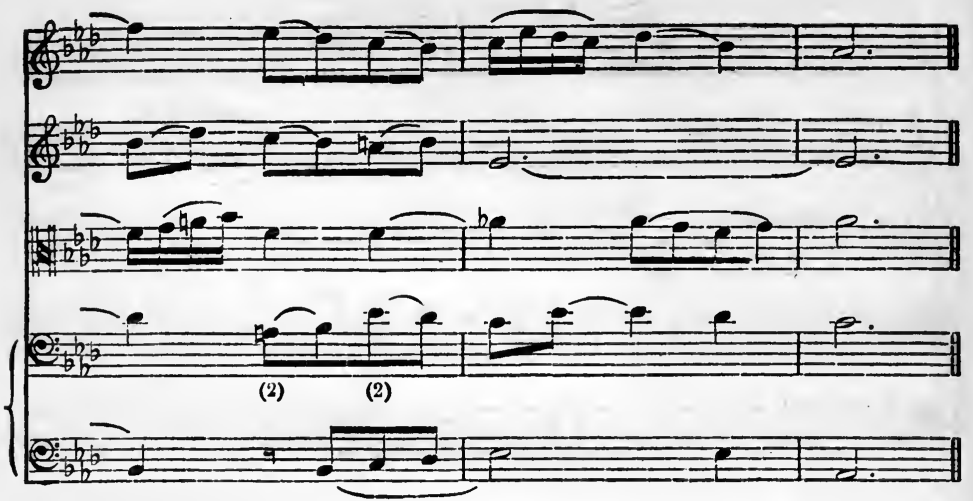

The first two bars are given in the form of Scholastic Counterpoint.

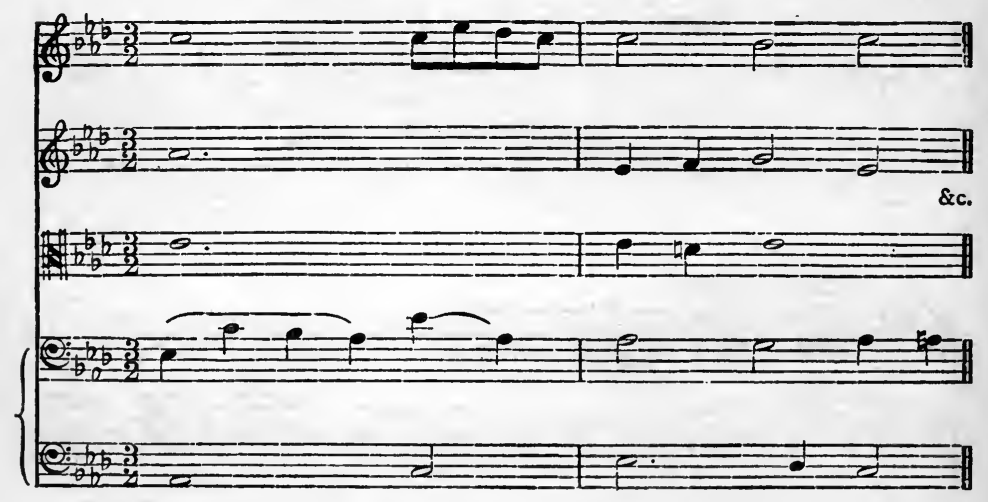

In the next example the Ist Violin part is developed by 'exhaustion', that is, after repeating the formula which consists of a two bar phrase (bars I-4), one bar of it is repeated (bars 5 and 6) and then one third of one bar (bar 7). 
Third Mus. B. Oxon., May, 1905.
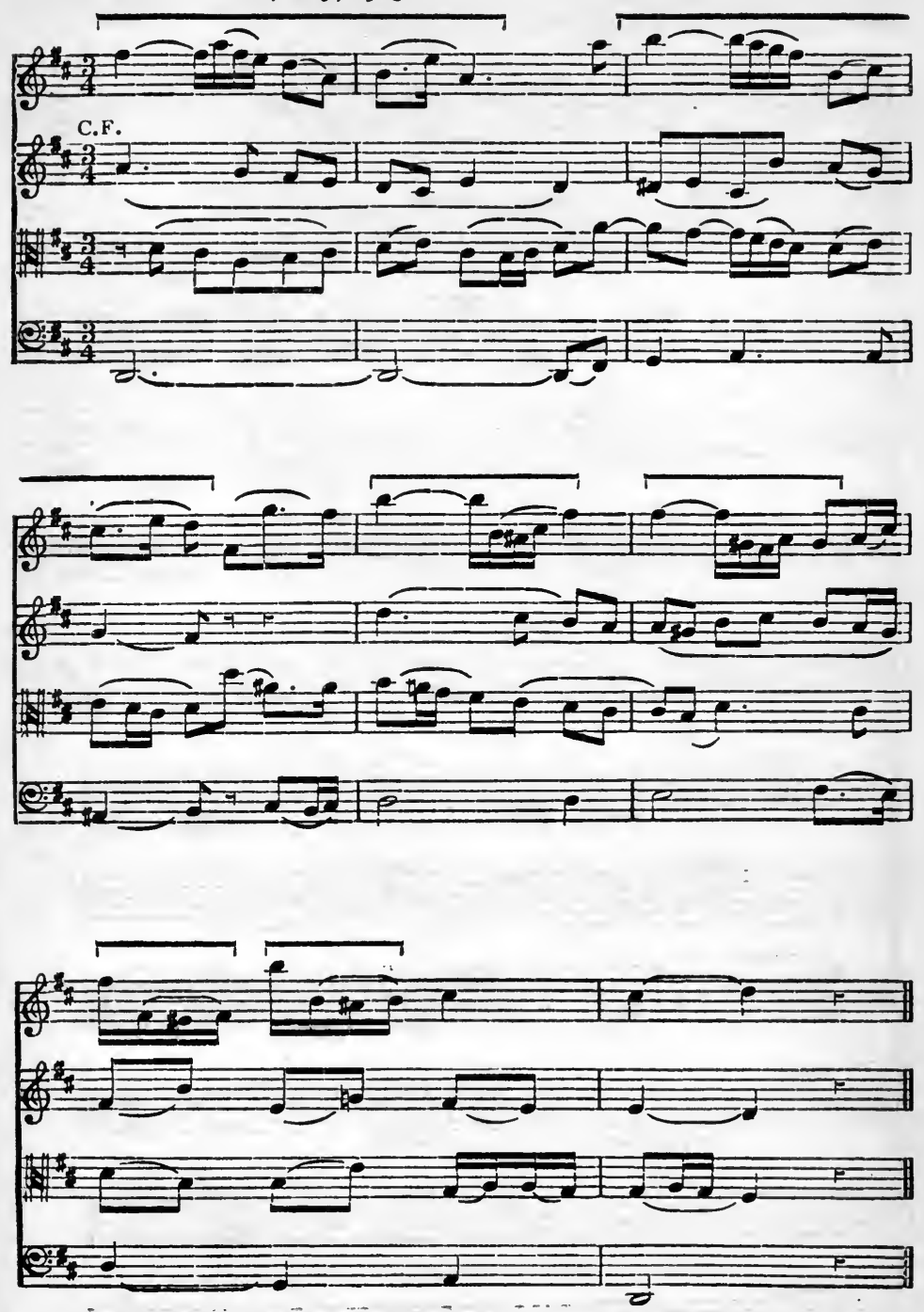
The next example offers no difficulty.

Mus. D. Oxon., Nov., rgo4.

Each Crotchet represents a bar of Counterpoint.

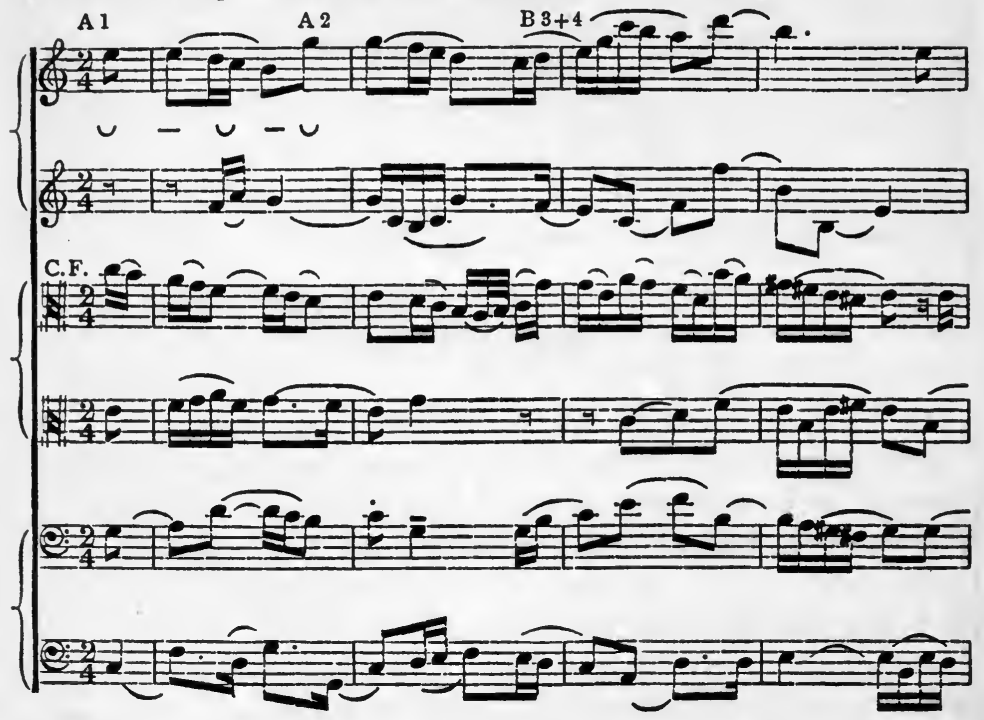

A $5 \quad A 6$

C $7+8$

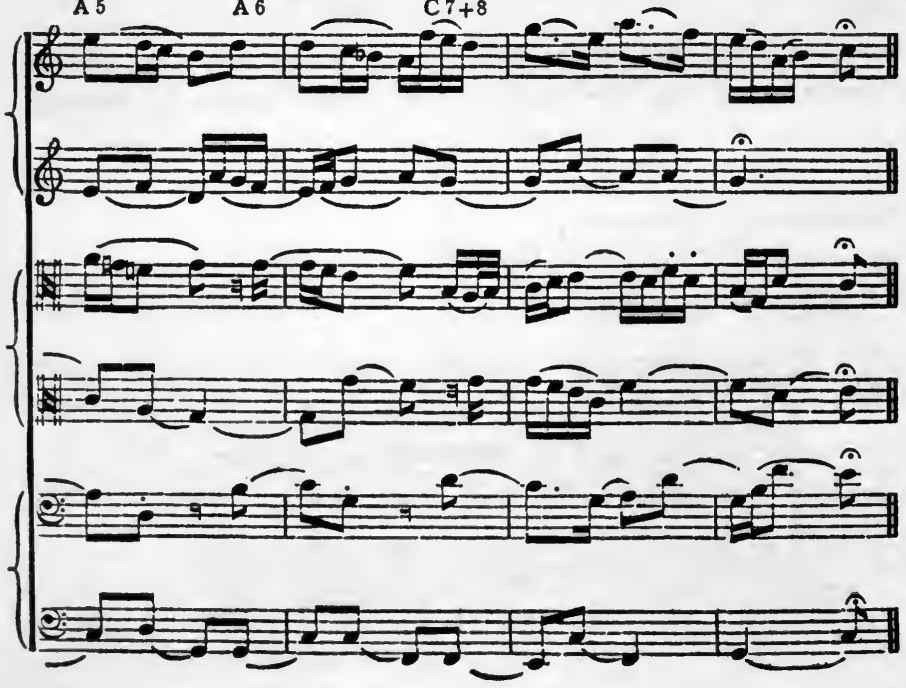


Contrapuntal Analysis.

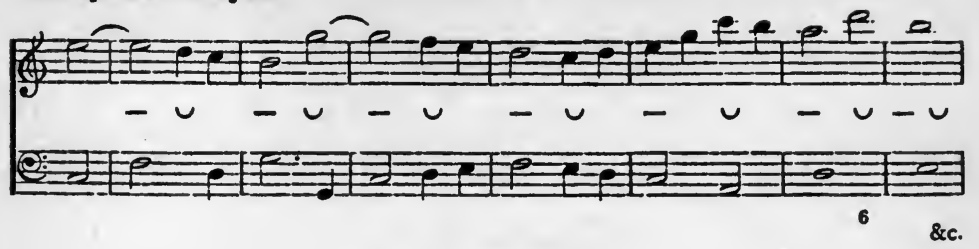

\section{Section IV.}

The working of problems in modulation, the opening bars being given.

Method of working.

(I) The given bars should be reduced to chordal centres.

(2) A period consisting of the plain centres should be constructed, introducing the various modulations, with due attention to proportion, balance, key distribution and cadential effect. A course such as that outlined in the previous chapter will have given the student the necessary technique.

(3) Good melody cannot exist without pre-supposed good harmony; and although we are only dealing here with purely harmonic progression, yet it will be well at the outset to have an idea of the rhythm of the melody. This is therefore placed above the harmonic progression. No melody ought to be conceived without due consideration of its harmonic basis, and vice versa.

\section{Problem.}

Begin as follows :-

Third Mus. B. Oxon., May, 1903.

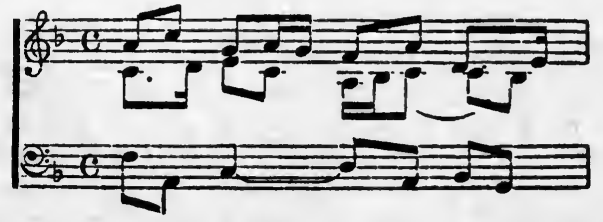

and modulate to $\mathrm{D}$ minor, $\mathrm{B}$ flat, $\mathrm{G}$ flat, $\mathrm{D}$ flat, and back to $\mathrm{F}$. 
It is felt at once that there are four strong accents in this bar.

Now complete the sentence.

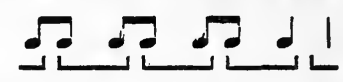

A crotchet is used here to give the cadential effect, see pp. 254-5. Next, write a parallel sentence, and we have four bars, thus-

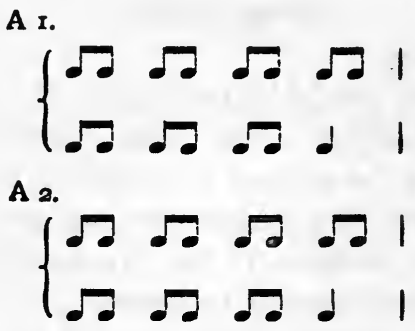

This constitutes the end of the first half. Since A I and A 2 are parallel sentences, this must be brought out in the melodic curve, so that the harmonic rhythm and melodic curve will be exactly balanced.

Now, we can only get to $\mathrm{D}$ minor by the end of bar four, as the other keys are on the flat side of $\mathrm{F}$; therefore the cadence at the end of bar two should be a half close in the key of F, and that at bar four a full close in D minor. It is necessary to avoid-

(a) harmonic monotony;

(b) repeating the idiom of the modulation, or anticipating its completion at the cadence.

A I.

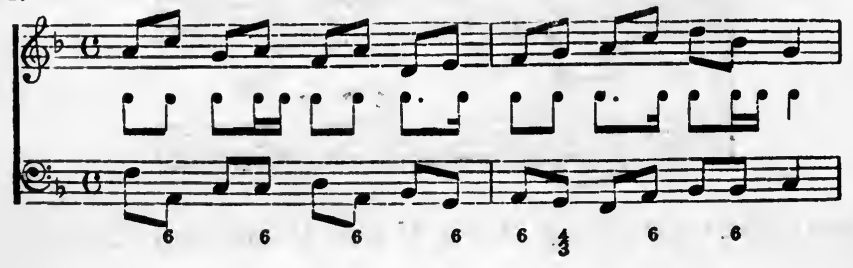




\section{APPLIED COUNTERPOIN'T}

A 2.

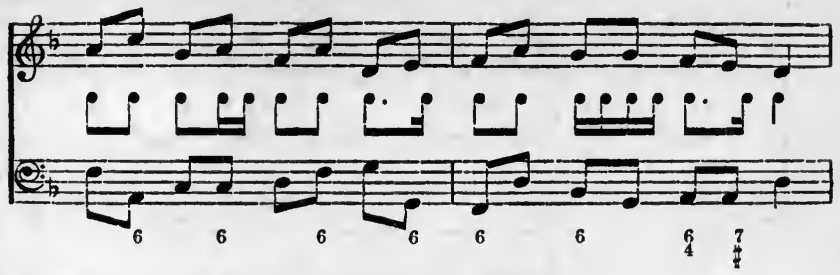

It will be noted that the melodic rhythms of bars two and four are not identical; variety is needed.

Note. Bar four would have been very faulty in style thus-

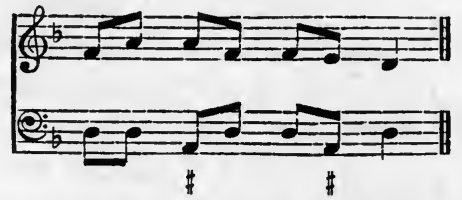

The third phrase is the place for frequent modulation, and if the first and second phrases have been parallel, three and four will also be parallel, but usually containing sub-phrases, e. g.--

B r.

B 2.
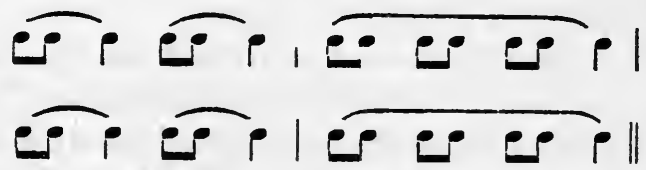

These crotchets imply cadential effect, and the same chord should be used for the parts of the two motives which they dovetail.

We must also manage to get back to the Tonic in sufficient time to re-establish the key satisfactorily.

Sequence should be employed in this section.

B r.

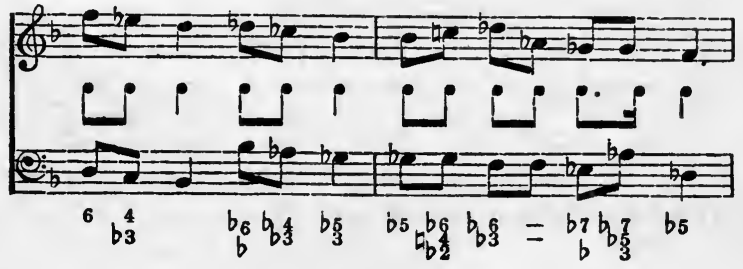


B 2.

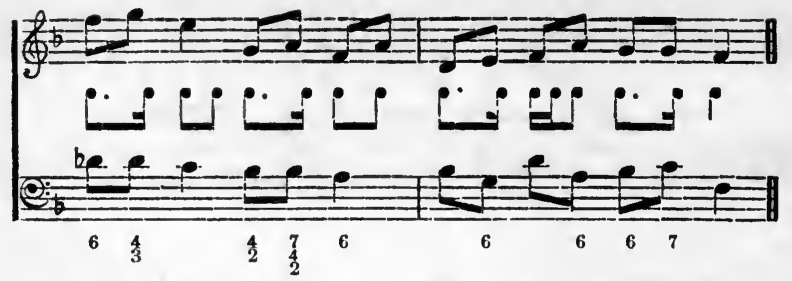

The melodic rhythm of $\mathrm{B}_{2}$ is varied, in order to refer to $\mathrm{A}$.

We can now employ contrapuntal methods, regarding each part of a motive as an accent in Counterpoint, implying one chordal centre. It is important to remember that the C.F. now ceases to be an actual note, and becomes an implied chordal centre, which may be decorated in various ways, without losing its predominating effect as a centre. All the previous work has been a course of training leading up to this. In other words, the student must now build up his score with a mental frame; for although the actual frame in the form of a C.F. in semibreves has disappeared, the principles remain.

The decoration of a chordal centre will often produce various changes of harmony, and the study of Combined Counterpoint will have provided the student with the necessary technique.

It is hardly necessary to point out that in actual composition such a process of analysis and synthesis is never systematically utilized, but it will be found that good composition will bear this dissection, and it has been our province to show the principles underlying it.

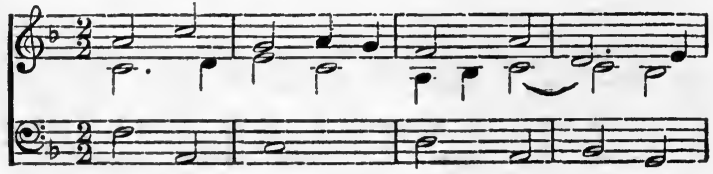

The working of the problem :- 


\section{APPLIED COUNTERPOINT}

Third Mus. B. Oxon., May, 1903.
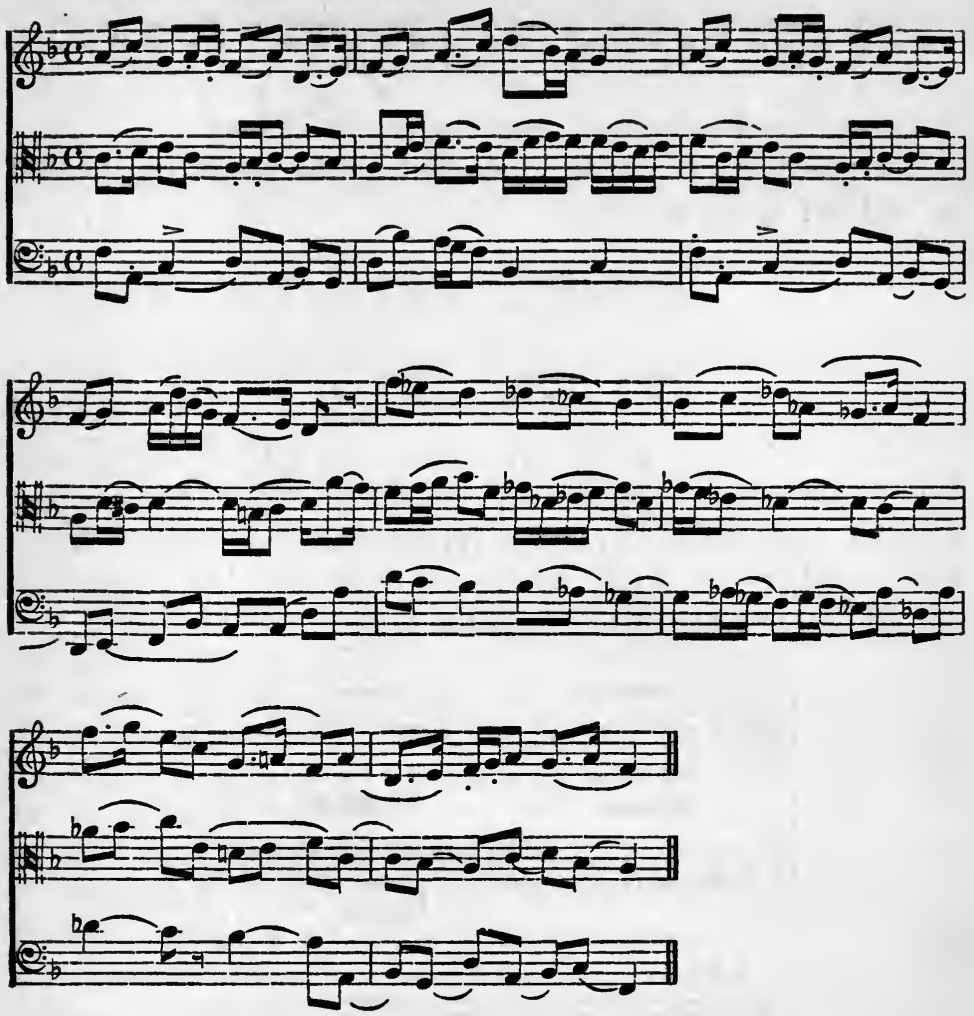

We now have an example in triple time. Begin as follows, and modulate to D min., B flat maj., G min., C maj., and back to $\mathrm{F}$.

First Mus. B. Oxon., Nov., 1900.

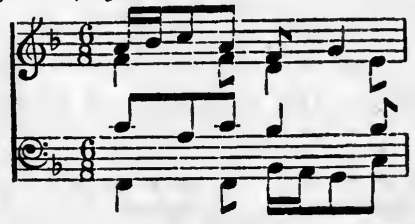

Centres here are obviously quavers. 
(a)

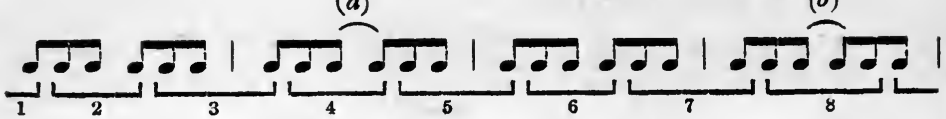

This completes the first sentence, just as before.

But this must be made intelligible by the use of cadences at $(a)$ and $(b)$; so we get-

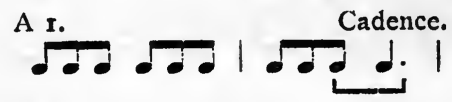

A 2.

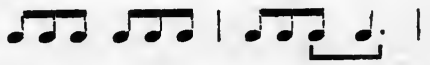

Now, as the given bar forms only one half of $A$, the next bar should differ rhythmically, else $\mathrm{A}_{\mathrm{I}}$ and $\mathrm{A}_{2}$ will form four parallel rhythms, and create a very monotonous effect. Again we can only reach $\mathrm{D}$ min. by the end of the first half as the next key ( $\mathrm{B}$ flat) is on the flat side of the tonic.

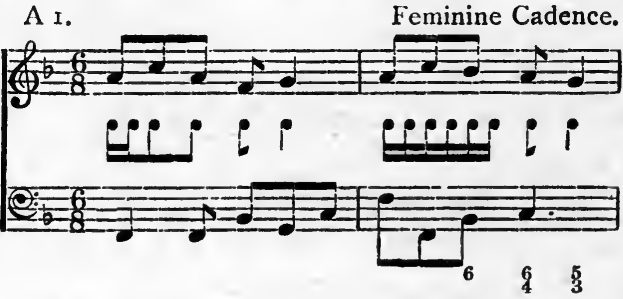

A 2.

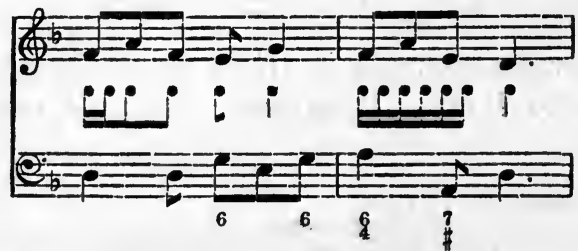

The second sentence will contain exactly the same number of motives. Sub-phrases are used in the first half, and the last phrase is indivisible.

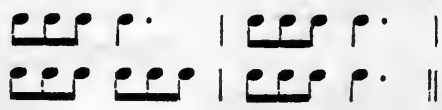




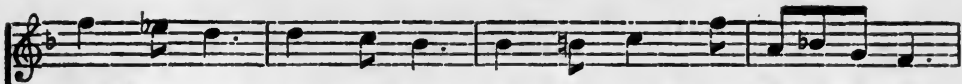

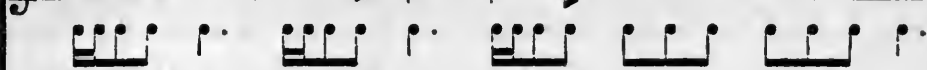

$\frac{{ }_{6}^{4}}{b_{3}^{4}}$

The following is the Scholastic Analysis :-

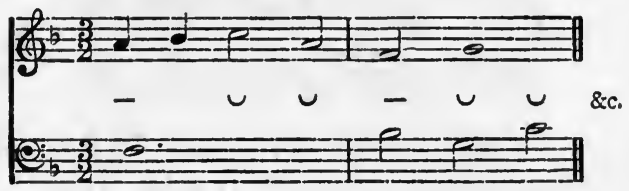

The working of the problem :-

A r.

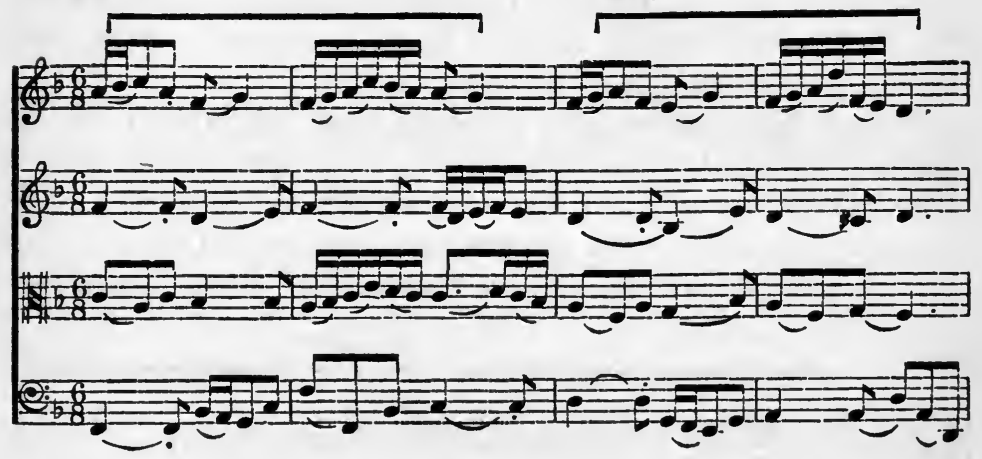

Fifere Sub-phrase $r$. 2.

$f^{2}=1,10+2$

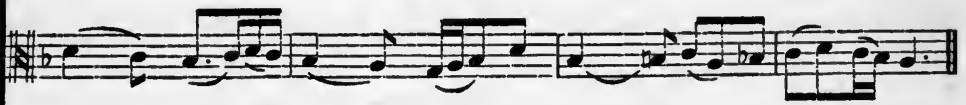
Oi: 


\section{Problem.}

Begin as follows and modulate to $\mathrm{B}$ min., $\mathrm{E}$ min., $\mathrm{F} \#$ min., A maj., F maj., D min., B flat maj., D maj., B min., D major.

Third Mus. B. Oxon., Nov., Igoz.

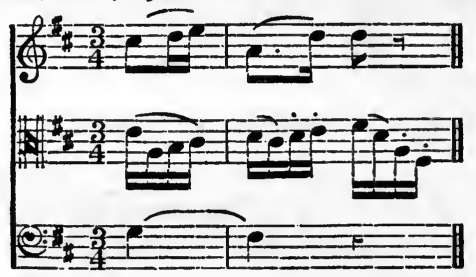

If the course of the opening ( $\mathrm{D}$ maj., $\mathrm{B}$ min., $\mathrm{E}$ min.) be compared with the ending (D maj., B min., D maj.) it will be seen that it is intended that the last few bars should be a recapitulation of the opening, with the modulation turned so as to end in the Tonic, after the manner of Corelli, Scarlatti, Bach and Handel (see Grove, Article on Form).

We have here another case of Compound Counterpoint. Each crotchet represents an accent, that is, the centres form Counterpoint in triple time. Then, when the centres are decorated, each crotchet is regarded as the equivalent of one bar of Counterpoint in duple time. First we draw up the chordal centres in terms of Scholastic Counterpoint.

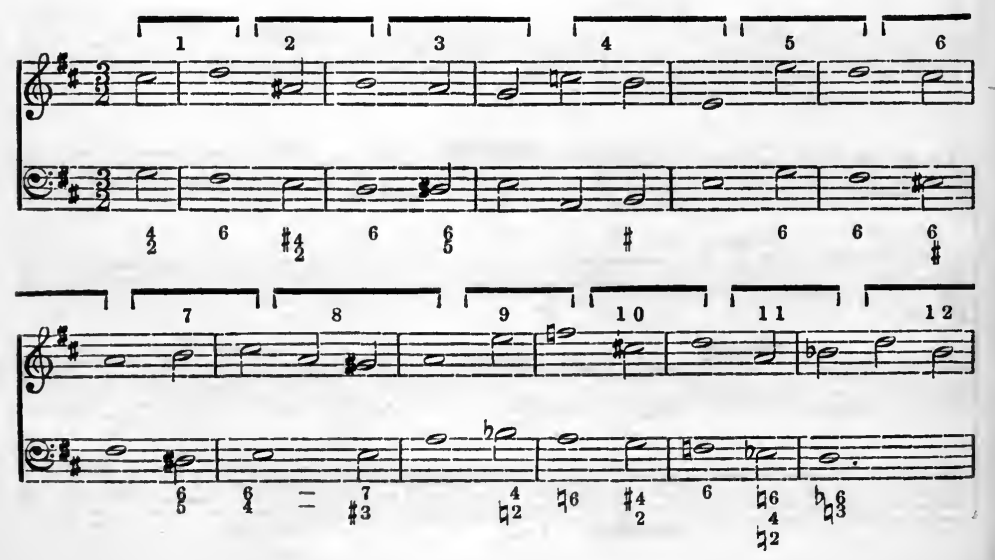




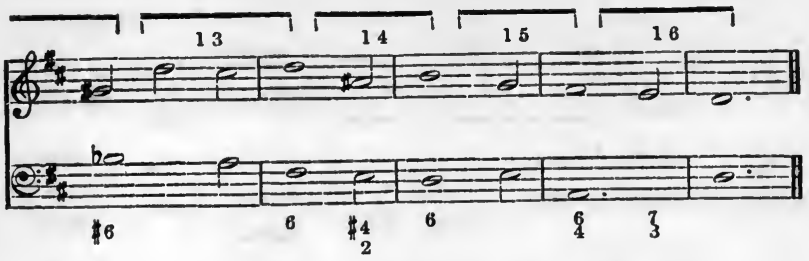

Next we put this in correct terms for decoration.

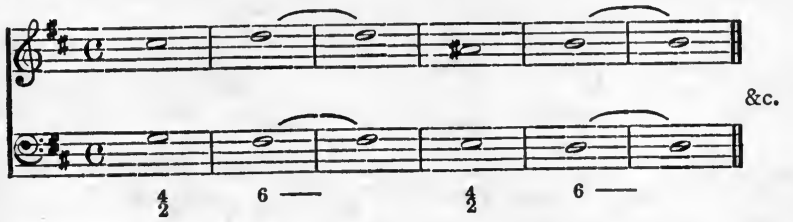

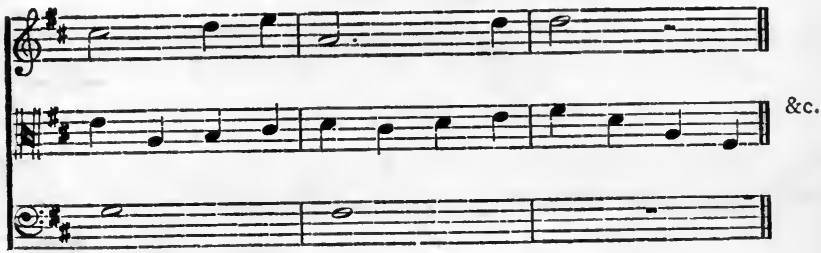

The working of the problem :-

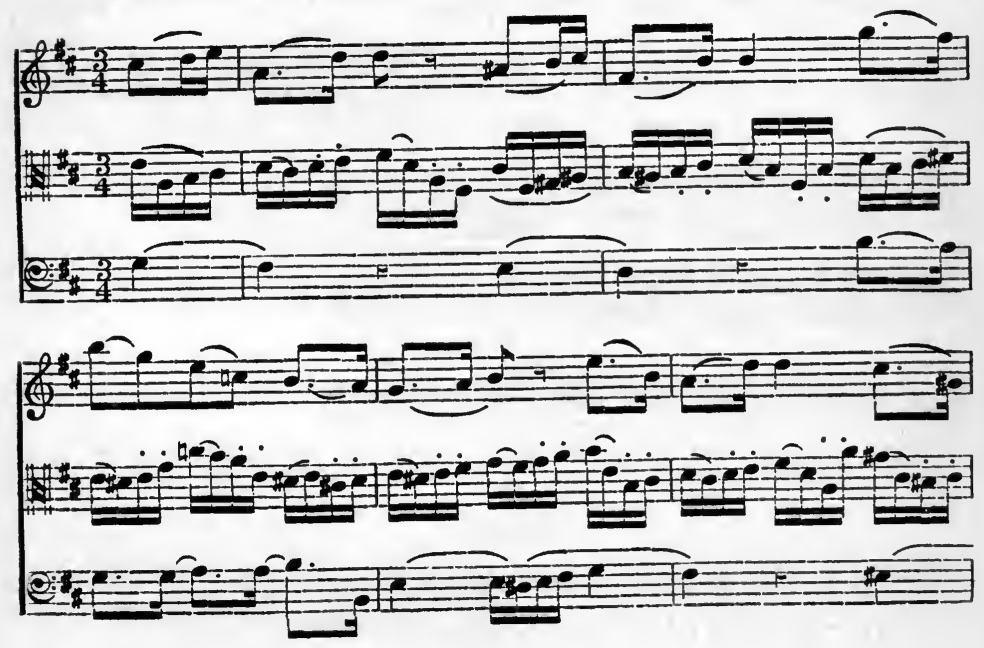




\section{THE ART OF COUNTERPOINT}

Cf. bar 8 .

(2)

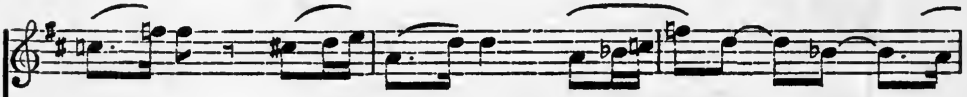

H.pjef

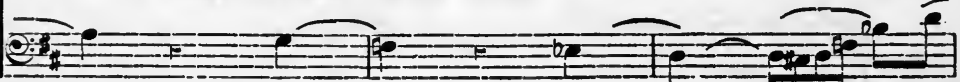

(2)

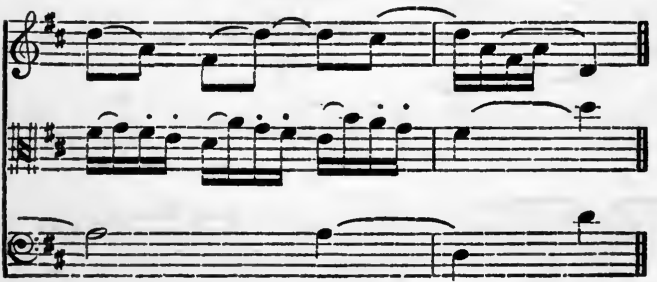

Problem.

Begin as follows, and modulate to D maj, $F \#$ min., $F \#$ maj., $\mathrm{G}$ min., $\mathrm{E}$ flat maj., $\mathrm{D}$ maj., $\mathrm{E}$ min., and back to $\mathrm{B}$ min. 


\section{APPLIED COUNTERPOINT}

Schereando

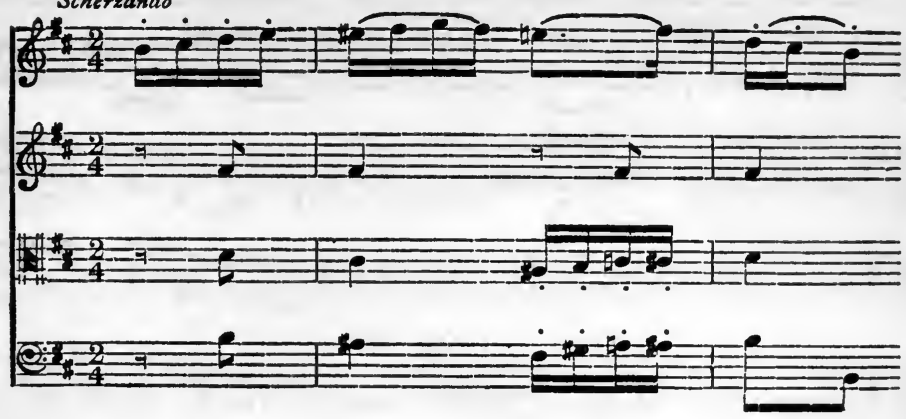

The student may be left to analyse the working for himself. Mus. D. Oxon., Igo3.
A I.
A 2.

$\longrightarrow$

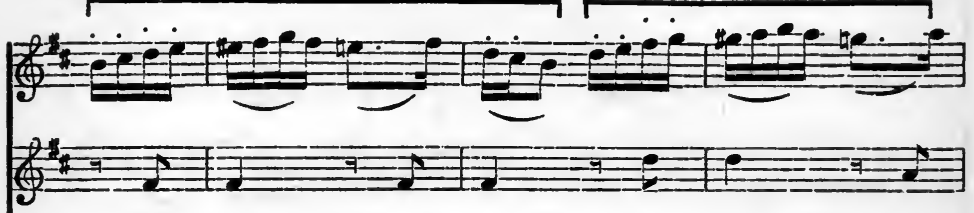

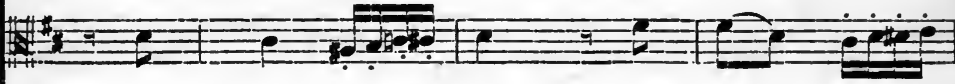

Fin

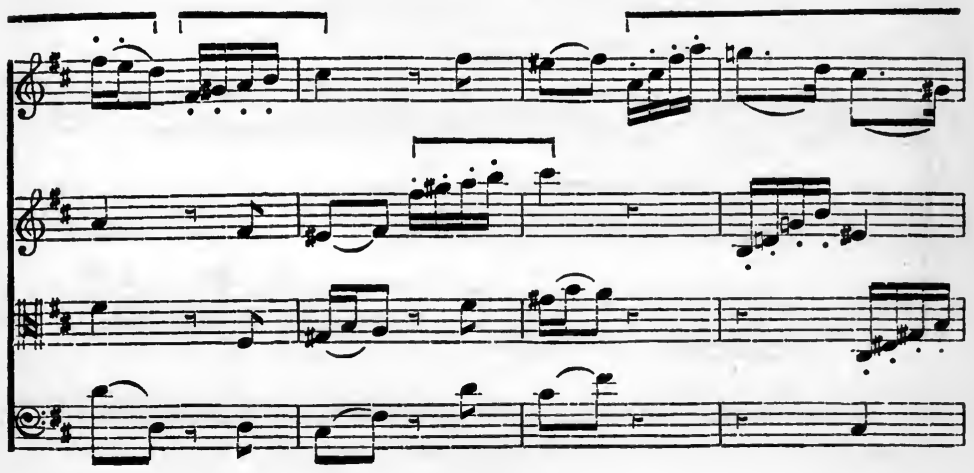





The following are two cases deserving of attention :-

(I) 'Continue the following for about twelve bars,' \&c. :-

First Mus. B. Oxon., May, rgo4.

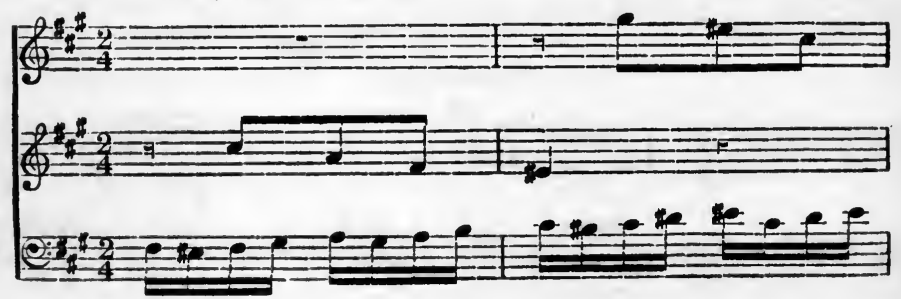

Now, at first sight, it would seem that the chordal centres were crotchets- 


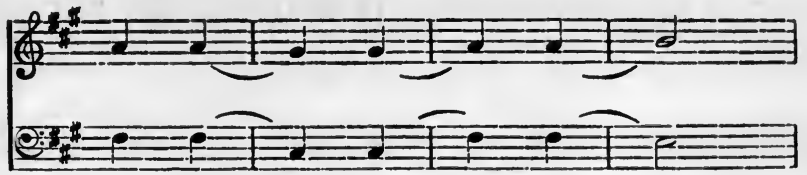

But, elaborate this, and the proportion is all wrong.

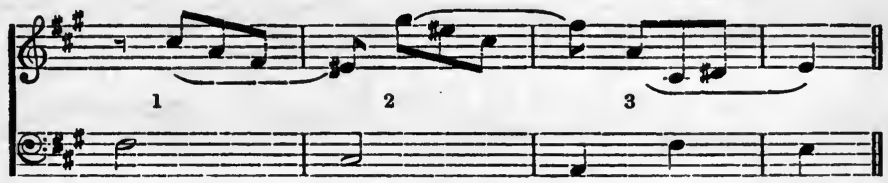

for we only get three groups of the formula. It is evident that another is wanted, but this produces a further difficulty -

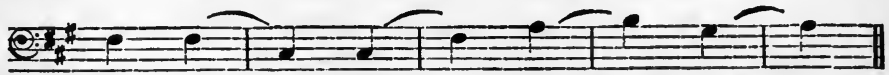

Here we have five bars. The fact is the centres are quavers -

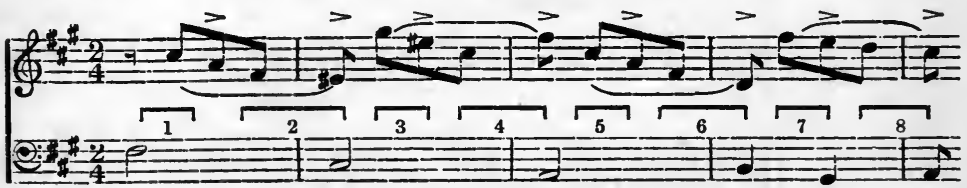

and the first beat of the first bar counts for nothing, so that it takes just four bars to complete the phrase (e.g. counting by motives and not bar lines); and the second phrase will end not in bar ten but on the first quaver of bar nine-

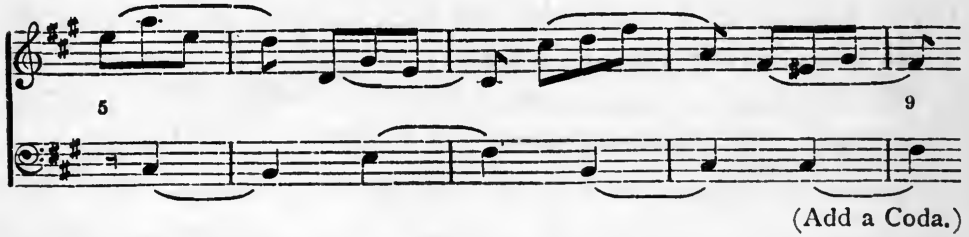

The reason that we have the appearance of an irregular sentence is that the statement

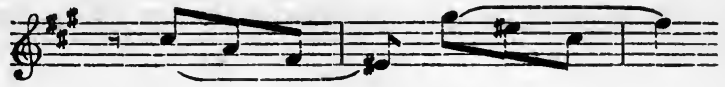


demands a parallel response. If the given bars had been as follows :

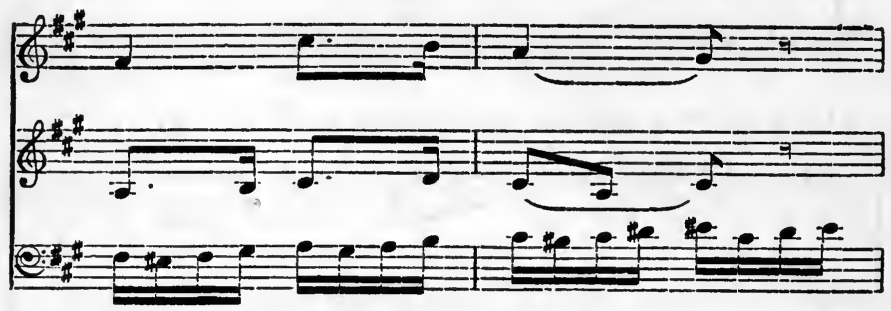

the barring of the sentence would have been quite normal.

(2) Begin as follows, and modulate to A min., G min., B flat maj., the dominant of $\mathrm{F}$ min., and back to $\mathrm{F}$ maj. :-

Third Mus. B. Oxon., Nov., 1904.

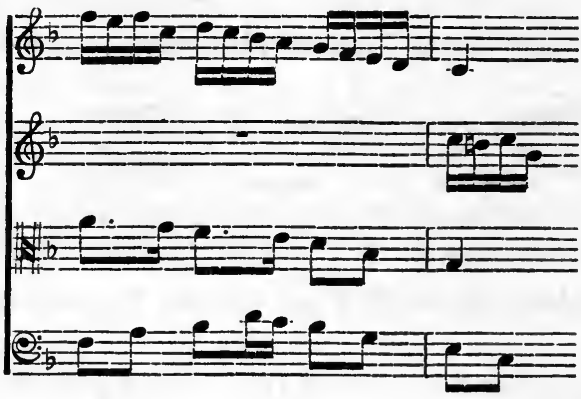

The expression 'dominant of $\mathrm{F}$ minor' implies two modulations, $\mathrm{F}$ min. and $\mathrm{C}$ maj., else the latter could not be called the dominant of the former, as a key.

The important thing to note is that the rhythm of the first two bars implies the displaced accent as a characteristic feature.

A I. A 2.

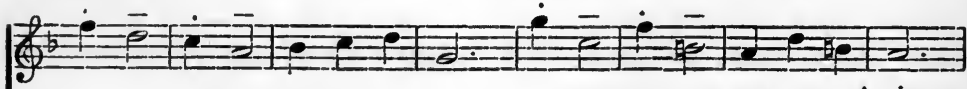

A min.

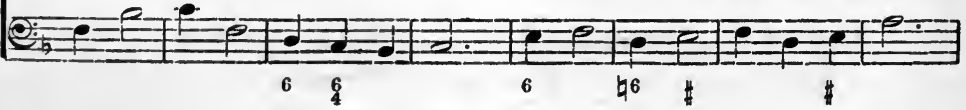



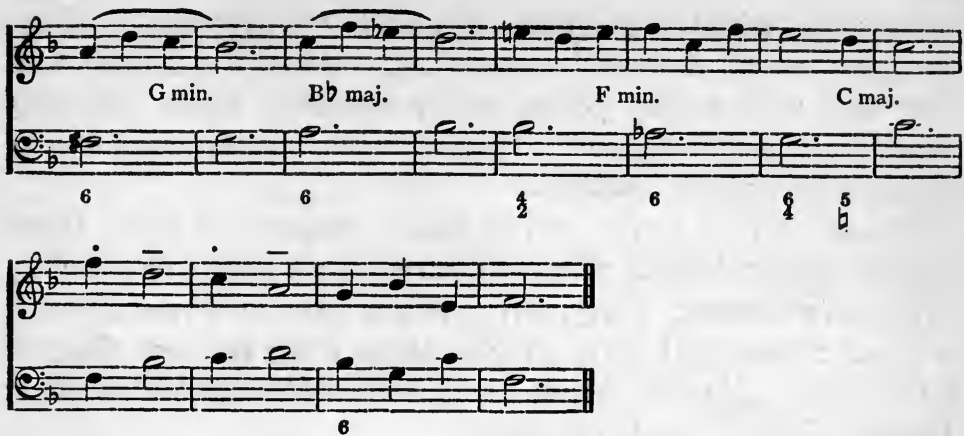

It is strongly urged that along with the study of Counterpoint, and harmonic progression, the student should pursue the following course of training :-

I. Analytical. Florid modern work should be reduced to plain chordal centres. This will teach harmonic framework, and the proper treatment of unessential notes, when harmonizing a melody. All that has to be found out is the time value of the accents, and each is to be regarded as an accent in Counterpoint.

2. Synthetical. Given: The chordal centres, or a component florid part. Required: To build up a florid score. If the component part be given, the analytical process should be gone through first, then, the chordal centres having been found, the score should be built up.

3. Constructive. The course of a problem being broadly outlined, by indicating the form, style, modulations, and the use of figure, \&c., the whole of the framework to be built up.

It is quite astounding to find how many students are unable to write plain harmonic progressions (chordal centres) in any intelligible way. As a rule the form is chaotic, the modulations are introduced in no logical way, and the progressions themselves are appallingly crude. If a student cannot write plain hymn tune progressions smoothly, he cannot do more elaborate work. In other words, if the four walls of the house are out, it is useless to attempt to proceed with the interior.

It has been seen how Counterpoint can be applied in modern work as a decorative principle, but textbooks on harmony 
merely deal with the grammatical aspect of progressions. Some few give so-called melodies to be harmonized, but inasmuch as they deal with special points under classified heads, they only serve that special purpose. The following recommendations as to study may prove useful :-

Melodic Form : Parry, Art of Music, chapter on Folk Music (Kegan Paul) ; Wood, twenty-five Irish Folk Songs (Boosey).

Form in General: Parry, Art of Music; art. on Form (Grove); art. on Sonata (ib.); art. on Symphony (ib.); art. on Concerto (ib.) ; Prout, Musical Form (Augener); Applied Forms (ib.); Hadow, Sonata Form (Novello).

Decorative Principles: Parry, art. on Variations (Grove); Prout, Variations (Applied Form).

The student should read various classic examples, such as Beethoven's Variations on Diabelli's Valse; Brahms's Variations on a theme of Handel, and on an original Theme; Schumann's Variations for two Pianofortes, \&c.

It has been impossible within the limits of a single chapter to deal at all adequately with a subject of such wide scope. But it is to be hoped that at any rate the foregoing remarks may have served to show the student the proper lines on which to direct the course of his investigations. 


\section{CHAPTER XVIII}

COUNTERPOINT ON A CHORALE

I.

In the Strict Style.

Add an Alto, Tenor, and Bass in Florid Counterpoint.

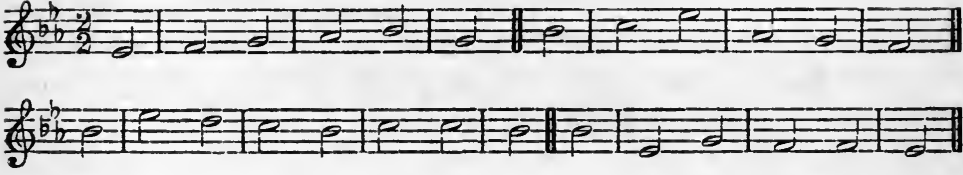

This is a form of question closely linked with the Scholastic process. Every semibreve is equivalent to a semibreve of Scholastic Counterpoint, that is, the minims are to be regarded as Second Species, in reference to melodic rhythm; the other parts must generally conform to the idioms of Florid Counterpoint, e.g. :-

(a) A minim on the second half of the bar, if preceded by notes shorter than itself, should generally be tied into the next bar.

(b) Suspensions should generally be resolved on the third rather than the second crotchet; the latter procedure may be occasionally employed.

(c) Quavers must be approached and quitted conjunctly.

The student is referred to the rules of Florid Counterpoint for further details.

The Counterpoint differs from the Scholastic process in the following ways :-

(a) The Counterpoints begin before the entry of the Chorale, introducing 'points' founded on the first line, varied of course in time value, generally by diminution in part. 
(b) When the Chorale reaches the end of the first line (double bar) a few rests are introduced in the part that has the C.F., to allow the Counterpoints to introduce imitations based on the next line, and so forth to the end. Full closes may be used with discretion. Thus, each line of the Chorale is marked off by the use of rests, the Counterpoints meanwhile sustaining the movement. The Counterpoints may adopt for imitative purposes independent figures, such as the Nota Cambiata used in Gibbons's Hosanna, but the former method is the more usual.

(c) Greater latitude is allowed in reference to change of harmony. It may be changed three or even four times in a scholastic bar; e.g. on each crotchet.

Palestrina, Sanctus, Aeterna Christi Muncra.

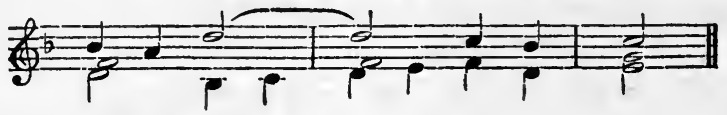

Dignity will be lost if the change of harmony be too frequent. It is hardly necessary to point out that the imitations need not be strict (the melodic curve of the points being roughly followed) and that strict imitation or ingenuity in device will never justify crude harmony.

Example. (The addition of words is optional.)

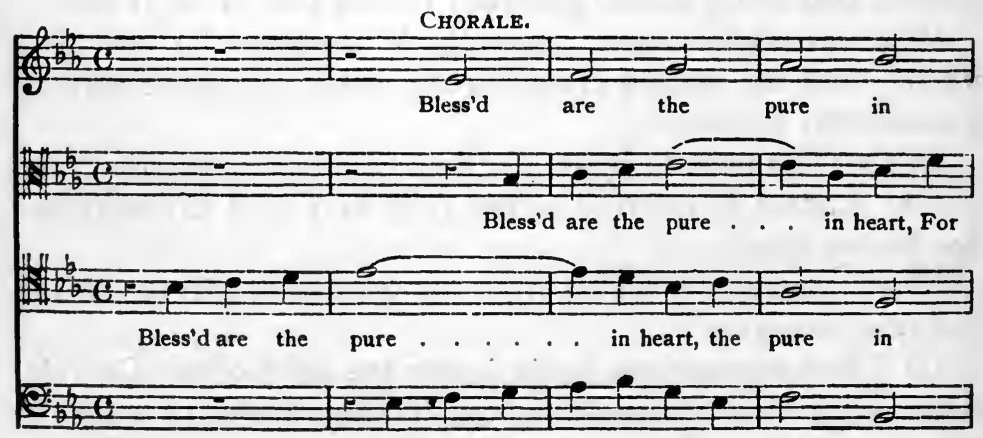

Bless'd are the pure in heart, the pure in 

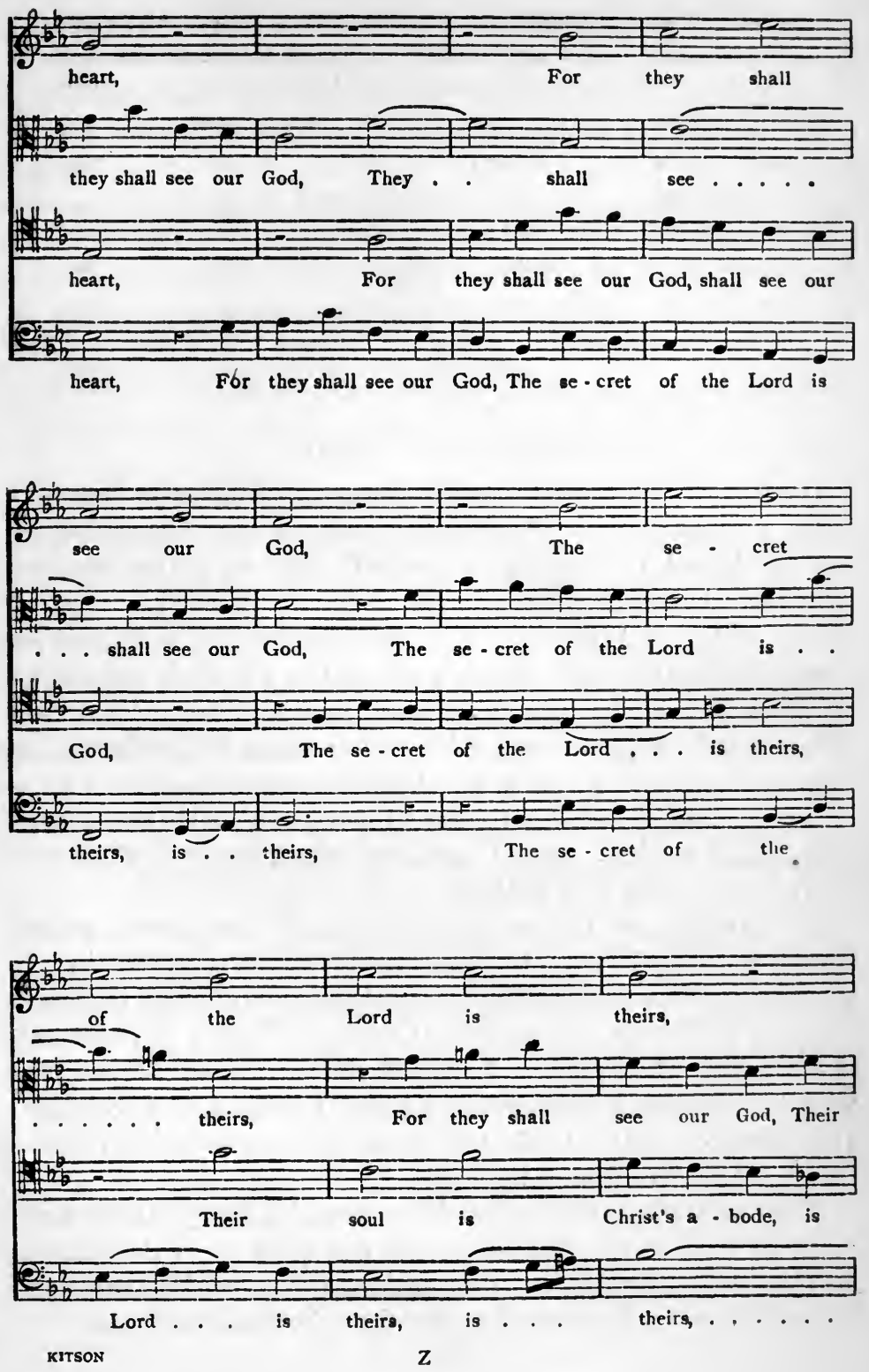


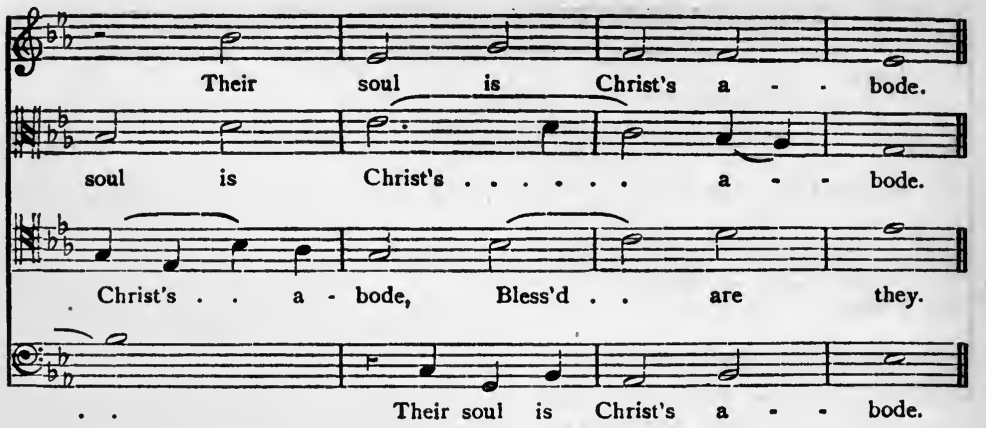

2.

IN the Free Style.

In work of this kind, the strict idioms of Scholastic Counterpoint are discarded. Unprepared discords and chromaticisms are used, and the student is entirely free as to his melodic rhythms. Counterpoints may be added in a vocal or instrumental style ; and they may be either independent or framed on phrases of the Chorale. Bach's two settings of Valet will ich dir geben are excellent examples of both methods (Choral Preludes). The Chorale may be sung, while other parts are added in an instrumental style, forming an elaborate accompaniment; for a beautiful example of this treatment see Bach's Cantata Wachet auf. The student should examine the Choral Preludes of Buxtehude, Bach, and Brahms.

An example of independent modern Counterpoint woven round a Chorale may be seen in Harwood's Capriccio for the Organ. As a combination of vocal and instrumental Counter. point on a Chorale, the opening Chorus of Bach's Wachet auf, is a magnificent example. Students should acquaint themselves with the Church Cantatas and Choral Preludes of J. S. Bach. It is infinitely better to do this than to gain a superficial knowledge of the form from a textbook.

The limits of this treatise would be exceeded if any attempt were made to deal at all adequately with the question of decoration, and the use of formulae. The student should analyse the Bach Choral Preludes for himself under the following headings :- 


\section{COUNTERPOINT ON A CHORALE}

(i) Counterpoints formed from the C.F.

(ii) independent of the C.F.

(iii) A catalogue of the various formulae or figures should be drawn up, with special reference to the harmonic sub-structure; and it would prove of immense benefit to copy out sections in bars of Scholastic Counterpoint. It is of course to be remembered that it is only the principles of decoration from a harmonic point of view that remain. The student will find some figures built upon the sub-structure of only one scholastic bar, some on two, or three, and so forth.

A few examples are given:

(r) Formulae built upon the basis of one scholastic bar.

(a) Wir Christenleut.
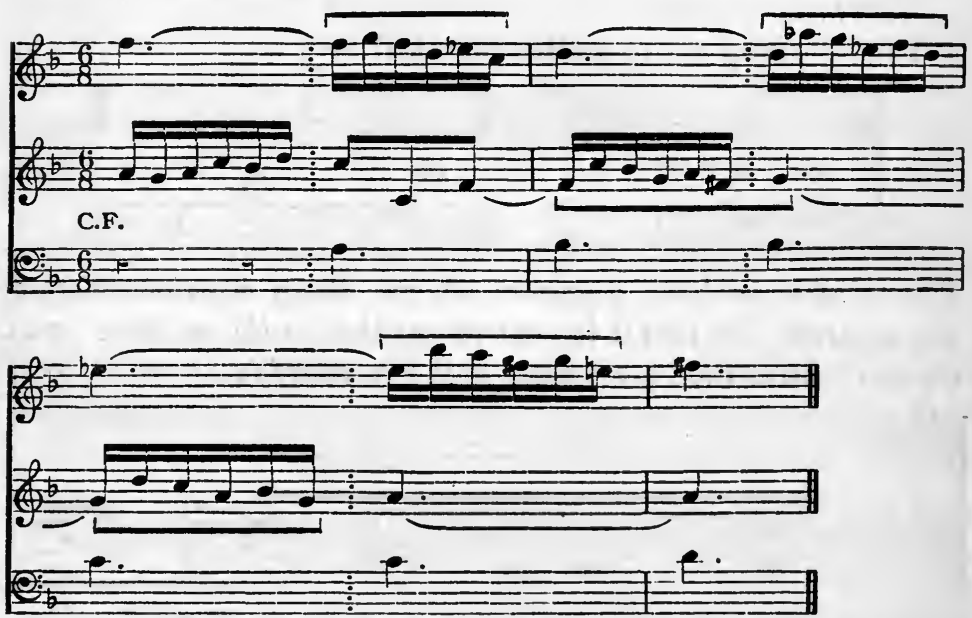

Each half bar corresponds to one bar of Scholastic Counterpoint in Triple Time.

(b) Wer nur den lieben Gott lässt walten.

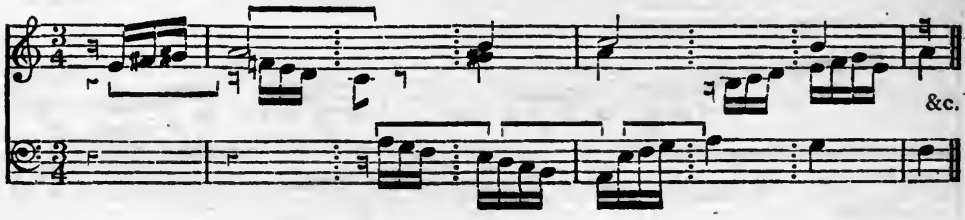


Each crotchet corresponds to one bar of Scholastic Counterpoint.

(c) Jesu, meine Freude.

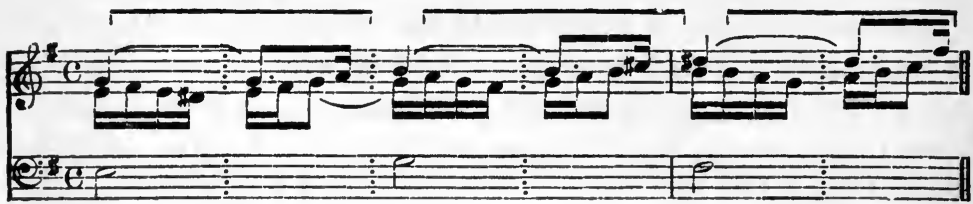

Here each crotchet corresponds to one bar of Scholastic Counterpoint, but each group of two bars has the same C.F. (eight notes to one).

(2) Formulae built upon the basis of two compound scholastic bars.

Jesu, meine Freude.

The following are in outline identical:

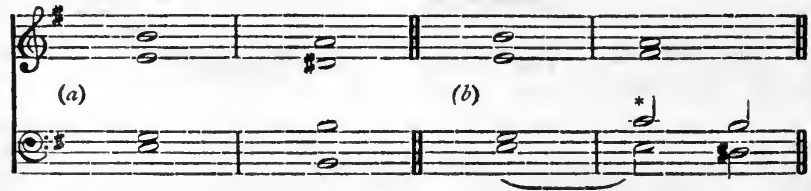

that is at ${ }^{*}$ we have a passing chord, which does not disturb the centres. So that it is true to say that at $(b)$ we have as at (a) two centres (not necessarily only two chords).
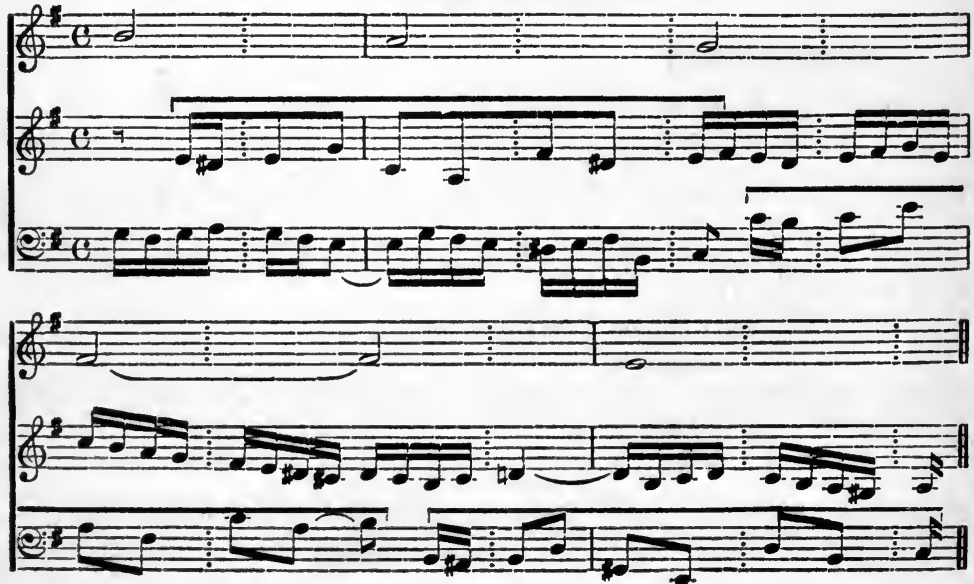


\section{COUNTERPOINT ON A CHORALE}

Each crotchet corresponds to a bar of Scholastic Counterpoint, but we have here really a compound time, e.g. eight notes to one.

(3) Formulae based on three scholastic bars.

Wo soll ich fliehen hin.

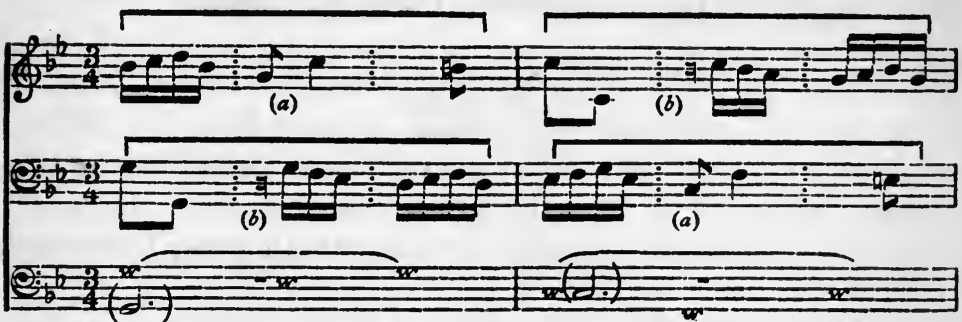

Implied C.F.

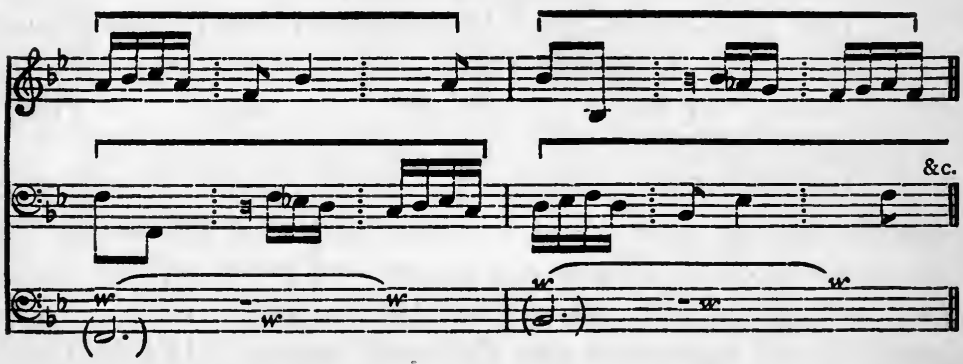

The foregoing will have served to show how much may be learned from a study of these Preludes alone.

It is of the highest importance that the student should go to the root of the matter and find out the preconceived harmonic basis in the mind of the composer, as he weaved his Counterpoints. And it is only by developing this feeling for harmonic sub-structure that any progress in this direction can be made.

(iv) The formal side of the question in all its aspects.

All decorative work is based on such principles as have been indicated.

Two points are vital :-

(a) The chordal centres must be quite smooth and spontaneous.

(b) The harmonic seams must be satisfactory. 
When the mastery of this technique is so complete that the result is not merely a piece of clever craftsmanship, but an expression of the emotions, and when the style has become influenced by wide reading, the student may consider himself equipped, so far as the technique of decoration is concerned, for his first attempts at composition in a modern style.

\section{Conclusion.}

A few words, in conclusion, on a further study of Counterpoint may be added, in the hope that they may induce the student to obtain a deeper knowledge of the subject than a mere perusal of a textbook can provide.

I. The system of the ecclesiastical modes should be studied. Information on this point may be found in :-

(a) The Oxford History of Music, vols. i and ii ; (b) Grove, article on The Ecclesiastical Modes; (c) Rockstro, The Rules of Counterpoint ; (d) Pearce, Composers' Counterpoint; (e) Prout, Harmony.

2. Having gained a knowledge of the modal system, the student should proceed to a deeper study of Palestrina. It would be excellent practice to copy out one of Palestrina's parts, then add others, and compare with the original, writing in every detail in the manner of the sixteenth century. It is only by going to the root of matters in this way that any real grasp of the history of the evolution of music can be obtained. Then the third volume of the Oxford History of Music should be studied, showing the gradual change from the old order to the new. The enlargement of harmonic resource also provides a very engaging study (see article on Harmony in Grove, and Dr. Sawyer's recent paper on Modern Harmony).

For variety in working scholastic problems, the student may take as his C.F. a part from an already solved problem in any Species other than the First, and add parts in various Species, together with one in the First Species, which should have been the original C.F.

It is almost superfluous to invent any Canti Fermi for the use 
of students; but a few of the time-honoured C.F.'s are added for the use of those students who may wish to work without the aid of a teacher.

It is hoped that this book may help those students who have found the discrepancies between various textbooks perplexing, and place those who are beginners in the art on a path which is at any rate historic, and which has as its goal not only an acquaintance with the methods of the past, but also a prepara. tion for the possibilities of the Harmonic School.

The Counterpoint of Palestrina has formed the harmony of Bach; the Counterpoint of Bach has enlarged the harmonic resource of the modern diatonic school. The Counterpoint of this school has formed the harmony of Wagner, Dvořak, Elgar, and Strauss. In this way has the unessential merged into the essential; and the student may await with interest further developments of this nature.

Canti Fermi for the use of Students.

These may be written in any clef, and transposed to a key convenient to the voice.

The indication of strongly accented bars is for guidance in writing uncombined Counterpoint.

In the Major Mode.

I.

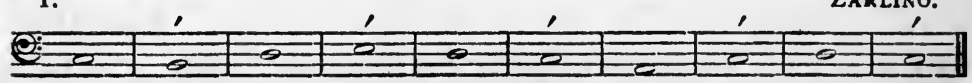

2.

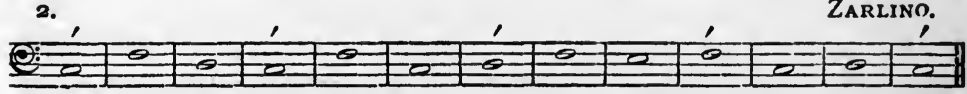

3.

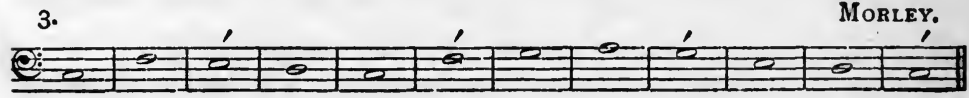

4.

MORLEY.

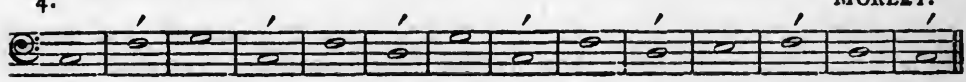


5.
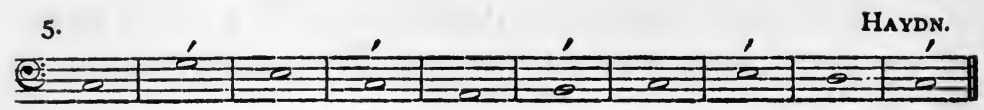

6.
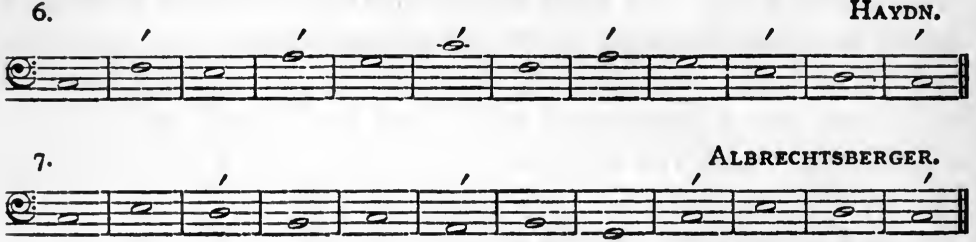

8.

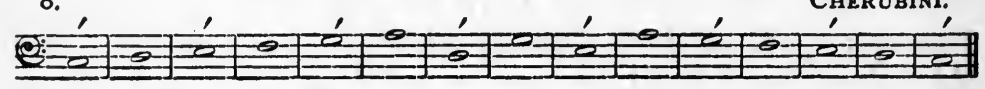

Cherubini.

9.

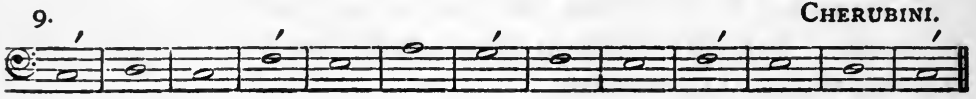

Io.

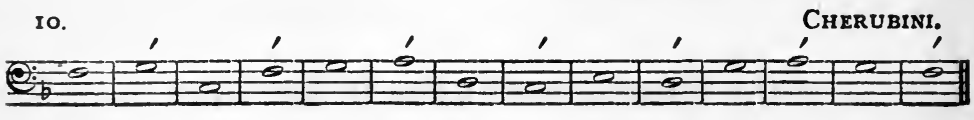

II.

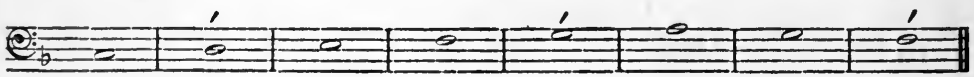

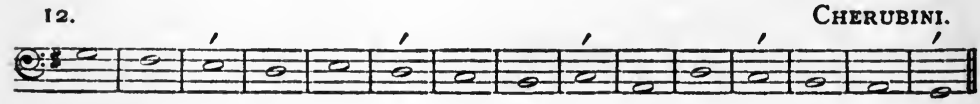

(3.)

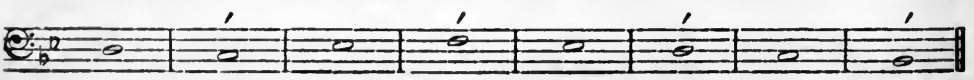

14.

Albrechtsberger.

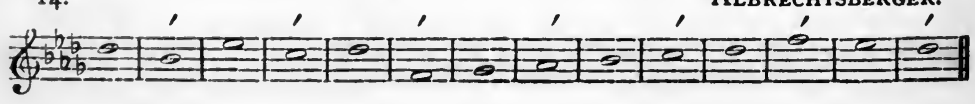


I5.

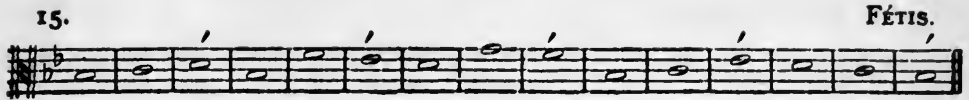

I6.

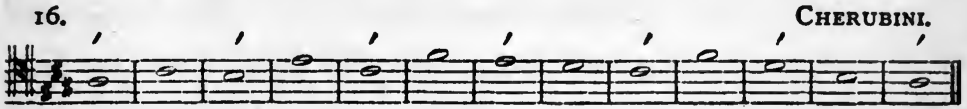

For variety, hymn-tunes may be taken thus :First Species in two parts.

Franconia (Müller's Choralbuch, 1754).

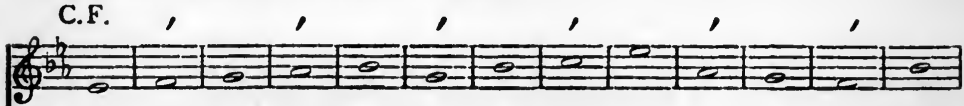

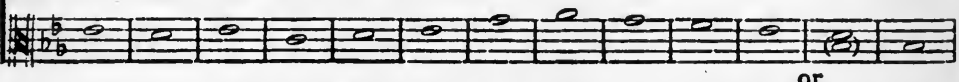
or

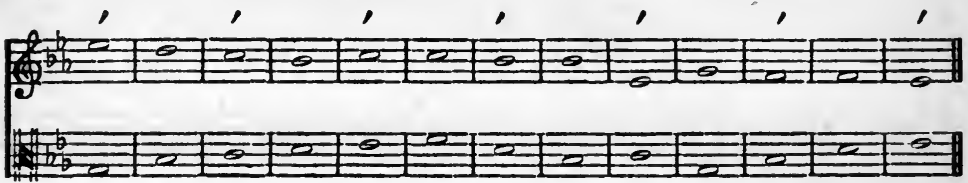

Second Species in two parts.
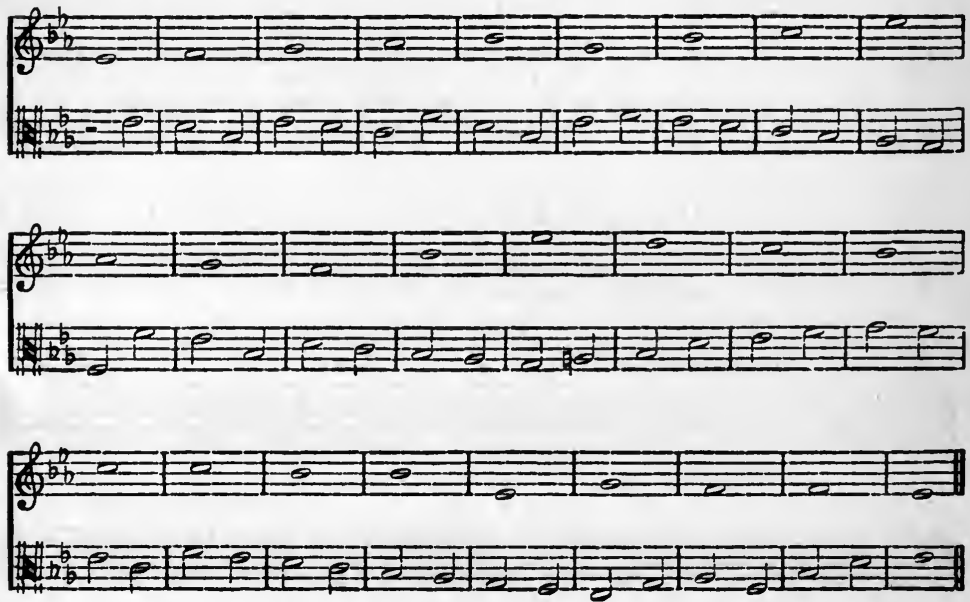


\section{6 \\ THE ART OF COUNTERPOINT}

In the Minor Mode.

S:

2.

HAYDN.

OQ:-0

3.

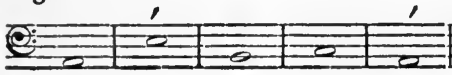

Albrechtsberger.

4

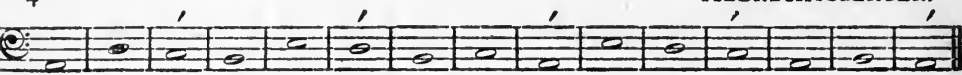

(0)

6

Cherubini.

20:50

7.

(0.0.

+

9.

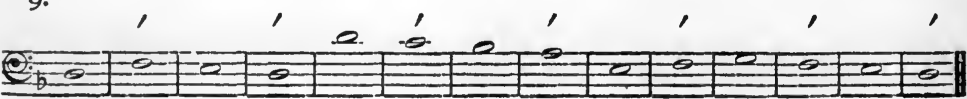

Io.

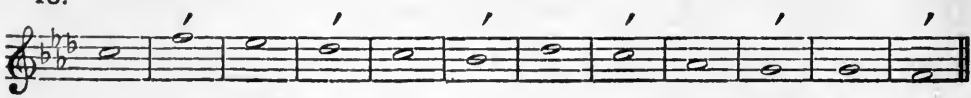

II.

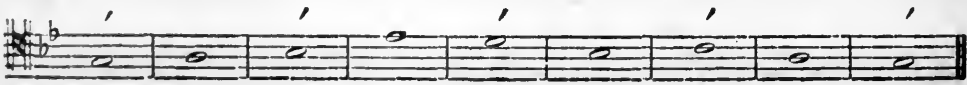




\section{CANTI FERMI}
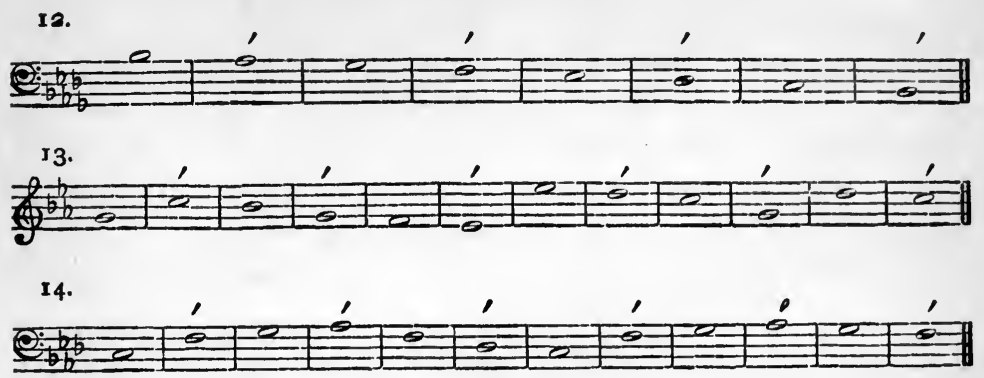



\section{N D E X I}

Accents, 136, 290.

displaced, $257,332$.

Aeolian mode, 2r.

Anapaest, II, 253.

Antiphony, 2.

Appoggiatura, I54, 280, 314 .

Augmented Sixth, origin of, 145 .

Auxiliary notes, 7I, 72.

ВасH, 4, 6.

treatment of unessential notes, 144 , I45.

choral preludes, 338 et seq.

Cadence, 12.

Two parts: Second Species, 31, 46 ; Triple time, 104 ; Third Species, 50, 72 ; Fourth Species, 84 ; Fifth Species, 93.

Three parts: First Species, I20; Second Species, 122 ; Third Species, 127; Fourth Species, 132 ; Combined Counterpoint, 158. anticipation of, 27, 255, 259, 282.

Feminine, 257, 28I.

Perfect, I 10, 253.

Caesura, 254.

Cantus Firmus, I.

Change of Harmony, 33, 6r, 83, 92, $94,96,104,153,167,177,301$, 336.

Changing notes, $5 \mathrm{r}$ et seq.

Chordal centre, 6, 290.

Clausula vera, 12.

Concords, 10, II, 18, 19, 32, 97.

Consecutives :

Two parts : First Species, 25 ; Second Species, 43; Third Species, 66 et seq. ; Fourth Species, 77.

Three parts: First Species, II 7 ; Second Species, 124 ; Third Species, 126 ; Fourth Species, 13r ; Combined Counterpoint, 157, 160, 167, r68 et seq., I89.
Consecutives (cont.) :

Four parts, 197, 198.

Five parts, 215.

Six parts, 227.

Exposed, Two parts, 25, 44, 107; Three parts, I 12 et seq., 164, I65; Four parts, 192.

Consonances, 3.

Crossing of parts, 26, I18, 221, 223, 224.

Diaphony, 2.

Discant, 2.

Dorian Sixth, 24, 29.

Dots, use of, 90,178 .

Exposed Consecutives, see Consecutives.

False Relation, 26, 44 .

Florid Counterpoint, 87, 179, 208, 225, $235,238,240$ et seq.

Fourth, II, 70, 80, III, 132, 156, I57, 208.

Ground Bass, 292.

Harmonic Progression, 18 et seq. ; 25 I et seq.

Iambus, II, 253.

Imitation, 229, 336 .

Incomplete harmony, IIx.

Ionian mode, 2I, I IO.

Leading note, doubling of :

Two parts, $25,44$.

Three parts, I 19, 126, I 72, 173 .

Four parts, 192.

Six parts, 227, 228.

Magadizing, 2.

Melodic Progression, 14 et seq.

Modes, the ecclesiastical, 342. 
Modulation, 9, 23, 26, 26I, 286. problems in, 3r9 et seq.

Motive, 12, 27, 253, 321, 322.

Musica Ficta, 9, 21, 26, I 10.

Nota Cambiata, $5^{1}$ et seq., 88.

Notation, 96.

Organalis, 2.

Organum, 2.

Ornamental resolution, see Resolution.

Oxford History of Music, $34^{2}$. quoted, 16,17 .

\section{Palestrina, 3, 9, i I.}

the study of, $95,230,342$.

his technique, 16,17 .

his treatment of consecutives, II2, I 7, r69.

Passing chords, $147,148,149,180$, $\mathrm{r} 82, \mathrm{r} 83,208,298,340$.

Passing notes, 43, 93, 94, 122, 127, I 4 I, I52, I53, I8I, I82, 208.

Pedal, I33, 134, 142.

Phrase, 253.

Prepared discords, $161,162,163,168$, I 77, I 78, 205.

Primary triads, 193, 25 I, 252, 268.

Principalis, 2.

Prolation, I03.

Quavers, use of, 89, 90, 92, 179, 180, 209, 335 .

Resolution, anticipation of, 9I, 214 . deferred, 40, 4I, 128.
Resolution, ornamental, 91, 95, ro7. $178,205$.

Rests, 229, 336.

Retardation, 81, 82, II4.

Secondary triads, 252.

Sentence, 269, 288. construction of, $3^{20}$ et seq., 33r.

Seventh, chords of, 282 .

Six-four, 10, 34, 70, 71, 8I, 185, 19I, $278,3^{14}$.

Sixth, leap of, major; $16,33,70$, 159, 216.

minor, 33, 70, 229.

Species, 7.

Sub-phrase, 321, 324 .

Suspensions, $76,78,79,81,82$, 130, 172, 199, 206, 214, 278, 335 . double, 177,204 .

Syncopation, 76 . broken, 83, 221. internal, 89, зго.

Syncopated Concords, 76, 132, 169, 206.

Tie, use of, $87,88,89,335$.

Tritone, 26, 44, 70, 83, I 18, 193.

Unessential notes :

Second Species, 35 et seq.

Third Species, 6r et seq.

Combined Counterpoint, $18 \mathrm{r}$. elimination of, 297, 309, 313 .

Unison, 25, 44, 7I, 84, 104, III, 122, 229. 


\section{N D E X I I}

\section{WORKINGS OF EXAMINATION QUESTIONS}

Unless otherwise stated, the index refers to papers set at Oxford.

$\begin{array}{lll}1897 & \text { Mus. D. } & \text { Harmony, 314. } \\ 1899 & \text { Mus. D. } & \text { Counterpoint, 243, } 246 .\end{array}$

1900 Nov. First Mus. B. Counterpoint, 188, 203.

First Mus. B. Harmony, 325.

Igor Nov. First Mus. B. Counterpoint, 173, 195, 2 ro.

Mus. D. Counterpoint, 235 .

1902 Nov. Third Mus. B. Counterpoint, 216.

Third Mus. B. Harmony, 327.

1903 May. First Mus. B. Counterpoint, 174.

First Mus. B. Counterpoint (Cambridge), 73, r21.

Third Mus. B. Harmony, 323 .

Nov. First Mus. B. Counterpoint, 194.

First Mus. B. Harmony, 296.

Third Mus. B. Counterpoint, 225.

Mus. D.

Counterpoint, 222, 232.

Mus. D.

Harmony, 329.

First Mus. B. Counterpoint (Durham), 209.

1904 May. First Mus. B. Harmony, 330. ${ }^{1}$

First Mus. B. Counterpoint, 202, 203, 2 II.

Third Mus. B. Counterpoint, 217.

First Mus. B. Counterpoint (Cambridge), 48, 84 .

Nov. First Mus. B. Counterpoint, 212.

Third Mus. B. Harmony, 302, 312, 332. ${ }^{1}$

Mus. D. Harmony, 318.

Mus. D. Counterpoint, 237.

1905 May. Third Mus. B. Counterpoint, 207.

Third Mus. B. Harmony, 293, 298, 317.

Nov. Third Mus. B. Harmony, 300, 307.

Third Mus. B. Counterpoint, 224.

Mus. D.

Harmony, 3 ro.

I906 May. Third Mus. B. Harmony, 303 .

Nov. Mus. D. Counterpoint, 240.

1 These questions are analysed, but the working is not given. 


\section{OXFORD}

PRINTED AT THE CLARENDON PRESS

BY HORACE HART, M.A.

PRINTER TO THE UNIVERSITY 


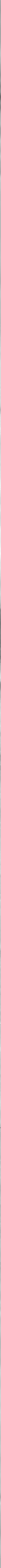




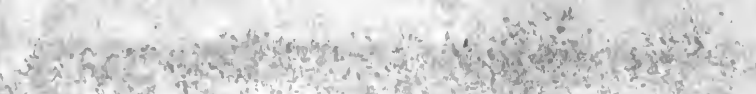

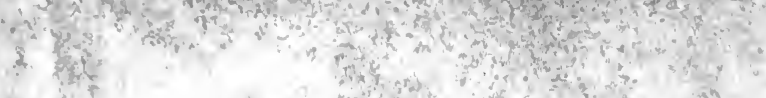
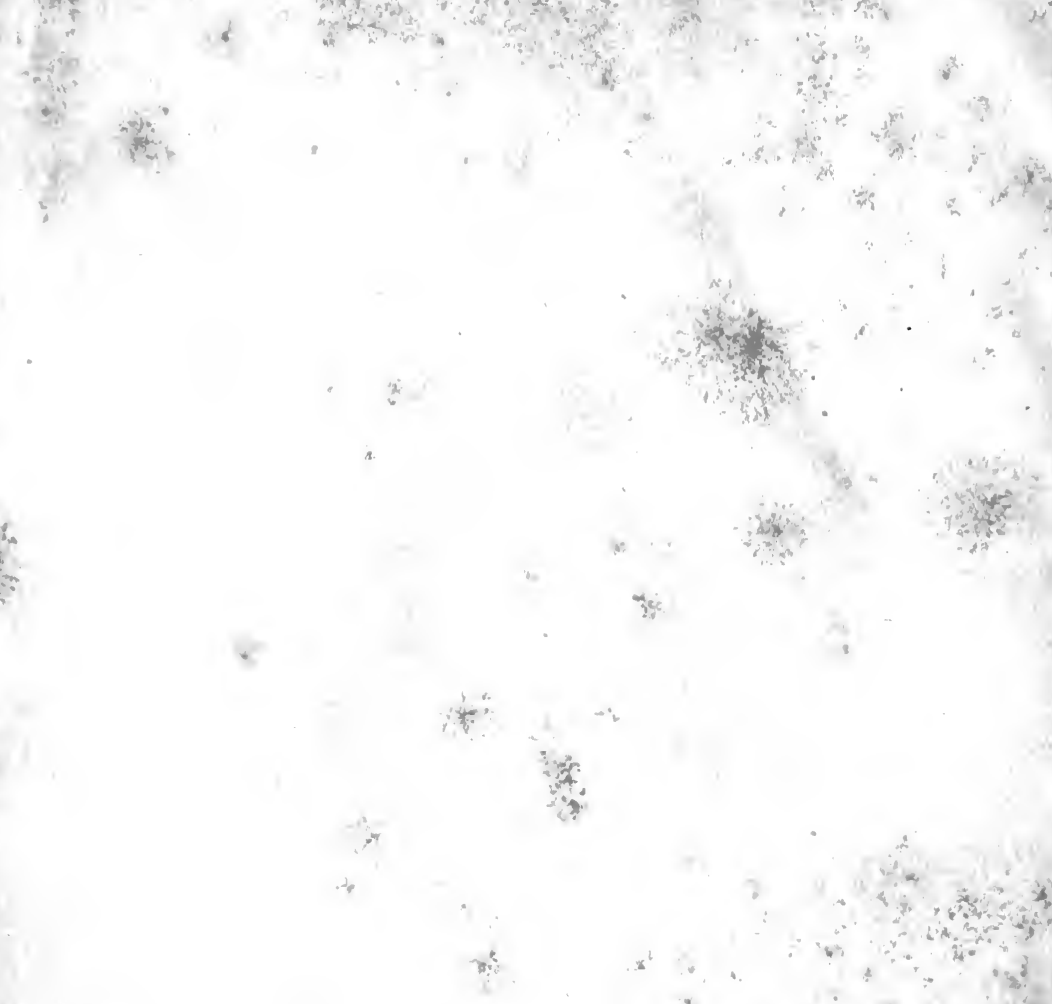

2
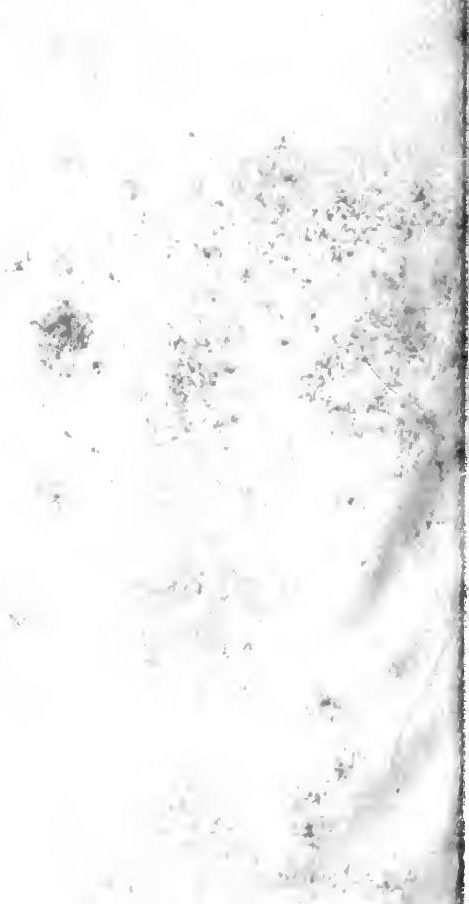


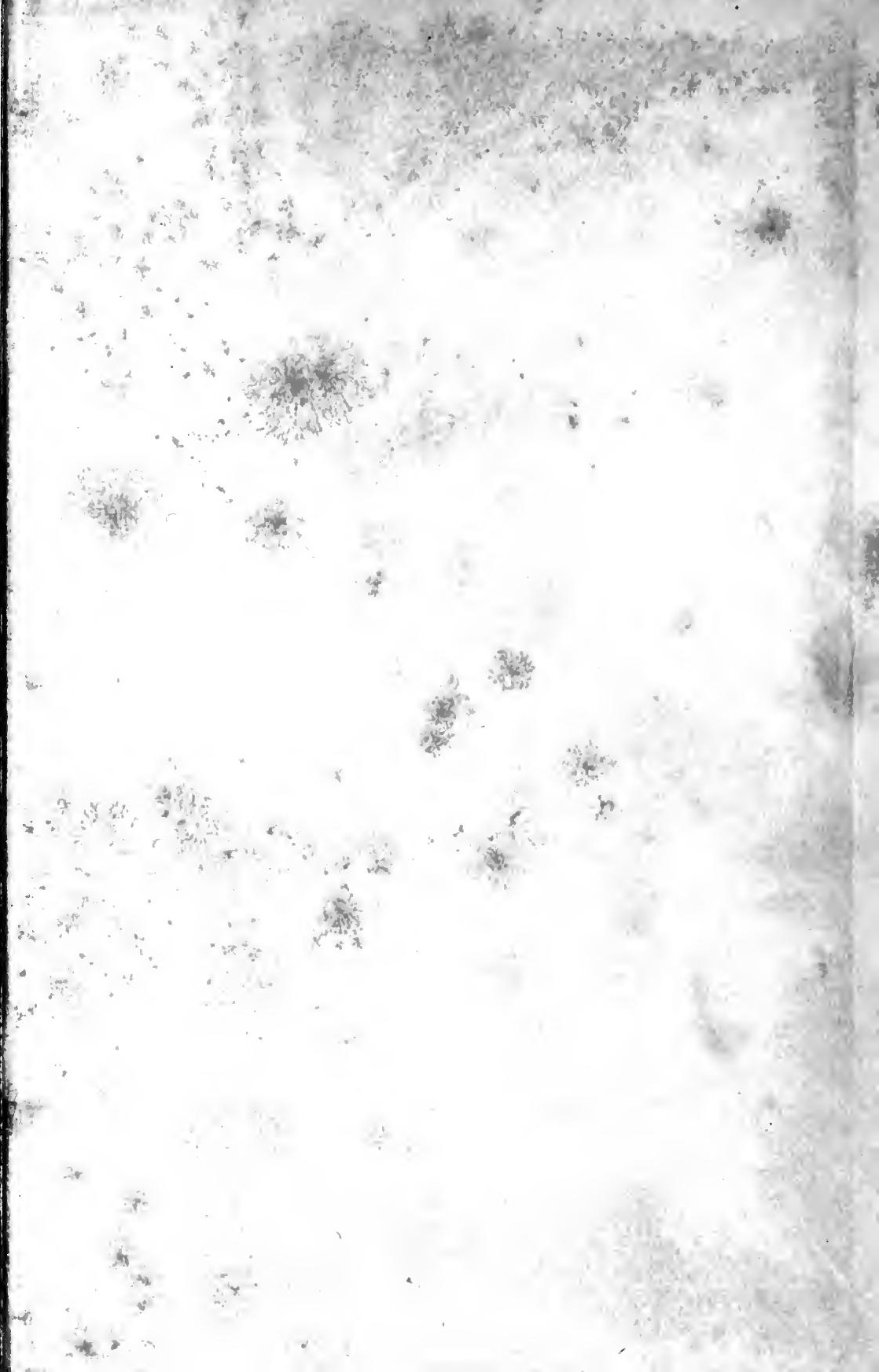


